

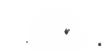


s. L. Clark, OP. 


$$
\text { • }
$$




\section{INTRODUCTION TO GENERAL CHEMISTRY}




\section{Me Graw-Fill Book Co. Tne PUBLISHERS OF BOOKS FOR}

Coal Age $\quad \nabla$ Electric Railway Journal Electrical World $\nabla$ Engineering News-Record American Machinist $\nabla$ Ingeniería Internacional Engineering 8 Mining Journal $\nabla$ Power Chemical $\&$ Metallurgical Engineering Electrical Merchandising 


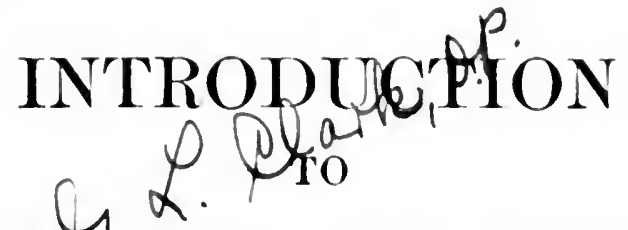 \\ GENERAL CHEMISTRY
}

ST. ALBERT'S COLLECE LIBRARY

\author{
BY \\ HERBERT N. McCOY \\ A.ND \\ ETHEL M. 'TERRY
}

Second Edition

SECUND IMPREASION

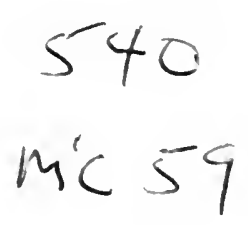

MCGRAW-HILL BOOK COMPANY, INC.

NEW YORK: 239 WEST 39TH STREET

LONDON: $6 \& 8$ BOUVERIE ST., E. C. 4

1920 . 
COPYRIGHT, I9I9, I $920, \mathrm{BY}$ Herbert N. MCCOY aNd Ethel M. TerRy 


\section{PREFACE}

This text has been in process of growth at the University of Chicago since 1913. At that time a synopsis of the first nine chapters was printed. This was followed, in $1916-17$, by the completed work of the first fifteen chapters. Chapters xvi-xix were added and used in class work during I9I 8 and 1919.

The book has been written for college Freshmen, and, as its title implies, it is intended to serve as an introduction to general chemistry. In consequence we have aimed to present a continuous and connected story in teachable form and have not attempted to give extensive descriptive and numerical data where such matter is of little interest to the student or is not needed for the development of important principles.

Inasmuch as the choice and arrangement of topics in the earlier part of this book depart noticeably from the familiar order, some explanation seems necessary. We shall therefore sketch briefly the plan of the less conventional chapters, together with the "philosophy of arrangement" which has resulted in the scheme presented.

The first chapter, which is brief, deals with the measurement of gases and the gas laws. In the next five chapters the most fundamental concepts of the science of chemistry are developed. These include: indestructibility of matter, idea of a pure substance, decomposition of pure substances, elements, analysis of substances and percentage composition, the law of definite composition, derivation of formulae.

Chapter $\mathrm{v}$ shows how chemical formulae are derived from a knowledge of percentage composition and gas or vapor density. This development keeps as close as possible to the arguments of Avogadro and Cannizzaro and shows how formulae are obtained by methods independent of the atomic-molecular hypothesis. Reference, at this stage, to combining weights and chemical equivalents is purposely avoided, for the reason that the history of chemistry between the time of Avogadro and 
that of the epoch-making paper of Cannizzaro (1858) shows the fallacy of trying to develop formulae and fix atomic (symbol) weights by any method other than that proposed by Avogadro and elucidated by Cannizzaro. The sixth chapter introduces the use of equations before the atomic-molecular hypothesis is studied. This plan has the great advantage of fixing in the student's mind the fundamental relationship between equations and the quantitative experimental data such equations represent.

Chapters ii to vi inclusive form a compact division of the subject, in which the argument, illustrated at every step by experimental data, is substantially continuous. In these chapters we have aimed at a logical development of the subject without the introduction of any matter that does not serve to illustrate the topics under discussion.

The next three chapters, vii, "Acids, Bases, and Salts-I"; viii, "Water and Solutions"; and ix, "Acids, Bases, and SaltsII," are introduced at this point for very definite reasons. In the first place, it is obvious to every teacher that much of the beginner's work will deal with acids, bases, and salts and their solutions. It is our opinion that a knowledge of these topics is best obtained by studying them directly and specifically, both in the classroom and in the laboratory. Our plan provides for laboratory work by the student, following closely the content of these three chapters. This laboratory work is interesting to students, since they like to make and crystallize a variety of salts. It also gives good training in technique and is not difficult either experimentally or theoretically, while at the same time it offers a wealth of material for practice in writing equations and solving problems. The most important reason, however, for the introduction of the early study of acids, bases, and salts is to supply the indispensable data needed later for the understanding of the ionic hypothesis.

Chapters $\mathrm{x}$ and $\mathrm{xi}$ present the kinetic-molecular and atomic hypotheses respectively. It will be noted that these subjects follow the development and use of formulae instead of preceding them. This emphasizes the generally overlooked fact that for- 
mulae are in no way, of necessity, dependent upon the molecularatomic hypothesis. At the same time the student is in a position to appreciate more readily the molecular-atomic hypothesis because it furnishes a plausible explanation of facts already familiar to him.

Since the early chapters of the text contain as much information about hydrogen and oxygen as the student needs, or can fully appreciate at the start, the formal discussion of these elements has been postponed to chapter xiv. Chapters xiii ("Chemical Equilibrium"), xv ("Oxidation and Reduction"), and xvi ("Heat and Energy") present important theoretical matters.

The extended discussion given of the ionic hypothesis needs no apology. In this connection we have introduced a new method of graphic representation of ionic equilibrium. In chapter $\mathrm{xx}$, on "Electrochemistry", the electronic conception of reactions, including oxidation and reduction, is discussed.

The arrangement from this point on needs little comment. The authors recognize, of course, that organic chemistry (xxv and xxvi), "Theory of Dilute Solutions" (xxvii), "Disperse Systems" (xxviii), and "Radioactivity" (xxxii) are optional studies for beginning general chemistry courses. We have included these subjects, since the interest which they have aroused has apparently justified so doing.

The 880 sections of the book have been not only titled but numbered, in order that frequent cross-references might be given. 'The student is thus constantly informed of the material on which each discussion is based. Instructors will find the references helpful in laying out work if they desire to skip certain portions of the text, for instance with classes of students who have had a secondary-school course in chemistry.

The great importance of close connection between the work of the laboratory and that in class has been kept constantly in mind in planning this text. A laboratory guide (A Laboratory Oulline for Cieneral Chemistry, by McCoy and Terry), which follows strictly the arrangement of the text, has been written to accompany the latter. 
In addition a pamphlet has been prepared containing those details of the lecture experiments which are important to the lecture assistant but of no interest to the elementary student. This pamphlet will be available for teachers using the text.

We are indebted to Professor W. D. Harkins, of the University of Chicago, for the contribution of sections 6 and 7 (pp. 3 and 4 ) of the text and for the use of Fig. II 7 ; to Mr. Leo Finkelstein for the drawings of Figs. I to 43 ; and to Dr. R. D. Mullenix for the seventy-seven additional drawings, as well as for valuable criticism.

Chicago, Ill.

The Authors.

July, I9I9

\section{NOTE TO SECOND IMPRESSION.}

We have taken advantage of the necessity of a second printing of this text to correct a number of misprints and to add a chapter on metallurgy. In the preparation of the latter we have found the following works of general assistance: Economic Geology, by Heinrich Ries (John Wiley \& Sons); Non-technical Chats on Iron and Steel, by LaVerne W. Spring (Frederick A. Stokes Co.); a bulletin entitled "Copper" published by the Anaconda Copper Mining Co., New York; The Mineral Industry in I9I8; Principles, Operation and Products of the Blast Furnace, by J. E. Johnson, Jr.; and the works of H. O. Hoffman on General Metallurgy, The Metallurgy of Lead, and The Metallurgy of Copper. (The last five books are all published by the McGraw-Hill Book Co.)

The Authors.

Chicago, Ill.,

January, I920. 


\section{TABLE OF CONTENTS}

CRAPTER

1. INTRODUCTION-LAWS OF GaSES . . . . . . . . I

11. The Burning of Substances-Oxigex . . . . . 6

III. Puke Substances-Elenents . . . . . . . . I6

IV. The Law of Definite Composition . . . . . . 26

V. Symbols and Chenical formulae. . . . . . 40

Vi. Chemical Equations . . . . . . . . . . 50

III. Acids, BASES, AND SAlts-1 . . . . . . . . . 57

Vili. Hater and Solutions . . . . . . . . . . 68

IX. Acins, Bases, and SAlts-II . . . . . . . . 86

X. The Kinetic Theory of Matter and the Molecular HYPOTHESIS . . . . . . . . . . . . . IO9

XI. The Atomic Hypothesis AND Atomic Weights . . 12 I

XII. The Halogens and Theik Compounds with Hydrogex AND MEtals . . . . . . . . . . . . I35

Xili. Chemical Equilibrium . . . . . . . . . ${ }_{5} \delta$

III. Hydrogen and OxYgen . . . . . . . . . I76

IV. Onidation and Reduction . . . . . . . . . 194

XII. Heat AND ENERGY . . . . . . . . . . . 214

XIII. The Ionic Hypothesis . . . . . . . . . . 227

Xilil. Applications of the lonic Hyothesis . . . . . 25 I

Mix. Applications of the Ionic Hipothesis. Reactions Involing Changes of State . . . . . . . 275

XX. Electrochemistry . . . . . . . . . . . . 296

XYi. Nitrogen and Ammonia . . . . . . . . . 323

X.ili. Nitric Acid and tile Oxines of Nitrogen . . . 341

XXil. Phosphorus and Its Compoundo . . . . . . . 363

Xist. Sulfur and Its Compoundo . . . . . . . 375

Xiv. Carbon and Carbon Compounds, Organic CompoundI . . . . . . . . . . . . 393 
CBAPTER

XXVI. ORganic Compounds-II Page

XXVII. Theory of Dilute Solutions . . . . . . . . $45^{2}$

XXVIII. Disperse Systems . . . . . . . . . . . . . 470

XXIX. The Atmosphere and Related Topics . . . . . 49 I

XXX. Some Additional Elements and Their Compounds . 5 I4

XXXI. Classification of the Elements. The Periodic System 539

XXXII. Radioactivity and the Nature of Matter. . . 568

XXXili. Methll.urgy . . . . . . . . . . . . . $59 \mathrm{I}$

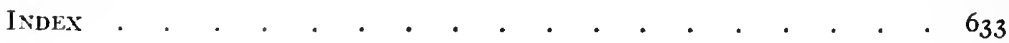




\section{CHAPTER I \\ INTRODUCTION-LAWS OF GASES}

I. A Knowledge of Physics Prerequisite for Chemistry.The sciences of physics and chemistry are so closely related that the latter may be considered an extension of the former. A knowledge of physics is therefore necessary for an adequate understanding of chemistry, and it is to be assumed that the student taking up chemistry has had at least a one-year highschool course in physics.

2. The Three Forms of Matter: Gases.-In his work in physics, the student will have learned the meaning of the term matter, which may be defined as anything which occupies space and has weight. He will have learned, also, that matter may exist in three forms: solid, liquid, and gaseous. Since gases are less tangible than solids and liquids, we shall first take up the study of air, the most familiar of all gases. That air has the two attributes just mentioned as belonging to all forms of matter may readily be shown by experiment.

3. Air Occupies Space and Has Weight.-If a drinking glass or beaker be thrust, mouth downward, into a vessel of water, the water does not enter until the glass is tilted to allow the air to escape. This shows that air occupies space.

That air has weight may be shown by weighing a flask, first empty and afterward filled with air. The flask (Fig. I) should be round-bottomed and have a capacity of 250 to 500 c.c. It is fitted with

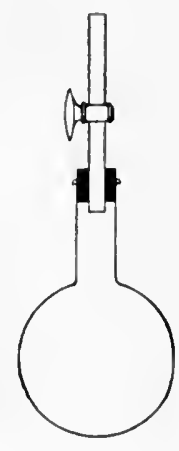

Fig. I a tight rubber stopper carrying a glass stopcock. The air is first pumped out by means of an efficient air pump; the stopcock is then closed and the flask counterbalanced with weights. When the stopcock is opened the inrush of air can be heard, and it is easy to observe that there is an appreciable increase 
in weight. Since air occupies space and has weight, it is undoubtedly a form of matter.

One liter of air weighs more than a gram and the air contained in a room 12 feet square and $\mathrm{I} 2$ feet high would weigh about 100 pounds. At the earth's surface air exerts a pressure of about I 5 pounds on every square inch of surface. The existence of this pressure may readily be shown by means of the following experiment. A tin can with a narrow neck (such as is often used for shipping alcohol, etc.) and of about I gallon capacity is fitted with a stopper carrying a glass tube, by means of which the air filling the can may be pumped out. Usually, before the exhaustion of the air is complete, the can is crushed by the pressure of the air on the outside-a pressure which is now no longer balanced by the equal and opposite pressure on the inside.

4. The Effect of Pressure on Volume: Boyle's Law.-The atmospheric pressure is measured by means of the barometer. At the sea-level the normal barometric pressure serves to support a column of mercury $76 \mathrm{~cm}$. high. The effect of pressure upon the volume of air was first studied by Robert Boyle in the seventeenth century. Boyle found that the volume of a given portion of air was inversely proportional to the pressure. This relation is known as Boyle's law. If we represent the pressure by $P$ and the volume by $V$, then $P V=$ a constant.

5. The Effect of Temperature on Volume: The Law of Charles.-In scientific work we use the Centigrade thermometer, the scale of which is so constructed that the freezing-point of water is $0^{\circ}$, while the boiling-point is $100^{\circ}$. The effect of temperature upon the volume of a given portion of air at a fixed pressure was studied over a century ago by Charles and by Gay Lussac. It was found that the volume of the air increased I/273 of its volume at zero for each increase of $\mathrm{I}^{\circ} \mathrm{C}$. This statement is known as the law of Charles, or sometimes also as the law of Gay Lussac.

6. The Gas Thermometer: Absolute Temperature.-An experiment will show that if 273 c.c. of air contained in a flask or cylinder at $0^{\circ} \mathrm{C}$. is heated to $100^{\circ} \mathrm{C}$. the volume will change to 
373 c.c. Such an apparatus is called an air thermometer and temperatures may be measured in this way instead of by the expansion of niercury, as in ordinary thermometers. At $I^{\circ} \mathrm{C}$. the volume of the air is 274 c.c.; at $2^{\circ}$ it equals 275 c.c. and thus the volumes in the following table correspond to the temperatures given.

TABLE I

\begin{tabular}{|c|c|c|c|c|c|c|}
\hline \multicolumn{2}{|c|}{$\begin{array}{l}\text { Volume } \\
\text { in c.c. }\end{array}$} & $\begin{array}{l}\text { Dezrees } \\
\text { Centigrade }\end{array}$ & \multicolumn{2}{|c|}{$\begin{array}{l}\text { Volume } \\
\text { in c.c. }\end{array}$} & \multicolumn{2}{|r|}{$\begin{array}{c}\text { Degrees } \\
\text { Centigrade }\end{array}$} \\
\hline 373 & minus & $273=100$ & 293 & minus & $273=$ & 20 \\
\hline 372 & " & $\because=99$ & $28_{3}$ & “" & $\because=$ & 10 \\
\hline $36_{3}$ & “" & $"=90$ & 27.3 & " & $"=$ & $=0$ \\
\hline 323 & " & $"=50$ & 263 & $" ،$ & $"=$ & $=-10$ \\
\hline 313 & $" 6$ & $"=40$ & 253 & “ & $"=$ & $=-20$ \\
\hline 303 & “ & $"=30$ & 243 & " & $" *=$ & $=-30$ \\
\hline
\end{tabular}

Since the zero of the Centigrade thermometer is arbitrarily chosen, being the temperature of the freezing of water (the student is already familiar with the Fahrenheit zero, which is at a lower temperature), it would be possible and convenient to use a temperature scale in which the volumes of the air in the air thermometer as described are taken as the temperatures. Since temperatures on the Centigrade scale are obtained by subtracting 273 from the corresponding air-thermometer temperatures, the zero of the air thermometer or gas scale must be 273 degrees lower than the Centigrade zero, or 273 degrees below the freezing-point. These air-thermometer temperatures are usually called the absolute temperatures; the absolute temperature may therefore be defined as the Centigrade temperature plus 273 degrees. Since most other gases act like air they may be used in the gas thermometer, and it is evident that if a certain amount of gas is used in such an experiment, no matter what its volume may be at the freezing-point of water, the volume will always vary with the temperature in the same ratio as the absolute temperature, provided the pressure on the gas is kept constant.

7. Problems.-We may now consider a few simple problems based on the two laws of gases just discussed. 
Problem I: The volume of a certain amount of air at $27^{\circ} \mathrm{C}$. is $\mathrm{I}, 000$ c.c. What would its volume be at $127^{\circ} \mathrm{C}$. if the pressure is kept constant?

Centigrade temperature $+273=$ absolute temperature

$$
\begin{aligned}
27^{\circ}+273 & =300 \\
127^{\circ}+273 & =400
\end{aligned}
$$

The volume of the gas must therefore increase in the ratio of 400 to 300 , or it will become

$$
1000 \text { c.c. } \times \frac{400}{300}=1333 \cdot 3 \text { c.c. }
$$

Problem 2: Let the original pressure on the gas in Problem I be $60 \mathrm{~cm}$. of mercury (or $\frac{60}{76}$ of the ordinary pressure of the atmosphere). What will be the final volume of the gas if the pressure is increased to $100 \mathrm{~cm}$. of mercury? An increase of pressure must decrease the volume of the gas, and in the ratio of the pressures, 60 to 100 , or by $\frac{60}{100}$.

a) Let the change of pressure come after the change of temperature as given in Problem I: then

$$
\text { I } 333: 3 \text { c.c } \times \frac{60}{100}=800 \text { c.c. (final volume) Ans. }
$$

b) Let the change of pressure take place first: I000 c.c $\times \frac{60}{100}=600$ c.c., volume after the pressure change.

The temperature change would then change the volume as follows:

$$
600 \text { c.c. } \times \frac{400}{300}=800 \text { c.c. (final volume) Ans. }
$$

It is thus seen that the same answer is obtained, no matter which step in the problem is worked first, so the whole problem、 I and 2 together, may be stated in one expression as follows:

$$
1000 \text { c.c. } \times \frac{400}{300} \times \frac{60}{100}=800 \text { c.c. Ans. }
$$


Problem 3: Suppose that I,000 c.c. of air at $20^{\circ} \mathrm{C}$. and $70 \mathrm{~cm}$. pressure is cooled to $0^{\circ}$ and that at the same time the pressure is increased to $76 \mathrm{~cm}$. Find the final volume.

When a gas is at the temperature of $\circ^{\circ} \mathrm{C}$. and under a pressure of $76 \mathrm{~cm}$. (the normal a tmospheric pressure at sca-level) it is said to be at standard conditions. Unless otherwise stated, numerical data for gases refer to the latter under standard conditions.

Problem 4: Find the volume at standard conditions of 400 c.c. of air measured at $30^{\circ}$ and $72 \mathrm{~cm}$.

8. Steam Is the Gaseous Form of Water.-It is well known that when water is heated it passes into steam. The white cloud which is frequently spoken of as steam is not really steam, but is composed of minute droplets of water. If we boil water in a glass flask the space above the water is filled with steam, but we notice that the steam is entirely invisible and that the visible cloud forms only when the steam cools and condenses to liquid droplets. Water in the form of steam is, like air, a gas. When we boil any liquid like alcohol or mercury the liquid passes into the state of a gas or vapor, as it is sometimes also called. The gas or vapor when cooled condenses to the liquid form of the substance.

\section{Change of Form of Matter with Change of Temperature.-} Just as water when cooled solidifies to ice, so every other liquid substance solidifies when sufficiently cooled. We speak of steam and ice as the gaseous and solid forms respectively of water. The substance known as moth-balls is called naphthalene by the chemist; it is a solid at ordinary temperatures, but when heated it melts to a colorless liquid, and when heated still hotter it boils, giving a colorless vapor, which is naphthalene in the form of a gas. When this gas is cooled it condenses to a liquid, which when cooled still further solidifies or freezes, giving solid naphthalene again. Behavior like that of water and naphthalene is met with in the case of very many other substances. They can exist in three different forms, gas, liquid, and solid, according to the temperature. 


\section{CHAPTER II}

\section{THE BURNING OF SUBSTANCES-OXYGEN}

10. Burning Substances Require Air.-The history of chemistry shows that the discovery of the real nature of the process of burning was one of the most important, if not the most important, in the development of the whole science. That air is needed for the burning of a substance is, in general, well known, and can easily be shown by many simple

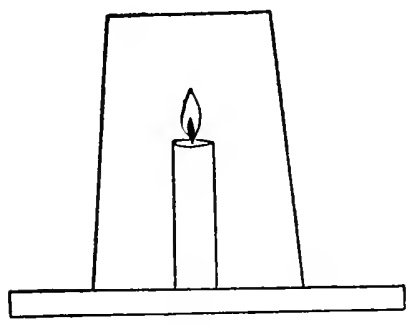

FIG. 2 experiments. For example, if we place an inverted drinking-glass over a burning candle standing on a table (Fig. 2), the flame quickly grows smaller and smaller and soon goes out, the glass having cut off the needed supply of air.

A still more interesting and instructive experiment may be made with phosphorus, a substance which burns very readily in the air, giving off clouds of white smoke. A piece of phosphorus of the size of a pea is placed on a cork floating on water and covered with a bell-jar (Fig. 3). When a heated wire passing through the tight-fitting stopper of the jar is brought in contact with the phosphorus the latter takes fire and burns with the production of light and heat and the formation of a cloud of white smoke. At the

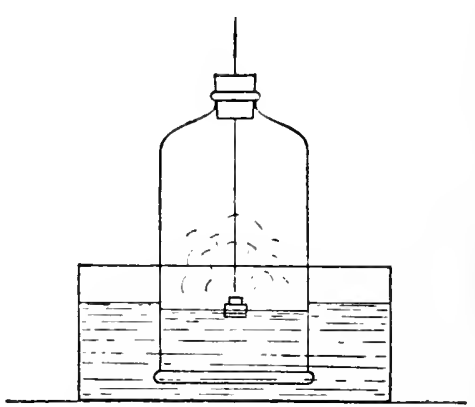

FIG. 3 same time the level of the water inside the bell-jar first falls a little and later rises; but while there is still a large volume of air left above the water on which the cork floats, the flame dies out and the burning ceases. By 
the time the bell-jar and its content have become cold, the cloud has disappeared and the water has risen on the inside so that the volume of the remaining air is seen to be about fourfifths of the original volume. It follows that about one-fifth by volume of the air has disappeared.

Further examination also shows that much of the phosphorus still remains unburned. Why, then, should the burning stop while there is still four-fifths by volume of the air left in the jar? The answer to this question may be made when we find that, try as we may, we cannot make phosphorus or anything else burn in the air remaining in the jar. We therefore conclude that the remaining air is different from common air. The correctness of this conclusion is supported by the fact that small animals, such as mice, suffocate at once if allowed to breathe this remaining portion of the air. The facts just considered make it seem probable that one-fifth of the air is different from the balance, and that it is this portion which takes part in the burning of substances and which is necessary for the respiration of animals.

Everyday experience would seem to indicate that wood, coal, paper, gasoline, etc., are completely destroyed when they are burned. Wood and coal leave a small amount of ash when burned, but nothing visible remains in the case of gasoline and other oils. Since we have found that water in the form of steam is invisible, it is possible that the substance burned may have passed into an invisible form and thus escaped notice.

There are many substances which burn very readily and in so doing leave behind large amounts of ash; the experimental study of the burning of such substances leads to important conclusions. We may now consider two typical cases of this sort.

I1. The Burning of Magnesium.-The metal magnesium, which is used in photographic flash lights, will burn very readily in air, either in the form of powder or thin ribbon. In either case we notice that a white ash is left. If we collect and weigh the ash from the burning of a weighed piece of magnesium ribbon we find that the ash weighs more than the original metal ribbon. The-actual experiment is best carried out by placing about one 
gram of magnesium, in the form of wire (Fig. 4) or ribbon, in a porcelain crucible, having a cover, and then weighing crucible and

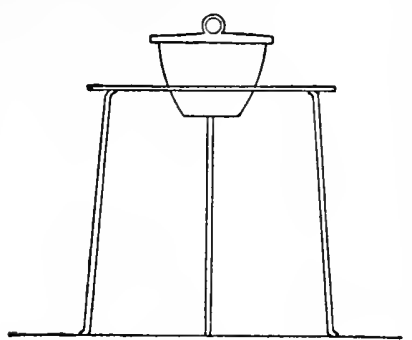

FIG. 4 contents. The magnesium is then ignited and the cover so adjusted that some air can enter, but that the dense cloud of white smoke is largely held back in the crucible. After the burning is finished and the crucible has cooled and the whole is again weighed, it will be found that there has been a considerable increase in weight.

I2. The Burning of Iron.-Iron powder or filings burn readily when thrown into a flame, and in a similar manner we find that the burned iron or iron ash, as we might possibly call it, is heavier than the original metal. In order to show this by experiment, we may suspend on one side of a balance (Fig. 5) a horseshoe

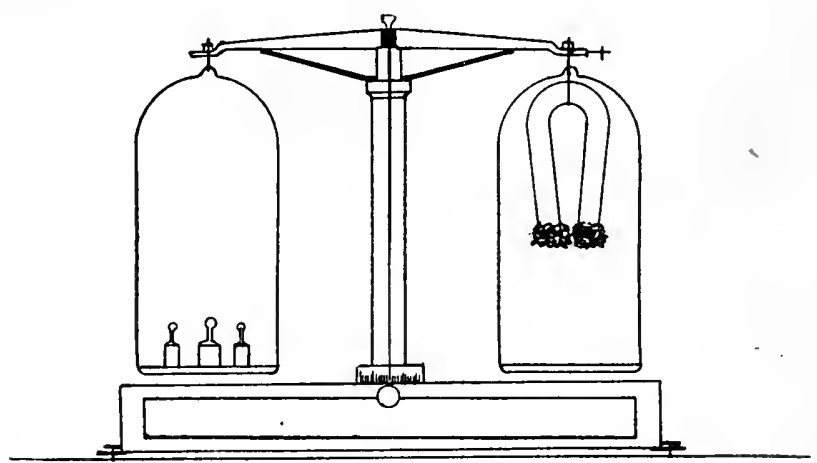

FIG. 5

magnet which has been dipped in iron filings, and counterpoise the magnet and adhering iron by adding small shot or sand to the other pan of the balance. By the application of a flame, the iron, which now presents a large surface to the air, may be ignited. As it burns with a dull glow we observe a gradual increase in its weight, and, while there is no noticeable change in its volume, the cold residue, which we may call iron ash, is 
seen to have lost its metallic luster and taken on a dead black color. We find, thus, that iron ash is heavier than the iron burned. If we seck the cause of this increase in weight, we may get a hint when we remember that for the burning of a candle air is required, and that, moreover, part of the air disappeared when phosphorus was burned in it. What, then, becomes of the weight of the one-fifth of the air that disappeared? Is it added to the weight of the iron, so as to increase the weight of its ash? The facts presented in the next paragraph will furnish the required answers.

13. Lavoisier's Experiment with Mercury。-An experiment which turned out to be one of the most important made in the early development of the science of chemistry was carried out by the great French chemist, Lavoisier, in the latter part of the eighteenth century. The arrangement in this classic experiment is shown in Fig. 6. The retort (the glass vessel with the long bent neck) was partly filled with mercury (quicksil-

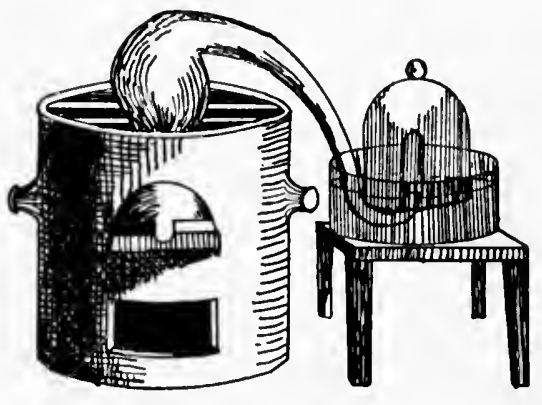

Fic. 6 ver); the space above the mercury contained ordinary air, which also filled the bell-jar with which the neck of the retort communicated. The bell-jar stood in a shallow vessel containing mercury, which served to prevent outside air from passing into or out of the jar. The mercury in the retort was now heated by means of a charcoal stove for a period of several days. The heating first caused an expansion of the air; but as time went on a gradual contraction occurred, which entirely ceased after several days, whereupon the heating was stopped. The volume of the air left in the entire apparatus when brought to its original temperature and pressure was practically four-fifths of what it had been at the start. The surface of the mercury in the retort was found to be covered with a red powder, which may be considered 
as analogous to the white ash formed in the burning of magnesium or the black ash formed by the burning of iron filings.

I4. Heating the Red Ash of Mercury.-If we take some of the red ash of mercury, place it in a glass test tube, and heat it very strongly (Fig. 7), we find that it changes in a remarkable way: first it turns black, and then at red heat it gradually grows smaller, until after a few minutes none of it remains. At the

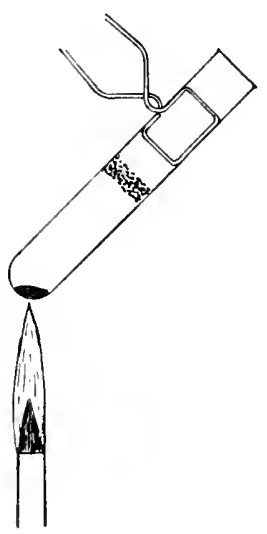

FIG. 7 same time, however, on the cooler part of the wall of the tube a silvery-looking coating has appeared, which when the tube has cooled may be brushed to the bottom of the tube, and is then readily seen to consist of drops of liquid mercury. Thus by heating the red powder to a higher temperature than that used in its formation, mercury is reproduced. But this is only half the story.

The more important part remains to be told. Lavoisier reasoned about the matter somewhat as follows: If burning substances require air; if a part of the air disappears (in some cases at least) during burning; if in the burning of metals like magnesium and iron the ash is heavier than the metal burned; if, as is indeed a fact, air has weight; is it not possible that the burning substance unites with a part of the air to form a new kind of substance, and that this new substance, for example, magnesium ash, is heavier than the substance burned because it contains not only the latter but also a part of the air? Perhaps also the red ash formed by the gentle heating of mercury in contact with air is also made up of mercury and something taken from the air. Perhaps the one-fifth of the air that vanished has combined with the mercury to form the red ash. If all these suppositions are true, perhaps when the red. ash was changed again into mercury by being strongly heated there was set free at the same time the part of the air which by originally uniting with the mercury produced the red ash. If all this were true, how could it be proved? Let us see. 
15. The Active Part of the Air: Oxygen.-The part of the air which disappeared may be just that part which causes substances to burn. If it were to be obtained pure, free from the inert fourfifths which does not support burning (combustion), it ought to support combustion far better than common air. This is a matter easily put to the test of experiment. Let us again heat some of the red powder in a test tube and at the same time thrust into the tube a burning wood splint. We see that it burns much more fiercely and brightly than in common air. Furthermore, if we have no flame, but only a tiny spark on the end of the splint, we see that when thrust into the tube above the heated red ash the spark bursts into a vigorous flame. The suppositions seem to be true. Lavoisier was led in this way to the discovery of the secret of the nature of burning. He called the gas formed by the heating of the red powder oxygen. This gas forms one fifth by volume of the air and is the part of the air which is necessary for the burning of substances. The other four-fifths by volume of the air is inert; it does not support combustion; neither does it support the respiration of animals. Lavoisier called it azote; we call it nitrogen.

I6. The Properties of Oxygen.-Oxygen is an invisible gas like air; it has no odor and it supports combustion far better than

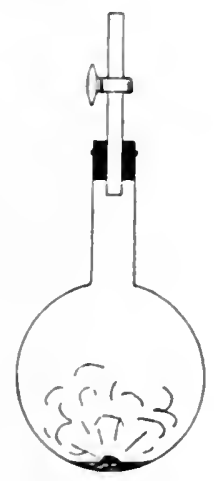

FIG. 8 does air. By the same method as that employed in the case of air (chap. i), we may readily find that I liter of oxygen at a temperature of $0^{\circ}$ and $76 \mathrm{~cm}$. pressure weighs I. $43 \mathrm{~g}$. It has, therefore, a somewhat greater density than air, of which I liter weighs I.29 g. Further evidence that the explanation of the nature of burning, given in the preceding paragraph, is the correct one is furnished by experiments which we may now consider.

17. Burning Iron in Oxygen.-If we place a gram or two of iron filings and a minute piece of phosphorus on a piece of asbestos paper in the bottom of a 300-c.c. round-bottomed flask filled with pure oxygen and fitted with a rubber stopper and a glass stopcock 
(Fig. 8), we shall find that the weight of the flask with its contents does not change if by heating we cause the iron to burn in the oxygen. Now, we know that when iron burns, the product weighs more than the original iron. We know also that oxygen has weight and that the total weight of the flask with its contents has not changed during the burning. What then is the cause of the increase of the weight of iron when burned? If we open the stopcock while its open end is held under water, we find that the water nearly fills the flask. We must conclude that the oxygen has disappeared. Is it not reasonable to suppose that the ash resulting from the burning of iron is composed of the iron originally taken and the oxygen which has disappeared?. Our experiment has shown that the weight of this ash is precisely the same as the combined weights of the iron and the oxygen which disappeared in the burning.

It will readily be seen that the experiment with iron is similar to that made by Lavoisier with mercury-with the difference that iron burns rapidly, whereas mercury changes but slowly in oxygen. Furthermore, the fact that the red ash of mercury when strongly heated gives again mercury and oxygen makes it practically certain that the red ash was formed by the combination or union of mercury with oxygen which composed part of the original air used in Lavoisier's experiment. Instead of iron, in the experiment described, we might have substituted magnesium or phosphorus, or indeed any one of a large number of other substances. In each case the result would have been similar to that in the case of iron and oxygen and a similar conclusion would have been forced upon us. In all such cases we would conclude that the process of burning consists in the combination or union of gaseous oxygen with the solid substance burned to form the product of the combustion.

18. Burning Charcoal in Oxygen.--If we put a piece of burning charcoal into a bottle containing oxygen we notice that it burns even more rapidly in oxygen than in the air. In this case there is but a trifling amount of ash left compared with the amount of charcoal burned. In order to see whether an invisible product may have been produced we may make the following experiment. If we pour a little limewater into a bottle contain- 
ing oxygen and shake the limewater with the oxygen we notice no change. If now we pour limewater into a bottle in which charcoal has been burned in oxygen and again shake the container, the limewater becomes milky in appearance. We must conclucle that some invisible substance, different from oxygen, has been produced in the latter case. If the burning of charcoal is thought to be analogous in nature to the burning of iron, then we might expect that the product would be something composed of carbon and oxygen and that its weight should be equal to the combined weights of the carbon burned and the oxygen taken up. We can get some evidence that this is the case by means of the following experiment.

19. Carbon Dioxide. $-A$ small quantity of charcoal is placed near one end of a hard glass tube, the other end of which contains pieces of caustic soda (Fig. 9). If we now weigh the tube, which

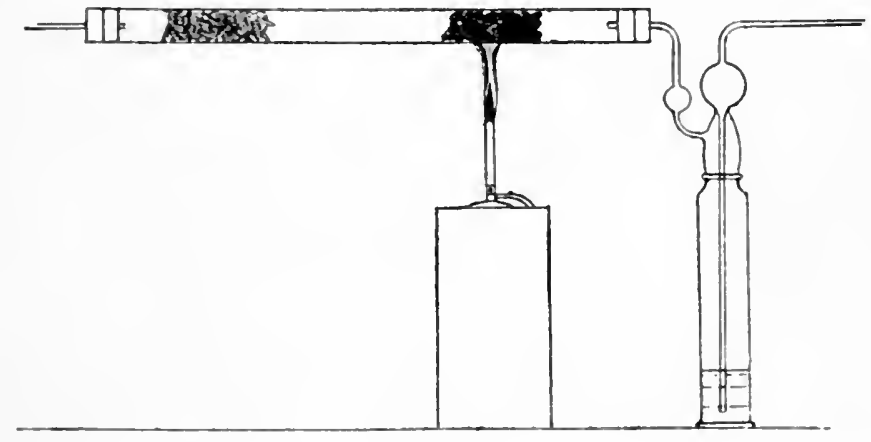

FIG. 9

may be fitted at the end containing the charcoal with a stopper and a small glass tube, and then cause the charcoal to burn in a stream of oxygen gas which we may pass through the tube, we shall find that there is an increase of weight, due to the fact that the product formed by burning the charcoal has been absorbed by the caustic soda in the tube. If we place some caustic soda in a beaker, dissolve it in water, and add some hydrochloric acid, we can see no marked change. If we treat the material from the charcoal experiment in the same way, we notice that a gas is given off when we pour the acid into the solution. A test of this gas with limewater shows that it behaves like that obtained 
when charcoal is burned directly in oxygen. The results of these experiments lead us to conclude that when charcoal is burned an nvisible gas is produced, and that this gas is heavier than the charcoal burned; and, in fact, if charcoal had been burned in a closed vessel with oxygen, we should find that the weight of vessel and contents had not changed during the burning, and would be forced to conclude that the weight of the invisible product was just equal to the sum of the weights of the charcoal burned and the oxygen which had united with it. This gaseous product of the burning of charcoal was formerly called carbonic acid gas, but is now usually called carbon dioxide.

20. Experiments with a Burning Candle.-We find by experiment that carbon dioxide is formed when wood, coal, illuminating gas, gasoline, etc., burn. We may easily show by the limewater test that it is also formed during the burning of a candle. We

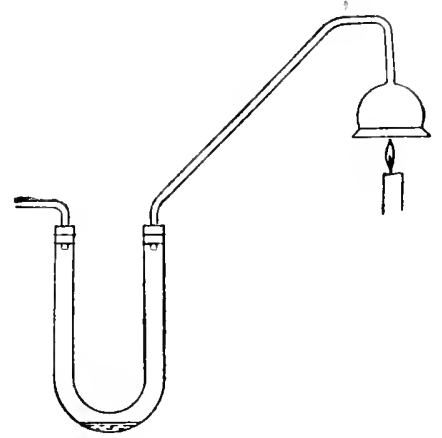

FIG Io. may also show that another wellknown substance is produced when the candle burns. If we burn the candle under an inverted funnel connected by means of a glass tube with a $U$-tube which is cooled by immersion in a vessel of mercury and draw air through the funnel and U-tube we find that a colorless liquid collects in the cold U-tube (Fig. Io). This liquid is water. The burning of the candle gives,

then, both carbon dioxide and water. We may readily show that the weight of the products of a burning candle, if these are suitably collected, is greater than the weight of the candle burned.

To do this we make use of the arrangement shown in Fig. 1 . The candle is inclosed in a glass cylinder, closed below by a cork having three or four holes for the admission of air. The top of the cylinder is filled with pieces of a solid substance (caustic potash) which readily absorbs both carbon dioxide and water, but not oxygen or nitrogen, the components of air. The apparatus thus arranged is suspended on one side of a balance and counterpoised. 
The candle is now lighted and allowed to burn for ten or fifteen minutes, whereupon it will be found that the apparatus has become appreciably heavier. The increase in weight is due to the fact that the carbon dioxide and water formed weigh more than the candle burned. In fact, the excess weight is exactly the weight of the oxygen which has been consumed in the burning. Under ordinary circumstances the carbon dioxide and water escape our notice because both, the latter being in the form of steam, are invisible gases.

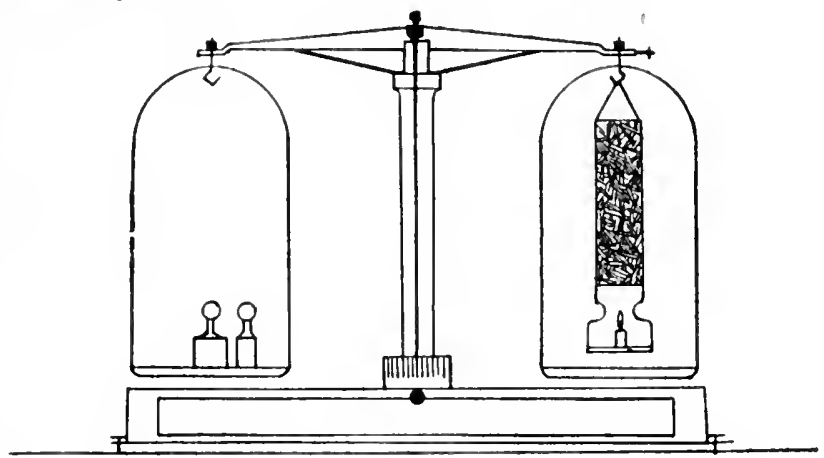

FIG, II

21. The Law of the Conservation of Matter.-By the study of such facts as those discussed in the preceding paragraph and many others of a similar nature, Lavoisier arrived at the conclusion that when a substance burns it unites with the oxygen of the air, and that the weight of the product is always exactly equal to the weight of the substance burned plus the weight of the oxygen which unites with the burned substance during the combustion. The product may be a solid, a liquid, or a gas. If it is a volatile liquid or a gas it usually escapes notice because it is invisible. Burning, therefore, consists in a union of the substance burned with oxygen. In this sense a substance which is burned is not destroyed; the material or matter composing it merely passes into another form, the quantity of matter in all cases being measured by its weight. These facts are briefly summed up in the statement that maller is indestructible, a statement which is frequently referred to as the Law of the Conservation of Matter. 


\section{CHAPTER III \\ PURE SUBSTANCES-ELEMENTS}

22. Bodies and Substances.-We use the words "substance" and "body" in chemistry in very definite senses. We speak of things like watches or knives as "bodies." We say that the blade of the knife is steel, the handle is pearl. We say that a watch has a case of gold and a watch crystal of glass. We call steel, pearl, gold, and glass "substances." A substance is thus a particular kind of material, a body is an object which may be composed of one or many kinds of substances. Water, salt, and sugar are further examples of substances in the sense of this definition.

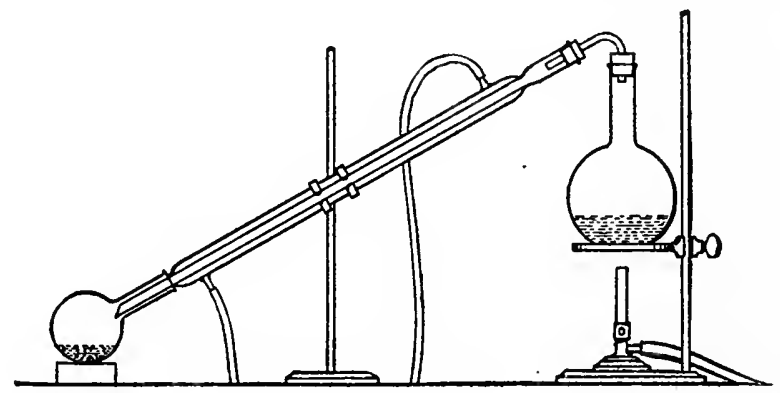

FIG, I2

23. Pure Substances.-We find that natural waters, as those of lakes, rivers, and springs, are not all alike. It now becomes important to discover the cause of the differences between waters from different sources. If we boil a quantity of lake water we find when the water has entirely disappeared that a solid residue is left. If the steam from the boiling water is condensed by cooling it, as by means of a condenser (Fig. I 2) through the outer tube of which a stream of cold water flows, we obtain what is called distilled water. If we now evaporate to dryness a quantity of this distilled water we find that no residue is left. If we prepare distilled water from any natural water we find that 
it will always evaporate completely, leaving no solid residue. We find further that different kinds of natural water leave different proportions of solid residue upon evaporation and that the nature of the solid material left also differs in different cases, but that the distilled water in one case cannot be distinguished in any way from that obtained in another. We say then that distilled water is pure water, a pure substance, and the natural waters are not pure water, but that they contain dissolved foreign substances. If the natural water is muddy, that is, if it is not

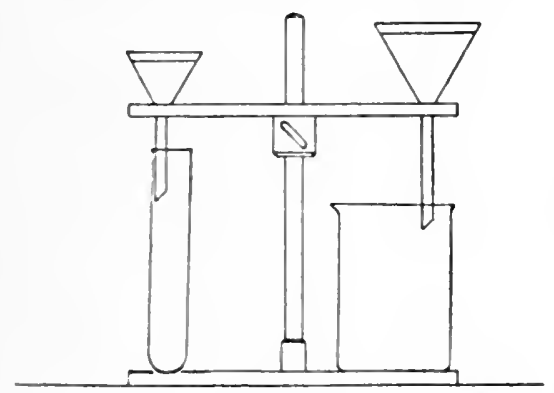

FIG. I3 clear, the foreign material which causes it to appear muddy can be separated by filtration (Fig. 13), a process in which the liquid is allowed to seep through a piece of filter paper folded so as to fit snugly into a funnel. The mud remains on the filter paper. However, filtration will not remove any of the dissolved material, but only that which is suspended in the water.

24. Pure Salt Made from Rock Salt.-Common salt is found in nature as a mineral known as rock salt. We find that different samples of rock salt differ in color, taste, specific gravity, and in other ways. If we mix rock salt with water we find that a large part dissolves in the water. In general a small amount of material, sand, etc., will not dissolve, even though we take a large amount of water. If we filter the solution we separate the water and dissolved material from the part which has not dissolved. That which runs through the filter paper is called the filtrate; it is the solution of the salt in water. If we boil away the water we find that the salt is left in the solid form and that the material is now free from color, that is, that it is white, and that it will dissolve completely in water. The salt so prepared is purer than the rock salt taken. Just as it is possible to prepare pure water from any natural water, so analogously it is possible to prepare 
pure salt from any natural salt. Pure salt is always exactly the same in taste, color, specific gravity, etc., from whatever source it may have been obtained. The process for the purification of salt, described in the statement above, gives in all cases a much purer product than the original rock salt-pure enough for table use, but not a perfectly pure substance. It still contains very small amounts of some foreign substances; but even these can be removed by well-known methods which the student will learn later. A pure substance is a substance which consists of one sort of material. It always has definite physical properties, from whatever source it may be obtained.

25. Decomposition of Substances. - It was found that the red ash formed by heating mercury in contact with air was changed, upon being heated still more, into mercury and oxygen. We say in this case that the red ash of mercury has been decomposed into

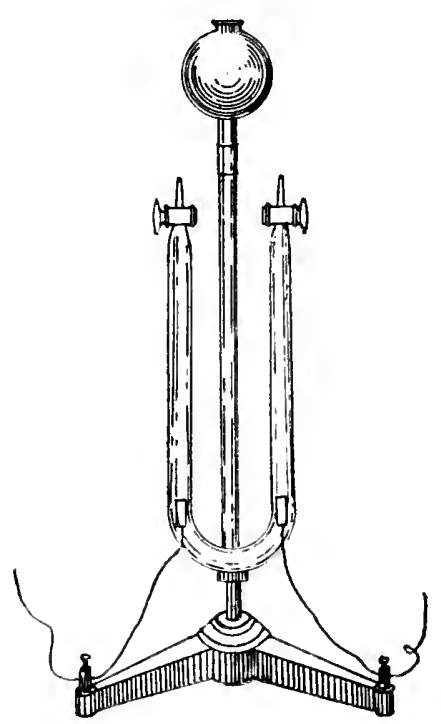

FIG. ${ }^{4}$ mercury and oxygen. We can accomplish the decomposition of many substances in an equally simple fashion.

We will now consider a few such cases as illustrations.

26. Decomposition of Sal Soda.-If we place in a test tube a crystal of common washing-soda, also known as sal soda, and heat it gently over a Bunsen flame, we find that water is produced as steam, and that it condenses in the cold end of the test tube. An opaque solid is left in place of the clear crystal of sal soda taken. We say that the sal soda has been decomposed into dry soda and water. It would be easy to show that the weight of the water and dry soda formed is equal to the weight of sal soda taken. In other words, the sal soda has been decomposed into dry soda and water. 
27. Electrolysis of Water.-If we pass an electric current through some water (Fig. I4) to which we have added a few drops of sulfuric acid, we find that gases are produced at the platinum electrodes. The decomposition of a substance by an electric current is called electrolysis. If we collect each of these gases separately we find that one of them is oxygen. The other gas, the volume of which is double that of the oxygen, has quite different properties; it is called hydrogen. If we bring a lighted splinter into the oxygen, the splinter continues to burn with increased brilliancy and rapidity. If we repeat this test with hydrogen, we find that the hydrogen itself catches fire, just as illuminating gas would do, and that the splinter itself no longer burns in the hydrogen gas. These facts may be concisely stated by saying that oxygen supports combustion, while hydrogen burns but does not support combustion. It would be possible to show by experiment that the weight of the water decreases during the passage of the electric current through it, and that this decrease in weight is just equal to the combined weights of the oxygen and hydrogen formed. The total amount of sulfuric acid added to the water remains in the water at the end of the electrolysis and would serve to promote the decomposition of any desired amount of water. The complete explanation of the behavior of the sulfuric acid cannot be given at this point, but we know that the hydrogen and oxygen formed come exclusively from the water and not from the acid nor the platinum nor the glass of the vessel used. We conclude that water is decomposed by the electric current into hydrogen and oxygen. Therefore we may say that water is composed of hydrogen and oxygen or that water is a compound of hydrogen and oxygen. As a matter of fact, when hydrogen burns in air water is formed. If a cold beaker is held over a jet of burning hydrogen, water will be seen to condense in a mist on the surface of the beaker.

28. Magnesium Burned in Steam.-That water is composed of oxygen and hydrogen may be shown in many other ways, one of which is the following. When a piece of magnesium ribbon burns in air the magnesium unites with the oxygen of the air to form a white solid which we call magnesium oxide. Now, 
magnesium will also burn in steam (Fig. I5) nearly as readily as it does in the air or in pure oxygen, and we find that the white solid which is again formed is also magnesium oxide. In addition, hydrogen gas is produced and may easily be collected over water. Since magnesium oxide is composed of magnesium and oxygen, and we obtain from magnesium and water magnesium oxide and hydrogen, we are again led to the conclusion that water is composed of hydrogen and oxygen.

29. Steam Passed over Hot Iron.-An entirely analogous experiment may be carried out with iron and steam. In this

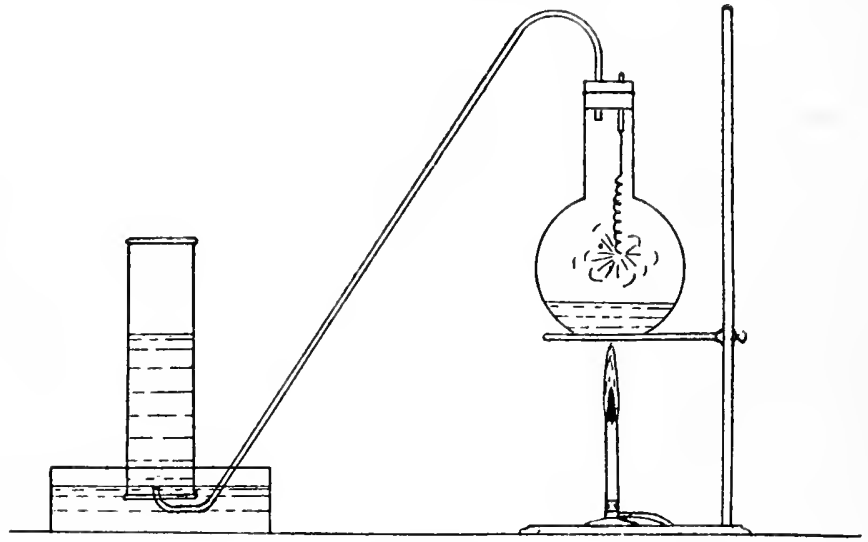

FIG. 15

case iron turnings or fine iron wire is strongly heated in an iron or glass tube (Fig. I6). When steam is passed through the tube, iron oxide and hydrogen are produced, a result which leads to the same conclusion as before regarding the composition of water.

30. Magnesium Burned in Carbon Dioxide.-The composition of carbon dioxide may be discovered by burning magnesium in this gas. We find that magnesium oxide and a product resembling charcoal are formed. The latter substance is carbon, of which charcoal is a nearly pure form. We conclude, therefore, that carbon dioxide is composed of carbon and oxygen or is a compound of carbon and oxygen.

The facts already considered lead to the conclusion that the red ash obtained when mercury is heated gently in air is com- 
posed of mercury and oxygen; briefly, that it is a compound of mercury and oxygen - a fact represented by the chemical name of the red ash, mercuric oxide.

31. Elements.-The substances mercury, oxygen, hydrogen, and carbon have never been decomposed into simpler substances. We say that hydrogen and oxygen are the elements of which water is composed; that carbon and oxygen are the elements composing carbon dioxide.

We may discover of what elements a substance is composed in two ways: either by the decomposition of the substance into the

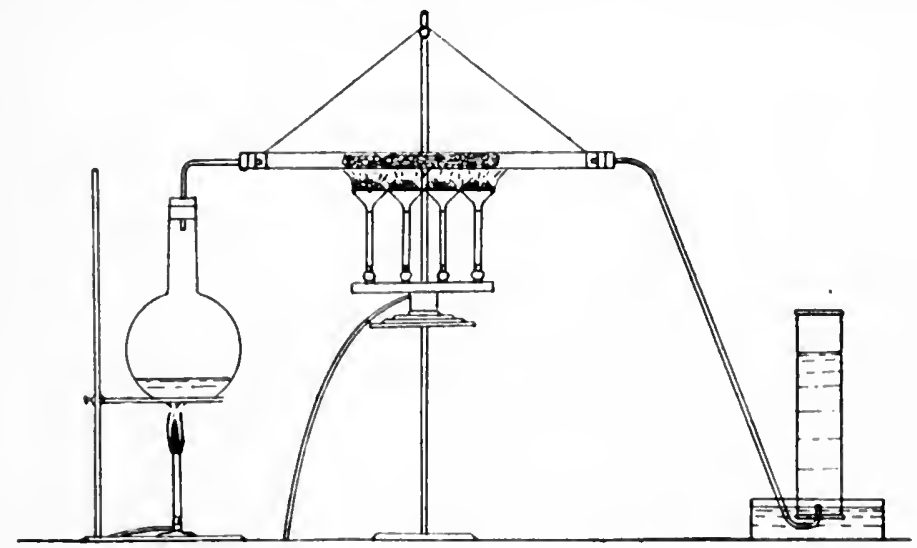

Fig. I6

simpler ones which compose it - the process called analysis, or by causing known elements to unite in the formation of the original-the process called synthesis. As a result of the electrolysis of water we have concluded that water is composed of hydrogen and oxygen. This conclusion may now be tested by seeing whether water can be obtained from hydrogen and oxygen. We found that hydrogen burns readily. If we burn a jet of hydrogen under an inverted funnel and draw the product through a cooled $U$-tube, as in the experiment with the candle, we shall find that liquid water collects in the $U$-tube and that the most careful search fails to reveal any other substance as the product of the burning of hydrogen in air or in pure oxygen. Water is, therefore, a compound of the elements hydrogen and 
oxygen. Since the burning of charcoal, which is a nearly pure form of the element carbon, gives carbon dioxide and nothing else, we know that carbon dioxide is a compound of the elements carbon and oxygen.

32. The Burning of Copper; Copper Oxide.-If the metal copper, in the form of fine wire or filings, is heated in air or in oxygen, it is slowly changed into a black substance quite different in appearance from metallic copper; but during this change we do not observe the production of any light. By means of the balance we may find that the black substance formed is heavier than the copper taken, and we at once suspect that the copper has united with oxygen to form a compound. If the heating of the copper had been carried out in a sealed glass vessel containing oxygen, as in the earlier experiment with iron powder, it would have been found that gaseous oxygen had disappeared and that the weight of the black product was exactly equal to the weight of the copper taken plus the weight of the gaseous oxygen which had disappeared. The black substance would seem, therefore, to be a compound of copper and oxygen. We know that when the red mercury oxide is strongly heated it is decomposed into mercury and oxygen. If we heat the black product from copper to the highest temperature we can obtain with the Bunsen burner, we find that it remains unaltered in weight and appearance and that no oxygen is given off. 'This fact might lead us to suspect that the black substance is not a compound of copper and oxygen, since its behavior is not analogous to that of mercury oxide. In this connection the following experiment will prove of interest.

33. Hydrogen Passed over Hot Copper Oxide.-If we put two or three grams of the black copper product in a porcelain boat in a "hard" or difficultly fusible glass tube, heat the tube and contents by means of a Bunsen flame, and then pass a current of hydrogen through the tube, we observe that the solid glows or seems to burn (Fig. I 7 ). At the same time we notice that liquid water condenses in the colder part of the glass tube. After a few minutes the glow has disappeared, even though the stream of hydrogen has continued. At this point the heating may be 
discontinued and the solid which is left in the boat allowed to cool in the stream of hydrogen gas. We now observe that the solid has the appearance and properties of metallic copper, which in fact it is. However, the copper is not in a single compact lump, for a reason which must be evident. Metallic copper can be melted, but the melting-point is a very much higher temperature than that attained in the preceding experiment. Only by heating the copper to a point above this melting temperature could the material be obtained in a single lump. This could easily be accomplished by directing an intense blowpipe flame upon the metal particles contained in the porcelain boat.

We may now consider the nature of the changes which occured in this experiment. Since we obtained water and copper, and

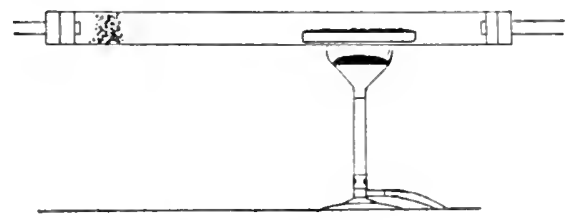

FIG. I 7

since we know that water is a compound of the element hydrogen with oxygen, we conclude that the oxygen was originally united with the copper and that the black substance must have been a compound of copper and oxygen. This substance is called copper oxide. We might express the result in the following simple fashion: Copper Oxide + Hydrogen $\rightarrow$ Water + Copper; or instead of "Water" we might write "Hydrogen Oxide," the true chemical name for water. This statement would then show at a glance the nature of the chemical change which had occurred.

34. Discovery of the Elements Composing a Substance: the Analysis of Malachite.-There is an almost innumerable variety of bodies on and in the earth, but these are composed of a very much smaller number of definite chemical substances. However, the number of definite substances is still very great, many thousands having been carefully described. Chemistry has for its object the systematic study of pure substances, their properties, 
and their behavior toward one another. Happily the study of this immense number of substances is greatly simplified by the fact that they are all made up of a relatively small number of elements. The way in which the elements composing a substance of unknown composition are discovered may be illustrated by means of an experiment with the mineral known as malachite. Malachite is a beautiful crystalline substance often used as an ornamental stone and also as one of the sources from which a familiar metal is obtained. If we place in a test tube, fitted with a cork and a bent glass tube, a few grams of malachite and heat the substance gently in a flame, we notice that a change in color from green to black occurs and at the same time that water condenses in the colder part of the glass tube and a gas is also given off. If we pass this gas into limewater we find that it behaves like carbon dioxide, which in fact it is. By means of the balance we might find that the combined weights of the carbon dioxide, water, and black product equal the weight of the original malachite. Since we know of what elements carbon dioxide and water are composed, it only remains to find the composition of the black substance in order to have a complete knowledge of the elements composing malachite. If this black substance were heated in a stream of hydrogen, it would be found to yield water and a red metallic-looking substance which could easily be recognized as copper. Therefore, the black substance must have been copper oxide. The results may then readily be interpreted. Malachite when heated is decomposed into carbon dioxide, water, and copper oxide. Knowing as we do the elements composing each of these three products, we are led to the conclusion that malachite is a compound of the elements carbon, oxygen, hydrogen, and copper. Chemists have so far been unable to decompose copper into anything simpler. It is, therefore, known as an elementary or simple substance, and we say that malachite is a compound of the four elements, carbon, hydrogen, oxygen, and copper.

35. Some Common Elements.-The total number of known elements is about eighty-five, of which less than thirty are common. In the following partial list of commoner elements, the 
student will find the names of ten or twelve familiar metals. Carbon and sulfur, which are well known to everyone, are not metals; they are classed as non-metals.

\section{A FEW COMMON ELEMENTS}

$\begin{array}{llll}\text { Silver } & \text { Copper } & \text { Nickel } & \text { Carbon } \\ \text { Gold } & \text { Lead } & \text { Magnesium } & \text { Sulfur } \\ \text { Platinum } & \text { Tin } & \text { Zinc } & \text { Oxygen } \\ \text { Iron } & \text { Aluminum } & \text { Mercury } & \text { Hydrogen }\end{array}$




\section{CHAPTER IV}

\section{THE LAW OF DEFINITE COMPOSITION}

\section{The Percentage Composition of Water.-We have}

already seen that when an electric current was passed through water, the latter was decomposed into two gases, hydrogen and oxygen. It was found that the volume of the hydrogen was double that of the oxygen obtained in the electrolysis. This was not a matter of accident, for it is always found that the same result is obtained whenever water is electrolyzed. Since water is composed only of hydrogen and oxygen, we may calculate the percentages of hydrogen and oxygen by weight if we know the weight of a liter of each of these gases. Direct weighing of the gases has shown that I liter of hydrogen weighs $0.090 \mathrm{~g}$. and I liter of oxygen weighs I.429 g., the gases being weighed at $0^{\circ}$ and $76 \mathrm{~cm}$. pressure. From these figures it is easy to calculate that water is composed of II. 2 per cent of hydrogen and 88.8 per cent of oxygen by weight. Pure water prepared from any source whatever always has exactly this composition.

The percentage composition of water may also be found in another way. It was found in section 33 that water and copper are formed when hydrogen is passed over heated copper oxide. If this experiment be carried out with a weighed quantity of copper oxide, and the weight of copper which remains after the experiment is found, the difference in the two weights will represent the weight of oxygen contained in the water which has been formed. If the weight of the water is determined, then the percentage of oxygen in water may readily be calculated. In this case we find precisely the same result as that given in the preceding paragraph.

The details of the experiment are as follows.

37. The Quantitative Synthesis of Water.-About one gram of pure copper oxide is placed in a weighed porcelain boat and heated sufficiently to drive off the moisture which it may con- 
tain. ${ }^{x}$ The boat and contents are weighed as soon as cool and placed at once in a hard glass tube. This tube (Fig. I8) is connected at each end with $U$-tubes filled with calcium chloride, a substance that absorbs water with great readiness. One of these $\mathbf{U}$-tubes is connected with a source of hydrogen gas and serves to remove all moisture (water vapor) from the hydrogen. The other $U$-tube will serve to absorb the water formed in the chemical reaction between the copper oxide and the hydrogen.

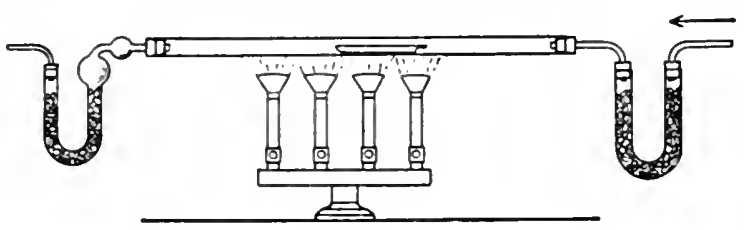

Fig. 18

This second $U$-tube is accurately weighed at the beginning of the experiment.

When all is ready, the stream of hydrogen is started and continued until all air is driven from the tubes. The tube containing the boat is now heated until the reaction begins, and kept hot enough beyond the boat to prevent the condensation of the steam formed, which is carried by the stream of hydrogen into the weighed $U$-tube.

When all the copper oxide has been changed into copper and the water has all been driven over into the $U$-tube, the heating is discontinued and the copper allowed to cool in a stream of hydrogen. 'The hydrogen is then driven out by a stream of air, and the U-tube detached and weighed. The object in replacing the hydrogen by air is readily understood when one recalls that hydrogen is far lighter than air. Therefore the weight of the tube filled with hydrogen would be appreciably less than if it is filled with air. The increase in weight is the weight of the water formed. The boat containing the copper is also weighed. The loss in weight is the weight of oxygen contained

1 Most substances, esprecially if porous or in the form of powder, absorb more or less moisture from the air. 
in the water formed. The results of an actual experiment were as follows:

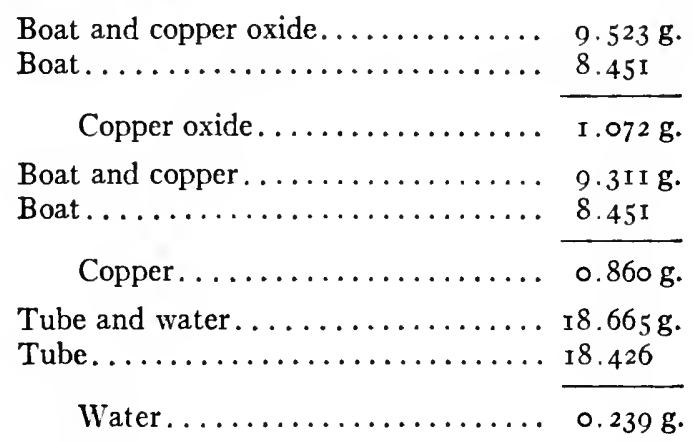

Since $\mathrm{I} .072 \mathrm{~g} .-0.860 \mathrm{~g} .=0.2 \mathrm{I} 2 \mathrm{~g}$., we conclude that $0.239 \mathrm{~g}$. of water was formed from $0.212 \mathrm{~g}$. of oxygen, which at the beginning was in combination with the copper in the form of copper oxide. Therefore water consists of 0.212 g. $\div 0.239$ g. $=0.887$ $=88.7$ per cent oxygen. The difference between the weight of water formed and that of the oxygen used is the weight of hydrogen, which is $0.239 \mathrm{~g}$. $-0.2 \mathrm{I} 2 \mathrm{~g} .=0.027 \mathrm{~g}$. This is readily found to be II.3 per cent of the weight of the water. Very carefully performed experiments, made in this way, show that water contains 88.8 per cent by weight of oxygen and I I. 2 per cent of hydrogen; the difference of 0.1 per cent between the values found in the lecture experiment quoted and those obtained in the most accurate experiments made by skilled chemists working with greatest care and under ideal conditions is due to the experimental errors in the rather crude lecture experiment.

38. The Percentage Composition of Copper Oxide.-It is also easy to see that we may find the percentage composition of copper oxide from the data just considered. Thus $\mathrm{I} .072 \mathrm{~g}$. of copper oxide gave $0.860 \mathrm{~g}$. of copper by loss of $0.212 \mathrm{~g}$. of oxygen; from which we find that copper oxide is composed of 80.2 per cent copper and I9.8 per cent oxygen. The most accurate experiments made in this way give 79.9 per cent copper and 20 . I per cent oxygen, the difference being due to experimental error in the lecture experiment. Pure copper oxide always has exactly the composition shown by these figures. 
39. The Percentage Composition of Carbon Dioxide.-We have found that carbon in the form of charcoal burns readily in air or in oxygen with the formation of a colorless gas called carbon dioxide. The percentage composition of carbon dioxide may be found by burning a known weight of pure carbon in oxygen gas and finding the weight of carbon dioxide formed. It will be recalled that carbon dioxide is easily absorbed by solid caustic soda. It is also readily absorbed by a solution of caustic potash in water, while neither oxygen nor air is absorbed by such a solution. If the gases formed by the burning of carbon in a stream of oxygen are passed through a suitable bulb containing caustic potash solution, all of the carbon dioxide will be retained by the solution and the oxygen will pass through unabsorbed. The increase in weight of the bulb will represent the weight of the carbon dioxide formed by the burning of the carbon.

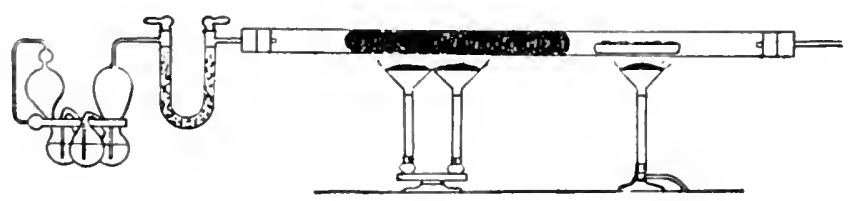

FIG. 19

The arrangement of the apparatus is shown in Fig. I9. About $0.2 \mathrm{~g}$. of pure carbon, made from sugar, is contained in a porcelain boat which is placed in a hard glass tube connected at one end with a supply of pure oxygen and at the other with a calcium chloride tube and a weighed potash bulb, which contains a 30 per cent solution of caustic potash. The middle part of the tube should contain a column of copper oxide, to insure the complete conversion of the carbon into carbon dioxide. The calcium chloride tube serves to catch any moisture present. The carbon is ignited by heating the tube with a gas burner; after the carbon has completely burned and all of the carbon dioxide formed has been driven over into the potash bulb by the stream of oxygen, a slow stream of air is blown or drawn through the apparatus to replace the oxygen by air. 'The potash bulb is then detached and weighed. In an actual lecture experiment 0. 194 g. of carbon yielded 0.70 I g. of carbon dioxide; 
from which we find that this gas contains 27.6 per cent of carbon and 72.4 per cent of oxygen.' The most accurate experiments of skilled chemists show the correct percentages to be $27.3 \mathrm{per}$ cent carbon and 72.7 per cent oxygen.

40. The Action of Sodium on Water: Caustic Soda.-It is a matter of importance to know the exact percentage composition of pure substances and a great variety of methods must be employed in the making of such determinations. It often happens that the method which would seem to be most direct and desirable is not practicable because the violence of the interaction of the elements which we bring together would cause loss of some of the material taken. This may be illustrated by an experiment with the element sodium. If we throw a piece of this metal upon water, we observe that the action is a violent one which ordinarily ends in an explosion that throws part of the substance out of the beaker in which it was contained. We may carry out the same reaction without loss of material and obtain precisely the same product if the piece of sodium is exposed to water vapor instead of being thrown upon liquid water. In this case the reaction requires much more time, but it proceeds quietly and without loss of material. The white solid so obtained is caustic soda.

4r. The Action of Hydrochloric Acid on Caustic Soda: Common Salt.-If we add to a solution of caustic soda contained in a beaker a sufficient amount of pure hydrochloric acid and evaporate the resulting solution to dryness, we find that the product is one with which we are well acquainted. It is nothing more nor less than common salt, and if the materials used are all pure the product will be chemically pure salt. We discover in this way that the metal sodium is one of the constituents of common salt. In fact, metallic sodium may be obtained by the electrolysis of molten salt, although this is not the most satisfactory method of making this metal. The percentage of sodium in salt may readily be found if the weights of sodium taken and of salt obtained are determined.

42. The Percentage of Sodium in Common Salt.-In an actual experiment $0.483 \mathrm{~g}$. of metallic sodium was weighed in a 
stoppered test tube (to prevent action of the moisture of the air). The sodium was placed on a strip of silver foil which rested on the edges of a small porcelain dish containing about io c.c. of water, and covered with a beaker. In the course of a few hours the sodium had reacted completely with the water vapor to form a solution of caustic soda which dripped into the dish. A little of the solution adhering to the foil was rinsed into the dish with a little water. Sufficient pure hydrochloric acid was then added and the solution evaporated by steam heat in the manner shown in Fig. 20. The beaker contained ordinary water. By. this mode of evaporation of the solution in the dish we avoid loss by spattering that would occur if we should boil the solution by heating the dish directly with the flame. When the salt appeared to be dry, the dish was heated very cautiously with the direct flame, to drive off the small amount of remaining water. When cold, the dish and contents were weighed. It was found in this way that $0.483 \mathrm{~g}$. of sodium gave I.2 $7 \mathrm{~g}$. of common salt, which indicated that salt

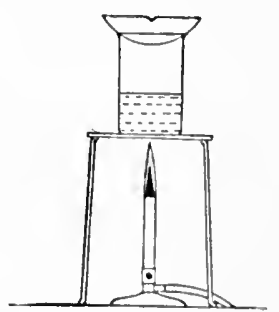

FIG. 20 contains 39.7 per cent of sodium. 'The correct result is 39.4 per cent.

43. The Electrolysis of Hydrochloric Acid: Chlorine.-It is, of course, obvious that the sodium in common salt must be combined with one or more elements and the student will readily guess that a clue to the other constituents of common salt may be gained by a knowledge of the constituents of hydrochloric acid. If we pass an electric current through a concentrated solution of hydrochloric acid contained in the apparatus shown in Fig. 2r, we find that two gaseous products are obtained, the volumes of which are practically equal. One of these is colorless. It is lighter than air and burns with a hot but non-luminous flame and in so doing yields water; these properties show the colorless gas to be hydrogen. The other gas is pale yellow in color; it is heavier than air, one liter weighing $3.22 \mathrm{~g}$., and has an exceedingly disagrecable, irritating odor. This gas is known 
as chlorine. Inasmuch as chlorine has never been separated into simpler substances, we conclude that it is an element.

44. The Union of Hydrogen and Chlorine: Hydrogen Chloride Gas.-Since the hydrochloric acid which was electrolyzed contained water, we should not be warranted in concluding that hydrogen is a constituent of hydrochloric acid;

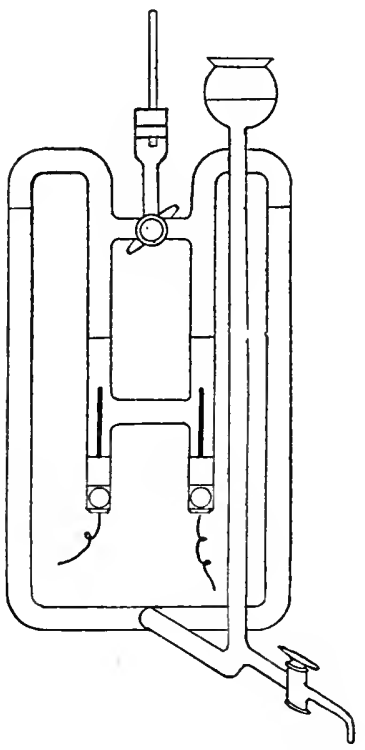

FrG. 2 I.-Brownlee's apparatus. for, as we know, hydrogen is also one of the constituents of water. If we bring together equal volumes of the gases hydrogen and chlorine and allow them to mix, and if we allow the vessel to stand in diffused light for a day or two, we notice that the yellow color of the chlorine has disappeared. We find that a colorless gas remains which dissolves with the greatest ease in water, and that neither hydrogen nor chlorine is left. Since water which has dissolved the gas has all of the properties of a solution of pure hydrochloric acid, we interpret the results as showing that equal volumes of hydrogen and chlorine gases combine to form a new gas which we call hydrogen chloride gas, and that the latter when dissolved in water constitutes hydrochloric acid. Hydrogen chloride gas may be distinguished from the other gases which we have met in several ways, notably by its marked, choking odor, by the fact that it fumes or gives a white cloud in moist air, and it dissolves with great ease in water, as well as in several other ways.

45. Salt a Compound of Sodium and Chlorine.-The fact that hydrochloric acid is known to be a compound of chlorine suggests that common salt may also contain this element. This is in fact the case. It can readily be shown by experiment that common salt results from the union of chlorine gas with metallic sodium. Inasmuch as nothing else is needed and no other 
product than salt is formed, we must conclude that salt is a compound of the elements sodium and chlorine. This fact is indicated by the chemical name of common salt, sodium chloride. Since salt contains 39.4 per cent of sodium, the percentage of chlorine must be 60.6 .

46. The Law of Definite Composition.-The preceding paragraphs of this chapter are intended to illustrate how we may arrive at a knowledge of the nature and percentage by weight of each element entering in to the composition of a pure substance. It is possible, by well-known methods, to do this for all pure substances. As a result of countless thousands of such quantitative experiments made by chemists, the conclusion has been reached that the percentage composition of every pure substance is perfectly definite for that substance and is found to be the same by whatever method we may make the determination. This is one of the most important laws of chemistry. It is usually spoken of as the Law of Definite Composition or of Definite Proportions. This explains why a pure substance always has definite properties, from whatever source it may be obtained.

47. Hydrogen and Its Gaseous Compounds. - We have already become acquainted with hydrogen and one of its gaseous compounds hydrogen chloride, a water solution of which is known as hydrochloric acid. Hydrogen forms many compounds which are gaseous at ordinary temperatures. We shall now take up a study of some of these, with the object in view, first, of discovering the nature of the other element combined with the hydrogen; secondly, of discovering the percentage composition; and, finally, of disclosing a very remarkable relation between the weights of hydrogen contained in equal volumes of these gases.

48. Hydrogen Chloride. - We have found that equal volumes of hydrogen and chlorine combined to form hydrogen chloride gas. Since we know that I liter of hydrogen weighs $0.090 \mathrm{~g}$. and that I liter of chlorine weighs $3.220 \mathrm{~g}$., we find by calculittion that hydrogen chloride contains 2.76 per cent by weight of hydrogen. By direct weighing of pure hydrogen chloride gas it is found that I liter weighs $1.642 \mathrm{~g}$. Since 2.76 per cent of I. $642 \mathrm{~g}$. is $0.045 \mathrm{~g}$., it follows that I liter of hydrogen chloride 
gas contains $0.045 \mathrm{~g}$. of combined hydrogen. It has already been stated that I liter of hydrogen gas weighs $0.090 \mathrm{~g}$., which weight we see is exactly double the weight of hydrogen in I liter of hydrogen chloride gas.

49. Acetylene: a Compound of Carbon and Hydrogen.Let us next consider the gas acetylene which is extensively used for illumination. This gas is obtained by allowing water to drop on calcium carbide. We find that it is a colorless gas with a peculiar odor. Everyone knows that it burns in air, giving an exceedingly bright flame. If we collect and test the products coming from the acetylene flame we find carbon dioxide and water. We find the same products and no others when acetylene is burned in pure oxygen gas, and therefore conclude that carbon

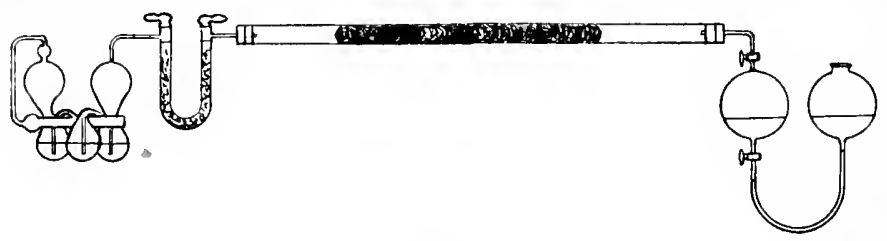

FIG. 22

and hydrogen are constituents of acetylene; but the experiment obviously does not decide whether oxygen is or is not also a constituent of acetylene. This question could be decided if we knew the percentages of carbon and hydrogen in the gas.

50. The Analysis of Acetylene.-We may find the percentages of carbon and hydrogen by means of the following experiment. A tube of hard glass a centimeter or more in diameter and $30 \mathrm{~cm}$. long (Fig. 22) is partly filled with pure dry copper oxide. The tube is then heated red hot and a measured volume of acetylene at a known temperature and pressure is caused to pass through the tube and over the heated copper oxide. It is found that carbon dioxide and water are formed and that part of the copper oxide is changed into metallic copper. A U-tube filled with calcium chloride, for the absorption of the water formed, is attached to the exit of the hard glass tube. Beyond this, attached by rubber tubing, we have a bulb containing caustic potash solution to absorb the carbon dioxide. After 
all of the acetylene has been driven over into the combustion tube holding the copper oxide, by allowing mercury from the attached reservoir slowly to displace the acetylene, a slow stream of pure dry oxygen is passed into the combustion tube to insure the complete burning of the carbon of the acetylene. Finally, the oxygen is displaced by a stream of air.

The increase in weight of the calcium chloride tube represents the weight of water formed. Similarly the increase in weight of the caustic potash bulb represents the weight of carbon dioxide obtained. Now we know that water contains I I. 2 per cent of hydrogen and that carbon dioxide contains 27.3 per cent of carbon. We may then calculate the weights of hydrogen and carbon corresponding to the weights of water and carbon dioxide obtained. If we know that $\mathrm{I}$ liter of acetylene under standard conditions, that is, at $0^{\circ}$ and $76 \mathrm{~cm}$. $P$, weighs $I .190 \mathrm{~g}$., we have all the data needed to enable us to calculate the percentages of hydrogen and carbon in acetylene. In an actual lecture experiment 200 c.c. of pure dry acetylene at $18^{\circ}$ and $75.4 \mathrm{~cm}$. gave $0.150 \mathrm{~g}$. of water and $0.75 \mathrm{I} \mathrm{g}$. of carbon dioxide. From the data above we find that the weight of the acetylene taken was $0.222 \mathrm{~g}$, and that the weights of hydrogen and carbon contained in the water and carbon dioxide respectively were $0.0168 \mathrm{~g}$. and 0.205 g., respectively. Therefore acetylene contains (according to this analysis) 7.5 per cent of hydrogen and 92.3 per cent of carbon. The correct percentages are 7.7 and 92.3 respectively; and since the sum of these percentages is 100, we know that hydrogen and carbon are the only elements contained in acetylene. We may also calculate from the same data the weight of combined hydrogen in one liter of acetylene under standard conditions. We find in this way $0.090 \mathrm{~g}$. of hydrogen.

5I. Ammonia.-Let us next take up the study of ammonia. Common household ammonia, which is familiar to everyone, is a solution in water of the substance, ammonia, which is a gas at ordinary temperature and pressure. If we warm such a solution of ammonia, a gas having an intense odor is given off. When this gas, ammonia, is strongly compressed, it condenses to a colorless liquid which we speak of as liquid ammonia. This is 
a commercial article which is shipped in heavy steel cylinders six feet long and a foot in diameter. The liquid ammonia exists under considerable pressure in such cylinders. If the valve of the cylinder is opened gaseous ammonia escapes. We may use a small cylinder of liquid ammonia as a convenient source of ammonia gas.

If we fill a glass cylinder with mercury, invert it in a dish of mercury, and allow ammc: a gas to escape under the mouth of the cylinder, the mercury is displaced by the ammonia gas. We notice that the gas is invisible, like air. It is to be distinguished from air, however, by its intense odor, as well as in other ways. If we dip the mouth of the cylinder, which has been closed by a glass plate, into a vessel of water, we find that the water rushes into the cylinder almost as readily as if the space were a vacuum. An examination of the water now shows that it has new properties. The water now has the odor of ammonia, it has a peculiar disagreeable taste, and changes the color of immersed red litmus paper blue. If we bring a burning candle into a cylinder of ammonia the flame of the candle is extinguished but the ammonia does not take fire. These properties distinguish ammonia from oxygen, hydrogen, and acetylene.

52. Ammonia a Compound of Nitrogen and Hydrogen.-We may now inquire, What is the chemical composition of ammonia? Is it an elementary substance or a compound, and, if a compound, of what elements is it composed? If ammonia gas is passed through a heated glass tube containing copper oxide we observe that a colorless liquid condenses in the cold part of the tube. This liquid proves to be water. We find also that a colorless, odorless gas is formed. If we pass this gas into limewater we observe no result and conclude, therefore, that this gas is not carbon dioxide. We find that the gas is not appreciably soluble in water, so that it cannot be unchanged ammonia gas. If we test the gas with a burning candle we find that it neither burns nor supports combustion. The student will doubtless recall (Io) that this gas has just those properties which the portion of the air left after the removal of oxygen by mercury or phosphorus possesses. It would seem, therefore, to be nitrogen. 
The identity of the gas with nitrogen is confirmed by a determination of the density; whereupon it is found that a liter weighs I.25I g. Since water and copper were formed from ammonia and copper oxide, we conclude that ammonia has furnished the hydrogen which united with the oxygen supplied by the copper oxide to form the water obtained in the preceding experiment. Ammonia must be a compound containing nitrogen and hydrogen. It has been shown in many ways by experiments, which we need not consider at present, that nitrogen and hydrogen are the only constituents of ammonia.

53. The Percentage Composition of Ammonia.-The percentage of hydrogen in ammonia may be found by carrying out the experiment above described with a known volume of ammonia measured at a known temperature and pressure. If we cause the ammonia to pass through the heated copper oxide tube, driving out water vapor completely by means of air after all of the ammonia has passed into the tube, and if the products are caused to pass through a calcium chloride tube connected to the copper oxide tube as in the determination of the composition of acetylene, the increase in weight of the calcium chloride tube gives us the weight of water formed from the hydrogen of the ammonia used. Knowing as we do the percentage of hydrogen in water, if we know the weight of a liter of ammonia gas $(0.772 \mathrm{~g}$.) we may calculate the percentage of hydrogen in ammonia and also the weight of combined hydrogen in I liter of ammonia gas measured under standard conditions. We find this latter weight to be $0.135 \mathrm{~g}$.

54. Methane, Another Compound of Carbon and Hydrogen.-The chief component of natural gas is a substance called methane. This same gas methane often escapes in bubbles when the decaying vegetable matter in marshes is disturbed. For this reason methane is also known as marsh gas. We may prepare methane artificially in the laboratory by methods which we need not now discuss. It may be collected over water, as its solubility in water is slight. We note that it is a colorless gas, that it is lighter than air, since the gas will escape rapidly from an open cylinder when the mouth of the cylinder is turned upward, 
but will not escape if the mouth is downward. One liter of methane weighs $0.72 \mathrm{I}$ g., which is but little more than half of the weight of the same volume of air. If we bring a lighted candle into a cylinder of methane we find that the gas burns with a slightly luminous flame but that the candle flame is extinguished.

55. The Quantitative Analysis of Methane.-If we examine the products of combustion from a methane flame we find water and carbon dioxide, from which we know that methane is a compound of carbon and hydrogen with or without other elements. We may determine the quantitative composition of methane by precisely the same method as that used for the quantitative analysis of acetylene, whereupon we find that methane contains 75. 0 per cent of carbon and 25.0 per cent of hydrogen by weight. Since the sum of these percentages is Ioo we know that methane must contain only the elements carbon and hydrogen. From the data obtained in the analysis of methane we may also calculate that I liter of methane under standard conditions contains o. I80 g. of combined hydrogen.

56. The Weight of Hydrogen in One Liter of Gaseous Hydrogen Compounds.-By a study of the composition of the four gases, hydrogen chloride, acetylene, ammonia, and methane, as well as of hydrogen itself, we have found the weight of hydrogen in I liter of each. These results may now be tabulated as in Table II. An inspection of the results given in the table reveals

TABLE II

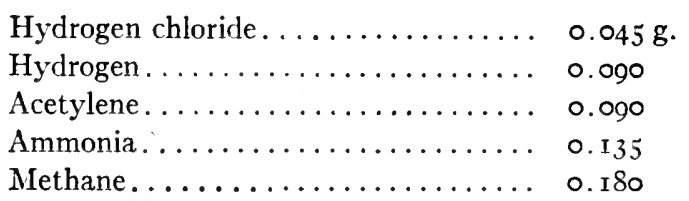

a remarkable fact. The weight of hydrogen in I liter of hydrogen chloride is less than that in any other case. The weight per liter of hydrogen gas itself is double the weight of hydrogen in I liter of hydrogen chloride. Likewise the weight of hydrogen in I liter of acetylene is exactly equal to the weight of a liter of free hydrogen and 
also donble the weight of hydrogen in I liter of hydrogen chloride. The a'eight of hydrogen in I liter of ammonia is three times that in I liter of hydrogen chloride, while in the case of methane the weight of hydrogen per liter is four times the weight of this element in the sume iolume of hydrogen chloride.

If we consider the weight of hydrogen in a liter of hydrogen chloride as unity, we find that the weights in the same columes of the other gases are expressed by the numbers 2,3 , or 4 . It is obvious that the relations we discussed would also hold equally well if we dealt with weights of hydrogen contained in any other fixed volume, as a cubic foot or a cubic meter. We could express the facts by saying that the weight of hydrogen contuined in a fixed zolume of any of these gases is in each case a multiple of the mini$m u m$ areight, which is found in the case of hydrogen chloride gas. Since I liter of hydrogen chloride gas contains $0.045 \mathrm{~g}$. of hydrogen, I g. of combined hydrogen would be contained in $\mathbf{2 2 . 4}$ liters ${ }^{\mathrm{I}}$ of hydrogen chloride. In the same solume of the other gases the weights of hydrogen would be $2 \mathrm{~g}$., $3 \mathrm{~g}$., or $4 \mathrm{~g}$.

I In reality $1 \div 0.045$ gives 22.2 instead of the correct value 22.4 liters. The discrepancy is caused by the fact that the numbers used are only approximate. This subject is discussed further in section 222 . 


\section{CHAPTER V}

\section{SYMBOLS AND CHEMICAL FORMULAE}

57. Gaseous Carbon Compounds.-We may now inquire whether the remarkable relations between the weights of hydrogen in equal volumes of compounds of hydrogen hold good in the case of compounds of other elements. We have already studied three gaseous compounds of carbon: carbon dioxide, acetylene, and methane, and have seen how the percentage composition of each is determined. Before discussing the results so obtained, let us consider two new gaseous compounds of carbon: propane and trimethylamine.

58. Propane: a Compound of Carbon and Hydrogen.-Propane is found in small amounts in the natural gas of some wells and also dissolved, in small quantities, in crude petroleum. It may also be obtained artificially by methods well known to the chemist, the nature of which we need not now consider. We observe that propane is a colorless, odorless gas which is somewhat heavier than air, I liter under standard conditions weighing I.97 g. We find that propane resembles methane in its chemical behavior, since it extinguishes a burning candle but takes fire itself at the same time, burning with a slightly luminous flame and yielding carbon dioxide and water as the only products of combustion. The analysis of propane may be carried out in precisely the same manner as our analysis of methane and acetylene. We find in this way that propane contains $8 \mathrm{I} .8$ per cent of carbon and 18.2 per cent of hydrogen. Since the sum of these percentages is 100 , it follows that carbon and hydrogen are the only constituents of propane.

59. Trimethylamine: a Compound of Carbon, Hydrogen, and Nitrogen.-Trimethylamine is a colorless gas about twice as heavy as air, I liter weighing $2.65 \mathrm{~g}$. Its odor is very powerful and somewhat disagreeable, but if inhaled in small quantities the gas is not poisonous nor irritating, as is, for example, chlorine gas. The odor is that of decaying fish. In fact, the gas can be 
obtained from products separated from herring brine. We find that the gas is very easily soluble in water and that the solution turns red litmus paper blue, just as ammonia does; but the gas may be distinguished from ammonia by the fact that it will burn, whereas ammonia will not. It is easy to discover that water and carbon dioxide are formed when trimethylamine is burned in air or in oxygen. If we pass trimethylamine through a tube containing heated copper oxide we obtain, in addition to water and carbon dioxide, a colorless, odorless, incombustible gas which can easily be identified as nitrogen. These facts show that trimethylamine contains the elements carbon, hydrogen, and nitrogen. We could determine the percentages of carbon and hydrogen by finding the weights of carbon dioxide and water formed by the action of the gas on hot copper oxide, as in analyses previously made. We might also find the percentage of nitrogen by finding the volume of nitrogen which we could obtain from a known volume of the gas. The percentages of carbon, hydrogen, and nitrogen would be found to be $61.0,1_{5} \cdot 3$, and 23.7 respectively.

60. The Weights of Carbon in I Liter and in 22.4 Liters of Gaseous Carbon Compounds. - Let us now consider the facts presented in Table III. The weight of I liter and the percentage

TABLE III

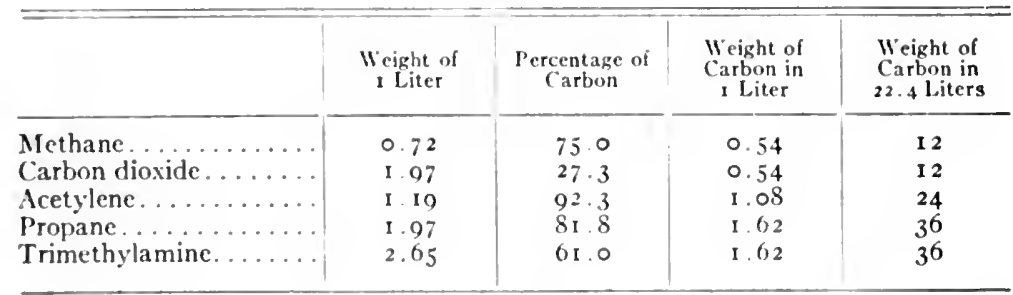

of carbon in each of the five gaseous compounds of carbon we have studied are given in the first and second columns of figures. The product of the weight of I liter of a gas by the percentage of carbon it contains gives the weight of combined carbon in I liter. These products are given in the third column. The weights of carbon in 22.4 liters, as given in the last column, are found by multiplying the corresponding weights in the third column by 22.4 . 
We see by a glance at the last column of the table that 22.4 liters of carbon dioxide and methane contain $\mathrm{I} 2 \mathrm{~g}$. of combined carbon, that the same volume of acetylene contains $24 \mathrm{~g}$. of carbon, while the weight of combined carbon in 22.4 liters of propane and trimethylamine is $36 \mathrm{~g}$., and therefore that the weight of carbon in 22.4 liters of any of these gases is either one, two, or three times $12 \mathrm{~g}$. In the case of gaseous hydrogen compounds, we found that the weight of hydrogen was either one, two, three, or four times I g., which was the minimum weight of this element found in any case. We thus find that in 22.4 liters of various pure gases the minimum weight of hydrogen is I $\mathrm{g}$. and the minimum weight of carbon $12 \mathrm{~g}$., and, further, that if $a$ greater weight of either of these elements is contained in this volume of any pure gas, the weight is a multiple of the minimum weight by a small whole number.

Let us now consider the weights of carbon and hydrogen contained in 22.4 liters of the three gaseous compounds which contain only carbon and hydrogen, namely, methane, acetylene, and propane. In 22.4 liters of methane we find $12 \mathrm{~g}$. of carbon combined with $4 \mathrm{~g}$. of hydrogen. In the same volume of acetylene, $24 \mathrm{~g}$. of carbon combined with $2 \mathrm{~g}$. of hydrogen, and in the case of propane $36 \mathrm{~g}$. of carbon combined with $8 \mathrm{~g}$. of hydrogen. Without considering at present the theoretical significance of the remarkable facts which these figures show, we may consider a practical application of the facts which will enable us to express the composition of these gases in a simple fashion.

The student must realize that since we have three compounds all consisting of carbon and hydrogen and having different properties, the difference in percentage composition must be an important factor in determining the properties of the substance. $\mathrm{He}$ will also understand that a knowledge of the percentage composition is a matter of prime importance for the chemist, and that any scheme by means of which a knowledge of the composition by weight could be easily memorized would be important.

6r. Symbols.-Suppose we represent $\mathrm{I}$ g. of hydrogen by a sign or symbol and choose the letter $\mathrm{H}$ for this purpose. We could, then, represent by $\mathrm{H}$ taken four times the weight of hydro-: 
gen contained in 22.4 liters of methane; by $\mathrm{H}$ taken twice, or $2 \mathrm{H}$, the amount of hydrogen in 22.4 liters of acetylene; and similarly by $8 \mathrm{H}$, the amount of hydrogen in 22.4 liters of propane. Suppose that, on the other hand, we represent $\mathrm{I} 2 \mathrm{~g}$. of carbon by the sign or symbol $\mathrm{C}$, then $\mathrm{C},{ }_{2} \mathrm{C}$, and ${ }_{3} \mathrm{C}$ will represent the weights of carbon in 22.4 liters of methane, acetylene, and propane respectively. The weights of carbon and hydrogen in 22.7 liters of methane may then be represented by wriling ${ }_{I} C$ logelher with ${ }_{4} H$. As a matter of convenience the multiples I for the $\mathrm{C}$ and 4 for the $\mathrm{H}$, are written as subscripts; so that instead of ${ }_{1} \mathrm{C}$ and ${ }_{4} \mathrm{H}$ we write $\mathrm{C}_{1} \mathrm{H}_{4}$. In practice no subscript is used when the multiple is $\mathrm{I}$. The composition of methane is represented simply by $\mathrm{CH}_{4}$.

62. Chemical Formulae.-In a similar way we may represent the weights of carbon and hydrogen in 22.4 liters of acetylene by $\mathrm{C}_{2} \mathrm{H}_{2}$ while the composition of the same volume of propane may' be represented by $\mathrm{C}_{3} \mathrm{H}_{8}$. We call $H$ the symbol for hydrogen, and for the present we may consider that $\mathrm{H}$ or $\mathrm{IH}$ represents $\mathrm{I} g$. of hydrogen and similarly that $\mathrm{C}$, the symbol for carbon, represents $12 \mathrm{~g}$. of that element. We call the expressions $\mathrm{CH}_{4}, \mathrm{C}_{2} \mathrm{H}_{2}$, and $\mathrm{C}_{3} \mathrm{H}_{8}$ the formulae of methane, acetylene, and propane respectively. We shall now proceed to show how this system may be extended to all gaseous compounds of any element whatever.

Chemists are familiar with a large number of gases in addition to those which we have already studied. Some of these are of much practical importance while others are chiefly of interest to the chemist for scientific reasons. In every case it is a simple matter to determine the weight of I liter of the gas under standard conditions, the method of making the determination being essentially the same in all cases. Furthermore, by methods which are well known to chemists we may determine what elements compose any gas, and by means of a quantitative analysis we may determine the percentage of each element in the gas. If we calculate in the case of each gas the weight of each element contained in 22.4 liters of the gas, we obtain results like those shown in Table IV. 
63. The Minimum Weights of Oxygen, Nitrogen, and Chlorine.-An inspection of the results given in Table IV shows that the same regularity in the weights of hydrogen and carbon holds in all cases, as we have observed it to hold in the few cases discussed in the preceding paragraphs. We notice also that the minimum weight of oxygen in 22.4 liters of any of its gaseous

TABLE IV

Weights of Constituents in 22.4 Liters of Gases

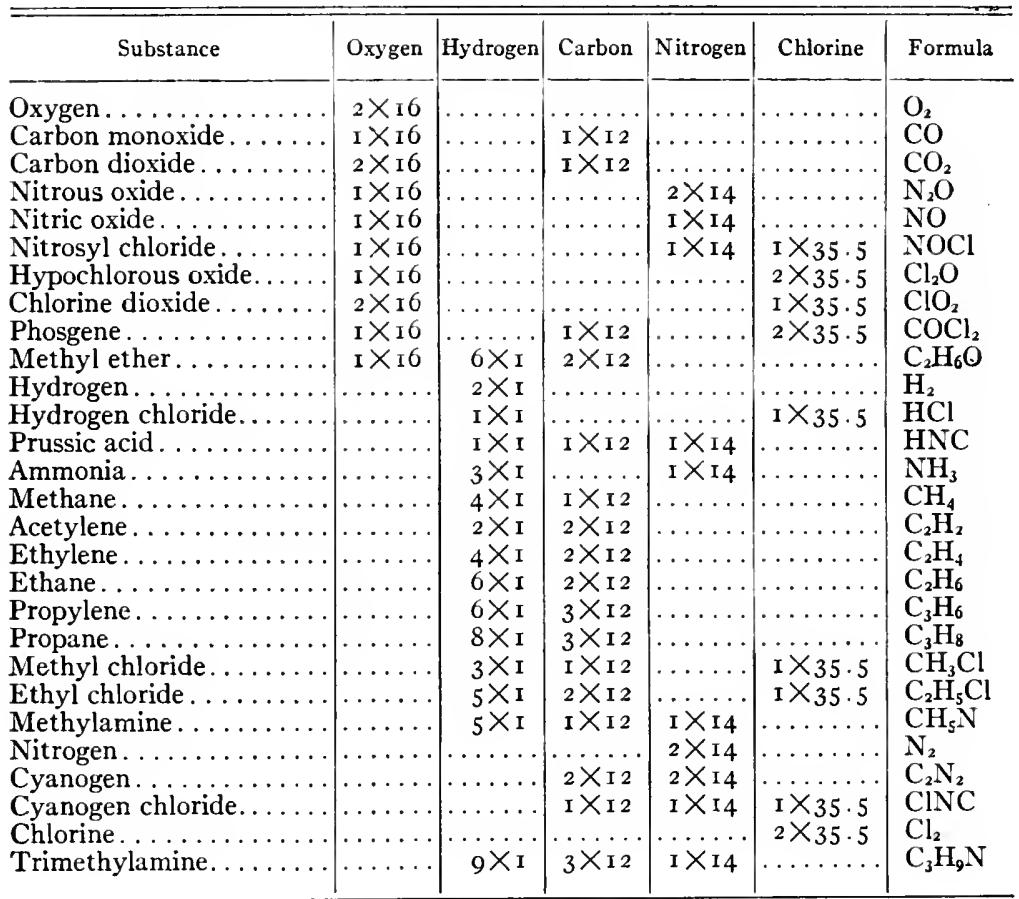

compounds is $\mathrm{r} 6 \mathrm{~g}$., and that this weight is found in many cases, while in others the weight is twice 16 . In the case of the compounds of nitrogen we note that the minimum weight is $14 \mathrm{~g}$. and that in other cases the weight is double this minimum weight. In the case of chlorine compounds the minimum weight of chlorine is $35.5 \mathrm{~g}$., while those compounds with a larger proportion of chlorine contain double the minimum weight. 
64. The Law of Minimum and Multiple Weights.-Entirely analogous regularities will be found if we consider the data obtained from a study of the gaseous compounds of any other elements. For each element we find a minimum weight in the zolume of 22.4 liters of any of its gaseous compounds under standard conditions and also find that the weight if greater than the minimum would be 2,3, or 4, or some small multiple of this minimum. This last statement may be called the Law of Minimum and Multiple Weights.

65. The Chemical Unit Volume: 22.4 Liters. - The volume 22. 4 liters thus becomes a kind of unit volume for the chemist, this particular volume having been chosen because it contains I g. of hydrogen in the case of those hydrogen compounds which contain the minimum weight of this element. In this volume no other element has a minimum weight as small as that of hydrogen.

66. Symbols Represent Minimum Weights.-In the same manner as that suggested in a preceding paragraph for hydrogen and carbon, we may represent the minimum weight of each of the other elements by a symbol. Table $\mathrm{V}$ shows the minimum weights of the five elements we have been considering, together with the corresponding symbols.

TABLE $\mathrm{V}$

Minime Weights in 22.4 Liters, and Symbols

\begin{tabular}{|c|c|c|}
\hline Hydrogen . . . . . . . . . . & I. O g. & II \\
\hline 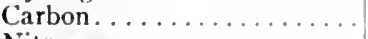 & 12.0 & C \\
\hline Nitrogen ................ & 14.0 & $N$ \\
\hline 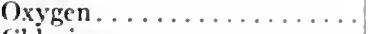 & 16.0 & 0 \\
\hline 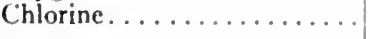 & $35 \cdot 5$ & $\mathrm{Cl}$ \\
\hline
\end{tabular}

67. Making Formulae.-We see from Table IV that 22.4 liters of carbon dioxide contain $12 \mathrm{~g}$. of carbon combined with $2 \times 16$ g. of oxygen. We may, therefore, represent the composition of the quantity of carbon dioxide in 22.4 liters by the formula $\mathrm{CO}_{2}$. In an analogous fashion we may obtain as the formula representing the composition of 22.4 liters of ammonia, $\mathrm{NH}_{3}$, and as the formula for hydrogen chloride, $\mathrm{HCl}$. By making 
use of this system the student will now have no difficulty in writing down at once the formula of each of the gases from the data contained in Table IV. He will also readily see that it is a much less difficult task to learn the formulae of such gases than to learn their percentage composition; that is to say, it is an easier tax upon the mind to remember the formula $\mathrm{HCl}$ than to remember that hydrogen chloride contains 2.76 per cent of hydrogen and 97.24 per cent of chlorine.

68. The Practical Use of Formulae.-A review of the methods employed in arriving at the results represented by the formula of any substance shows that in each case we have made use of the knowledge, first, of the weight of I liter of the gas, and, secondly, of the percentage of each of its elementary constituents. Conversely, if we know the weights which the symbols of the elements represent, and know the formula of a gas, we may by working backward find its percentage composition. For example, suppose that we remember that the formula of methane is $\mathrm{CH}_{4}$ and know that $\mathrm{H}$ stands for $\mathrm{I} g$. of hydrogen and $\mathrm{C}$ for $\mathrm{I} 2 \mathrm{~g}$. of carbon. Then 22.4 liters of methane contain $4 \mathrm{~g}$. hydrogen combined with I $2 \mathrm{~g}$. of carbon. The proportion of hydrogen is, therefore, $4 / 16$, or 25 per cent, and of carbon $12 / 16$, or 75 per cent, the weight of 22.4 liters being I 6 g., and I liter weighs $16 / 22.4=$ $0.72 \mathrm{~g}$. In calculating in this way the density and percentage composition of methane we are merely reproducing the results which originally were obtained by experiment. In order to find the formula of any gas, we must know its density and the percentage of each elementary constituent. We find by actual experience that we can represent by a formula, usually of a very simple character, the composition of 22.4 liters of any gaseous substance.

69. Formulae of Liquids and Solids. - The system which we have just considered is capable of extension to liquid and solid substances, in which case, however, the formula may have a slightly less definite meaning. We may illustrate this by considering the cases of water and mercury oxide. We have found that water is composed of II.2 per cent of hydrogen and 88.8 per cent of oxygen, from which we observe that the weight of 
the oxygen is 8 times the weight of the hydrogen with which it is united. This ratio of hydrogen and oxygen might be represented by $\mathrm{H}_{2} \mathrm{O}$, since this formula would mean that $2 \mathrm{~g}$. of hydrogen are united with $16 \mathrm{~g}$. of oxygen, which weights of hydrogen and oxygen are in the ratio of $\mathrm{I}$ to 8 , but the formulae $\mathrm{H}_{4} \mathrm{O}_{2}$ and $\mathrm{H}_{6} \mathrm{O}_{3}$ would also represent equally well the proportion of hydrogen and oxygen actually found in water.

70. The Formula of Water.-We may be led to choose a consistent formula for water by the consideration of the density of water vapor or steam; but in this case the density determination must be made at a temperature above the boiling-point of water, if we work at atmospheric pressure. Since the effect of changes of pressure and temperature upon the volume of a given quantity of steam are the same as upon an equal volume of any gas which would not liquify if cooled to $0^{\circ}$ at $76 \mathrm{~cm}$. pressure, we might calculate by the laws of Boyle and Charles what the volume of the known weight of steam measured at a high temperature and known pressure would be if the steam were under standard conditions, that is, at $0^{\circ}$ and $76 \mathrm{~cm}$. pressure. It has been found in this way that I liter of water vapor if it did not condense to a liquid would weigh $0.806 \mathrm{~g}$. under standard conditions, which corresponds to a weight of $18 \mathrm{~g}$. for 22.4 liters. Now, I I.2 per cent of $\mathrm{I} 8 \mathrm{~g}$. is $2 \mathrm{~g}$. and 88.8 per cent of $\mathrm{I} 8 \mathrm{~g}$. is $\mathrm{I} 6 \mathrm{~g}$. From these results we conclude that if water vapor could exist under standard conditions as a gas that 22.4 liters would contain $2 \mathrm{~g}$. of combined hydrogen and $\mathrm{I} 6 \mathrm{~g}$. of combined oxygen, which amounts would be exactly represented by the formula $\mathrm{H}_{2} \mathrm{O}$.

71. Formulae of Volatile Liquids and Solids. - In a perfectly analogous fashion we could find the formula for any other volatile substance, the density of whose vapor we could measure experimentally. Such a procedure would enable us to represent by a formula the composition of a great number of volatile chemical substances which are not gaseous, but are liquid or solid under ordinary conditions of temperature and pressure.

72. Formulae of Involatile Substances. - There are, however, many chemical substances which are not volatile or which cannot be volatilized at temperatures at which we could make 
experimental determinations of their vapor densities. There are other solids and liquids which would be decomposed if strongly heated. For such substances we could not find chemical formulae in the same way as for gases or volatile substances. However, we can and do represent by formulae the composition of such involatile substances.

73. The Formula of Red Oxide of Mercury.-The method of obtaining the formula of such a substance may be illustrated by the case of the red oxide of mercury, which, it will be remembered, is readily decomposed when heated into mercury and oxygen. We find by analysis that this compound contains 92.6 per cent of mercury and 7.4 per cent of oxygen. By the experimental study of volatile mercury compounds, as well as of mercury itself, we find that the minimum weight of mercury in 22.4 liters is $200 \mathrm{~g}$., and therefore represent this weight of mercury by the symbol $\mathrm{Hg}$. It now remains to discover what multiples of 200 for the mercury and of 16 for the oxygen are in the same ratio as the percentages of mercury and oxygen in mercury oxide. We find very easily that 200 is to $I 6$ as 92.6 is to 7.4 , and from this we write the formula $\mathrm{HgO}$.

We could of course represent the same proportions of mercury and oxygen by the formula $\mathrm{Hg}_{2} \mathrm{O}_{2}$. But we are not able to decide which of these to choose as in the case of a volatile substance where the formula represents the quantity of material in 22.4 liters of the gas or vapor under standard conditions. In such a case we choose the simpler formula, in this case $\mathrm{HgO}$, but we must bear in mind that the formula does not mean quite as much in such a case as in that of a gas or volatile substance, where it always represents in addition to the true proportion of the constituent elements the actual weights of each in 22.4 liters of the gas under standard conditions.

74. Symbol Weights and Formula Weights.-The letter or pair of letters which represents the minimum weight of an element in 22.4 liters of any of its gaseous compounds is called the symbol of that element and the weight which this symbol represents may then be called the symbol weight. Each of the eighty-five or more known elements has been assigned a definite 
symbol which represents a definite symbol weight. We have seen (62) how the quantities of each element in 22.4 liters of a compound gas may be represented by a formula made up of symbols, each symbol being multiplied by a factor which shows how many times the minimum weight of the element is present in 22.4 liters of the gaseous compound. The sum of the weights represented by the various symbols each multiplied by its factor is naturally the weight of 22.4 liters of the gas, represented by the formula. This weight is often spoken of as the formula weight. In the case of an involatile solid substance the formula weight is the weight represented by the formula but indicates only theoretically the weight which we should expect 22.4 liters of the substance to have if it were a gas under standard conditions.

75. The Formulae of Some Elementary Gases.-It is important to note that 22.4 liters of the gases hydrogen, oxygen, nitrogen, and chlorine weigh $2,32,28$, and $7 \mathrm{I}$ g. respectively (63, Table IV). These weights are for each element just double the minimum weights which we find in numerous compounds of the elements and therefore in each case just double the weight represented by the symbol. We must therefore write, as the formulae of these gases, $\mathrm{H}_{2}, \mathrm{O}_{2}, \mathrm{~N}_{2}$, and $\mathrm{Cl}_{2}$, respectively. The formula of an elementary gas in the free state will then represent the quantity of that gas in 22.4 liters. We must here point out that not every element in the form of gas or vapor is to be represented by a formula composed of its symbol taken twice. For example, the vapors of mercury and sodium have the single symbol formulae $\mathrm{Hg}$ and $\mathrm{Na}$, respectively; on the other hand, the formulae of the vapors of the elements phosphorus and sulfur are $\mathrm{P}_{4}$ and $\mathrm{S}_{8}$. 


\section{CHAPTER VI \\ CHEMICAL EQUATIONS}

76. Equations. - In this chapter we shall see how it is possible to represent in a very simple way the quantities of substances entering into and formed in a chemical reaction. Let us consider the case of hydrogen and chlorine which has already been studied experimentally. We have learned that hydrogen and chlorine unite to form hydrogen chloride (44). Furthermore we find by experiment that one volume of hydrogen and one volume of chlorine give two volumes of hydrogen chloride; so that if 22.4 liters of hydrogen united with 22.4 liters of chlorine we should obtain 44.8 liters of hydrogen chloride. Now we may represent 22.4 liters of hydrogen by the formula $\mathrm{H}_{2}$ and 22.4 liters of chlorine by $\mathrm{Cl}_{2}$, while for twice 22.4 liters of hydrogen chloride we put the coefficient, 2 , in front of the formula and write $2 \mathrm{HCl}$. We may then express the facts by stating that $\mathrm{H}_{2}$ plus $\mathrm{Cl}_{2}$ gives $2 \mathrm{HCl}$ or

$$
\mathrm{H}_{2}+\mathrm{Cl}_{2} \rightarrow{ }_{2} \mathrm{HCl} \text {. }
$$

which may also be written

$$
\mathrm{H}_{2}+\mathrm{Cl}_{2}={ }_{2} \mathrm{HCl} \text {. }
$$

We call either of these expressions the equation for the reaction between hydrogen and chlorine.

77. What an Equation Means.-The equation

$$
\mathrm{H}_{2}+\mathrm{Cl}_{2} \rightarrow{ }_{2} \mathrm{HCl}
$$

expresses the fact that the quantity of hydrogen represented by the formula $\mathrm{H}_{2}$ or $2 \mathrm{~g}$. unites with the quantity of chlorine: represented by $\mathrm{Cl}_{2}$ or $7 \mathrm{I} \mathrm{g}$. to give the quantity of hydrogen chloride represented by $2 \mathrm{HCl}$ or $73 \mathrm{~g}$. It also expresses the fact that 22.4 liters of hydrogen unite with 22.4 liters of chlorine to give $2 \times 22.4$ liters of hydrogen chloride, or in general that one volume of hydrogen and one volume of chlorine unite to give two volumes of hydrogen chloride, the volumes being those of 
the gases measured in all cases under standard conditions. In reactions involving gases the volume of each gas taken or formed is always shown by the coefficient in front of its formula in the equation for the reaction.

78. The Equation for the Burning of Carbon.-Some free elements like carbon are not sufficiently volatile to enable us to find the formula of the element from measurements of the vapor density of the free element, and in such a case we use the symbol of the element in writing equations involving its reactions. When carbon is burned we find that $12 \mathrm{~g}$. of carbon require $32 \mathrm{~g}$. of oxygen occupying a volume of 22.4 liters, and producing $44 \mathrm{~g}$. of carbon dioxide occupying also a volume of 22.4 liters. These facts may therefore be represented by the equation

$$
\mathrm{C}+\mathrm{O}_{2} \rightarrow \mathrm{CO}_{2} \text {. }
$$

Here the equation expresses directly the weights of carbon and oxygen which unite, as well as the weight of carbon dioxide formed. At the same time it also shows that 22.4 liters of oxygen when completely combined with sufficient carbon gives 22.4 liters of carbon dioxide, but since the carbon is not in the gaseous state the equation does not indicate anything regarding the volume of the solid carbon which unites with the volume of oxygen represented by the formula $\mathrm{O}_{2}$.

79. Solving Problems.-If we remember that the equation for the burning of carbon in oxygen is

$$
\mathrm{C}+\mathrm{O}_{2} \rightarrow \mathrm{CO}_{2}
$$

we may make use of the facts represented by the equation in the solution of problems such as the following: How many liters of oxygen are required for the burning of $5 \mathrm{~g}$. of carbon? To solve this problem we first write down the equation which represents the reaction. 'This shows that the quantity of carbon represented by the symbol C, namely, 2 g., requires for its combustion the volume of oxygen represented by the formula $\mathrm{O}_{2}$, namely, 22.4 liters. Therefore $5 \mathrm{~g}$. of carbon would require the volume determined by the proportion 
where $x$ is the number of liters of oxygen necessary for the combustion of $5 \mathrm{~g}$. of carbon. In an analogous manner we may calculate what volume of carbon dioxide is produced by the burning of a known weight of carbon.

We may also calculate what weight of oxygen is required or carbon dioxide produced in the burning of $5 \mathrm{~g}$. of carbon. If I $2 \mathrm{~g}$. of carbon require $32 \mathrm{~g}$. of oxygen, as our equation indicates, then we have only to solve the following proportions in order to find the weight of oxygen required for $5 \mathrm{~g}$. of carbon:

$$
\text { I } 2: 5:: 32: y \text {, }
$$

where $y$ is the required answer.

8o. The Burning of Magnesium.--Suppose we desire to find by experiment the formula of the product formed by burning magnesium in oxygen. It will be recalled that the metal magnesium in the form of wire or powder burns with great ease in oxygen, forming a white solid substance which we have called magnesium oxide (28). We find by experiment that ro g. of magnesium when burned yields $16.6 \mathrm{~g}$. of magnesium oxide. Let us suppose that we have discovered by careful experiment that magnesium oxide contains only the elements magnesium and oxygen. The difference between the weight of the magnesium oxide formed and the magnesium taken must represent the weight of oxygen which has combined with the $10 \mathrm{~g}$. of magnesium. This we find to be $6.6 \mathrm{~g}$.

Suppose we know that the symbol weight of magnesium is $24.3 \mathrm{~g}$. or

$$
\mathrm{Mg}=24 \cdot 3 \mathrm{~g} .
$$

It is now required to calculate the relative numbers of symbol weights of magnesium and oxygen that unite to form magnesium oxide. We know that rog. of magnesium unite with $6.6 \mathrm{~g}$. of oxygen. We may then make the proportion

$$
\text { I0:6.6::24.3:z, }
$$

from which we find that $z=16$. Therefore $16 \mathrm{~g}$. of oxygen represented bv $\mathrm{O}$, combine with the weight of magnesium repre- 
sented by the symbol $\mathrm{Mg}$, and consequently we may represent the composition of magnesium oxide by the formula $\mathrm{MgO}$ and write the equation for the burning of magnesium thus:

$$
\mathrm{Mg}+\mathrm{O} \rightarrow \mathrm{MgO}
$$

or better

$$
2 \mathrm{Mg}+\mathrm{O}_{2} \rightarrow 2 \mathrm{MgO}
$$

the latter equation having the advantage in that it shows the volume of oxygen, 22.4 liters, as well as its weight required for the burning of the weight of magnesium represented by $2 \mathrm{Mg}$. But since both magnesium and magnesium oxide are solid involatile substances the equation does not show the volumes of these solids entering into the reaction, as it would in the case of gaseous substances.

81. The Burning of Iron.-It will be recalled (I7) that iron burns in oxygen, giving iron oxide, the formula for which we may now calculate. In an experiment in which $12.6 \mathrm{~g}$. of iron was burned the weight of iron oxide produced was $\mathrm{I} 7.4 \mathrm{~g}$., from which we find, by subtracting the weight of the iron burned, the weight of the oxygen to be $4.8 \mathrm{~g}$. 'These weights of iron and oxygen must be in the same ratio that some number of times 56 , the symbol weight of iron, is to some number of times 16 where these numbers are small integers. Dividing 12.6 by 56 we get 0.225 . Dividing 4.8 by I6 we get 0.300 . Since these numbers 0.225 and 0.300 are not equal, the formula cannot be $\mathrm{FeO}$. It will, however, readily be found that 0.225 is to 0.300 as 3 is to 4 , and therefore that $12.6: 4.8:: 3 \times 56: 4 \times 16$, which shows that the formula of the oxide of iron formed by burning iron in oxygen is $\mathrm{Fe}_{3} \mathrm{O}_{4}$. We may then write the equaltion for the burning of iron as follows:

$$
{ }_{3} \mathrm{Fe}+{ }_{2} \mathrm{O}_{2} \rightarrow \mathrm{Fe}_{3} \mathrm{O}_{4} \text {. }
$$

82. The Action of Hydrogen on Copper Oxide.-It will be remembered that we found earlier that heated copper oxide and hydrogen give metallic copper and water (33). In a quantitative experiment it was found that $2.387 \mathrm{~g}$. of copper oxide yielded I. $907 \mathrm{~g}$. of copper and $0.54 \mathrm{~g}$. of water. From the weights of 
copper and copper oxide, together with a knowledge of the fact that copper oxide is composed of copper and oxygen only, we may discover very readily that the formula of copper oxide is $\mathrm{CuO}$, knowing the symbol weight of copper to be 63.6. Furthermore, since water contains only hydrogen and oxygen and $0.54 \mathrm{~g}$. of water has been formed from $2.387-\mathrm{I} .907$ or $0.48 \mathrm{~g}$. of oxygen, the weight of hydrogen present in the $0.54 \mathrm{~g}$. of water must have been $0.06 \mathrm{~g}$. Making a calculation analogous to that made in finding the formula for iron oxide, we find that $0: 06: 0.48:: 2 \times$ $I: I \times I 6$ and that therefore the composition of water is represented by the formula $\mathrm{H}_{2} \mathrm{O}$. We may now write, as the equation for the reaction between copper oxide and hydrogen,

$$
\mathrm{CuO}+\mathrm{H}_{2} \rightarrow \mathrm{Cu}+\mathrm{H}_{2} \mathrm{O} \text {. }
$$

83. The Action of Acetylene on Copper Oxide.-From what has preceded the student will understand that in order to be able to write the equation for any reaction we must know all of the substances entering into the reaction and all of the products. In addition we must know the formula of each substance. We may illustrate the method then employed by means of reaction between acetylene and copper oxide which we have already studied.

When acetylene is passed over heated copper oxide we obtain carbon dioxide and water, while metallic copper is left behind, these three substances being the sole products of the reaction $\left(5^{0}\right)$. The formula of acetylene is $\mathrm{C}_{2} \mathrm{H}_{2}(62)$. The quantity of carbon represented by $\mathrm{C}_{2}$ would give the quantity of carbon dioxide represented by ${ }_{2} \mathrm{CO}_{2}$; and the quantity of hydrogen represented by $\mathrm{H}_{2}$ would give the quantity of water represented by $\mathrm{H}_{2} \mathrm{O}$, so that the quantities of carbon and hydrogen represented by one formula weight of acetylene $\mathrm{C}_{2} \mathrm{H}_{2}$ would yield the quantities of carbon dioxide and water represented by ${ }_{2} \mathrm{CO}_{2}+\mathrm{H}_{2} \mathrm{O}$. The quantity of oxygen contained in the quantities of carbon dioxide and water represented by ${ }_{2} \mathrm{CO}_{2}+\mathrm{H}_{2} \mathrm{O}$ is represented by ${ }_{5} \mathrm{O}$, which quantity is contained in the amount of copper oxide represented by ${ }_{5} \mathrm{CuO}$. It will thus appear that the quantity of acetylene represented by $\mathrm{C}_{2} \mathrm{H}_{2}$ will require the quantity of 
copper oxide represented by $5 \mathrm{CuO}$, and there will be produced the quantities of the three products represented by

$$
{ }_{2} \mathrm{CO}_{2}+\mathrm{H}_{2} \mathrm{O}+{ }_{5} \mathrm{Cu} \text {. }
$$

The equation is therefore

$$
\mathrm{C}_{2} \mathrm{H}_{2}+{ }_{5} \mathrm{CuO} \rightarrow{ }_{2} \mathrm{CO}_{2}+\mathrm{H}_{2} \mathrm{O}+{ }_{5} \mathrm{Cu} \text {. }
$$

84. The Action of Ammonia on Copper Oxide.-In an analogous manner we may obtain as the equation for the reaction which occurs when ammonia gas is passed over heated copper oxide, in which case water, nitrogen, and metallic copper are formed,

$$
{ }_{2} \mathrm{NH}_{3}+{ }_{3} \mathrm{CuO} \rightarrow{ }_{3} \mathrm{H}_{2} \mathrm{O}+{ }_{3} \mathrm{Cu}+\mathrm{N}_{2} .
$$

85. The Meaning of an Equation.-Since chemists make extensive use of equations, it is of fundamental importance that the student should understand exactly how equations are obtained and what they mean. In every case before the equation for the reaction can be written the reaction must have been thoroughly investigated by experiment in the manner illustrated in the preceding examples. The equation then shows at a glance what substances enter into and are formed as a result of the reaction. It also shows the composition of each of the substances concerned and the proportions in which they take part in the reaction, it being assumed in all cases that we know the weight for which the symbol of each element stands.

86. An Equation Balances.-It is one of the most fundamental facts in chemistry that in chemical change no material is destroyed but that the elements merely change their forms of combination with one another. 'This important fact, which we know as the Law of the Indestructibility of Matter, is also represented in every chemical equation. For we notice that in each equation we have on each side the same number of symbol weights of each element. Thus in the equation

$$
\mathrm{C}_{2} \mathrm{H}_{2}+{ }_{5} \mathrm{CuO} \rightarrow{ }_{2} \mathrm{CO}_{2}+\mathrm{H}_{2} \mathrm{O}+{ }_{5} \mathrm{Cu}
$$

we see that there are on each side two symbol weights of carbon, two symbol weights of hydrogen, five symbol weights of copper, 
and five symbol weights of oxygen. This fact is usually expressed by saying that the equation balances.

All of the reactions which we have studied up to this time have been thoroughly investigated by chemists and for each the reaction equation has been discovered. We may now give, in Table VI, a list of such equations for purposes of reference. It is not to be expected, however, that the student should make great effort to memorize all of these equations, although such a task would not be very difficult, for, as a little inspection will show, there are certain regularities observable which make this a less difficult task than might at first sight seem to be the case.

TABLE VI

Equations of Other Reactions Studied

$$
\begin{aligned}
2 \mathrm{Hg}+\mathrm{O}_{2} & \rightarrow{ }_{2} \mathrm{HgO} \\
2 \mathrm{H}_{2}+\mathrm{O}_{2} & \rightarrow{ }_{2} \mathrm{H}_{2} \mathrm{O} \\
2 \mathrm{Na}+{ }_{2} \mathrm{H}_{2} \mathrm{O} & \rightarrow 2 \mathrm{NaOH}+\mathrm{H}_{2} \\
\mathrm{NaOH}+\mathrm{HCl} & \rightarrow \mathrm{NaCl}+\mathrm{H}_{2} \mathrm{O} \\
2 \mathrm{Na}+\mathrm{Cl}_{2} & \rightarrow 2 \mathrm{NaCl} \\
\mathrm{Mg}+\mathrm{H}_{2} \mathrm{O} & \rightarrow \mathrm{MgO}+\mathrm{H}_{2} \\
3 \mathrm{Fe}+\mathrm{H}_{2} \mathrm{O} & \rightarrow \mathrm{Fe}_{3} \mathrm{O}_{4}+{ }_{4} \mathrm{H}_{2} \\
\mathrm{CH}_{4}+\mathrm{O}_{2} & \rightarrow \mathrm{CO}_{2}+2 \mathrm{H}_{2} \mathrm{O} \\
\mathrm{C}_{3} \mathrm{H}_{8}+\mathrm{I} O \mathrm{CuO} & \rightarrow{ }_{3} \mathrm{CO}_{2}+{ }_{4} \mathrm{H}_{2} \mathrm{O}+{ }_{10 \mathrm{Cu}}
\end{aligned}
$$

\section{Problems}

I. What weight of mercury can be obtained by the decomposition of ro g. of mercuric oxide?

2. What volume of oxygen at $0^{\circ}$ and $76 \mathrm{~cm}$. can be made from $8 \mathrm{~g}$. of mercuric oxide?

3. What weight of sodium must be acted on by water to yield 500 c.c. of hydrogen at $0^{\circ}$ and $76 \mathrm{~cm}$.?

4. What weight of common salt can be made from ro g. of metallic sodium?

5. What volume of hydrogen at $20^{\circ}$ and $72 \mathrm{~cm}$. would be formed by the action of sufficient steam on $6 \mathrm{~g}$. of magnesium?

6. What weight of copper oxide would be required for the oxidation of 200 c.c. of propane measured at $25^{\circ}$ and $74 \mathrm{~cm}$.? (See last equation of Table VI above.)

What weight of water would be formed? 


\section{CHAPTER VII \\ ACIDS, BASES, AND SALTS-I}

88. Caustic Soda or Sodium Hydroxide.-Let us now consider the chemical changes which occurred in the formation of common salt from metallic sodium, which we have already studied experimentally. It will be recalled that sodium reacted violently with water, giving hydrogen and sodium hydroxide, the reaction being represented by the equation

$$
2 \mathrm{Na}+2 \mathrm{H}_{2} \mathrm{O} \rightarrow{ }_{2} \mathrm{NaOH}+\mathrm{H}_{2} \text {. }
$$

If we repeat the experiment and evaporate the water we find that sodium hydroxide (also known as caustic soda) is left as a white solid which is readily soluble in water. This solution feels "soapy" to the fingers and if greatly diluted with water is found to have an unpleasant "soapy" taste. (It must not be tasted unless greatly diluted with water, since the concentrated solution acts powerfully on the mucous membrane.) A piece of red litmus paper is turned blue if dipped in the solution. We know many other substances which have properties similar to those of sodium hydroxide. Such substances are called bases; they also have other characteristic properties, the most important of which we may now consider.

89. Bases Neutralize Acids. - We have learned (4I) that caustic soda and hydrochloric acid (which is a solution of hydrogen chloride in water) react to give common salt. 'The equation for this reaction is

$$
\mathrm{NaOH}+\mathrm{HCl} \rightarrow \mathrm{H}_{2} \mathrm{O}+\mathrm{NaCl} \text {. }
$$

If we add more than sufficient of the acid and then evaporate the solution to dryness, the excess of hydrogen chloride will pass off with the water and nothing but pure salt, the chemical name of which is sodium chloride, will remain. If we test hydrochloric acid with blue litmus we find that the latter is turned red, even by a very dilute solution. But we find that a solution of 
pure common salt in water has no effect on either blue or red litmus: it is neutral.

90. Properties of Acids. - If we again add, drop by drop, a solution of hydrogen chloride to one of sodium hydroxide to which a few drops of a solution of litmus have been added, we find that the change of color from blue to red is produced suddenly and not gradually, a single drop being sufficient to cause the change. If we stop adding hydrogen chloride at this point we find that the solution consists only of pure salt and water (with but a minute amount of litmus). It no longer has the taste of the sodium hydroxide, but only that of salty water. A diluted solution of hydrogen chloride has a rather agreeable sour taste, reminding one of vinegar or lemon juice. Our experiment has shown that both the taste and the behavior toward litmus of sodium hydroxide and hydrogen chloride have been changed in their interaction. We say that they have neutralized each other. We know very many substances which will neutralize sodium hydroxide; all of these have a sour taste and color litmus red. We call such substances acids, the common name of hydrogen chloride solution being hydrochloric acid.

91. Another Base: Ammonium Hydroxide.-As we have already seen $(5 \mathbf{I})$, ammonia gas dissolves readily in water, giving a solution which turns litmus blue, and we are not surprised to find that it neutralizes hydrochloric acid. If we evaporate the neutralized solution we obtain a white crystalline substance, the composition of which is represented by the formula $\mathrm{NH}_{4} \mathrm{Cl}$. Since ammonia gas has the formula $\mathrm{NH}_{3}$ and hydrogen chloride the formula $\mathrm{HCl}$, we might be inclined to write the equation

$$
\mathrm{NH}_{3}+\mathrm{HCl} \rightarrow \mathrm{NH}_{4} \mathrm{Cl},
$$

and, in fact, just this reaction takes place if we bring the two gases together, a dense white cloud of the solid product being formed. However, if a very concentrated solution of ammonia in water is cooled to a very low temperature, we may obtain crystals of a substance having a composition represented by the formula $\mathrm{NH}_{4} \mathrm{OH}$ and called ammonium hydroxide. This substance is formed thus:

$\mathrm{NH}_{3}+\mathrm{H}_{2} \mathrm{O} \rightarrow \mathrm{NH}_{4} \mathrm{OH}$. 
We might think to obtain it by the evaporation of the water solution of ammonia; but instead we get only ammonia gas and water vapor. In fact, the crystals of ammonium hydroxide obtained at a low temperature undergo a similar change if they are not kept very cold. We say that ammonium hydroxide dissociates readily into ammonia and water. Chemists think that in a water solution of ammonia part of the latter is combined with water to form ammonium hydroxide. It is this substance which is thought to act directly on red litmus, changing it to blue, and to act on hydrochloric acid as follows:

$$
\mathrm{NH}_{4} \mathrm{OH}+\mathrm{HCl} \rightarrow \mathrm{NH}_{4} \mathrm{Cl}+\mathrm{H}_{2} \mathrm{O} .
$$

We thercfore call ammonium hydroxide a base.

92. Ammonium Chloride, Salts. - The substance $\mathrm{NH}_{4} \mathrm{Cl}$ is called ammonium chloride. In appearance, taste, and other properties to be studied later, sodium chloride and ammonium chloride closely resemble one another. They are examples of an important class of chemical substances called salts.

A review of the two neutralizations just discussed will show that they have much in common: in each case a base reacls wilh an acid to form a sall and water. Somewhat later, other important facts regarding neutralization will be discovered. Before discussing such matters we will first become acquainted with a few other important acids, bases, and salts.

93. Sulfuric Acid.-One of the most important, if not the most important, of all acids is a substance which is known as oil of vitriol or sulfuric acid. It is manufactured in immense quantities and is very cheap, the commercial grade selling for less than one cent a pound. We shall not now consider the method of its manufacture further than to state that it is made from sulfur. Its composition is represented by the formula $\mathrm{H}_{2} \mathrm{SO}_{4}$. It is a colorless liquid of "oily" consistency, but is not really an oil, as it will mix with water in all proportions. It must be handled with caution, since it can cause bad burns if it is spilled on the skin. (In case of accident, wash off the acid in much running water, immedialcly.) When sulfuric acid is mixed with water, the mixture gets boiling hot, for which reason the acid 
should be added very slowly, with stirring, to the water, if a dilute solution is to be made.

94. Neutralization of Sulfuric Acid, Sodium Sulfate.-We find that the dilute solution has a sour taste and that it turns litmus red. We may next try whether it will neutralize a solution of sodium hydroxide, for which purpose we may add to a dilute solution of sulfuric acid a few drops of litmus solution and then run in sodium hydroxide solution drop by drop until neutrality is reached. If the neutral solution is now boiled until a solid begins to appear and then is left to evaporate at room temperature, large, transparent, glassy-looking crystals will be formed. These crystals dissolve readily in water to form a neutral solution, which does not have a sour taste.

If we allow the dry crystals to remain in the open air we find that they lose weight rapidly and turn white upon the surface, forming a fine white powder. Finally nothing is left of the large, clear, glassy crystals; only the powder remains, the weight of which is much less than that of the original material. What is the cause of this curious change? Let us put one of the large clear crystals into a dry test tube and heat gently the lower end of the tube containing the crystal, while the tube is held nearly horizontally. We soon see that water has collected in large amount in the cold end of the tube, while only a white powder is left behind. It is now easy to understand what occurred when the large crystal was exposed in the open air. It dissociated into the white powder and water which disappeared as vapor. The analysis of the thoroughly dried powder would show that it contains only sodium, sulfur, and oxygen, and in the proportions represented by $\mathrm{Na}_{2} \mathrm{SO}_{4}$, and since the clear crystals yielded only $\mathrm{Na}_{2} \mathrm{SO}_{4}$ and water, their composition must be represented by $\mathrm{Na}_{2} \mathrm{SO}_{4} \cdot x \mathrm{H}_{2} \mathrm{O}$, where $x$ is a whole number which must be found by means of a quantitative analysis. We call the original substance the hydrate of sodium sulfate, a hydrate of a salt being a compound of the salt with water.

We may now make the equation for the formation of this salt from sulfuric acid. We took $\mathrm{H}_{2} \mathrm{SO}_{4}$ and $\mathrm{NaOH}$ and got $\mathrm{Na}_{2} \mathrm{SO}_{4}$, from which we see that if two formula weights of water 
were formed from one formula weight of $\mathrm{H}_{2} \mathrm{SO}_{4}$ and two of $\mathrm{NaOH}$, the whole of the material taken would be accounted for thus:

$$
\mathrm{H}_{2} \mathrm{SO}_{4}+2 \mathrm{NaOH} \rightarrow \mathrm{Na}_{2} \mathrm{SO}_{4}+2 \mathrm{H}_{2} \mathrm{O} \text {. }
$$

This conclusion is rendered probable by the fact that in the other neutralizations we have studied water was always one of the products; it may be confirmed by mixing with dry sodium hydroxide pure sulfuric acid, whereupon water and $\mathrm{Na}_{2} \mathrm{SO}_{4}$ will result. The salt $\mathrm{Na}_{2} \mathrm{SO}_{4}$ is called sodium sulfate. Crystals of anhydrous sodium sulfate are different in form from those of the hydrate.

95. Quantitative Analysis of a Hydrate.-Let us now consider the quantitative composition of the large, glassy crystals which yielded $\mathrm{Na}_{2} \mathrm{SO}_{4}$ and water. If we weigh a crystal contained in a porcelain dish and allow it to stand a day or two at room temperature we find that only the white powder remains. If we now heat the dish and contents over a flame in order thoroughly to dry the powder, and let it cool and weigh it again, it is obvious that the loss of weight will represent the weight of water originally combined with the weight of dry $\mathrm{Na}_{2} \mathrm{SO}_{4}$ left in the dish.

96. Sodium Sulfate Decahydrate: $\mathrm{Na}_{2} \mathrm{SO}_{4} \cdot 10 \mathrm{H}_{2} \mathrm{O}$.- Now suppose that $5.796 \mathrm{~g}$. of the hydrate of sodium sulfate yielded $2.556 \mathrm{~g}$. of dried sodium sulfate, $\mathrm{Na}_{2} \mathrm{SO}_{4}$, what is the formula of the hydrate? In other words, what is the numerical value of $x$ in the formula $\mathrm{Na}_{2} \mathrm{SO}_{4} \cdot x \mathrm{H}_{2} \mathrm{O}$ ? The weight of water driven off was $5 \cdot 796-2 \cdot 55^{6}=3 \cdot 240 \mathrm{~g}$. We may therefore write the proportion, 2.556 is to 3.240 as the formula weight of sodium sulfate is to the $x$ times the formula weight of water. Now, the formula weight of sodium sulfate is $2 \times 23+32+4 \times 16=142$ and that of water is $2 X_{1}+16=18$. Therefore $2 \cdot 556: 3 \cdot 240:$ : I $42: 18 x$, from which we find that $x=10$, and are thus led to the conclusion that the hydrate of sodium sulfate has the formula $\mathrm{Na}_{2} \mathrm{SO}_{4} \cdot 10 \mathrm{H}_{2} \mathrm{O}$. If the reaction between sulfuric acid and sodium hydroxide is represented by the equation $\mathrm{H}_{2} \mathrm{SO}_{4}+$ $2 \mathrm{NaOH} \rightarrow \mathrm{Na}_{2} \mathrm{SO}_{4}+2 \mathrm{H}_{2} \mathrm{O}$, then the hydrate $\mathrm{Na}_{2} \mathrm{SO}_{4} \cdot 10 \mathrm{H}_{2} \mathrm{O}$ must have resulted from the union of the sodium sulfate with part of the water which formed the solution, thus:

$$
\mathrm{Na}_{2} \mathrm{SO}_{4}+\mathrm{IOH}_{2} \mathrm{O} \rightarrow \mathrm{Na}_{2} \mathrm{SO}_{4} \cdot 10 \mathrm{H}_{2} \mathrm{O} \text {. }
$$


This substance is called sodium sulfate decahydrate (deca meaning ten).

97. Hydrates.-Sodium sulfate forms other compounds with water, namely $\mathrm{Na}_{2} \mathrm{SO}_{4} \cdot{ }_{7} \mathrm{H}_{2} \mathrm{O}$ and $\mathrm{Na}_{2} \mathrm{SO}_{4} \cdot \mathrm{H}_{2} \mathrm{O}$; but the decahydrate is the common one. Many other salts form hydrates and some form a series of hydrates, as this salt does. But it must not be supposed that all salts form hydrates. For example, sodium chloride and ammonium chloride do not.

Solutions of the hydrated salt have exactly the same properties as those of solutions of the anhydrous salt.

98. Sodium Hydrogen Sulfate: $\mathrm{NaHSO}_{4}$.-If we exactly neutralize a definite quantity of sulfuric acid with a solution of sodium hydroxide, noting the volume of the latter used, and again add to a second portion of sulfuric acid, exactly equal to the first, exactly half as much sodium hydroxide solution as that used in the first case, we find that the first solution yields when evaporated pure sodium sulfate, $\mathrm{Na}_{2} \mathrm{SO}_{4}$; while the second gives crystals having a different shape and appearance, and different chemical properties. Analysis shows that the composition of these crystals is represented by the formula $\mathrm{NaHSO}_{4}$. The substance is called sodium hydrogen sulfate. The equation for the reaction in the second case is

$$
\mathrm{H}_{2} \mathrm{SO}_{4}+\mathrm{NaOH} \rightarrow \mathrm{NaHSO}_{4}+\mathrm{H}_{2} \mathrm{O} .
$$

99. The Law of Definite Composition Again.-We may now consider one of the most important and fundamental of all chemical questions, namely, whether the proportions of the elementary constituents of a substance are dependent upon the proportions which we take of the substances from which we form the product in question. For example, we may inquire whether we could get a sulfate of sodium with a somewhat larger or smaller percentage of sodium if we had used, in the preceding experiment, other proportions of acid and base. Experiment will show, however, that if we had added a little more or less sodium hydroxide we would still have been able to obtain much $\mathrm{NaHSO}_{4}$, but that in such cases there would also be some $\mathrm{Na}_{2} \mathrm{SO}_{4}$ formed or a little free sulfuric acid left after all 
the $\mathrm{NaHSO}_{4}$ had been separated from the water. Facts like these which are met with on every hand give a special significance to the Law of Definite Composition.

I00. Acid Properties of Sodium Hydrogen Sulfate.-We see that sodium sulfate, $\mathrm{Na}_{2} \mathrm{SO}_{4}$, contains exactly twice the weight of sodium for a given weight of sulfur and oxygen as does sodium hydrogen sulfate, $\mathrm{NaHSO}_{4}$. Moreover, we have become acquainted with the important fact that sulfuric acid can form two sorts of sodium salls. If we dissolve crystals of sodium hydrogen sulfate in water, we find that the dilute solution has a sour taste and it turns litmus red, for which reasons we should be inclined to say that it has acid propcrtics. In accord with this view, we find that the solution will readily neutralize a solution of sodium hydroxide, giving sodium sulfate and water, thus:

$$
\mathrm{NaHSO}_{4}+\mathrm{NaOH} \rightarrow \mathrm{Na}_{2} \mathrm{SO}_{4}+\mathrm{H}_{2} \mathrm{O} \text {. }
$$

IOI. Ammonium Sulfate and Ammonium Hydrogen Sulfate.-If we completely neutralize sulfuric acid with a solution of ammonium hydroxide, we obtain a salt called ammonium sulfate $\left(\mathrm{NH}_{4}\right)_{2} \mathrm{SO}_{4}$, thus:

$$
\mathrm{H}_{2} \mathrm{SO}_{4}+2 \mathrm{NH}_{4} \mathrm{OH} \rightarrow\left(\mathrm{NH}_{4}\right)_{2} \mathrm{SO}_{4}+2 \mathrm{H}_{2} \mathrm{O}
$$

while with half the proportion of ammonium hydroxide we obtain ammonium hydrogen sulfate, thus:

$$
\mathrm{H}_{2} \mathrm{SO}_{4}+\mathrm{NH}_{4} \mathrm{OH} \rightarrow \mathrm{NH}_{4} \mathrm{HSO}_{4}+\mathrm{H}_{2} \mathrm{O} \text {. }
$$

I02. Monobasic and Dibasic Acids: Acid Salts and Neutral Salts.-Hydrochloric acid reacts with sodium hydroxide only in one proportion, thus:

$$
\mathrm{HCl}+\mathrm{NaOH} \rightarrow \mathrm{NaCl}+\mathrm{H}_{2} \mathrm{O},
$$

for which reason we call it a monobasic acid; but since one formula weight of sulfuric can unite with a maximum of two formula weights of sodium hydroxide we call sulfuric acid a dibasic acid. Salts in which but half the maximum quantity of base has been neutralized are usually called acid salts, because they still have acid properties. 'Thus we frequently speak of sodium acid sulphate, meaning $\mathrm{NaHSO}_{4}$. Chemists know many 
other dibasic acids, all of which also can form acid salts as well as neutral salts, as salts like $\mathrm{Na}_{2} \mathrm{SO}_{4}$ are called.

103. Making Hydrochloric Acid from Common Salt.-If we place in a flask (Fig. 23) $5^{8} \mathrm{~g}$. of dry common salt and roo g. of sulfuric acid, to which $30 \mathrm{~g}$. of water have been added, and warm the mixture, a change occurs with the production of a colorless gas which dissolves in water very readily, giving a solution which we can easily recognize as hydrochloric acid. After the action of the sulfuric acid on the salt is complete, a white solid is left in the flask, which may easily be dissolved in water. By evaporating part of the water, and letting the solution stand a while, we

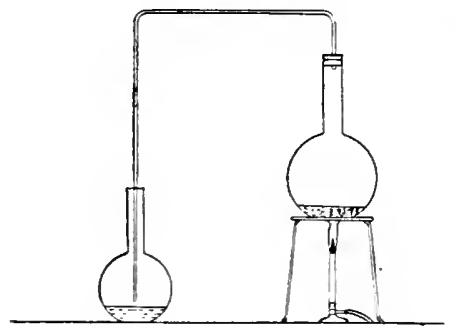

FIG. 23 may obtain colorless, transparent crystals of sodium hydrogen sulfate. The following equation represents the reaction:

$$
\mathrm{NaCl}+\mathrm{H}_{2} \mathrm{SO}_{4} \rightarrow \mathrm{NaHSO}_{4}+\mathrm{HCl} \text {. }
$$

We have to deal here with a new sort of chemical change-one in which an acid acts upon a salt of another acid to give a salt of the first acid and to produce the acid corresponding to the first salt. This is a very important kind of chemical reaction, which we shall frequently make use of, since by its means we may make acids from their salts.

I04. Making Nitric Acid from Chile Saltpeter.-We shall now use the method just described for the preparation of a new acid from a white, crystalline substance called Chile saltpeter, which is found in large quantities as a mineral substance in the desert region of Chile.

If we place $85 \mathrm{~g}$. of Chile saltpeter in a retort (Fig. 24), add $100 \mathrm{~g}$. of sulfuric acid, mixed with 30 c.c. of water, and then heat the mixture gently, a yellow-colored liquid may be collected in a cooled flask. This yellow liquid gives off a brown gas and becomes colorless when boiled a few minutes. Its analysis shows its formula to be $\mathrm{HNO}_{3}$ and it is called nitric acid. It is a colorless liquid which may be boiled and distilled in glass vessels. 
Pure or concentrated nitric acid is even more dangerous than sulfuric acid, causing serious burns and destroying clothing, and must be handled with greatest care. It will mix with water in all proportions, giving a solution which, when very dilute, has a sour taste and turns litmus red.

When nitric acid is mixed with sodium hydroxide solution the latter is neutralized, a salt of the composition $\mathrm{NaNO}_{3}$ and water being the only products, as represented by the equation

$$
\mathrm{HNO}_{3}+\mathrm{NaOH} \rightarrow \mathrm{NaNO}_{3}+\mathrm{H}_{2} \mathrm{O} \text {. }
$$

The salt, which is called sodium nitrate, is found to be identical with purified Chile saltpeter. The action of sulfuric acid on saltpeter leaves in the retort a white solid which closely resembles that left when salt is heated with sulfuric acid, and, in fact, the residue is easily found to be the same substance, sodium hydrogen sulfate, $\mathrm{NaHSO}_{4}$. The equation for the reaction is therefore

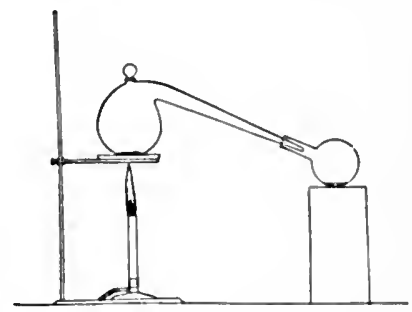

FIG. 24

$$
\mathrm{NaNO}_{3}+\mathrm{H}_{2} \mathrm{SO}_{4} \rightarrow \mathrm{NaHSO}_{4}+\mathrm{HNO}_{3} \text {. }
$$

105. The Action of Nitric Acid on Ammonium Hydroxide.We may now propose a question to be answered, not after direct experiment, but as a result of the general knowledge we have gained regarding the behavior of the acids and bases already studied. It is: What would be the result of mixing nitric acid and ammonium hydroxide? We recall that hydrochloric acid and sodium hydroxide, a base, give sodium chloride and water, thus:

$$
\mathrm{HCl}+\mathrm{NaOH} \rightarrow \mathrm{NaCl}+\mathrm{H}_{2} \mathrm{O} ;
$$

that the same acid gives with ammonium hydroxide, also a base, ammonium chloride and water, thus:

$$
\mathrm{HCl}+\mathrm{NH}_{4} \mathrm{OH} \rightarrow \mathrm{NH}_{4} \mathrm{Cl}+\mathrm{H}_{2} \mathrm{O} \text {. }
$$

Furthermore, we have just seen (104) that nitric acid and sodium hydroxide give sodium nitrate and water, thus:

$$
\mathrm{HNO}_{3}+\mathrm{NaOH} \rightarrow \mathrm{NaNO}_{3}+\mathrm{H}_{2} \mathrm{O},
$$


and we would certainly expect that nitric acid and ammonium hydroxide would behave analogously and give ammonium nitrate and water, thus:

$$
\mathrm{HNO}_{3}+\mathrm{NH}_{4} \mathrm{OH} \rightarrow \mathrm{NH}_{4} \mathrm{NO}_{3}+\mathrm{H}_{2} \mathrm{O} .
$$

Now this is precisely what takes place when we test our prediction by experiment. We seem, therefore, to have discovered the secret of the way in which acids and bases act toward each other. It may be summed up in the statement, An acid and a base neutralize each other, forming a salt and water.

I06. A New Base: Caustic Potash or Potassium Hydroxide. -Let us now take up the study of a new base, caustic potash, which closely resembles caustic soda (sodium hydroxide). It will be remembered that the metal sodium reacts violently with water, giving sodium hydroxide and hydrogen gas, thus:

$$
2 \mathrm{Na}+{ }_{2} \mathrm{H}_{2} \mathrm{O} \rightarrow{ }_{2} \mathrm{NaOH}+\mathrm{H}_{2} .
$$

Now, chemists know another metallic element, potassium, which closely resembles sodium. Like sodium, it is a silver-white metal, soft enough to be cut easily with a knife and tarnishing very rapidly in the air. For a reason that we shall soon learn it is kept covered with oil in a carefully stoppered bottle. If we throw a small bit of potassium into a beaker of water, it bursts into a flame of lavender color, spinning and darting to and fro on the surface of the water and completely disappearing in a few moments. Examination of the water shows that it will turn litmus blue, that it has a "soapy" taste, like a very dilute solution of sodium hydroxide, and that a white solid is left when the solution is evaporated to dryness. This solid is found by suitable methods of analysis to contain the elements potassium, oxygen, and hydrogen in the proportion represented by the formula $\mathrm{KOH}$, and is called potassium hydroxide.

If instead of throwing the bit of potassium on the surface of the water we bring it under the mouth of an inverted cylinder filled with water, with the mouth immersed in a vessel of water, the potassium rises to the top of the water in the cylinder, producing a gas which displaces the water in the cylinder, but does 
not take fire. The gas is easily identified as hydrogen, while the water contains dissolved potassium hydroxide as before. 'The equation for the reaction in the cylinder is

$$
2 \mathrm{~K}+2 \mathrm{H}_{2} \mathrm{O} \rightarrow 2 \mathrm{KOH}+\mathrm{H}_{2} .
$$

When the action takes place in the open beaker, the heat produced sets fire to the hydrogen, which burns, together with a small portion of the potassium.

I07. Potassium Salts.- On account of the behavior of a solution of potassium hydroxide toward litmus and also because of its "soapy" feel and taste, we should conclude that it is a base and if so that it should form salts with acids. We might even venture to predict the formulae of the salts it would be expected to form with hydrochloric, sulfuric, and nitric acids, and to write the equations as follows:

$$
\begin{aligned}
\mathrm{HCl}+\mathrm{KOH} & \rightarrow \mathrm{KCl}+\mathrm{H}_{2} \mathrm{O} \\
\mathrm{H}_{2} \mathrm{SO}_{4}+2 \mathrm{KOH} & \rightarrow \mathrm{K}_{2} \mathrm{SO}_{4}+2 \mathrm{H}_{2} \mathrm{O} \\
\mathrm{H}_{2} \mathrm{SO}_{4}+\mathrm{KOH} & \rightarrow \mathrm{KHSO}_{4}+\mathrm{H}_{2} \mathrm{O} \\
\mathrm{HNO}_{3}+\mathrm{KOH} & \rightarrow \mathrm{KNO}_{3}+\mathrm{H}_{2} \mathrm{O} .
\end{aligned}
$$

And in every case these predictions would be found by experiment to be correct! The potassium salts so formed are all white crystalline solids and are all soluble in water. All except potassium hydrogen sulfate give solutions which are neutral to litmus, while this salt has acid properties like those of sodium hydrogen sulfate. 


\section{CHAPTER VIII}

\section{WATER AND SOLUTIONS}

108. Water.-We have already learned that pure water is readily obtained by the distillation of natural waters (23), and that it is a compound of hydrogen and oxygen, the composition of which is represented by the formula $\mathrm{H}_{2} \mathrm{O}(\mathbf{7 0})$. In describing a substance we shall often mention its physical and chemical properties. The properties of a substance embrace: the state (whether solid, liquid, or gaseous); crystalline form, if solid; specific gravity or density; color; odor; taste; conductivity for heat and electricity; boiling-point; freezing-point, etc. The chemical properties of a substance are those which it exhibits in its typical chemical reactions.

I09. The Physical Properties of Water: Color.-We know that according to the temperature water can exist as solid, liquid, or gas. The color of liquid water is a very faint blue; so faint, in fact, that it cannot be noticed in a glass of water, but is obvious in a white bathtub full of clear water. The color of large bodies of clear water is usually blue, but it may be of any other shade if dissolved or suspended impurities (mud) are present. The yellow color of the waters of many rivers is due to suspended clay; such water is not clear, but muddy or turbid. Streams and lakes in hemlock forests often contain perfectly clear water having the color of tea, due to coloring-matter dissolved from the hemlock. The clear green color of some waters is usually the result of the blending of the natural blue color of the water with the yellow light reflected from the sand beneath.

II0. Specific Gravity or Density.-At the temperature of $4^{\circ}$ C., I C.c. of water weighs I g. Since the specific gravity or density of any substance may be defined as the weight of I c.c., it follows that water has a specific gravity of $\mathrm{I} .000$ at $4^{\circ} \mathrm{C}$. Or, we may say that the specific gravity or density of a substance is found by dividing its weight by the weight of an equal volume of water. Water has its greatest density at $4^{\circ}$; if a given volume 
of water at $4^{\circ}$ is either heated or cooled, it expands and therefore decreases in density.

III. Specific Heat.- The quantity of heat required to raise the temperature of $\mathrm{I} g$. of water $\mathrm{I}^{\circ} \mathrm{C}$. is by definition called one calorie. Water is said to have a specific heat of one or unity. The spccific heat of any substance is the quantity of heat in calorics required to raise the temperature of one gram of it one degree. Nearly all substances have specific heats less than unity.

I12. Vapor Pressure.-Water contained in an open vessel evaporates at all temperatures, but the more rapidly in proportion as the temperature is higher, other things being equal. If water evaporates into an evacuated space the pressure within the space increases to a value which is dependent only upon the temperature, being greater in proportion as the temperature is higher. The pressure so produced is called the vapor pressure of water; it may easily be demonstrated by means of a barometer tube filled with mercury. If we prepare two such tubes (Fig. 25) and introduce a few drops of water into one by means of a suitably shaped glass tube, the water will rise until it floats on the surface of the

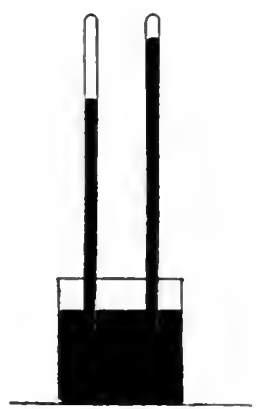

FIG. 25 mercury. At the same time the level of the mercury will fall 2 or $3 \mathrm{~cm}$., showing that a pressure has been produced above the mercury in the space which has been a vacuum. If the tube into which the water is introduced has a glass jacket into which warm water can be poured, it will be found that the higher the temperature is, the higher the iupor pressure will be. If we should raise the temperature to $100^{\circ}$, the level of the mercury in the barometer tube would sink to that of the surface of the mercury in the dish in which the tube stands, thus showing that the vapor pressure at $100^{\circ}$ is equal to the pressure of the almosphere. Table VII shows the vapor pressure of water at various temperatures between $0^{\circ}$ and $100^{\circ}$.

When the atmospheric pressure is $760 \mathrm{~mm}$, water boils at $100^{\circ}$. Now, we see from the table that at $100^{\circ}$ the vapor 
pressure is $760 \mathrm{~mm}$., therefore the boiling-point is that temperature at which the vapor pressure becomes just equal to the normal atmospheric pressure, $760 \mathrm{~mm}$., which is the average pressure at sea-

TABLE VII

\begin{tabular}{c|c||c|l}
\hline \hline Temperature & Pressure & Temperature & \multicolumn{1}{|c}{ Pressure } \\
\cline { 2 - 3 } $0^{\circ} \ldots \ldots$ & $4.6 \mathrm{~mm}$. & $60^{\circ} \ldots \ldots$ & I $49.2 \mathrm{~mm}$. \\
$10 \ldots \ldots$ & 9.2 & $70 \ldots \ldots$ & 233.8 \\
$20 \ldots \ldots$ & 17.4 & $80 \ldots \ldots$ & 355.5 \\
$30 \ldots \ldots \ldots$ & 31.6 & $90 \ldots \ldots$ & 526.0 \\
$40 \ldots \ldots \ldots$ & 55.0 & $99 \ldots \ldots$ & 733.2 \\
$50 \ldots \ldots \ldots$ & 92.2 & $100 \ldots \ldots$ & 760.0 \\
\hline
\end{tabular}

level. At higher altitudes, at which the atmospheric pressure is less than $760 \mathrm{~mm}$., water boils at temperatures lower than $100^{\circ}$. Thus if the pressure is $733.2 \mathrm{~mm}$., the boiling-point is $99^{\circ}$. Since the atmospheric pressure at a given place is variable through a range of $20 \mathrm{~mm}$. or more, the boiling-point at this place is not constant, but varies with the rise and fall of the barometer.

II3. Correction of the Voluine of a Gas for Vapor Pressure.Gases like hydrogen and oxygen, which are not very soluble in

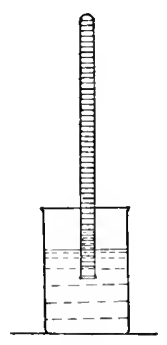

FIG. 26 water, are often measured in tubes in which the gases are confined by means of water. Such gases always contain water vapor, and part of the total pressure exerted by the gas is due to the vapor pressure of the water. The part of the pressure (partial pressure) exerted by the gas itself is found by subtracting from the total pressure the vapor pressure of the water. For example, suppose that some hydrogen is collected over water in a graduated glass tube (Fig. 26). If the position of the tube is adjusted so that the level of the water is the same inside the tube as outside, the total pressure within must be exactly equal to the atmospheric pressure, as shown by the barometer. Suppose that the barometric pressure is $748.6 \mathrm{~mm}$. and the temperature $20^{\circ}$. Table VII shows that at $20^{\circ}$ the vapor pressure of water is $17.4 \mathrm{~mm}$, therefore the pressure due to the 
hydrogen is $748.6-17.4=73 \mathrm{I} .2 \mathrm{~mm}$. If the observed volume was 30 c.c., the volume, $V$, at standard conditions would be

$$
V=\frac{30 \times 731.2 \times 273}{760 \times 293}=26.9 \text { c.c. }
$$

114. Vapor Pressure of Liquids and Solids in General.Liquids in general readily pass into the form of vapor, and just as in the case of water, a given pure liquid has, at each temperature, a definite vapor pressure; but the vapor pressure of one liquid-say alcohol-is not in general the same at a given temperature as that of another liquid-say water. In every case, however, the boiling-point of the liquid is that temperature at which its a apor pressure equals $760 \mathrm{~mm}$. Many solids, for example, camphor and naphthalene (moth-balls), have appreciable vapor pressures at room temperature; but the vapor pressures of most solids at such temperature are too small to be noticeable.

II5. Latent Heat of Evaporation.-If it is true that water boils at $100^{\circ}$ because at this temperature the vapor pressure of water just equals the normal atmospheric pressure, it may be asked why the whole of the water does not change at once into steam as soon as its temperature is raised to $100^{\circ}$. We know, of course, that this does not occur, and, further, that the rapidity with which water boils away is greater, the greater the amount of heat applied. The explanation is found in the fact that it requires a large amount of heat to change water at $100^{\circ}$ into steam at the same temperature. In fact, 540 calories of heat are required for the conversion of $\mathrm{I} g$. of water at $100^{\circ}$ into steam. The heat so used up does not raise the temperature of the substance. It is consumed in changing the liquid water into the gaseous state; it is said to become latent, and in consequence we say that the latent heat of evaporation of water is 5 fo calories. Every pure liquid has a latent heat of evaporation. This differs from one substance to another.

r16. Use of Steam for Heating.-When steam cools to $100^{\circ}$ it begins to condense to liquid water, and for every gram of steam that condenses 540 calories of heat are given out. The heat 
so given out may be considered to be that which became latent when the water was, by being heated, converted into steam. It is on account of the latent heat given out upon condensation that steam is so effective in the heating of buildings: every gram of steam that condenses in the radiator liberates 540 calories of heat. Of course, the further cooling of the water in the radiator gives out some additional heat.

II7. Burns Produced by Steam.-It is a well-known fact that serious burns result when steam comes in contact with the skin. At first thought, this result seems to be out of harmony with the fact that air at $100^{\circ}$ can be borne by the hand without discomfort. The explanation of this difference is found in the fact that gases (including the vapors of boiling liquids) are very poor conductors of heat as compared with liquids. Steam at $100^{\circ}$ partly condenses on striking the skin and wets it with a layer of boiling-hot water, which is a good conductor of heat. Furthermore, since 540 calories of heat are given out by every gram of steam condensed to water, the latter is kept at $100^{\circ}$ as long as steam is present. On the other hand, air is so poor a conductor of heat that the skin is not burned by a brief exposure to it at $100^{\circ}$.

I 18. Latent Heat of Fusion of Ice.-Ice melts at $0^{\circ}$; but all of a given mass of ice does not melt immediately when its temperature is raised to zero. Just as heat is required to change liquid water into vapor, so also heat is needed to change ice at zero into water at the same temperature. The heat so absorbed is called the latent heat of fusion of ice. It requires 79 calories to melt I $\mathrm{g}$. of ice; therefore the latent heat of fusion of ice is 79 calories. Every solid has a definite and characteristic latent heat of fusion.

II9. The Density of Ice.-The density or specific gravity of ice is $0.9 \mathrm{I} 7$. It is for this reason that ice floats on water. The expansion which occurs when water freezes exerts very great pressure, illustrations of which are often seen in the bursting of water pipes and other vessels when water freezes in them. Not all liquids expand upon freezing; in many cases contraction occurs, thereby giving rise to solids which sink in the corresponding liquids. 
I20. Solutions and Suspensions.-The mixture which results upon dissolving salt in water is called a solution of salt in water. The terms "dissolve" and "solution" are used in chemistry with definite meanings. If, upon mixing a solid with a liquid, the former partly or wholly disappears and the resulting liquid is still clear and transparent and not cloudy or muddy, and if, moreover, upon allowing the liquid to evaporate we regain the unchanged solid substance, we say that the solid had dissolved in the liquid to form a solution. Either or both of the substances may be colored and still a clear (although colored) solution may result. The liquid in which a substance is dissolved is called the solvent.

If we stir up some common clay with water, much of the clay fails to settle out of the water at once, and we get a cloudy or muddy fluid, like the water of a muddy river. In this case we do not say that the clay has dissolved in the water or that we have a true solution of the clay. We say that the clay is suspended in the water, and call the muddy water a suspension. Clay suspended in water will settle out very slowly and finally leave clear water above a layer of mud.

I2I. The Concentration of Solutions.-A solution containing a small proportion of a dissolved substance is said to be dilute, while one containing a large proportion is called concentrated. We dilute a concentrated solution by adding solvent to it, and concentrate a dilute solution by evaporating the solvent. We use the term concentration in discussing the relative amount of dissolved substances in a solution.

I22. Solubility of Substances: Saturated Solutions. - It is casy to discover that the amount of a substance which will dissolve in a given amount of water, say roo c.c., depends upon the nature of the substance and upon the temperature. If we mix some common salt with about double its weight of water and stir or shake the mixture a sufficient length of time (usually one to two hours), keeping the temperature constant all the while, and then, after allowing any suspended crystals to settle, draw off a portion of the clear solution,' weigh it, and evaporate the water, we get the salt dissolved in the portion of the solution 
taken. By weighing the salt we can readily find the weight of salt dissolved in a given weight of water at the temperature at which the experiment was made. We find in this way that Io0 g. of water at $25^{\circ}$ dissolves $37.6 \mathrm{~g}$. of salt.

To make such a solubility determination we must observe several precautions: First, the amount of solid substance must be considerably greater than the amount of water taken will dissolve; secondly, the shaking must be continued as long as more substance dissolves - this is easily ascertained by prolonging the shaking and making additional determinations of the concentration of the solution; thirdly, the temperature must be kept constant.

A solution which at a fixed temperature will dissolve no more of a given substance is called a saturated solution. When we speak of the solubility of a substance we mean the amount of substance dissolved in a given amount of water in the case of the saturated solution. The following brief table gives the solubilities in water at $25^{\circ}$ of several salts.

TABLE VIII

Grams of Substance in ioo G. OF Water at $25^{\circ}$

\begin{tabular}{l|l||l|l}
\hline \hline $\mathrm{NaCl} \ldots \ldots$ & 37 g. & $\mathrm{KCl}_{2} \ldots \ldots \ldots$ & $34 \mathrm{~g}$. \\
$\mathrm{Na}_{2} \mathrm{SO}_{4} \cdot \mathrm{IOH}_{2} \mathrm{O}$ & 27 & $\mathrm{~K}_{2} \mathrm{SO}_{4} \ldots \ldots \ldots$ & I 2 \\
$\mathrm{NaNO}_{3} \ldots \ldots$ & $9^{2}$ & $\mathrm{KNO}_{3} \ldots \ldots \ldots$ & 37 \\
\hline
\end{tabular}

I23. Supersaturated Solutions.-At $25^{\circ} \mathrm{IOO}$ g. of water will dissolve $27 \mathrm{~g}$. of sodium sulfate decahydrate, $\mathrm{Na}_{2} \mathrm{SO}_{4} \cdot \mathrm{IoH}_{2} \mathrm{O}$, while at $30^{\circ}$ the same amount of water will dissolve $40 \mathrm{~g}$. of the salt. If we make a saturated solution of the salt at $30^{\circ}$, having an excess of crystals of the salt present, and then cool the whole to $25^{\circ}$, and keep it at $25^{\circ}$, stirring or shaking it for an hour or two, more solid is deposited and there results a solution which contains just the same weight of the salt in Ioo g. of water as a saturated solution at $25^{\circ}$, namely, $27 \mathrm{~g}$.

A slight change in the procedure gives a very different result and brings to light a new phenomenon. If the solution of sodium sulfate which is saturated at $30^{\circ}$ is freed from every particle of the 
solid crystalline substance and then allowed to cool to $25^{\circ}$ or even lower, without being stirred or shaken, it remains perfectlyclear and does not deposit any crystals. Such a solution contains at $25^{\circ}$ much more sodium sulfate than a saturated solution prepared at $25^{\circ}$ in the manner described in the preceding paragraph. This more concentrated solution is called a supersaturated solution. If we now drop into the supersaturated solution a crystal of sodium sulfate (and for this purpose an almost invisible fragment of the crystalline dust will be sufficient), the formation of crystals will begin at once and proceed until the amount of dissolved substance per roog. of water is reduced exactly to that of a saturated solution at the existing temperature.

Experience has shown that a supersaturated solution can only be obtained in the complete absence of the solid substance, and that a supersaturated solution begins to deposit its excess of dissolved substance when a crystal of this same substance is brought into the solution. The deposition of crystals by a supersaturated solution can also often be started by shaking or stirring the solution or by adding a crystal of another substance having the same crystalline form.

Not all substances form supersaturated solutions equally readily. The presence of impurities favors supersaturation. Syrups, preserves, and honey are often supersaturated with respect to the sugar dissolved in the water present. When such solutions "turn to sugar," this is only the crystallization of the excess of sugar above that required to make a saturated solution.

124. Solubility of Liquids in Liquids. - It is proverbial that "oil and water will not mix." On the other hand, some pairs of liquids will mix completely in all proportions; examples of such combinations are water and alcohol and water and sulfuric acid. We know other pairs of liquids that will not dissolve one another in all proportions, but that will dissolve one another partially. Water and ether belong to this class; I00 c.c. of water will dissolve 8 c.c. of ether, and roo c.c. of ether will dissolve 3 c.c. of water. If we pour ether into water, we find that the former floats on the surface of the latter. If equal volumes of ether and 
water are thoroughly shaken together, the former soon separates from the latter, and two distinct layers result as before. If, now, we examine each layer, we find that the water contains some dissolved ether and the ether some dissolved water. This is a case of partial miscibility.

125. Solubility of Gases in Liquids.-We have already learned that hydrogen chloride (44) and ammonia (5I) are both very soluble in water. At $0^{\circ}$ water dissolves 550 times its own volume of the first gas and I,I 50 times its volume of the second. No gas which we have studied is completely insoluble in water; for example, roo c.c. of water dissolves 2 . I c.c. of hydrogen and 4.8 c.c. of oxygen. Fishes depend for their existence upon the oxygen dissolved in water; by means of their gills they take from the water the oxygen they require.

126. Henry's Law.-The solubility of all gases decreases with rise of temperature. At a fixed temperature the weight of gas dissolved by a given volume of water of other liquid is dependent upon the pressure of the gas and is, in general, directly proportional to the pressure. This statement is known as Henry's Law. The law does not apply to very soluble gases, like ammonia, dissolving in water-probably because chemical union occurs, since we know that $\mathrm{NH}_{4} \mathrm{OH}$ is formed in this case (9r).

r27. Heat of Solution.-If we shake some potassium nitrate or ammonium chloride, or indeed any one of many salts, with water, we find that as the substance dissolves the solution becomes appreciably colder. This indicates that heat is rcquired to change the solid into the dissolved state. This phenomenon is analogous to that met with when a solid, like ice, melts. It requires 79 calories to melt I g. of ice, while I 5 calories are absorbed when I g. of potassium nitrate dissolves. That is, we must supply I 5 calories to $\mathrm{I} g$. of the salt, and sufficient water, in order to prevent a fall of temperature when solution takes place. The heat so required is called heat of solutior.

When any substance whatever melts, heat is required, or is absorbed, and we might expect, similarly, that heat will always be absorbed when a substance dissolves; but this is not the case. Many substances, upon dissolving, give out heat. In the case of a 
few substances the absorption or evolution of heat upon dissolving is very small. Common salt dissolves in water with very small heat absorption.

I28. Boiling-Point of Solutions. - It is very easy to show that a solution of a solid substance, like salt or sugar in water, boils at a higher temperature than pure water. This is an invariable rule for solutions of substances which are not readily volatile at the boiling-point of water. Now, we have in the first part of this chapter ( 112 ) considered the relationship between boilingpoints and vapor pressures, and it will easily be understood that a solution will boil at the temperature at which the pressure of its vapor is equal to the atmospheric pressure.

129. The Lowering of the Vapor Pressure by Dissolved Substances.-If a solution must be heated above $100^{\circ}$ to raise its vapor pressure to that which water has at $100^{\circ}$, it is clear that at this latter temperature the solution has a lower vapor pressure than pure water. It is also a fact that at every lower temperature the vapor pressure of a solution of an involatile substance is less than that of the pure solvent at the same temperature. This is a very important universal law. The law applies to solutions formed from all kinds of solvents.

I30. Deliquescence.-In the case of a very soluble substance, like caustic soda, the vapor pressure of the saturated solution may be so small that it is below the partial pressure exerted by the vapor usually present in the air. If such a solution is exposed to the air, water vapor from the air will condense in it until the solution has become so dilute that its vapor pressure is just equal to the partial pressure of the water vapor in the air. Moreover, if such a very soluble substance is exposed to air containing moisture, water will condense on the solid, thus converting it slowly, first into a saturated solution, and finally into a dilute solution. This action is called deliquescence. We say caustic soda is a deliquescent substance. A little thought will lead to the conclusion that deliquescence is the result of two concurrent conditions; first, the possibility of the formation, by a substance, of a saturated solution which has a very small vapor pressure as compared with pure water-a condition usually 
accompanying great solubility; and, secondly, the presence in the air of a sufficiently great water-vapor content. No substance is deliquescent in a perfectly dry atmosphere, while every soluble substance exhibits this property in air saturated with water vapor. Deliquescence is, therefore, not a fixed property of a substance. Thus common salt is usually decidedly deliquescent at the seashore, where the air contains much water vapor; but it never shows this property in a desert region.

In several experiments we have used caustic soda or calcium chloride to dry air or other gases or to absorb water vapor formed in the burning of hydrogen $(39,50)$. These drying agents are among the most deliquescent substances known.

I3I. Efflorescence.-In paragraph 94 the peculiar behavior of sodium sulfate decahydrate, $\mathrm{Na}_{2} \mathrm{SO}_{4}, \mathrm{IOH}_{2} \mathrm{O}$, when exposed to the open air was described. We are now in a position to understand more about this spontaneous loss of water. If a crystal of the hydrate is floated on the surface of mercury in a vaccum tube like one of those shown in Figure 25, the mercury level is depressed more than can be accounted for by the weight of the crystal. Apparently the latter is giving off water vapor and attempting to establish a saturation pressure. This pressure is called the vapor pressure of the hydrate. As a matter of fact all hydrates show this same behavior, with the difference that each has its own characteristic vapor pressure at a given temperature. With increased temperature the vapor pressure rises. If a hydrate is exposed to air in which the partial pressure of water vapor is less than the vapor pressure of this substance, the latter will give off water to the air just as a water surface does to air in which the partial pressure of water vapor is below the saturation value for water. Along with the loss of water, the crystals of the decomposing hydrate crumble to a powder. This process is called efflorescence. It is obvious that whether or not a given hydrate effloresces depends not only upon its own vapor pressure but upon the moisture content of the air surrounding it.

132. Effect of Temperature on Solubility.-The solubility of a substance, that is, the amount of the substance which dissolves 
(to form a saturated solution) in a given amount of water, is dependent upon the temperature. Most substances are more soluble at a higher than at a lower temperature; but this is not always the case, as the solubility of some substances decreases with rise of temperature. In fact, gases are always less soluble at a higher temperature.

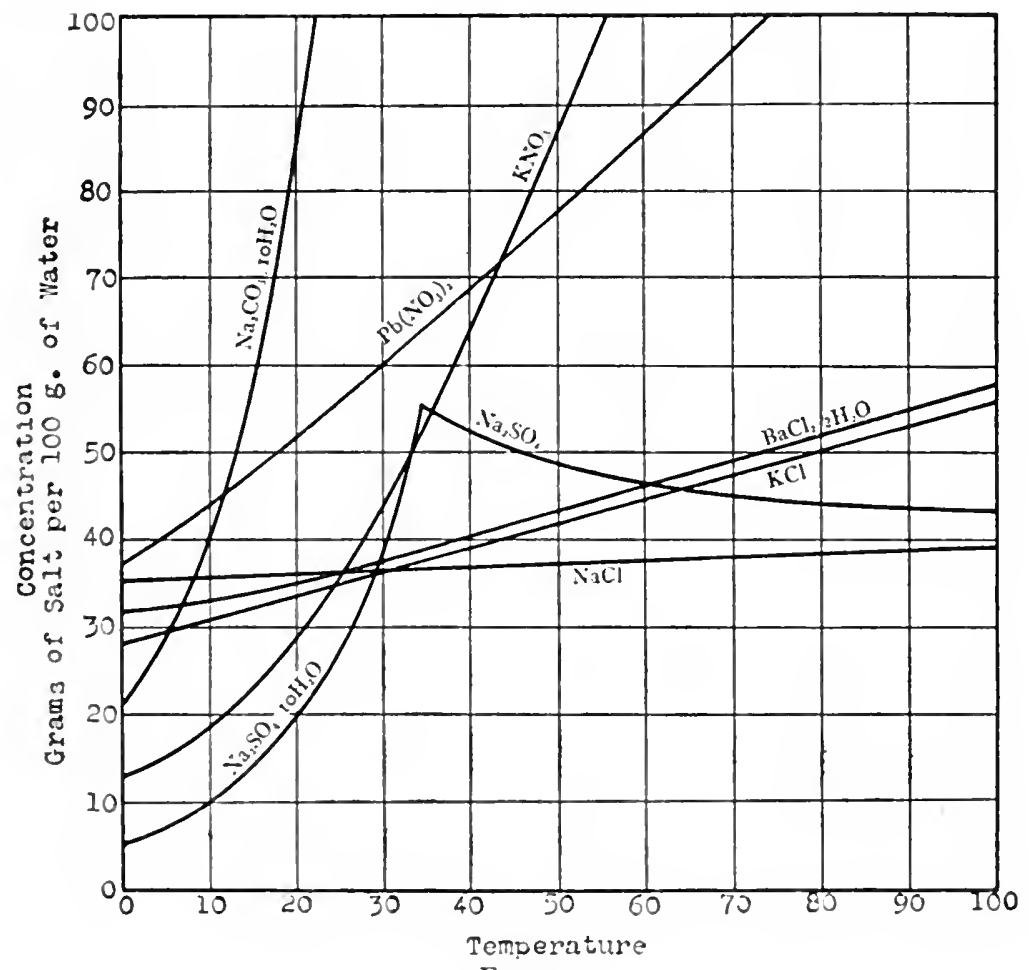

FIG. 27

The change of solubility with change of temperature can most easily be expressed graphically, that is, by means of so-called solubility curves. 'The accompanying diagram (Fig. 27) illustrates the method and gives the curves for water solutions of several substances.

133. Effect of Crystalline Form on Solubility.-Sodium sulfate has the formula $\mathrm{Na}_{2} \mathrm{SO}_{4}$. By the action of water we may 
readily obtain the hydrate $\mathrm{Na}_{2} \mathrm{SO}_{4} \cdot 10 \mathrm{H}_{2} \mathrm{O}(96)$, which can easily be recrystallized from water, as described under "Supersaturated Solutions" in this chapter. We see that the solubility curve for $\mathrm{Na}_{2} \mathrm{SO}_{4} \cdot \mathrm{IOH}_{2} \mathrm{O}$ rises rapidly until a temperature of $33^{\circ}$ is reached. At this temperature the crystals melt and at the same time decompose into $\mathrm{Na}_{2} \mathrm{SO}_{4}$ and $\mathrm{H}_{2} \mathrm{O}$, thus:

$$
\mathrm{Na}_{2} \mathrm{SO}_{4} \cdot 10 \mathrm{H}_{2} \mathrm{O} \rightarrow \mathrm{Na}_{2} \mathrm{SO}_{4}+10 \mathrm{H}_{2} \mathrm{O} \text {. }
$$

Above $33^{\circ}$ we have the solubility curve of anhydrous $\mathrm{Na}_{2} \mathrm{SO}_{4}$, which is a different chemical substance from its hydrate. Thus we see that there are for the anhydrous salt and its hydrate two distinct solubility curves, and that these intersect at a point for which the temperature is that at which the hydrate changes into the anhydrous substance. This is a typical case. Each hydrate of a substance has its own solubility curve; but these always intersect at the point corresponding to the temperature at which one substance changes into the other. The difference in solubility is due to the fact that each has its own characteristic crystalline form.

134. Heat of Solution and Changes of Solubility with Temperature.-A question which will now very naturally occur to the student is: Why should the solubility of various substances change with temperature in different ways? Although a complete and satisfying answer cannot be given to this question, it is possible to find a connection between the shape of the solubility curve of a substance and another fundamental property. It will be recalled that potassium nitrate absorbs much heat upon dissolving in water, and we notice that its solubility curve rises rapidly with temperature. Sodium chloride dissolves with but slight absorption of heat, and its curve is nearly horizontal. Finally, when it is known that anhydrous sodium sulfate, $\mathrm{Na}_{2} \mathrm{SO}_{4}$, dissolves at temperatures above $33^{\circ}$ with production of heat, and that its curve falls with rising temperature, the general law becomes apparent. These are typical cases. If any substance dissolves with absorption of heat, its solubility curve rises with rise of temperature. If it dissolves with evolution of heat, then the curve falls with rise of temperature. The frac- 
tional change of solubility with rise of $\mathrm{I}^{\circ}$ of temperature is in general proportional to the heat of solution. In eicry case that change of solubility which will absorb heat will take place when the temperature is raised. This will involve a decrease of solubility with rise of temperature, in the case of a substance like $\mathrm{Na}_{2} \mathrm{SO}_{4}$, above $33^{\circ}$, since, if heat is evolved when the substance dissolves, heat is absorbed in equal amount when the same weight of the substance crystallizes out of a solution.

In some cases where heat is evolved when a substance is dissolved, the observed heat is the result of the union of the solid with water to form a hydrate, which may dissolve with a small absorption of heat. In such cases the solubility of the hydrate increases with rise of temperature in strict accord with the law. For example when anhydrous calcium chloride is dissolved in water the mixture gets very hot. The saturated solution deposits crystals of $\mathrm{CaCl}_{2}, 6 \mathrm{H}_{2} \mathrm{O}$ on cooling. This hydrate dissolves in water with absorption of heat and its solubility increases with a rise in temperature. The heat given out on dissolving the anhydrous salt is the excess of the heat produced in the reaction

$$
\mathrm{CaCl}_{2}+6 \mathrm{H}_{2} \mathrm{O}=\mathrm{CaCl}_{2}, 6 \mathrm{H}_{2} \mathrm{O}
$$

above the lieat absorbed in the dissolving of the hydrate $\mathrm{CaCl}_{2}$, $6 \mathrm{H}_{2} \mathrm{O}$.

135. Two Apparent Kinds of Solubility.-In cases of ordinary solubility, evaporation of the water leads to the recovery unchanged of the substance originally dissolved. In other cases, evaporation of the solution obtained by the apparent dissolving of a substance leaves an entirely different substance. For example, if we throw a piece of sodium on water the former soon disappears and a solution results $(40,88)$. We might be inclined to say that the sodium has dissolved in the water; but there is another way of looking at the matter. We know that in this case a chemical change has occurred, as represented by the equation

$$
2 \mathrm{Na}+{ }_{2} \mathrm{H}_{2} \mathrm{O} \rightarrow 2 \mathrm{NaOH}+\mathrm{H}_{2} \text {. }
$$

Furthermore, we know that by evaporation of the solution we get sodium hydroxide and not sodium; for this reason it seems 
more logical to say that sodium and water react to give sodium hydroxide, which then dissolves in water, than to say that sodium itself is soluble in water. In fact, we know nothing about the solubility of sodium in water, since the two react as soon as they are brought into contact. We know a very great number of cases analogous to this one, and in all of them we recognize that we have to deal with chemical changes which give rise to soluble products.

136. Normal Solutions.-In the neutralization of hydrochloric acid by sodium hydroxide, which takes place according to the equation

$$
\mathrm{HCl}+\mathrm{NaOH} \rightarrow \mathrm{NaCl}+\mathrm{H}_{2} \mathrm{O},
$$

one formula weight of the acid $(36.5 \mathrm{~g}$.) requires one formula weight of the base ( $40 \mathrm{~g}$.). If we make a solution of the acid of such concentration that I liter contains $36.5 \mathrm{~g}$. of hydrogen chloride, and also make a solution of the base containing $40 \mathrm{~g}$. of sodium hydroxide per liter, then upon mixing the liter of the acid solution with the liter of the basic solution exact neutralization will take place. It follows, of course, that, to neutralize a given volume of such an acid solution, exactly the same volume of the basic solution will be required. We call such solutions normal solutions.

If we wish to make a solution of nitric acid of such concentration that I liter of it will exactly neutralize I liter of normal sodium hydroxide, we see, in accord with the equation

$$
\mathrm{HNO}_{3}+\mathrm{NaOH} \rightarrow \mathrm{NaNO}_{3}+\mathrm{H}_{2} \mathrm{O},
$$

that one formula weight of $\mathrm{HNO}_{3}$ must be contained in I liter of the solution. This gives a normal solution of nitric acid.

Now the case is a little different if a normal solution of sulfuric acid is to be made, since in this case we have

$$
\mathrm{H}_{2} \mathrm{SO}_{4}+2 \mathrm{NaOH} \rightarrow \mathrm{Na}_{2} \mathrm{SO}_{4}+{ }_{2} \mathrm{H}_{2} \mathrm{O} \text {. }
$$

We see that one formula weight of sulfuric acid neutralizes two formula weights of sodium hydroxide, so that to neutralize I liter of normal sodium hydroxide, which contains but one formula weight of the base, only one-half a formula weight 
( $\frac{1}{2}$ of $98 \mathrm{~g}$. or $49 \mathrm{~g}$.) of sulfuric acid is required. Therefore if we dissolve $49 \mathrm{~g}$. of the acid in sufficient water to make a liter of solution, this liter of acid solution will just neutralize I liter of normal sodium hydroxide. We call the sulfuric acid solution so made also a normal solution.

A normal solution of potassium hydroxide, $\mathrm{KOH}$, would contain one formula weight $(56 \mathrm{~g}$. per liter (106). A normal solution of any acid. aliwys neutralizes an exactly equal volume of a normal solution of any base. The term "normal" is usually abbreviated $\mathrm{N}$, so that for a normal solution by hydrochloric acid we write $\mathrm{N} \mathrm{HCl}$.

Normal solutions are of great importance in practical work. Suppose we wish to know the concentration of a given solution of sodium hydroxide. We take, with a pipette (Fig. 2S), a carefully measured volume, say 20 c.c., add to it sufficient

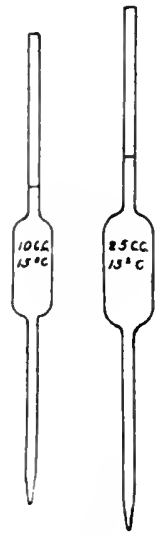

FIG. 28 litmus solution to produce a pale blue color, and then from a measuring tube, called a burette (Fig. 29), run in a normal solution of hydrochloric or other acid until the color just changes from blue to red. A little practice enables one to find, to within

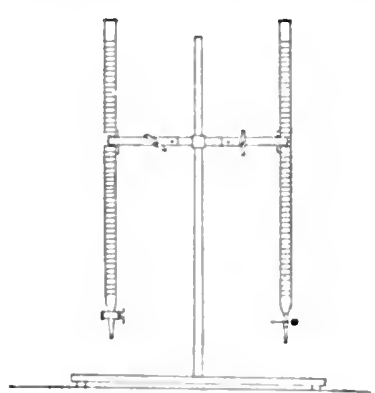

FIG. 29 one drop or less, the volume of acid required. Let us say 42 c.c. of $\mathrm{N} \mathrm{HCl}$ was required for the 20 c.c. of $\mathrm{NaOH}$ solution of unknown concentration. Our problem is to find the weight of sodium hydroxide in the 20 c.c. uf solution taken. Now, 42 c.c. of $\mathrm{N}$ acid will neutralize 42 c.c. of $\mathrm{N}$ sodium hydroxide, of which I liter ( $=\mathrm{I}, 000$ c.c.) contains $40 \mathrm{~g}$. of sodium hydroxide. Therefore the weight of sodium hydroxide in the 20 c.c. taken $=0.042 \times$ $40 \mathrm{~g} .=\mathrm{I} .68 \mathrm{~g}$. We also see that the sodium hydroxide solution is $42 / 20=2$. I times as concentrated as a normal solution of this base. We express its concentration by saying that it is 2 . I times normal in concentration. 
It is often convenient in practice to use solutions of $\frac{1}{2}, \frac{1}{5}, \frac{1}{10}$, or some other fraction of normal; we call these half-normal $\left(\frac{N}{2}\right)$, one-fifth normal $\left(\frac{N}{5}\right)$, and one-tenth or deci-normal $\left(\frac{\mathrm{N}}{\mathrm{IO}}\right)$, respectively.

137. Acidimetry and Alkalimetry.-The analyses of acids and bases by means of normal solutions are called respectively acidimetry and alkalimetry. The act of running in a solution from a burette until the neutral or end-point is reached is called titration. The volume of solution used is called the titer. Instead of litmus we may use some other colored substance to indicate the end-point; such a substance is called an indicator. Other useful indicators are methyl orange, phenolphthalein, and Congo red.

I38. Problems.-

r. How many c.c. of $\frac{\mathrm{N}}{\mathrm{IO}}$ nitric acid are required to neutralize 50 c.c. of normal potassium hydroxide? (I07)

2. How many c.c. of $\frac{\mathrm{N}}{5}$ sodium hydroxide are required to neutralize $2 \circ$ c.c. of $\frac{\mathrm{N}}{5}$ sulfuric acid?

3. If I6 c.c. of a solution of sulfuric acid of unknown concentration requires for its neutralization 36 c.c. of $\frac{\mathrm{N}}{2}$ potassium hydroxide, $(a)$ what is the weight of sulfuric acid in the I6 c.c. taken? (b) what is the weight of sulfuric acid in I liter of this acid? (107)

139. The Formation of Water.-We have, in earlier chapters, learned various ways in which water can be formed chemically. We may enumerate these by way of review.

Water is formed-

I. By the burning of hydrogen:

$$
{ }_{2} \mathrm{H}_{2}+\mathrm{O}_{2} \rightarrow{ }_{2} \mathrm{H}_{2} \mathrm{O} \text {. }
$$

2. By the burning of a compound of hydrogen, for example, methane:

$$
\mathrm{CH}_{4}+{ }_{2} \mathrm{O}_{2} \rightarrow \mathrm{CO}_{2}+2 \mathrm{H}_{2} \mathrm{O} \text {. }
$$


3. By the oxidation of hydrogen or its compounds by means of combined oxygen, as, for example, when ammonia is passed over hot copper oxide:

$$
{ }_{2} \mathrm{NH}_{3}+{ }_{3} \mathrm{CuO} \rightarrow{ }_{3} \mathrm{H}_{2} \mathrm{O}+{ }_{3} \mathrm{Cu}+\mathrm{N}_{2} .
$$

4. By the union of acids and bases, whereby a salt and water are always formed; for example:

$$
\mathrm{HCl}+\mathrm{NaOH} \rightarrow \mathrm{NaCl}+\mathrm{H}_{2} \mathrm{O} .
$$

5. By the decomposition or dissociation of various unstable compounds, as, for example, sodium sulfate decahydrate into the anhydrous salt and water:

$$
\mathrm{Na}_{2} \mathrm{SO}_{4} \cdot 10 \mathrm{H}_{2} \mathrm{O} \rightarrow \mathrm{NaSO}_{4}+\mathrm{IoH}_{2} \mathrm{O} \text {. }
$$

140. The Chemical Reactions of Water.-We have also studied some of the important kinds of reactions in which water takes part. We may now summarize these as follows:

I. Water unites with salts to form hydrates, thus:

$$
\mathrm{Na}_{2} \mathrm{SO}_{4}+\mathrm{IOH}_{2} \mathrm{O} \rightarrow \mathrm{Na}_{2} \mathrm{SO}_{4} \cdot 10 \mathrm{H}_{2} \mathrm{O} \text {. }
$$

2. Ammonia and water unite to form ammonium hydroxide:

$$
\mathrm{NH}_{3}+\mathrm{H}_{2} \mathrm{O} \rightarrow \mathrm{NH}_{4} \mathrm{OH} \text {. }
$$

4. Water acts upon some metals to give hydroxides and hydrogen. Thus, sodium and cold water react very easily, giving sodium hydroxide and hydrogen:

$$
2 \mathrm{Na}+2 \mathrm{H}_{2} \mathrm{O} \rightarrow 2 \mathrm{NaOH}+\mathrm{H}_{2} .
$$

Magnesium does not act readily on cold water, but burns vigorously in steam giving the hydroxide and hydrogen:

$$
\mathrm{Mg}+{ }_{2} \mathrm{H}_{2} \mathrm{O} \rightarrow \mathrm{Mg}(\mathrm{OH})_{2}+\mathrm{H}_{2} .
$$




\section{CHAPTER IX}

\section{ACIDS, BASES, AND SALTS.-II}

I4I. New Acids, Bases, and Salts.-The present chapter will treat of three new acids, carbonic, $\mathrm{H}_{2} \mathrm{CO}_{3}$, acetic, $\mathrm{C}_{2} \mathrm{H}_{4} \mathrm{O}_{2}$, and phosphoric, $\mathrm{H}_{3} \mathrm{PO}_{4}$, and the bases derived from the elements magnesium, calcium, barium, zinc, iron, aluminum, copper, silver, lead, and mercury, together with the more important salts which these bases form with the three acids studied in the first chapter on acids, bases, and salts, as well as with the three acids above-mentioned.

I42. The Action of Water on Magnesium Oxide: Magnesium Hydroxide, $\mathrm{Mg}(\mathrm{OH})_{2}$. - All of the three bases studied in the first chapter on "Acids, Bases, and Salts" are readily soluble in water. We shall next consider one which dissolves in water only to a very slight extent. If we shake, with water, a little magnesium oxide ( $\mathrm{I}, 80$ ), obtained by burning magnesium, we find that the solution will turn red litmus blue, although but a small amount of the magnesium oxide has dissolved in the water, the larger part having remained undissolved. It has been found by careful experiment that magnesium oxide and water unite when brought together, giving a single new compound, the composition of which is represented by $\mathrm{MgO}_{2} \mathrm{H}_{2}$, which we may also write $\mathrm{Mg}(\mathrm{OH})_{2}$, and call magnesium hydroxide. This is a white substance, with which the student may already be familiar under the name of "milk of magnesia." It is extensively used in medicine. It is to be classified as a base, since, like sodium hydroxide, it colors litmus blue and neutralizes hydrocloric acid. The equation for the action of water on the oxide is

$$
\mathrm{MgO}+\mathrm{H}_{2} \mathrm{O} \rightarrow \mathrm{Mg}(\mathrm{OH})_{2} \text {. }
$$

I43. The Action of Hydrochloric Acid on Magnesium Hydroxide: Magnesium Chloride, $\mathrm{MgCl}_{2}$.-Magnesium hydroxide is but very slightly soluble in water. However, if we add hydrochloric acid to the magnesium hydroxide formed from the mag- 
nesium oxide and water until the solution just turns litmus red, we find that all of the solid dissolves, giving a clear, colorless solution which if left to evaporate in an open vessel will deposit colorless crystals. An investigation of this new substance shows that it is a compound of magnesium, chlorine, hydrogen, and oxygen, in the proportion indicated by the formula $\mathrm{MgCl} \cdot 6 \mathrm{H}_{2} \mathrm{O}$. This hydrate of magnesium chloride is formed as a result of the following two reactions:

$$
\begin{aligned}
\mathrm{MIg}(\mathrm{OH})_{2}+2 \mathrm{HCl} & \rightarrow \mathrm{MgCl}_{2}+{ }_{2} \mathrm{H}_{2} \mathrm{O}, \\
\mathrm{IgCl}_{2}+6 \mathrm{H}_{2} \mathrm{O} & \rightarrow \mathrm{MgCl}_{2} \cdot 6 \mathrm{H}_{2} \mathrm{O} .
\end{aligned}
$$

144. Magnesium Sulfate, $\mathrm{MgSO}_{4}$. - If we now add diluted sulfuric acid to some magnesium hydroxide mixed with water, until all of the solid has dissolved and litmus shows the solution to be neutral, we may obtain from the solution by careful cvaporation crystals of magnesium sulfate having the formula $\mathrm{MgSO}_{4} \cdot{ }_{7} \mathrm{H}_{2} \mathrm{O}$, a substance much used in medicine and known as Epsom salts. The reaction occurs according to the equation:

$$
\mathrm{H}_{2} \mathrm{SO}_{4}+\mathrm{Mg}(\mathrm{OH})_{2} \rightarrow \mathrm{MgSO}_{4}+{ }_{2} \mathrm{H}_{2} \mathrm{O},
$$

the $\mathrm{MgSO}_{4}$ then combining with water from the solution, thus:

$$
\mathrm{IgSO}+{ }_{7} \mathrm{H}_{2} \mathrm{O} \rightarrow \mathrm{MgSO}_{4} \cdot 7 \mathrm{H}_{2} \mathrm{O},
$$

to form the hydrate. The latter, when heated, readily dissociates into $\mathrm{MgSO}_{4}$ and ${ }_{7} \mathrm{H}_{2} \mathrm{O}$, a fact which may be expressed thus:

$$
\mathrm{MgSO}_{4} \cdot{ }_{7} \mathrm{H}_{2} \mathrm{O} \rightarrow \mathrm{MgSO}_{4}+{ }_{7} \mathrm{H}_{2} \mathrm{O} \text {. }
$$

This, as we see, is just the reverse of the preceding reaction. The reactions of hydrates in solution are of course the same as those of the anhydrous salts, since solutions of the two cannot be distinguished. In what follows, the discussion of the hydrates formed will be omitted except in a single important instance.

145. Magnesium Nitrate, $\mathrm{Mg}\left(\mathrm{NO}_{3}\right)_{2}$. - Magnesium hydroxide is readily neutralized by nitric acid, with the formation of magnesium nitrate, which forms white crystals very easily soluble in water:

$$
\mathrm{Mg}(\mathrm{OH})_{2}+2 \mathrm{HNO}_{3} \rightarrow \mathrm{Mg}\left(\mathrm{NO}_{3}\right)_{2}+2 \mathrm{H}_{2} \mathrm{O} .
$$


Magnesium oxide and dilute hydrochloric acid react to give magnesium chloride, which is the same compound as that formed from magnesium hydroxide and the same acid. The equation for the reaction is

$$
\mathrm{MgO}+2 \mathrm{HCl} \rightarrow \mathrm{MgCl}_{2}+\mathrm{H}_{2} \mathrm{O} .
$$

The corresponding reactions take place with sulfuric and with nitric acid, and are represented by the equations

$$
\begin{aligned}
\mathrm{MgO}+\mathrm{H}_{2} \mathrm{SO}_{4} & \rightarrow \mathrm{MgSO}_{4}+\mathrm{H}_{2} \mathrm{O}, \\
\mathrm{MgO}+2 \mathrm{HNO}_{3} & \rightarrow \mathrm{Mg}\left(\mathrm{NO}_{3}\right)_{2}+\mathrm{H}_{2} \mathrm{O} .
\end{aligned}
$$

146. Monacid and Diacid Bases: Valence.-If we compare the formula of magnesium chloride, $\mathrm{MgCl}_{2}$, with that of sodium chloride, $\mathrm{NaCl}$, or potassium chloride, $\mathrm{KCl}$, we see that, in the first case, one symbol weight of the metal is combined with two symbol weights of chlorine, while in the other two cases one symbol weight of metal is combined with but one symbol weight of chlorine. In the cases of the neutralization of the hydroxides by hydrochloric acid we found that one formula weight of magnesium hydroxide required two formula weights of hydrochloric acid (143); while one formula weight of the hydroxide of either sodium or potassium required but one of hydrochloric acid (I02, 107). For this reason we call sodium and potassium hydroxides monacid bases, and magnesium hydroxide a diacid base. We also make use of the term valence in referring to facts like those just mentioned, saying that the valence of sodium or potassium is one, while that of magnesium is two, or that sodium and potassium are univalent, while magnesium is a bivalent element. Since hydrogen chloride has the formula $\mathrm{HCl}$, we say that hydrogen has a valence of one, and we also say that the valence of chlorine is one.

147. Radicals and Their Valence.-We have already become acquainted with several ammonium compounds, as, for example, the chloride $\mathrm{NH}_{4} \mathrm{Cl}(9 \mathrm{I})$ and the nitrate $\mathrm{NH}_{4} \mathrm{NO}_{3}$ (I05). We call the combination $\mathrm{NH}_{4}$ the ammonium radical; it has never been obtained as a separate substance, but is known only as a component of ammonium compounds. We know of many other such radicals, one of which is met with in sulfuric acid and sul- 
fates, where we have found that sulfur and oxygen are always present in the ratio represented by $\mathrm{SO}_{4}$. Here again we have a radical which is found in many salts, the sulfates, but is not known as a separate chemical substance. In nitric acid and the nitrates we have the radical $\mathrm{NO}_{3}$. A radical is composed of two or more elements united in a definite proportion; consequently the composition of a radical can always be represented by a formula. We may consider that the combination of nitrogen and hydrogen, $\mathrm{NH}_{4}$, taken as a radical, has a valence of one, since the weight of nitrogen and hydrogen represented by $\mathrm{NH}_{4}$ taken once unites with one symbol weight of chlorine, giving $\mathrm{NH}_{4} \mathrm{Cl}$. Since sulfuric acid, $\mathrm{H}_{2} \mathrm{SO}_{4}$, forms such salts as $\mathrm{Na}_{2} \mathrm{SO}_{4}$, $\mathrm{K}_{2} \mathrm{SO}_{4}$ and $\left(\mathrm{NH}_{4}\right)_{2} \mathrm{SO}_{4}$, we say that the sulfate radical, $\mathrm{SO}_{4}$, has a valence of two, a fact which is also shown by the existence of such salts as $\mathrm{NaHSO}_{4}$, etc. Now if magnesium has a valence of two and the sulfate radical has also the valence of two, we see in the fact that magnesium sulfate has the formula $\mathrm{MgSO}_{4}$, a broader meaning of the term valence. And so chemists of ten speak of the two valences of magnesium being satisfied by the two valences of the sulfate radical. The subject of valence will be considered again at the end of this chapter ( 183 ).

148. Zinc and Its Salts. - Zinc is an element and a very important metal; it is known in commerce as spelter, and is used in enormous amounts in making galvanized iron, which is iron coated with metallic zinc, in making brass, whose other component is copper, and for many other purposes. Zinc will burn when strongly heated in the air or in oxygen, giving a white oxide, the reaction being represented by the equation

$$
2 \mathrm{Zn}+\mathrm{O}_{2} \rightarrow 2 \mathrm{ZnO} \text {. }
$$

Zinc oxide is used extensively in making white paint.

It will be recalled that magnesium burns, giving an oxide (II, 80), and that this oxide reacts with acids giving salts, thus:

$$
\mathrm{MgO}+{ }_{2} \mathrm{HCl} \rightarrow \mathrm{MgCl}_{2}+\mathrm{H}_{2} \mathrm{O} \text {. }
$$

$(143,145)$

Zinc oxide behaves like magnesium oxide when treated with hydrochloric acid, giving zinc chloride, thus:

$$
\mathrm{ZnO}+2 \mathrm{HCl} \rightarrow \mathrm{ZnCl}_{2}+\mathrm{H}_{2} \mathrm{O} \text {. }
$$


Zinc chloride is a salt which dissolves very readily in water, giving a clear, colorless solution. Zinc oxide gives zinc sulfate and zinc nitrate as follows:

$$
\begin{aligned}
\mathrm{ZnO}+\mathrm{H}_{2} \mathrm{SO}_{4} & \rightarrow \mathrm{ZnSO}_{4}+\mathrm{H}_{2} \mathrm{O}, \\
\mathrm{ZnO}+2 \mathrm{HNO}_{3} & \rightarrow \mathrm{Zn}\left(\mathrm{NO}_{3}\right)_{2}+\mathrm{H}_{2} \mathrm{O} .
\end{aligned}
$$

These are white salts, also easily soluble in water.

149. The Action of Hydrochloric Acid on Zinc.-If we pour some hydrochloric acid on zinc we observe a vigorous reaction; the zinc dissolves and a gas which proves to be hydrogen is given off. If, after the zinc has all dissolved, we evaporate the solution, we obtain a white solid which is found to be zinc chloride. The reaction is represented thus:

$$
\mathrm{Zn}+{ }_{2} \mathrm{HCl} \rightarrow \mathrm{ZnCl}_{2}+\mathrm{H}_{2} \text {. }
$$

Comparing this equation with that for the action of zinc oxide on hydrochloric acid, we see that in the latter case the hydrogen of the acid, instead of passing off as gas, unites with the oxygen of the zinc oxide, giving water.

We might expect that metallic magnesium and hydrochloric acid would act thus:

$$
\mathrm{Mg}+{ }_{2} \mathrm{HCl} \rightarrow \mathrm{MgCl}_{2}+\mathrm{H}_{2},
$$

and it is easy to show by experiment that this is the case. With dilute sulfuric acid these metals behave as follows:

$$
\begin{aligned}
\mathrm{Mg}+\mathrm{H}_{2} \mathrm{SO}_{4} & \rightarrow \mathrm{MgSO}_{4}+\mathrm{H}_{2}, \\
\mathrm{Zn}+\mathrm{H}_{2} \mathrm{SO}_{4} & \rightarrow \mathrm{ZnSO}_{4}+\mathrm{H}_{2} .
\end{aligned}
$$

In making hydrogen in the laboratory we usually use zinc and hydrochloric acid.

I50. Marble and Other Compounds of the Element Calcium.-Let us now consider the chemical behavior of marble. If we place some lumps of marble in a hard glass tube and heat strongly, a gas is given off, while the lumps change but little in appearance. This gas causes limewater to turn milky; it is carbon dioxide (I9). If the lumps left after heating the marble are moistened with water, they grow very hot, swell up, and crumble to a white powder. It is evident therefore that the 
marble has been changed chemically by the heating. The solid left after the heating is the common substance, quicklime. The action of water upon quicklime is called slaking. If the slaked lime is shaken with a large amount of water, not much seems to dissolve; but if we filter the mixture, a clear, colorless solution is obtained. If some carbon dioxide gas is run into this clear solution it turns milky, because this solution is limewater (18), of which we have so often made use. If we test the limewater with litmus we find that it turns the latter blue, showing the limewater to be a solution of a base. This base reacts with acids to form salts. All of these products contain an element called calcium, whose symbol is $\mathrm{Ca}$. Calcium is a brassy-looking metal, which will readily burn with a bright light if heated in air or oxygen, giving calcium oxide:

$$
{ }_{2} \mathrm{Ca}+\mathrm{O}_{2} \rightarrow{ }_{2} \mathrm{CaO} \text {. }
$$

Calcium oxide, $\mathrm{CaO}$, is quicklime; but the latter is never made practically in this way, because metallic calcium is too expensive, and because the oxide is made very cheaply by heating marble or, more often, limestone, which is an impure form of the same compound as marble. By heating marble, $\mathrm{CaO}$ and $\mathrm{CO}_{2}$ are formed, and nothing else. By finding the percentage of each we can easily calculate the formula for marble to be $\mathrm{CaCO}_{3}$, which is called calcium carbonate; the effect of the heating is, therefore, represented thus:

$$
\mathrm{CaCO}_{3} \rightarrow \mathrm{CaO}+\mathrm{CO}_{2} \text {. }
$$

I5I. Calcium Hydroxide, $\mathrm{Ca}(\mathrm{OH})_{2}$. - As has been stated, when water acts on calcium oxide or quicklime, we get calcium hydroxide or slaked lime, a solution of which is called limewater:

$$
\mathrm{CaO}+\mathrm{H}_{2} \mathrm{O} \rightarrow \mathrm{Ca}(\mathrm{OH})_{2} \text {. }
$$

This reaction is analogous to the action of water on magnesium oxide, which was studied earlier (142). The action of hydrochloric acid on calcium hydroxide gives calcium chloride and water:

$$
\mathrm{Ca}(\mathrm{OH})_{2}+2 \mathrm{HCl} \rightarrow \mathrm{CaCl}_{2}+2 \mathrm{H}_{2} \mathrm{O} \text {. }
$$


We are now in position to understand the cause of the milkiness produced when carbon dioxide acts on limewater. The white solid formed is really calcium carbonate, $\mathrm{CaCO}_{3}$. The equation is

$$
\mathrm{Ca}(\mathrm{OH})_{2}+\mathrm{CO}_{2} \rightarrow \mathrm{CaCO}_{3}+\mathrm{H}_{2} \mathrm{O} .
$$

152. Carbonic Acid, $\mathrm{H}_{2} \mathrm{CO}_{3}$. - If we pass carbon dioxide into water, a solution results which has faint acid properties. This solution is in fact the well-known plain soda served at soda fountains. The dissolved carbon dioxide and water partially combine to form an acid called carbonic acid:

$$
\mathrm{CO}_{2}+\mathrm{H}_{2} \mathrm{O} \rightarrow \mathrm{H}_{2} \mathrm{CO}_{3} \text {. }
$$

Therefore we may then consider that it is this acid which neutralizes the base calcium hydroxide, thus:

$$
\mathrm{Ca}(\mathrm{OH})_{2}+\mathrm{H}_{2} \mathrm{CO}_{3} \rightarrow \mathrm{CaCO}_{3}+{ }_{2} \mathrm{H}_{2} \mathrm{O} \text {. }
$$

Calcium carbonate is a salt which is almost insoluble in water. In fact, salts exhibit all degrees of solubility in water. Some, like zinc chloride, dissolve in less than their own weight of water; others, like common salt, are much less soluble; while many, like calcium carbonate, are very nearly insoluble in water.

I53. Calcium Sulfate, $\mathrm{CaSO}_{4}$ - - Calcium hydroxide and sulfuric acid form calcium sulfate and water:

$$
\mathrm{Ca}(\mathrm{OH})_{2}+\mathrm{H}_{2} \mathrm{SO}_{4} \rightarrow \mathrm{CaSO}_{4}+{ }_{2} \mathrm{H}_{2} \mathrm{O} \text {. }
$$

We find by experiment that the calcium sulfate so formed dissolves very slightly in water, Ioo c.c. of water dissolving but one-fourth of a gram of the salt. On the other hand, calcium chloride is very soluble in water. If we add to a solution containing, say, 5 or ro per cent of calcium chloride, a sufficient amount of sulfuric acid, we observe that a large amount of a white powder forms in the mixed solutions and soon settles to the bottom of the vessel, leaving a clear, colorless liquid above. The white powder proves to be calcium sulfate, which is formed thus:

$$
\mathrm{CaCl}_{2}+\mathrm{H}_{2} \mathrm{SO}_{4} \rightarrow \mathrm{CaSO}_{4}+2 \mathrm{HCl} \text {. }
$$


154. Precipitation.-We often encounter chemical reactions in which, as in the action between calcium chloride and sulfuric acid, a solid is formed upon bringing together two solutions. A solid so thrown down is called a precipitate; and we speak of the precipitation of calcium sulfate. The formation of insoluble calcium carbonate by the action of carbon dioxide on limewater is another example of precipitation.

155. Gypsum and Plaster of Paris.-Calcium sulfate occurs in nature as the mineral gypsum, $\mathrm{CaSO}_{4} \cdot{ }_{2} \mathrm{H}_{2} \mathrm{O}$. If part of the water of hydration is driven off by heat, gypsum is converted into the well-known plaster of Paris:

$$
{ }_{2} \mathrm{CaSO}_{4} \cdot{ }_{2} \mathrm{H}_{2} \mathrm{O} \rightarrow{ }_{2} \mathrm{CaSO}_{4} \cdot \mathrm{H}_{2} \mathrm{O}+{ }_{3} \mathrm{H}_{2} \mathrm{O} \text {. }
$$

When powdered plaster of Paris is mixed with enough water to form a paste, it sets in the course of an hour into a solid mass which retains the form of the vessel or mold which holds it. Plaster casts are made in this way. The setting is due to the formation of interlacing crystal filaments of the hydrate $\mathrm{CaSO}_{4} \cdot 2 \mathrm{H}_{2} \mathrm{O}$, formed by a reversal of the action by which plaster of Paris is formed from gypsum:

$$
{ }_{2} \mathrm{CaSO}_{4} \cdot \mathrm{H}_{2} \mathrm{O}+{ }_{3} \mathrm{H}_{2} \mathrm{O} \rightarrow{ }_{2} \mathrm{CaSO}_{4} \cdot{ }_{2} \mathrm{H}_{2} \mathrm{O} \text {. }
$$

156. Calcium Bicarbonate and Hard Water.-A very interesting and important reaction occurs when carbon dioxide is passed for a long time into a sufficiently dilute solution of calcium hydroxide (limewater). At first a milkiness appears, due to the formation of calcium carbonate:

$$
\mathrm{Ca}(\mathrm{OH})_{2}+\mathrm{CO}_{2} \rightarrow \mathrm{CaCO}_{3}+\mathrm{H}_{2} \mathrm{O} \text {. }
$$

If we continue to pass in carbon dioxide, the precipitate slowly dissolves, giving finally a perfectly clear solution. If this solution is now boiled, carbon dioxide gas is given off and a white precipitate is formed. These facts are explained in the following way. Carbonic acid, $\mathrm{H}_{2} \mathrm{CO}_{3}$, like sulfuric acid, is a dibasic acid (I02) and can form acid salts as wcll as neutral salts. Just as sulfuric acid yields $\mathrm{Na}_{2} \mathrm{SO}_{4}$ and $\mathrm{NaHSO}_{4}$, so carbonic acid gives $\mathrm{Na}_{2} \mathrm{CO}_{3}$ and $\mathrm{NaHCO}_{3}$, sodium carbonate and sodium acid carbonate, also known as bicarbonate (baking-soda). 
The calcium salts corresponding to sodium carbonate and bicarbonate are $\mathrm{CaCO}_{3}$ and $\mathrm{Ca}\left(\mathrm{HCO}_{3}\right)_{2}$. The difference in the formulae of the sodium and calcium salts is due to the fact that the valence of calcium is two, while that of sodium is one. Now when carbon dioxide, in excess, acts on calcium carbonate, calcium acid carbonate, called also bicarbonate, is formed, and this being soluble in water the precipitate goes into solution:

$$
\begin{aligned}
\mathrm{CO}_{2}+\mathrm{H}_{2} \mathrm{O} & \rightarrow \mathrm{H}_{2} \mathrm{CO}_{3} \\
\mathrm{CaCO}_{3}+\mathrm{H}_{2} \mathrm{CO}_{3} & \rightarrow \mathrm{Ca}\left(\mathrm{HCO}_{3}\right)_{2} .
\end{aligned}
$$

When the clear solution so obtained is boiled the following reaction occurs:

$$
\mathrm{Ca}\left(\mathrm{HCO}_{3}\right)_{2} \rightarrow \mathrm{CaCO}_{3}+\mathrm{H}_{2} \mathrm{O}+\mathrm{CO}_{2} \text {. }
$$

These reactions take place extensively in nature. Natural waters, e.g., those of springs and rivers, contain dissolved carbon dioxide, and therefore carbonic acid. Such waters passing over limestone, impure $\mathrm{CaCO}_{3}$, dissolve it and take the $\mathrm{Ca}\left(\mathrm{HCO}_{3}\right)_{2}$ into solution, forming so-called hard water. When boiled, as in a teakettle, it gives off carbon dioxide and deposits the calcium carbonate.

157. Vinegar: Acetic Acid, $\mathrm{C}_{2} \mathrm{H}_{4} \mathrm{O}_{2}$. - Acetic acid is the principal ingredient, other than water, in vinegar, of which it constitutes about 4 per cent. The formula of acetic acid is $\mathrm{C}_{2} \mathrm{H}_{4} \mathrm{O}_{2}$. It neutralizes sodium hydroxide according to the equation

$$
\mathrm{C}_{2} \mathrm{H}_{4} \mathrm{O}_{2}+\mathrm{NaOH}=\mathrm{NaC}_{2} \mathrm{H}_{3} \mathrm{O}_{2}+\mathrm{H}_{2} \mathrm{O} \text {. }
$$

The salt $\mathrm{NaC}_{2} \mathrm{H}_{3} \mathrm{O}_{2}$, sodium acetate, is the only sodium salt which can be made from this acid. Therefore the acid radical of acetic acid and its salts is $\mathrm{C}_{2} \mathrm{H}_{3} \mathrm{O}_{2}$ and we may write the formula of the acid $\mathrm{HC}_{2} \mathrm{H}_{3} \mathrm{O}_{2}$ to indicate that only one of the four hydrogen atoms of a molecule is replaceable in salt formation.

Pure acetic acid is a colorless liquid, miscible with water in all proportions. It is monobasic and forms with most bases salts called acetates.

158. Bone Ash: Calcium Phosphate, $\mathrm{Ca}_{3}\left(\mathrm{PO}_{4}\right)_{2}$.-When bones are burned only the gelatinous matter and connective tissue are removed; the white material which is left is called 
bone ash and consists essentially of calcium phosphate, $\mathrm{Ca}_{3}\left(\mathrm{PO}_{4}\right)_{2}$. If powdered bone ash, which is practically insoluble in water, is stirred with somewhat diluted sulfuric acid, the following reaction occurs:

$$
\mathrm{Ca}_{3}\left(\mathrm{PO}_{4}\right)_{2}+{ }_{3} \mathrm{H}_{2} \mathrm{SO}_{4} \rightarrow{ }_{3} \mathrm{CaSO}_{4}+2 \mathrm{H}_{3} \mathrm{PO}_{4} .
$$

The calcium sulfate formed is difficultly soluble in water and may be filtered out, giving a clear, colorless filtrate containing dissolved phosphoric acid, $\mathrm{H}_{3} \mathrm{PO}_{4}$.

159. Phosphoric Acid: a Tribasic Acid.-This acid is a white crystalline solid, which is very soluble in water, frequently coming on the market in the form of a very concentrated solution of syrupy consistency. Its dilute solution has a pleasant sour taste and turns litmus red. With suitable proportions of sodium hydroxide it yiclds the three salts, $\mathrm{Na}_{3} \mathrm{PO}_{4}$, trisodium phosphate, $\mathrm{Na}_{2} \mathrm{HPO}_{4}$, disodium hydrogen phosphate, and $\mathrm{NaH}_{2} \mathrm{PO}_{4}$, sodium dihydrogen phosphate. The latter is a typical acid salt, having a sour taste and acid action on litmus. Phosphoric acid is therefore a tribasic acid. It forms with bases three series of salls, corresponding to those of sodium. To distinguish these classes of salts from one another they are called primary, secondary, and tertiary, that with the smallest proportion of base being the primary and that in which all hydrogen is replaced being the tertiary.

I60. The Practical Importance of Calcium Phosphate.Since calcium phosphate, $\mathrm{Ca}_{3}\left(\mathrm{PO}_{4}\right)_{2}$, constitutes the mineral matter of bones, it is, of course, a substance of very great importance. Phosphates in small amounts are also indispensable constituents of most plants, and it is from these, especially from the seeds, like wheat, oats, and corn, that men and animals get their needed supply. Plants, in turn, get their phosphates from the soil, and do not thrive on soil deficient in phosphates. Such infertile soil may be greatly improved by the use of fertilizers containing phosphates. For this purpose, bone ash is often employed; but since bone ash is almost insoluble in water, it is not directly a vailable for plant use. In order to make it available it is treated with sufficient sulfuric acid to convert it into 
$\mathrm{Ca}\left(\mathrm{H}_{2} \mathrm{PO}_{4}\right)_{2}$, usually known as calcium superphosphate, which is soluble in water:

$$
\mathrm{Ca}_{3}\left(\mathrm{PO}_{4}\right)_{2}+2 \mathrm{H}_{2} \mathrm{SO}_{4} \rightarrow \mathrm{Ca}\left(\mathrm{H}_{2} \mathrm{PO}_{4}\right)_{2}+{ }_{2} \mathrm{CaSO}_{4} \text {. }
$$

Immense deposits of calcium phosphate occur in Florida and Tennessee, as phosphate rock. These deposits have doubtless been formed in past geological ages from the bones of marine animals. Phosphate rock, after treatment with sulfuric acid as in the case of bone ash, is used in enormous quantities as a fertilizer.

I6I. Sodium Carbonate and Bicarbonate.-The carbonates of sodium which were referred to above (156) may be obtained by passing carbon dioxide into sodium hydroxide solution; we get in this way either the carbonate $\mathrm{Na}_{2} \mathrm{CO}_{3}$, or the bicarbonate $\mathrm{NaHCO}_{3}$, according to the proportion of carbon dioxide used. We may consider that the gas first unites with water to form carbonic acid, which then reacts with sodium hydroxide according to the two following equations:

$$
\begin{aligned}
{ }_{2} \mathrm{NaOH}+\mathrm{H}_{2} \mathrm{CO}_{3} & \rightarrow \mathrm{Na}_{2} \mathrm{CO}_{3}+{ }_{2} \mathrm{H}_{2} \mathrm{O}, \\
\mathrm{NaOH}+\mathrm{H}_{2} \mathrm{CO}_{3} & \rightarrow \mathrm{NaHCO}_{3}+\mathrm{H}_{2} \mathrm{O} .
\end{aligned}
$$

These carbonates of sodium are manufactured in immense quantities, as they are very important substances. In practice they are not made according to the reactions given, but by more economical processes, which will be considered later.

162. Potassium Carbonate and Bicarbonate.-Potassium also forms analogous carbonates, $\mathrm{K}_{2} \mathrm{CO}_{3}$ and $\mathrm{KHCO}_{3}$; the former, commonly known as potash, is contained in wood ashes, from which it may be dissolved by water. Upon boiling down the solution known popularly as lye, a residue of crude potassium carbonate, $\mathrm{K}_{2} \mathrm{CO}_{3}$, remains. This, when more strongly heated to burn out brown tarry matters, gives white potash, so called from the fact that the evaporation of the lye is carried out in an iron pot. This lye is extensively used in the preparation of a crude soft soap. A purer form of potash is used in manufacturing liquid soaps. Common hard soap is made from sodium carbonate and fats of various kinds. 
163. The Action of Acids on Carbonates.-If some hydrochloric acid is poured on a piece of marble (150), the liquid appears to boil, although the temperature does not rise noticeably. It is easy to show that the apparent boiling, called effervescence, is due to the escape of carbon dioxide gas. The marble dissolves completely if sufficient acid is used, and the evaporated solution leaves a residue of calcium chloride (I5I). The reaction is as follows:

$$
\mathrm{CaCO}_{3}+{ }_{2} \mathrm{HCl} \rightarrow \mathrm{CaCl}_{2}+\mathrm{H}_{2} \mathrm{O}+\mathrm{CO}_{2} \text {. }
$$

Similar reactions take place between calcium carbonate and nitric and sulfuric acids:

$$
\begin{aligned}
\mathrm{CaCO}_{3}+2 \mathrm{HNO}_{3} & \rightarrow \mathrm{Ca}\left(\mathrm{NO}_{3}\right)_{2}+\mathrm{H}_{2} \mathrm{O}+\mathrm{CO}_{2}, \\
\mathrm{CaCO}_{3}+\mathrm{H}_{2} \mathrm{SO}_{4} & \rightarrow \mathrm{CaSO}_{4}+\mathrm{H}_{2} \mathrm{O}+\mathrm{CO}_{2} .
\end{aligned}
$$

In fact, the carbonates of other elements all show this kind of a reaction with these acids; for example:

$$
\begin{aligned}
\mathrm{NaHCO}_{3}+\mathrm{HCl} & \rightarrow \mathrm{NaCl}+\mathrm{H}_{2} \mathrm{O}+\mathrm{CO}_{2}, \\
\mathrm{~K}_{2} \mathrm{CO}_{3}+2 \mathrm{HNO}_{3} & \rightarrow 2 \mathrm{KNO}_{3}+\mathrm{H}_{2} \mathrm{O}+\mathrm{CO}_{2} .
\end{aligned}
$$

In general, carbonates are decomposed by acids.

I64. Barium Sulfate: a Test for Sulfates.-The element barium resembles calcium (150) very closely in its behavior. Let us consider just one of its reactions at present, leaving a study of the others until a later time. Barium sulfate, $\mathrm{BaSO}_{4}$, is a white solid which is as insoluble in water as glass; barium chloride, $\mathrm{BaCl}_{2}$, is about as soluble as common salt. If we pour some sulfuric acid into a clear, colorless solution of barium chloride, a white precipitate of barium sulfate forms at once:

$$
\mathrm{BaCl}_{2}+\mathrm{H}_{2} \mathrm{SO}_{4} \rightarrow \mathrm{BaSO}_{4}+2 \mathrm{HCl} \text {. }
$$

We should observe the similarity of this equation to that for the action of sulfuric acid on calcium chloride (153).

If we add to a solution of barium chloride a solution of sodium sulfate, or of magnesium sulfate, or in fact of any sulfate whatsoever, a precipitate of barium sulfate is formed. For example, with magnesium sulfate we have

$$
\mathrm{BaCl}_{2}+\mathrm{MgSO}_{4} \rightarrow \mathrm{BaSO}_{4}+\mathrm{MgCl}_{2} \text {. }
$$


Unlike other common barium salts the sulfate is insoluble not only in water but also in acids. If barium chloride is added to a solution and a white precipitate appears which has these properties, a sulfate is proved to be present.

I65. Copper and Its Compounds. - The important, familiar metal copper is an element. We have already learned that when heated in air or oxygen it unites with oxygen to form copper oxide $(32,33,82)$, a black solid:

$$
{ }_{2} \mathrm{Cu}+\mathrm{O}_{2} \rightarrow{ }_{2} \mathrm{CuO} \text {. }
$$

Copper oxide reacts with the corresponding acids to form the chloride, nitrate, and sulfate, thus:

$$
\begin{aligned}
\mathrm{CuO}+2 \mathrm{HCl} & \rightarrow \mathrm{CuCl}_{2}+\mathrm{H}_{2} \mathrm{O} \\
\mathrm{CuO}+2 \mathrm{HNO}_{3} & \rightarrow \mathrm{Cu}\left(\mathrm{NO}_{3}\right)_{2}+\mathrm{H}_{2} \mathrm{O} \\
\mathrm{CuO}+\mathrm{H}_{2} \mathrm{SO}_{4} & \rightarrow \mathrm{CuSO}_{4}+\mathrm{H}_{2} \mathrm{O} .
\end{aligned}
$$

These salts are all easily soluble in water, giving blue solutions.

It will be recalled that calcium oxide unites with water to form the hydroxide, thus:

$$
\mathrm{CaO}+\mathrm{H}_{2} \mathrm{O} \rightarrow \mathrm{Ca}(\mathrm{OH})_{2} \text {. }
$$

On the other hand, if we bring copper oxide and water together, no union takes place. This might be taken to indicate that copper hydroxide, which we might expect to have the formula $\mathrm{Cu}(\mathrm{OH})_{2}$, cannot be formed or does not exist. This, however, is not the case; it is a well-known substance which is easily obtained in another way. If we add to a solution of copper sulfate a solution of sodium hydroxide, a blue precipitate of copper hydroxide forms. This is a blue solid which is very nearly insoluble in water. Its formation takes place thus:

$$
\mathrm{CuSO}_{4}+2 \mathrm{NaOH} \rightarrow \mathrm{Cu}(\mathrm{OH})_{2}+\mathrm{Na}_{2} \mathrm{SO}_{4} .
$$

We may also get copper hydroxide by the interaction of solutions of copper chloride or nitrate with sodium hydroxide:

$$
\mathrm{CuCl}_{2}+2 \mathrm{NaOH} \rightarrow \mathrm{Cu}(\mathrm{OH})_{2}+2 \mathrm{NaCl} \text {. }
$$

If the copper hydroxide formed in the last reaction is heated by boiling the mixture, the blue precipitate turns black. This 
change in color is due to a change of part of the hydroxide into the oxide and water:

$$
\mathrm{Cu}(\mathrm{OH})_{2} \rightarrow \mathrm{CuO}+\mathrm{H}_{2} \mathrm{O} \text {. }
$$

I66. The Preparation of Difficultly Soluble Hydroxides. Many hydroxides of elements are nearly insoluble in water. In such cases, the hydroxides are formed from a solution of a salt of the element by adding sodium hydroxide, or potassium hydroxide, or in many cases ammonium hydroxide, as illustrated by the following equations:

$$
\begin{gathered}
\mathrm{CaCl}_{2}+2 \mathrm{KOH} \rightarrow \mathrm{Ca}(\mathrm{OH})_{2}+2 \mathrm{KCl}, \\
\mathrm{IgSO}_{4}+2 \mathrm{NaOH} \rightarrow \mathrm{Mg}(\mathrm{OH})_{2}+\mathrm{Na}_{2} \mathrm{SO}_{4} .
\end{gathered}
$$

Such difficultly soluble hydroxides separate as precipitates, which may be filtered out.

167. Lead and Its Compounds.--The well-known metal lead is an element, which is used extensively in metallic form and also in the form of compounds. Lead unites with oxygen directly when heated in air or oxygen, giving, under suitable conditions, the pale yellow oxide $\mathrm{PbO}$, known as litharge. This oxide, like those of magnesium, zinc, and copper, reacts with acids to form salts. Thus with nitric acid we get lead nitrate:

$$
\mathrm{PbO}+{ }_{2} \mathrm{HNO}_{3} \rightarrow \mathrm{Pb}\left(\mathrm{NO}_{3}\right)_{2}+\mathrm{H}_{2} \mathrm{O} \text {. }
$$

This salt forms large, white crystals which dissolve readily in water to form a colorless solution. If hydrochloric acid is added to a solution of lead nitrate, a white precipitate of lead chloride is obtained:

$$
\mathrm{Pb}\left(\mathrm{NO}_{3}\right)_{2}+2 \mathrm{HCl} \rightarrow \mathrm{PbCl}_{2}+2 \mathrm{HNO}_{3} \text {. }
$$

Lead chloride is only slightly soluble in cold water, but is much more soluble in hot water, from which, upon cooling, it separates again in white needle-shaped crystals. Lead chloride is also obtained from litharge and hydrochloric acid:

$$
\mathrm{PbO}+{ }_{2} \mathrm{HCl} \rightarrow \mathrm{PbCl}_{2}+\mathrm{H}_{2} \mathrm{O} \text {. }
$$


Upon adding dilute sulfuric acid to a solution of lead nitrate, a white precipitate of lead sulfate forms:

$$
\mathrm{Pb}\left(\mathrm{NO}_{3}\right)_{2}+\mathrm{H}_{2} \mathrm{SO}_{4} \rightarrow \mathrm{PbSO}_{4}+{ }_{2} \mathrm{HNO}_{3} \text {. }
$$

Lead sulfate is nearly insoluble in hot or cold water.

Metallic lead is acted upon very slowly by hydrochloric acid. With the cold dilute acid no appreciable action takes place; with boiling, concentrated acid, a very slow reaction occurs, thus:

$$
\mathrm{Pb}+2 \mathrm{HCl} \rightarrow \mathrm{PbCl}_{2}+\mathrm{H}_{2} \text {. }
$$

By methods to be considered later, it is possible to prepare an oxide of lead containing double the proportion of oxygen present in $\mathrm{PbO}$, namely $\mathrm{PbO}_{2}$, or lead dioxide. This oxide does not react with dilute nitric acid. When it is heated with hydrochloric acid it gives lead chloride and chlorine:

$$
\mathrm{PbO}_{2}+{ }_{4} \mathrm{HCl} \rightarrow \mathrm{PbCl}_{2}+{ }_{2} \mathrm{H}_{2} \mathrm{O}+\mathrm{Cl}_{2} \text {. }
$$

If we compare this reaction with the following,

$$
\mathrm{PbO}+{ }_{2} \mathrm{HCl} \rightarrow \mathrm{PbCl}_{2}+\mathrm{H}_{2} \mathrm{O},
$$

we see that the excess of oxygen in $\mathrm{PbO}_{2}$ above that in $\mathrm{PbO}$ oxidizes the hydrochloric acid, forming water and sctting free chlorine.

Lead acetate, $\mathrm{Pb}\left(\mathrm{C}_{2} \mathrm{H}_{3} \mathrm{O}_{2}\right)_{2} \cdot 3 \mathrm{H}_{2} \mathrm{O}$, is formed by dissolving litharge, $\mathrm{PbO}$, in acetic acid. It forms colorless prismatic crystals, which are readily soluble in water. It is a poisonous salt and is called sugar of lead on account of its sweetish taste.

168. Silver and Its Compounds.-Silver is so familiar a metal that we need not describe its properties. It is an element which is most extensively used in the metallic form, but which forms several compounds of great practical importance. The metal is not readily acted upon by dilute acids, with the exception of nitric acid, with which it undergoes a complex reaction represented by the equation

$$
{ }_{3} \mathrm{Ag}+{ }_{4} \mathrm{HNO}_{3} \rightarrow{ }_{3} \mathrm{AgNO}_{3}+\mathrm{NO}+{ }_{2} \mathrm{H}_{2} \mathrm{O} .
$$

We need not consider this reaction critically at this time, although it is well worth careful study; but note that silver 
nitrate is an casily soluble salt, forming a colorless solution. The solid salt forms large white crystals.

r69. Silver Chloride, $\mathrm{AgCl}$. - The addition of hydrochloric acid to a solution of silver nitrate produces at once a heavy white precipitate of silver chloride, which is almost insoluble in water:

$$
\mathrm{AgNO}_{3}+\mathrm{HCl} \rightarrow \mathrm{AgCl}+\mathrm{HNO}_{3} \text {. }
$$

By adding an excess of hydrochloric acid practically all of the silver in a solution is precipitated. The precipitate does not dissolve appreciably in any of the common acids. It is, however, "cry casily soluble in ammonia solution, from which it is again thrown down if the solution is acidificd with nitric or hydrochloric acid. If any solution of unknown nature gives with hydrochloric acid a white precipitate which is insoluble in an excess of the acid, but easily soluble in ammonia, from which solution it is thrown down by acidifying the solution with hydrochloric acid, it is safe to conclude that the original solution contained a salt of silver. This series of reactions constitutes a test for silver in the form of a dissolved salt.

I70. Silver Sulfate, $\mathrm{Ag}_{2} \mathrm{SO}_{4}$.- This salt is formed as a whitc crystalline precipitate when sulfuric acid is added to a concentrated solution of silver nitrate. It is not very soluble, I g. requiring about 200 c.c. of cold water for its solution. The same salt is also formed by the action of hot, concentrated sulfuric acid on metallic silver.

I7r. Silver Phosphate, $\mathrm{Ag}_{3} \mathrm{PO}_{4}$. - This salt is formed as a yellow precipitate when sodium phosphate, $\mathrm{Na}_{3} \mathrm{PO}_{4}$, or some other soluble phosphate is added to a solution of silver nitrate:

$$
3 \mathrm{AgNO}_{3}+\mathrm{Na}_{3} \mathrm{PO}_{4} \rightarrow \mathrm{Ag}_{3} \mathrm{PO}_{4}+3 \mathrm{NaNO}_{3} \text {. }
$$

This yellow precipitate is readily soluble in dilute nitric acid, forming a colorless solution. It also dissolves easily in aqueous ammonia, giving a colorless solution, from which it is again thrown down when the solution is exactly neutralized with nitric acid.

Silver may be distinguished from lead most easily by reason of the solubility of lead chloride in hot water, in which silver chloride is insoluble. 
172. Silver Oxide, $\mathrm{Ag}_{2} \mathrm{O}$.-The addition of sodium hydroxide to a solution of silver nitrate gives a black precipitate of silver oxide, $\mathrm{Ag}_{2} \mathrm{O}$.' We might expect silver hydroxide, $\mathrm{AgOH}$. to be formed thus:

$$
\mathrm{AgNO}_{3}+\mathrm{NaOH} \rightarrow \mathrm{AgOH}+\mathrm{NaNO}_{3} .
$$

Possibly this is what first happens, but, if so, the hydroxide formed changes at once in to the oxide,

$$
2 \mathrm{AgOH} \rightarrow \mathrm{Ag}_{2} \mathrm{O}+\mathrm{H}_{2} \mathrm{O} .
$$

It will be recalled that copper hydroxide is decomposed at the temperature of boiling water into the oxide and water (I65). In the case of silver hydroxide the change takes place at room temperature.

I73. Iron and Its Compounds.-The element iron is the most important of all metals. It unites directly with oxygen at a red heat, forming the oxide $\mathrm{Fe}_{3} \mathrm{O}_{4}(8 \mathrm{I})$. It can also form two other oxides, $\mathrm{FeO}$ and $\mathrm{Fe}_{2} \mathrm{O}_{3}$. The oxide $\mathrm{Fe}_{3} \mathrm{O}_{4}$ is magnetic and is called magnetic iron oxide; $\mathrm{FeO}$ is called ferrous oxide (from ferrum, iron), while $\mathrm{Fe}_{2} \mathrm{O}_{3}$ is called ferric oxide. Ferrous oxide gives, with the corresponding acids, ferrous chloride, $\mathrm{FeCl}_{2}$, and ferrous sulfate, $\mathrm{FeSO}_{4}$. These salts are also formed from iron by the following reactions:

$$
\begin{aligned}
& \mathrm{Fe}+{ }_{2} \mathrm{HCl} \rightarrow \mathrm{FeCl}_{2}+\mathrm{H}_{2}, \\
& \mathrm{Fe}+\mathrm{H}_{2} \mathrm{SO}_{4} \rightarrow \mathrm{FeSO}_{4}+\mathrm{H}_{2} .
\end{aligned}
$$

In all ferrous compounds the valence of iron is two.

The action of hydrochloric acid on ferric oxide takes place thus:

$$
\mathrm{Fe}_{2} \mathrm{O}_{3}+6 \mathrm{HCl} \rightarrow{ }_{2} \mathrm{FeCl}_{3}+{ }_{3} \mathrm{H}_{2} \mathrm{O} .
$$

The salt $\mathrm{FeCl}_{3}$ is called ferric chloride. It is a dark-yellow substance which dissolves easily in water to form a yellow solution. On the other hand, ferrous chloride, $\mathrm{FeCl}_{2}$, is pale green and forms a pale-green solution. We cannot get $\mathrm{FeCl}_{3}$ from iron and hydrochloric acid, but we do get the salt by the action of chlorine on ferrous chloride,

$$
2 \mathrm{FeCl}_{2}+\mathrm{Cl}_{2} \rightarrow 2 \mathrm{FeCl}_{3},
$$


or on iron,

$$
{ }_{2} \mathrm{Fe}+{ }_{3} \mathrm{Cl}_{2} \rightarrow{ }_{2} \mathrm{FeCl}_{3} \text {. }
$$

In ferric chloride the valence of the iron is three. We also know ferric nitrate, $\mathrm{Fe}\left(\mathrm{NO}_{3}\right)_{3}$, and ferric sulfate, $\mathrm{Fe}_{2}\left(\mathrm{SO}_{4}\right)_{3}$. There are, therefore, two series of iron salts-the ferrous, in which the valence of iron is two, and the ferric, in which the valence is three.

We can obtain the two hydroxides of iron, both of which are nearly insoluble in water, by the action of sodium hydroxide on solutions of ferrous and ferric salts:

$$
\begin{aligned}
& \mathrm{FeCl}_{2}+2 \mathrm{NaOH} \rightarrow \mathrm{Fe}(\mathrm{OH})_{2}+2 \mathrm{NaCl}, \\
& \mathrm{FeCl}_{3}+3 \mathrm{NaOH} \rightarrow \mathrm{Fe}(\mathrm{OH})_{3}+{ }_{3} \mathrm{NaCl} .
\end{aligned}
$$

Ferrous hydroxide is white if pure, but is usually obtained as a dirty-green precipitate; this is due to partial oxidation by the action of oxygen of the air, with which it readily unites. Ferric hydroxide is a brown precipitate. These hydroxides unite with acids to form salts:

$$
\begin{aligned}
& \mathrm{Fe}(\mathrm{OH})_{2}+{ }_{2} \mathrm{HCl} \rightarrow \mathrm{FeCl}_{2}+{ }_{2} \mathrm{H}_{2} \mathrm{O}, \\
& \mathrm{Fe}(\mathrm{OH})_{3}+{ }_{3} \mathrm{HCl} \rightarrow \mathrm{FeCl}_{3}+{ }_{3} \mathrm{H}_{2} \mathrm{O} .
\end{aligned}
$$

I74. Aluminum and Its Compounds. - The common metal aluminum is an element. As is well known, the metal is not acted upon by air or water. It reacts easily with dilute hydrochloric acid, giving aluminum chloride, $\mathrm{AlCl}_{3}$, and hydrogen:

$$
2 \mathrm{Al}+6 \mathrm{HCl} \rightarrow 2 \mathrm{AlCl}_{3}+3 \mathrm{H}_{2} .
$$

Upon evaporation, the solution deposits white crystals of the compound $\mathrm{AlCl}_{3} \cdot 6 \mathrm{H}_{2} \mathrm{O}$. It is not possible to obtain the anhydrous salt, $\mathrm{AlCl}_{3}$ by heating these crystals, for the purpose of driving off water, since they decompose thus:

$$
2 \mathrm{AlCl}_{3} \cdot 6 \mathrm{H}_{2} \mathrm{O} \rightarrow \mathrm{Al}_{2} \mathrm{O}_{3}+6 \mathrm{HCl}+{ }_{3} \mathrm{H}_{2} \mathrm{O} .
$$

The anhydrous chlorile is formed by the action of dry chlorine gas on aluminum:

$$
2 \mathrm{Al}+{ }_{3} \mathrm{Cl}_{2} \rightarrow 2 \mathrm{AlCl}_{3} .
$$


Aluminum chloride is easily soluble in water, forming a colorless solution. This solution gives with ammonia a white precipitate of aluminum hydroxide, $\mathrm{Al}(\mathrm{OH})_{3}$, which is insoluble in water:

$$
\mathrm{AlCl}_{3}+{ }_{3} \mathrm{NH}_{4} \mathrm{OH} \rightarrow \mathrm{Al}(\mathrm{OH})_{3}+3 \mathrm{NH}_{4} \mathrm{Cl} \text {. }
$$

When heated, the hydroxide gives the oxide and water:

$$
2 \mathrm{Al}(\mathrm{OH})_{3} \rightarrow \mathrm{Al}_{2} \mathrm{O}_{3}+{ }_{3} \mathrm{H}_{2} \mathrm{O} \text {. }
$$

Rubies and sapphires are natural forms of aluminum oxide. Emery, which is a valuable abrasive, is an impure form of the same substance.

I75. Various Aluminum Salts.-The hydroxide is a base which reacts with acids to give the corresponding salts, thus:

$$
\begin{aligned}
\mathrm{Al}(\mathrm{OH})_{3}+3 \mathrm{HCl} & \rightarrow \mathrm{AlCl}_{3}+{ }_{3} \mathrm{H}_{2} \mathrm{O}, \\
\mathrm{Al}(\mathrm{OH})_{3}+3 \mathrm{HNO}_{3} & \rightarrow \mathrm{Al}\left(\mathrm{NO}_{3}\right)_{3}+{ }_{3} \mathrm{H}_{2} \mathrm{O}, \\
2 \mathrm{Al}(\mathrm{OH})_{3}+{ }_{3} \mathrm{H}_{2} \mathrm{SO}_{4} & \rightarrow \mathrm{Al}_{2}\left(\mathrm{SO}_{4}\right)_{3}+6 \mathrm{H}_{2} \mathrm{O} .
\end{aligned}
$$

The nitrate and sulfate are easily soluble in water, giving colorless solutions. The well-known substance alum is potassium, aluminum sulfate, $\mathrm{KAl}\left(\mathrm{SO}_{4}\right)_{2} \cdot \mathrm{I}_{2} \mathrm{H}_{2} \mathrm{O}$. It is obtained in large, colorless crystals when a solution made from potassium sulfate and aluminum sulfate is allowed to evaporate. The corresponding sodium and ammonium salts are well known, and have the formulae $\mathrm{NaAl}\left(\mathrm{SO}_{4}\right)_{2} \cdot \mathrm{I}_{2} \mathrm{H}_{2} \mathrm{O}$ and $\mathrm{NH}_{4} \mathrm{Al}\left(\mathrm{SO}_{4}\right)_{2} \cdot \mathrm{I}_{2} \mathrm{H}_{2} \mathrm{O}$, respectively. All such compounds are known as double salts; chemists are familiar with a great variety of these. Other examples of well-known double salts are ammonium ferrous sulfate, $\left(\mathrm{NH}_{4}\right)_{2}$ $\mathrm{Fe}\left(\mathrm{SO}_{4}\right)_{2} \cdot 6 \mathrm{H}_{2} \mathrm{O}$, and potassium cupric chloride, $\mathrm{K}_{2} \mathrm{CuCl}_{4} \cdot 2 \mathrm{H}_{2} \mathrm{O}$.

176. Acid Reaction of Aluminum Salts.-Solutions of the chloride, nitrate, and sulfate of aluminum, and also of alum, are not neutral, as we might expect, but are distinctly acid in reaction. They also have a sour taste. On the other hand, we find that moist aluminum hydroxide, if it has been carefully washed free from the ammonia used in precipitating it, has no action on either blue or red litmus. It is also tasteless. Nevertheless, we call the hydroxide a base, because it unites with acids to form salts. We say, however, that it is a weak base; and we find in general 
that weak bases, of which many are known, give salts whose solutions are acid in reaction. This is an important matter which will have to be studied carefully later.

177. Acid Properties of Aluminum Hydroxide.-If we add sodium hydroxide to a solution of an aluminum salt, a white precipitate of aluminum hydroxide is first formed, just as with ammonia:

$$
\mathrm{AlCl}_{3}+3 \mathrm{NaOH} \rightarrow \mathrm{Al}(\mathrm{OH})_{3}+3 \mathrm{NaCl} .
$$

However, upon adding an excess of sodium hydroxide, we find that the precipitate goes into solution. If pure aluminum hydroxide is dissolved in a solution of sodium hydroxide and the resulting solution evaporated, crystals of sodium aluminate, $\mathrm{NaAlO}_{2}$, are obtained. This substance is easily soluble in water and is, in reality, a salt. It thus appears that aluminum hydroxide acts as an acid in this case, and we might write the equation for the action of sodium hydroxide upon it thus:

$$
\mathrm{HAlO}_{2} \cdot \mathrm{H}_{2} \mathrm{O}+\mathrm{NaOH} \rightarrow \mathrm{NaAlO}_{2}+2 \mathrm{H}_{2} \mathrm{O} \text {. }
$$

We find that the solution of sodium aluminate is strongly alkaline toward litmus, and say, therefore, that, although aluminum hydroxide has some acid properties, it is a very weak acid.

Thus we see that a substance may be both a base and an acid. Such a substance is said to be amphoteric. Several metallic hydroxides are amphoteric. 'Thus zinc hydroxide, $\mathrm{Zn}(\mathrm{OH})_{2}$, forms with hydrochloric acid, $\mathrm{ZnCl}_{2}$, and with sodium hydroxide, $\mathrm{Na}_{2} \mathrm{ZnO}_{2}$, sodium zincate. It is of interest to note that aluminum hydroxide does not react with carbonic acid, and in fact no carbonate of aluminum has ever been made. Now, carbonic acid is a very weak acid, and aluminum hydroxide is a very weak base. In general, we find that very weak bases do not form salts with very weak acids.

Compounds of aluminum are very abundant in the earth. Common clay and numerous kinds of common rocks are compounds of aluminum.

I78. Mercury and Its Compounds. - We have already learned something of the chemical behavior of mercury and mercuric oxide, $\operatorname{HgO}\left(1_{3}, 1_{4}, 86\right)$. The oxide, which is insoluble in water, 
dissolves in dilute hydrochloric acid, giving mercuric chloride, $\mathrm{HgCl}_{2}$, and in nitric acid, giving mercuric nitrate, $\mathrm{Hg}\left(\mathrm{NO}_{3}\right)_{2}$ :

$$
\begin{gathered}
\mathrm{HgO}+2 \mathrm{HCl} \rightarrow \mathrm{HgCl}_{2}+\mathrm{H}_{2} \mathrm{O} . \\
\mathrm{HgO}+2 \mathrm{HNO}_{3} \rightarrow \mathrm{Hg}\left(\mathrm{NO}_{3}\right)_{2}+\mathrm{H}_{2} \mathrm{O} .
\end{gathered}
$$

These salts form white crystals which are soluble in water. The soluble salts of mercury are all extremely poisonous when taken internally. Mercuric chloride is familiarly known as bichloride of mercury or corrosive sublimate, and is extensively used as a powerful germicide and antiseptic.

179. The Formation of Mercuric Salts.-The nitrate can be made by the action of warm, concentrated nitric acid upon metallic mercury:

$$
{ }_{3} \mathrm{Hg}+8 \mathrm{HNO}_{3} \rightarrow{ }_{3} \mathrm{Hg}\left(\mathrm{NO}_{3}\right)_{2}+2 \mathrm{NO}+{ }_{4} \mathrm{H}_{2} \mathrm{O} .
$$

Hydrochloric acid does not act appreciably upon mercury; but the chloride can be obtained by the action of chlorine on the metal:

$$
\mathrm{Hg}+\mathrm{Cl}_{2} \rightarrow \mathrm{HgCl}_{2} \text {. }
$$

It is also made by heating a mixture of mercuric sulfate and common salt:

$$
\mathrm{HgSO}_{4}+2 \mathrm{NaCl} \rightarrow \mathrm{HgCl}_{2}+\mathrm{Na}_{2} \mathrm{SO}_{4} .
$$

The mercuric chloride formed is readily volatile and is separated by sublimation; hence the old name "corrosive sublimate." The process of vaporization of a solid and the condensation of its vapor directly to the crystalline form is called sublimation. The sulfate is made by strongly heating mercury with concentrated sulfuric acid:

$$
\mathrm{Hg}+{ }_{2} \mathrm{H}_{2} \mathrm{SO}_{4} \rightarrow \mathrm{HgSO}_{4}+\mathrm{SO}_{2}+{ }_{2} \mathrm{H}_{2} \mathrm{O} .
$$

I80. Mercurous Salts.- - The action of cold, dilute nitric acid on an excess of mercury gives rise to a solution of a salt having the formula $\mathrm{HgNO}_{3}$ and called mercurous nitrate. A solution of this salt gives with hydrochloric acid a white precipitate of mercurous chloride, $\mathrm{HgCl}$, and with dilute sulfuric acid also a white precipitate of mercurous sulfate. $\mathrm{Hg}_{2} \mathrm{SO}_{4}$; both of these 
precipitates are practically insoluble in water. Thus mercury, like iron, forms two series of salts: the mercurous, in which the element has a valence of one, or is univalent, and the mercuric, in which it has a valence of two, or is bivalent.

181. The Two Oxides of Mercury.-A solution of mercuric nitrate gives with a solution of sodium hydroxide a yellow precipitate of mercuric oxide, $\mathrm{HgO}$ :

$$
\mathrm{Hg}\left(\mathrm{NO}_{3}\right)_{2}+2 \mathrm{NaOH} \rightarrow \mathrm{HgO}+2 \mathrm{NaNO}_{3}+\mathrm{H}_{2} \mathrm{O} .
$$

The hydroxide of mercury, like that of silver, cannot be obtained; we might say that it is so unstable that it changes into the oxide and water as soon as it is formed; in this respect it resembles the corresponding compound of silver. The yellow oxide, formed in this way, seems to differ from the red oxide, obtained by heating mercury in the air or in oxygen, only in being made up of very much smaller particles.

A solution of mercurous nitrate gives with sodium hydroxide a nearly black precipitate of mercurous oxide, $\mathrm{Hg}_{2} \mathrm{O}$.

182. Calomel.-Mercurous chloride, $\mathrm{HgCl}$, which is commonly called calomel, is extensively used in medicine. It is a remarkable but well-known fact that the usual medicinal dose of calomel contains many times as much mercury asdoes a fatal dose of mercuric chloride. This great difference in physiological effect is in part due to the fact that while mercuric chloride is easily soluble in water, mercurous chloride is nearly insoluble.

183. The Valencies of Radicals. - Now that we have studied a considerable additional number of acids, bases, and salts, we may again revert to the study of valence $(146,147)$ since it furnishes a key to the casy mastery of formulae, an undertaking which is as necessary to the study of chemistry as learning to spell is in the mastery of a language. To write the formula of a chloride of a metal it is only necessary to group with the symbol of the latter as many chlorine symbols as the metal in question has units of valence; thus the formulae of barium chloride and of aluminum chloride are $\mathrm{BaCl}_{2}$ and $\mathrm{AlCl}_{3}$ respectively. If we wish to write the formulae of the nitrates we group the nitrate 
radical with the metal symbol in question according to the same rule, thus $\mathrm{Ba}\left(\mathrm{NO}_{3}\right)_{2}$ and $\mathrm{Al}\left(\mathrm{NO}_{3}\right)_{3}$. To write the formulae of sulfates, we must again group the symbols of the radical and the metal so that the total valence of each satisfies that of the other. Since the sulfate radical is bivalent, we may have to use more than one symbol weight of either the sulfate or the metal. Thus the formula of sodium sulfate is $\mathrm{Na}_{2} \mathrm{SO}_{4}$; that of barium sulfate is $\mathrm{BaSO}_{4}$; while that of aluminum sulfate is $\mathrm{Al}_{2}\left(\mathrm{SO}_{4}\right)_{3}$. Since $\mathrm{PO}_{4}$ is trivalent, we know at once that the formula of sodium phosphate must be $\mathrm{Na}_{3} \mathrm{PO}_{4}$, that of barium phosphate must be $\mathrm{Ba}_{3}\left(\mathrm{PO}_{4}\right)_{2}$, and that of aluminum. $\mathrm{AlPO}_{4}$. These examples are sufficient to show how a knowledge of valence simplifies the writing of formulae. In Table IX the

TABLE IX

\begin{tabular}{|c|c|c|c|c|}
\hline$\stackrel{\text { I }}{\text { Univalent }}$ & $\begin{array}{l}\mathrm{H} \ldots \ldots \ldots \\
\mathrm{Na} \ldots \ldots \\
\mathrm{K} \ldots \ldots \\
\mathrm{NH}_{4} \ldots \ldots \\
\mathrm{Ag} \ldots \ldots \\
\mathrm{Hg} \ldots \ldots\end{array}$ & $\begin{array}{l}\mathrm{Cl} \\
\mathrm{NO}_{3} \\
\mathrm{OH}_{3} \\
\mathrm{C}_{2} \mathrm{H}_{3} \mathrm{O}_{2}\end{array}$ & $\begin{array}{l}\mathrm{HCl} \\
\mathrm{NaCl} \\
\mathrm{HC}_{2} \mathrm{H}_{3} \mathrm{O}_{2} \\
\mathrm{NH}_{4} \mathrm{Cl} \\
\mathrm{AgCl} \mathrm{HgCl} \\
\mathrm{HgCl}\end{array}$ & $\begin{array}{l}\mathrm{HOH}\left(\mathrm{H}_{2} \mathrm{O}\right) \\
\mathrm{HNO} \\
\mathrm{NaOH}_{3} \\
\mathrm{NH}_{4} \mathrm{OH} \\
\mathrm{Ag}^{2} \mathrm{O} \\
\mathrm{Na}_{2} \mathrm{SO}_{4}\end{array}$ \\
\hline $\begin{array}{c}\text { II } \\
\text { Bivalent }\end{array}$ & $\begin{array}{l}\mathrm{Mg} \ldots \ldots \ldots \\
\mathrm{Ca} \ldots \ldots \\
\mathrm{Ba} \ldots \ldots \\
\mathrm{Zn} \ldots \ldots \\
\mathrm{Fe} \ldots \ldots \\
\mathrm{Cu} \ldots \ldots \\
\mathrm{Pb} \ldots \ldots \\
\mathrm{Hg} \ldots \ldots\end{array}$ & $\begin{array}{l}\mathrm{SO}_{4} \\
\mathrm{CO}_{3} \\
\mathrm{O} \\
\cdots \cdots \\
\cdots \cdots \\
\cdots \cdots \\
\cdots \cdots \\
\cdots \cdots\end{array}$ & $\begin{array}{l}\mathrm{MgCl}_{2} \\
\mathrm{CaCl}_{2} \\
\mathrm{BaCl}_{2} \\
\mathrm{ZnCl}_{2} \\
\mathrm{FeCl}_{2} \\
\mathrm{CuCl}_{2} \\
\mathrm{PbCl}_{2} \\
\mathrm{HgCl}_{2}\end{array}$ & $\begin{array}{l}\mathrm{H}_{2} \mathrm{SO}_{4} \\
\mathrm{H}_{2} \mathrm{CO}_{3} \\
\mathrm{CaCO}_{3} \\
\mathrm{BaSO}_{4} \\
\mathrm{CuSO} \\
\mathrm{Ca}(\mathrm{OH})_{2} \\
\mathrm{MgO} \\
\mathrm{HgO}\end{array}$ \\
\hline$\underset{\text { Trivalent }}{\text { III }}$ & $\begin{array}{l}\mathrm{Al} . \ldots \ldots \\
\mathrm{Fe} . . . .\end{array}$ & $\begin{array}{l}\mathrm{PO}_{4} \\
\mathrm{~N}\end{array}$ & $\begin{array}{l}\mathrm{AlCl}_{3} \\
\mathrm{FeCl}\end{array}$ & $\begin{array}{l}\mathrm{H}_{3} \mathrm{PO}_{4} \\
\mathrm{AlPO}_{4} \\
\mathrm{NH}_{3} \\
\end{array}$ \\
\hline $\begin{array}{c}\text { IV } \\
\text { Quadrivalent }\end{array}$ & & $\mathrm{C}$ & $\begin{array}{l}\mathrm{CCl}_{4} \\
\mathrm{CO}_{2}\end{array}$ & $\begin{array}{l}\mathrm{CH}_{4} \\
\ldots .\end{array}$ \\
\hline
\end{tabular}

various elements and radicals studied are classified with respect to their valencies, which vary from one to four. In the column headed by hydrogen we have the metals together with the ammonium radical. In the next column, headed by chlorine, are the elements and radicals that unite as a rule with those of the first column. The last two columns contain the formulae of some typical compounds. 


\section{CHAPTER X}

\section{THE KINETIC THEORY OF MATTER AND THE MOLECULAR HYPOTHESIS}

184. An Old Greek Hypothesis.-The question whether a portion of a given substance, say a drop of water, could be subdivided to an unlimited extent, and whether the smallest particle so produced would still differ in no way except in size from the original, was one which was much debated by the Greek philosophers centuries before chemistry became a science. Anaxagoras (B.C. 500), who held that there was no limit to the divisibility of matter, was opposed by Democritus (B.C. 470), who taught that in the imagined process of continued subdivision minute particles would finally be encountered which could not be cut in two without destroying or completely changing the nature of the substance; these particles were called atoms (ätopos, a body which cannot be cut in two). This idea may be illustrated by the following analogy. If we take a bushel of wheat, we may divide it into pecks, quarts, gills, etc., and yet each measure of the material will be a quantity of wheat; we may go still farther, but we will ultimately reach the single grains, which are still grains of wheat; but if we cut these grains in two the resulting parts may no longer be called wheat, since they would no longer possess the most remarkable property of wheat, which is that of growing if planted. At present we use the term molecule (from Latin molecula, the diminutive of moles, a mass) to mean essentially the same as the term "atom," as used by the Greeks, and speak therefore of the molecular theory of matter and the molecular hypothesis. The present chapter gives an account of this hypothesis and aims to show how it furnishes us an explanation of many important facts.

185. The Molecules of Water.-According to the molecular hypothesis, a drop of water can be subdivided, and still remain water, only until the single molecules are reached, and no further. The splitting up of the molecules of water might separate the 
smallest particles of oxygen from those of hydrogen; but the result would be the decomposition of the water into its elementary constituents. The particles of the elements oxygen and hydrogen of which the molecules of water are made up are now called atoms. It is supposed that all of the molecules of water are alike in every respect, each being made up of one atom of oxygen and two atoms of hydrogen. The reason for this last conclusion is discussed in the next chapter. In general, each molecule of a given pure substance is just like every other molecule of that substance. The nature of a substance is determined by the nature of its molecules; and this is, in turn, determined by the number and kind of atoms composing the molecules. We imagine molecules to be very small, since they cannot be seen with the aid of a microscope of the highest power. A cubic centimeter of air may contain an almost inconceivable number of these tiny particles.

I86. The Molecular Hypothesis Applied to Gases.-Let us first consider the known facts concerning gases and try to see how these facts can be connected with the supposition that gases are made up of small particles, the molecules. In the first place, we know that a confined gas tends to expand and exerts a pressure on the walls of the vessel which holds it. If we increase the pressure, the volume of the gas is diminished, and by applying a great pressure the decrease in volume may be made very great. We may explain this in either of two ways: first, that the molecules, like rubber balloons, are themselves compressible; or, second, that the molecules, which may not be appreciably compressible, are at considerable distances from one another, but are brought closer together when the gas as a whole is compressed. Let us follow up this second idea and see to what it leads. Two important questions now present themselves: (I) Why are the molecules not in contact - that is, why should they be at considerable distances from one another? (2) Why does a gas exert a pressure in all directions on the walls of the vessel which contains it?

I87. Are Molecules at Rest or in Motion?-Would it make a difference in the state of affairs whether the molecules were at 
rest or in motion? Suppose they are in rapid motion: what would follow? Let us recall Newton's first law of motion: A body at rest remains at rest, and a body in motion continues to more with constant aclocity in a straight line, unless acted upon by some extcrnal unbalanced forcc. Now, if molecules are in motion, and if they behave in the same manner as other bodies, and if, further, they are elastic - that is, if they, like rubber or ivory balls, can rebound from one another or from the walls of the containing vessel, then they will tend to continue in motion. Of course, in such a case as the one imagined, the molecules of the gas would acry frequently strike one another and also the walls of the containing vessel; but, being elastic, they would rebound and continuc in motion in a new direction; and although the velocity of an individual molecule might be increased or decreased as the result of a collision with another molecule, on the whole the average sclocity of all the molecules would be constant.

The various elaborations of the ideas here presented constitute the Kinetic Theory of Matter. This hypothesis is the most important corollary of the molecular hypothesis. It has proved enormously fruitful in explaining very diverse phenomena and in suggesting new lines of investigation.

188. The Cause of Gas Pressure; Boyle's Law.-The striking of a gas molecule against the wall of the vessel would deliver a little blow; and if millions of molecules struck each square centimeter every second the effect would be to tend to push back the surface. But what is this but the exertion of pressure? If the molecules strike of ten enough, regularly enough, and close enough together, this pressure would seem constant and uniform. Now, suppose the gas to be compressed until it occupies half its original volume. In each cubic centimeter there would now be double the original number of molecules, and on each square centimeter of the wall of the container twice as many molecules would strike per second as before; so that, as the mass of each molecule and also its velocity have remained unchanged, we should expect just double the pressure per square centimeter; and, in fact, this is just what we find by experiment. Thus we see that by imagining a gas to be made of numerous small, rapidly moving, 
elastic particles, the molecules, we get an explanation of gas pressure and of Boyle's law. We also see how it would be possible for the molecules to be at considerable distances (compared with their own diameters) from one another without tending to fall together into a mass in which the molecules would all be permanently in contact.

189. The Effect of Temperature on Molecular Velocity.-We may next consider why it is that the molecules are in motion and whether the average velocity of the molecules of a given gas can ever be changed. We know, of course, that, for a constant volume, the pressure exerted by a gas increases with rise of temperature, so that if we are to explain gas pressure as due to the momenta (mass $\times$ velocity) of the molecules which strike the walls of the container, we must suppose that an increase of temperature increases either the mass of a molecule or its velocity, or both. Naturally, the number of collisions with the wall could not be increased unless the velocity increased. Now, it would seem more reasonable to think of rise of temperature as causing an increase in velocity than to think of it as causing an increase in mass; so we have only to imagine that a rise in temperature causes the average velocity of the molecules to increase in order to get a simple and satisfying explanation of the effect of temperature on the pressure of a gas.

On the other hand, a decrease in temperature is accompanied by a decrease in pressure; and, indeed, the pressure is at all times proportional to the absolute temperature. This would imply that the pressure would be zero at the absolute zero of temperature. But zero pressure could only result if the molecules were completely at rest. We may suppose, therefore, that at absolute zero there is no molecular motion. A body when hot differs from the same body when cold only by reason of the more rapid motion of its molecules. In short, according to this way of looking at the matter, heat is merely the outward manifestation of molecular motion.

I90. The Mixing of Gases.-We have already learned (122-124) that, as a rule, liquids and solids do not form perfect mixtures (solutions) in all proportions; that is to say, a solid or a 
liquid will dissolve in a second liquid only to a limited extent. Not so with gases: ciery gas will form with any proportion of any other gas a perfectly uniform mixture. Air, for example, is a perfectly homogeneous mixture of several gases. Of course, if some of the gases which are brought together react chemically, new liquid or solid compounds might be formed which would separate from the gaseous mixture. But for gases that do not react chemically we find that all gases mix perfectly in all proportions.

191. The Diffusion of Gases.-If we bring two gases into the same vessel without attempting to mix them we find, after a time, that a perfectly uniform mixture is present in the vessel. We also know that if a gas like ammonia is liberated at one place in a closed room its odor is soon perceptible everywhere in the room. The process of the spontaneous mixing of gases is called diffusion, and we say that the ammonia has diffused through the air of the whole room. It is now casy to understand how this diffusion takes place. The molccules of ammonia are moving in all directions with high velocities; the same is true also of the molecules of the air; their complete and uniform intermingling is therefore inevitable.

192. The Law of Partial Gas Pressures.-It is easily found by experiment that, if portions of various gases are brought together in the same vessel, the tolal pressure exerted by the gas mixture is the sum of the pressures that would be exerted, at the same temperature, by the same portions of these gases if each occupied the space alone. 'This is known as Dalton's Law of Partial Pressures. It is not difficult to explain this law, since, in a mixture of gases, the molecules of a given sort will strike a given area of the wall just as often in the presence of other unlike molecules as in their absence. Each kind of molecule will therefore produce the same partial pressure as if the others were absent.

193. Avogadro's Hypothesis. - The student must already have been impressed by the fact that all gases show greal similarily in physical behazior. They all conform to the laws of Boyle and Charles. This fact, together with others which we shall consider later, led Avogadro, then professor of physics in Turin, Italy, to 
suggest in I8I I that it is probable that all gases contain the same number of molecules per cubic centimeter. Although this suggestion received some support for the first twenty years after its proposal, it was then nearly forgotten until about I860, since which time its importance and probability have been impressed more and more deeply on the minds of physicists and chemists, so that during the last fifty years it has become one of the most fundamental principles of chemistry. It may be stated concisely thus: Equal volumes of every gas or vapor at the same temperature and pressure contain the same number of molecules.

Further reasons for accepting Avogadro's hypothesis will be given in the next chapter. Indeed, the evidence from so many independent sources for the truth of this view is now so convincing that the hypothesis is looked upon by many as a statement of fact, and in consequence is referred to as Avogadro's Law.

194. Gas Statistics. - Within the last few years methods have been found by means of which the number of molecules in I c.c. of a gas has been found with a high degree of probability and accuracy. At $0^{\circ}$ and $76 \mathrm{~cm}$., I c.c. of any gas has been found to contain $2.7 \times 10^{19}$ molecules, with a probable error of less than $\mathrm{I}$ per cent. The number of molecules in 22.4 liters of a gas under standard conditions is therefore $22,400 \times 2.7 \times 10^{19}=6.06 \times 10^{23}$. The number of molecules in I c.c., twenty-seven millions of millions of millions, is so immense that it is difficult for the mind to get any tangible conception of its magnitude. However, if we think of the molecules in I c.c. as at rest for the moment, and uniformly distributed in rows and layers, we should then have in each row of $\mathrm{I} \mathrm{cm}$. length $\mathrm{r}^{3} \overline{27 \times 10^{18}}=3 \times 10^{6}$, or three million molecules, a number which, although large, is at least comprehensible. Then in each layer there would be three million of these rows, and, in the whole cubic centimeter, three million such layers.

I95. A Cubic Mile of Sand.-Another mental picture of the case may be got if we imagine I c.c. of gas to have been expanded until it occupied a cubic mile. Then each row of molecules would be a mile long and would contain three million molecules, 
spaced about $\frac{1}{50}$ of an inch apart. Now, a grain of fine sand is about $-\frac{1}{3}$ of an inch in diameter; and three million such grains placed side by side would extend one mile. Therefore, a cubic mile of such sand would contain $3 \times 10^{6}$ cubed or $27 \times 10^{18}$ grains, which is the number of molecules in I c.c. of gas at standard pressure and temperature. Since the number of molecules per cubic centimeter has been determined by several independent methods which give closely agreeing results, we may safely accept the value given above as being correct within I per cent.

196. Some Further Conclusions.-It may now be of some interest to note a few additional conclusions that have been reached in the study of gases. Let us illustrate by means of the gas oxygen. We know the weight of I c.c. of oxygen and the number of molecules in I c.c. at standard conditions; dividing the first by the second gives the weight of a single molecule of this gas; this comes out $5.3 \times 10^{-23}$ gram. The size of a molecule has also been approximately determined and in the case of oxygen it turns out that the diameter of a molecule is approximately $2.5 \times 10^{-8} \mathrm{~cm}$. We have already scen that the average distance between molecules is about $\frac{\mathrm{I}}{3,000,000}$ of a cm. $=3 \cdot 3 \times$ $10^{-7} \mathrm{~cm}$. We may now ask: How far, on the average, will a molecule travel in a straight line before it strikes another molecule? This result can be calculated when the diameter and average distance apart of the molecules are known, and is called the free path; for oxygen it is $1.3 \times 10^{-5} \mathrm{~cm}$. Thus we see that, on the average, a molecule, after one collision will travel about 40 times $\left(1.3 \times 10^{-5} \div 3.3 \times 10^{-7}=40\right)$ the average distance between two neighboring molecules before striking a second molecule. This is not surprising when we note that the average distance between molecules is about $I_{3}$ times their diameters $\left(3.3 \times 10^{-7} \div 2.5 \times 10^{-8}=13\right)$.

197. The Velocity of Molecular Motion.-The average velocity with which molecules travel between collisions can be calculated with a high degree of certainty. The velocity varies with the mass of the molecule and its temperature but is independent of the pressure. Molecules of equal masses have equal velocities 
at the same temperatures, while for those with different masses the velocities are inversely proportional to the square root of the mass. At $0^{\circ}$ the velocity of the oxygen molecules is $10^{4} \mathrm{~cm}$. per second, or about I 5 miles per minute. But since the free path of a molecule of oxygen is only $\mathrm{I} .3 \times 1 \mathrm{IO}^{-5} \mathrm{~cm}$., it will experience many thousands of collisions in progressing $\mathrm{I} \mathrm{cm}$. At each collision its direction of travel will change so that its actual progress from a given position is far slower than its high velocity would indicate if no collisions occurred.

198. The Liquid State.-As a gas is compressed at constant temperature its molecules are brought closer together, but otherwise conditions remain nearly unchanged. The mass, diameter, and velocity of each molecule will not be altered; only the average distances between the molecules and their free paths will be shortened. It seems probable, in fact, that the average kinetic energy of a molecule, which is equal to one-half the product of its mass and the square of its velocity $\left(\frac{1}{2} m v^{2}\right)$, remains unchanged, however much the gas is compressed. If we accept this view, we may easily extend it to cover the liquid state, in which we may imagine that the molecules have the same velocities and therefore the same kinetic energies as the molecules of the vapor of the liquid have at the same temperature, but that the crowding of the molecules is so great that their free paths are short compared with their diameters. However, we may think of the molecules as able to progress slowly from one place to another, although the motion will be very irregular, like that of persons moving about in a dense crowd.

I99. Vaporization of a Liquid.--It has already been stated that all of the molecules of a given gas cannot have equal velocities; nor can a given molecule always have the same velocity, since at every one of the frequent collisions the velocity will be changed. It is only the average velocity of all the molecules that remains unchanged as long as the temperature remains constant. The velocities of the molecules of a liquid also are not all the same at a given instant; some will be moving much slower, others much faster, than the average. If a fast-moving molecule approaches the free surface of the liquid, it may escape 
into the space above the liquid, whereas a slow-moving molecule, under the same conditions, might not be able to escape. Now the passage of molecules from the liquid to the space above it is nothing but the evaporation of the liquid. Moreover, we see that the rate of escape of the molecules, and therefore the rate of evaporation, will be greater in proportion as the average velocity of the molecules is increased. Since molecular velocity increases with rise of temperature, we get in this way a simple explanation as to why heating a liquid hastens its evaporation. When the evaporation of a liquid goes on with a poor supply of heat, as when water evaporates in an open vessel, the liquid becomes cooler. Obviously this is due to the lowering of the average velocity of the molecules of the liquid because of the escape of the faster-moving ones.

200. Vapor Pressure.-If a liquid is placed in a closed vessel which it does not completely fill, it will evaporate only until the pressure exerted by the vapor attains a certain value which is definitely determined by the temperature. For example, at $20^{\circ}$ the vapor pressure of water is equal to that exerted by $17.4 \mathrm{~mm}$. of mercury; at $25^{\circ}$ it equals $23.6 \mathrm{~mm}$. Does the water cease to pass into vapor when these pressures are reached? If so, does this mean that molecules of water no longer pass from the liquid to the space above it? This would seem strange. Let us look at the question from another point of view. Suppose we have a vessel full of steam and allow it to cool. We know that most of the steam will condense; only a little will remain as vapor. If we try to picture how this occurs, we must think of some of the molecules of vapor, that is, gaseous water, coming together first to form liquid droplets; these fall to the bottom and soon form a layer of liquid; other molecules then strike this liquid and remain as a part of it. Finally, when the temperature of the room, say $20^{\circ}$, has been reached, the pressure within the vessel will have fallen to $17.4 \mathrm{~mm}$. of mercury, and most of the water, but not all, will have condensed to the liquid state. It is important to note that at a given temperature, say $20^{\circ}$, the same final vapor pressure is reached whether steam condenses or water evaporates. 
201. Equilibrium between Liquid and Vapor.-A very important question now confronts us: Do water molecules cease to pass from the vapor into the liquid when at $20^{\circ}$ the pressure reaches $17.4 \mathrm{~mm}$.? If so, Why? Would it not seem more reasonable to suppose that for every molecule that passes from the vapor into the liquid there is another that leaves the liquid and passes into the vapor? This supposed state of affairs would correspond to that in which the number of customers in a large shop remains substantially constant during a given hour of the day, by reason of the fact that in each minute as many persons enter the shop as leave it. When at constant temperature the vapor pressure of a liquid has reached a constant value, we say there is equilibrium between liquid and vapor; and it would seem from the discussion above that this condition does not represent a state of rest or inaction, but one in which two opposing actions exactly counteract one another.

202. Molecular Attraction.-If we think of the matter critically, we may wonder why molecules of cooling water vapor collect into drops. Perhaps there is a sort of attraction between the molecules that holds them together. If so, why should it seem to be more effective at lower than at higher temperatures? If, in reality, one molecule has some attraction for another, must we suppose that this attraction increases with fall of temperature? Would it not be sufficient to assume a constant attraction of each molecule of water for every other? Suppose, now, the vapor of water is very hot; then the molecules will be moving with such great velocities that if two of them collide they will rebound, exactly as a rubber ball, thrown downward, will rebound on striking the floor, although gravitational attraction tends to keep it on the floor. But suppose the vapor to be cooled; its molecules will then have smaller velocities and some may be moving so slowly that upon collision they remain in contact. Other slow-moving molecules, striking by accident a pair of molecules so formed, may add themselves to it, and in this way the droplet of water could be formed. There are also other reasons for assuming that molecules attract one another. 
203. The Solid State.-The most striking physical difference between a solid and a liquid is the rigidity of the former. This property of solids can most easily be accounted for by assuming that the molecules are not free to move about as in the case of a liquid, where the freedom of motion is comparable to that of people in a crowd, but that each molccule remains in its place with respect to the whole solid, as well as to its neighboring molecules. It is not necessary to think of the molecules as being absolutely at rest. It is more likely that each molecule has a ribrating motion at all temperatures above absolute zero and that, in fact, its kinetic energy is as great as it would be if the molecule were in the vapor state at the same temperature.

204. Crystals.-Pure chemical substances in the solid state usually form crystals. The crystals of a given substance all have the same general form. Thus, for example, the crystals of common salt, when perfect, are all cubical in form, while those of quartz occur as hexagonal prisms. If we think of a crystal as built up of molecules, it is natural to wonder whether the molecules are present in haphazard fashion, like potatoes in a barrel, or if they may not perhaps be arranged in some systematic manner, like bricks in a wall or balls in a regular pile. The probability that the molecules of a crystal are arranged in a definite and regular manner is greatly increased when it is known that there are exactly as many types of crystalline form as there are possible regular arrangements of points in space.

Within the last few years it has become possible by means of photographic studies made by the use of $\mathrm{X}$-rays to obtain very precise information regarding the arrangement of molecules forming a crystal. As a result we now know quite definitely the molecular structure of a number of crystals.

205. The Melting of Crystals.-Pure crystalline substances have definite melting temperatures; thus, ice melts at $0^{\circ}$, and potassium nitrate at $339^{\circ}$. Increase of temperature must increase the intensity of molecular vibration; at some temperature (the melting-point) this vibration seems to become so great that the systematic structure of the crystal is wrecked, leaving only an irregularly mixed mass of molecules, forming the resulting 
liquid. Crystals cannot be heated above their melting-points. Ice, for example, although it may be melting on the surface, is never hotter than zero.

206. Supercooling.--On the other hand, water may be cooled 2 or 3 degrees below zero without freezing, if it is kept quiet and is not in contact with ice. Such supercooled water immediately begins to freeze if touched with a piece of ice. This phenomenon is a common one and is easily explained. In order that the formation of a crystal can start, a certain minimum number of molecules must come together in the proper positions. But this exact arrangement of the several molecules necessary may not readily occur, especially as immediately above the freezingpoint (which is the same as the melting-temperature) the molecules are vibrating so fast that they are just able to shake apart this regular arrangement (that is, to melt the crystal). At a little lower temperature the molecular motion is less and therefore the conditions are more favorable for the starting of crystallization. However, if a crystal of the substance is present, then supercooling does not occur, but the liquid at once begins to crystallize (freeze) at the temperature of its melting-point. The reason is obvious: now each molecule that touches the crystal can find its proper lodging-place, and so crystalline growth can continue.

207. Solutions.-In a solution the molecules of the dissolved substance must be very uniformly distributed; it would seem, therefore, that they may be moving about freely among the molecules of the solvent, being carried from place to place by their own.motions. The process of dissolving of a substance would closely resemble that of evaporation, and the crystallization of a solid from its solution would correspond to the condensation of a vapor to a liquid. In fact, we may imagine that in the case of a saturated solution in contact with the crystals of a substance we have a state of equilibrium as a result of the passage of molecules into and out of the solution at exactly equal rates. 


\section{CHAPTER XI}

\section{THE ATOMIC HYPOTHESIS AND ATOMIC WEIGHTS}

208. Dalton's Atomic Hypothesis.-The application of the Atomic-Molecular Hypothesis to the explanation of chemical phenomena was first made by John Dalton of Manchester in I 803 . Long before this time Bernoulli had proposed the KineticMolecular Hypothesis as an explanation of the physical behavior of gases, and Dalton, knowing this view of the nature of matter, sought to explain the difference in solubility in water of different gases as due to a possible difference in size of their molecules. But how could this imagined difference be discovered? At this date, 1803 , the theory of the indestructibility of matter and the doctrine of elements were well established, owing to the work of Lavoisier, a quarter of a century earlier, as well as the labors of many able chemists of the intervening period. It was generally accepted that the formation of a substance was due to the union of the elements composing it and in many cases the proportions of the elements in a compound were already known-not very accurately, it is true, but at least approximately. Dalton wished to discover the relative weights of the ultimate particles of gases; but in order to do this he would have to know, in the case of hydrogen and oxygen, for example, in addition to knowing the weight of oxygen that would combine with a given weight of hydrogen, the relative numbers of ultimate particles of the two gases that combine with one another in the formation of water. As Dalton had no experimental means of discovering the information he lacked, he simply assumed that one ultimate particle of hydrogen united with one ultimate particle of oxygen to give one ultimate particle of water, meaning by the expression ultimate particle essentially the same as the Greeks and later philosophers meant by the terms "atom" or "molecule," that is, the smallest possible particle of the substance.

209. Finding the Relative Weights of Atoms.-Now, since I g. of hydrogen unites with $8 \mathrm{~g}$. of oxygen to form $9 \mathrm{~g}$. of water, 
the ultimate particle or atom of oxygen must weigh eight times as much as the ultimate particle or atom of hydrogen; and the ultimate particle of water, in this case the molecule, must weigh nine times as much as an atom of hydrogen. In making such suppositions Dalton also assumed that all of the atoms of hydrogen were exactly alike in size, weight, and all other properties; that each atom of oxygen was exactly like every other atom of this element, but entirely different from an atom of any other element. Dalton knew that the same pair of elements often form two or more compounds in which the constituents are present in different proportions. This forced him to assume also that in such cases the atoms unite, not only one to one, but also one to two, or one to three, etc. In order that the student may have a perfectly clear notion of the matter, we may summarize by stating that Dalton assumed that a molecule of water is composed of one atom of hydrogen and one atom of oxygen, and then reached the conclusion that an atom of oxygen was eight times, and a molecule of water nine times, as heavy as an atom of hydrogen. But Dalton did not know, as we can see clearly, whether one atom of hydrogen unites with one atom of oxygen or with two or three of oxygen, or whether two or perhaps three atoms of hydrogen unite with one of oxygen to form a molecule of water: it was all a guess. But it must also be clear that if we could discover the numbers of atoms of hydrogen and oxygen in a molecule of water we could find the relative weights of the two atoms, knowing the percentages of hydrogen and oxygen in water. Now the question is: How can we discover the number of atoms of each kind in a molecule of a substance?

210. The Application of Avogadro's Hypothesis.-Suppose we accept Avogadro's suggestion that equal volumes of all gases at the same temperature and pressure contain the same number of molecules, and see to what conclusion we are led. Let us represent by $N$ the number of molecules in 22.4 liters of any gas under standard conditions. Now according to Dalton's suggestion one molecule of a given substance will contain one, two, three, or some small whole number of atoms of a given element, but cannot, by reason of the assumed indivisible nature 
of an atom, contain a fraction of an atom. Let us consider the gas ammonia as an example. Ammonia is composed of 17.8 per cent of hydrogen and 82.2 per cent of nitrogen, and nothing else. One molecule of ammonia, according to Dalton's suggestion, contains one, two, three, or four, or at least some small number of atoms of hydrogen. Now, if 22.4 liters of ammonia gas under standard conditions contain $N$ molecules, then this volume of the gas must contain some small whole number of times $N$ atoms of hydrogen. The least number of hydrogen atoms that could possibly be contained in 22.4 liters of ammonia is $N$, but the true number may be $2 \times N$, which we may write $2 N$, or it may be greater, as $3 N$ or $4 N$, so far as we know; only it must be $N$ or some small whole number of times $N$ if we assume that there are $N$ molecules in 22.4 liters of the gas and also assume, with Dalton, that each molecule of the gas contains one, two, three, or some small number of atoms of hydrogen. In the case of any other gaseous compound of hydrogen we should conclude, according to Avogadro, that 22.4 liters of the gas contained $N$ molecules and that each molecule contained one, two, three, or some other small number of hydrogen atoms, the smallest possible number being one atom of hydrogen to the molecule, and therefore that 22.4 liters of the gas would contain $N, 2 N$, or $3 N$, etc., atoms of combined hydrogen.

211. The Number of Atoms and Weight of Hydrogen in 22.4 Liters.-According to Dalton all hydrogen atoms are alike and each has a definite weight, so that the weight of $N$ atoms of hydrogen would be a perfectly definite weight of this element. The weight of $2 \mathrm{~N}$ atoms of hydrogen would, of course, be twice that of $N$ atoms, etc. It seems reasonable to think that it would be likely to happen that in some of the gaseous compounds of hydrogen the molecules would contain but one atom of hydrogen each. In such a case 22.4 liters would contain $N$ atoms of combined hydrogen, having a definite weight. Now as such gases contain the minimum possible number of atoms of hydrogen in each molecule, namely, one, and as we assume that all gases contain $N$ molecules in 22.4 liters, then such gases would contain the minimum possible weight of hydrogen in this volume. 
As a matter of fact we actually find that in 22.4 liters of the various gaseous compounds of hydrogen the weight of this element is in no case less than $\mathrm{I} g$. In this volume of any definite gas there is either no combined hydrogen or there is at least I g.: the minimum weight of hydrogen is $I \mathrm{~g}$.

212. The Explanation of the Laws of Minimum and Multiple Weights.-In other gaseous compounds of hydrogen we find in 22.4 liters larger weights of combined hydrogen, but these weights are then either 2 g., 3 g., or some whole multiple of the minimum weight. Is it not logical then to think that in such gases as hydrogen chloride, where the minimum weight, I g., of combined hydrogen is contained in 22.4 liters of the compound gas, the molecule contains but one atom of hydrogen, and that in acetylene, where $2 \mathrm{~g}$. of hydrogen are found in 22.4 liters each molecule contains two atoms of hydrogen, while in methane with $4 \mathrm{~g}$. of hydrogen in the same volume, there are four atoms of hydrogen per molecule? Undoubtedly so. We see then that we have in the assumptions made by Avogadro and Dalton the basis of an explanation of the remarkable Laws of Minimum and Multiple Weights, which have been discovered by experiment; and, because of the agreement between theory and fact, we are inclined to think that perhaps the views of Avogadro and Dalton are correct. In any case we cannot fail to see that these hypotheses are useful, and that, indeed, is the criterion by which the worth of any hypothesis should be judged.

213. Application of the Explanation to Other Elements.--The question now arises whether the simple explanations of the laws of minimum and multiple weights may be applied to elements other than hydrogen, and a very little thought will show that this must be the case. The minimum number of atoms of any given element in 22.4 liters of any of its gaseous compounds is again $N$, the number found in the case of those gases the molecules of which contain but one atom each of the given element. The minimum weight of this element is, of course, the weight of $N$ atoms of the element. For example, we find that in 22.4 liters of gaseous carbon compounds the minimum weight of carbon is $\mathrm{r} 2 \mathrm{~g}$. Since this minimum weight is found in the 
gases carbon dioxide and methane, we conclude that but one atom of carbon is contained in a molecule of each. On the other hand, 22.4 liters of acetylene contain $24 \mathrm{~g}$. of combined carbon, which is twice the minimum weight, from which we conclude that in a molecule of this gas there are two atoms of carbon. As we have now reached the conclusion that a molecule of melhane contuins four atoms of hydrogen and one of curbon we see that we have developed a method whereby we can solve the problem first suggested by Dalton, that of discovering the number of atoms of each sort in a molecule of a given substance at least in the case where the substance is a gas, since it is evident that the method used for methane is applicable to any gaseous substance.

214. The Number of Atoms of Each Kind in a Molecule.--To illustrate by further examples, we may consider the cases of a few of the gases we have already studied. We see, by reference to 'Table IV, that in 22.4 liters of hydrogen chloricle there are found the mininum weights of both hydrogen and chlorine and conclude that the molecule of this gas is made up of one atom each of hydrogen and chlorine. We also see by Table IV that the unit volume of ammonia contains the minimum weight of nitrogen and three times the minimum weight of hydrogen, and decide that in a molecule of ammonia one atom of nitrogen must be united with three atoms of hydrogren. In all other cases the reasoning is equally simple, so that the student will have no trouble in deciding upon the number of atoms of each kind in a molecule of each of the gases mentioned in 'Table IV.

215. The Number and Kind of Atoms in a Molecule Shown by the Formula. - We are now in position to notice a most remarkable fact, which the following examples will illustrate. One molecule of hydrogen chloride conlains one atom of hydrogen and one of chlorine, and ils formula is $\mathrm{IICl}$; one molecule of ammonia contains three atoms of hydrogen and one atom of nitrogen, and its formula is $\mathrm{NH}_{3}$; one molecule of methane contains four atoms of hydrogen and one atom of carbon, and its formula is $\mathrm{CH}_{4}$; in each case the number of atoms of each clement is the same as the number of symbol weights of that element in the formula of the substance! And that the same thing is true for all gases of Table IV 
may readily be found by considering each separate case in the same way as we did those of three of the gases. In every case, therefore, the formula shows not only the weight of each element in 22.4 liters of the gas but also the number of atoms of each element in one molecule of the substance.

216. Symbol Weights and Atomic Weights.-But we may now inquire, Why should this be true? To answer this question, we will recall that the minimum weight of any element in 22.4 liters of its gaseous compounds is the weight of $N$ atoms of that element. If $N$ atoms of hydrogen weigh I g. and $N$ atoms of carbon weigh I $2 \mathrm{~g}$., then one atom of carbon must be 12 times as heavy as an atom of hydrogen. In a similar way we are led to conclude that an atom of nitrogen is 14 times, and an atom of oxygen 16 times, as heavy as an atom of hydrogen. Analogous relations must likewise exist in the cases of all other elements; and, therefore, taking the weight of one atom of hydrogen as one or unity, the weight of an atom of any other element is represented by exactly the same number as its symbol weight. For this reason a table of symbol weights is also called a table of Atomic Weights; and symbol weights are usually referred to as atomic weights. But we must remember that the symbol weights may be found by simple and direct experiments, independently of all suppositions and hypotheses, while atomic weights are to be represented by the same set of numbers only when we assume that matter is made up of atoms which unite in simple ratios to form molecules of which all gases are assumed to contain equal numbers in equal volumes. Briefly stated, symbol weights are natural constants, but atomic weights are the probable relative weights of the atoms of which we imagine matter to be made $u p$. We now may answer the question proposed in the first sentence of this paragraph. The number of atoms of any sort in a molecule is the same as the number of symbol weights of that element because the absolute weight of an atom of any element is proportional to its symbol weight. In this chapter we have seen how the problem which Dalton set for himself over a century ago is to be solved, at least as definitely as chemists know, up to the present time, how to solve it. The key to the solution was 
the hypothesis of Avogadro, which was suggested in $\mathrm{I} 8 \mathrm{I} \mathrm{r}$, only three years after Dalton's views first appeared in print, and which was rejected by Dalton himself, and was only accepted by the chemical world at large half a century later.

2I7. Formula Weights and Molecular Weights.-It is of course obvious that the weight of a molecule may also be expressed in terms of the weight of one atom of hydrogen which is taken as unity. For example, if one atom each of hydrogen and chlorine compose a molecule of hydrogen chloride and if, as we have seen, an atom of chlorine weighs 35.5 times as mich as an atom of hydrogen, then a molecule of the compound must weigh 36.5 times as much as an atom of hydrogen; and we say therefore that the Molecular Weight of hydrogen chloride is 36.5. The molecular weight of a gas has consequently the same mumerical value as its formula weight, the weight of an atom of hydrogen being in all cases taken as unity. The conclusion that the relative weights of the molecules of gases are proportional to their respective formula weights follows at once from the assumption of Avogadro's hypothesis. But we now see also that the weights of gaseous molecules, briefly their molecular weights, are all represented by the same numbers as their formula weights if we choose the atomic weight of hydrogen as unity; for this reason it seemed logical to discuss atomic weights before molecular weights. It is also evident that the molecular weight of a substance must be equal to the sum of the atomic weights indicated by the formula.

218. The Formulae of Some Elementary Gases.-We are now in position to consider the meaning of the fact that the formulae $\mathrm{H}_{2}, \mathrm{O}_{2}, \mathrm{~N}_{2}$, and $\mathrm{Cl}_{2}$ were found for the four elementary gases studied. If we accept Arogadro's hypothesis for these as well as for compound gases, then the unit volume of each gas must also contain $N$ molecules. But we know also that the weight in each case is twice the minimum weight of $N$ atoms; for this reason we are forced to conclude that the unit volume of each gas contains $2 N$ atoms, and hence that each molecule contains two atoms. The same rule that applies to compound gases applies here also: the number of atoms of each element in any 
molecule is the same as the number of symbol weights of that element in the formula of the substance. The obvious meaning of these facts is that an atom of hydrogen, for example, can unite with another atom of hydrogen as well as with one of chlorine or some other element. There remains the question: Do we have any other evidence of the truth of this conclusion? Let us see.

219. The Union of Hydrogen and Chlorine by Volume.-It will be recalled that when hydrogen and chlorine gases unite to form gaseous hydrogen chloride, one volume of each of the elementary gases combines to give two volumes of the product. Now in two unit volumes of 22.4 liters each of hydrogen chloride there must be $2 \mathrm{~N}$ atoms of hydrogen and $2 \mathrm{~N}$ atoms of chlorine, since we cannot have less than one atom of each element in a molecule of the compound; but the two unit volumes of hydrogen chloride are formed from one unit volume of hydrogen and one of chlorine each containing $N$ molecules; and again we are led to the conclusion that $N$ molecules of hydrogen or chlorine contain $2 . V$ atoms in each case, or that each molecule of either elementary gas contains two atoms.

220. Gay Lussac's Law of Combining Volumes. - The simple volumetric relation between hydrogen, chlorine, and hydrogen chloride, I:I:2, is not an exceptional case; other gases also exhibit similar simple relations. Thus, two volumes of hydrogen and one volume of oxygen unite and, if the temperature at which the experiment is carried out is so high that the water remains in the form of steam, the latter measures two volumes; so that the volume relations are $2: 1: 2$. In the burning of methane one volume of the gas requires two volumes of oxygen and gives one volume of carbon dioxide and two volumes of steam, the measurements all being made at a sufficiently high temperature in this case also to keep the steam from condensing. Or, when ammonia gas is decomposed, as it may be by means of electric sparks, two volumes of ammonia yieid one volume of nitrogen and three volumes of hydrogen. The fact that gases and vapors of volatile substances always react in simple ratios by volume was discovered by Gay Lussac in I808, and is known as Gay Lussac's Law of Combining Volumes. 
221. Explanation of the Law of Combining Volumes.-The explanation of this law will appear if we write the equations of the reactions mentioned:
$\mathrm{H}_{2}+\mathrm{Cl}_{2} \rightarrow 2 \mathrm{HCl}$
$\mathrm{CH}_{4}+2 \mathrm{O}_{2} \rightarrow \mathrm{CO}_{2}+2 \mathrm{H}_{2} \mathrm{O}$
I vol. I vol. 2 vols.
I vol. 2 vols. I vol. 2 vols.
${ }_{2} \mathrm{H}_{2}+\mathrm{O}_{2} \rightarrow 2 \mathrm{H}_{2} \mathrm{O}$
2 vols. I vol. 2 vols.

$$
\begin{aligned}
& 2 \mathrm{NH}_{3} \rightarrow{ }_{3} \mathrm{H}_{2}+\mathrm{N}_{2} \\
& 2 \text { vols. } 3 \text { vols. I vol. }
\end{aligned}
$$

Again we see, as we did earlier $(76,77)$, that the volumes are the same as the coefficients of the formulae in the equations, and this for the fundamental reason that one formula weight always represents one unit volume, in the case of a gaseous or volatile substance. Moreover, we now understand, to cite the first example, that one unit volume of hydrogen containing $N$ molecules and $2 \mathrm{~N}$ atoms will require $2 \mathrm{~N}$ atoms of chlorine or $N$ molecules, which, according to Arogadro's hypothesis, will be found in one unit volume of chlorine. The reaction will then produce $2 N$ molecules of hydrogen chloride, which according to the same hypothesis will occupy two unit volumes. Similar reasoning may be applied to all other cases.

222. The Degree of Accuracy of Symbol Weights.-Before leaving the discussion of symbol and atomic weights we must consider the degree of accuracy of the statements of numerical results made in early chapters and summarized in Table IV. It is perhaps needless to point out that statements of lengths, areas, volumes, weights, etc., whether they refer to scientific or other matters, are in general more or less approximate, the degree of accuracy aimed at being determined by the requirements of the case. Thus, if a stranger in the city inquires the distance from the City Hall to the University and is told by a policeman that it is seven miles, the answer is quite as accurate as necessary. But such approximate statements of distance would not satisfy the requirements of a surveyor who wished to make an accurate map of the city. Up to about twenty-five years ago the most accurate analyses of water indicated that $2 \mathrm{~g}$. of hydrogen were combined with $55.96 \mathrm{~g}$. of oxygen. As all chemists know that in every analysis there is inevitably some experimental error 
of greater or less magnitude, it was thought that the true weight of oxygen combined with exactly $2 \mathrm{~g}$. of hydrogen was exactly I6 $\mathrm{g}$. It then became apparent from the new researches of a number of chemists that the error in the accepted results was greater than suspected, and, moreover, that the true proportion of oxygen in water was less instead of greater than the value found earlier, the new experiments leading to a ratio of 2 to $\mathrm{I}_{5} .88$, with a probable error of less than $0.0 \mathrm{Ig}$. in the weight of oxygen combined with exactly $2 \mathrm{~g}$. of hydrogen.

223. $O=16.000$, the Real Basis for Symbol and Atomic Weights.-An annoying difficulty now arose from the fact that far more symbol weights had been found by the analysis of oxygen compounds than by the analysis of compounds with hydrogen, owing to the greater accuracy with which the former analyses could be made; so that it then became necessary for chemists to decide whether they should change the symbol weights of oxygen and all elements whose symbol weights had been found by the analysis of their oxygen compounds, or whether they should change the symbol weights of hydrogen and a few other elements. After much debate the former policy was adopted and the symbol weight of oxygen, $\mathrm{O}=\mathrm{r} 6.000$, kept unchanged, although this made it necessary to change the symbol weight of hydrogen to I.008. Our most accurate knowledge of the composition of water is expressed by the statement that $2 \times 1.008 \mathrm{~g}$. of hydrogen are combined with $16.000 \mathrm{~g}$. of oxygen in $18.016 \mathrm{~g}$. of water, a fact which is also expressed by the formula $\mathrm{H}_{2} \mathrm{O}$, when we consider that $\mathrm{H}=\mathrm{I} .008 \mathrm{~g}$. of hydrogen and $\mathrm{O}=\mathrm{I} 6.000 \mathrm{~g}$. of oxygen. Oxygen with a symbol weight of 16.000 has thus become the real basis of the system of symbol and atomic weights rather than hydrogen with a symbol weight of unity.

224. The Method of Finding Symbol Weights.-The symbol weights, and therefore also the atomic weights, of all other elements are now based upon that of oxygen taken as 16.000 ; but we see by a comparison of the values given in a table of exact atomic weights that in no case does the exact value based on $\mathrm{O}=\mathrm{I} 6.000$ differ greatly from the approximate value we have previously used. Just as more accurate analyses led to a change 
in the symbol weight of hydrogen, so also newer analyses have led and will continue in the future to lead to a more exact knowledge of the symbol weights of other elements. We do not expect, however, that the values accepted at present for the commoner elements will be changed by more than a few units in the second decimal place. Concisely stated, the matter stands thus: Approximate symbol weights are found in the manner described in chap. v, while the more exact values are fixed by the most painstaking analyses and syntheses, being computed on the basis of $O=16.000$.

225. Inexactness of the Gas Laws.-The gas laws of Boyle, Charles, and Avogadro are also only closely approximate statements of the facts. For example, if the pressure on 1,000 c.c. of oxygen under standard conditions be exactly doubled, the volume will become 499.3 c.c. instead of exactly 500, as Boyle's law would indicate. The deviations from the simple laws are thought to be due to attractions between the molecules, on the one hand, tending to diminish the volume, and, on the other hand, to the fact that part of the space occupied by the gas is filled with the molecules themselves, so that the free space is reduced to less than half if the volume of the gas is, by increase of pressure, reduced to half. The actual deviations from the simple law, $P V=$ a constant, become negligible if gases are under low pressures. Then the three great laws express almost exactly the behavior of all gases. In other words, if the barometric pressure at sea-level were $0.0 \mathrm{I}$ of its actual value, so that our standard of atmospheric pressure would be $0.76 \mathrm{~cm}$. of mercury instead of $76 \mathrm{~cm}$., then we should find that not only would the laws of Boyle and Charles express with a high degree of accuracy the behavior of gases under pressures of this order of magnitude, but that for all gases the law of Avogadro would also hold good with as great a degree of accuracy as experiment would enable us to determine.

226. Exactness of Avogadro's Law for Corrected Gas Volumes.-Now, instead of trying to weigh and measure gases under such low pressures in attempts to study them more accurately, chemists have worked at ordinary pressures and then corrected 
the data so obtained so as to give the results that would theoretically have been obtained for the weights of I liter if the measurements had been made at very low pressures and the calculations made for a pressure of $76 \mathrm{~cm}$. exactly according to Boyle's law. Working in this way, it was found that the corrected volume of $32 \mathrm{~g}$. of oxygen, the weight represented by $\mathrm{O}_{2}$, is $22.4 \mathrm{I}$ liters at $0^{\circ}$. It was then discovered that exactly this (corrected) volume of any other gas at $0^{\circ}$ contains, as nearly as the determinations could be made, just the weight of the gas which its formula indicates, this weight being calculated from the most exact symbol weights. In others, Avogadro's law would hold exactly at low pressures or also at ordinary pressure if the attractions of the molecules for each other did not exist, and if their own volumes were negligible as compared with the total space occupied by the gas.

227. A Little Explanation and Advice.-It is not necessary nor desirable that the beginner in chemistry should pay much attention to the matters discussed in the three preceding paragraphs. The approximate symbol weights and the gas laws in their simplest forms are sufficiently exact for his use. It is much better that he should see clearly the general fundamental principles than that he should be perplexed and confused by the details and refinements that are of importance only to the specialist. If the beginner continues his study of chemistry he will be sure to encounter later these interesting topics, when he will be better able to appreciate and understand them; while if he should not go farther than the first course, he may feel assured that he has become acquainted with the principles of most fundamental importance. These matters are discussed here in order to explain why the symbol or atomic weights given in Tables of Atomic Weights (see inside of back cover of this book) are not exactly the same as those we have used in the earlier chapters.

228. Means of Discovering Symbol Weights.-The student will doubtless have received the impression from the study of the foregoing chapters that we can discover the approximate symbol weight of any element by finding the minimum weight of the element in the unit volume of its gaseous or vaporized com- 
pounds; and this, in fact, is true for a large number of elements in addition to the five included in Table IV. We shall now consider some facts leading to a knowledge of the symbol weights of a dozen elements other than the five already studied. These twelve elements all form volatile compounds, the densities of which may be determined by making experiments at sufficiently high temperatures and then calculating, by the laws of Boyle and Charles, for the standard conditions, the weight of the compound in 22.4 liters. Multiplication of the weight so found by the percentage of the element in question in the compound gives the weight of the element in 22.4 liters of the vapor, as recorded in Table $\mathrm{X}$.

TABLE $\mathrm{X}$

\begin{tabular}{|c|c|c|c|c|}
\hline $\begin{array}{l}\text { Volatile Compounds of } \\
\text { Various Elements }\end{array}$ & $\begin{array}{l}\text { Weight of } \\
\text { Elements in } \\
22.4 \text { Liters }\end{array}$ & $\begin{array}{l}\text { Symbol and } \\
\text { Symbol Weight }\end{array}$ & Specific Heat & $\begin{array}{c}\text { Product of } \\
\text { Symbol Weight } \\
\text { and Specific Heat }\end{array}$ \\
\hline Antimony trichloride. . & $119 \cdot 5$ & $\mathrm{Sb}=\mathrm{t} 20.2$ & 0.0503 & 6.0 \\
\hline Arsenic trichloride... & $75 \cdot 4$ & $A s=75.0$ & 0.0830 & 6.2 \\
\hline Bismuth trichloride. & 217.0 & $\mathrm{Bi}=208.5$ & 0.0303 & 0.3 \\
\hline Cadmium. . . . $\ldots \ldots$ & 114.0 & $\mathrm{Cd}=112.4$ & $0.055^{1}$ & 6.2 \\
\hline Chromium oxychloride. & 55.0 & $\mathrm{Cr}=5^{2} \cdot \mathrm{I}$ & $0.112 \mathrm{I}$ & $5 \cdot 8$ \\
\hline Hydrogen iordide. . . . . & 127.7 & $I=127.9$ & $0.054 \mathrm{I}$ & 6.9 \\
\hline Iron carbonyl. . & 53.2 & $\mathrm{Fe}=55.9$ & 0.1162 & 6.5 \\
\hline Lead chloride.. & 207.2 & $\mathrm{~Pb}=206.9$ & 0.0304 & 6.3 \\
\hline Mercury........ & 202.2 & $\mathrm{Hg}=200.0$ & 0.0308 & 6.1 \\
\hline Nickel carbonyl........ & $59 \cdot 7$ & $\mathrm{Ni}=58.7$ & 0. 1084 & 6.4 \\
\hline Phosphorus trichloride. . & $3 \mathrm{I} \cdot 0$ & $P=31.0$ & 0.2020 & 6.3 \\
\hline Zinc chloride.......... & $63 \cdot 5$ & $\mathrm{Zn}=65.4$ & 0.0935 & 6.1 \\
\hline
\end{tabular}

In all cases the compounds are such as contain the minimum weight of the element the symbol of which appears in the table; that is to say, we do not know any other volatile compounds in the respective cases containing appreciably smaller weights in the unit volume. 'The weights so found are, therefore, approximately the symbol weights in each case. The exact symbol weight in any case is then calculated from the accurately determined percentage composition of some compound of the element with an element of exactly known symbol weight.

229. The Product of Specific Heats and Symbol Weights. There are a great many elements which do not form gaseous compounds, or compounds which are sufficiently volatile without 
decomposition, to enable us to find their symbol weights in the manner above indicated. Very fortunately other methods have long been known by which the desired end can be attained. We shall now consider one of these methods.

A very simple relation was discovered nearly, a century ago, by Dulong and Petit, between symbol weights and specific heats of solid free elements. The amount of heat required to raise the temperature of a given weight of iron $I^{\circ}$ would raise the temperature of an equal weight of water only $0.1162^{\circ}$; and we say, therefore, that the specific heat of iron is 0.1162 . The specific heats of the other elements of Table $\mathrm{X}$ are given in the fourth column. If, now, we multiply the specific heat of an element by its symbol weight we get the remarkable series of products contained in the last column of the table, where we see that the values are nearly the same in all cases. Does it not seem probable that the law which we find applying to the elements of Table $\mathrm{X}$ would also hold good for other solid elements even though they do not form easily volatile compounds? If so, it is clear that in order to find the approximate symbol weight of an element we have only to divide 6.4 by its specific heat, which latter constant can in general be found by a simple, direct experiment. As a matter of fact, this method has been of much service in just this way.

230. Interpretation of the Law of Dulong and Petit.- The law of Dulong and Petit is, moreover, of the greatest interest and importance when viewed from the theoretical standpoint. The product of the specific heat and symbol weight is obviously the quantity of heat required to raise the temperature of the symbol weight of an element one degree; and this amount of heat is the same for one element as for another. But the symbol weights of various elements are the weights of equal numbers of atoms, and we see, therefore, that it requires equal amounts of heat to raise the temperature of equal numbers of various kinds of atoms by one degree! The products of symbol weights and specific heats are generally called Atomic Heats; so that the Law of Dulong and Petit may be stated thus: The atomic heats of the solid elements are equal. 


\section{CHAPTER XII}

\section{THE HALOGENS AND THEIR COMPOUNDS WITH HYDROGEN AND METALS}

231. The Halogens:-The elements fluorine, chlorine, bromine, and iodine bear a close resemblance to one another in their properties and chemical behavior; collectively they are called the halogens (from halite, the scientific name for rock salt). In the present chapter we shall first briefly review what has already been learned about chlorine and some of its compounds, and then after a more extensive consideration of the chemistry of chlorine take up a study of the remaining members of this important group of elements.

232. Résumé of Facts Already Learned.-We know that common salt, $\mathrm{NaCl}$, is the most abundant compound of chlorine; it forms the raw material from which all other compounds of chlorine as well as the free element are made. The action of sulfuric acid on salt (103) yields hydrochloric acid which, by electrolysis (43) or by the action of lead dioxide, gives free chlorine (167). With bases or metallic oxides hydrochloric acid yields chlorides, as illustrated by the following reactions:

$$
\begin{aligned}
\mathrm{KOH}+\mathrm{HCl} & \rightarrow \mathrm{KCl}+\mathrm{H}_{2} \mathrm{O} \\
\mathrm{MgO}+2 \mathrm{HCl} & \rightarrow \mathrm{MgCl}_{2}+\mathrm{H}_{2} \mathrm{O} .
\end{aligned}
$$

Chlorides also result when carbonates are treated with hydrochloric acid (I63):

$$
\begin{aligned}
\mathrm{Na}_{2} \mathrm{CO}_{3}+{ }_{2} \mathrm{HCl} & \rightarrow 2 \mathrm{NaCl}+\mathrm{CO}_{2}+\mathrm{H}_{2} \mathrm{O} \\
\mathrm{CaCO}_{3}+2 \mathrm{HCl} & \rightarrow \mathrm{CaCl}_{2}+\mathrm{CO}_{2}+\mathrm{H}_{2} \mathrm{O} .
\end{aligned}
$$

It will be recalled that the chlorides of silver, lead, and univalent mercury are almost insoluble in water $(167, \mathbf{1 6 9}, \mathbf{1 8 2})$; these salts are easily obtained by the action of solutions of hydrochloric acid or any soluble chloride on solutions of soluble salts of these metals, thus:

$$
\begin{aligned}
\mathrm{AgNO}_{3}+\mathrm{HCl} & \rightarrow \mathrm{AgCl}+\mathrm{HNO}_{3} \\
\mathrm{~Pb}\left(\mathrm{NO}_{3}\right)_{2}+2 \mathrm{NaCl} & \rightarrow \mathrm{PbCl}_{2}+2 \mathrm{NaNO}_{3} .
\end{aligned}
$$


The metals which react with hydrochloric acid set free hydrogen and are themselves converted into chlorides, for example:

$$
\begin{gathered}
\mathrm{Zn}+2 \mathrm{HCl} \rightarrow \mathrm{ZnCl}_{2}+\mathrm{H}_{2} \\
2 \mathrm{Al}+6 \mathrm{HCl} \rightarrow 2 \mathrm{AlCl}_{3}+3 \mathrm{H}_{2} .
\end{gathered}
$$

Chlorides also result from the direct union of chlorine with other elements:

$$
\begin{array}{r}
\mathrm{H}_{2}+\mathrm{Cl}_{2} \rightarrow{ }_{2} \mathrm{HCl} \\
2 \mathrm{Al}+\mathrm{Cl}_{2} \rightarrow{ }_{2} \mathrm{AlCl}_{3} .
\end{array}
$$

\section{The Occurrence of Chlorine Compounds in Nature.-} Free chlorine does not occur in nature. If free chlorine were present in nature it would very soon unite with other substances to form compounds. Common salt is by far the most abundant natural compound of the element. It occurs as a mineral, rock salt (halite), and as dissolved salt in sea-water and the waters of salt lakes and springs. Sea-water contains about 3 per cent, while the water of Great Salt Lake in Utah contains about 20 per cent, of salt. Rock salt has doubtless been formed in past geological times by the slow, natural evaporation of seawater. Other chlorides, particularly those of potassium, $\mathrm{KCl}$; magnesium, $\mathrm{MgCl}_{2}$; silver, $\mathrm{AgCl}$; and lead, $\mathrm{PbCl}_{2}$, are also found in nature.

234. The Discovery of Chlorine.-Free chlorine was first made by the Swedish chemist Scheele, in I774, and therefore practically at the same time that Lavoisier in France discovered the true explanation of burning. Scheele made chlorine by the action of hydrochloric acid on manganese dioxide, a mineral having the formula $\mathrm{MnO}_{2}$, and therefore an oxide of the metallic element, manganese. The reaction occurs thus:

$$
{ }_{4} \mathrm{HCl}+\mathrm{MnO}_{2} \rightarrow \mathrm{MnCl}_{2}+\mathrm{Cl}_{2}+2 \mathrm{H}_{2} \mathrm{O} .
$$

Chlorine was not thought to be an element until nearly forty years after its discovery, but was believed to be an oxide of hydrochloric acid, until a famous English chemist, Sir Humphrey Davy, showed by conclusive experiments that it did not contain oxygen and was really an elementary substance. 
235. The Preparation of Chlorine from Hydrochloric Acid.We have already seen (I67) that chlorine is formed when lead dioxide is warmed with hydrochloric acid:

$$
{ }_{4} \mathrm{HCl}+\mathrm{PbO}_{2} \rightarrow \mathrm{PbCl}_{2}+\mathrm{Cl}_{2}+{ }_{2} \mathrm{H}_{2} \mathrm{O} .
$$

'This reaction is entirely analogous to the one between hydrochloric acid and manganese dioxide mentioned in the preceding paragraph, and since the last substance is cheaper than lead dioxide it is the one commonly used in the laboratory for the preparation of chlorine. The experimental method consists in adding to, say, Ioog. of granular manganese dioxide contained in a flask about 300 c.c. of concentrated hydrochloric acid and warming gently:

$$
{ }_{4} \mathrm{HCl}+\mathrm{MnO}_{2} \rightarrow \mathrm{MnCl}_{2}+\mathrm{Cl}_{2}+2 \mathrm{H}_{2} \mathrm{O} \text {. }
$$

Manganese chloride is an easily soluble salt which forms pink crystals of a hydrate: $\mathrm{MnCl}_{2} \cdot{ }_{4} \mathrm{H}_{2} \mathrm{O}$.

An excellent, though expensive, method of making small amounts of chlorine for experimental work in the

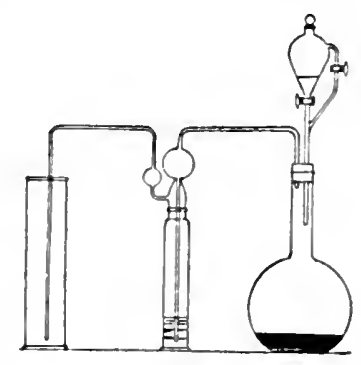

FII. 30 laboratory consists in allowing concentrated hydrochloric acid to drop slowly on to solid potassium permanganate, $\mathrm{KMnO}_{4}$ (Fig. 30 ). The latter substance is one of the most powerful oxidizing agents and reacts rapidly in the cold with hydrochloric acid, thus:

$$
16 \mathrm{HCl}+2 \mathrm{KMnO}_{4} \rightarrow 2 \mathrm{KCl}+2 \mathrm{MnCl}_{2}+{ }_{5} \mathrm{Cl}_{2}+8 \mathrm{H}_{2} \mathrm{O} .
$$

Since the rate of production of chlorine is easily regulated by control of the rate of flow of the acid, the method is a very convenient one for the lecture table.

236. Chlorine, a Poisonous Gas. - The chlorine which is given off is a heavy, yellowish, poisonous gas having an exceedingly violent action on all mucous membranes. It is the gas which was first used with such frightful effect in the trenches in the European war. Great care must be exercised to prevent the escape of appreciable amounts of chlorine into the air of the laboratory 
and to avoid as far as possible inhalation of the gas. Waste chlorine is easily absorbed when passed into a solution of caustic soda.

237. The Electrolytic Preparation of Chlorine.-We have already learned (43) that chlorine is formed when hydrochloric acid is electrolyzed. By means of the Brownlee apparatus shown in Fig. $2 \mathrm{I}$ it is found that equal volumes of hydrogen and chlorine are formed when the concentrated acid is used. If, however, very dilute acid is used, then the products are largely hydrogen and oxygen formed by the decomposition of the water, and very little chlorine is set free. A complete explanation of this curious fact is not possible until certain matters treated in a following chapter have been considered; but it may be stated that hydrochloric acid is more easily decomposed than water by the electric current, and that if much of the former is present in a water solution it is decomposed by preference to the water. In the electrolysis apparatus the poles or electrodes are sticks of carbon. The hydrogen is liberated at the negative pole, the chlorine at the positive pole.

238. The Electrolysis of Common Salt.-The electrolysis of a concentrated solution of common salt is by far the most important practical method for the manufacture of chlorine. It is a process which is carried out on a very large scale, as at Niagara Falls, where electrical power is cheap and yields not only chlorine but also hydrogen and caustic soda. We might expect the products of the electrolysis of salt to be sodium and chlorine,

$$
{ }_{2} \mathrm{NaCl} \rightarrow 2 \mathrm{Na}+\mathrm{Cl}_{2},
$$

but when we recall that sodium reacts at once with water to form hydrogen and sodium hydroxide (caustic soda), the actual result appears reasonable. A more complete explanation must be deferred until later. As in the case of the electrolysis of hydrochloric acid the chlorine is set free at the positive electrode, which is a carbon plate, while the sodium and hydrogen are formed at the negative electrode.

239. Deacon's Process.-Before the electrical method just described was used practically, a process invented by Deacon 
was the cheapest technical method of making chlorine. This process is based on the fact that a mixture of hydrogen chloride gas and oxygen react at a high temperature to form chlorine and water,

$$
{ }_{4} \mathrm{HCl}+\mathrm{O}_{2} \rightarrow{ }_{2} \mathrm{Cl}_{2}+{ }_{2} \mathrm{H}_{2} \mathrm{O} \text {. }
$$

This reaction scarcely takes place at all at ordinary temperatures, and even at the most favorable high temperature it takes place very slowly. Deacon discovered that the reaction could be greatly hastened if the heated mixture of hydrogen chloride and oxygen were passed over broken bricks coated with copper chloride, $\mathrm{CuCl}_{2}$. A small amount of this substance is able to promote the reaction of almost unlimited amounts of the reacting gases without itself being permanently changed or destroyed. A substance that behaves in this way is called a catalytic agent. Catalytic agents of various sorts are extensively employed in chemistry. In the Deacon process air, which is essentially a mixture of oxygen and nitrogen, may be used instead of pure oxygen, which would be too expensive for practical purposes.

240. A Remarkable Phenomenon: Chemical Equilibrium.It is a remarkable fact that even under the most favorable conditions the reaction between hydrogen chloride and oxygen does not go to completion, but stops while the gaseous mixture still contains some of both of these gases. The cause is discovered when we find that steam and chlorine react at about $400^{\circ}$ to give some hydrogen chloride and oxygen:

$$
{ }_{2} \mathrm{Cl}_{2}+{ }_{2} \mathrm{H}_{2} \mathrm{O} \rightarrow{ }_{4} \mathrm{HCl}+\mathrm{O}_{2} \text {. }
$$

This is, in facl, exactly the reverse of the reaction we have been considering. It is plain, therefore, that the failure of the reaction between hydrogen chloride and oxygen to go to completion is due to the interaction of the products, chlorine and water, to form again some of the first pair of gases.

If a mixture of hydrogen chloride and oxygen in the proportions shown in the equation is heated to a constant temperature, say $400^{\circ}$, a mixture finally results in which all four of the substances are present in definite proportions. A mixture having exactly the same proportions of each of the four substances 
results if the starting substances are chlorine and water, taken also in the proportions indicated by the equation. In the mixture which finally results, the four substances are said to be in a state of chemical equilibrium. The subject of chemical equilibrium is a very important one which is to be studied in detail in the next chapter.

24I. The Physical Properties of Chlorine.-Chlorine is a pale-yellow gas, having a density about two and a half times as great as air. Under standard conditions one liter weighs $3.22 \mathrm{~g}$. Chlorine is rather soluble in water, Ioo c.c. of water at $20^{\circ}$ dissolving 226 c.c. of the gas. For this reason the gas is not easily

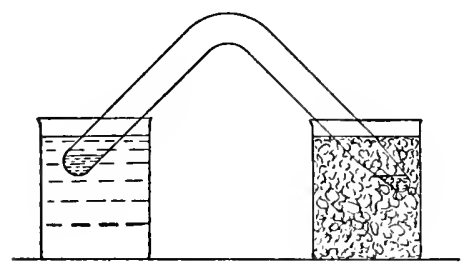

FIG. 3I collected over water; on account of its high density it is easily collected by the downward displacement of air. If a water solution of chlorine is cooled nearly to $\circ^{\circ}$, yellow crystalline chlorine hydrate, having the formula $\mathrm{Cl}_{2} \cdot 8 \mathrm{H}_{2} \mathrm{O}$, is formed.

This hydrate is very unstable and decomposes slowly at room temperature and rapidly at higher temperatures into chlorine gas and water.

242. The Liquefaction of Chlorine.-A very interesting and important experiment was once made with this hydrate by the great English physicist and chemist Faraday, who was at the time assistant to Sir Humphrey Davy (234). Crystals of chlorine hydrate were sealed up in one end of a bent glass tube, as shown in Fig. 3I; when the hydrate was gently warmed while the other end of the tube was cooled with ice a yellow liquid formed in the cold end of the tube. This liquid proved to be liquefied chlorine. It is a heavy, mobile liquid, which is easily obtained from chlorine gas either by cooling the latter to about $40^{\circ}$ below zero at atmospheric pressure, or by compressing it to about four atmospheres' pressure at about $0^{\circ}$. Under one-atmosphere pressure liquid chlorine boils at $-34^{\circ}$. This work of Faraday in liquefying chlorine was of very great importance, since it was the beginning of the epoch-making 
experiments in which he succeeded in liquefying all known gases except five, among which were hydrogen, oxygen, and nitrogen.

243. The Union of Chlorine and Hydrogen.-Chlorine and hydrogen do not react at an appreciable rate at room temperature if kept in complete darkness, but do unite with explosive violence if exposed to a bright light, hydrogen chloride being formed, thus:

$$
\mathrm{H}_{2}+\mathrm{Cl}_{2} \rightarrow 2 \mathrm{HCl} \text {. }
$$

In order to demonstrate this interesting phenomenon a thinwalled glass bulb is filled with a mixture of equal volumes of the two gases; the bulb is then covered with a thick-walled bell jar (Fig. 32) and strongly illuminated either by direct sunlight

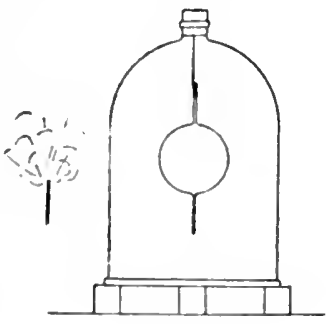

FIG. 32

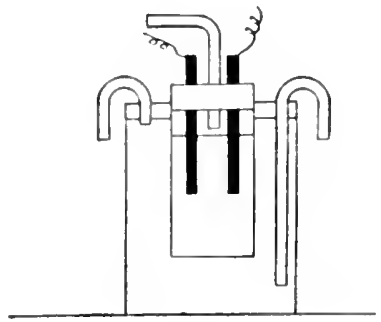

Fig. 33

or by the rays from burning magnesium ribbon. The sharp explosion which follows reduces the glass bulb to a powder, but does no damage to the bell jar. The mixture of chlorine and hydrogen is best obtained by the electrolysis of concentrated hydrochloric acid in the apparatus shown in Fig. 33. The inner vessel has two carbon electrodes. It is surrounded by a larger vessel, through which water flows to prevent rise of temperature. During the filling of the bulb and up to the time all is ready for the explosion it must be shielded from bright light. The union of chlorine with hydrogen takes place slowly, without explosion, if the mixture of the two gases is exposed for a sufficient length of time to moderate light (44).

244. The Burning of Hydrogen in Chlorine.-If a jet of hydrogen burning in air is lowered into a jar of chlorine it continues 
to burn with a pale flame (Fig. 34). The flame is the result of the intense heat produced by the union of the two gases to form hydrogen chloride.

245. The Action of Chlorine on Water.-Water dissolves about two or three times its own volume of chlorine at room

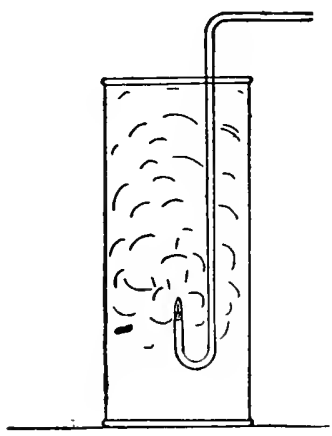

FIG. 34 temperature, giving a yellowish solution known as chlorine water. This solution smells strongly of chlorine and is often used in the laboratory in place of chlorine gas. If chlorine water is exposed to light it soon loses its color and odor, and at the same time a colorless, odorless gas, which proves to be oxygen, is given off. The experiment may readily be carried out in the manner shown in Fig. 35. A cylinder filled with chlorine water is inverted in a dish or beaker and exposed to bright light for a day or two. The gas produced will be found to be oxygen, formed according to the equation

$$
{ }_{2} \mathrm{Cl}_{2}+{ }_{2} \mathrm{H}_{2} \mathrm{O} \rightarrow{ }_{4} \mathrm{HCl}+\mathrm{O}_{2} \text {. }
$$

This is the reversal of the reaction by which chlorine is made by Deacon's process. While chlorine gas and steam react only partially at a high temperature, as already stated, chlorine dissolved in water and exposed to light reacts slowly, but completely, at room temperature to form hydrochloric acid and oxygen. This curious difference in behavior may be traced to the fact that while gaseous hydrogen chloride and oxygen react to the extent of about 80 per cent at $400^{\circ}$, oxygen gas does not act at all on a solution of

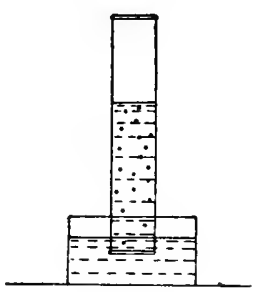

FIG. 35 hydrochloric acid at room temperature. No chlorine and water, therefore, can be reproduced in cold water solution from the products of the action of these two substances, and so the main reaction goes on to completion. Much more is known 
about the action of chlorine on water than is contained in this paragraph, and the subject will be taken up again in the following chapter.

246. The Union of Chlorine with Metals.-Chlorine unites directly with many metals forming chlorides. In many cases the reaction takes place at once, with the production of heat and even in some cases of light, upon bringing the metal into chlorine gas. Thin pieces of copper in the form of dutch metal take fire when dropped into a jar of chlorine, forming copper chloride,

$$
\mathrm{Cu}+\mathrm{Cl}_{2} \rightarrow \mathrm{CuCl}_{2} \text {. }
$$

The metal antimony (symbol Sb), in the form of powder, also unites with chlorine, with the production of light and heat, if sifted into a cylinder of the gas, antimony trichloride being formed,

$$
{ }_{2} \mathrm{Sb}+{ }_{3} \mathrm{Cl}_{2} \rightarrow{ }_{2} \mathrm{SbCl}_{3} \text {. }
$$

Chlorine also unites directly with sodium, potassium, magnesium, zinc, iron, aluminum, mercury, and many other metals to form the corresponding chlorides.

247. The Union of Chlorine and Phosphorus.-The element phosphorus is a white, waxy solid which can be made from calcium phosphate, bone ash (I58). We have already seen (Io) that phosphorus burns readily in the air. In so doing it unites with oxygen, thus:

$$
{ }_{4} \mathrm{P}+{ }_{5} \mathrm{O}_{2} \rightarrow{ }_{2} \mathrm{P}_{2} \mathrm{O}_{5},
$$

forming a white, solid product, phosphorus pentoxide. Phosphorus also unites directly with chlorine to form either phosphorus trichloride, $\mathrm{PCl}_{3}$, or phosphorus pentachloride, $\mathrm{PCl}_{5}$. The preparation of the trichloride may be carried out in a retort as shown in Fig. 36. About $20 \mathrm{~g}$. of dry phosphorus are placed in the retort and a stream of chlorine, dried by passing it through a wash bottle containing concentrated sulfuric acid, is passed in by means of the glass tube which passes through the stopper of the retort. As soon as the chlorine reaches the phosphorus, union takes place with the formation of much heat and the appearance of a pale flame. The course of the reaction is readily con trolled by regulating the rate of flow of the gas and by moving 
the gas inlet tube up or down in the retort. If the contents get too hot so that phosphorus begins to distil, the temperature can be lowered by raising the tube. On the other hand, if yellowish crystals of the pentachloride appear in the retort, the temperature is too low and the tube should be lowered. The reaction occurs thus:

$$
{ }_{2} \mathrm{P}+{ }_{3} \mathrm{Cl}_{2} \rightarrow{ }_{2} \mathrm{PCl}_{3} \text {. }
$$

Phosphorus trichloride distils over and condenses to a liquid in the cooled receiver. It may be purified by being distilled

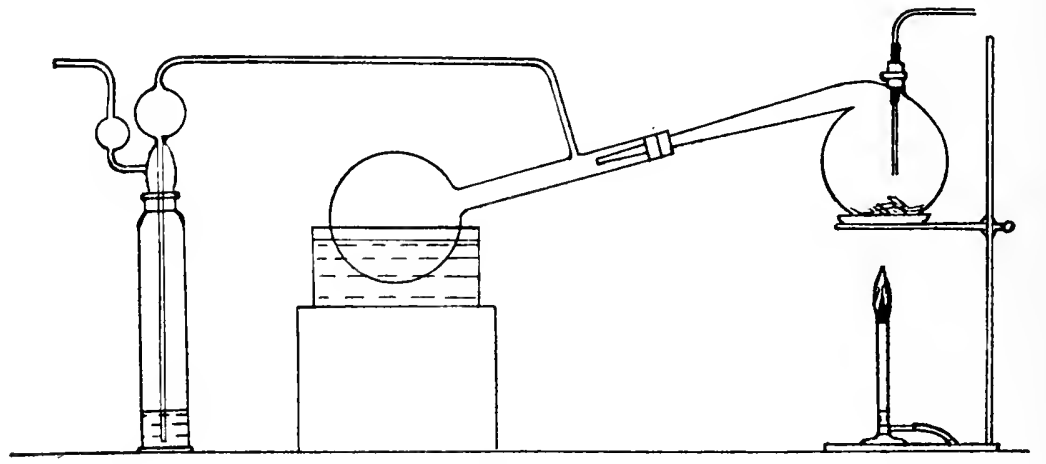

Fig. 36

from a clean, dry retort. It is a colorless liquid which boils at $74^{\circ}$. It readily unites with more chlorine, forming solid crystalline pentachloride, $\mathrm{PCl}_{5}$ :

$$
\mathrm{PCl}_{3}+\mathrm{Cl}_{2} \rightarrow \mathrm{PCl}_{5} \text {. }
$$

The chlorides of phosphorus are not salts. Both compounds are acted upon vigorously by water, according to the following equations:

$$
\begin{aligned}
& \mathrm{PCl}_{3}+{ }_{3} \mathrm{H}_{2} \mathrm{O} \rightarrow \mathrm{H}_{3} \mathrm{PO}_{3}+{ }_{3} \mathrm{HCl} \\
& \mathrm{PCl}_{5}+{ }_{4} \mathrm{H}_{2} \mathrm{O} \rightarrow \mathrm{H}_{3} \mathrm{PO}_{4}+{ }_{5} \mathrm{HCl} .
\end{aligned}
$$

The products are hydrochloric acid and in the first case phosphorous acid, $\mathrm{H}_{3} \mathrm{PO}_{3}$, and in the second case phosphoric acid, $\mathrm{H}_{3} \mathrm{PO}_{4}$ (159). 
248. Chlorine and Turpentine.-Turpentine is a colorless liquid having the formula $\mathrm{C}_{\mathbf{r 0}} \mathrm{H}_{16}$. It reacts violently with chlorine, thus:

$$
\mathrm{C}_{10} \mathrm{H}_{16}+8 \mathrm{Cl}_{2} \rightarrow 10 \mathrm{C}+{ }_{\mathrm{I}} 6 \mathrm{HCl} \text {. }
$$

The reaction is best shown by bringing a strip of filter paper which has been dipped in turpentine into a cylinder of chlorine; a flash of flame occurs accompanied by a dense, black smoke, due to the finely divided carbon formed. This reaction, as well as that between chlorine and water, shows the great tendency of chlorine to unite with hydrogen even if the hydrogen is in the form of a compound.

249. Practical Uses of Chlorine.-A piece of litmus paper dipped into chlorine water becomes colorless. Many other vegetable colors are also bleached in the same way. 'The process is of great practical importance. All white cotton goods have been bleached by a modification of this process, which will be described in another chapter (35I).

In recent years a new and important use for chlorine has been found as a reagent for the sterilization of municipal water supplies. The effectiveness of chlorine is due to the fact that it is a powerful germicide by reason of its great chemical activity. The chlorine is dissolved in the water at the pumping stations and during the interval required for the water to flow through the mains it reacts with the germs present and is itself reduced to harmless chlorides. The water supply of the city of Chicago is purified in this way.

250. The Preparation of Hydrochloric Acid.-We have already learned that hydrogen chloride is made by the action of sulfuric acid on common salt. The best laboratory method is that described earlier (103), the reaction taking place according to the following equation:

$$
\mathrm{NaCl}+\mathrm{H}_{2} \mathrm{SO}_{4} \rightarrow \mathrm{NaHSO}_{4}+\mathrm{HCl} .
$$

If, however, double the proportion of salt indicated by this equation is taken and the temperature is finally raised sufficiently, the following reaction will take place:

$2 \mathrm{NaCl}+\mathrm{H}_{2} \mathrm{SO}_{4} \rightarrow \mathrm{Na}_{2} \mathrm{SO}_{4}+{ }_{2} \mathrm{HCl}$. 
By the last reaction a given quantity of sulfuric acid will produce double the quantity of hydrogen chloride as in the first; it is therefore the more economical and is the one used in the commercial production of hydrochloric acid.

The union of hydrogen and chlorine to form hydrogen chloride has already been discussed $(44,243)$. In recent years, since chlorine has become available in immense quantities as a by-product of the manufacture of caustic soda, some hydrochloric acid has been produced commercially in this way.

The old name for hydrochlorie acid was muriatic acid, and this is the name by which the crude acid is still commonly known in trade.

25I. The Physical Properties of Hydrogen Chloride.Hydrogen chloride is a colorless gas, having a choking odor and forming a cloud of white fumes in moist air. Its density is somewhat greater than that of air; one liter weighs $\mathrm{I} .642 \mathrm{~g}$. The gas is very soluble in water; at room temperature water dissolves about $45^{\circ}$ times its volume of the gas, giving a concentrated solution of hydrochloric acid. Considerable heat is produced when the gas dissolves in water, so that the solution becomes decidedly warm. In general, when gases dissolve in water heat is produced. So-called chemically pure hydrochloric acid has a specific gravity of $I .2$ and contains about 37 per cent of hydrogen chloride, the balance being water.

When the 37 per cent acid is heated, hydrogen chloride gas is given off, together with some water vapor, and the remaining solution becomes less concentrated. Finally the temperature rises to $110^{\circ}$ before the liquid boils; by this time the concentration has decreased to 20 per cent. As the solution continues to boil, its concentration, 20 per cent, and boilingpoint, $110^{\circ}$, remain constant; the condensed vapor, the so-called distillate, also has a concentration of 20 per cent.

On the other hand, if very dilute hydrochloric acid is boiled it loses water chiefly and becomes more concentrated; finally, when the concentration has reached 20 per cent the boiling temperature has become $110^{\circ}$, after which both concentration and boiling-point remain constant. 
252. The Chemical Properties of Hydrochloric Acid.-The most important chemical properties of hydrochloric acid have already been studied. These may be briefly reviewed in this paragraph. Hydrochloric acid is perhaps the most typical of all acids; it turns litmus red and its very dilute solution, say I per cent, has a pleasant sour taste; it neutralizes the hydroxides and oxides of metals, forming chlorides and water, for example:

$$
\begin{aligned}
\mathrm{NaOH}+\mathrm{HCl} & \rightarrow \mathrm{NaCl}+\mathrm{H}_{2} \mathrm{O} \\
\mathrm{CuO}+2 \mathrm{HCl} & \rightarrow \mathrm{CuCl}_{2}+\mathrm{H}_{2} \mathrm{O} .
\end{aligned}
$$

It acts on many metals forming chlorides and hydrogen, thus:

$$
\mathrm{Fe}+{ }_{2} \mathrm{HCl} \rightarrow \mathrm{FeCl}_{2}+\mathrm{H}_{2} \text {. }
$$

The addition of hydrochloric acid to solutions of salts of silver (I69), lead (I67), and univalent mercury (I82) gives precipitates of insoluble chlorides, thus:

$$
\mathrm{AgNO}_{3}+\mathrm{HCl} \rightarrow \mathrm{AgCl}+\mathrm{HNO}_{3} \text {. }
$$

Oxidizing agents, such as oxygen gas at a high temperature and higher oxides of the metals like manganese dioxide, liberate chlorine:

$$
\begin{gathered}
{ }_{4} \mathrm{HCl}+\mathrm{O}_{2} \rightarrow{ }_{2} \mathrm{Cl}_{2}+{ }_{2} \mathrm{H}_{2} \mathrm{O} \\
{ }_{4} \mathrm{HCl}+\mathrm{MnO}_{2} \rightarrow \mathrm{Cl}_{2}+\mathrm{MnCl}_{2}+{ }_{2} \mathrm{H}_{2} \mathrm{O} .
\end{gathered}
$$

Hydrochloric acid is an almost indispensable chemical reagent. It is used extensively both in scientific and in technical work. It is manufactured in large quantities and is an important article of commerce.

253. The Action of Hydrochloric Acid on Sodium Hydrogen Sulfate.-If concentrated hydrochloric acid is added slowly, with stirring, to a concentrated solution of sodium hydrogen sulfate, a white crystalline precipitate is formed, which, when filtered out, washed with a little water, and dried, is found to consist of pure sodium chloride. The reaction is represented thus:

$$
\mathrm{HCl}+\mathrm{NaHSO}_{4} \rightarrow \mathrm{NaCl}+\mathrm{H}_{2} \mathrm{SO}_{4} .
$$

This is seen to be just the reverse of the reaction by which hydrogen chloride is made from salt. It is therefore a reversible 
reaction. The direction which the reaction will take depends upon the amount of water present and the temperature. Dry salt and anhydrous (water-free) sulfuric acid react practically completely to form hydrogen chloride and sodium hydrogen sulfate; while sufficiently dilute sulfuric acid and salt do not give off any hydrogen chloride gas. The reason is simple: the gas is very soluble in water, and even if it were formed it would remain dissolved in the water present. The fact that concentrated 'solutions of hydrogen chloride and sodium hydrogen sulfate give a precipitate of solid sodium chloride shows clearly that the reaction has a tendency to reverse. It seems probable that in the presence of much water, that is, in dilute solution, all four of the substances are present in any solution that is made by bringing either pair of substances together. In such a solution we may say that there exists a state of equilibrium as the result of each pair of substances on the same side of the equation continuously reacting to form the pair on the opposite side, thus:

$$
\mathrm{H}_{2} \mathrm{SO}_{4}+\mathrm{NaCl} \leftrightarrows \mathrm{NaHSO}_{4}+\mathrm{HCl}
$$

254. Bromine.-The element bromine (symbol Br) resembles chlorine more closely than does any other element. It does not occur free in nature. Its salts, the bromides, are frequently found in small amounts associated with chlorides. Sea-water contains a small proportion of bromides. Large quantities of bromides are obtained from deposits accompanying those of sodium nitrate in the desert regions of Chile. The brines from salt springs in Michigan also furnish bromides in commercial quantities.

255. Sodium bromide, $\mathrm{NaBr}$, potassium bromide, $\mathrm{KBr}$, and magnesium bromide, $\mathrm{MgBr}_{2}$, are the commonest salts directly obtainable from natural salt deposits and brines. From any of these the element is readily set free by the action of chlorine, thus:

$$
{ }_{2} \mathrm{KBr}+\mathrm{Cl}_{2} \rightarrow 2 \mathrm{KCl}+\mathrm{Br}_{2} \text {. }
$$

Upon passing chlorine gas into a solution of potassium bromide, the solution turns brown and when heated gives off reddish-brown vapors of bromine, which when cooled condense to liquid 
bromine. Bromine is a reddish-brown liquid which has a density over three times that of water. It boils at $58^{\circ}$ and readily volatilizes at ordinary temperatures. The vapor is, if anything, more irritating to mucous membranes than chlorine, and the liquid produces deep burns when brought into contact with the skin. Bromine must be handled with extreme caution. In case of accident wash off the bromine with water immediately; then consult an instructor regarding further treatment.

Bromine dissolves in water to the extent of about 3 per cent tc form a light-brown solution, known as bromine water.

256. Hydrobromic Acid, $\mathrm{HBr}$.- Hydrogen bromide, the water solution of which is known as hydrobromic acid, can be made by the direct union of its constituent elements:

$$
\mathrm{H}_{2}+\mathrm{Br}_{2} \rightarrow{ }_{2} \mathrm{HBr} \text {. }
$$

The best method of making hydrogen bromide is based on the fact that bromine unites with phosphorus to form a tribromide or a pentabromide, thus,

$$
\begin{aligned}
& { }_{2} \mathrm{P}+{ }_{3} \mathrm{Br}_{2} \rightarrow 2 \mathrm{PBr}_{3} \\
& { }_{2} \mathrm{P}+{ }_{5} \mathrm{Br}_{2} \rightarrow 2 \mathrm{PBr}_{5} .
\end{aligned}
$$

These compounds are entirely analogous to $\mathrm{PCl}_{3}$ and $\mathrm{PCl}_{5}(247)$. The bromides of phosphorus also resemble the chlorides in their reactions with water, thus:

$$
\begin{aligned}
& \mathrm{PBr}_{3}+{ }_{3} \mathrm{H}_{2} \mathrm{O} \rightarrow \mathrm{H}_{3} \mathrm{PO}_{3}+{ }_{3} \mathrm{HBr} \\
& \mathrm{PBr}_{5}+{ }_{4} \mathrm{H}_{2} \mathrm{O} \rightarrow \mathrm{H}_{3} \mathrm{PO}_{4}+{ }_{5} \mathrm{HBr} .
\end{aligned}
$$

The preparation of hydrobromic acid is carried out in the apparatus shown in Fig. 37.

Ten grams of red phosphorus, ro c.c. of water, and 20 to $25 \mathrm{~g}$. of quartz sand are placed in a 250 c.c. flask and 15 c.c. of bromine, contained in the dropping funnel, are allowed to run in slowly, drop by drop. 'The U-tube contains some pieces of broken glass or brick or similar inert

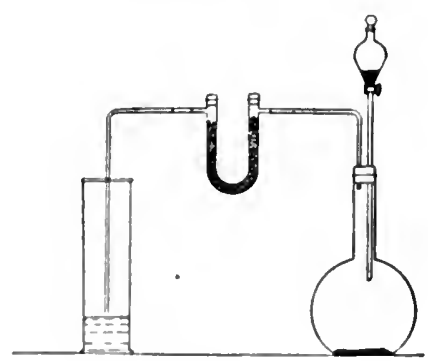

Fis. 37 material mixed with 3 or $4 \mathrm{~g}$. of red phosphorus, the object of 
the glass or brick being to distribute the phosphorus so that it will present the maximum of surface. The hydrogen bromide given off is freed from accompanying bromine vapor by the phosphorus in the $\mathrm{U}$-tube and is absorbed by water contained in the cylinder. The delivery tube should not dip into the water in the cylinder, since the gas is so soluble that there would be danger of water getting back into the $U$-tube and flask.

257. The Properties of Hydrogen Bromide.-Hydrogen bromide is a colorless gas with a choking odor; it gives white fumes in moist air and dissolves abundantly in water to form a solution known as hydrobromic acid. This is a colorless liquid which closely resembles hydrochloric acid in its properties. It neutralizes bases and unites with metallic oxides to form salts called bromides, for example:

$$
\begin{aligned}
\mathrm{NaOH}+\mathrm{HBr} & \rightarrow \mathrm{NaBr}+\mathrm{H}_{2} \mathrm{O} \\
\mathrm{MgO}+ & \mathrm{HBr} \rightarrow \mathrm{MgBr}_{2}+\mathrm{H}_{2} \mathrm{O} \\
\mathrm{CuO}+{ }_{2} \mathrm{HBr} & \rightarrow \mathrm{CuBr}_{2}+\mathrm{H}_{2} \mathrm{O} \\
\mathrm{Al}(\mathrm{OH})_{3}+{ }_{3} \mathrm{HBr} & \rightarrow \mathrm{AlBr}_{3}+{ }_{3} \mathrm{H}_{2} \mathrm{O} .
\end{aligned}
$$

The bromides of silver, lead, and univalent mercury are almost insoluble in water, as are the chlorides of these same metals (252). All other bromides are easily soluble. The addition of hydrobromic acid or any soluble bromide to a solution of a salt of silver, lead, or univalent mercury gives a white precipitate of the insoluble bromide, thus:

$$
\mathrm{Pb}\left(\mathrm{NO}_{3}\right)_{2}+{ }_{2} \mathrm{HBr} \rightarrow \mathrm{PbBr}_{2}+{ }_{2} \mathrm{HNO}_{3} \text {. }
$$

258. The Oxidation of Hydrobromic Acid.-Hydrogen bromide and oxygen gases react when heated to form bromine and water,

$$
{ }_{4} \mathrm{HBr}+\mathrm{O}_{2} \rightarrow{ }_{2} \mathrm{Br}_{2}+{ }_{2} \mathrm{H}_{2} \mathrm{O} \text {. }
$$

This reaction is analogous to that between hydrogen chloride and oxygen (239), but takes place far more completely, indicating that hydrogen bromide is more easily oxidized than hydrogen chloride. Other oxidizing agents, such as manganese dioxide, readily set free bromine:

$$
{ }_{4} \mathrm{HBr}+\mathrm{MnO}_{2} \rightarrow \mathrm{MnBr}_{2}+\mathrm{Br}_{2}+{ }_{2} \mathrm{H}_{2} \mathrm{O} .
$$


In the technical preparation of bromine by means of this reaction sodium bromide is treated with dilute sulfuric acid and manganese dioxide. In this case all of the available bromine is set free.

$2 \mathrm{NaBr}+2 \mathrm{H}_{2} \mathrm{SO}_{4}+\mathrm{MnO}_{2} \rightarrow \mathrm{Na}_{2} \mathrm{SO}_{4}+\mathrm{MnSO}_{4}+\mathrm{Br}_{2}+2 \mathrm{H}_{2} \mathrm{O}$.

259. The Action of Chlorine on Bromides. - A solution of any bromide reacts with chlorine to form a chloride and free bromine,

$$
2 \mathrm{KBr}+\mathrm{Cl}_{2} \rightarrow 2 \mathrm{KCl}+\mathrm{Br}_{2} \text {. }
$$

Similarly, hydrobromic acid and chlorine give hydrochloric acid and bromine. These reactions are nearly complete, that is, they are not reversible to any marked extent, so that we may conclude that the metals and hydrogen form by preference compounds with chlorine rather than with bromine. This fact may also be expressed by saying that chlorine has greater affinity than bromine for metals and hydrogen. Using this mode of expression, we should also say that oxygen has grcater affinity than bromine for hydrogen, since hydrogen bromide and oxygen give water and free bromine.

260. The Uses of Bromine and Its Compounds. - Potassium and sodium bromides are used extensively in medicine as sedatives. Silver bromide is the light-sensitive substance of photographic plates. The free element is extensively used in the manufacture of important coal-tar dyes.

26r. Iodine. - The element iodine (symbol I), bears almost the same relation to bromine that the latter bears to chlorine. It does not occur free in nature, but is readily prepared from its compounds, the iodides of sodium or potassium, which are obtained from two principal natural sources.

Certain seaweeds contain small amounts of combined iodine which has been taken up from sea-water in which a minute quantity is present. The ashes left upon burning the dried seaweed yield by extraction with water sodium iodide, $\mathrm{NaI}$, and potassium iodide, KI. Iodine compounds are also obtained as by-products in the purification of the sodium nitrate found 
in Chile (r04). Iodine is set free from iodides by the action of chlorine, thus:

$$
{ }_{2} \mathrm{NaI}+\mathrm{Cl}_{2} \rightarrow{ }_{2} \mathrm{NaCl}+\mathrm{I}_{2} .
$$

It is also liberated by the action of manganese dioxide and sulfuric acid.

262. The Physical Properties of Iodine.-Iodine is an almost black, crystalline substance, having a density of nearly five. It melts at $\mathrm{II} 4^{\circ}$ and boils at a somewhat higher temperature, producing a vapor having a magnificent violet color. At a temperature slightly below its melting-point iodine has so great a vapor pressure that by cautious heating it may be volatilized completely without being melted. If the vapor is allowed to strike a cold surface crystals of iodine deposit directly without preliminary formation of liquid iodine. The sublimation (I79) of iodine in this way is an important step in the purification of this element.

Iodine is very slightly soluble in water, giving a faintly brownish solution. It dissolves abundantly in water solutions of potassium or sodium iodide. It dissolves easily in alcohol, forming a dark-brown solution called by druggists tincture of iodine. Iodine also dissolves easily in ether, forming a brown solution, and in chloroform and carbon disulfide, forming violet-colored solutions.

263. Iodine and Starch. - If a dilute solution of iodine is added to water containing a little starch paste, made by boiling starch with 50 to roo times its weight of water, a deep bluecolored solution results. This reaction is a characteristic and very delicate test for free iodine. Iodides, like $\mathrm{KI}$, do not give this test; but by adding chlorine to a solution of an iodide the element is set free and can then be recognized by the starch test. An excess of chlorine interferes with this test.

264. Hydrogen Iodide, HI.--Iodine and hydrogen unite slowly at a temperature of $400^{\circ}$ to form hydrogen iodide, thus:

$$
\mathrm{I}_{2}+\mathrm{H}_{2} \rightarrow 2 \mathrm{HI} \text {. }
$$

The product is a colorless gas, analogous to hydrogen chloride and hydrogen bromide. Like these latter gases it dissolves abundantly in water, and forms fumes in moist air. 
Hydrogen iodide is easily made by a reaction resembling that used for making hydrogen bromide. Iodine forms with phosphorus a tri-iodide, $\mathrm{PI}_{3}$. This reacts with water to form phosphorous and hydriodic acids thus:

$$
\mathrm{PI}_{3}+{ }_{3} \mathrm{H}_{2} \mathrm{O} \rightarrow \mathrm{H}_{3} \mathrm{PO}_{3}+{ }_{3} \mathrm{HI} \text {. }
$$

The process of making hydrogen iodide is carried out by placing a mixture of powdered iodine and red phosphorus in a flask and running in water, drop by drop from a dropping funnel, care being taken not to use more water than is necessary, since an excess of water would dissolve the gas and so prevent its escape from the flask. The apparatus used for making hydrogen bromide, Fig. 37, may be used in this case. The U-tube containing red phosphorus serves here to remove iodine vapor. The hydrogen iodide gas may be collected by downward displacement of air or it may be dissolved in water to form a solution of hydriodic acid.

265. Hydriodic acid is colorless when pure, but is brown if it contains free iodine, which it dissolves readily. It neutralizes bases and so yields salts called iodides, for example:

$$
\begin{aligned}
\mathrm{HI}+\mathrm{NaOH} & \rightarrow \mathrm{NaI}+\mathrm{H}_{2} \mathrm{O} \\
{ }_{2} \mathrm{HI}+\mathrm{Ca}(\mathrm{OH})_{2} & \rightarrow \mathrm{CaI}{ }_{2}+{ }_{2} \mathrm{H}_{2} \mathrm{O} .
\end{aligned}
$$

Hydriodic acid acts on metals similarly to hydrochloric acid, giving iodides and hydrogen, thus:

$$
{ }_{2} \mathrm{HI}+\mathrm{Zn} \rightarrow \mathrm{ZnI}_{2}+\mathrm{H}_{2} \text {. }
$$

Hydriodic acid is much more easily oxidized than is hydrobromic acid, which in turn is more easily oxidized than hydrochloric acid; while all three acids are oxidized by powerful oxidizing agents such as manganese dioxide and lead dioxide; hydriodic acid, even in dilute solution, is oxidized slowly by atmospheric oxygen, which has no action whatever on dilute hydrochloric acid:

$$
{ }_{4} \mathrm{HI}+\mathrm{O}_{2} \rightarrow 2 \mathrm{H}_{2} \mathrm{O}+2 \mathrm{I}_{2} .
$$

The iodine which is slowly liberated according to the equation given above remains dissolved in the unchanged acid and gives it a brown color. 
266. Uses of Iodine and Iodides.-Iodine is used extensively in certain processes of analysis and also in the preparation of important compounds containing the element carbon, so-called organic compounds. Iodine in the form of tincture of iodine, which is a solution of iodine in alcohol, is used externally as an antiseptic and also as a counterirritant in medicine. The iodides of potassium, sodium, and ammonium are of great importance for internal administration in medicine.

267. Fluorine.-The element fluorine (symbol F), is classed among the halogens, although it is less closely related to the other three halogens, chlorine, bromine, and iodine, than these three are to one another. The atomic weights of these elements are: fluorine, 19; chlorine, 35.5; bromine, 80; iodine, I27. Fluorine has, therefore, the smallest atomic weight of any of the halogens. We might expect it to resemble chlorine more closely than it does bromine and iodine and, in fact, such is the case. It is a pale-yellow gas which is very active chemically and never occurs free in nature. Its most abundant natural compound is calcium fluoride or fluor-spar, $\mathrm{CaF}_{2}$. It also occurs as cryolite, sodium aluminum fluoride, ${ }_{3} \mathrm{NaF} \cdot \mathrm{AlF}_{3}$. These substances are salts of hydrofluoric acid. We might expect that free fluorine could be made by oxidizing hydrofluoric acid with manganese dioxide, thus:

$$
{ }_{4} \mathrm{HF}+\mathrm{MnO}_{2} \rightarrow \mathrm{MnF}_{2}+\mathrm{F}_{2}+{ }_{2} \mathrm{H}_{2} \mathrm{O} ;
$$

but we find, in fact, that hydrofluoric acid is entirely unacted upon by the most powerful oxidizing agents. The free element was first made by Moissan, by the electrolysis of anhydrous liquid hydrogen fluoride, in which some potassium fluoride, $\mathrm{KF}$, was dissolved to make it conduct electricity readily. The products of the electrolysis were fluorine and hydrogen:

$$
{ }_{2} \mathrm{HF} \rightarrow \mathrm{F}_{2}+\mathrm{H}_{2} \text {. }
$$

Fluorine is one of the most active of all elements. It rapidly attacks glass and also most metals, and it reacts at once with water forming hydrofluoric acid and oxygen:

$$
{ }_{2} \mathrm{~F}_{2}+{ }_{2} \mathrm{H}_{2} \mathrm{O} \rightarrow 4 \mathrm{HF}+\mathrm{O}_{2} \text {. }
$$


The preparation of fluorine is a matter of great difficulty, for which reason it is very seldom made.

268. Hydrogen Fluoride, HF.-Hydrogen fluoride, a gas whose water solution is called hydrofluoric acid, is the most important compound of fluorine. It is formed by the action of concentrated sulfuric acid on powdered calcium fluoride:

$$
\mathrm{H}_{2} \mathrm{SO}_{4}+\mathrm{CaF}_{2} \rightarrow \mathrm{CaSO}_{4}+2 \mathrm{HF} \text {. }
$$

It is a colorless gas with a choking odor. At temperatures of $100^{\circ}$ and higher its density shows that the gas has the formula $\mathrm{HF}$; at room temperature the density is more than double that expected for a gas with the formula HF. This fact leads to the conclusion that the single molecules have become associated, probably to form double or triple molecules such as $\mathrm{H}_{2} \mathrm{~F}_{2}$ and $\mathrm{H}_{3} \mathrm{~F}_{3}$. Hydrogen fluoride gas is condensed to a liquid merely by cooling it with ice; colorless liquid hydrogen fluoride, so obtained, boils at $19^{\circ}$.

269. Hydrofluoric Acid and Its Salts. - A 30 per cent solution of hydrofluoric acid is an important article of commerce. The acid has several practical uses. These include the etching and polishing of glass, the removal of sand from castings, and the preparation of its salts and also of hydrofluosilicic acid, $\mathrm{H}_{2} \mathrm{SiF}_{6}$.

Hydrofluoric acid forms with bases salts called fluorides. The soluble fluorides are very effective preservatives, since they inhibit the growth of bacteria, molds, etc. But their use in foodstuffs is prohibited because of their interference with digestion.

Ammonium fluoride, $\mathrm{NH}_{4} \mathrm{~F}$, is used as a disinfectant for utensils used in breweries. Sodium fluoride, $\mathrm{NaF}$, is extensively used as a vermin exterminator for poultry.

270. The Action of Hydrogen Fluoride on Quartz. - We must now digress a little from the subject in hand in order to be able fully to understand one of the most interesting reactions of hydrogen fluoride. The substance called quartz is the oxide of an element silicon (symbol $\mathrm{Si}$ ) and has the formula $\mathrm{SiO}_{2}$. Common sand is more or less pure quartz. Glass, which is 
made by melting together sand, sodium carbonate, and slaked lime, may be considered a mixture of sodium silicate, $\mathrm{Na}_{2} \mathrm{SiO}_{3}$, and calcium silicate, $\mathrm{CaSiO}_{3}$. Hydrofluoric acid and quartz react very readily to form gaseous silicon fluoride, $\mathrm{SiF}_{4}$, and water, thus:

$$
{ }_{4} \mathrm{HF}+\mathrm{SiO}_{2} \rightarrow \mathrm{SiF}_{4}+{ }_{2} \mathrm{H}_{2} \mathrm{O} .
$$

This is a very characteristic reaction; none of the other halogen acids have any action on quartz.

Glass, which is almost unaffected by the other halogen acids, is rapidly attacked by either hydrogen fluoride gas or hydrofluoric acid solution. The fluorine unites, not only with the silicon, as in the case of quartz, forming silicon fluoride, but also with the sodium and calcium forming sodium fluoride, $\mathrm{NaF}$, and calcium fluoride, $\mathrm{CaF}_{2}$, the reactions being:

$$
\begin{aligned}
\mathrm{Na}_{2} \mathrm{SiO}_{3}+6 \mathrm{HF} & \rightarrow \mathrm{SiF}_{4}+2 \mathrm{NaF}+{ }_{3} \mathrm{H}_{2} \mathrm{O} \\
\mathrm{CaSiO}_{3}+6 \mathrm{HF} & \rightarrow \mathrm{SiF}_{4}+\mathrm{CaF}_{2}+{ }_{3} \mathrm{H}_{2} \mathrm{O} .
\end{aligned}
$$

The result is that glass dissolves very easily in hydrofluoric acid, in consequence of which this acid cannot be kept in glass bottles. Paraffine and other waxes, which are not attacked, are used for bottles for this acid, while larger containing vessels are made of lead.

271. Etching Glass with Hydrogen Fluoride.-The etching of glass may be illustrated by coating a glass plate with a thin layer of paraffine, and after making a design or inscription by means of a hard pencil which will cut through the paraffine and thus expose the surface of the glass, exposing the plate to the action of hydrogen fluoride gas. The gas is easily made by mixing a few grams of powdered fluor spar with concentrated sulfuric acid in a shallow lead dish. The latter is covered with the glass plate and set aside for ten or fifteen minutes. Upon removing the paraffine, the design will be found to have been etched upon the glass.

272. Hydrofluosilicic Acid, $\mathrm{H}_{2} \mathrm{SiF}_{6}$.-Hydrogen fluoride and silicon tetrafluoride unite readily in the presence of water to form a solution of hydrofluosilicic acid:

$$
{ }_{2} \mathrm{HF}+\mathrm{SiF}_{4} \rightarrow \mathrm{H}_{2} \mathrm{SiF}_{6} .
$$


The solution is a colorless, odorless liquid which does not attack glass appreciably. It has well-characterized acid properties: it reddens litmus, has a sour taste, and neutralizes bases to form salts. This acid is important technically. It is made, in practice, by the action of hydrofluoric acid solution on quartz sand:

$$
6 \mathrm{HF}+\mathrm{SiO}_{2} \rightarrow \mathrm{H}_{2} \mathrm{SiF}_{6}+2 \mathrm{H}_{2} \mathrm{O} \text {. }
$$

The acid is used for the preparation of its sodium, magnesium, and lead salts. Sodium fluosilicate, $\mathrm{Na}_{2} \mathrm{SiF}_{6}$, is extensively used in making white enameled ware and also white, or so-called milk, glass. It is remarkable in being one of the very few nearly insoluble salts of sodium. It is obtained as a white precipitate when solutions of common salt and hydrofluosilicic acid are mixed.

$$
2 \mathrm{NaCl}+\mathrm{H}_{2} \mathrm{SiF}_{6} \rightarrow \mathrm{Na}_{2} \mathrm{SiF}_{6}+{ }_{2} \mathrm{HCl} \text {. }
$$

Magnesium fluosilicate, $\mathrm{MgSiF}_{6}$, easily soluble in water, is used to harden concrete. Lead fluosilicate, $\mathrm{PbSiF}_{6}$, also easily soluble in water, is made as an intermediate product in refining lead (Betts's process). 


\section{CHAPTER XIII \\ CHEMICAL EQUILIBRIUM}

273. Incomplete Physical Processes.-While many physical processes are seemingly complete, there are others which stop far short of completion. Thus, for example, if a small bulb of water is broken in a large closed bottle, evaporation of the water will start at once, but will apparently cease as soon as the pressure of the vapor reaches a value which is definite for a definite temperature, although much liquid may still remain (II2).

If we add to some water an equal weight of common salt, the latter will at once start to dissolve and will continue to do so until the solution has, for a given temperature, a certain definite concentration; then, although much solid salt is still present, no further increase in concentration will take place (122).

When water in a closed vessel, which it fills but partially, reaches its maximum vapor pressure for a given temperature, we believe (20r) that for every molecule that passes from liquid to vapor there is one that passes from vapor to liquid. We say that there is equilibrium between liquid and vapor. We believe that a similar condition exists when a solid apparently stops dissolving in a solution (207). The apparent state of rest or inaction in both cases is very probably one in which two opposing actions exactly counteract the effects of each other.

274. Incomplete Chemical Reactions.-Just as in the case of physical processes, there are also some chemical reactions that do not go to completion. We have already studied some reactions of this kind and must now consider the matter more fully, as it is one of great importance.

The reaction between hydrogen chloride and oxygen at $400^{\circ}$ has been considered $(\mathbf{2 3 9}, \mathbf{2 4 0})$ under the heading "Deacon's Process." It has been pointed out (245) that only 80 per cent of the hydrogen chloride is oxidized when a mixture of this gas 
is heated with oxygen in the proportion indicated in the following equation:

$$
{ }_{4} \mathrm{HCl}+\mathrm{O}_{2}=2 \mathrm{Cl}_{2}+{ }_{2} \mathrm{H}_{2} \mathrm{O} \text {. }
$$

On the other hand, when a mixture of two formula weights each of chlorine and water is also heated to $400^{\circ}$, 80 per cent of the chlorine remains unchanged, while 20 per cent is converted into hydrogen chloride. It thus happens that whether we start with the pair of gases on the left side of the foregoing equation or the pair on the right, taking in each case the amounts indicated in this equation, there results a mixture of the four gases which has exactly the same amount of each gas present in the two cases. It is easy to see that the cause of each reaction being incomplete is found in the fact that the products of either reaction again react in the opposite direction. In the mixture of the four gases which finally results we say that a state of equilibrium exists and that the apparent stopping of further change is really the result of the formation of hydrogen chloride and oxygen at just the same rate as that at which these two gases change into chlorine and water.

275. Velocity of Chemical Change.-The idea that a state of chemical equilibrium is the result of two opposing changes which take place continuously at such rates or with such velocities that for every molecule of a given substance formed one also disappears would imply that chemical changes take place gradually and possibly at definite speeds or velocities.

It is well known that certain reactions, as for example the burning of a candle or the action of an acid on a metal, certainly do take place gradually. It is not so plain that if the reaction takes place between two perfectly mixed gases or between two substances completely dissolved as a uniformly mixed solution that time is required for the reaction to take place. Nevertheless it is probable that no reaction, even an explosion, however rapid it may be, is absolutely instantaneous.

The speed or velocity of reaction in a uniformly mixed solution may be beautifully and convincingly demonstrated by means of the following experiment: 
To 800 c.c. of water contained in a flask there is added 25 c.c. of starch solution (made by boiling $2 \mathrm{~g}$. of starch with Ioo c.c. of water) and $\mathrm{I}_{5}$ c.c. of a 3 per cent solution of iodic acid, $\mathrm{HIO}_{3}$. The solution is then well mixed and 5 c.c. of a 3 per cent solution of sulfur dioxide, $\mathrm{SO}_{2}$, is added and the contents of the flask are at once thoroughly mixed by being shaken. The time of adding the sulfur dioxide solution is accurately noted-best with a stop watch. No change will be seen in the colorless solution for about 60 seconds, then the whole solution will suddenly turn deep blue. The result is startling!

If the experiment is repeated, using the same amounts of water and of each of the three solutions, and if the temperature is also the same, it will be found that the time required for the change to occur is always the same. If, however, we increase the amount of sulfur dioxide solution added from 5 c.c. to Io c.c., everything else remaining the same, the time required for the change will be decreased to about 30 seconds. The increased velocity is the result of the increase in concentration of the sulfur dioxide.

276. The Effect of Temperature on Reaction Velocity.-The effect on the velocity of increasing the temperature is easily shown by starting with water at $25^{\circ}$ instead of at $20^{\circ}$, when it will be clear that at the higher temperature the velocity is decidedly greater.

277. The Action of Sulfur Dioxide on Iodic Acid.-The chemical changes involved in the reaction just described need not greatly concern the student at this time, as they are of less importance than the main facts of reaction velocity that they serve here to illustrate. But as it is only natural to wonder what has happened in such a striking experiment, the equations for the reactions may now be given. In the first place, sulfur dioxide, $\mathrm{SO}_{2}$, and water form sulfurous acid, $\mathrm{H}_{2} \mathrm{SO}_{3}$,

$$
\mathrm{SO}_{2}+\mathrm{H}_{2} \mathrm{O} \rightarrow \mathrm{H}_{2} \mathrm{SO}_{3} \text {. }
$$

The latter reacts with the iodic acid, forming hydriodic and sulfuric acids, thus:

$$
\mathrm{HIO}_{3}+{ }_{3} \mathrm{H}_{2} \mathrm{SO}_{3} \rightarrow \mathrm{HI}+{ }_{3} \mathrm{H}_{2} \mathrm{SO}_{4} \text {. }
$$


But hydriodic acid can also react with iodic acid to form free iodine and water,

$$
\mathrm{HIO}_{3}+{ }_{5} \mathrm{HI} \rightarrow{ }_{3} \mathrm{I}_{2}+{ }_{3} \mathrm{H}_{2} \mathrm{O},
$$

and then the iodine set free acts on the starch to produce the blue color. Now this reaction between iodic and hydriodic acid does not take place until all the sulfurous acid has disappeared. The time observed for the appearance of the blue color is therefore essentially that required for the complete oxidation of the sulfurous acid.

278. The Kinetic Hypothesis Applied to Reaction Velocity.The application of the kinetic-molecular hypothesis (chap. $\mathrm{x}$ ) leads to a simple and reasonable explanation of reaction velocity.

Let us suppose that two gases, $A$ and $B$, can unite to form a compound $A B$, and let the reaction be represented by the equation

$$
A+B \rightarrow A B \text {. }
$$

Let us also suppose that the reaction takes place rather slowly after the two gases have been thoroughly mixed. We may now consider what determines the rate at which $A$ and $B$ unite. It is obvious that union can occur only when a molecule of $A$ comes in contact with a molecule of $B$. Such collisions will frequently occur by reason of the rapid motion of both kinds of molecules. Now as these collisions are matters of chance it is very easy to see that if more molecules of one or both kinds are brought into a given space the number of collisions of $A$ molecules with $B$ molecules will be increased. On the other hand, decreasing the number of one or both kinds of molecules will surely decrease the possible collisions of $A$ with $B$ molecules.

Probably not every collision of an $A$ with a $B$ molecule will result in a union of the two to form $A B$; but if, on the average, a certain definite fraction of the collisions result in union, then we can say that the greater the number of $A$ and $B$ molecules present in a given volume, say I c.c., of the gas mixture, the greater will be the number of $A B$ molecules formed per second. If we start with a mixture of equal numbers of $A$ and $B$ 
molecules there will be for a definite pressure and temperature a certain number of $A B$ molecules formed per second. After a short time the number of $A$ and $B$ molecules will have decreased appreciably, so that now fewer $A B$ molecules will be formed per second, and as time goes on, owing to continual decrease in the numbers of $A$ and $B$ molecules present, there will be fewer and fewer $A B$ molecules formed per second. The result will be that the rate of formation of $A B$ molecules will be greatest at the start and will gradually decrease, until finally, if the reaction is not reversible, all $A$ and $B$ molecules will have united.

279. The Kinetic Hypothesis Applied to Chemical Equilibrium.-Let us next consider, in the light of the kinetic-molecular hypothesis, the state of affairs if a reaction between gases is reversible. The case of hydrogen chloride and oxygen will serve as a good illustration. The equation is

$$
{ }_{4} \mathrm{HCl}+\mathrm{O}_{2} \leftrightarrows{ }_{2} \mathrm{Cl}_{2}+{ }_{2} \mathrm{H}_{2} \mathrm{O}
$$

This reaction takes place with moderate velocity at $400^{\circ}$, finally reaching a state of equilibrium in which all four of the substances are present.

Suppose we bring into a closed vessel at $400^{\circ}$ a mixture of hydrogen chloride and oxygen in the proportion indicated by the equation; that is, four molecules of the first gas to one of the second. The reaction will begin at a certain velocity, molecules of hydrogen chloride and oxygen disappearing by uniting to form molecules of chlorine and water vapor. As time goes on there will be fewer and fewer hydrogen chloride and oxygen molecules present, so that the number of each uniting per second and also the number of chlorine and water molecules formed per second will continuously decrease. On the other hand, the molecules of chlorine and water which have been formed begin to reunite to form hydrogen chloride and oxygen. As the total numbers of chlorine and water molecules present will increase as time goes on, so the numbers of these molecules which react and so disappear per second will also increase. The final result will be that in each second there will be just as many molecules 
of chlorine and water disappearing as the numbers of each formed. The same sort of thing will be true for the hydrogen chloride and oxygen-as many molecules of each will finally be produced per second as the numbers that disappear. When this condition is reached no further change in the number of any of the four sorts of molecules will take place, although chemical change will go on continuously. The system is then in a state of equilibrium.

We may now take up a study of a number of reversible reactions which reach a state of equilibrium.

280. Ferric Chloride and Ammonium Sulfocyanate.-If we add to a very dilute solution of ferric chloride, $\mathrm{FeCl}_{3}$, which is faintly yellow in color, a dilute solution of ammonium sulfocyanate, $\mathrm{NH}_{4} \mathrm{NCS}$, which is colorless, a blood-red solution results. This red substance is ferric sulfocyanate, $\mathrm{Fe}(\mathrm{NCS})_{3}$, which is formed thus:

$$
\mathrm{FeCl}_{3}+{ }_{3} \mathrm{NH}_{4} \mathrm{NCS} \rightarrow \mathrm{Fe}(\mathrm{NCS})_{3}+{ }_{3} \mathrm{NH}_{4} \mathrm{Cl} \text {. }
$$

Let us now consider how we may discover whether this reaction is complete when the two substances on the left-hand side of the equation are mixed in the indicated proportion or whether a state of equilibrium results. The experiment may be carried out on the lecture table in the following manner:

Tó 2 liters of water we add 20 c.c. of a decinormal solution of ferric chloride and 20 c.c. of a decinormal solution of ammonium sulfocyanate, which is just the amount indicated by the equation as required for the amount of ferric chloride present. Let us now divide the red solution into four equal portions, which we may place in four similar cylinders or beakers. Suppose we now add to the solution in one of the cylinders 20 c.c. more of ammonium sulfocyanate solution. The solution will be seen to become deeper red in color, which means that more red ferric sulfocyanate has been formed. Now this fact may be explained in either of two ways: first, that we had by mistake used, in the first place, less than the correct proportion of ammonium sulfocyanate indicated by the equation; or, secondly, that a state of equilibrium existed in the solution and that the increased concentration of ammonium sulfocyanate had shif ted the equilibrium so as to form more ferric sulfocyanate. 
We can test the truth or falsity of the first supposition very easily. If the original mixture contained less than the correct proportion of ammonium sulfocyanate, then there would be an excess of ferric chloride, and the addition of more of this salt would not increase the amount of ferric sulfocyanate and so increase the depth of red color. Let us add, therefore, 20 c.c. more ferric chloride to the solution in the second cylinder. It becomes deeper red! This seems to show that we are dealing with a condition of equilibrium as indicated by the double arrows of the following equation:

$$
\mathrm{FeCl}_{3}+{ }_{3} \mathrm{NH}_{4} \mathrm{NCS} \leftrightharpoons \mathrm{Fe}(\mathrm{NCS})_{3}+{ }_{3} \mathrm{NH}_{4} \mathrm{Cl} \text {. }
$$

If such is the case, then the addition of ammonium chloride to the solution in the third cylinder should cause a partial fading of the red color by reason of the partial disappearance of the red ferric sulfocyanate. Now this is actually what happens when the experiment is carried out, as can be seen by comparison with the color of the solution in the fourth cylinder.

It is clear, therefore, that we have here a case of chemical equilibrium in which all four of the substances represented in the equation can coexist in the same solution. When we added more ammonium sulfocyanate to the solution in the first cylinder we increased the number of molecules of this salt and so increased the chances of collision of ferric chloride molecules with ammonium sulfocyanate molecules and this increased the number of ferric sulfocyanate molecules formed per second. This caused an increase in the total amount of the latter salt, and thus gave rise very quickly to a new state of equilibrium in which the proportion of ferric salt in the form of red sulfocyanate was greater than at first.

The addition of more ferric chloride to the solution in the second cylinder caused a similar shift of equilibrium for analagous reasons. It is a general rule that increasing the concentration of either of the reacting substances on the same side of an equation causes a shift in equilibrium so as to form more of the substances on the other side of the equation. This rule is also illustrated by the fact that when more ammonium chloride was added to the 
solution in the third cylinder the color partially faded; this showed that some of the red ferric sulfocyanate had disappeared, and thus indicated that more ferric chloride and ammonium sulfocyanate had been formed.

281. Hydrogen and Iodine. -We have already seen (264) that hydrogen unites with iodine vapor with appreciable speed at about $400^{\circ}$. The reaction is not complete, but reaches a state of equilibrium while there are still considerable uncombined substances present. The equation is

$$
\mathrm{H}_{2}+\mathrm{I}_{2} \leftrightarrows 2 \mathrm{HI} \text {. }
$$

That the reaction is reversible is easily shown by heating hydrogen iodide gas, when the purple vapors of iodine appear. If the temperature is $370^{\circ}$, equilibrium is reached when one-fifth of the hydrogen iodide has dissociated into free iodine and free hydrogen. This means that out of every 1,000 molecules of hydrogen iodide taken, 200 have dissociated and 800 remain when the state of equilibrium is reached. The equation shows that one molecule of hydrogen and one of iodine are formed by the dissociation of two molecules of hydrogen iodide. Therefore for every 200 molecules of the compound dissociated there would be formed 100 molecules of hydrogen and roo of iodine. The equilibrium mixture resulting from every $\mathrm{I}, 000$ molecules of hydrogen iodide taken consists, therefore, of 800 molecules of hydrogen iodide, roo molecules of hydrogen, and roo molecules of iodine.

If we bring together in a closed vessel equal numbers of molecules of hydrogen and iodine and heat at $370^{\circ}$ until equilibbrium is reached we shall find that for every 500 molecules of hydrogen and 500 molecules of iodine taken there result 800 molecules of hydrogen iodide, roo molecules of hydrogen, and Ioo of iodine. In other words, just the same proportion as would be obtained by starting with pure hydrogen iodide gas.

282. The Criterion of Equilibrium. - In all cases of reactions reaching a condition of equilibrium the resulting mixture has the same proportions of all substances, whether we start with the substances on one side of the equation or with equivalent 
amounts of those on the other side. Therefore, if we wish to know whether a given reaction has reached equilibrium we bring together the substances which would be the products of the first reaction. If the resulting reaction then gives a mixture of the same composition as that obtained in the first case we conclude that both reactions have reached equilibrium.

283. Equilibrium Constant.-In the hydrogen and iodine reaction

$$
\mathrm{H}_{2}+\mathrm{I}_{2} \leftrightarrows 2 \mathrm{HI}
$$

the rate of union of hydrogen and iodine, which we may call the speed of the reaction from left to right, will depend on the numbers of molecules of these two elements present in each c.c. It would seem probable that for a fixed number of hydrogen molecules per c.c. the speed of union would vary directly as the number of iodine molecules, and vice versa; so that this speed should be proportional to the product of the number of hydrogen molecules $N_{\mathrm{x}}$ and the number of iodine molecules $N_{2}$ present in each c.c. of the gas mixture. That is, the speed of union, $S_{\mathbf{I}}$, is proportional to $N_{\mathrm{I}}$ times $N_{2}$; or, algebraically,

$$
S_{\mathrm{r}}=k_{\mathrm{I}} \times N_{\mathrm{r}} \times N_{2} \text {, }
$$

where $k_{\mathrm{I}}$ is a constant proportionality factor.

On the other hand, the reverse change involves the formation. of hydrogen and iodine from hydrogen iodide, and we see by referring to the equation that two molecules of hydrogen iodide must react in order that one molecule of hydrogen and one molecule of iodine may be formed. This fact would make it seem necessary for two molecules of hydrogen iodide to collide in order that the change could occur. If so, increasing the number of $\mathrm{HI}^{\mathbf{I}}$ molecules in each c.c. would increase for each molecule the chances per second of collision and, in fact, doubling the number of molecules of this gas per c.c. would increase the total number of the chances per second fourfold, etc. In other words, the number of collisions per second of $\mathrm{HI}$ molecules with

${ }^{\mathbf{I}}$ It has become customary in chemical literature to use formulae of simple substances as abbreviations for the names of these substances; especially in cases of frequent repetition. 
one another will be proportional to the square of the number of molecules of this sort in each c.c. The details of the method of arriving at this conclusion need not be considered at present. If we call the speed of change of hydrogen iodide into hydrogen and iodine $S_{2}$ and call the number of HI molecules in I c.c. $N_{3}$, then it is plain that this speed is proportional to $N_{3}{ }^{2}$, or

$$
S_{2}=k_{2} N_{3}{ }^{2} \text {, }
$$

where $k_{2}$ is a constant proportionality factor.

Let us now think of the state of affairs when equilibrium has resulted. The speed of formation of hydrogen iodide which is equal to the speed of union of hydrogen and iodine, $S_{x}$, is now just equal to the speed of dissociation, $S_{2}$, of the hydrogen iodide. 'This must be the case, as otherwise further changes in the proportions of the three substances would still be taking place and the mixture would not be in equilibrium. For the state of equilibrium, therefore, we may write

$$
S_{\mathrm{I}}=S_{2}
$$

and hence

$$
k_{1} \times N_{1} \times N_{2}=k_{2} \times N_{3}^{2}
$$

or

$$
\frac{\mathrm{N}_{3}^{2}}{N_{1} \times N_{2}}=\frac{k_{1}}{k_{2}}
$$

Now $k_{1}$ and $k_{2}$ are both constant quantities for the reaction under consideration if the temperature is fixed, and therefore their quotient is a constant, so that we may write

$$
\frac{k_{1}}{k_{2}}=K,
$$

a constant. Therefore,

$$
\frac{N_{3}^{2}}{N_{1} \times N_{2}}=K
$$

This algebraic equation means that for the condition of equilibrium at a fixed temperalure the square of the number of molecules per c.c. of $\mathrm{HI}$ divided by the product of the numbers of molecules of $\mathrm{H}_{2}$ and $I_{2}$ is a fixed or constant quantily. This matter can perhaps be made a little plainer by use of a numerical example. We have 
seen that at $370^{\circ}$ the equilibrium mixture which results from I,, 00 original $\mathrm{HI}$ molecules consists of 800 molecules of $\mathrm{HI}$, I00 of $\mathrm{H}_{2}$, and $\mathrm{IOO}$ of $\mathrm{I}_{2}$. In each c.c. of such an equilibrium mixture the total number of molecules will be very great; but, of course, the numbers of each kind will be in the same proportion as for a total of 1,000 molecules, and therefore

$$
K=\frac{800^{2}}{100 \times 100}=64 .
$$

If we start with unequal instead of equal numbers of molecules of hydrogen and iodine we can calculate by means of the equation

$$
\frac{N_{3}^{2}}{N_{1} \times N_{2}}=64
$$

what the state of equilibrium will be. For example, suppose we start with a mixture of hydrogen and iodine containing four times as much hydrogen as would theoretically be necessary for the iodine taken; that is, four molecules of hydrogen for one of iodine. Calculation shows that if we start with 800 molecules of hydrogen and 200 molecules of iodine, when equilibrium is reached, out of a total of $\mathrm{I}, 000$ molecules 392 will be hydrogen iodide, 604 will be free hydrogen, and 4 will be free iodine.

284. Ammonia and Water.-Several reactions already studied reach a condition of equilibrium; three of the most familiar of these may now be considered as additional examples of the subject under discussion. Ammonia gas, $\mathrm{NH}_{3}$, dissolves abundantly in water, giving a solution which turns litmus blue and forms salts with acids. The solution contains ammonium hydroxide, formed by the union of ammonia with water (9I):

$$
\mathrm{NH}_{3}+\mathrm{H}_{2} \mathrm{O} \rightarrow \mathrm{NH}_{4} \mathrm{OH} \text {. }
$$

The solution smells strongly of ammonia and if it is boiled a short time all of the gas is given off. This shows that ammonium hydroxide easily dissociates into its constituents. It seems highly probable that in the water solution a condition of equilibrium exists, as indicated in the equation

$$
\mathrm{NH}_{3}+\mathrm{H}_{2} \mathrm{O} \leftrightarrows \mathrm{NH}_{4} \mathrm{OH},
$$


both free ammonia and ammonium hydroxide being present. Heating such a solution renders the free ammonia less soluble, and as this partially escapes, the rate of formation of ammonium hydroxide falls farther and farther behind the rate of dissociation of this compound until finally all of the latter has disappeared.

285. Carbon Dioxide and Water.-A water solution of carbon dioxide, $\mathrm{CO}_{2}$, contains carbonic acid, $\mathrm{H}_{2} \mathrm{CO}_{3}$. But such a solution easily gives off carbon dioxide, especially if warmed; which leads us to conclude that the reaction is a reversible one, and that in the solution there is a state of equilibrium as represented by the equation

$$
\mathrm{CO}_{2}+\mathrm{H}_{2} \mathrm{O}=\mathrm{H}_{2} \mathrm{CO}_{3} \text {. }
$$

286. Sulfur Dioxide and Water.-Sulfur dioxide, $\mathrm{SO}_{2}$, which is formed when sulfur burns, is a colorless gas with a suffocating odor:

$$
\mathrm{S}+\mathrm{O}_{2} \rightarrow \mathrm{SO}_{2} \text {. }
$$

It is easily soluble in water, giving a solution which smells strongly of the gas and has acid properties. The solution contains a compound, sulfurous acid, $\mathrm{H}_{2} \mathrm{SO}_{3}$. This solution gives off all of its sulfur dioxide when boiled, and we conclude, therefore, that the acid easily decomposes into its constituents, water and sulfur dioxide, and that in the solution we have a state of equilibbrium, as represented by the equation

$$
\mathrm{SO}_{2}+\mathrm{H}_{2} \mathrm{O} \rightarrow \mathrm{H}_{2} \mathrm{SO}_{3} \text {. }
$$

287. The Effect of Pressure on a System in Equilibrium.Suppose we have, say, I liter of water saturated with a gas, say oxygen, at a fixed temperature and at one-atmosphere pressure. 'To say that the water is saturated with the gas means that a condition of equilibrium exists between solution and gas. Let us suppose the solution and gas are contained in a cylinder fitted with a gastight piston (Fig. 38) and that the volume of the undissolved oxygen gas above the solution is I liter. If now we double the pressure on the gas

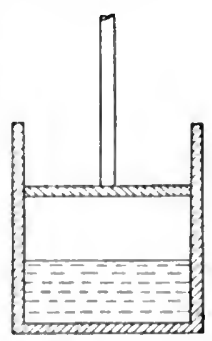

FIG. 38 more of the gas passes into solution, finally producing a new 
state of equilibrium. By reason of the fact that part of the gas dissolved when the pressure was doubled the volume of the remaining gas will not be half a liter, as we should expect if no additional quantity of oxygen dissolved in the water present, but appreciably less than half a liter. The effect, therefore, of increasing the pressure on the system is to cause its volume to become smaller than would be the case if no shift of equilibrium had occurred. This is the way in which an increase of pressure always affects a system in equilibrium: the state of equilibrium shifts in such a way as to cause a greater decrease in volume than would be the case if no change in the state of equilibrium occurred.

Let us consider another case. We may inquire how the equilibrium represented by the equation

$$
{ }_{4} \mathrm{HCl}+\mathrm{O}_{2} \leftrightarrows 2 \mathrm{Cl}_{2}+2 \mathrm{H}_{2} \mathrm{O}
$$

would be affected by increase of pressure. We see by reference to the equation that four volumes of $\mathrm{HCl}$ and one of $\mathrm{O}_{2}$ give two volumes of $\mathrm{Cl}_{2}$ and two of $\mathrm{H}_{2} \mathrm{O}$; that is, that when the reaction takes place from left to right there is a decrease in volume from 5 to 4 . We should expect, therefore, that by increasing the pressure the equilibrium would shift somewhat from left to right; that is, that more chlorine and water would be formed at the expense of the hydrogen chloride and oxygen; and this is exactly what actually happens.

The effect of increase of pressure on any system in equilibrium is, in all cases, to shift the equilibrium so as to favor the formation of substances occupying a smaller volume. In case no change of volume accompanies a chemical reaction, then the state of equilibrium is not affected by change of pressure. The reaction

$$
\mathrm{H}_{2}+\mathrm{I}_{2} \leftrightarrows 2 \mathrm{HI}
$$

is an example of this sort. Here one volume of hydrogen and one volume of iodine vapor react to form two volumes of hydrogen iodide, so that no change of volume occurs when the reaction takes place. It has been found by careful investigation that the equilibrium proportion of the three substances is not changed 
by altering the pressure, as long as the temperature remains constant.

288. Effect of Temperature on a System in Equilibrium.-We have already learned (II2) that the vapor pressure of water increases with increase of temperature. We know also that a large amount of heat is absorbed when water is evaporated; at $100^{\circ}$ it requires 540 calories to change one gram of water into steam. This is the so-called latent heat of vaporization. If we have, in a closed vessel, water in equilibrium with its vapor, and then apply heat, two effects are produced: the temperature is raised and the vapor pressure is increased. The increase in pressure is caused by the evaporation of some water, and this evaporation absorbs some of the heat which has been applied. This is a typical case, for we alway's find that when we apply heat to any system in equilibrium that the state of equilibrium shifts in such a way that heat is absorbed in the change. As heat is absorbed when water evaporates, heating causes increased vapor pressure.

The effect of temperature on the solubility of substances has already been studied (134). We have learned that heat is either absorbed or produced when a substance dissolves; this is the so-called heat of solution. Substances which dissolve with absorption of heat become more soluble with rise of temperature, while those which dissolve with evolution of heat, like anhydrous sodium sulfate, $\mathrm{Na}_{2} \mathrm{SO}_{4}$, decrease in solubility as the temperature is raised (134, Fig. 27). If a substance like the last named dissolves with evolution of heat, its crystallization out of a solution is accompanied by absorption of heat. In every case raising the temperalure causes that change of solubility to occur which involies an absorption of heat.

The state of chemical equilibrium is shifted in all cases by a change of temperature. Now we find that eiery chemical reaction either gives out or absorbs heat. When substances burn, the heat given out is very great. In many other reactions the heat produced is considerable, while in still others an absorption of heat occurs. If a reaction is reversible (all reactions that reach a state of equilibrium are, of course, of this class) and produces 
heat when it goes in one direction, it absorbs an equal amount of heat for the same quantity of materials transformed when it goes in the opposite direction.

If the reaction

$$
\mathrm{H}_{2}+\mathrm{I}_{2} \leftrightarrows 2 \mathrm{HI}
$$

has reached a state of equilibrium at $370^{\circ}$, out of every $1, \infty 00$ molecules present 800 will be $\mathrm{HI}, 100 \mathrm{H}_{2}$, and $100 \mathrm{I}_{2}$. If the temperature is then raised to $440^{\circ}$ and held constant until a new state of equilibrium is reached, the gas mixture will consist of 780 molecules of $\mathrm{HI}$, I IO of $\mathrm{H}_{2}$, and I Io of $\mathrm{I}_{2}$. Part of the $\mathrm{HI}$ has changed to $\mathrm{H}_{2}$ and $\mathrm{I}_{2}$, and the equilibrium may be said to have shifted from right to left. At temperatures between $370^{\circ}$ and $440^{\circ}$ the change of $\mathrm{HI}$ into $\mathrm{H}_{2}$ and $\mathrm{I}_{2}$ takes place with absorption of heat. We see, then, that raising the temperature causes the equilibrium to shift in the direction that involves an absorption of heat. Now this is a perfectly general law for chemical changes, just as it is also for physical changes like the vaporizing of a liquid and dissolving of a solid.

289. The Effect of Removing One Product of a Reaction.The reaction represented by the equation

$$
\mathrm{NaCl}+\mathrm{H}_{2} \mathrm{SO}_{4} \leftrightarrows \mathrm{NaHSO}_{4}+\mathrm{HCl}
$$

has already been studied rather fully. We may summarize the facts briefly, as follows: The action of concentrated sulfuric acid on dry salt gives sodium hydrogen sulfate, $\mathrm{NaHSO}_{4}$, and hydrogen chloride gas, the reaction going nearly to completion in the direction of the lower arrow in the equation given above if the mixture is warmed. On the other hand, if a cold saturated solution of sodium hydrogen sulfate is mixed with concentrated hydrochloric acid-that is, a saturated solution of hydrogen chloride in water-an abundant precipitate of solid salt, $\mathrm{NaCl}$, is formed. This reaction is, we see, just the reverse of the other. If now we mix a dilute solution of salt with dilute sulfuric acid, we see no visible change. We also see no change upon mixing a dilute solution of sodium hydrogen sulfate with dilute hydrochloric acid. 
We are now in position to explain all the facts of the foregoing paragraph from the standpoint of chemical equilibrium. If we bring together dilute solutions of either pair of substances in the reaction

$$
\mathrm{NaCl}+\mathrm{H}_{2} \mathrm{SO}_{4} \cdot \mathrm{NaHSO}_{4}+\mathrm{HCl},
$$

the resulling solution probably contains all four substances, side by side, in a state of cquilibrium. But we cannot notice any effect of the mixing, because in the presence of much water all four are held completely in solution, since all four are more or less readily soluble in water. If, however, but little water is present, the least soluble of the four substances, common salt, may partially separate. This is the case when a concentrated solution of $\mathrm{NaHSO}_{4}$ is mixed with concentrated $\mathrm{HCl}$. The reason is a simple onc: the substances taken react partially to form $\mathrm{NaCl}$ and $\mathrm{H}_{2} \mathrm{SO}_{4}$ in the sense of the upper arrow; but the amount of $\mathrm{NaCl}$ so formed is more than the water present can hold in solution; so the excess $\mathrm{NaCl}$ separates out in the solid form. This separation of $\mathrm{NaCl}$ continues until the four substances in the solution have reacherl amounts which can and do exist in equilibrium with one another. Removing the solid $\mathrm{NaCl}$ which has separated, or adding morc solid salt, will in no way alter the amounts of any of the four substances contained in the solution.

When concentrated $\mathrm{H}_{2} \mathrm{SO}_{4}$ is mixed with dry $\mathrm{NaCl}, \mathrm{NaHSO}_{4}$ and $\mathrm{HCl}$ begin to be formed. Now $\mathrm{HCl}$ is but slightly soluble in concentrated $\mathrm{H}_{2} \mathrm{SO}_{4}$ and, being a gas, it at once escapes from the mixturc. Warming the mixture also promotes the escape of the $\mathrm{HCl}$, since the higher the temperature the smaller the solubility of the gas in the concentrated $\mathrm{H}_{2} \mathrm{SO}_{4}$. The escape of the $\mathrm{HCl}$ gas also has another fundamental effect on the reaction. In order that any reaction may reach a state of equilibrium it must be reversible; but this reaction cannot go in the reverse direction if the $\mathrm{HCl}$ escapes from the reacting mixture as fast as it is formed. The result is that if no water is present, concentrated $\mathrm{H}_{2} \mathrm{SO}_{4}$ and dry $\mathrm{NaCl}$ react practically completely, giving solid $\mathrm{NaHSO}_{4}$ and $\mathrm{HCl}$ gas. 
290. The Action of Steam on Iron and the Reverse Action.When steam is passed over heated iron (29, Fig. I6) hydrogen and an oxide of iron are formed. On the other hand, if hydrogen is passed over the heated oxide, $\mathrm{Fe}_{3} \mathrm{O}_{4}$, the products are iron and water. The equation for these two reactions, of which one is the reverse of the other, is

$$
{ }_{3} \mathrm{Fe}+{ }_{4} \mathrm{H}_{2} \mathrm{O} \leftrightarrows \mathrm{Fe}_{3} \mathrm{O}_{4}+{ }_{4} \mathrm{H}_{2} \text {. }
$$

If we bring together either pair of substances in a closed vessel and heat them for some time, a state of equilibrium is reached in which all four substances are present. The iron and iron oxide are solids, while the water, as steam, is a gas. For the condition of equilibrium the relative amounts of steam and hydrogen are always the same for a given temperature, no matter what proportions of either pair of substances have been used. This is the state of affairs if the reaction occurs in a closed vessel. But the results are entirely different if the reactions take place in a tube holding the solids, through which either steam in the one case or hydrogen in the other is passed. If steam is passed through a tube containing iron, then the hydrogen which is formed is carried along with the excess of steam and has no chance to act on the iron oxide which has been formed. There is therefore no chance for iron to be formed again, once it has been changed to iron oxide. As long as unchanged iron remains and the current of steam is continued, the reaction from left to right continues. The inevitable result is the complete change of the iron to the oxide. On the other hand, if a current of hydrogen is passed over heated iron oxide contained in a tube, the substances react in the direction from right to left of the equation. The steam which is formed passes along with the excess of hydrogen, and once having left the tube cannot possibly act on the iron to convert it back into oxide, so that this change also continues as long as the stream of hydrogen is kept up and comes to an end only when all of the iron oxide has been reduced to metallic iron.

29r. Conclusions.-We see, therefore, that a chemical reaction like the one just discussed may reach a state of equilib- 
rium, if the reverse reaction tends to take place noticeably, and if none of the substances involved escape from the vessel in which the change takes place; or it may go to completion in one direction or the other if one of the products of either reaction is allowed to escape from the scene of action.

Whether a given reaction reaches a state of equilibrium or goes to completion in one direction or the other of ten depends upon the conditions. In the preparation or manufacture of chemical substances it is usually very important to cause equilibrium reactions to take place as completely as possible in order to obtain the maximum possible yields of the desired products. 


\section{CHAPTER XIV \\ HYDROGEN AND OXYGEN}

292. Hydrogen.-Hydrogen was first recognized in $I_{7} 66$ as a distinct substance by Cavendish, a celebrated English chemist, who called the gas inflammable air and prepared it by the action of acids on metals. It was not until ten years after Cavendish's discovery that Lavoisier explained the rôle played by oxygen in combustion and stated the law of the indestructibility of matter (2I) and thus laid the foundation for the doctrine of the elements in its present form. For this reason the classification of hydrogen as an element was not possible at the time of its discovery. In $\mathrm{I}_{78 \mathrm{I}}$ Cavendish showed that nothing but water is formed when hydrogen burns and thus proved that water is a compound of hydrogen and oxygen. The name hydrogen means water-former.

The element occurs in but minute amounts in the free form in nature. Water is its most abundant compound; but it is also a constituent of all dry animal and vegetable tissues, forming therein principally compounds with carbon, oxygen, and nitrogen. Petroleum and natural gas are compounds of hydrogen with carbon; coal also contains considerable combined hydrogen.

293. Preparation of Hydrogen.-We have already learned several methods by which free hydrogen can be obtained. These may now be briefly reviewed. Hydrogen is formed:

I. By the electrolysis of water (27),

$$
2 \mathrm{H}_{2} \mathrm{O} \rightarrow 2 \mathrm{H}_{2}+\mathrm{O}_{2} \text {; }
$$

2. By the action of water on some metals, as by (a) the burning of magnesium wire in steam (28, Fig. 15),

$$
\mathrm{Mg}+\mathrm{H}_{2} \mathrm{O} \rightarrow \mathrm{MgO}+\mathrm{H}_{2} \text {, }
$$

(b) the passage of steam over heated iron turnings (29, Fig. 16),

$$
\begin{aligned}
{ }_{3} \mathrm{Fe}+{ }_{4} \mathrm{H}_{2} \mathrm{O} & \rightarrow \mathrm{Fe}_{3} \mathrm{O}_{4}+{ }_{4} \mathrm{H}_{2}, \\
& \text { I } 76
\end{aligned}
$$


(c) the action of sodium or potassium on water $(40,86$, Table VI, 106),

$$
\begin{aligned}
& 2 \mathrm{Na}+{ }_{2} \mathrm{H}_{2} \mathrm{O} \rightarrow 2 \mathrm{NaOH}+\mathrm{H}_{2} \\
& 2 \mathrm{~K}+{ }_{2} \mathrm{H}_{2} \mathrm{O} \rightarrow 2 \mathrm{KOH}+\mathrm{H}_{2} ;
\end{aligned}
$$

3. By the action of hydrochloric or sulfuric acid on zinc, magnesium, iron, or aluminum, as well as on several other metals,

$$
\begin{gathered}
\mathrm{Zn}+2 \mathrm{HCl} \rightarrow \mathrm{ZnCl}_{2}+\mathrm{H}_{2} \\
\mathrm{Fe}+\mathrm{H}_{2} \mathrm{SO}_{4} \rightarrow \mathrm{FeSO}_{4}+\mathrm{H}_{2} .
\end{gathered}
$$

294. Making Hydrogen in the Laboratory. - The best laboratory method of making hydrogen consists in treating zinc with hydrochloric acid in some form of specially constructed gas generator. The Kipp apparatus, Fig. 39, is the form most extensively used. The solution used is made from equal volumes of concentrated hydrochloric acid and water. 'The action of this generator is very simple in principle. Upon opening the stopcock gas escapes and allows the acid to rise into the middle compartment, where it acts upon the zinc and so produces a steady flow of hydrogen. When the cock is closed the gas formed

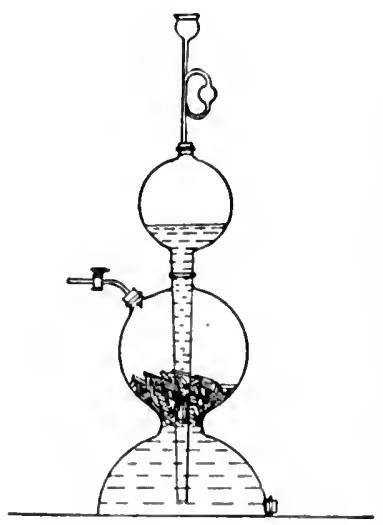

FIG. 39 forces the acid downward and causes it to flow from the lower into the upper compartment. As soon as the acid is out of contact with the zinc all action stops, and no more gas is produced until the cock is again opened. The Kipp apparatus has one unfortunate defect: since there is but little circulation of the solution the acid in contact with the zinc is soon exhausted, causing the action to stop while there is still a large supply of almost unchanged acid in other parts of the apparatus. To start the action again it is necessary to empty all the solution and refill with fresh acid; much acid is thus wasted. 
The McCoy apparatus, shown in Fig. 40, has several ad. vantages over the Kipp apparatus. The lowest compartment is filled as full as possible with granulated or stick zinc, on which hydrochloric acid drops at just the rate required to keep up the stream of hydrogen that is being drawn from the apparatus. When the stopcock is closed the gas which is formed from the small excess of acid in the zinc compartment forces the acid from the middle to the upper compartment and thus stops the further

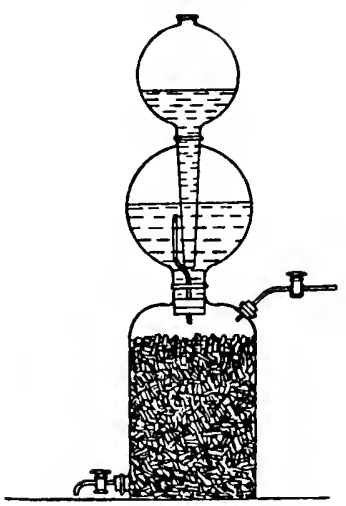

FIG. 40 flow of acid upon the zinc. This apparatus is also conveniently used for generating other gases.

295. The Electrolysis of Water.Compared with metals, pure water is a very poor conductor of electricity. The addition of a little sulfuric acid increases the electrical conductivity of water enormously. The sulfuric acid so added is not permanently destroyed in the course of the electrolysis (27), so that very little will serve to promote the electrolysis of a large amount of water. The exact way in which the acid behaves will be discussed later. Ordinarily, poles or electrodes of the elementary metal platinum are employed, since most other metals would be attacked chemically. The electrode at which the hydrogen is liberated is called the negative electrode, or cathode; the other, at which the oxygen appears, is the positive electrode, or anode.

One of the important technical methods of making hydrogen, which yields at the same time oxygen, consists in the electrolysis of water in which sodium hydroxide is dissolved to make it a good conductor. Here the cathode is of iron and the anode of carbon. Hydrogen is also obtained in commercial quantities as a by-product in the manufacture of caustic soda by the electrolysis of a solution of common salt.

Hydrogen is often made for use in balloons by the action of dilute sulfuric acid on scrap iron. 
296. The Physical Properties of Hydrogen.-We have already learned that hydrogen is colorless; when perfectly pure it is also odorless and tasteless. One liter of the gas at $0^{\circ}$ and $76 \mathrm{~cm}$. pressure weighs $0.0899 \mathrm{~g}$.; and 22.4 liters, $2 \mathrm{~g}$. approximately. It is the lightest of all gases. It can be liquefied, giving a colorless liquid which boils at $-253^{\circ}$, or only $20^{\circ}$ above absolute zero. At a somewhat lower temperature the liquid freezes to a colorless solid.

Hydrogen is but slightly soluble in water: Ioo c.c. of water dissolves about 2 c.c. of the gas at room temperature.

The speed of diffusion of hydrogen is greater than that of any other gas (rgr).

297. The Chemical Properties of Hydrogen.-The most important chemical properties of hydrogen have already been studied, but may now be briefly reviewed. Hydrogen burns with an almost non-luminous flame, which is, however, very much hotter than that obtained from ordinary fuel or illuminating gas. Water is the product of the reaction. Hydrogen reacts readily with hot copper oxide, forming water and copper:

$$
\mathrm{H}_{2}+\mathrm{CuO} \rightarrow \mathrm{H}_{2} \mathrm{O}+\mathrm{Cu} \text {. }
$$

Hydrogen also acts on other metallic oxides at a red heat, for example:

$$
{ }_{4} \mathrm{H}_{2}+\mathrm{Fe}_{3} \mathrm{O}_{4} \rightarrow{ }_{4} \mathrm{H}_{2} \mathrm{O}+{ }_{3} \mathrm{Fe} \text {. }
$$

Hydrogen and chlorine, if mixed in equal volumes and ignited, or exposed to a bright light, unite with explosive violence, forming hydrogen chloride,

$$
\mathrm{H}_{2}+\mathrm{Cl}_{2} \rightarrow 2 \mathrm{HCl} \text {. }
$$

A jet of burning hydrogen lowered into a jar of chlorine continues to burn by reason of the union of the two elements (244).

Hydrogen unites with bromine to form hydrogen bromide (256) and with iodine to form hydrogen iodide (264).

298. The Union of Hydrogen and Nitrogen.-A mixture of hydrogen and nitrogen does not react at all under ordinary conditions. If electric sparks are passed through the mixed 
gases contained in a eudiometer, Fig. 4I, a small amount of ammonia is formed:

$$
3 \mathrm{H}_{2}+\mathrm{N}_{2} \rightarrow 2 \mathrm{NH}_{3} \text {. }
$$

The reaction soon reaches a state of equilibrium, because under the same conditions ammonia is very largely decomposed into its elements. As the result of the reverse reaction, a state of equilibrium is reached when less than I per cent of the elementary gases has been converted into ammonia. If the

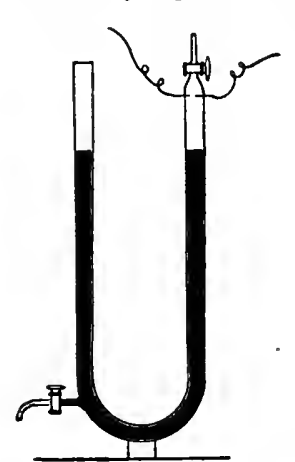

FIG. 4 I ammonia is absorbed in some suitable way, as by union with sulfuric acid, as fast as it is formed, then, with continued sparking, the formation of ammonia goes on until all the hydrogen and nitrogen have united. The practical method of making ammonia by this reaction will be discussed in chapter xxi.

299. Heat of Reaction and Flame Temperature.-When I g. of hydrogen burns, about 34,000 calories of heat are produced; this is sufficient to heat 340 c.c. of water from $0^{\circ}$ to $100^{\circ}$. By reason of the great amount of heat produced, the flame of hydrogen burning in air has a very high temperature. When hydrogen burns in pure oxygen instead of in air, the flame is much hotter, but not because a greater amount of heat is produced by the burning of a given amount of hydrogen, since the quantity of heat is the same in the two cases. When hydrogen burns in air, the nitrogen, which forms four-fifths by volume of the air, is heated to the flame temperature along with the steam formed. But in pure oxygen no nitrogen is present, and so the temperature reached by the flame is much higher, as there is far less material to be heated.

300. The Oxyhydrogen Blowpipe.-Fig. 42 shows an oxyhydrogen blowpipe. The two gases mix in the proper proportions before issuing from the jet. The temperature of the flame is high enough to melt platinum, which cannot be melted in a Bunsen flame supplied with fuel or illum:nating gas. 
301. The Limelight. - A very bright light is produced when an oxyhydrogen flame strikes a stick of quicklime, by reason of the bright white heat to which the lime is raised. 'This is the so-called limelight, which was very extensively used before the electric arc light was perfected and which is still frequently used in rural communities. In place of lime other difficultly fusible white oxides may be employed. For this purpose thorium oxide containing I per cent of cerium oxide is much superior to lime.

302. Ignition Temperature. - A mixture of hydrogen and oxygen in their combining proportion remains unchanged for any

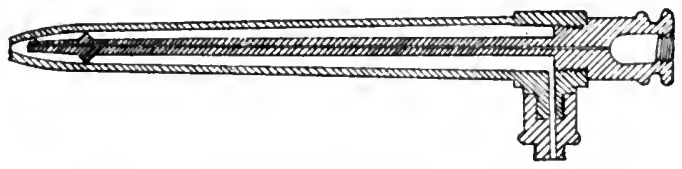

FIG. 42

length of time at room temperature, but if brought in contact with a flame or electric spark it explodes violently. 'The explosion is due to the great increase in volume of the reaction product, steam, caused by the almost instantaneous reaction, with its attendant heat production. But we may inquire why a reaction which does not take place at room temperature can become explosive. Investigation shows that a mixture of the two gases reacts perceptibly at $450^{\circ}$, and that the formation of water goes on faster the higher the temperature, but that the mixture does not become explosive until the temperature reaches about $600^{\circ}$. It is easy to see why explosion finally occurs when the temperature is raised. While the reaction is taking place slowly, heat is being produced by the chemical change; below $600^{\circ}$ the rate of change is so slow that heat is lost by the gas mixture faster than it is produced. Above $600^{\circ}$ the reaction goes faster, so that heat is produced more rapidly than it is lost, and this causes the gas mixture to grow hotter; and the hotter it gets the faster the reaction goes, until soon it proceeds with enormous rapidity, and this constitutes an explosion. For any combustible substance there is some temperature to which it must be heated before its 
rate of production of heat by union with oxygen exceeds its rate of loss of heat; if heated to this temperature the substance takes fire and continues to burn. This point is called the ignition temperature.

Hydrogen, issuing from a jet, burns quietly in air when ignited. This is because the actual union with oxygen can occur only as fast as the two gases can reach one another by diffusion (I9I), one from the jet, the other from the surrounding air. The flame is the reacting gas mixture, which is raised to incandescence by the great heat produced by the union.

303. Platinum as a Catalytic Agent.-The element platinum can be deposited on asbestos as a thin, spongy coating by dipping a bit of fibrous asbestos in a solution of platinum chloride, $\mathrm{PtCl}_{4}$, drying the material and holding it in a Bunsen flame for a minute. The salt decomposes into its elements thus:

$$
\mathrm{PtCl}_{4} \rightarrow \mathrm{Pt}+2 \mathrm{Cl}_{2} \text {. }
$$

If a jet of cold hydrogen gas is directed on the cold platinized asbestos, the latter gets red-hot and sets fire to the hydrogen. Spongy platinum absorbs gases to a marked extent. Heat is produced in this way, and this ignites the intimate mixture of hydrogen and oxygen condensed on the surface of the metal. The platinum itself is entirely unchanged and will continue active in this way indefinitely. A substance which initiates or promotes a chemical reaction without itself being changed is called a catalytic agent (239).

304. The Use of Hydrogen in Balloons.-The lifting power of a balloon filled with hydrogen can easily be calculated. Since I liter of air under standard conditions weighs I.29 g. and I liter of hydrogen weighs $0.09 \mathrm{~g}$., the difference, I.2 g., represents the lifting power per liter of capacity of a balloon. At higher temperature and lower pressure the lifting power is smaller. If a Zeppelin has a capacity of 5,000,000 liters its lifting power will be about 6,000 kilos, or more than 13,000 pounds.

305. Oxygen.--Oxygen in the form of compounds makes up about one-half by weight of the matter forming the crust of the 
earth. It also constitutes 89 per cent by weight of water and 2I per cent by volume of the air. The oxygen of the air is not chemically combined but is only mixed with nitrogen and small amounts of other gases present. Oxygen was first prepared by Priestley, in England (I 774), by heating mercuric oxide,

$$
2 \mathrm{HgO} \rightarrow 2 \mathrm{Hg}+\mathrm{O}_{2} \text {. }
$$

At practically the same time Scheele, in Sweden, made oxygen by this method and also by heating potassium nitrate,

$$
2 \mathrm{KNO}_{3} \rightarrow 2 \mathrm{KNO}_{2}+\mathrm{O}_{2} \text {. }
$$

The salt $\mathrm{KNO}_{2}$ is called potassium nitrite. 'The name oxygen, which means acid-former, was given to the gas by Lavoisier, who believed that it was a necessary constituent of all acids. At that time hydrochloric acid, which was called muriatic or marine acid, was thought to contain oxygen. We now know many other acids which do not contain oxygen.

306. The Preparation of Oxygen. - We have already learned several ways by which oxygen may be made. 'The heating of mercuric oxide and the electrolysis of water $(14,295)$ have already been fully studied. We have also seen (245) that oxygen is formed when chlorine water is exposed to sunlight:

$$
{ }_{2} \mathrm{Cl}_{2}+{ }_{2} \mathrm{H}_{2} \mathrm{O} \rightarrow \mathrm{O}_{2}+{ }_{4} \mathrm{HCl} \text {. }
$$

The heating of certain salts which are rich in o:ygen is also a simple way of making the gas. The behavior of potassium nitrate, $\mathrm{KNO}_{3}$, is given in the preceding paragraph. Potassium chlorate, $\mathrm{KClO}_{3}$, is easily decomposed by heat according to the following equation:

$$
2 \mathrm{KClO}_{3} \rightarrow 2 \mathrm{KCl}+3 \mathrm{O}_{2} \text {. }
$$

This last reaction is the one usually employed in making small amounts of oxygen in the laboratory. It can be carried out in a test tube, a small flask, or a retort. 'The crystals of potassium chlorate first melt, and at a little higher temperature the liquid seems to boil, by reason of the oxygen given off. 
The change of the chlorate, $\mathrm{KClO}_{3}$, into chloride, $\mathrm{KCl}$, does not take place completely in one step. The first stage of the reaction is probably represented by the equation,

$$
\mathrm{IoKClO}_{3} \rightarrow 4 \mathrm{KCl}+6 \mathrm{KClO}_{4}+3 \mathrm{O}_{2} .
$$

The salt $\mathrm{KClO}_{4}$, called potassium perchlorate, can also be decomposed by heat, thus:

$$
\mathrm{KClO}_{4} \rightarrow \mathrm{KCl}+2 \mathrm{O}_{2} \text {. }
$$

This last reaction requires a higher temperature than the first. If the heating of the chlorate is stopped when about one-fifth of its total oxygen has been given off, $\mathrm{KClO}_{4}$ will be found in the residue.

In making oxygen from potassium chlorate two precautions should be observed: first, the material must be entirely free from bits of wood, paper, etc., which are easily combustible; and, secondly, the heating must be gentle, as otherwise the decomposition may occur explosively.

When powdered potassium chlorate is mixed with about half its weight of manganese dioxide, $\mathrm{MnO}_{2}$, it will give off its oxygen rapidly at a temperature far below that at which the pure chlorate starts to decompose. Since manganese dioxide alone does not give off any of its oxygen until a rather high temperature is reached, and is not changed itself in promoting the decomposition of the potassium chlorate, we must consider that the former substance acts only as a catalytic agent in promoting the decomposition of potassium chlorate.

307. Oxygen from Sodium Peroxide.-Sodium peroxide, $\mathrm{Na}_{2} \mathrm{O}_{2}$, is a solid made by burning metallic sodium,

$$
2 \mathrm{Na}+\mathrm{O}_{2} \rightarrow \mathrm{Na}_{2} \mathrm{O}_{2} \text {. }
$$

The trade name of the material is oxone; it is supplied in the form of lumps or sticks. Water acts on it as follows:

$$
{ }_{2} \mathrm{Na}_{2} \mathrm{O}_{2}+{ }_{2} \mathrm{H}_{2} \mathrm{O} \rightarrow{ }_{4} \mathrm{NaOH}+\mathrm{O}_{2} .
$$

By dropping water on lumps of oxone contained in a suitable apparatus (236, Fig. 30) a steady stream of oxygen is obtained. 
The method is rather expensive, but it is very convenient, since the action stops when the supply of water is turned off and can be started again at will.

308. Oxygen from Other Oxides. - Lead dioxide, $\mathrm{PbO}_{2}$, when strongly heated gives oxygen and lead monoxide, or litharge, $\mathrm{PbO}$ :

$$
{ }_{2} \mathrm{PbO}_{2} \rightarrow 2 \mathrm{PbO}+\mathrm{O}_{2} \text {. }
$$

Manganese dioxide is also decomposed at a high temperature, thus:

$$
3 \mathrm{MnO}_{2} \rightarrow \mathrm{Mn}_{3} \mathrm{O}_{4}+\mathrm{O}_{2} \text {. }
$$

309. Technical Methods of Making Oxygen.-The electrolysis of water is an important technical method of making oxygen. It also yields hydrogen and has already been described. By far the larger part of the oxygen of commerce is made from liquid air. This substance is a mixture of liquid oxygen and liquid nitrogen. The latter boils about $\mathrm{II}^{\circ}$ lower than the former, which boils at $-183^{\circ}$. Therefore nitrogen distils off first when liquid air is allowed to evaporate, leaving nearly pure oxygen. This is stored under pressure in steel tanks and brought on the market.

310. Brin's Process.-Brin's process, formerly used technically, is a method of obtaining oxygen from the air by means of barium oxide, $\mathrm{BaO}$. 'This oxide unites with more oxygen at a red heat, forming barium peroxide:

$$
2 \mathrm{BaO}+\mathrm{O}_{2} \leftrightharpoons 2 \mathrm{BaO}_{2} \text {. }
$$

This is a reversible reaction, which at a constant temperature will go in one direction or the other with change of pressure. In practice, air is pumped under pressure into a vessel containing $\mathrm{BaO}$ at $700^{\circ}$, and when all the oxide has been changed into $\mathrm{BaO}_{2}$ the nitrogen present is allowed to escape. By reducing the pressure with a vacuum pump the $\mathrm{BaO}_{2}$ is caused to decompose completely, yielding nearly pure oxygen.

311. Oxygen from Plants. - Growing plants absorb carbon dioxide from the air. They also take up water through their roots. In some manner, not fully understood, carbon dioxide and water react under the influence of sunlight to form such 
principal plant constituents as starch, cellulose, and sugar, together with oxygen, which is given off to the air. The percentage of oxygen in the air would soon decrease if it were not maintained by growing plants.

312. The Physical Properties of Oxygen.-It is, of course, obvious that oxygen is colorless, odorless, and tasteless. One liter weighs I.429 g. and 22.4 liters about $32 \mathrm{~g}$., corresponding to the formula $\mathrm{O}_{2}$. Liquid oxygen is pale blue in color; it boils at $-\mathrm{I} 83^{\circ}$. At $\circ^{\circ}$, IOo c.c. of water dissolves about 5 c.c. of oxygen; at $20^{\circ}$, about 3 C.c. (r25).

313. The Chemical Properties of Oxygen.-We have already learned that combustion was first explained by Lavoisier in I 774 as due to union of the burning substance with the oxygen of the air (I3-I5). All the elements so far studied, except fluorine, form oxides. This does not mean that all these elements burn, sincè some oxides, like those of chlorine and silver, can only be made indirectly (I72). The oxides of metallic elements, by union with water, form hydroxides which are bases, for example:

$$
\mathrm{CaO}+\mathrm{H}_{2} \mathrm{O} \rightarrow \mathrm{Ca}(\mathrm{OH})_{2} \text {. }
$$

The oxides of non-metallic elements, including carbon, sulfur, nitrogen, phosphorus, and the halogens (except flourine), give, with water, acids. The following equations will serve as illustrations of such reactions, some of which have already been studied; the others will be studied later.

$$
\begin{gathered}
\mathrm{CO}_{2}+\mathrm{H}_{2} \mathrm{O} \rightarrow \mathrm{H}_{2} \mathrm{CO}_{3} \text {, carbonic acid, } \\
\mathrm{SO}_{3}+\mathrm{H}_{2} \mathrm{O} \rightarrow \mathrm{H}_{2} \mathrm{SO}_{4} \text {, sulfuric acid, } \\
\mathrm{N}_{2} \mathrm{O}_{5}+\mathrm{H}_{2} \mathrm{O} \rightarrow{ }_{2} \mathrm{HNO}_{3} \text {, nitric acid, } \\
\mathrm{P}_{2} \mathrm{O}_{5}+{ }_{3} \mathrm{H}_{2} \mathrm{O} \rightarrow{ }_{2} \mathrm{H}_{3} \mathrm{PO}_{4} \text {, phosphoric acid, } \\
\mathrm{I}_{2} \mathrm{O}_{5}+\mathrm{H}_{2} \mathrm{O} \rightarrow{ }_{2} \mathrm{HIO}_{3} \text {, iodic acid. }
\end{gathered}
$$

An oxide which by union with water forms an acid is often called the anhydride of the acid.

314. Respiration.--Animals breathe air in order to obtain oxygen. The blood contains a complex substance, haemoglobin, which forms with oxygen a compound, oxyhaemoglobin, which easily decomposes reversibly into oxygen and haemo- 
globin. This is a typical equilibrium reaction: when oxygen, at the pressure at which it exists in the air, comes in contact with the blood in the lungs the compound is formed, I g. of haemoglobin uniting with I. 3 c.c. of oxygen; when the blood reaches the tissues, which take up oxygen, the compound decomposes and the haemoglobin is carried by the blood back to the lungs, where it again takes up fresh oxygen from the air.

315. Uses of Oxygen. The Oxyacetylene Torch.-The use of oxygen in the oxyhydrogen blowpipe has already been mentioned. By substituting acetylene for hydrogen in a blowpipe similarly constructed we get the oxyacetylene torch, which gives an intensely hot flame. It is extensively used for welding and for cutting iron and steel.

Oxygen is used in several analytical processes, such as those studied in chapter iv.

Deposits of carbon in the cylinders of gasoline engines are often removed by burning out with oxygen. Since iron burns also rather readily in a stream of oxygen, care must be taken to avoid injuring the cylinder in this way.

316. Ozone.-When a silent electric discharge passes through oxygen a very remarkable change is produced; there is a decrease in volume, and a gas having a powerful irritating odor is produced. The new gas is ozone. The simplest form of apparatus

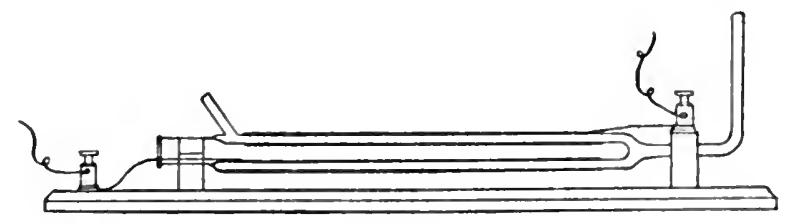

FIG. 43

used for making ozone is shown in Fig. 43. It is a double-walled glass tube having the outside of the outer tube and the inside of the inner tube coated with tin foil. These coatings are connected by wires to the terminals of an induction coil. When the coil is set in action and a slow stream of oxygen is passed through the space between the outer and the inner tubes, the issuing gas is found to contain ozone. The peculiar odor of the air in the 
neighborhood of powerful electrical machinery is due to ozone. Ozone is very much more active as an oxidizing agent than oxygen. Mercury shaken with ozone is very quickly oxidized. Ozone also sets iodine free from a solution of an iodide.

If nothing but oxygen is needed to produce ozone-and such is actually the case-what then is the cause of the remarkable change in properties? In the first place it was noticed that a decrease in volume occurs when ozone is formed from oxygen. On the other hand, when ozone is changed to oxygen, as may be done by heating the former, the volume of the oxygen, when it is again cooled to the original temperature, is greater than that of the ozone. In fact, three volumes of oxygen give exactly two of ozone and vice versa. The density of ozone is one-half greater than that of oxygen. While 22.4 liters of oxygen weigh $32 \mathrm{~g}$., the same volume of ozone weighs $48 \mathrm{~g}$. Ozone is oxygen in another form. If for oxygen we write the formula $\mathrm{O}_{2}$, we must write $\mathrm{O}_{3}$ as the formula of ozone. The molecules of ozone differ from those of oxygen by containing three instead of two atoms. We may write the reversible equation for the relation between oxygen and ozone thus:

$$
3 \mathrm{O}_{2} \leftrightarrows 2 \mathrm{O}_{3} \text {. }
$$

When ozone acts on mercury, for example, the action is as follows:

$$
\mathrm{Hg}+\mathrm{O}_{3} \leftrightarrows \mathrm{HgO}+\mathrm{O}_{2} \text {. }
$$

Only one-third of the oxygen of ozone is active, the balance changing into ordinary oxygen. Iodides are oxidized by ozone, thus:

$$
{ }_{2} \mathrm{KI}+\mathrm{O}_{3}+\mathrm{H}_{2} \mathrm{O} \leftrightharpoons 2 \mathrm{KOH}+\mathrm{I}_{2}+\mathrm{O}_{2} .
$$

The liberated iodine may be recognized by its action on starch. Minute amounts of ozone may be recognized in this way, although the test is not conclusive proof of the presence of ozone, since many other substances also set iodine free from iodides.

3I7. Ozone as a Germicide.-Since ozone is a very powerful ozidizing agent, it is not surprising that it should readily destroy germs. It has been found that impure water containing even $\mathbf{I}, 000,000$ bacteria per c.c. is completely sterilized by intimate 
contact with an equal volume of air containing $2 \mathrm{~g}$. of ozone per cubic meter. In a number of important cities of Europe the entire municipal water supply is purified by means of ozone. Disinfection by chlorine is more popular.

318. Hydrogen Peroxide, $\mathrm{H}_{2} \mathrm{O}_{2}$.- The well-known household antiseptic and disinfectant, hydrogen peroxide, $\mathrm{H}_{2} \mathrm{O}_{2}$, is a 3 per cent solution of this substance in water. Some hydrogen peroxide is formed by the action of sodium peroxide on ice water, thus:

$$
\mathrm{Na}_{2} \mathrm{O}_{2}+{ }_{2} \mathrm{H}_{2} \mathrm{O} \leftrightharpoons \mathrm{H}_{2} \mathrm{O}_{2}+2 \mathrm{NaOH} \text {. }
$$

If water is dropped on sodium peroxide the material becomes very hot, and oxygen and water instead of hydrogen peroxide are formed (307). This is because the latter substance easily decomposes if hot, especially in the presence of caustic soda:

$$
{ }_{2} \mathrm{H}_{2} \mathrm{O}_{2} \leftrightarrows 2 \mathrm{H}_{2} \mathrm{O}+\mathrm{O}_{2} \text {. }
$$

319. Preparation of Hydrogen Peroxide.-Barium peroxide, $\mathrm{BaO}_{2}$, the formation of which was discussed in connection with Brin's process of making oxygen (3ro), reacts with dilute sulfuric acid to form hydrogen peroxide and barium sulfate:

$$
\mathrm{BaO}_{2}+\mathrm{H}_{2} \mathrm{SO}_{4} \leftrightharpoons \mathrm{H}_{2} \mathrm{O}_{2}+\mathrm{BaSO}_{4} \text {. }
$$

Since barium sulfate is insoluble in water a very pure solution of hydrogen peroxide is easily obtained. The reaction is best carried out by adding finely powdered barium peroxide, suspended in water, very gradually to ice-cold, diluted sulfuric acid. The precipitate of barium sulfate is allowed to settle, leaving a clear solution of hydrogen peroxide. This solution must be made as nearly neutral as possible, otherwise it will decompose more or less rapidly into oxygen and water.

By cautious evaporation, at a moderate temperature in a partial vacuum, a dilute solution of hydrogen peroxide may be freed from most of its water; the resulting concentrated solution when cooled to $-10^{\circ}$ deposits crystals of $\mathrm{H}_{2} \mathrm{O}_{2}$.

320. Properties of Hydrogen Peroxide.-At ordinary temperatures pure hydrogen peroxide is a colorless liquid which will 
mix with water in all proportions. It freezes at $-2^{\circ}$. It does not boil without decomposition, and when strongly heated it is liable to explode, water and oxygen being the products. The speed of decomposition of hydrogen peroxide at ordinary temperatures is greatly influenced by the presence of other substances which act as catalytic agents. Finely divided metals like platinum and gold cause hydrogen peroxide to decompose rapidly. Manganese dioxide behaves similarly. In these reactions neither the metals nor the manganese dioxide are changed. They are catalytic agents (303).

Hydrogen peroxide seems to have the property of an acid, since it combines with some bases to form compounds which may be considered salts. For example, with barium hydroxide, $\mathrm{Ba}(\mathrm{OH})_{2}$, it reacts thus:

$$
\mathrm{H}_{2} \mathrm{O}_{2}+\mathrm{Ba}(\mathrm{OH})_{2}+6 \mathrm{H}_{2} \mathrm{O} \leftrightarrows \mathrm{BaO}_{2} \cdot 8 \mathrm{H}_{2} \mathrm{O}
$$

The product consists of white crystals, rather difficultly soluble in water.

Hydrogen peroxide is often used as a bleaching agent for plant and animal substances, such as hair, feathers, silk, ivory, and straw.

The most characteristic property of hydrogen peroxide is its great tendency to give up oxygen and thus to act on substances capable of reacting with oxygen. For example, with hydriodic acid it gives iodine and water:

$$
\mathrm{H}_{2} \mathrm{O}_{2}+2 \mathrm{HI} \leftrightarrows \mathrm{I}_{2}+2 \mathrm{H}_{2} \mathrm{O}
$$

The free iodine can easily be recognized by the blue color which it gives with a starch solution. Instead of using hydriodic acid we may use a solution of potassium or sodium iodide to which hydrochloric acid has been added, since the solution will then contain some hydriodic acid formed as follows:

$$
\mathrm{KI}+\mathrm{HCl} \leftrightarrows \mathrm{KCl}+\mathrm{HI}
$$

32I. Detection of Hydrogen Peroxide.-A very delicate reaction which serves to detect small quantities of hydrogen 
peroxide is that which occurs when a solution of this substance is mixed with a little sulfuric acid and a very dilute solution of potassium dichromate, $\mathrm{K}_{2} \mathrm{Cr}_{2} \mathrm{O}_{7}$. The latter substance contains the element chromium, $\mathrm{Cr}$, as one of its constituents. The solution turns blue, and when a little ether is added and shaken up with the blue solution the ether dissolves the blue substance. If the mixture is allowed to stand a minute or two the blue ether solution separates from the water solution, on which it floats as a blue layer.

Other reactions of hydrogen peroxide are discussed in the following chapter $(347,348)$.

322. Peroxides and Dioxides. - We have just learned that hydrogen peroxide is formed by the action of dilute acids on $\mathrm{Na}_{2} \mathrm{O}_{2}$ and $\mathrm{BaO}_{2}$, and we might therefore be inclined to expect that we should also get $\mathrm{H}_{2} \mathrm{O}_{2}$ by the action of acids on $\mathrm{PbO}_{2}$ and $\mathrm{InO}_{2}$. But this is not the case; no $\mathrm{H}_{2} \mathrm{O}_{2}$ can be obtained in any way from these last-mentioned oxides. For this reason these oxides of lead and manganese are called dioxides to distinguish them as a class from those which yield $\mathrm{H}_{2} \mathrm{O}_{2}$ and which are called peroxides. Thus we call $\mathrm{BaO}_{2}$ barium peroxide and $\mathrm{PbO}_{2}$ lead dioxicle.

323. Graphic Formulae.-We have so far considered that oxygen has a valence (183) of two, or is bivalent, since in water two symbol weights of hydrogen are united with one of oxygen. But what then is the valence of oxygen in $\mathrm{H}_{2} \mathrm{O}_{2}$ ? In order to be able to answer this question, we must consider the matter of valence from the standpoint of the atomic-molecular hypothesis. We have learned (221) that the molecule of water is made up of two atoms of hydrogen and one of oxygen. Since all the molecules of water are made up in just this fashion, it would seem to follow that the three atoms must be related to one another in some very definite way. We may think of them as being joined to one another, in which case there are the two possibilities indicated by the following graphic formulae in which the lines joining the symbols are called bonds.
(I) $\mathrm{H}-\mathrm{H}-\mathrm{O}$
(2) $\mathrm{H}-\mathrm{O}-\mathrm{H}$. 
The first graphic formula indicates that one of the hydrogen atoms is attached on the one hand to the atom of oxygen and on the other to the second atom of hydrogen. Formula (2) indicates that it is the oxygen atom which is attached on either hand to an atom hydrogen. It is obvious that the second formula is the more consistent, since in it both atoms of hydrogen are attached by single bonds to the atom of oxygen which holds an atom of hydrogen by each of its two bonds. In formula (I) the middle hydrogen atom is represented as having two bonds, while the other hydrogen atom and also the atom of oxygen are shown as having but one bond each. Since we think of all atoms of hydrogen as being alike, we must reject the first formula in favor of the second.

When viewed in the above-mentioned manner the valence of an element is seen to be the holding capacity of its atoms for atoms of hydrogen or other univalent elements like chlorine. We may therefore think of an atom of oxygen which is bivalent as having two valence bonds, each of which can hold one atom of a univalent element.

324. The Graphic Formulae of Peroxides.-We are now prepared to consider the question of the valence of oxygen in $\mathrm{H}_{2} \mathrm{O}_{2}$ and the graphic formula of this substance. At the outset it may be stated that the valence of hydrogen is considered by chemists to be invariably one. If the valence of oxygen is taken to be two, there is but one possible graphic formula, namely:

$$
\mathrm{H}-\mathrm{O}-\mathrm{O}-\mathrm{H} \text {. }
$$

This is the commonly accepted formula. For sodium peroxide we then have the formula,

$$
\mathrm{Na}-\mathrm{O}-\mathrm{O}-\mathrm{Na} \text {, }
$$

and for barium peroxide,<smiles></smiles> 
In their dioxides, manganese and lead have without doubt a valence of four, or are tetravalent, and oxygen is, as usual, bivalent. We therefore write for these oxides the formulae

$$
\mathrm{O}=\mathrm{Mn}=\mathrm{O} \text { and } \mathrm{O}=\mathrm{Pb}=\mathrm{O} \text {, }
$$

thereby indicating that each oxygen atom is attached to an atom of manganese or lead by two bonds, or in other words by a double bond.

The monoxides of manganese, $\mathrm{MnO}$, and lead, $\mathrm{PbO}$, have their atoms doubly bound, thus:

$$
\mathrm{Mn}=\mathrm{O} \text { and } \mathrm{Pb}=\mathrm{O} \text {. }
$$

In these oxides both metals are bivalent. 


\section{CHAPTER XV \\ OXIDATION AND REDUCTION}

325. Oxidation.-When a substance unites with oxygen it is said to be oxidized. Hydrogen when burned is oxidized, giving water. Metals are said to be oxidized when they combine with oxygen; for example,

$$
{ }_{2} \mathrm{Cu}+\mathrm{O}_{2} \rightarrow 2 \mathrm{CuO} \text {. }
$$

By certain indirect methods a lower oxide of copper, cuprous oxide, $\mathrm{Cu}_{2} \mathrm{O}$, can be made. This oxide can unite with more oxygen if heated in air or in oxygen and form the common oxide, $\mathrm{CuO}$, which is known also as cupric oxide, in order to distinguish it from the lower oxide:

$$
{ }_{2} \mathrm{Cu}_{2} \mathrm{O}+\mathrm{O}_{2} \rightarrow{ }_{4} \mathrm{CuO} \text {. }
$$

We say in this case that cuprous oxide has been oxidized to cupric oxide.

The action of oxygen gas on hydrogen chloride at a high temperature (239) proceeds according the equation

$$
{ }_{4} \mathrm{HCl}+\mathrm{O}_{2} \rightarrow{ }_{2} \mathrm{Cl}_{2}+2 \mathrm{H}_{2} \mathrm{O} \text {, }
$$

and in consequence we say that the hydrogen chloride has been oxidized.

326. Oxidizing Agents.--Very often substances may be oxidized by compounds of oxygen as well as by oxygen itself. For example, heated copper oxide, $\mathrm{CuO}$, oxidizes hydrogen and all its compounds, such as ammonia, acetylene, etc. We say, therefore, that copper oxide is an oxidizing agent. Any substance which oxidizes another is called an oxidizing agent. Lead dioxide and manganese dioxide are powerful oxidizing agents, as shown by the fact that each is able to oxidize hydrochloric acid,

$$
\mathrm{MnO}_{2}+{ }_{4} \mathrm{HCl} \rightarrow \mathrm{MnCl}_{2}+\mathrm{Cl}_{2}+{ }_{2} \mathrm{H}_{2} \mathrm{O} .
$$


Potassium permanganate, $\mathrm{KMnO}_{4}$, which also easily oxidizes hydrochloric acid, is one of the most powerful of all oxidizing agents. It acts according to the equation

$$
2 \mathrm{KMnO}_{4}+16 \mathrm{HCl} \rightarrow 2 \mathrm{KCl}+2 \mathrm{MnCl}_{2}+5 \mathrm{Cl}_{2}+8 \mathrm{H}_{2} \mathrm{O} \text {. }
$$

Nitric acid and its salts (104), the nitrates, are good oxidizing agents. Gunpowder is a mixture of finely powdered potassium nitrate, charcoal, and sulfur. The explosion of gunpowder is due to the extremely rapid oxidation of the charcoal (carbon) and sulfur to carbon dioxide, $\mathrm{CO}_{2}$, and sulfur dioxide, $\mathrm{SO}_{2}$, the oxygen being furnished by the potassium nitrate, $\mathrm{KNO}_{3}$. The other products of the explosion are nitrogen and potassium sulfide, $\mathrm{K}_{2} \mathrm{~S}$.

Potassium chlorate, $\mathrm{KClO}_{3}$, is a powerful oxidizing agent, readily giving up all its oxygen to oxidizable substances and leaving the chloride, $\mathrm{KCl}$.

Sulfuric acid (93) is capable of oxidizing some substances; hot sulfuric acid acts on charcoal thus:

$$
{ }_{2} \mathrm{H}_{2} \mathrm{SO}_{4}+\mathrm{C} \rightarrow \mathrm{CO}_{2}+2 \mathrm{SO}_{2}+2 \mathrm{H}_{2} \mathrm{O} \text {. }
$$

It is probable that each molecule of sulfuric acid first loses one atom of oxygen giving a molecule of sulfurous acid, $\mathrm{H}_{2} \mathrm{SO}_{3}$, and that this substance, which is unstable, then decomposes into sulfur dioxide and water:

$$
\mathrm{H}_{2} \mathrm{SO}_{3} \rightarrow \mathrm{SO}_{2}+\mathrm{H}_{2} \mathrm{O} \text {. }
$$

327. Reduction and Reducing Agents.-When hydrogen is oxidized by hot copper oxide, thus:

$$
\mathrm{CuO}+\mathrm{H}_{2} \rightarrow \mathrm{Cu}+\mathrm{H}_{2} \mathrm{O} \text {, }
$$

the copper oxide is said to be reduced to metallic copper. In consequence we call hydrogen a reducing agent. Any substance, as, for example, acetylene or methane, which can reduce copper oxide is also called a reducing agent. In any reaction, if one substance is oxidized the oxidizing agent is by necessity reduced; oxidation and reduction alway's go on together. All substances which are acted upon by oxidizing agents are, of course, reducing agents. 
328. Carbon as a Reducing Agent.-Since charcoal, which is nearly pure carbon, burns readily, it is capable of taking up oxygen from oxidizing agents and is therefore a good reducing agent. A mixture of powdered copper oxide and charcoal reacts vigorously, if strongly heated, giving copper and carbon dioxide:

$$
{ }_{2} \mathrm{CuO}+\mathrm{C} \rightarrow{ }_{2} \mathrm{Cu}+\mathrm{CO}_{2} \text {. }
$$

In this reaction the copper oxide is the oxidizing agent and the charcoal (carbon) the reducing agent.

Many other metallic oxides can be reduced in a similar manner by carbon. In place of charcoal, coke or coal, which are largely carbon, may be used. Thus ferric oxide, $\mathrm{Fe}_{2} \mathrm{O}_{3}$, which in the form of the mineral hematite is the most important ore of iron, is reduced by coke at a white heat to metallic iron. The result may be represented by the equation

$$
{ }_{2} \mathrm{Fe}_{2} \mathrm{O}_{3}+{ }_{3} \mathrm{C} \rightarrow{ }_{4} \mathrm{Fe}+{ }_{3} \mathrm{CO}_{2},
$$

although it is very probable that the reaction is less simple under the conditions actually met with in practice.

329. Carbon Monoxide as a Reducing Agent.-When carbon is burned in a deficient supply of air, carbon monoxide, $\mathrm{CO}$, is formed instead of dioxide:

$$
{ }_{2} \mathrm{C}+\mathrm{O}_{2} \rightarrow{ }_{2} \mathrm{CO} \text {. }
$$

This is a colorless, odorless, and very poisonous gas which will burn with a nearly non-luminous flame to form carbon dioxide,

$$
{ }_{2} \mathrm{CO}+\mathrm{O}_{2} \rightarrow{ }_{2} \mathrm{CO}_{2} \text {. }
$$

Some oxidizing agents are able to oxidize carbon only to monoxide and not to dioxide. Zinc oxide behaves in this way:

$$
\mathrm{ZnO}+\mathrm{C} \rightarrow \mathrm{Zn}+\mathrm{CO} \text {. }
$$

This is the reaction by which zinc is made from its ores. The reaction between ferric oxide and carbon can also give carbon monoxide,

$$
\mathrm{Fe}_{2} \mathrm{O}_{3}+{ }_{3} \mathrm{C} \rightarrow{ }_{2} \mathrm{Fe}+{ }_{3} \mathrm{CO} \text {. }
$$

But carbon monoxide can also reduce ferric oxide:

$$
\mathrm{Fe}_{2} \mathrm{O}_{3}+{ }_{3} \mathrm{CO} \rightarrow{ }_{2} \mathrm{Fe}+{ }_{3} \mathrm{CO}_{2} \text {. }
$$


The last two equations doubtless represent the steps by which ferric oxide and carbon react to give iron and carbon dioxide (328).

330. Aluminum as a Reducing Agent.-Metallic aluminum unites vigorously with oxygen at a white heat, although it has no tendency to oxidize in the air at ordinary temperatures. The burning of aluminum occurs thus:

$$
4 \mathrm{Al}+{ }_{3} \mathrm{O}_{2} \rightarrow 2 \mathrm{Al}_{2} \mathrm{O}_{3} \text {. }
$$

When a mixture of powdered aluminum and ferric oxide is strongly heated a very violent reaction takes place, giving iron and aluminum oxide:

$$
2 \mathrm{Al}+\mathrm{Fe}_{2} \mathrm{O}_{3} \rightarrow{ }_{2} \mathrm{Fe}+\mathrm{Al}_{2} \mathrm{O}_{3} .
$$

The mixture of aluminum and ferric oxide has been given the trade name of thermite by its inventor, Goldschmidt, who uses it to make small quantities of molten iron for the repair of broken iron castings, etc.

Many other metallic oxides can also be reduced by aluminum.

33I. Oxidation Considered as a Change of Valence.-We have already learned (173) that iron forms two series of compounds, ferrous and ferric, as illustrated by the following formulae:

Ferrous Compounds
$\mathrm{FeO}$
$\mathrm{Fe}(\mathrm{OH})_{2}$
$\mathrm{FeCl}$
$\mathrm{FeBr}$
$\mathrm{Fe}\left(\mathrm{NO}_{3}\right)_{2}$
$\mathrm{FeSO}$

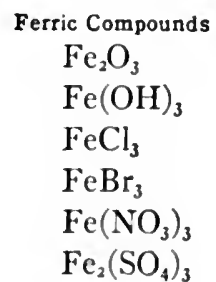

The valence of iron is two in ferrous compounds and three in ferric. According to this usage of the term valence we should be forced to say that the valence of free or uncombined iron is zero.

If free iron is changed into ferrous oxide,

$$
2 \mathrm{Fe}+\mathrm{O}_{2} \rightarrow 2 \mathrm{FeO},
$$


it is oxidized, and its valence is increased from zero to two. Moreover, if ferrous oxide is changed into ferric oxide,

$$
{ }_{4} \mathrm{FeO}+\mathrm{O}_{2} \rightarrow 2 \mathrm{Fe}_{2} \mathrm{O}_{3},
$$

it is also plain that the iron is further oxidized, and that its valence has increased from two to three. It is customary to say that ferric oxide is a higher oxide of iron than ferrous oxide; or that iron in ferric oxide is in a higher state of oxidation than in ferrous oxide.

In the case of iron and its oxides we see that the oxidation of iron and the increase in its valence go hand in hand.

With respect to other elements that unite with oxygen we also find that their oxidation results in an increase in their valence. A few additional examples will help to illustrate this point. In the change of copper into cuprous oxide, $\mathrm{Cu}_{2} \mathrm{O}(325)$, the oxidation of the copper is accompanied by an increase $\mathrm{cf}$ its valence from zero to one. In cuprous oxide copper is univalent (146). In the oxidation of cuprous oxide to cupric oxide,

$$
{ }_{2} \mathrm{Cu}_{2} \mathrm{O}+\mathrm{O}_{2} \rightarrow{ }_{4} \mathrm{CuO} \text {, }
$$

the valence of copper is increased from one to two. In cupric oxide copper is bivalent (146).

When carbon is oxidized to carbon monoxide,

$$
{ }_{2} \mathrm{C}+\mathrm{O}_{2} \rightarrow 2 \mathrm{CO},
$$

the valence of carbon is increased from zero to two (carbon is bivalent in carbon monoxide). In the oxidation of carbon monoxide to carbon dioxide,

$$
{ }_{2} \mathrm{CO}+\mathrm{O}_{2} \rightarrow{ }_{2} \mathrm{CO}_{2},
$$

the valence of carbon is increased to four, carbon becoming quadrivalent.

332. A Broader Meaning of the Term Oxidation.-Since in the change of any ferrous compound into the corresponding ferric compound ( $173,33 I)$ the valence of iron always increases from two to three, all such changes may well be considered to be of the same class. It has become the custom among chemists 
to call such increase of valence of iron an oxidation of the iron irrespective of the nature of the element or radical combined with the iron. Thus in the reaction

$$
{ }_{2} \mathrm{FeCl}_{2}+\mathrm{Cl}_{2} \rightarrow{ }_{2} \mathrm{FeCl}_{3} \text {, }
$$

whereby ferrous chloride is changed to ferric chloride, we say that the iron has been oxidized. The only question that should arise here is: Why call this increase in valence of iron an oxidation in cases where no oxygen is involved? We can only say that it is a custom sanctioned by long and universal usage.

By way of further illustration of the use of the term oxidation in its broader sense we may cite the following examples. When metallic sodium is changed into chloride, $\mathrm{NaCl}$, or nitrate, $\mathrm{NaNO}_{3}$, its valence is increased from zero to one, and we say that the sodium has been oxidized. When zinc is changed into oxide, $\mathrm{ZnO}$; sulfate, $\mathrm{ZnSO}_{4}$; chloride, $\mathrm{ZnCl}_{2}$; or nitrate, $\mathrm{Zn}\left(\mathrm{NO}_{3}\right)_{2}$ (I48), we say that the zinc has undergone oxidation; and furthermore, since in all these compounds zinc is bivalent, we say that zinc in all these compounds is in the same state or stage of oxidation. In fact, zinc in its compounds is always bivalent.

333. Review of Other Elements with Variable Valence.Iron is not the only clement having a variable valence. We have already seen (I79, I80) that mercury also forms two series of compounds, the mercurous, in which the element has a valence of one, and the mercuric, where the valence is two, as illustrated by the following formulae:

$\begin{array}{cc}\text { Mercurous Compounds } & \text { Mercuric Compounds } \\ \mathrm{Hgg} & \mathrm{HgO} \\ \mathrm{HgCl} & \mathrm{HgCl}_{2} \\ \mathrm{HgI} & \mathrm{HgI}_{2} \\ \mathrm{HgNO}_{3} & \left.\mathrm{Hg}_{\mathrm{NO}_{3}}\right)_{2} \\ \mathrm{Hg}_{2} \mathrm{SO}_{4} & \mathrm{HgSO}_{4}\end{array}$

Mercurous compounds are converted into mercuric by oxidation, and mercuric into mercurous by reduction.

Copper also forms two series of compounds, cuprous and cupric. We know cuprous oxide, $\mathrm{Cu}_{2} \mathrm{O}$, and cuprous chloride, $\mathrm{CuCl}$, as well as the commoner cupric compounds, such as cupric 
oxide, $\mathrm{CuO}$, cupric chloride, $\mathrm{CuCl}_{2}$, cupric sulfate, $\mathrm{CuSO}_{4}$, etc. ( 165 ).

It will be noted that compounds representing the lower state of oxidation have names ending in ous, while those corresponding to the higher state of oxidation end in ic.

334. Another Class of Oxidation Reactions.--We have given as one illustration of an oxidation reaction the action of manganese dioxide on hydrochloric acid:

$$
\mathrm{MnO}_{2}+{ }_{4} \mathrm{HCl} \rightarrow \mathrm{MnCl}_{2}+\mathrm{Cl}_{2}+{ }_{2} \mathrm{H}_{2} \mathrm{O}
$$

In this reaction the manganese dioxide is the oxidizing agent. The chlorine of the hydrochloric acid in being set free has its valence decreased from one to zero. The valence of the hydrogen remains unchanged in the reaction: it is neither oxidized nor reduced. Now we see that when combined chlorine, as in a chloride, is set free its valence is decreased, and that in this change the chlorine is oxidized. In the case of metallic elements oxidation involves increase of valence (33r). We see, therefore, that in the case of chlorine, a non-metallic element, its oxidation in cases like that cited involves a decrease in its valence. Other non-metallic elements, like bromine, iodine, and sulfur, when set free by oxidizing agents from their compounds with hydrogen or metals are oxidized, while at the same time there occurs a decrease in valence.

335. Reduction and Change of Valence.-That valence changes accompany reduction, as well as oxidation, will be at once apparent by the consideration of any reaction in which reduction takes place. Take, for example, the simple case of the reduction of cupric oxide by hydrogen,

$$
\mathrm{CuO}+\mathrm{H}_{2} \rightarrow \mathrm{Cu}+\mathrm{H}_{2} \mathrm{O} \text {. }
$$

The copper is reduced to the free state, its valence changing from two to zero, while the oxygen merely changes partners without change of valence and is therefore neither oxidized nor reduced. When any oxide of a metal is reduced by hydrogen, carbon, or any other reducing agent, the valence of the metal is also reduced or lowered. 
On the other hand, in the reaction

$$
\mathrm{Cl}_{2}+\mathrm{H}_{2} \rightarrow 2 \mathrm{HCl}
$$

we see that the reduction of the non-metallic element chlorine is accompanied by an increase in its valence from zero to one. In general, when a non-melallic clement like a halogen, sulfur, or oxygen itself unites with hydrogen or a metal, the non-metal is reduced, while concurrently its valence is increased.

336. Classification of Valence Changes.-In analyzing the foregoing cases we have seen that there is a difference in the behavior of metals and non-metals. If we consider the many compounds which we have studied, we shall see that metals and hydrogen unite with non-metals more or less readily but do not unite with each other, at least in the cases studied. On the other hand non-metals not only unite with metals but also unite among themselves, as in the case of carbon and oxygen or sulfur and oxygen (286).

In all but the rarest cases the valence of an atom in combination may be found directly or indirectly from the following rules, which are established by experience: hydrogen (sodium and potassium) are univalent in all their compounds; oxygen is bivalent in all its compounds except the peroxides. The latter have definite characteristics and may be identified. The classification of the changes of valence may be made systematically on the basis of the change of the valence of an atom toward a metal or non-metal as indicated in the table below. Whether the atom in question is a metal or a non-metal does not matter.

\begin{tabular}{c|c|c}
\hline $\begin{array}{c}\text { For the Atom in } \\
\text { Question }\end{array}$ & In Oxidation & In Reduction \\
\hline $\begin{array}{r}\text { Valence toward } \\
\text { non-metals... }\end{array}$ & Increases & Decreases \\
$\begin{array}{r}\text { Valence toward } \\
\text { metals or hy- } \\
\text { drogen ...... }\end{array}$ & Iecreases & Increases \\
\hline
\end{tabular}

If the reactions of reagents are classified according to this scheme those which are found to undergo oxidation or reduction, and 
so are themselves either reducing agents or oxidizing agents, will be found actually to have similar chemical activities.

337. Two Important Kinds of Reactions.-But very few of the reactions of substances in solution studied in chapters prior to the present involve oxidation and reduction. In such reactions as

$$
\begin{aligned}
\mathrm{HCl}+\mathrm{NaOH} & \rightarrow \mathrm{NaCl}+\mathrm{H}_{2} \mathrm{O}, \\
\mathrm{AgNO}_{3}+\mathrm{HCl} & \rightarrow \mathrm{AgCl}+\mathrm{HNO}_{3},
\end{aligned}
$$

and

$$
\mathrm{FeCl}_{3}+3 \mathrm{NaOH} \rightarrow \mathrm{Fe}(\mathrm{OH})_{3}+3 \mathrm{NaCl},
$$

neither oxidation nor reduction occurs. These are called doubledecomposition reactions. In such reactions no element changes its valence.

If oxidation and reduction take place, the reaction is of a distinctly different kind. In such reactions two or more elements change valence.

338. Intensity of Activity of Oxidizing and Reducing Agents. -Both oxidizing and reducing agents differ greatly in their intensity of activity. For example, manganese dioxide will oxidize cold, dilute hydrochloric acid, but oxygen gas will not. In consequence we say that manganese dioxide is a stronger or more powerful oxidizing agent than oxygen itself. When we find, as we may readily do by experiment, that dilute nitric acid will oxidize a ferrous salt to a ferric salt and that dilute sulfuric acid will not do so, we conclude that nitric acid is a stronger or better oxidizing agent than sulfuric acid. When we know that hydriodic acid will reduce sulfuric acid, but that hydrochloric acid will not do so, we conclude that hydriodic acid is a better reducing agent than hydrochloric acid.

339. Hydrogen Sulfide, $\mathbf{H}_{2} \mathbf{S}$.- - Iron and sulfur unite directly at a red heat to form ferrous sulfide, $\mathrm{FeS}$ :

$$
\mathrm{Fe}+\mathrm{S} \rightarrow \mathrm{FeS} \text {. }
$$

This is a black solid, which is insoluble in water. It reacts readily with hydrochloric acid to give ferrous chloride and hydrogen sulfide:

$\mathrm{FeS}+{ }_{2} \mathrm{HCl} \rightarrow \mathrm{FeCl}_{2}+\mathrm{H}_{2} \mathrm{~S}$. 
Hydrogen sulfide is a colorless gas, of which water dissolves three to four times its own volume. It has a very disagreeable odor, resembling rotten eggs, and is extremely poisonous. Fatal accidents have often occurred from breathing the gas. Hydrogen sulfide is a very powerful reducing agent. In water solution it is easily oxidized by atmospheric oxygen, giving sulfur and water:

$$
2 \mathrm{H}_{2} \mathrm{~S}+\mathrm{O}_{2} \rightarrow 2 \mathrm{~S}+2 \mathrm{H}_{2} \mathrm{O} \text {. }
$$

A water solution of hydrogen sulfide reacts rapidly with iodine to form hydriodic acid (265) and sulfur:

$$
\mathrm{H}_{2} \mathrm{~S}+\mathrm{I}_{2} \rightarrow 2 \mathrm{HI}+\mathrm{S} \text {. }
$$

This reaction furnishes a very good practical method for making hydriodic acid. We have only to pass hydrogen sulfide gas into water containing powdered iodine. When all the iodine has been reduced, the solid sulfur can be filtered out, giving a clear, colorless filtrate which contains only hydriodic acid and water.

Hydrogen sulfide readily reduces dilute sulfuric acid, which is but a very mild oxidizing agent capable of oxidizing only the most active reducing agents; the products are sulfurous acid, $\mathrm{H}_{2} \mathrm{SO}_{3}$, sulfur, and water:

$$
\mathrm{H}_{2} \mathrm{~S}+\mathrm{H}_{2} \mathrm{SO}_{4} \rightarrow \mathrm{H}_{2} \mathrm{SO}_{3}+\mathrm{S}+\mathrm{H}_{2} \mathrm{O} \text {. }
$$

Hydrogen sulfide can be made by the action of sulfuric acid on ferrous sulfide, thus:

$$
\mathrm{FeS}+\mathrm{H}_{2} \mathrm{SO}_{4} \rightarrow \mathrm{FeSO}_{4}+\mathrm{H}_{2} \mathrm{~S}
$$

but this is not advisable in practice because of the interaction of the sulfuric acid with the hydrogen sulfide. Hydrochloric acid is the best acid to use in making hydrogen sulfide.

Hydrogen sulfide is oxidized by all except the very mildest oxidizing agents. As a final example of its behavior, its action on ferric salts may be given. These are reduced to ferrous salts, thus:

$$
{ }_{2} \mathrm{FeCl}_{3}+\mathrm{H}_{2} \mathrm{~S} \rightarrow 2 \mathrm{FeCl}_{2}+\mathrm{S}+{ }_{2} \mathrm{HCl} .
$$


340. Sulfurous Acid, $\mathrm{H}_{2} \mathrm{SO}_{3}$.-When sulfur burns, it forms sulfur dioxide, $\mathrm{SO}_{2}$, a colorless gas, with a strong odor:

$$
\mathrm{S}+\mathrm{O}_{2} \rightarrow \mathrm{SO}_{2} \text {. }
$$

Sulfur dioxide is very soluble in water, with which it unites partially to form sulfurous acid:

$$
\mathrm{SO}_{2}+\mathrm{H}_{2} \mathrm{O} \leftrightarrows \mathrm{H}_{2} \mathrm{SO}_{3}
$$

This reaction is reversible; by boiling a solution of sulfurous acid the latter can be completely decomposed and all sulfur dioxide driven off.

Sulfurous acid is a reducing agent which, when oxidized, is converted into sulfuric acid. It reacts slowly with atmospheric oxygen, thus:

$$
2 \mathrm{H}_{2} \mathrm{SO}_{3}+\mathrm{O}_{2} \rightarrow 2 \mathrm{H}_{2} \mathrm{SO}_{4} \text {. }
$$

It is rapidly oxidized by chlorine, which is thereby reduced to hydrochloric acid:

$$
\mathrm{H}_{2} \mathrm{SO}_{3}+\mathrm{Cl}_{2}+\mathrm{H}_{2} \mathrm{O} \rightarrow \mathrm{H}_{2} \mathrm{SO}_{4}+2 \mathrm{HCl} \text {. }
$$

Manganese dioxide and sulfurous acid react as follows:

$$
\mathrm{MnO}_{2}+\mathrm{H}_{2} \mathrm{SO}_{3} \rightarrow \mathrm{MnSO}_{4}+\mathrm{H}_{2} \mathrm{O} \text {. }
$$

Ferric salts are reduced to ferrous salts by sulfurous acid, as illustrated by the following equation:

$$
\mathrm{Fe}_{2}\left(\mathrm{SO}_{4}\right)_{3}+\mathrm{H}_{2} \mathrm{SO}_{3}+\mathrm{H}_{2} \mathrm{O} \rightarrow 2 \mathrm{FeSO}_{4}+{ }_{2} \mathrm{H}_{2} \mathrm{SO}_{4} .
$$

34I. Hydrochloric, Hydrobromic, and Hydriodic Acids as Reducing Agents.-These acids in water solution can all be oxidized, and are therefore to be considered as reducing agents. Hydriodic acid is the most easily oxidized of the three, and is therefore the best or most powerful reducing agent. It is oxidized by atmospheric oxygen (265), which has no action whatever on a water solution of hydrochloric acid. The latter substance acts as a reducing agent only with respect to the most powerful oxidizing agents, such as manganese (234) and lead dioxides (235) and potassium permanganate (236). Hydro- 
bromic acid is a better reducing agent than hydrochloric acid, but not as powerful as hydriodic acid.

Hydrobromic acid, as a reducing agent, reacts with concentrated sulfuric acid, as an oxidizing agent, as follows,

$$
2 \mathrm{HBr}+\mathrm{H}_{2} \mathrm{SO}_{4} \rightarrow \mathrm{Br}_{2}+\mathrm{SO}_{2}+2 \mathrm{H}_{2} \mathrm{O},
$$

forming free bromine, sulfur dioxide, and water.

Hydriodic acid reacts even more vigorously with concentrated sulfuric acid. In this case the products vary according to the proportions taken, but the reduction of the sulfuric acid may go as far as the formation of free sulfur and hydrogen sulfide. The possible reactions are represented in the following equations:

$$
\begin{aligned}
& \mathrm{H}_{2} \mathrm{SO}_{4}+{ }_{2} \mathrm{HI} \rightarrow \mathrm{I}_{2}+\mathrm{SO}_{2}+{ }_{2} \mathrm{H}_{2} \mathrm{O}, \\
& \mathrm{H}_{2} \mathrm{SO}_{4}+6 \mathrm{HI} \rightarrow{ }_{3} \mathrm{I}_{2}+\mathrm{S}+{ }_{4} \mathrm{H}_{2} \mathrm{O}, \\
& \mathrm{H}_{2} \mathrm{SO}_{4}+8 \mathrm{HI} \rightarrow{ }_{4} \mathrm{I}_{2}+\mathrm{H}_{2} \mathrm{~S}+{ }_{4} \mathrm{H}_{2} \mathrm{O} .
\end{aligned}
$$

It will now be understood why roundabout methods are used to prepare hydrogen bromide and hydrogen iodide instead of the simple reaction with concentrated sulfuric acid and a salt, as is done in the preparation of hydrogen chloride.

342. Manganese and Its Compounds.-Manganese, $\mathrm{Mn}$, is a metallic element which in the free form resembles iron rather closely. Its principal ore is the dioxide, $\mathrm{MnO}_{2}$, called by mineralogists pyrolusite. Manganese forms a series of salts corresponding to the salts of ferrous iron; among such we have manganous chloride, $\mathrm{MnCl}_{2}$, manganous nitrate, $\mathrm{Mn}\left(\mathrm{NO}_{3}\right)_{2}$, and manganous sulfate, $\mathrm{MnSO}_{4}$. These salts are pale pink in color and are easily soluble in water. The dilute solutions, which are almost colorless, give with sodium hydroxide white precipitates of manganous hydroxide:

$$
\mathrm{MnCl}_{2}+2 \mathrm{NaOH} \rightarrow \mathrm{Mn}(\mathrm{OH})_{2}+2 \mathrm{NaCl} .
$$

This hydroxide corresponds to an oxide, $\mathrm{MnO}$ :

$$
\mathrm{Mn}(\mathrm{OH})_{2} \rightarrow \mathrm{MnO}+\mathrm{H}_{2} \mathrm{O} \text {. }
$$

In all these cumpounds except the dioxide, $\mathrm{MnO}_{2}$, manganese is bivalent; in the dioxide it is quadrivalent. 
Manganese forms a variety of compounds of a very different character from the ones just mentioned; of these the most important is potassium permanganate.

343. Potassium Permanganate, $\mathrm{KMnO}_{4}$. - This substance is the potassium salt of permanganic acid, $\mathrm{HMnO}_{4}$, in which manganese acts as an acid-forming element. The salt is made from manganese dioxide and potassium hydroxide by complicated reactions which need not be considered at present. It forms dark-purple crystals which dissolve in water to form a purple solution having nearly the color of the vapor of iodine. It is a very important substance and is one of the most powerful of all oxidizing agents.

We have already learned that potassium permanganate oxidizes hydrochloric acid, thus:

$$
{ }_{2} \mathrm{KMnO}_{4}+\mathrm{I} 6 \mathrm{HCl} \rightarrow 2 \mathrm{KCl}+2 \mathrm{MnCl}_{2}+{ }_{5} \mathrm{Cl}_{2}+8 \mathrm{H}_{2} \mathrm{O} .
$$

It can also oxidize almost any substance which is capable of being oxidized in solution. Two additional examples may be given as illustrations:

${ }_{2} \mathrm{KMnO}_{4}+{ }_{5} \mathrm{H}_{2} \mathrm{SO}_{3} \rightarrow \mathrm{K}_{2} \mathrm{SO}_{4}+2 \mathrm{MnSO}_{4}+{ }_{2} \mathrm{H}_{2} \mathrm{SO}_{4}+{ }_{3} \mathrm{H}_{2} \mathrm{O}$, ${ }_{2} \mathrm{KMnO}_{4}+\mathrm{IOFeSO}_{4}+8 \mathrm{H}_{2} \mathrm{SO}_{4} \rightarrow \mathrm{K}_{2} \mathrm{SO}_{4}+2 \mathrm{MnSO}_{4}+{ }_{5} \mathrm{Fe}_{2}\left(\mathrm{SO}_{4}\right)_{3}+8 \mathrm{H}_{2} \mathrm{O}$.

In the last reaction the sulfuric acid acts neither as a reducing nor an oxidizing agent, but is used to keep the solution acid. 'The sulfate radical is not decomposed in the reaction.

From the foregoing equations it is apparent that when two molecules of permanganate change to manganese sulfate or chloride, they change the valence of ten atoms (chlorine in hydrochloric acid or iron in a ferrous salt) by one unit of valence each, or of five atoms by two units of valence each (sulfur in sulfurous acid). This relationship exists because the valence of manganese, which is seven in permanganate, changes to two in manganese sulfate or chloride.

344. Chromium and Its Compounds.-The element chromium, $\mathrm{Cr}$, is a hard metal, resembling iron in appearance. It forms a series of salts of which chromic chloride, $\mathrm{CrCl}_{3}$, and chromic sulfate, $\mathrm{Cr}_{2}\left(\mathrm{SO}_{4}\right)_{3}$, are typical examples. Solutions of 
chromic salts are either green or violet in color, according to the method of preparation. These solutions give with ammonium hydroxide bluish precipitates of chromic hydroxide:

$$
\mathrm{CrCl}_{3}+3 \mathrm{NH}_{4} \mathrm{OH} \rightarrow \mathrm{Cr}(\mathrm{OH})_{3}+3 \mathrm{NH}_{4} \mathrm{Cl} \text {. }
$$

The hydroxide when strongly heated gives chromic oxide:

$$
{ }_{2} \mathrm{Cr}(\mathrm{OH})_{3} \rightarrow \mathrm{Cr}_{2} \mathrm{O}_{3}+{ }_{3} \mathrm{H}_{2} \mathrm{O} \text {. }
$$

It will be seen that chromic salts are analogous to ferric salts and that in these compounds chromium is trivalent.

345. Chromates and Dichromates. - When chromic oxide is fused with sodium nitrate or sodium peroxide, sodium chromate, $\mathrm{Na}_{2} \mathrm{CrO}_{4}$, is formed. This is a bright-yellow crystalline salt, readily soluble in water. It may be considered as derived from chromic acid, $\mathrm{H}_{2} \mathrm{CrO}_{4}$. Potassium chromate, $\mathrm{K}_{2} \mathrm{CrO}_{4}$, is also a yellow crystalline salt which is readily made by methods similar to those that give the sodium salt.

A solution of potassium chromate, which is bright yellow in color, turns deep orange when mixed with sulfuric acid. The solution contains potassium dichromate, $\mathrm{K}_{2} \mathrm{Cr}_{2} \mathrm{O}_{7}$, which has been formed thus:

$$
2 \mathrm{~K}_{2} \mathrm{CrO}_{4}+\mathrm{H}_{2} \mathrm{SO}_{4} \rightarrow \mathrm{K}_{2} \mathrm{Cr}_{2} \mathrm{O}_{7}+\mathrm{K}_{2} \mathrm{SO}_{4}+\mathrm{H}_{2} \mathrm{O} .
$$

Potassium dichromate forms orange-colored crystals, which dissolve in water to form an orange-colored solution. In the foregoing reaction we might have expected to get potassium hydrogen chromate, $\mathrm{KHCrO}_{4}$; but if this salt is first formed it decomposes at once, as follows:

$$
2 \mathrm{KHCrO}_{4} \rightarrow \mathrm{K}_{2} \mathrm{Cr}_{2} \mathrm{O}_{7}+\mathrm{H}_{2} \mathrm{O} \text {. }
$$

Sodium dichromate, $\mathrm{Na}_{2} \mathrm{Cr}_{2} \mathrm{O}_{7}$, orange-colored crystals, can be made in a similar manner from sodium chromate.

346. Chromates and Dichromates as Oxidizing Agents.Solutions of either chromates or dichromates are oxidizing agents. More commonly a strongly acid solution is used. Thus with hydrogen sulfide an atcid solution of potassium chromate 
(potassium dichromate) reacts to form sulfur and chromium salts:

$$
{ }_{2} \mathrm{~K}_{2} \mathrm{CrO}_{4}+{ }_{3} \mathrm{H}_{2} \mathrm{~S}+{ }_{10 H C l} \rightarrow{ }_{4} \mathrm{KCl}+{ }_{2} \mathrm{CrCl}_{3}+8 \mathrm{H}_{2} \mathrm{O}+{ }_{3} \mathrm{~S} \text {; }
$$

or if the equation is written for the dichromate we have

$$
\mathrm{K}_{2} \mathrm{Cr}_{2} \mathrm{O}_{7}+{ }_{3} \mathrm{H}_{2} \mathrm{~S}+8 \mathrm{HCl} \rightarrow 2 \mathrm{KCl}+{ }_{2} \mathrm{CrCl}_{3}+{ }_{7} \mathrm{H}_{2} \mathrm{O}+{ }_{3} \mathrm{~S} \text {. }
$$

With sulfurous acid as a reducing agent the reaction yields sulfuric acid and chromium sulfate:

$$
{ }_{2} \mathrm{~K}_{2} \mathrm{CrO}_{4}+{ }_{3} \mathrm{H}_{2} \mathrm{SO}_{3}+2 \mathrm{H}_{2} \mathrm{SO}_{4} \rightarrow 2 \mathrm{~K}_{2} \mathrm{SO}_{4}+\mathrm{Cr}_{2}\left(\mathrm{SO}_{4}\right)_{3}+{ }_{5} \mathrm{H}_{2} \mathrm{O} \text {. }
$$

Apparently two molecules of potassium chromate (or one of the dichromate) can cause six units of valence change on other atoms, three of sulfur in $\mathrm{H}_{2} \mathrm{~S}$ if free sulfur is the product, or three of sulfurous acid to form sulfuric acid.

It is plain that permanganates are more powerful oxidizers than chromates or dichromates, since the first can oxidize hydrochloric acid and the second cannot, except in very concentrated acid solution.

347. Hydrogen Peroxide as an Oxidizing Agent.-We have already learned (3I8) that hydrogen peroxide easily decomposes into water and oxygen, and that for this reason it acts as an oxidizing agent. Its action on hydriodic acid was shown to take place thus:

$$
\mathrm{H}_{2} \mathrm{O}_{2}+{ }_{2} \mathrm{HI} \rightarrow \mathrm{I}_{2}+{ }_{2} \mathrm{H}_{2} \mathrm{O} \text {. }
$$

Sulfurous acid is readily oxidized to sulfuric acid:

$$
\mathrm{H}_{2} \mathrm{O}_{2}+\mathrm{H}_{2} \mathrm{SO}_{3} \rightarrow \mathrm{H}_{2} \mathrm{SO}_{4}+\mathrm{H}_{2} \mathrm{O} \text {. }
$$

Ferrous salts are oxidized to ferric salts as the following equation will illustrate:

$$
\mathrm{H}_{2} \mathrm{O}_{2}+{ }_{2} \mathrm{FeSO}_{4}+\mathrm{H}_{2} \mathrm{SO}_{4} \rightarrow \mathrm{Fe}_{2}\left(\mathrm{SO}_{4}\right)_{3}+{ }_{2} \mathrm{H}_{2} \mathrm{O} \text {. }
$$

Lead forms with sulfur lead sulfide, $\mathrm{PbS}$, a black substance, almost insoluble in water. It is obtained as a black precipitate by the action of hydrogen sulfide on a solution of a lead salt:

$$
\mathrm{Pb}\left(\mathrm{NO}_{3}\right)_{2}+\mathrm{H}_{2} \mathrm{~S} \rightarrow \mathrm{PbS}+2 \mathrm{HNO}_{3} \text {. }
$$


Hydrogen peroxide oxidizes lead sulfide to lead sulfate (I67):

$$
\mathrm{PbS}+{ }_{4} \mathrm{H}_{2} \mathrm{O}_{2} \rightarrow \mathrm{PbSO}_{4}+{ }_{4} \mathrm{H}_{2} \mathrm{O} \text {. }
$$

Since lead sulfate is white, the effect of the action is easily scen. The blackening of old oil paintings is due to the gradual conversion of the lead compounds that have served as ingredients of the paint into lead sulfide by the action of sulfur compounds occurring in the air. Blackened paintings are often restored to their original colors by treating them with hydrogen peroxide, which converts the black lead sulfide into white lead sulfate.

It has already been mentioned that animal and vegetable substances are bleached by hydrogen peroxide. The exact nature of the changes that occur in such reactions is not in general known, but it is safe to conclude that they are processes of oxidation which convert colored into colorless substances.

348. The Reducing Action of Hydrogen Peroxide.-The action of hydrogen peroxide on silver oxide yields free silver, and we may say that the silver oxide has been reduced.

$$
\mathrm{H}_{2} \mathrm{O}_{2}+\mathrm{Ag}_{2} \mathrm{O} \rightarrow 2 \mathrm{Ag}+\mathrm{H}_{2} \mathrm{O}+\mathrm{O}_{2} \text {. }
$$

Another important reaction of this class is found in the action of hydrogen peroxicle on potassium permanganate in acid solution, which takes place thus:

$$
{ }_{5} \mathrm{H}_{2} \mathrm{O}_{2}+2 \mathrm{~K}_{4} \mathrm{MnO}_{4}+{ }_{3} \mathrm{H}_{2} \mathrm{SO}_{4} \rightarrow \mathrm{K}_{2} \mathrm{SO}_{4}+2 \mathrm{MnSO}_{4}+8 \mathrm{H}_{2} \mathrm{O}+{ }_{5} \mathrm{O}_{2} \text {. }
$$

The products are the colorless solution of the sulfates of potassium and manganese in addition to free oxygen.

349. Hypochlorous Acid, $\mathrm{HClO}$. - It is probable that chlorine reacts reversibly with water in which it is dissolved to form hydrochloric acid and hypochlorous acid, $\mathrm{HClO}$, thus:

$$
\mathrm{Cl}_{2}+\mathrm{H}_{2} \mathrm{O}=\mathrm{HCl}+\mathrm{HClO} \text {. }
$$

Since this is a reversible reaction, all four substances are contained in equilibrium in a solution of chlorine in water. Hypochlorous acid is very unstable, that is, it casily decomposes, and for this reason it cannot be obtained except in the form of a dilute water solution. It has only very weak acid properties 
and cannot even decompose calcium carbonate, which is acted upon by almost all other acids. As is well known, hydrochloric acid reacts with calcium carbonate as follows:

$$
{ }_{2} \mathrm{HCl}+\mathrm{CaCO}_{3} \rightarrow \mathrm{CaCl}_{2}+\mathrm{CO}_{2}+\mathrm{H}_{2} \mathrm{O} .
$$

As a matter of fact, when calcium carbonate is added to chlorine water it reacts as follows:

$$
\mathrm{CaCO}_{3}+{ }_{2} \mathrm{Cl}_{2}+\mathrm{H}_{2} \mathrm{O} \rightarrow{ }_{2} \mathrm{HClO}+\mathrm{CaCl}_{2}+\mathrm{CO}_{2} \text {. }
$$

From the resulting solution hypochlorous acid mixed with much water vapor can be driven off by cautious heating; the condensed vapor forms a dilute solution of hypochlorous acid. This reaction seems to prove that chlorine and water react to form hydrochloric and hypochlorous acids.

350. Hypochlorites. - If chlorine is passed into a cold, dilute solution of sodium hydroxide, sodium chloride and sodium hypochlorite, $\mathrm{NaClO}$, are formed:

\section{$\mathrm{Cl}_{2}+2 \mathrm{NaOH} \rightarrow \mathrm{NaCl}+\mathrm{NaClO}+\mathrm{H}_{2} \mathrm{O}$.}

This is exactly what we should expect if both acids which result from the action of chlorine on water are neutralized by the sodium hydroxide. Chlorine and potassium hydroxide react similarly:

$$
\mathrm{Cl}_{2}+2 \mathrm{KOH} \rightarrow \mathrm{KCl}+\mathrm{KClO}+\mathrm{H}_{2} \mathrm{O} .
$$

35I. Bleaching Powder.-The action of chlorine gas on solid slaked lime, calcium hydroxide, takes place thus:

$$
\mathrm{Cl}_{2}+\mathrm{Ca}(\mathrm{OH})_{2} \rightarrow \mathrm{CaCl}(\mathrm{OCl})+\mathrm{H}_{2} \mathrm{O} \text {. }
$$

The product of the reaction is a white powder known as chloride of lime or bleaching powder. It is a mixed salt, a chloride and hypochlorite of calcium. It is extensively used in the bleaching of cotton goods and for a variety of other purposes. Before taking up the chemical behavior of hypochlorous acid and hypochlorites it will be of interest to consider the formation of these substances from the standpoint of oxidation and reduction. 
352. The Oxidation Products of Chlorine.-By the action of chlorine gas on dry mercuric oxide, $\mathrm{HgO}$, chlorine monoxide, $\mathrm{Cl}_{2} \mathrm{O}$, a brownish-yellow gas, is obtained. It is obvious that in this reaction the chlorine has been oxidized. Now this oxide of chlorine unites with water to form hypochlorous acid,

$$
\mathrm{Cl}_{2} \mathrm{O}+\mathrm{H}_{2} \mathrm{O} \rightarrow 2 \mathrm{HClO} \text {. }
$$

The relation between chlorine monoxide and hypochlorous acid is similar to that between sulfur dioxide and sulfurous acid:

$$
\mathrm{SO}_{2}+\mathrm{H}_{2} \mathrm{O} \rightarrow \mathrm{H}_{2} \mathrm{SO}_{3} \text {. }
$$

Chlorine and sulfur also show similar behavior in that each forms compounds with hydrogen and metals, namely chlorides and sulfides.

353. The Formation of Chlorates.-Hypochlorites are very unstable salts. A warm, concentrated solution of sodium hypochlorite changes more or less rapidly into sodium chloride and sodium chlorate, $\mathrm{NiClO}_{3}$, according to the equation,

$$
3 \mathrm{NaClO} \rightarrow 2 \mathrm{NaCl}+\mathrm{NaClO}_{3} \text {. }
$$

Potassium hypochlorite changes in a similar fashion, yielding potassium chlorate, $\mathrm{KClO}_{3}$.

Sodium and potassium chlorates are powerful oxidizing agents, since they contain large proportions of easily liberated oxygen. When the dry crystals are heated they decompose finally into chlorides and oxygen:

$$
2 \mathrm{KClO}_{3} \rightarrow 2 \mathrm{KCl}+{ }_{3} \mathrm{O}_{2} \text {. }
$$

This reaction takes place in two stages (306). The first change gives rise to at perchlorite, $\mathrm{KClO}_{4}$, thus:

$$
10 \mathrm{KClO}_{3} \rightarrow 4 \mathrm{KCl}+6 \mathrm{KClO}_{4}+{ }_{3} \mathrm{O}_{2} \text {. }
$$

Until recently potassium chlorate was used extensively, and sodium chlorate was rarely seen. The reason was twofold: in the first place potassium chlorate was made very largely in Germany, where potassium compounds are cheap on account of the immense potash reposits found in that country; and in 
the second place sodium chlorate, being more soluble, is more difficultly purified than the potassium salt. Since the war began there has been a shortage of potash, because no other country besides Germany has much easily accessible potash. As a consequence the manufacture of sodium salts has been stimulated, and since I9I5 there has been an abundant supply of sodium chlorate. This can be used advantageously in place of potassium chlorate for nearly all purposes.

354. Chloric Acid and Chlorine Dioxide.-Potassium and sodium chlorates are salts of chloric acid, $\mathrm{HClO}_{3}$. This is a very unstable acid, which is known only in dilute solution. Upon evaporation of the solution the acid decomposes, giving chlorine dioxide, $\mathrm{ClO}_{2}$, and other products.

If a few drops of concentrated sulphuric acid are poured on a small crystal of sodium chlorate in a dry test tube, a yellow gas forms, which explodes with violence a few seconds later. This dangerous experiment should be performed with great caution. The yellow gas is chlorine dioxide, $\mathrm{ClO}_{2}$, which was formed by the decomposition of the chloric acid set free, thus:

$$
\mathrm{NaClO}_{3}+\mathrm{H}_{2} \mathrm{SO}_{4} \rightarrow \mathrm{NaHSO}_{4}+\mathrm{HClO}_{3} \text {. }
$$

The explosion of chlorine dioxide is due to decomposition into its elements:

$$
{ }_{2} \mathrm{ClO}_{2} \rightarrow \mathrm{Cl}_{2}+2 \mathrm{O}_{2} \text {. }
$$

Chloric acid is a powerful oxidizing agent. For example, it changes lead sulfide to lead sulfate ( 167$)$. This operation is usually carried out by adding a few crystals of sodium chlorate and dilute hydrochloric acid to the black lead sulfide. The dark color is seen to change slowly to the white of the sulfate:

$$
{ }_{3} \mathrm{PbS}+{ }_{4} \mathrm{HClO}_{3} \rightarrow{ }_{3} \mathrm{PbSO}_{4}+{ }_{4} \mathrm{HCl} \text {. }
$$

355. Perchlorates and Perchloric Acid.-Perchlorates are formed by heating chlorates gently $(306,353)$.

Sodium perchlorate, $\mathrm{NaClO}_{4}$, and potassium perchlorate, $\mathrm{KClO}_{4}$, are white crystalline salts. They decompose completely into chlorides and oxygen at dull-red heat. For example,

$$
\mathrm{NaClO}_{4} \rightarrow \mathrm{NaCl}+2 \mathrm{O}_{2} \text {. }
$$


Ammonium perchlorate, $\mathrm{NH}_{4} \mathrm{ClO}_{4}$, is made by neutralizing perchloric acid, $\mathrm{HClO}_{4}$, with ammonia. It is used as an oxidizing agent and as a very powerful explosive.

When powdered sodium or potassium perchlorate is mixed with concentrated sulfuric acid and cautiously heated in a small retort (104, Fig. 24), perchloric acid, $\mathrm{HClO}_{4}$, is distilled from the mixture. This experiment should not be made by the student, as it might result in an explosion in unskilled hands.

$$
\mathrm{NaClO}_{4}+\mathrm{H}_{2} \mathrm{SO}_{4} \rightarrow \mathrm{NaHSO}_{4}+\mathrm{HClO}_{4} \text {. }
$$

Perchloric acid is a colorless liquid. It is a violent oxidizing agent, as shown by the fact that a drop of the acid will set fire to filter paper. The diluted acid is now coming into use in laboratories as an oxidizing agent, and also for the purpose of precipitating potassium perchlorate in the quantitative analysis of potassium. 


\section{CHAPTER XVI}

\section{HEAT AND ENERGY}

356. Heat of Combustion.--Since coal, wood, and fuel gas are burned ordinarily in order to produce heat rather than as a means of obtaining their products of combustion, carbon dioxide and water, it becomes a matter of importance to discover

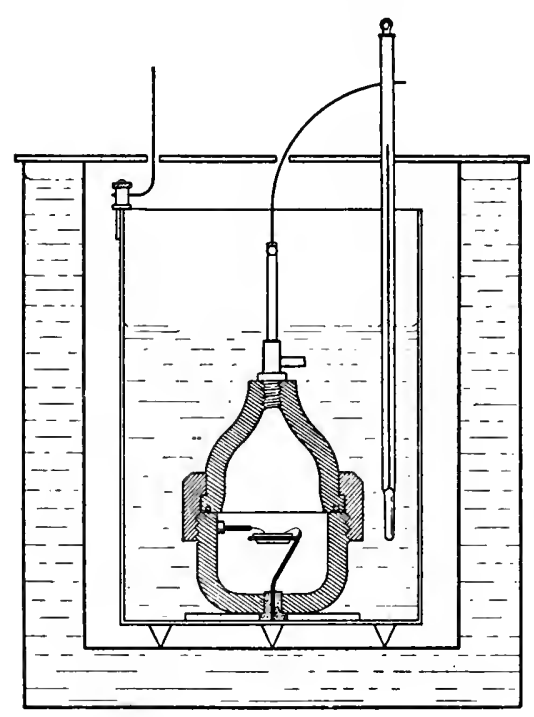

FIG. 44 how much heat is produced in the burning of a known weight of a given substance.

The unit of heat is the calorie (III), which is the amount of heat required to raise the temperature of one gram of water one degree centigrade. The amount of heat produced by the burning of one formula weight of a pure substance is called its heat of combustion. The heat of combustion of a solid is determined by burning a known weight of it within an apparatus of special design, called a bomb calorimeter.

357. The Bomb Calorimeter.--This apparatus, illustrated in Fig. 44, consists of a heavy-walled metallic bomb with a gastight cover, surrounded by a vessel of water. The latter is contained in a larger vessel with walls of heat-insulating material. A weighed amount of substance whose heat of combustion is to be found is placed in the crucible of the bomb, which is filled with oxygen gas. The substance is then ignited by heat from a wire which carries an electric current. The temperature of the water surrounding the bomb is measured accurately before 
and after the burning, and the number of calories of heat produced is calculated from the rise of temperature and the weight of water actually heated, plus the water equivalent of the bomb, etc. The water equivalent is the amount of water which has the same heat capacity as the bomb and other heated parts of the apparatus. Some typical results of measurements of heats of combustion are shown in Table XI. The values are given to the nearest hundred, since this is about the limit of accuracy in such measurements.

TABLE XI

\begin{tabular}{|c|c|c|c|}
\hline Substance & Calories per gram & Formula & Heat of Combustion \\
\hline 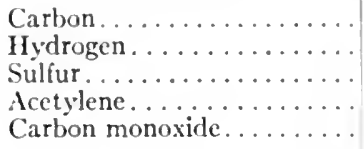 & $\begin{array}{r}8,130 \\
34,400 \\
2,200 \\
11,900 \\
2,430\end{array}$ & $\begin{aligned} \mathrm{C} & =\mathrm{I} 2 \mathrm{~g} . \\
\mathrm{H}_{2} & =2 \mathrm{~g} . \\
\mathrm{S} & =32 \mathrm{~g} . \\
\mathrm{C}_{2} \mathrm{H}_{2} & =26 \mathrm{~g} . \\
\mathrm{CO} & =28 \mathrm{~g} .\end{aligned}$ & $\begin{array}{r}97,600 \\
68,800 \\
70,400 \\
3 I 5,400 \\
68,200\end{array}$ \\
\hline
\end{tabular}

Since one formula weight of a gascous substance has a volume 22.4 liters, the heats of combustion of $\mathrm{H}_{2}, \mathrm{C}_{2} \mathrm{H}_{2}$, and $\mathrm{CO}$ are the amounts of heat produced in the burning of equal volumes of these gases. It will be seen that the heat of combustion of $\mathrm{C}_{2} \mathrm{H}_{2}$ is very large (nearly five times that of hydrogen). This accounts in part for the very high temperature of the oxyacetylene flame (315).

358. The British Thermal Unit, B.T.U.-In engineering practice quantities of heat are measured in British Phermal Units (B.T.U.) instead of in calories. This unit is the amount of heat requircd to raise the tcmperalure of one pound of water onc degree Fahrcnheit. Since one pound equals 453 g., and $\mathrm{I}^{\circ} \mathrm{F}=5 / 9$ of $\mathrm{I}^{\circ} \mathrm{C}$., it follows that $\mathrm{I}$ B.T.U. $=\mathbf{2 5 2}$ calories. The heat produced in burning coal, coke, and fuel gas is called its calorific power. It is usually stated in terms of B.T.U. per pound of fuel.

359. Composition and Calorific Power of Fuel.--Since the value of fuel is directly dependent on its calorific power, the testing of fuel is a matter of great practical importance. In testing coal it is customary to determine the moisture, volatile 
matter, "fixed carbon," and ash in addition to the calorific power. The "fixed carbon" is the non-volatile residue left when all volatile matter is driven off at a bright-red heat in the absence of air, less the ash contained therein. The calorific power is usually expressed in terms of B.T.U. per pound of fuel, or per cubic foot in the case of gases. Table XII gives some results for a variety of solid fuels.

TABLE XII

\begin{tabular}{|c|c|c|c|c|c|}
\hline \multirow[b]{2}{*}{ Kind of Fued } & \multicolumn{3}{|c|}{ Percentage Composition } & \multicolumn{2}{|c|}{ Calorific Power } \\
\hline & $\begin{array}{l}\text { Volatile } \\
\text { Matter }\end{array}$ & $\begin{array}{l}\text { Fixed } \\
\text { Carbon }\end{array}$ & Ash & $\begin{array}{l}\text { Calories } \\
\text { per gram }\end{array}$ & $\begin{array}{l}\text { B. T. U. } \\
\text { per pound }\end{array}$ \\
\hline Lackawanna anthracite coal & 5 & 84 & I I & 7,724 & I 3,900 \\
\hline Pocahontas coal. ......... & I8 & 74 & 7 & 8,760 & I 5,680 \\
\hline Indiana bituminous coal. . . & 35 & 50 & 6 & 8,080 & I 4,540 \\
\hline Coke................. & 0.5 & 90 & 9 & 7,900 & 14,200 \\
\hline Lignite. . . . . . . . . . . & $3^{8}$ & $5 \mathrm{I}$ & 4 & 7,200 & 13,000 \\
\hline Oak wood $\ldots \ldots \ldots$ & $\cdots$ & & 0.4 & 4,600 & 8,300 \\
\hline Pine wood (resinous). & & & 0.4 & 5,000 & 9,100 \\
\hline Crude petroleum.... & & & $\ldots$. & II, 520 & 20,736 \\
\hline
\end{tabular}

Table XIII gives the calorific power of some typical fuel gases.

\section{TABLE XIII}

Calorific Power in B.T.U. per Cubic Foot

Kokomo, Indiana, natural gas .............. I,000

Pittsburgh, Pennsylvania, natural gas ..........., 50

Coal gas..............................650

City of Chicago gas......................600

360. The Evaporation of Water and the Production of Steam.-We can easily calculate the amount of fuel theoretically needed to change water at ordinary temperature into steam. If one gram of water at $20^{\circ}$ is heated to $100^{\circ}$, 80 calories of heat are required, and in addition 540 calories are needed to change this hot water into steam. The total is 620 calories. Since the burning of one gram of coal produces about 8,000 calories, if all this heat were utilized it would be sufficient to evaporate $(8,000 \div 620)$ I $3 \mathrm{~g}$. of water. In practice much heat is lost to the surroundings, as well as in the hot smoke which goes up the 
smokestack. Engineers consider that it is good practice to evaporate $8 \mathrm{~g}$. of water with I $\mathrm{g}$. of coal. Therefore one pound of good coal will change $8 \mathrm{lb}$., or about I gal. $(8.3 \mathrm{lb})$ of water at ordinary temperature into steam at $100^{\circ}$.

36r. Heat of Reaction and Heat of Formation.-We have already frequently observed that numerous reactions other than combustions in oxygen (air) produce much heat. Among such are the reactions of chlorine with hydrogen (244), phosphorus (247), antimony (246), and turpentine (248); and water with sulfuric acid (93), potassium (106), and calcium oxide (150). The heat produced in these and other reactions may be measured in suitably constructed calorimeters and the results expressed most conveniently by stating the amount of heat given out in the reaction of formula weights of the uniting substances; or in the formation of one formula weight of the product. Thus the heat of reaction of $\mathrm{CaO}$ and $\mathrm{H}_{2} \mathrm{O}$ may be written

$$
\mathrm{CaO}+\mathrm{H}_{2} \mathrm{O} \rightarrow \mathrm{Ca}(\mathrm{OH})_{2}+5, \mathrm{r} 00 \mathrm{cal} .
$$

and the heat of formation of water from its elements

$$
\mathrm{H}_{2}+\frac{1}{2} \mathrm{O}_{2} \rightarrow \mathrm{H}_{2} \mathrm{O}+68,500 \text { cal. }
$$

362. Heat of Neutralization. - The union of acids and bases to form salts and water always gives out heat. In fairly dilute solutions the amount of heat given out when one formula weight of water is so formed is almost exactly the same for many acids and bases. For example.

$$
\begin{aligned}
& \mathrm{HCl}, \quad \mathrm{NaOH}=\mathrm{I}_{3}, 700 \mathrm{cal} . \\
& \mathrm{HCl}, \quad \mathrm{KOH}=\mathrm{I}_{3}, 700 " \\
& \mathrm{HNO}_{3}, \mathrm{NaOH}=\mathrm{I}_{3}, 700 " \\
& \mathrm{HNO}_{3}, \mathrm{KOH}=\mathrm{I}_{3}, 700 "
\end{aligned}
$$

This regularity is indeed striking and must mean close similarity in the processes of these reactions. How chemists interpret this phenomenon will be considered in chapter xviii.

363. The Law of Constant Heat Summation.-Let us now consider the following question: If equal quantities of a given substance can be changed into the same product by two different 
ways, will the amounts of heat produced be the same in the two cases? Carbon, for example, gives carbon dioxide when it is burned,

$$
\mathrm{C}+\mathrm{O}_{2} \rightarrow \mathrm{CO}_{2}
$$

but in a deficiency of oxygen the product is carbon monoxide,

$$
{ }_{2} \mathrm{C}+\mathrm{O}_{2} \rightarrow{ }_{2} \mathrm{CO} \text {. }
$$

Carbon monoxide is a colorless gas which burns readily, giving carbon dioxide,

$$
2 \mathrm{CO}+\mathrm{O}_{2} \rightarrow 2 \mathrm{CO}_{2} \text {. }
$$

Therefore it is possible to change given weights of carbon and oxygen into carbon dioxide in two different ways. The heats of combustion are as follows:

$$
\begin{gathered}
\text { FIRST WAY } \\
\mathrm{C}+\frac{1}{2} \mathrm{O}_{2} \rightarrow \mathrm{CO}+29.400 \mathrm{cal} . \\
\mathrm{CO}+\frac{1}{2} \mathrm{O}_{2} \rightarrow \mathrm{CO}_{2}+6 \mathrm{~S}, 200 \mathrm{cal} . \\
\text { Sum } 9 \pi, 600 \mathrm{cal} . \\
\text { SECOND } \mathrm{WAY} \\
\mathrm{C}+\mathrm{O}_{2} \rightarrow \mathrm{CO}_{2}+97.600 \mathrm{cal} .
\end{gathered}
$$

These results show that if $\mathrm{I} 2 \mathrm{~g}$. of carbon $(\mathrm{C}=\mathrm{I2})$ unite with $32 \mathrm{~g}$. of oxygen $\left(\mathrm{O}_{2}=32\right.$ and $\left.\frac{1}{2} \mathrm{O}_{2}=16\right)$ the total heat produced is the same no matter in which way the union occurs.

Another illustration is found in the formation of a solution of ammonium chloride, $\mathrm{NH}_{4} \mathrm{Cl}$, from $\mathrm{NH}_{3}$ and $\mathrm{HCl}$ gases. This reaction can take place in tro ways:

FIRST WAY

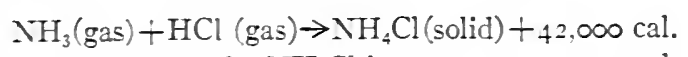

Heat absorbed in dissolving the $\mathrm{NH}_{4} \mathrm{Cl}$ in water $=-3,900 \mathrm{cal}$.

Excess of heat produced over heat absorbed $=38,100 \mathrm{cal}$.

$$
\text { SECOAD WAY }
$$

Heat of solution of $\mathrm{NH}_{3}$ in water

Heat of solution of $\mathrm{HCl}$ in water

$$
\begin{aligned}
& =\quad 8,400 \mathrm{cal} . \\
& =\quad 17,300 \mathrm{cal} .
\end{aligned}
$$

Heat of neutralization of the two solutions $=$ I $2,400 \mathrm{cal}$.

$$
\text { Total heat produced }=\overline{3^{8}, \mathrm{ro0} \mathrm{cal} \text {. }}
$$


Innumerable cases like the two here given in illustration have led to the Law of Constant Heat Summation (Law of Hess). The heat produced or absorbed in the change of gicen substances into the same final products (in the same physical state) is the same, by whateier w'y the changes occur.

That the heat of a given reaction is dependent on the physical state of the reacting substances and products is illustrated by the following example:

$$
\begin{array}{r}
\mathrm{CaO}+\mathrm{H}_{2} \mathrm{O} \text { (liquid) } \rightarrow \mathrm{Ca}(\mathrm{OH})_{2} \text { (solid) }+\mathrm{I}_{5}, 100 \mathrm{cal} . \\
\mathrm{CaO}+\mathrm{H}_{2} \mathrm{O} \text { (ice) } \rightarrow \mathrm{Ca}(\mathrm{OH})_{2} \text { (solid) }+1_{3,700 \mathrm{cal} .} \\
\text { Difference }=1,400 \mathrm{cal} .
\end{array}
$$

The difference, I,400 cal., is due to the fact that it requires this amount of heat to change one formula weight of ice into water ( $18 \times 79=1,422)$ (I I 8$)$.

364. Heat Produced in Slow Oxidation. Spontaneous Combustion.-Numerous experiments have proved that the amount of heat formed in a given reaction is just the same whether the change takes place slowly or rapidly. The decay of wood leads ultimately to the production of carbon dioxide and water, the same products as those formed when wood is burned. During the decay of wood, heat is produced so slowly that its formation is usually not perceptible by ordinary observation. Coal also, when exposed to the air, slowly oxidizes. In so doing it often loses an appreciable part of its heating value before it is burned. 'The depreciation on this account in the value of stored coal is a matter of considerable importance.

If coal (especially bituminous coal) in small lumps and containing much dust is heaped in immense piles, such as are seen in coal yards, the heat produced by the slow oxidation does not escape readily from the bottom layers of the pile. The result is a gradual rise of temperature. At the higher temperature oxidation and therefore heat production go on still faster, since usually enough air can diffuse in to keep up the supply of oxygen. Finally the temperature may rise so high that the pile of coal actually takes fire at the surface, where there is of course an unlimited supply of oxygen. Fire originating in this way is 
said to be due to spontaneous combustion. The loss of coal through such fires was a very serious feature of the "coal famine" of I9I7-I8. Some smoke is seen issuing from the majority of large piles of low-grade coal in the Chicago district, thus indicating more or less fire beneath. It is almost impossible to extinguish fire in a very large coal pile. The best way to prevent serious rise of temperature in coal piles is to provide numerous air shafts in the pile, by means of which warm air can escape. This does not entirely prevent oxidation but keeps the temperature down to a point where the oxidation is not dangerously fast.

It is a popularly known fact that "greasy" rags will often catch fire spontaneously. As a matter of fact such fires originate usually in rags soaked in oils used in paint or varnish, especially linseed oil or turpentine. The "drying" of paint and varnish is not a process of evaporation as much as one of oxidation of the oil used. These paint and varnish oils readily unite with oxygen to form solid products. In this process heat is produced. In a pile of rags, etc., covered with such oils sufficient rise of temperature may occur to cause spontaneous combustion. For this reason greasy rags, etc., should never be left where they can do damage if they take fire.

365. Dust Explosions. - When the air is filled with the dust of coal, wood, flour, or other combustible substance a flame will often start a combustion which will spread with explosive rapidity. Appalling explosions have occurred from such causes in coal mines, wood-working factories, and flour mills. Even dust which is at rest in such places is blown into the air by the on-coming explosion wave and is thus changed to an explosive dust and air mixture. It is easy to see that a dust explosion is due to the extremely rapid burning of minute particles, each surrounded by an abundance of oxygen. Dust explosions are best prevented by keeping mines, mills, etc., free from accumulations of dust.

366. Modes of Heat Production in Physical and Chemical Changes.-We have now learned that heat is produced (or absorbed) in a variety of physical and in all chemical changes. 
The following seven modes of heat production (or absorption) have been studied:

I. Latent heat of fusion (melting) (118).

2. Latent heat of evaporation (II5).

3. Heat of solution (127).

4. Heat of combustion (356).

5. Heat of formation $(36 r)$.

6 . Heat of reaction $(36 \mathrm{I})$.

7. Heat of neutralization (362).

The first three modes have to do with physical changes of the sort known as changes of state; the last four are due to chemical changes. All changes of state and many chemical changes are reversible processes. In every reversible process, if heat is given out when the change proceeds in one direction, heat is absorbed in equal amount when the change proceeds to an equal extent in the opposite direction. A change which results in the production of heat is called an exothermic change; one in which heat is absorbed is an endothermic change.

367. Heat Production and Equilibrium.-In chapter xiii (288) the effect of temperature on equilibrium was discussed briefly. With respect to the change of solubility it was stated that raising the temperature causes that change of solubility to occur which involies an absorption of heat. We also saw (288) that for chemical equilibrium raising the temperature causes the equilibrium to shift in the direction that involves an absorption of heat. These laws are entirely general and apply to all changes of state and all chemical changes.

In the shift of equilibrium which occurs with change of temperature the fraction of the reacting substances transformed to new products is determined, in a given case, by the change of temperature (measured in degrees). The amount of heat (in calories) absorbed (if the temperature is raised) or given out (if the temperature is lowered) is determined by the amount of material transformed. An example will make the matter clearer.

Hydrogen and iodine vapor react partially in the neighborhood of $400^{\circ}$ to give hydrogen iodide $(264,281,288)$ :

$$
\mathrm{H}_{2}+\mathrm{I}_{2}=2 \mathrm{HI}+\mathrm{I}, 000 \mathrm{cal} \text {. }
$$


This equation means that the formation of two formula weights of $\mathrm{HI}$ from $\mathrm{H}_{2}$ and $\mathrm{I}_{2}$ (vapor) at about $400^{\circ}$ takes place with the liberation of $1,000 \mathrm{cal}$. of heat or $500 \mathrm{cal}$. for each formula weight of $\mathrm{HI}$ produced. The following table shows the proportions of molecules in the equilibrium mixture at $370^{\circ}$ and $440^{\circ}$ :

$\begin{array}{ccccc} & \mathrm{H}_{2} & \mathrm{I}_{2} & \mathrm{HI} & \text { Total } \\ 370^{\circ} & \text { IOO } & \text { IOO } & 800 & \text { I, } 000 \\ 440^{\circ} & \text { I IO } & \text { IIO } & 780 & \text { I, } 000\end{array}$

We see that if the temperature is raised from $370^{\circ}$ to $440^{\circ}, 20$ molecules of $\mathrm{HI}$ out of a total of I,000 molecules (2 per cent of the whole) change into $\mathrm{H}_{2}$ and $\mathrm{I}_{2}$. If the total amount of material in the mixture is that resulting from one formula weight each of $\mathrm{H}_{2}$ and $\mathrm{I}_{2}$ (equivalent to two formula weights of $\mathrm{HI}$ ), and if 2 per cent of the whole number of molecules change into $\mathrm{H}_{2}$ and $\mathrm{I}_{2}$, the heat absorbed is $0.02 \times \mathrm{r}, 000 \mathrm{cal} .=20 \mathrm{cal}$.

368. Work and Energy.-The terms work and energy have very definite meanings in science. The subject of physics is largely concerned with these very important matters; and since it is assumed that the student has already studied physics, an elementary discussion of these very important topics is unnecessary. We may, however, briefly summarize some of the more prominent points. The typical example of work in the physical sense is the lifting of a weight. The scientific unit of work is the gram centimeter, which is the work required to lift one gram one centimeter. The amount of work done in lifting a weight is the product of the force required (which in this case is equal to the weight in grams) and the vertical distance measured in centimeters. Thus the lifting of $600 \mathrm{~g}$. to a height of $30 \mathrm{~cm}$. requires the doing of $600 \times 30=18,000 \mathrm{~g} . \mathrm{cm}$. of work. The weight of $600 \mathrm{~g}$., having been lifted $30 \mathrm{~cm}$., could do work to the extent of $18,000 \mathrm{~g} . \mathrm{cm}$. in descending $30 \mathrm{~cm}$. It is said to have the power to do this amount of work. Now power to do work is called energy, and therefore it has $18,000 \mathrm{~g} . \mathrm{cm}$. of energy. Two kinds of energy are recognized: potential energy, as possessed by a weight which may do work on descending, and kinetic energy, or the energy of a body in motion. It requires 
work to set a body in motion, and conversely a body in motion is able to do work.

369. The Mechanical Equivalent of Heat.-Heat is also a form of energy, because heat is able to do work. A steam engine is merely a machine which converts the heat of burning coal into kinetic energy. The change of kinetic energy into heat may be observed on every hand: anything that restrains or stops the motion of a moving body converts part or all of its kinetic energy into heat. We measure energy in gram centimeters and heat in calories, and if heat is a form of energy then the calorie, like the gram centimeter, must be an energy unit. It will at once

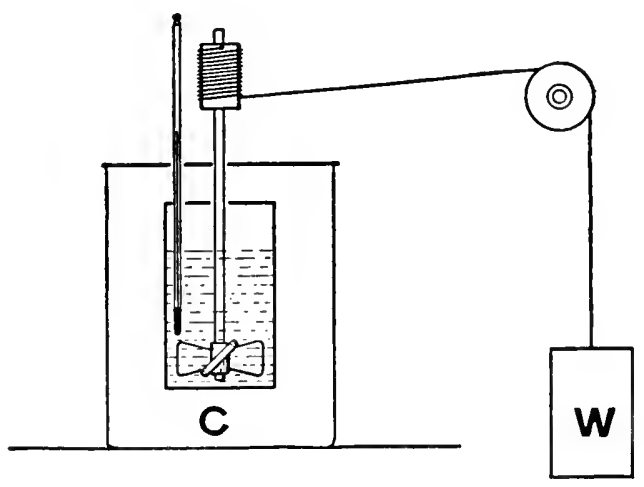

FIG. 45

be asked: Do these units represent equal amounts of energy? In other words, will one gram centimeter of work produce one calorie of heat? If not, how many gram centimeters are required to produce one calorie? This question was first answered by Joule in 1840 .

370. Joule's Experiment.-In Joule's experiment, with apparatus shown in Fig. 45, a weight, $W$, attached to a cord wound on a cylinder, in slowly descending turns a stirrer which is surrounded by water in a calorimeter, $C$. The water, which restrains the motion of the stirrer, becomes warmer, owing to the change of work into heat. The amount of work in gram centimeters done in heating the water is the product of the mass in grams of the weight and the distance of its descent in 
centimeters. The amount in calories of heat produced is the product of the rise in temperature in degrees C. and the mass, in grams, of water plus the water equivalent of the heated parts of the calorimeter.

By means of this apparatus Joule found pretty closely the number of gram centimeters of work equivalent to one calorie of heat. More refined work since then has shown that one calorie is equal to $42,700 \mathrm{~g} . \mathrm{cm}$. This ratio is called the mechanical equivalent of heat. This means, for example, that one gram falling $42,700 \mathrm{~cm}$. (a little over a quarter of a mile) produces one calorie.

37r. The Conservation of Energy.-At the time Joule began his experiments in 1840 it was not at all clear that the amount of heat produced by a given amount of work (kinetic or potential energy) was definite. It seemed possible, if not probable, that different modes of changing work into heat would give different values for the mechanical equivalent. So Joule used not only the method and apparatus already described but also two others. His three methods and the mechanical equivalent of one calorie were as follows: (I) stirring water in a brass vessel with a brass paddle, 42,400 g.cm.; (2) stirring mercury in an iron vessel with an iron paddle, 42,500 g.cm.; (3) rubbing two iron rings together under mercury, $42,500 \mathrm{~g} . \mathrm{cm}$.

The very close agreement of the results of the three expcriments led Joule to conclude that the amount of heat produced by a given amount of work is always the same, by whatever way the work is changed into heat. This result has been amply confirmed by all later experiments and experience. When work of any kind (mechanical energy, either kinetic or potential) is changed into heat there is no real loss or destruction of energy, since the heat produced is also energy in another form and exactly equal in amount to the work done in producing it. This conclusion is concisely stated in the Law of the Conservation of Energy: Energy is indestructible.

Just as the law of the conservation (indestructibility) of matter (2I) is the foundation stone of the science of chemistry, so, similarly, this law of the conservation (indestructibility) 
of energy is the solid rock upon which the whole structure of the science of physics rests.

372. Other Forms of Energy.-We have defined the term energy as the power of doing work; and since heat is also a form of energy, we might extend the definition so as to read: Energy is the power to do work or produce heat. According to this definition of energy it is obvious that light and even sound and especially electric currents are also forms of energy, since each of these by appropriate means can produce work or heat.

373. Chemical Energy.-For the chemist an important question now arises: What shall be said of the source of energy that produces the great heat of a burning substance? This question is somewhat like the one, What is the source of energy of a "wound-up" watch spring? To wind up the spring a certain amount of work must be done. Is it not reasonable to say that the energy used in winding up the spring has been "stored up" in the coiled spring? If so, we may say that this energy is changed into potential energy, just as we say that the energy required to lift a weight is changed into potential energy and can be regained as useful work then the weight is allowed to descend. Reasoning somewhat similarly, we may conclude that the energy given out as heat in the burning of hydrogen, for which we have

$$
\mathrm{H}_{2}+\frac{1}{2} \mathrm{O}_{2} \rightarrow \mathrm{H}_{2} \mathrm{O}+68,800 \text { cal., }
$$

comes from some form of potential energy which has been stored up in the two gases. This conclusion is rendered highly probable by reason of the fact that by means of an electric current (electrical energy) we can decompose water into hydrogen and oxygen. Since the electrical energy disappears and very little heat is formed, we may very reasonably conclude that it has been changed into some sort of potential energy stored up in the two gases formed from the water. The form of potential energy stored up in chemical substances and liberated when they react is called chemical energy.

374. The Sun as a Source of Energy.--It will be interesting to trace some familiar form of energy through various transformations back to its source. 'Take, as an example, the energy 
given out as light and heat by an electric lamp. The energy comes to the lamp as an electric current having electrical energy. This electrical energy was produced in a dynamo or generator, the armature (the moving part) of which was turned by a steam engine. The kinetic energy of the engine was derived from hot, compressed steam produced from water by the burning of coal which has resulted from the slow transformation of vegetable matter.

Plants derive nearly all of their substance from water and the carbon dioxide of the air under the influence of the light and heat of the sun. A great deal of energy is taken up by plants as light and heat and is stored as chemical energy in the substances composing them, as well as in the oxygen which is set free by the growing plant. Recapitulating, we see that the light and heat from the sun are changed by growing plants into chemical energy; this energy is largely conserved when plants are changed into coal. When the coal burns, its chemical energy, supplemented by that of the oxygen of the air, is changed into heat, which is in turn changed into kinetic energy in the steam engine. The kinetic energy of the engine is then changed by a dynamo into electrical energy. and the latter produces in the lamp heat and light. 


\section{CHAPTER XVII}

\section{THE IONIC HYPOTHESIS}

375. The Ionic Hypothesis.-This chapter will treat of the properties and behavior of acids, bases, and salts and aims to show how a supposition called the ionic hypothesis furnishes a satisfactory explanation of many facts.

376. The Two Parts of a Salt.-It must have been noticed that a salt is made up of two parts, the metallic or basic part and the non-metallic or acidic part. The latter may be an element like chlorine in sodium chloride; or it may be a radical (147) like $\mathrm{SO}_{4}$, which is contained in every sulfate. The name of a salt always indicates the parts of which it may be considered as being made up. Thus potassium nitrate, $\mathrm{KNO}_{3}$, is composed of potassium and nitrate radical, $\mathrm{NO}_{3}$; and calcium carbonate, $\mathrm{CaCO}_{3}$, of calcium and carbonate radical, $\mathrm{CO}_{3}$.

377. The Two Parts of an Acid.-Every acid may also be considered as made up of two parts, one of which is hydrogen and the other the characteristic acid radical of that acid. For example, sulfuric acid, $\mathrm{H}_{2} \mathrm{SO}_{4}$, may be considered to consist of hydrogen and sulfate radical, $\mathrm{SO}_{4}$; and phosphoric acid, $\mathrm{H}_{3} \mathrm{PO}_{4}$, to consist of hydrogen and phosphate radical, $\mathrm{PO}_{4}$. For this reason $\mathrm{SO}_{4}$ and $\mathrm{PO}_{4}$ may be called acidic radicals. Dilute solutions of pronounced acids have a sour taste. Since hydrogen is the only constituent which all acids have in common, we may reasonably conclude that the sour taste is due to the II radical.

378. The Two Parts of a Base.-A base is usually the hydroxide of a metallic element, and it may therefore be considered as made up of two parts, the metal and the hydroxyl radical, $\mathrm{OH}$. Thus sodium hydroxide, $\mathrm{NaOH}$, consists of sodium and hydroxyl radical, $\mathrm{OH}$. Ammonium hydroxide, $\mathrm{NH}_{4} \mathrm{OH}$, may be considered as made up of ammonium radical, $\mathrm{NH}_{4}$, and hydroxyl. Consequently the ammonium radical may 
be called a basic radical. It is the only basic radical that we have studied, although many others are known.

379. The Process of Neutralization.-The two following equations represent typical cases of neutralization:

$$
\begin{aligned}
\mathrm{NaOH}+\mathrm{HCl} & \rightarrow \mathrm{NaCl}+\mathrm{H}_{2} \mathrm{O} ; \\
\mathrm{NH}_{4} \mathrm{OH}+\mathrm{HNO}_{3} & \rightarrow \mathrm{NH}_{4} \mathrm{NO}_{3}+\mathrm{H}_{2} \mathrm{O} .
\end{aligned}
$$

We notice that in each case the salt which is formed is made up of two parts, one of which comes from the base, the other from the acid. The remaining parts of acid and base, $\mathrm{H}$ and $\mathrm{OH}$, unite to form water. We might call water hydrogen hydroxide and think of it as being made up of two parts hydrogen and hydroxyl radical. The process of neutralization consists, therefore, merely of an exchange of partners, so to speak, on the part of the base and the acid.

As a matter of fact, not only can neutralization be represented in this way, but most reactions in water solution between acids, bases, and salts which do not involve oxidation or reduction may be regarded as an exchange of the partners of the reagents initially used. This will be made clear by the following examples.

380. Reactions of Barium Salts with Sulfates. - If we add dilute sulfuric acid to a dilute solution of barium chloride a white precipitate of barium sulfate is formed,

$$
\mathrm{H}_{2} \mathrm{SO}_{4}+\mathrm{BaCl}_{2} \rightarrow \mathrm{BaSO}_{4}+2 \mathrm{HCl} \text {. }
$$

A precipitate of barium sulfate also results when a solution of any barium salt is added to a solution of any sulfate, as illustrated by the following equations:

$$
\begin{aligned}
\mathrm{K}_{2} \mathrm{SO}_{4}+\mathrm{Ba}\left(\mathrm{NO}_{3}\right)_{2} & \rightarrow \mathrm{BaSO}_{4}+2 \mathrm{KNO}_{3}, \\
\mathrm{CuSO}_{4}+\mathrm{BaBr}_{2} & \rightarrow \mathrm{BaSO}_{4}+\mathrm{CuBr}_{2} .
\end{aligned}
$$

This is so generally true that the formation of a precipitate of barium sulfate upon the addition of a solution of a barium salt to some other solution shows that this second solution contains the sulfate radical, $\mathrm{SO}_{4}$, in the form either of a sulfate or of sulfuric acid. We say therefore that the formation of a precipitate of barium sulfate when a solution of a barium salt is added to another solution is a test for the sulfate radical. It is 
important to note that it is the $\mathrm{SO}_{4}$ radical, and not sulfur or oxygen or a combination of the two in some other proportion, that responds to this test. A solution of hydrogen sulfide, $\mathrm{H}_{2} \mathrm{~S}$ (339), which may be considered as being made up of two parts, hydrogen and sulfur, does not give a precipitate of any sort with a solution of a barium salt. Furthermore, pure dilute sulfurous acid, $\mathrm{H}_{2} \mathrm{SO}_{3}$ (340), which is made up of hydrogen and sulfite radical, $\mathrm{SO}_{3}$, does not give a precipitate when mixed with a barium salt solution.

381. Reactions of Simple Lead Salts.-Lead sulfate, $\mathrm{PbSO}_{4}$, is also a white insoluble salt. If we add a solution of any simple lead salt to a dilute solution of sulfuric acid or any soluble sulfate, we obtain a white precipitate of lcad sulfate,

$$
\mathrm{Na}_{2} \mathrm{SO}_{4}+\mathrm{Pb}\left(\mathrm{NO}_{3}\right)_{2} \rightarrow \mathrm{PbSO}_{4}+2 \mathrm{NaNO}_{3} \text {. }
$$

In this casc, just as in the precipitation of barium sulfate, it is the sulfate radical, $\mathrm{SO}_{4}$, which has united with the lead to form the precipitate.

It is also of equal interest to note that if the nitrate of barium or of lead is used, the nitrate radical, $\mathrm{NO}_{3}$, is left in combination with the basic element or radical which was originally combined with the sulfate radical.

382. The Reaction of Silver Salts with Chlorides. - We have already learned that a solution of silver nitrate reacts with hydrochloric acid or a chloride to give a white precipitate of silver chloride :

$$
\mathrm{AgNO}_{3}+\mathrm{NaCl} \rightarrow \mathrm{AgCl}+\mathrm{NaNO}_{3} .
$$

A solution of any simple silver salt reacts similarly with hydrochloric acid or any chloride, so that we may think of the reaction as characteristic and call it a test for silver salts. This reaction is specifically that of the chloride radical; for if we add silver nitrate solution to a solution of potassium chlorate, $\mathrm{KClO}_{3}$ (353), no apparent change is observed; certainly no silver chloride is formed, otherwise the latter, being insoluble, would separate out as a white precipitate. This shows that chlorine in the chlorate radical, $\mathrm{ClO}_{3}$, behaves entirely differently 
from chlorine in the form of a chloride. We also find that solutions of perchlorates, of which potassium perchlorate, $\mathrm{KClO}_{4}$ (355), is an example, do not give precipitates with solutions of silver salts. It is possible to make both silver chlorate, $\mathrm{AgClO}_{3}$, and silver perchlorate, $\mathrm{AgClO}_{4}$, by methods which we need not consider at present, and it is found that these salts are entirely different from silver chloride, and that both are easily soluble in water.

This brief review of reactions, most of which have already been studied, is sufficient to illustrate the subject in hand, but many other examples of the same principle will be found in the previous chapters.

383. Summary and Conclusions.-The observations which we have made are typical for all acids, bases, and salts. Each may be shown to be made up of two parts. In the examples we have studied these are, on the one hand, hydrogen, a metal, or the ammonium radical, and, on the other, hydroxyl, a halogen, sulfur, or an acid radical. Hydrogen is one of the two parts of every acid, and hydroxyl one of the two parts of every base. In chemical reactions between acids and bases, acids and salts, bases and salts, and between two salts (where oxidation and reduction do not occur) the two substances simply exchange parts. This kind of chemical change is called double decomposition (337) or metathesis. The chemical reactions which acids, bases, and salts give are in reality only the reactions of their parts.

Finally it should be noted that the recombination of these parts always takes place between the basic or metallic part on the one hand and the acidic or the non-metallic part on the other. Double decompositions in water solutions never give compounds such as $\mathrm{KNa}$ or $\mathrm{ClSO}_{4}$. This is a striking observation, and the fact that we have as yet no explanation for it warns us at once that we must go farther in our observations to understand even the most commonplace of these reactions.

384. Double Decomposition and Electrical Conductivity.Along with the ability to undergo double decompositions, acids, bases, and salts in their water solutions have the property of conducting the electric current. If we set up a battery, a 
galvanometer, and a salt solution in the manner shown in Fig. 46, using platinum electrodes and a sufficient number of dry cells or other source of current to give a suitable deflection of the galvanometer, we shall find that if we replace the solution by distilled water practically no current will be indicated by the galvanometer. We also find that if dry salt is substituted for the solution no current will pass. If now we pour distilled water on the salt while the latter is still in contact with the electrodes, a current begins to pass through the solution of salt which is quickly formed.

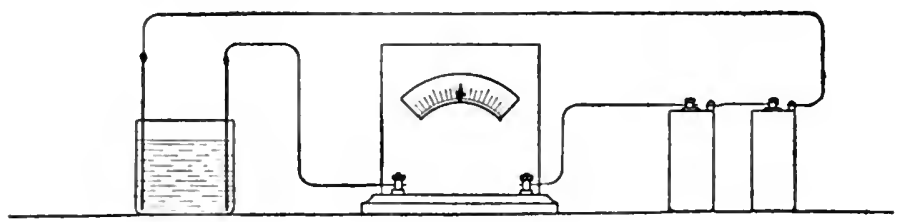

FIG. ${ }_{4} 6$

These results lead to the conclusion that neither pure water nor dry salt conducts the current appreciably compared with the solution formed from salt and water. All other soluble salts behave similarly. It is also easily discovered by experiment that dry (water-free) bases and acids are no better conductors than dry salts, although water solutions of acids and bases are good conductors. Water solutions of other substances than acids, bases, and salts do not conduct electricity any better than does pure water.

The close connection which always exists between electrical conductivity and the ability of a mixture to undergo double decomposition is illustrated by the following experiment. Ferric sulfate and sodium carbonate can be mixed dry without any apparent change; but let the mixture once be wet with water, immediately a violent evolution of gas follows and a red precipitate of ferric hydroxide appears. That the mixture of the dry substances is a non-conductor is shown by placing it in the dry beaker, Fig. 46. No current passes, but when water is added the substances dissolve, and the solution so formed conducts the current. At the same time the chemical reaction 
begins vigorously. Since chemical reactivity and electrical conductivity seem therefore to go hand in hand, we shall next study the behavior of solutions of acids, bases, and salts when an electric current is passed through them.

385. The Electrolysis of Solutions. - We have already learned that the electrolysis of concentrated hydrochloric acid sets free hydrogen and chlorine (43), and that the electrolysis of common salt (238) yields these same gases and in addition forms sodium hydroxide. In the case of hydrochloric acid, electrolysis simply causes the separation of the two constituents,

$$
\mathrm{HCl} \rightarrow \mathrm{H}+\mathrm{Cl} \text {. }
$$

On being set free the atoms of the two elements each form diatomic molecules, thus,

$$
{ }_{2} \mathrm{H} \rightarrow \mathrm{H}_{2} \text {, and } 2 \mathrm{Cl} \rightarrow \mathrm{Cl}_{2} \text {. }
$$

These last reactions are doubtless secondary; and for the sake of brevity, in the examples of electrolysis that follow, reactions of this kind will be indicated by separate equations without further comment.

In the case of the electrolysis of common salt it seems possible, as already explained (238), that the first change is a separation into sodium and chlorine, thus:

$$
\begin{aligned}
\mathrm{NaCl} & \rightarrow \mathrm{Na}+\mathrm{Cl}, \\
2 \mathrm{Cl} & \rightarrow \mathrm{Cl}_{2} .
\end{aligned}
$$

The sodium then reacts with the water present to form sodium hydroxide and hydrogen:

$$
\begin{aligned}
& \mathrm{Na}+\mathrm{H}_{2} \mathrm{O} \rightarrow \mathrm{NaOH}+\mathrm{H}, \\
& 2 \mathrm{H} \rightarrow \mathrm{H}_{2} .
\end{aligned}
$$

Whether this is the only possible explanation of the way the changes take place can best be discussed later; but it can be pointed out here that the foregoing equation would demand that the sodium hydroxide should be formed at the electrode at which the hydrogen is given off; and this is, in fact, the case. When hydrogen is set free in the electrolysis of any substance it always 
appears at the negative electrode or cathode, while chlorine is liberated only at the positive electrode or anode.

386. The Electrolysis of Copper Salts.-If a solution of cupric chloride, $\mathrm{CuCl}_{2}$, is electrolyzed between platinum poles or electrodes, copper is deposited on the negative pole and chlorine gas is set free at the positive pole. Here again, as in the case of hydrochloric acid, we have the simplest possible kind of a change, as represented by the following equation:

$$
\begin{aligned}
\mathrm{CuCl}_{2} & \rightarrow \mathrm{Cu}+{ }_{2} \mathrm{Cl}, \\
2 \mathrm{Cl} & \rightarrow \mathrm{Cl}_{2} .
\end{aligned}
$$

If copper sulfate, $\mathrm{CuSO}_{4}$, is electrolyzed, a plating of metallic copper is again formed on the negative electrode, while from the positive electrode oxygen gas is given off. Examination of the products after electrolysis shows that sulfuric acid is contained in the solution surrounding the positive electrode. In fact, if the electrolysis is continued until all the copper in the original solution is deposited, all the sulfate radical of the original copper sulfate is changed into sulfuric acid, and this acid is contained in the part of the solution surrounding the positive electrode. The formation of sulfuric acid and oxygen may be explained by supposing the copper sulfate to be separated by the electric current into copper and sulfate radical, $\mathrm{SO}_{4}$, and that the latter reacts with water to form sulfuric acid and oxygen:

$$
\begin{array}{rl}
\mathrm{SO}_{4}+\mathrm{H}_{2} \mathrm{O} & \rightarrow \mathrm{H}_{2} \mathrm{SO}_{4}+\mathrm{O}, \\
2 & \mathrm{O} \rightarrow \mathrm{O}_{2} .
\end{array}
$$

387. The Electrolysis of Silver Nitrate.-If an electric current is passed through a solution of silver nitrate, $\mathrm{AgNO}_{3}$, silver is deposited on the negative electrode and oxygen and nitric acid appear at the positive electrode. Probably silver nitrate is first separated into silver and nitrate radical, $\mathrm{NO}_{3}$; the latter then reacts with water to form nitric acid and oxygen:

$$
\begin{aligned}
2 \mathrm{NO}_{3}+\mathrm{H}_{2} \mathrm{O} & \rightarrow{ }_{2} \mathrm{HNO}_{3}+\mathrm{O}, \\
2 \mathrm{O} & \rightarrow \mathrm{O}_{2} .
\end{aligned}
$$

388. Summary.-In Table XIV we have summarized the results just discussed, leaving out of consideration the secondary 
changes that often take place between the substance set free and the water. We see that the parts into which a substance is separated by electrolysis are the same as those which change partners in double decomposition reactions.

\section{TABLE XIV}

Immediate Products of Electrolysis

\begin{tabular}{c|c|c}
\hline $\begin{array}{c}\text { Liberated at } \\
\text { Negative Plate }\end{array}$ & $\begin{array}{c}\text { Original } \\
\text { Substance }\end{array}$ & $\begin{array}{c}\text { Liberated at } \\
\text { Positive Plate }\end{array}$ \\
\cline { 1 - 2 } & $\mathrm{HCl}$ & $\mathrm{Cl}$ \\
$\mathrm{Ha}$ & $\mathrm{NaCl}$ & $\mathrm{Cl}$ \\
$\mathrm{Cu}$ & $\mathrm{CuCl}$ & $2 \mathrm{Cl}$ \\
$\mathrm{Cu}$ & $\mathrm{CuSO}_{4}$ & $\mathrm{SO}_{4}$ \\
$\mathrm{Ag}$ & $\mathrm{AgNO}_{3}$ & $\mathrm{NO}_{3}$ \\
\hline
\end{tabular}

389. The Terms Used in Electrolysis.-The phenomena of electrolysis were very carefully studied about I834 by Faraday, who, as we shall see, discovered many important facts. It was Faraday also who invented the terms electrolysis, electrolyze, electrode, anode, and cathode. He called the solution the electrolyte, but at present we use this term to mean the dissolved substance. That part of the electrolyte which during electrolysis is deposited or set free at the anode or positive electrode he called the anion. The other part, which goes to the cathode, he called the cathion. Frequently he had occasion to speak of anions and cathions together, and then he referred to them as the ions of the electrolyte. For example, the ions of copper sulfate are said to be copper and sulfate radical, because in electrolysis copper passes to and is deposited on one electrode, while the sulfate radical goes to the other. Of course at some time or other the radicals or the partners of the original electrolyte must have broken apart, otherwise they could not have arrived at poles distant from each other.

39o. Hydrogen and Metals as Cathions.-We may next consider how the composition of the ions of a substance can be discovered.

In the case of such a simple substance as $\mathrm{HCl}$ it is obvious that the ions are hydrogen and chlorine, hydrogen being the cathion and chlorine the anion. Since all acids upon electrolysis 
give off hydrogen at the cathode, we may conclude that hydrogen is the cathion of all acids.

When salts are electrolyzed the metal is either deposited in metallic form on the cathode, as in the case of copper and silver salts, or it collects in the solution about the cathode in the form of hydroxide, as when common salt is used. These facts lead to the conclusion that the basic or metallic elements of salts are cathions.

391. Acid Radicals as Anions.-On the other hand, the acid elements or radicals of acids and salts accumulate at the anode and a re either given off as free elements, as in the case of chlorine, bromine, and iodine, or they react with water to form acids and oxygen, as in the case of sulfate and nitrate radicals.

392. Ions and Chemical Reactions.-It would thus appear, from what has just been stated, that the ions of an acid or salt are the same as the two parts of which its chemical reactions show it to be composed. It may be added that there is good reason for thinking that the same statement also applies to bases. The cathion of a base is usually a metal; the anion is the hydroxyl radical.

393. Positive and Negative Ions. - The cathode is the electronegative electrode; to it go the cathions. Since it is well known that a negatively charged body repels another negatively charged body and attracts one which is positively charged, it is not unreasonable to attribute the movement of ions to electrical attraction, and to conclude that cathions are electropositively charged. Since the anode is electropositive, the anions are thought to be charged electronegatively. It is customary to call cathions positive ions, and anions negative ions.

394. The Cause of the Union of Ions.-Attention has been called to the fact that in reactions in solution basic or metallic radicals unite only with acidic or non-metallic radicals (383), and that unions of basic radicals with one another never occur; that is, double decompositions in water solutions never give compounds such as $\mathrm{KNa}$ and $\mathrm{ClSO}_{4}$. We are now in position to explain these important facts. We have learned that the radicals of acids, bases, and salts are identical with their ions, and that 
the ions are probably electrically charged, the basic or metallic ones being positively, the acidic or non-metallic negatively, charged. We can therefore summarize by stating that in reactions only ions of unlike electric charges unite with one another. The reason for this is that ions with unlike electric charges attract each other, and that those with like charges repel each other. The chemical union of ions is an electrical phenomenon and is due to the attractive force of unlike electric charges carried by the ions.

395. Colors of Ions.-The student has doubtless already observed that, although most salts and their solutions are colorless, a considerable number are colored. A little investigation will show that very dilute solutions of equal concentration of all cupric salts of colorless acids are of the same shade and intensity of blue color. This fact leads us to believe that the blue color is due to copperions, which is the only substance which all the solutions have in common. Moreover, we find that the colors of all dilute solutions of colored acids, bases, and salts can be ascribed to the colors of their ions.

If the dilute solution of any acid, base, or salt is colorless, like pure water, we may conclude that its positive and negative ions are both colorless. If a dilute colored solution of an electrolyte has one colorless ion we conclude that the observed color is that of the other ion. Thus we find that all dilute ferrous solutions $(\mathbf{I 7 3}, 33 \mathbf{I})$ are pale green and conclude that ferrous ion is pale green. Manganous salts (342) (for example, $\mathrm{MnCl}_{2}$ and $\mathrm{MnSO}_{4}$ ) give pale pink solutions, therefore positive $M n$ ion is pale pink. On the other hand, dilute solutions of all permanganates (343) are, like $\mathrm{KMnO}_{4}$, deep purple, and we conclude that negative $\mathrm{MnO}_{4}$ ion is purple. Similar reasoning leads us to conclude that negative $\mathrm{CrO}_{4}$ ion is yellow (345) and negative $\mathrm{Cr}_{2} \mathrm{O}_{7}$ ion is orange (345), while positive $\mathrm{Cr}$ ion is violet (344). The color of a dilute solution is usually an indication of the nature of one of its ions.

396. Colors of Molecules. Dissociation of Molecules into Free Ions.-Although dilute solutions of all cupric salts are blue the solid salts and also their concentrated solutions are in 
several cases of a different color. 'Thus cupric chloride, $\mathrm{CuCl}_{2}$, in solid form and in concentrated solution is green, and cupric bromide. $\mathrm{CuBr}_{2}$, which forms almost black crystals, gives a concentrated solution which is dark brown; but if this brown solution is sufficiently diluted the color gradually changes to blue, finally reaching the same shade of color as that of any other equally dilute cupric solution. A simple explanation of these color changes is found in the assumption that the dark brown color is that of the molecules, $\mathrm{CuBr}_{2}$, while the blue color is due to $\mathrm{Cu}$ ions. From the fact that many dilute solutions of bromides are colorless we conclude that $\mathrm{Br}$ ions are colorless. By following up this idea we are led to a very remarkable conclusion, namely, that moleculcs of $\mathrm{CuBr}$ zexist only in the solid state and in concentralcd solutions but not to an appreciable extent in very dilute solutions. This is accounted for if we assume as the concentrated solution is diluted molecules gradually split up or dissociate into ions, thus:

$$
\mathrm{CuBr}_{2} \rightarrow \mathrm{Cu}+2 \mathrm{Br},
$$

so that in a dilute solution the substance exists largely as free Cu and $\mathrm{Br}$ ions. If we evaporate the dilute blue solution we again obtain a brown concentrated solution and finally brown crystals. We must therefore assume that the change is a reversible one, the ions reuniting to form molecules as the solution is evaporated. Further evidence of the existence of free ions is afforded by the experiments next to be considered.

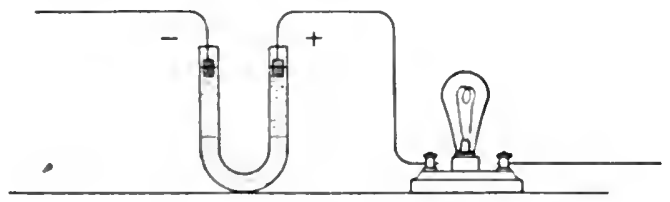

FIG. 47

397. The Migration of Ions. - Let us take advantage of the color of ions to discover their behavior during the process of electrolysis. In the U-tube, Fig. 47, we may put a solution of a colored electrolyte in the lower layer and colorless electrolytes 
in the layers next to the electrodes. As colored electrolytes we may use copper nitrate or potassium permanganate. When the current is turned on, the boundary of each colored electrolyte slowly moves up into one of the colorless layers above it, just as we would expect if the colored materal is the free ion which carries a charge of electricity. Thus positive copper ion migrates toward the negativ'e electrode, and negative permanganate ion migrates toward the positive electrode. We can carry out an experiment with a mixture of these two colored salts in the lower layer. The purple layer now shows on the side of the positive electrode, and the blue layer shows on the side of the negative electrode just as before. Thus we find that each ion migrates just as though the other were not there; and this, in fact, is just what we should expect if a dilute solution contains free ions formed by dissociation of its molecules.

398. The Mechanism of Electrolysis. - We can now make a fairly complete picture of the mechanism of the conduction of the current through a solution and of the accompanying electrolysis. We shall assume that in the dilute solution the dissolved substance is partially dissociated into positive and

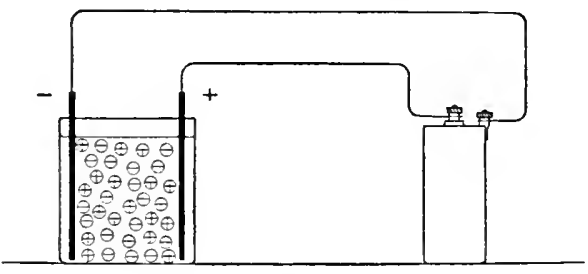

FIG. 48 negative ions. Fig. 48 represents diagrammatically such a solution in which the two electrodes are dipped, connected with a pair of dry cells. The cells charge the electrodes, one positively, the other negatively. The influence of these charges is felt by the ions in the solution. The positive ions are attracted by the negative electrode and repelled by the positive electrode and in consequence migrate toward the former. The negative ions move in the opposite direction for similar reasons.

When ions come into contact with the electrodes of unlike sign they give up their charges to the electrodes. This tends to discharge the latter, but the battery keeps them charged by 
continuously sending a current of electricity through the wires. A more detailed description of the mechanism of electrolysis, in terms of the newer views of the nature of electricity, will be given in chapter $\mathrm{xx}$.

399. Faraday's Laws of Electrolysis. - As the result of careful experimental investigation of the quantities of substances liberated during electrolysis, Faraday arrived at the following conclusions:

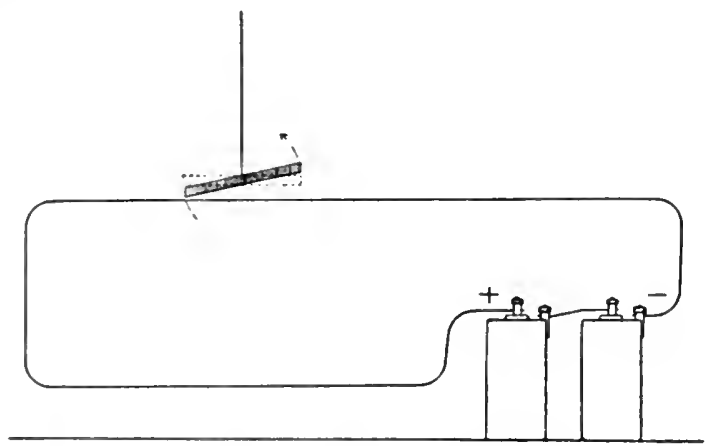

FIG. 49

I. The amount of a given substance, say hydrogen, set free by electrolysis is directly proportional to the quantity of clectricity ahich is passed through the solution.

2. The amount of a substance, hydrogen for example, which is liberated by a fixed quantity of clectricity is the same, whatever the nature of the solution electrolyzed, provided that this substance and nothing else is liberated at the given electrode. These two statements are known as Faraday's Laws of Electrolysis.

400. Two Electrical Units.-To understand these laws fully we must review bricfly some fundamental facts about the electrical current so that we can appreciate what is meant by quantity of electricity. In the first place we know that if a current passes through a wire there is produced around the wire a magnetic field. If we attach a thread to the middle of a magnetized steel needle and suspend the latter above and parallel to a wire, then as soon as we cause a current of electricity to pass through the wire the needle sets itself at an angle to the former, Fig. 49. The greater the angle between the 
needle and the wire the stronger is the magnetic field, and the stronger the current is said to be. It is on this principle that instruments are built to measure current strength. Of course, to measure anything we must first adopt some fundamental unit by comparison with which the measurement can be made. This was done in the case of the electric current on the basis of the strength of the magnetic field about a conductor, and this

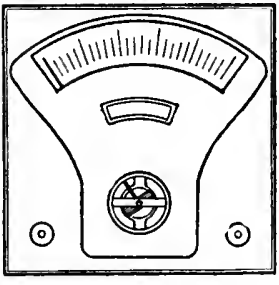

Fig. 50 unit was called the ampere. The ammeter (Fig. 50) allows us to read, from the position occupied by the needle on its scale, just how many amperes of current are passing.

The amperage tells us the strength of the current, but we must also know the time during which the current is allowed to pass if we are to know the amount of electricity delivered at the terminals of the conductor, say at two electrodes. If a current of one ampere is allowed to flow one second it is said to deliver a unit quantity of electricity, and this unit is called the coulomb.

40r. Illustration of Faraday's Laws.-The following facts will serve to illustrate the meaning of Faraday's laws. By the electrolysis of dilute acids hydrogen is set free at the negative electrode. In all cases the passage of 96,500 coulombs of electricity is required for the liberation of one gram of hydrogen. Since a current of one ampere delivers one coulomb per second, 96,500 coulombs will be given by a current of one ampere in 96,500 seconds, or 26.8 hours. A current of two amperes for the same length of time will liberate $2 \mathrm{~g}$. of hydrogen, or one gram molecular weight of hydrogen $\left(\mathrm{H}_{2}\right)$, which as we know has a volume of 22.4 liters at $0^{\circ}$ and $76 \mathrm{~cm}$.

402. Discussion. - It is not surprising that if a one-ampere current will liberate I g. of hydrogen in 26.8 hours, a two-ampere current will liberate $2 \mathrm{~g}$. of hydrogen in the same time, for this is the type of regularity which we have become accustomed to expect in nature. It is surprising, however, that the same amount of hydrogen is liberated by the same amount of electricity from a solution of any dilute acid, and the fact that this 
is so must reflect some regularity in the phenomena of electrolysis, the cause of which we have still to discover.

403. Faraday's Laws of Electrochemical Equivalents. - Let us now turn to cases of the liberation by electrolysis of elements other than hydrogen. Very careful experimentation has shown that by the passage of 96,500 coulombs of electricity through various solutions certain weights of elements are set free. These are given in Table XV. 'This table shows a most striking regu-

TABLE XV

Electochemical Equivalents

\begin{tabular}{|c|c|c|c|c|}
\hline Element & $\underset{\text { Weight }}{\text { Gram }}$ & bialence & $\begin{array}{l}\text { Weight Liberated } \\
\text { in Grams }\end{array}$ & $\begin{array}{c}\text { Gram Atomic } \\
\text { Weight } \div \text { Valence }\end{array}$ \\
\hline 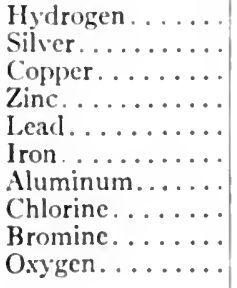 & $\begin{array}{l}1 \\
108 \\
64 \\
66 \\
208 \\
56 \\
27 \\
35 \cdot 5 \\
80 \\
16\end{array}$ & $\begin{array}{l}1 \\
1 \\
2 \\
2 \\
2 \\
2 \\
3 \\
1 \\
1 \\
2\end{array}$ & $\begin{array}{c}1 \\
108 \\
32 \\
33 \\
104 \\
28 \\
9 \\
35 \cdot 5 \\
80 \\
8\end{array}$ & $\begin{array}{c}1 \\
108 \\
32 \\
33 \\
104 \\
28 \\
9 \\
35 \cdot 5 \\
80 \\
8\end{array}$ \\
\hline
\end{tabular}

larity: The weight of an clement liberated in clectrolysis by the passage of 96,500 coulombs of clcctricity is equal to the gram atomic weight of that elcment divided by its valence (col. 5). This weight is called the electrochemical equivalent of a given element or, more bricfly, its equivalent weight. The electrochemical equivalents of the various elements are seen to be proportional to the weights of these elements which unite chemically with one another when union is possible; for example, I $\mathrm{g}$. of hydrogen, ro4 g. of lead, or $9 \mathrm{~g}$. of aluminum unite with $35.5 \mathrm{~g}$. of chlorine, or $8 \mathrm{~g}$. of oxygen. 'The discovery of facts such as those given in the table was made by Faraday, who stated his conclusion as the Law of Electrochemical Equivalents: The amounts of different substances liberaled by the same quantity of electricity are proportional to their cquicalcnt weights.

404. The Electric Charges of Ions. - The facts covered by Faraday's laws allow us to draw some interesting and significant 
conclusions regarding the guantities of electricity composing the charges on single ions. If the 96,500 coulombs of electricity supplied at the negative electrode to release one gram of hydrogen ion are used simply to neutralize the charge on sne gram of that ion, we may conclude at once that the charge carried by the one gram of hydrogen ion is not only opposite in sign but equal in amount to the electricity required. In general then one formula weight of a univalent ion must carry a total charge cqual to 96,500 coulombs. One formula weight of an ion of any valence will carry one, two, three, four, etc., times this charge, according to whether its valence is one, two, three, or four, etc. If we assume that one formula weight of any one ion represents the same number of free ions as a formula weight of any other ion (and this is in strict accord with our accepted definition of the term formula weight), we come at once to the conclusion that all univalent ions carry equal charges. We call this a unit charge; each bivalent ion carries two unit charges, each trivalent ion carries three unit charges, etc. In writing the symbols or formulae of free ions it is customary to add one or more + or - signs to indicate the number of positive or negative unit electric charges carried by the ion, for example, $\mathrm{H}^{+}, \mathrm{Cu}^{++}, \mathrm{Al}^{+++}, \mathrm{Cl}^{-}$, $\mathrm{SO}_{4}^{--}, \mathrm{PO}_{4}^{---}$.

405. Equilibrium between Molecules and Ions.--The facts already studied (396), together with a great volume of other evidence which we shall take up in turn, led the Swedish chemist Svante Arrhenius to the conclusion that in concentrated solutions of acids, bases, and salts a considerable part of the dissolved substance is present as molecules; but that as the solution is diluted, more and more of the molecules dissociate into free ions, until in very dilute solutions (at least in many cases) the dissociation is nearly complete. On the other hand, when a dilute solution is evaporated the ions undoubtedly gradually unite to form molecules, until, when complete dryness is reached, only molecules are present. In any given solution a state of equilibrium exists between molecules and ions, as represented in the case of common salt by the equation

$$
\mathrm{NaCl} \leftrightarrows \mathrm{Na}^{+}+\mathrm{Cl}^{-} \text {. }
$$


At a definite concentration a definite proportion of the salt will be present as ions; this proportion we call the fraction ionized or the degree of ionization. We shall next take up the important problem of determining the fraction ionized for any solution of an electrolyte. Since we believe that the current in a solution is carried by the ions present, the ability of a solution to conduct a current, or, briefly, its conductivity, must be an indication of the extent to which its molecules are dissociated into ions.

406. Effect of Dilution on Conductivity. - We have already learned from the color changes produced by diluting solutions that ionization is promoted by dilution. Let us now consicler the question, What influence, if any, does the volume of water in which a given quantity of an acid, base, or salt is dissolved

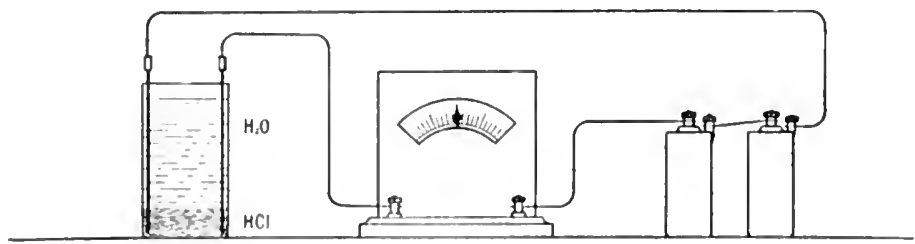

FIG. 5 I

have on its electrical conductivity? We may study this question experimentally by means of the apparatus shown in Fig. $5 \mathrm{r}$. The rectangular glass vessel of about I liter capacity is provided with two large copper electrodes, as shown in the figure. The vessel is first filled about three-fourths full of distilled water, and the electrical connections are made. No appreciable current passes. Next about 200 c.c. of concentrated hydrochloric acid are introduced below the water, without mixing, in such a way as to form a separate layer. This may be done by the use of a dropping funnel, the stem of which reaches the bottom of the vessel.

The vessel now contains two distinct layers-a lower layer of concentrated hydrochloric acid and an upper layer of water. The galvanometer indicates that a considerable current is passing, and we conclude that this is all passing through the acid in the lower layer and not through the upper water layer. 
If next we mix the acid and water thoroughly and so dissolve the acid in a much larger volume of water, we note that a large increase in the current takes place. This leads us to conclude that the conductivity of the hydrochloric acid present is greater when it is dissolved in the larger volume of water. We may now ask, however, whether there is a limit to the increase in conductivity when a given amount of acid is dissolved in larger and larger volumes of water, the conductivity being measured under such conditions that the solution is all contained between parallel plates at a fixed distance apart.

If, in the experiment described, the vessel were much deeper, but otherwise the same, and the electrodes extended all the way up the sides as before, it would be found that a given amount of hydrochloric acid diluted with double the amount of water used in the first experiment would show appreciably greater conductivity than in the first case. Or if the acid were diluted with three, or four, or still more times as much water, greater and greater conductivity would have been observed; but with increasing dilution the increase in conductivity would become smaller each time more water was added, so that finally a maximum conductivity would be reached. Beyond this limit further dilution would cause no increase in conductivity.

These same experiments could be repeated with many other electrolytes with similar results.

407. Definition of Molecular Conductivity.-If one formula weight (called also one gram molecular weight) of an acid, base, or salt is contained in a solution which is wholly included between two parallel electrodes I cm. apart, we call the electrical conductivity of this solution its molecular conductivity. To find the molecular conductivity experimentally we measure its electrical resistance in ohms. The reciprocal of the resistance so found is by definition the molecular conductivity. The conclusions of the paragraph on the effect of dilution on conductivity may now be summarized as follows: The molecular conductivity of every electrolyte increases as its solution is diluted and finally attains a maximum which has a definite numerical valuo for each substance (the temperature being fixed). Table XVI shows the 
change of molecular conductivity of hydrochloric acid as the volume in which one formula weight of acid is contained is increased.

\section{TABLE XVI}

The Molecular Conductivity of Hydrocilloric ACid at is $S^{\circ}$ (Noyes and CoOper)

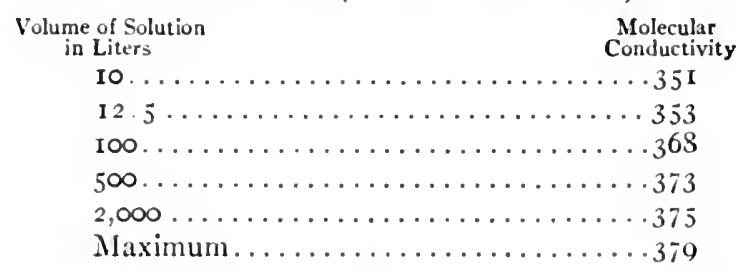

408. Determination of the Degree of Ionization-When a solution of a substance is so dilute that it has its maximum molecular conductivity, it is assumed that all of its molecules have dissocialed into ions. In a more concentrated solution, for which the molecular conductivity is less than the maximum, the fraction which its observed molecular conductivity forms of its maximum molecular conductivity is consequently equal to the fraction which the number of ions present in that particular solution form of the total number of ions in the completely dissociated (completely ionized) solution of the same quantity of that substance. This fraction is therefore the fraction ionized or the degree of ionization. Thus for decinormal hydrochloric acid the degree of ionization is $35 \mathrm{I} \div 379=93$ per cent.

This method of determining the degree of ionization was proposed by Arrhenius in the year 1887 . His reasoning ran thus: The passage of a current through a solution is accomplished by the migration of positive ions in one direction and negative ions in the other. These transport electricity through the solution between the electrodes. Since the molecular conductivity of a substance is the measure of its rate of transporting electricity, it is plain that the molecular conductivity will depend on the number of ions present, the charge on each, and the velocity of migration. Now under the conditions used in measuring resistance, and therefore also of measuring molecular conductivity, the velocity of migration of its ions will be the same 
for all concentrations of solutions of a given substance (except for very concentrated solutions). The charges of the individual ions of a given substance are also the same, whether the solution is dilute or concentrated. Therefore the molecular conductivities of solutions of a given substance are directly proportional to the numbers of ions present. Consequently the ratio of the molecular conductivity for a given concentration to the maximum molecular conductivity for this substance is the fraction ionized, since it is assumed that a very dilute solution having maximum molecular conductivity is completely ionized.

409. Results of Determination. - The degree of ionization of some common electrolytes is shown in Table XVII.

4I0. Discussion of Table XVII.-A study of the table leads to the very important generalization that in solutions of most salts a large percentage of the substance is in the form of ions; in consequence, we say that such solutions are highly ionized. It also appears that dilute solutions of hydrochloric and nitric acid are even more highly ionized than salt solutions of like concentration. On the other hand, decinormal acetic acid is only I. 3 per cent ionized, while the degree of ionization of decinormal carbonic acid is very much less, namely 0.17 per cent. In general, the extent to which acids are ionized, in solutions of equal concentration, varies enormously. Bases also differ greatly in their degrees of ionization. For example, decinormal sodium hydroxide is ionized 90 per cent, while the same concentration of ammonium hydroxide is only $\mathrm{r} .3$ per cent ionized. We have already learned that every substance is more highly ionized in dilute than in more concentrated solutions. The percentage of ionization of a substance as shown in the table applies only to the indicated concentration and temperature.

4II. Résumé of the Ionic Hypothesis.-We have already developed enough of the ionic hypothesis to go far into the understanding of double decomposition reactions. Let us therefore review in brief the ideas already brought out, and then, after a short critical survey of the fundamental assumptions, proceed in chapters xviii and xix to the application of the hypothesis to practical examples. 
According to the ionic hypothesis, as soon as an acid base or salt is dissolved in water it is immediately dissociated to some extent into ions which prove to be the parts of those substances which we have found active in double decomposition. The basic

\section{TABLE XVII}

V'alues of the Degree of Ionization of Some Common Electrolytes in Water Solution at i $8^{\circ}$

(Degree of ionization at the normality indicated at the head of the column)

\begin{tabular}{|c|c|c|c|c|}
\hline & o or & 0.05 & 0.1 & 1.0 \\
\hline \multicolumn{5}{|l|}{ SALTS: } \\
\hline $\mathrm{NaCl} \ldots \ldots \ldots \ldots$ & 0.04 & 0.88 & 0.85 & 0.74 \\
\hline $\mathrm{KCl} \ldots \ldots \ldots \ldots \ldots$ & .9 .4 & .88 & .86 & .74 \\
\hline $\mathrm{KBr} \ldots \ldots \ldots \ldots \ldots \ldots$ & .04 & .88 & .86 & $\ldots \ldots \ldots \ldots$ \\
\hline KI $\ldots \ldots \ldots \ldots \ldots \ldots$ & .94 & .89 & .87 & .73 \\
\hline $\mathrm{NaNO}_{3} \ldots \ldots \ldots \ldots \ldots$ & .93 & .87 & .83 & .66 \\
\hline $\mathrm{KNO}_{3} \ldots \ldots \ldots \ldots \ldots$ & .94 & .87 & .82 & .61 \\
\hline $\mathrm{AgNO} \mathrm{NO}_{3} \ldots \ldots \ldots \ldots$ & .03 & .86 & .81 & .62 \\
\hline $\mathrm{KClO}_{3} \ldots \ldots \ldots \ldots$ & .03 & .87 & .83 & $\ldots \ldots \ldots \ldots$ \\
\hline $\mathrm{BaCl}_{2} \ldots \ldots \ldots \ldots \ldots$ & .88 & .80 & .76 & .64 \\
\hline $\mathrm{CaCl}_{2} \ldots \ldots \ldots \ldots \ldots$ & .88 & .80 & .76 & .66 \\
\hline $\mathrm{MgCl}_{2} \ldots \ldots \ldots \ldots \ldots$ & .88 & .80 & .77 & .67 \\
\hline $\mathrm{PbCl}_{2} \ldots \ldots \ldots \ldots \ldots$ & .81 & .63 & $\ldots \ldots \ldots \ldots$ & $\ldots \ldots \ldots \ldots$ \\
\hline $\mathrm{Sr}\left(\mathrm{NO}_{3}\right)_{2} \ldots \ldots \ldots \ldots \ldots$ & .87 & .77 & .72 & .51 \\
\hline $\mathrm{Ba}\left(\mathrm{NO}_{3}\right)_{2} \ldots \ldots \ldots \ldots$ & .86 & .74 & .68 & $\ldots \ldots \ldots$ \\
\hline $\mathrm{K}_{2} \mathrm{SO}_{4} \ldots \ldots \ldots \ldots \ldots$ & .87 & .77 & .72 & .59 \\
\hline $\mathrm{Ag}_{2} \mathrm{SO}_{4} \ldots \ldots \ldots \ldots \ldots$ & .84 & $\ldots \ldots$ & $\ldots \ldots \ldots$ & $\cdots \ldots \ldots$ \\
\hline $\mathrm{MgSO}_{4} \ldots \ldots \ldots \ldots \ldots$ & .67 & $.5 \mathrm{I}$ & .45 & .35 \\
\hline $\mathrm{ZnSO}_{4} \ldots \ldots \ldots \ldots \ldots$ & .6 .3 & .46 & $.4 \mathrm{I}$ & $.3 I$ \\
\hline $\mathrm{CuSO}_{4} \ldots \ldots \ldots \ldots \ldots$ & .63 & .46 & .40 & 0.31 \\
\hline \multicolumn{5}{|l|}{ BASES: } \\
\hline $\mathrm{NaOH} \ldots \ldots \ldots \ldots \ldots \ldots$ & .96 & .93 & .90 & $\ldots \ldots \ldots$ \\
\hline $\mathrm{Ba}(\mathrm{OH})_{2} \ldots \ldots \ldots \ldots \ldots$ & .93 & .86 & $.8 \mathrm{r}$ & $\ldots \ldots \ldots$ \\
\hline $\mathrm{NH}_{4} \mathrm{OH} \ldots \ldots \ldots \ldots$ & .0 .4 & .017 & .013 & $\ldots \ldots \ldots$ \\
\hline \multicolumn{5}{|l|}{ AcIDs: } \\
\hline $\mathrm{HCl} \ldots \ldots \ldots \ldots \ldots \ldots$ & .97 & .94 & .93 & $\ldots \ldots \ldots$ \\
\hline $\mathrm{HNO}_{3} \ldots \ldots \ldots \ldots$ & .07 & .9 .1 & .93 & $\ldots \ldots \ldots$ \\
\hline $\mathrm{H}_{2} \mathrm{C}_{2} \mathrm{H}_{3} \mathrm{O}_{2} \ldots \ldots \ldots \ldots \ldots$ & .042 & .020 & .013 & $\ldots \ldots \ldots$ \\
\hline $\mathrm{H}_{3} \mathrm{PO}_{4}=\mathrm{H}^{+}+\mathrm{H}_{2} \mathrm{PO}_{4}^{-} \ldots$ & .60 & $\cdot 36$ & .29 & $\ldots \ldots \ldots$ \\
\hline $\mathrm{H}_{2} \mathrm{SO}_{4}=2 \mathrm{II}^{+}+\mathrm{SO}_{4}-\ldots$ & .01 & $\cdot 3^{8}$ & .31 & $\ldots \ldots \ldots$ \\
\hline $\mathrm{H}_{2} \mathrm{CO}_{3}=\mathrm{II}++\mathrm{ICO}_{3}^{-} \ldots$ & $0 . \infty 5$ & 0.002 & 0.0017 & $\ldots \ldots \ldots$ \\
\hline
\end{tabular}

part carries a positive charge and the acidic part a negative charge. The charge carried by any univalent ion is called a unit charge; ions having greater valence carry as many unit charges as they have valence. Since the solution of any electrolyte is always electrically neutral, the total quantity of positive electricity carried by the positive ions equals the total 
quantity of negative electricity carried by the negative ions. The more dilute the solution the greater is the proportion of the electrolyte transformed into ions, or, in other words, the greater is its degree of ionization.

When we put two electrodes, connected with dry cells or other source of current, into a solution of an electrolyte, the current is found to flow in the outside circuit, because of the discharging of the charge on the electrodes, due to the arrival at their surface of oppositely charged ions from the solution. The ions in the solution move up to the plates because each ion is attracted toward one plate and repelled from the other, owing to the fact that it also carries a charge of electricity. The sign of the charge on the ion determines the direction of the latter's movement. On coming in contact with the electrodes the ions become discharged by having their charges electrically neutralized by equal amounts of the opposite kind of electricity furnished by the electrode. Metallic ions after discharge are either deposited as metallic platings on the cathode or react with water to form hydroxides and hydrogen. Non-metallic ions such as hydrogen, oxygen, and chlorine are released as single atoms which then unite to form diatomic molecules of the gases $\mathrm{H}_{2}, \mathrm{O}_{2}, \mathrm{Cl}_{2}$, etc. Nitrate and sulfate ions never remain discharged at the electrodes, but instead we find there the products of their reactions with water-nitric acid and oxygen, and sulfuric acid and oxygen, respectively.

If a given quantity of electrolyte is kept between plates which are parallel to each other and carry a constant charge per unit area, at different dilutions the conductivity of this electrolyte will vary in proportion to the number of free ions present. As a consequence the proportion of an electrolyte which has been transformed into ions at any dilution can be determined by dividing the conductivity at the dilution in question by the maximum conductivity found after continuous dilution of this same quantity of electrolyte, provided the two measurements are made in the manner described (408). Values so determined show us that as a rule all salts are highly ionized substances, but acids and bases have very different degrees of ionization, 
some being even more highly ionized than salts, but others being very little ionized indeed.

4I2. Criticisms of the Ionic Hypothesis. - The idea that ions exist in solution as independent chemical substances has come to be known as the Ionic Hypothesis. It will be surprising if the student who learns of this hypothesis for the first time and thinks critically about the matter is not ready to offer at once several good reasons for doubting the truth of the conclusions. In the first place, the hypothesis seems to assume that in a solution of common salt, for example, a large part of its elements are present in a free state. Now the student who knows anything of the properties of metallic sodium and of chlorine gas will find it hard to believe that either of these elements can be present in a salt solution; because sodium reacts violently with water, forming sodium hydroxide and hydrogen, and chlorine has a horrible smell and a yellow color. Plain'y there is something incompatible with the obvious facts in the statement that a solution of salt contains free sodium and free chlorine.

A closer study of the hypothesis shows, however, that it is not assumed that the elements sodium and chlorine are present as ordinary atoms, for each atom is said to be electrically charged. 'Those who uphold the hypothesis will point out that a charged brass ball has very different properties from the same ball if uncharged. True, say the critics, but even a charged brass ball is still a brass ball; to which the opponents reply that the quantity of electricity on the ball is a matter of enormous importance.

If then the ions are so highly charged, why do the positive ions not unite with and so electrically discharge the negative ions, since the solution is a conductor? It may be said in reply that it is assumed that jons of unlike sign are constantly uniting, at a rate just equal to the rate of dissociation, with the result that a state of equilibrium is produced.

In spite of the foregoing criticisms and many others the ionic hypothesis with all its apparent inconsistencies has proved itself highly useful in explaining and correlating many facts and phenomena. 
Before passing final judgment on this remarkable hypothesis it will be better to consider its further applications and then, in chapter $\mathrm{xx}$, to take up the matter again in the light of newer discoveries, which have led to essential modifications of the views as originally proposed by Arrhenius.

Finally it may be urged that Arrhenius himself was not certain of the truth of his theory until he became acquainted with the wonderful work of Van't Hoff on the so-called osmotic pressures of dissolved substances (chap. xxvii). This work will be discussed as soon as we have progressed far enough to understand and interpret the experiments which we must then study. 


\section{CHAPTER XVIII}

\section{APPLICATIONS OF THE IONIC HYPOTHESIS}

413. Double Decomposition.-In the foregoing chapter it was pointed out that the probable cause of the union of two unlike ions is the attraction of their unlike electric charges. In general, every kind of positive ion can unite with any kind of negative ion. Therefore, if any two electrolytes (provided they have no ion in common) are mixed in solution, at least some double decomposition must take place, simply because new combinations of positive and negative ions are made possible. Let us first consider the important case in which the two starting materials, as well as the two products of the reaction, are easily soluble and highly ionized.

414. Class I. Equilibrium between Four Easily Soluble and Highly Ionized Electrolytes. - If dilute solutions of two imaginary clectrolytes $A B$ and $C D$, which ionize thus

$$
\begin{aligned}
& A B \leftrightharpoons A^{+}+B^{-}, \\
& C D \leftrightharpoons C^{+}+D^{-},
\end{aligned}
$$

are mixed, we may predict, without knowing anything more about these substances, that the following reactions are possible,

$$
\begin{aligned}
& A^{+}+D^{-} \leftrightharpoons A D, \\
& C^{+}+B^{-} \leftrightharpoons C B,
\end{aligned}
$$

III

IV

and that a double decomposition reaction,

$$
A B+C D=A D+C B,
$$

will take place to a greater or less extent. Since all four of the substances $A B, C D, A D$, and $C B$ are assumed to be highly ionized, it is plain that the mixed solution will contain chiefly the four kinds of ions, $A^{+}, B^{-}, C^{+}$, and $D^{-}$, and relatively few molecules. Since each of the four kinds of molecules present must be in equilibrium with its own two kinds of ions, the four 
equilibrium reactions (marked I, II, III, and IV) must be interrelated in the manner shown by the following arrangement of equations I, II, III, and IV:

$$
\begin{array}{rc}
\text { III } \quad \text { IV } \\
A B \leftrightarrows A^{+}+B^{-} \\
C D \leq D^{-}+\mathrm{C}^{+} \\
\downarrow \uparrow & \Downarrow \uparrow \\
A D & C B
\end{array}
$$

Equations I and II read horizontally, while III and IV read vertically. We may call this the compound equation of the reaction

$$
A B+C D \leftrightarrows A D+C B .
$$

The compound equation shows that the four molecular substances are in equilibrium with each other because each molecular substance is in direct equilibrium with its own pair of ions. Now if all of the four molecular substances are assumed to have exactly equal tendencies to ionize, then we must conclude that for the condition of equilibrium equal numbers of the four kinds of molecules will be present, if we have taken equivalent amounts of substances. We may summarize Class I as follows: If both starting substances and both products of a double decomposition reaction $A B+C D \leftrightarrows A D+C B$ are easily soluble and highly and equally ionizable, an equilibrium mixture will result in which (I) most of the dissolved material is present as free ions, (2) little of the material is present as molecules, and (3) if equivalent amounts are taken the four kinds of molecules are present in equal numbers.

415. An Example of Class I. The Reaction between Ferric Chloride and Ammonium Sulfocyanate.-The reaction

$$
\mathrm{FeCl}_{3}+{ }_{3} \mathrm{NH}_{4} \mathrm{NCS} \leftrightharpoons \mathrm{Fe}(\mathrm{NCS})_{3}+{ }_{3} \mathrm{NH}_{4} \mathrm{Cl},
$$

studied earlier (280), is a good illustration of Class I, since all four salts are easily soluble and highly ionized. It was shown by experiment that this reaction does not take place completely, but that it reaches equilibrium while there is still much of the material not converted into ferric sulfocyanate and ammonium chloride. 
4I6. Other Examples of Class I.-Numerous additional examples of Class I might be given. The following will serve as illustrations:

$$
\begin{aligned}
\mathrm{KCl}+\mathrm{NaNO}_{3} & \leftrightharpoons \mathrm{KNO}_{3}+\mathrm{NaCl}, \\
\mathrm{Na}_{2} \mathrm{SO}_{4}+2 \mathrm{KNO}_{3} & \leftrightharpoons \mathrm{K}_{2} \mathrm{SO}_{4}+2 \mathrm{NaNO}_{3}, \\
\mathrm{~K}_{2} \mathrm{CO}_{3}+\mathrm{Na}_{2} \mathrm{SO}_{4} & \leftrightarrows \mathrm{K}_{2} \mathrm{SO}_{4}+\mathrm{Na}_{2} \mathrm{CO}_{3}, \\
\mathrm{NaNO}_{3}+\mathrm{HCl} & \leftrightharpoons \mathrm{NaCl}+\mathrm{HNO}_{3} .
\end{aligned}
$$

In each case the mixed solution contains largely the four kinds of ions, together with small proportions of the four kinds of molecules in approximately equivalent amounts. In Class I the two substances taken react only partially, and therefore the reaction is always incomplete.

4I7. A Graphic Method of Representing Degrees of Ionization.-An acid, base, or salt, not in solution, exists wholly in the form of molecules (no ions are present).

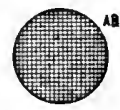

FIG. 52 We may represent such an un-ionized substance by a crosshatched circle, Fig. 52. When this substance, whose formula we may call $A B$, is dissolved in water it will partially ionize, thus:

$$
A B=A^{+}+B^{-} \text {. }
$$

This condition is represented by Fig. 53. Let us suppose that the solution is 80 per cent ionized; then 20 per cent is present as un-ionized molecules. In Fig. 53 the left-hand circle has a

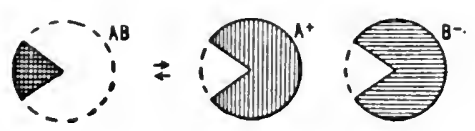

FIG. 53 cross-hatched sector which. is just 20 per cent of the area of the whole circle. This will represent the fact that 20 per cent of the substance is present as un-ionized molecules. The middle circle, of which 80 per cent is shaded with vertical lines, will represent the fact that 80 per cent of the total $A$ radical is in the form of free positive ions. In similar fashion the right-hand circle shows that 80 per cent of the total $B$ radical is in the form of free negative ions. Furthermore, if we take the area of the circle, Fig. 52, as proportional to the whole number of molecules in one formula weight of the substance before it is dissolved, then the area of the 
cross-hatched sector of the left-hand circle of Fig. 53 will be proportional to the number of un-ionized molecules in one formula weight of the dissolved substance. Since each $A B$ molecule,
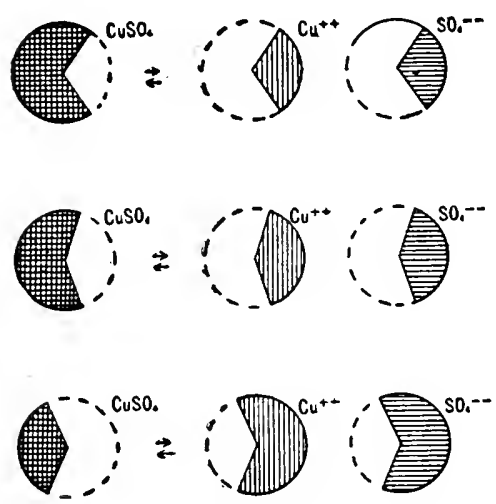

Fig. 54

when it ionizes, gives one $A^{+}$ ion and one $B^{-}$ion, the areas of the shaded portions of the middle and right-hand circles will be directly proportional to the numbers of $A^{+}$and $B^{-}$ ions respectively. By means of a figure like Fig. 53 the relative concentrations of ions and molecules of a dissolved electrolyte can be seen at a glance. By way of further illustration the condition of normal, one-tenth-normal, and one one-hundred th-normal copper sulfate solution is shown in Fig. 54 .

418. Graphic Representation of Class I.-Let us now turn to the graphic representation of a double decomposition reaction of the type just studied under Class I, where all four substances concerned are easily soluble and highly ionized. We again represent the reaction by

$$
A B+C D \leftrightarrows A D+C B .
$$

Figure 55 shows the condition of solutions of $A B$ and $C D$ before they are mixed, on the supposition that each is 85 per cent ionized in $\mathrm{N} /$ Io solution. When equal volumes of the two $\mathrm{N} / \mathrm{io}$ solutions are mixed, the reaction represented by the compound equation

$$
\begin{array}{r}
A B \leftrightarrows A^{+}+B^{-} \\
C D \leftrightharpoons D^{-}+C^{+} \\
\Downarrow \Uparrow \quad{ }^{+} \\
A D \quad C B
\end{array}
$$
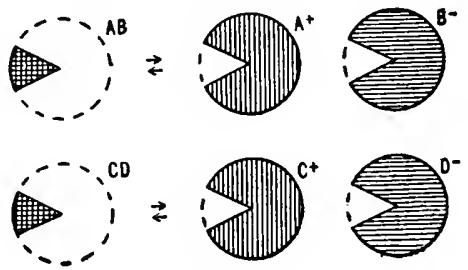

FIG. 55

takes place and very quickly reaches the condition of equilibrium shown graphically in Fig. 56, in which the proportions of 
molecules and ions have been calculated on the additional assumption that $A D$ and $C B$ both have the same tendency to ionize as have $A B$ and $C D$ (when each is separately dissolved in water). Comparison of Figs. 55 and 56 shows us that the areas representing the numbers of molecules and ions of the materials taken are not greatly changed as the result of the mixing. Consequently we say that the reaction is incomplete. All examples of Class I would be represented by similar graphs.

419. A Second Type of Double Decomposition: Class II.-Class II will comprise double decomposition reactions

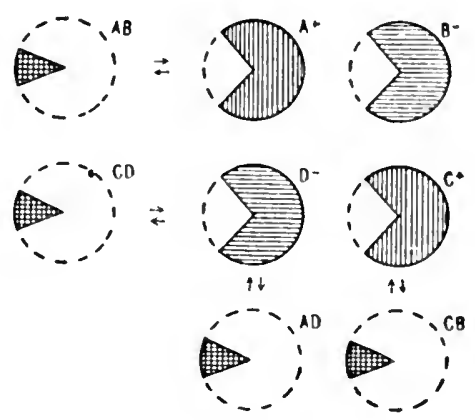

Fig. 56 in which two easily soluble and highly ionized substances give two easily soluble products, one of which is highly ionized, the other little ionized. 'The simplest example of Class II is found in a neutralization reaction such as

$$
\mathrm{HCl}+\mathrm{NaOH} \Rightarrow \mathrm{NaCl}+\mathrm{H}_{2} \mathrm{O},
$$

since all the substances except the water are highly ionized.

420. The Ionization of Water.-'The ionization of water may be determined from conductivity measurements, for though it is a very much poorer conductor of the current than is a salt solution, still, as we have already said, it conducts much better than glass or hard rubber. According to the ionic theory it is ionized thus:

$$
\mathrm{H}_{2} \mathrm{O}=\mathrm{H}^{+}+\mathrm{OH}^{-} .
$$

In one liter of pure water there is present about one ten-millionth of a gram of ionic hydrogen and the equivalent amount of hydroxyl.

If then we attempt to represent the proportion of ions in pure water by a graphic scheme, a single dot in the center of an otherwise empty circle would have too large an area to represent correctly the proportion of ions present if the rest of the circle 
represented the molecules of water. In cases of this kind we shall use a single dotted radius to indicate that the number of ions is too small to be accurately represented. The graph of

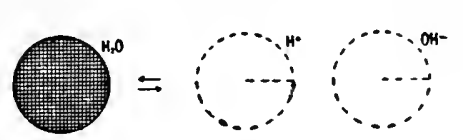

FIG. 57 water will then be that shown in Fig. 57 .

That there are so few ions present in a liter of pure water means that the ionic equilibrium is established only when all but a minute fraction of the total material is in the form of water molecules. Accordingly, when hydroxyl and hydrogen ions are brought together in solution we must expect them to combine almost completely to form molecules.

42I. Neutralization.- - If we mix equivalent amounts of solutions of $\mathrm{HCl}$ and $\mathrm{NaOH}$ the resulting reaction may be represented as follows:

$$
\begin{array}{cc}
\mathrm{HCl} \leftrightarrows & \mathrm{H}^{+}+\mathrm{Cl}^{-} \\
\mathrm{NaOH} \leftrightarrows & \mathrm{OH}^{-}+\mathrm{Na}^{+} \\
\Downarrow & \Downarrow \\
\mathrm{H}_{2} \mathrm{O} & \mathrm{NaCl} .
\end{array}
$$

The $\mathrm{H}^{+}$and $\mathrm{OH}^{-}$ions present unite almost completely to form molecules of $\mathrm{H}_{2} \mathrm{O}$. The removal of $\mathrm{H}^{+}$ions causes a shift of the reaction

$$
\mathrm{HCl} \leftrightarrows \mathrm{H}^{+}+\mathrm{Cl}^{-}
$$

to the right, and as the $\mathrm{H}^{+}$ions produced in this way are almost immediately taken up by new $\mathrm{OH}^{-}$ions formed by a shift to the right of the reaction

$$
\mathrm{NaOH} \leftrightarrows \mathrm{OH}^{-}+\mathrm{Na}^{+},
$$

the final result is the practically complete dissociation of both $\mathrm{HCl}$ and $\mathrm{NaOH}$ molecules and therefore the disappearance of these substances. Molecules of $\mathrm{H}_{2} \mathrm{O}$, once formed, dissociate very little into $\mathrm{H}^{+}$and $\mathrm{OH}^{-}$ions, and so the final equilibrium solution will contain no more free $\mathrm{H}^{+}$and $\mathrm{OH}^{-}$ions than an equal volume of pure water. The $\mathrm{Na}^{+}$and $\mathrm{Cl}^{-}$ions unite partially to form molecules

$$
\mathrm{Na}^{+}+\mathrm{Cl}^{-} \leftrightarrows \mathrm{NaCl}
$$


but this reaction does not proceed far in dilute solution, as common salt is a highly ionized substance. In fact, the solution resulting from the neutralization of $\mathrm{HCl}$ by $\mathrm{NaOH}$ is exactly the same as, and differs in no way from, that made by dissolving common salt in water to produce a solution of equal concentration. All the facts just stated are shown by a comparison of the two graphs, Figs. $5^{8}$ and 59. Thus it can be seen (Fig. 59) that the areas representing the numbers of molecules of $\mathrm{HCl}$ and $\mathrm{NaOH}$ respectively have been reduced to negligible dimensions; the same is also true of the areas for $\mathrm{H}^{+}$and $\mathrm{OH}^{-}$ions. But

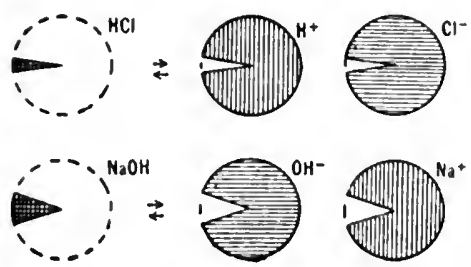

FIc. $5^{8}$

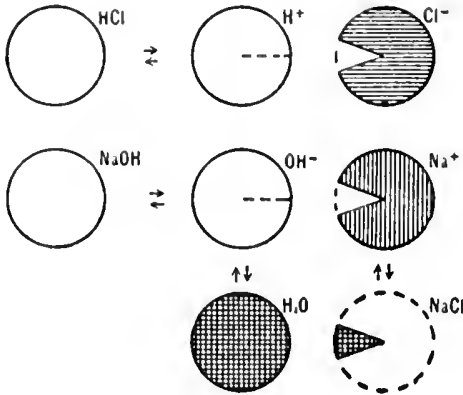

Fic, 59

the $\mathrm{Cl}^{-}$and $\mathrm{Na}^{+}$ion areas are not greatly clianged in the second grapl. Compared with these areas, that representing $\mathrm{NaCl}$ molecules is small. The circle representing the number of molecules of $\mathrm{H}_{2} \mathrm{O}$ is completely shaded, thus showing that the yield of molecular $\mathrm{H}_{2} \mathrm{O}$ is practically ioo per cent.

422. The Simplified Equation of Neutralization.--To sum up the matter, it may be said that acids and bases neutralize one another because of the tendency of $\mathrm{H}^{+}$and $\mathrm{OH}^{-}$ions to unite almost completely to form water. 'This almost complete union of $\mathrm{H}^{+}$and $\mathrm{OH}^{-}$ions takes place because $\mathrm{H}_{2} \mathrm{O}$ is but very slightly ionized. In a very dilute solution, where the acid and base taken are almost completely ionized at the instant of mixing, the principal change that takes place is the union of $\mathrm{H}^{+}$and $\mathrm{OH}^{-}$ions to form $\mathrm{H}_{2} \mathrm{O}$ molecules, since in the very dilute solution the $\mathrm{Na}^{+}$and $\mathrm{Cl}^{-}$ions remain largely uncombined. We may 
therefore write as the simplified equation of neutralization in dilute solution

$$
\mathrm{H}^{+}+\mathrm{OH}^{-} \leftrightarrows \mathrm{H}_{2} \mathrm{O} .
$$

423. Experimental Confirmation of the Theory of Neutralization.-The process of neutralization can be followed experimentally with the help of an apparatus somewhat like that shown in Fig. 5I (406); but having a small electric lamp in the place of the galvanometer. The solution layers in the cell shown in the figure are made by first putting into the cell a layer of one-tenth-normal hydrochloric acid, and then allowing an equal layer of sodium hydroxide to run under this first layer by introducing it at the bottom of the cell through a dropping funnel. As represented in the figure the two parallel electrodes are in contact with the two layers, which can be seen very nicely if a little litmus is put into the acid and base respectively before the layers are made. If now the key is closed the current flows through both layers, and the lamp glows. Hydrogen and sodium ions are arriving at one electrode, and chlorine and hydroxyl ions are arriving at the other. Of these ions the hydrogen and hydroxyl travel much more rapidly under the attraction from a given charge per unit area of the electrode, and so they are neutralizing their charges on the plates more quickly than are the other ions. As a result most of the current passing in the outside circuit is due to their discharge. If the two layers of acid and base are next mixed, the lamp no longer glows. Half of the carriers of the current and the most efficient ones have been used to form water molecules, and in the cell there remains only the slow-moving sodium ions and chlorine ions. If the acid and base in the respective layers were not quite equivalent in amount, a slight excess of one or the other will be shown by the litmus color, but the important part of the experiment, the serious loss of ions, will still be unmistakable from the great decrease in the conductivity of the solution between the plates.

424. A Second Example of Class II: Action of $\mathrm{HCl}$ on Sodium Acetate.- It will be recalled that acetic acid, $\mathrm{HC}_{2} \mathrm{H}_{3} \mathrm{O}_{2}$, neutralizes $\mathrm{NaOH}$, forming sodium acetate, thus:

$$
\mathrm{HC}_{2} \mathrm{H}_{3} \mathrm{O}_{2}+\mathrm{NaOH} \leftrightarrows \mathrm{NaC}_{2} \mathrm{H}_{3} \mathrm{O}_{2}+\mathrm{H}_{2} \mathrm{O} \text {. }
$$


Acetic acid is a monobasic acid, only one of the four hydrogen atoms of each molecule being ionizable:

$$
\mathrm{HC}_{2} \mathrm{H}_{3} \mathrm{O}_{2} \leftrightarrows \mathrm{H}^{+}+\mathrm{C}_{2} \mathrm{H}_{3} \mathrm{O}_{2}^{-} \text {. }
$$

This acid is but little ionized in normal solution, the degree of ionization being only 0.4 per cent. On the other hand, solutions of its salts, like $\mathrm{NaC}_{2} \mathrm{H}_{3} \mathrm{O}_{2}$, are highly ionized:

$$
\mathrm{NaC}_{2} \mathrm{H}_{3} \mathrm{O}_{2} \rightarrow \mathrm{Na}^{+}+\mathrm{C}_{2} \mathrm{H}_{3} \mathrm{O}_{2}^{-} \text {. }
$$

If we mix equivalent amounts of $\mathrm{HCl}$ and $\mathrm{NaC}_{2} \mathrm{H}_{3} \mathrm{O}_{2}$ in solution we cannot see that any chemical change occurs; but that a reaction has occurred we may show convincingly with the help of the electrolytic cell, which is used to discover the change in conductivity during ncutralization. In the lower layer this time we shall have sodium acetate and in the upper hydrochloric acid. As before, the lamp glows-both solutions are good conductors; the first by means of $\mathrm{Na}^{+}$and $\mathrm{C}_{2} \mathrm{H}_{3} \mathrm{O}_{2}-$ ions, the second by means of $\mathrm{H}^{+}$and $\mathrm{Cl}^{-}$ions. When we mix the two layers the decrease in brightness of the lamp shows that the conductivity has dropped off greatly, thus proving that many of the ions have been changed into non-conducting molecules. The compound equation is

$$
\begin{array}{rl}
\mathrm{HCl} & \leftrightarrows \mathrm{H}^{+}+\mathrm{Cl}^{-} \\
\mathrm{NaC}_{2} \mathrm{H}_{3} \mathrm{O}_{2} & \Rightarrow \mathrm{C}_{2} \mathrm{H}_{3} \mathrm{O}_{2}-+\mathrm{Na}^{+} \\
\| & \downarrow \\
\mathrm{HC}_{2} \mathrm{H}_{3} \mathrm{O}_{2} & \mathrm{NaCl}
\end{array}
$$

'The graphs are shown in Figs. 60 and 61. Since of the four substances concerned all but the acetic acid are highly ionized, while the latter is but little ionized, the reaction falls under Class II. When $\mathrm{HCl}$ and $\mathrm{NaC}_{2} \mathrm{H}_{3} \mathrm{O}_{2}$ solutions are mixed, the $\mathrm{H}^{+}$and $\mathrm{C}_{2} \mathrm{H}_{3} \mathrm{O}_{2}-$ ions will unite far more completely than will any other pair of ions, and at the same time the molecules of $\mathrm{HCl}$ and $\mathrm{NaC}_{2} \mathrm{H}_{3} \mathrm{O}_{2}$ will continue to ionize until but very few remain (Fig. 6I). Also $\mathrm{Na}^{+}$and $\mathrm{Cl}^{-}$ions will unite partially to form molecules of $\mathrm{NaCl}$. Therefore the equilibrium mixture will contain largely free acetic acid, for the most part un-ionized, 
together with common salt and its ions. Very little $\mathrm{HCl}$ and $\mathrm{NaC}_{2} \mathrm{H}_{3} \mathrm{O}_{2}$ will be present.

\section{Comparison of the First and Second Examples of Class} II.-Fig. 60 shows the conditions of the solutions of hydrochloric acid and of sodium acetate before they are mixed, while Fig. 6I shows the condition of the equilibrium mixture. These figures are almost a reproduction of Figs. 58 and 59, representing neutralization. In place of $\mathrm{NaOH}$ we have in the second case $\mathrm{NaC}_{2} \mathrm{H}_{3} \mathrm{O}_{2}$, which is also highly ionized; and in place of water we have $\mathrm{HC}_{2} \mathrm{H}_{3} \mathrm{O}_{2}$, which, like water, is but little ionized. In Fig. 6I the circle representing molecular acetic acid is nearly completely

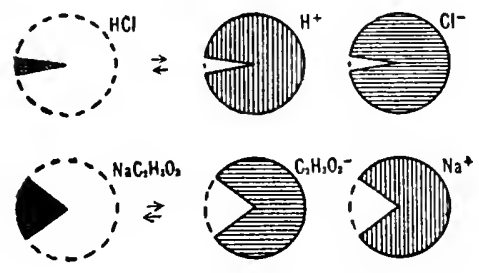

FIG. 60
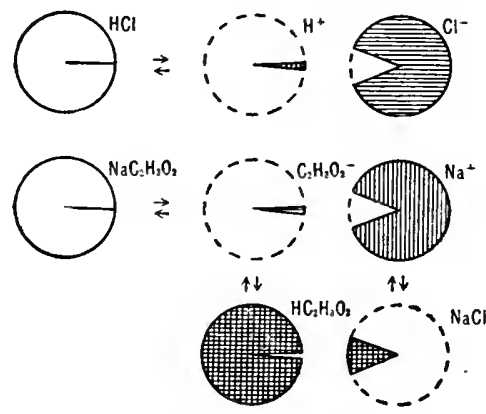

FIG. 6I

cross-hatched, showing that the yield of this substance is nearly Ioo per cent. Although the reactions represented by Figs. 59 and $6 \mathrm{I}$ are so nearly alike, there is a small difference due to the fact that acetic acid is ionized more than water. In consequence the formation of molecular acetic acid falls short of roo per cent by a small fraction of I per cent.

426. A Third Example of Class II: Action of $\mathrm{NaOH}$ on $\mathrm{NH}_{4} \mathbf{C l}$.-Another important example of Class II is found in the action of sodium hydroxide and ammonium chloride. The addition of dilute $\mathrm{NaOH}$ to a solution of $\mathrm{NH}_{4} \mathrm{Cl}$ does not produce any visible effect; but evidence that the reaction

$$
\mathrm{NaOH}+\mathrm{NH}_{4} \mathrm{Cl} \leftrightarrows \mathrm{NaCl}+\mathrm{NH}_{4} \mathrm{OH}
$$

takes place may be obtained in two ways: first, by finding a great decrease in conductivity on mixing superimposed layers 
of the two solutions; and secondly, by noting the odor of ammonia given off by reason of the partial dissociation of the $\mathrm{NH}_{4} \mathrm{OH}$ present in the solution

$$
\mathrm{NH}_{4} \mathrm{OH} \leftrightharpoons \mathrm{NH}_{3}+\mathrm{H}_{2} \mathrm{O} \text {. }
$$

The compound equation of the reaction follows:

$$
\begin{aligned}
& \mathrm{NaOH}=\mathrm{Na}^{+}+\mathrm{OH}^{-} \\
& \mathrm{NH}_{4} \mathrm{Cl} \leftrightharpoons \mathrm{Cl}^{-}+\mathrm{NH}_{4}+
\end{aligned}
$$

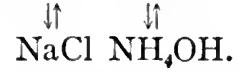

Since sodium hydroxide and ammonium chloride are highly ionized, and ammonium hydroxide is little ionized, this reaction is completely analogous to that between $\mathrm{HCl}$ and $\mathrm{NaC}_{2} \mathrm{H}_{3} \mathrm{O}_{2}$ :

$$
\mathrm{HCl}+\mathrm{NaC}_{2} \mathrm{H}_{3} \mathrm{O}_{2} \leftrightarrows \mathrm{NaCl}+\mathrm{HC}_{2} \mathrm{H}_{3} \mathrm{O}_{2}
$$

Each reaction takes place nearly completely from left to right because one product is but little ionized. The graphs for this reaction, Figs. 62 and 63 , are closely similar to those for

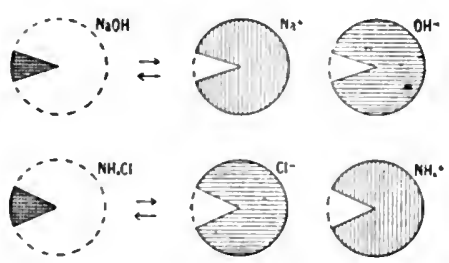

Fig. 62
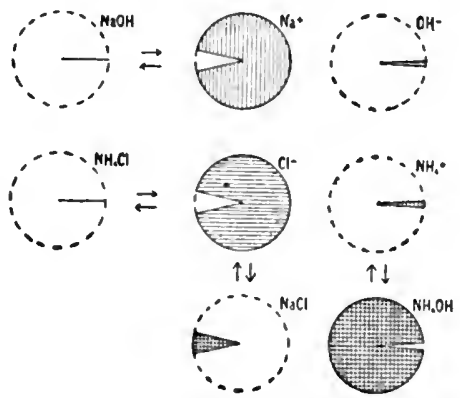

FIG. $6_{3}$

neutralization, Figs. $5^{8}$ and 59 , and for the reaction between $\mathrm{HCl}$ and $\mathrm{NaC}_{2} \mathrm{H}_{3} \mathrm{O}_{2}$, Figs. 60 and $6 \mathrm{I}$.

427. Summary of Class II Reactions.-As we have pointed out, all these reactions are alike, in that two highly ionized electrolytes react to form one highly ionized electrolyte and one little ionized electrolyte. Invariably reactions of this class are nearly complete. The smaller the degree of ionization of the little ionized product, the more completely the reaction takes. 
In the resulting mixture the little ionized substance is present, of course almost wholly in the molecular form.

428. Strength of Acids. - Since all salts are highly ionized, the reaction between any highly ionized acid and a salt of a little ionized acid must belong to Class II. We may therefore predict that, as in the second example studied, such reactions will give nearly Ioo per cent yields of their products, and that in the resulting solution there will be present the little ionized acid instead of the highly ionized acid originally used. The highly ionized acid may be said to have displaced the little ionized acid from its salt. As a result, we may call the former a strong acid and the latter a weak acid, and may say that $a$ strong acid always displaces a weak acid from its salts.

429. Strength of Bases.-Just as we call a highly ionized acid a strong acid and a little ionized acid a weak acid, so we may call a highly ionized base a strong base and a little ionized base a weak base. Since all reactions between strong bases and the salts of weak bases (see third example, 426) are examples of Class II, we can predict that the yield of weak base and salt of the strong acid will be nearly Ioo per cent. In other words, $a$ strong base will always displace a weak base from its salt.

430. Two Useful Laws.-The foregoing law and that given in the paragraph on the strength of acids (428) have been of very great practical convenience to chemists. These laws fail only when the salt of the weak acid is little ionized, a case so rare that the usefulness of the rules is virtually unaffected. The laws are of course only special cases of the fundamental one that if two highly ionized substances react to form one little ionized substance and one highly ionized substance, the reaction will be nearly complete.

43I. Suppression of the Ionization of a Weak Acid or a Weak Base.-Since the strength of an acid or a base is determined by its tendency to ionize, any factor that has an influence on this tendency will affect the strength or weakness of the acid or base. We must now consider this important subject and will begin by studying the action of $\mathrm{NH}_{4} \mathrm{Cl}$ on a solution of the weak base $\mathrm{NH}_{4} \mathrm{OH}$. 
If we add a little phenolphthalein to very dilute $\mathrm{NH}_{4} \mathrm{OH}$ a bright, red-colored solution results. This shows that the solution is alkaline, and therefore that it contains an abundance of $\mathrm{OH}^{-}$ions. Upon addition of a little $\mathrm{NH}_{4} \mathrm{Cl}$ to this red solution the color disappears almost completely. This proves that the number of $\mathrm{OH}^{-}$ions present has been very greatly decreased. In order to understand how this has happened, we must consider the matter from the standpoint of ionic equilibrium. A solution of $\mathrm{NH}_{4} \mathrm{OH}$ is ionized to a small extent, thus:

$$
\mathrm{NH}_{4} \mathrm{OH} \leftrightharpoons \mathrm{NH}_{4}++\mathrm{OH}^{-} \text {. }
$$

Ammonium chloride, on the other hand, is very highly ionized:

$$
\mathrm{NH}_{4} \mathrm{Cl} \leftrightarrows \mathrm{NH}_{4}++\mathrm{Cl}^{-} \text {. }
$$

If then we add an equivalent amount of $\mathrm{NH}_{4} \mathrm{Cl}$ to a dilute solution of $\mathrm{NH}_{4} \mathrm{OH}$, the number of $\mathrm{NH}_{4}^{+}$ions per cubic centimeter will be increased many fold. The $\mathrm{OH}^{-}$ions present will therefore collide with $\mathrm{NH}_{4}^{+}$ions and combine with them far more frequently than before. Since the rate of dissociation of $\mathrm{NH}_{4} \mathrm{OH}$ molecules into ions is not affected by the presence of the $\mathrm{NH}_{4} \mathrm{Cl}$, this increased rate of union of $\mathrm{NH}_{4}^{+}$and $\mathrm{OH}^{-}$ions causes a great shift to the left of the equilibrium

$$
\mathrm{NH}_{4} \mathrm{OH} \leftrightharpoons \mathrm{NH}_{4}^{+}+\mathrm{OH}^{-} \text {. }
$$

For example, it has been found, by methods that we need not consider here, that the addition of $\mathrm{I} g$. of $\mathrm{NH}_{4} \mathrm{Cl}$ to 100 c.c. of
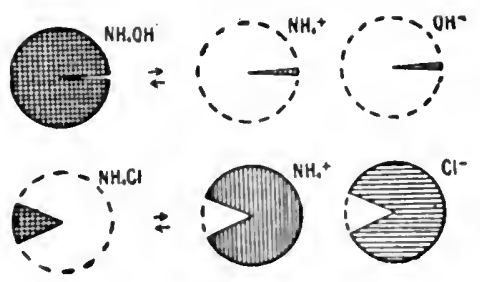

Fic. 64
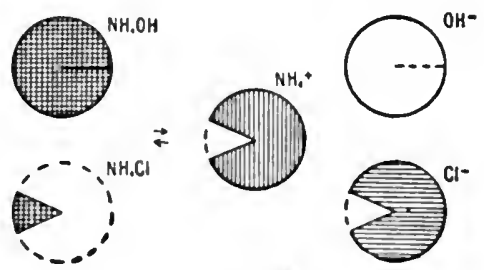

Fic. $6 \%$

decinormal $\mathrm{NH}_{4} \mathrm{OH}$ will decrease the number of $\mathrm{OH}^{-}$ions present about one hundred fold. In other words, the ionization of the base will be decreased one hundred fold (see Figs. $6_{4}$ and $6_{5}$ ). We may now state the general law of which the case just 
studied is a typical example: The ionization of a weak base is greatly suppressed by the addition of a salt of the base. This means that a weak base is made still weaker by the addition of its soluble salts.

In a similar manner the ionization of a weak acid is greatly suppressed by the addition of any of its soluble salts; that is, a weak acid is made still weaker by adding one of its salts. For example, the addition of $\mathrm{NaC}_{2} \mathrm{H}_{3} \mathrm{O}_{2}$ to a red solution of acetic acid, $\mathrm{HC}_{2} \mathrm{H}_{3} \mathrm{O}_{2}$, containing litmus changes the color from red to purple, thus showing a great decrease in the number of $\mathrm{H}^{+}$ions, and therefore a great decrease in ionization of the acid.

432. The Common Ion Law.-A base and any one of its salts must of necessity have one ion in common. (The $\mathrm{NH}_{4}^{+}$ ion is common to $\mathrm{NH}_{4} \mathrm{OH}$ and $\mathrm{NH}_{4} \mathrm{Cl}$.) An acid also must have one ion in common with any of its salts. We may therefore state the principle of the foregoing laws as follows: Suppression of the ionization of a little ionized substance occurs when we add to its solution a highly ionized substance having a common ion. This is the Common Ion Law, a very important generalization. The examples already cited are by no means the only ones of importance. For example, it is plain that the ionization of $\mathrm{NH}_{4} \mathrm{OH}$ must be suppressed by the addition of $\mathrm{NaOH}$ or $\mathrm{KOH}$ because of the increase in concentration of the common $\mathrm{OH}^{-}$ion; and that the ionization of $\mathrm{HC}_{2} \mathrm{H}_{3} \mathrm{O}_{2}$ must likewise be suppressed by the addition of any strong acid like $\mathrm{HCl}$ or $\mathrm{HNO}_{3}$. The effect of a highly ionized substance on the ionization of another highly ionized substance having one ion in common is of the same type but very much smaller in degree than when the second substance is slightly ionized.

We shall next consider the application of the Common Ion Law to solutions of acids and bases and thus obtain a definition of the term neutrality.

433. Neutrality Defined.-We have already learned (420) that water is slightly ionized, thus,

$$
\mathrm{H}_{2} \mathrm{O} \leftrightarrows \mathrm{H}^{+}+\mathrm{OH}^{-} \text {. }
$$

Each cubic centimeter of pure water must therefore contain exactly as many $\mathrm{H}^{+}$as $\mathrm{OH}^{-}$ions. Since all acids give $\mathrm{H}^{+}$ 
ions, the addition of an acid to water, in accord with the common ion law, will greatly suppress the ionization of water. Therefore acid solutions will contain far less $\mathrm{OH}^{-}$ions per cubic centimeter than pure water. In an acid solution the number of $\mathrm{H}^{+}$ions greatly exceeds the number of $\mathrm{OH}^{-}$ions. The ionization of water is also greatly suppressed by the addition of a base, since all bases have $\mathrm{OH}^{-}$ions in common with water. In basic solutions the number of $\mathrm{H}^{+}$ions per cubic centimeter is far less than in pure water and therefore the number of $\mathrm{OH}^{-}$ions greatly exceeds the number of $\mathrm{H}^{+}$ions. Since we may consider water a typically neutral substance we may define a neutral solution as one in which the number of $\mathrm{H}^{+}$ions equals the number of $\mathrm{OH}^{-}$ ions. Since, as we have already learned, a strong acid completely neutralizes a strong base, as for example in the reaction

$$
\mathrm{HCl}+\mathrm{NaOH} \leftrightharpoons \mathrm{NaCl}+\mathrm{H}_{2} \mathrm{O},
$$

we conclude that in the resulting solution the number of $\mathrm{H}^{+}$ions is just equal to the number of $\mathrm{OH}^{-}$ions: this is the criterion of complete neutrality.

434. First Example of Class III: The Action of a Weak Acid on a Strong Base.-Under Class III we shall study reactions in which one little ionized and one highly ionized substance give products, one of which is little ionized, the other highly ionized. As the first example we shall study the reaction between little ionized acetic acid (a weak acid) and highly ionized sodium hydroxide (a strong base). These react thus:

$$
\mathrm{HC}_{2} \mathrm{H}_{3} \mathrm{O}_{2}+\mathrm{NaOH} \leftrightharpoons \mathrm{H}_{2} \mathrm{O}+\mathrm{NaC}_{2} \mathrm{H}_{3} \mathrm{O}_{2} \text {. }
$$

Of the products, water is very. slightly ionized, while sodium acetate, $\mathrm{NaC}_{2} \mathrm{H}_{3} \mathrm{O}_{2}$, is highly ionized. If we mix equal volumes of normal solutions of $\mathrm{HC}_{2} \mathrm{H}_{3} \mathrm{O}_{2}$ and $\mathrm{NaOH}$, that is, if we add to the $\mathrm{NaOH}$ solution exactly that quantity of acetic acid that would neutralize it if the reaction were complete, we find that the resulting mixture is not neutral but is still alkaline to litmus. The fact that the mixture is alkaline means that the number of $\mathrm{HO}^{-}$ions is greater than the number of $\mathrm{H}^{+}$ions present. The 
cause of this condition is most easily understood by aid of the compound equation

$$
\begin{aligned}
\mathrm{HC}_{2} \mathrm{H}_{3} \mathrm{O}_{2} & \leftrightarrows \mathrm{H}^{+}+\mathrm{C}_{2} \mathrm{H}_{3} \mathrm{O}_{2}^{-} \\
\mathrm{NaOH} \leftrightarrows & \mathrm{OH}^{-}+\mathrm{Na}^{+} \\
& \stackrel{\uparrow}{\uparrow} \\
& \mathrm{H}_{2} \mathrm{O} \quad \mathrm{NaC}_{2} \mathrm{H}_{3} \mathrm{O}_{2}
\end{aligned}
$$

and Figs. 66 and 67 . At the instant of mixing, the solution contains an abundance of $\mathrm{OH}^{-}$ions (Fig. 66); these reduce greatly the number of $\mathrm{H}^{+}$ions present by forming $\mathrm{H}_{2} \mathrm{O}$ molecules:

$$
\mathrm{H}^{+}+\mathrm{OH}^{-} \leftrightarrows \mathrm{H}_{2} \mathrm{O} \text {. }
$$

The removal of $\mathrm{H}^{+}$ions disturbs the equilibrium

$$
\mathrm{HC}_{2} \mathrm{H}_{3} \mathrm{O}_{2} \leftrightarrows \mathrm{H}^{+}+\mathrm{C}_{2} \mathrm{H}_{3} \mathrm{O}_{2}^{-} \text {, }
$$

which shifts greatly to the right, thus producing both $\mathrm{H}^{+}$and $\mathrm{C}_{2} \mathrm{H}_{3} \mathrm{O}_{2}^{-}$ions. While the former unite with $\mathrm{OH}^{-}$almost (but

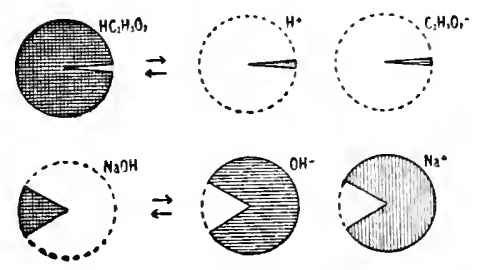

Frg. 66

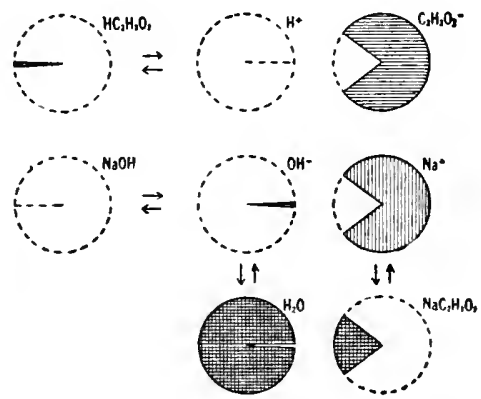

Fig. 67

not quite) completely, the latter remain for the larger part free in the solution, and by their great tendency to unite again with $\mathrm{H}^{+}$ions to form little ionized $\mathrm{HC}_{2} \mathrm{H}_{3} \mathrm{O}_{2}$ serve still further to diminish the number of free $\mathrm{H}^{+}$ions. In the final equilibrium mixture, shown in Fig. 67 , the number of $\mathrm{OH}^{-}$ions is greater than the number of $\mathrm{H}^{+}$ions because of the great tendency of the latter to unite readily with either $\mathrm{C}_{2} \mathrm{H}_{3} \mathrm{O}_{2}^{-}$ions or $\mathrm{OH}^{-}$ions. That the $\mathrm{OH}^{-}$ions get by far the lion's share of the $\mathrm{H}^{+}$ions is owing to the fact that water is much less ionized than acetic acid. 
Since the mixture contains more $\mathrm{OH}^{-}$than $\mathrm{H}^{+}$ions (see Fig. 67 ) it is not neutral but alkaline.

435. The Action of Water on Sodium Acetate. - In the foregoing paragraph we studied the equilibrium

$$
\mathrm{HC}_{2} \mathrm{H}_{3} \mathrm{O}_{2}+\mathrm{NaOH} \leftrightharpoons \mathrm{NaC}_{2} \mathrm{H}_{3} \mathrm{O}_{2}+\mathrm{H}_{2} \mathrm{O} \text {. }
$$

The composition of the equilibrium solution was shown in Fig. 67 . It must be plain from the deduction of $28 \mathrm{I}$, that exactly the same equilibrium solution would be obtained if we should dissolve in the same quantity of water pure sodium acetate, $\mathrm{NaC}_{2} \mathrm{H}_{3} \mathrm{O}_{2}$, in exactly the amount that would be produced by the complete union of all the $\mathrm{HC}_{2} \mathrm{H}_{3} \mathrm{O}_{2}$ and $\mathrm{NaOH}$ used in the first case. As a matter of fact we find that a solution of pure sodium acetate is not neutral but alkaline to litmus. The action of $\mathrm{H}_{2} \mathrm{O}$ on $\mathrm{NaC}_{2} \mathrm{H}_{3} \mathrm{O}_{2}$ takes place thus: the salt first dissolves and at once ionizes highly to form many $\mathrm{Na}^{+}$and $\mathrm{C}_{2} \mathrm{H}_{3} \mathrm{O}_{2}{ }^{-}$ions. Water, although but slightly ionized, contains some $\mathrm{H}^{+}$and $\mathrm{OH}^{-}$ions. Occasional collisions of $\mathrm{H}^{+}$and $\mathrm{C}_{2} \mathrm{H}_{3} \mathrm{O}_{2}^{-}$ions will occur, and part of these collisions will result in unions to form $\mathrm{HC}_{2} \mathrm{H}_{3} \mathrm{O}_{2}$ molecules; and as the latter have but little tendency to ionize, the result is a great decrease in the number of $\mathrm{H}^{+}$ions present. This in turn disturbs the equilibrium

$$
\mathrm{H}_{2} \mathrm{O} \leftrightharpoons \mathrm{H}^{+}+\mathrm{OH}^{-},
$$

which in consequence shifts to the right and so brings more $\mathrm{OH}^{-}$ ions into the solution. A few but not many of the $\mathrm{OH}^{-}$ions unite with $\mathrm{Na}^{+}$ions to form molecules of $\mathrm{NaOH}$, but most of the $\mathrm{OH}^{-}$ions remain free, thus producing in the solution a decided excess of $\mathrm{OH}^{-}$ions over $\mathrm{H}^{+}$ions (see Fig. 67 ), and so making the solution alkaline to litmus. Briefly stated, water acts on sodium acetate to a small extent, thus,

$$
\mathrm{NaC}_{2} \mathrm{H}_{3} \mathrm{O}_{2}+\mathrm{H}_{2} \mathrm{O} \leftrightharpoons \mathrm{HC}_{2} \mathrm{H}_{3} \mathrm{O}_{2}+\mathrm{NaOH} \text {, }
$$

and since $\mathrm{NaOH}$ is highly ionized, while $\mathrm{HC}_{2} \mathrm{H}_{3} \mathrm{O}_{2}$ is little ionized, the reaction of the solution is alkaline. The composition of a water solution of sodium acetate is that shown in Fig. 67 .

436. Hydrolysis of Salts. - The soluble salts of all weak acids with the strong bases sodium, potassium, calcium, or barium 
hydroxide give alkaline solutions when dissolved in water. In every case the reason is the same as that given for the alkaline reaction of sodium acetate solution. The effect of water on the salt of a weak acid and a strong base is an example of the type of reaction called hydrolysis (or also hydrolytic dissociation). Hydrolysis may be defined as a double decomposition reaction in which water is one of the reacting substances. The solutions of salts of all weak acids and strong bases are alkaline in reaction. Other things being equal, the weaker the acid from which the salt is derived the greater the extent of the hydrolysis; that is, the greater the alkalinity of the solution.

On the other hand, some salts (other than acid salts like $\mathrm{NaHSO}_{4}$ ) give solutions that have an acid reaction (176). Among such are the chlorides, sulfates, and nitrates of copper, lead, iron, zinc, aluminum, etc. Experiments show that the hydroxides of all these elements are weak bases. It would therefore seem probable that the acidity of solutions of the salts of these bases with strong acids is due to hydrolysis, and that the behavior of such salts with water is the counterpart of the behavior of salts of weak acids with strong bases.

437. A Second Example of Class III: The Action of a Strong Acid on a Weak Base.- The action of a strong acid on a weak base is plainly the reverse of that just discussed: the action of water on the salt of a strong acid and weak base. It follows that a weak base does not react completely with the theoretical or chemically equivalent amount of a strong acid, and in consequence the resulting mixture is still acid in its reaction. The action of $\mathrm{HCl}$ on the weak base $\mathrm{NH}_{4} \mathrm{OH}$ will serve as a simple illustration:

$$
\begin{aligned}
& \mathrm{NH}_{4} \mathrm{OH} \leftrightharpoons \mathrm{OH}^{-}+\mathrm{NH}_{4}{ }^{+} \\
& \mathrm{HCl} \leftrightharpoons \mathrm{H}^{+}+\mathrm{Cl}^{-} \\
& \downarrow \downarrow \\
& \mathrm{H}_{2} \mathrm{O} \quad \mathrm{NH}_{4} \mathrm{Cl} \text {. }
\end{aligned}
$$

Comparison of this reaction with that for $\mathrm{HC}_{2} \mathrm{H}_{3} \mathrm{O}_{2}$ and $\mathrm{NaOH}$ where we have a weak acid and strong base will bring out complete analogy. Experiment shows that a solution of $\mathrm{NH}_{4} \mathrm{Cl}$ in water is not neutral but slightly acid in reaction. Briefly 
stated, $\mathrm{NH}_{4} \mathrm{OH}$ does not completely neutralize an equivalent amount of $\mathrm{HCl}$ because it is a weak base. Conversely, water acts on pure $\mathrm{NH}_{4} \mathrm{Cl}$ to form some free $\mathrm{HCl}$ and $\mathrm{NH}_{4} \mathrm{OH}$.

438. Class IV: The Action of a Weak Acid and a Weak Base.-Under Class IV we shall include reactions between two little ionized substances, which give as products one little ionized and one highly ionized substance. The only reactions of importance in Class IV are those between a weak acid and a weak base, the products being water and a salt. Acetic acid and ammonium hydroxide are both moderately weak (but not extremely weak). They react thus:

$$
\mathrm{HC}_{2} \mathrm{H}_{3} \mathrm{O}_{2}+\mathrm{NH}_{4} \mathrm{OH} \leftrightharpoons \mathrm{NH}_{4} \mathrm{C}_{2} \mathrm{H}_{3} \mathrm{O}_{2}+\mathrm{H}_{2} \mathrm{O} \text {. }
$$

The reaction is not complete, as in the case of the action of a strong acid and a strong base, but reaches equilibrium when a few tenths of I per cent of the free un-ionized acid and free un-ionized base are still present in the solution. The conditions before and after the reactions are shown in Figs. 68 and 69.

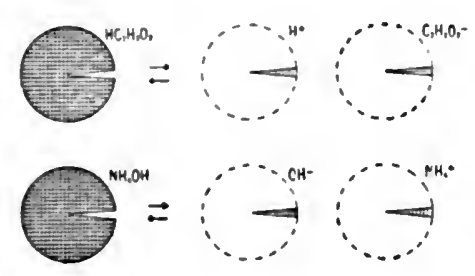

FIG. 68

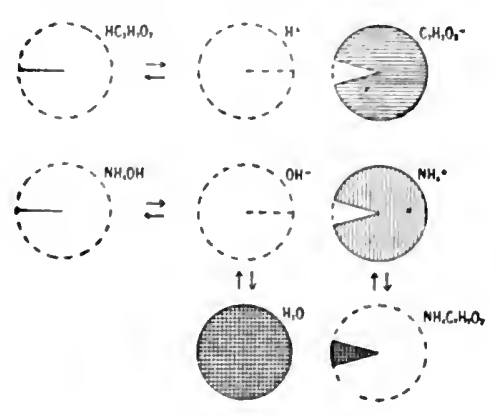

Fic. 69

If on the other hand the solution is made by dissolving solid $\mathrm{NH}_{4} \mathrm{C}_{2} \mathrm{H}_{3} \mathrm{O}_{2}$ in water partial hydrolysis takes place, giving a mixture the composition of which is also represented by Fig. 69 .

If both acid and base are extremely weak the extent of the hydrolysis will be much greater than in the case of $\mathrm{NH}_{4} \mathrm{C}_{2} \mathrm{H}_{3} \mathrm{O}_{2}$. In fact, in such cases hydrolysis may be so nearly complete that we may say that extremely weak acids in water solution do not form salts with extremcly weak bases. 
439. Heat of Ionization.-The heat liberated or absorbed by the complete dissociation into its ions of one formula weight of a dissolved electrolyte is called its heat of ionization. In some cases heat is absorbed, in other cases it is liberated, when the substances are ionized, but in the great majority of cases the heat of ionization is very small. For practical purposes we may say that the heat of ionization of readily ionizable electrolytes is almost negligible. Little ionized substances often have appreciable heats of ionization. This is notably the case with water, for which we have the following,

$$
\mathrm{H}^{+}+\mathrm{OH}^{-} \rightarrow \mathrm{H}_{2} \mathrm{O}+\mathrm{I}_{3}, 700 \text { cal. }
$$

It was stated earlier $(362)$ that the heat of neutralization of a strong acid by a strong base is almost the same in all cases, namely 13,700 calories. The reason can now be seen. We know that in the neutralization of a strong acid by a strong base in dilute solution the principal change is the union of $\mathrm{H}^{+}$and $\mathrm{OH}^{-}$ ions to form water. In other words, the simplified equation of neutralization is

$$
\mathrm{H}^{+}+\mathrm{OH}^{-} \rightarrow \mathrm{H}_{2} \mathrm{O} \text {. }
$$

Since strong acids and bases, as well as most salts, have negligible heats of ionization; and since, moreover, very little dissoçiation or union of ions, other than $\mathrm{H}^{+}$and $\mathrm{OH}^{-}$, occurs in neutralization (42 $\mathrm{r}$, Fig. 59), the heat produced in the reaction is simply that due to the formation of water from its ions. It is for this reason that heats of neutralization are practically the same for all strong acids and bases: 13,700 cal. for one formula weight (I8 g.) of water formed.

The heat of neutralization of ammonium hydroxide by a strong acid is 12,300 calories. The difference, $I 3,700-I 2,300=$ $\mathrm{I}, 400 \mathrm{cal}$, is the heat of ionization of the weak base.

In reactions between solutions of two highly ionized salts which form by interaction two other highly ionized and easily soluble salts no appreciable heat change is observed. This is because in such reactions very little change takes place (4I8, Fig. 55), and such changes as do occur are accompanied by nearly negligible heats of ionization. 
440. Indicators. - In addition to litmus, which is used so often to indicate the acidity or alkalinity of solutions, a number of other colored substances are also employed. These are called indicators. The more important indicators besides litmus are phenolphthalein and methyl orange. The former is a colorless substance which gives a bright red solution with alkalies. Methyl orange is orange color in neutral solution, pink in acid, and yellow in alkaline solution. In general, indicators are very complex chemical substances whose formulae need not be considered at present.

Since acid solutions always contain $\mathrm{H}^{+}$ions and alkaline solutions $\mathrm{OH}^{-}$, ions, we may say that an indicator is a substance which has one color in the presence of an excess of $\mathrm{H}^{+}$ions and a different color in the presence of an excess of $\mathrm{OH}^{-}$ions. We might expect that every indicator would show its transition shade of color in an exactly neutral solution; that is, in a solution where the number of $\mathrm{H}^{+}$ions equals the number of $\mathrm{OH}^{-}$ ions. This, however, is not the case. In other words, most indicators do not indicate perfect neutrality. Litmus is a nearly perfect indicator, but phenolphthalein shows a change of color when the number of $\mathrm{OH}^{-}$ions equals eighty times the number of $\mathrm{H}^{+}$ions; that is, if a solution contains more than eighty times as many $\mathrm{OH}^{-}$as $\mathrm{H}^{+}$ions it colors phenolphthalein red (the alkaline color); if it contains less than eighty times as many $\mathrm{OH}^{-}$ions as $\mathrm{H}^{+}$ions it leaves phenolphthalein colorless. On the other hand, methyl orange shows an orange color (its intermediate shade between pink, the acid color, and yellow, the alkaline color) when the number of $\mathrm{H}^{+}$ions is about a million times the number of $\mathrm{OH}^{-}$ions. Anomalous as it may seem at first thought, it is really fortunate that many of our indicators do not indicate perfect neutrality; for suppose we wish to discover how much acetic acid a certain solution contains. We may titrate it accurately with normal or decinormal sodium hydroxide or other strong base if we use the right indicator (137). Now we have learned that when acetic acid is mixed with exactly the theoretically equivalent amount of $\mathrm{NaOH}$ the resulting solution is not perfectly neutral but in reality slightly alkaline 
(434). In accord with this we found that a solution of $\mathrm{NaC}_{2} \mathrm{H}_{3} \mathrm{O}_{2}$ was slightly alkaline to litmus, showing that the number of $\mathrm{OH}^{-}$ ions was greater than the number of $\mathrm{H}^{+}$ions. Therefore we must use as a titration indicator one which shows its change of color when the number of $\mathrm{OH}^{-}$is greater than the number of $\mathrm{H}^{+}$ ions. We find that phenolphthalein proves to be just right for the purpose. In general, ie use phenolphthalein as indicator in titrating all moderately weak acids.

If we wish to titrate $\mathrm{NH}_{4} \mathrm{OH}$ with $\mathrm{HCl}$ we cannot use phenolphthalein, because a solution of $\mathrm{NH}_{4} \mathrm{Cl}$ contains more $\mathrm{H}^{+}$ than $\mathrm{OH}^{-}$ions. Such a solution "seems" acid to this indicator. We must use one which changes color when the number of $\mathrm{H}^{+}$ ions exceeds the number of $\mathrm{OH}^{-}$ions, and for this case we find methyl orange satisfactory. In general, we use methyl orange in titrating moderately weak bases. The acid used in such titrations must be a strong one. In titrating a strong base with a strong acid any of these indicators gives sufficiently accurate results. Table XVIII gives the colors of indicators in solutions

TABLE XVIII

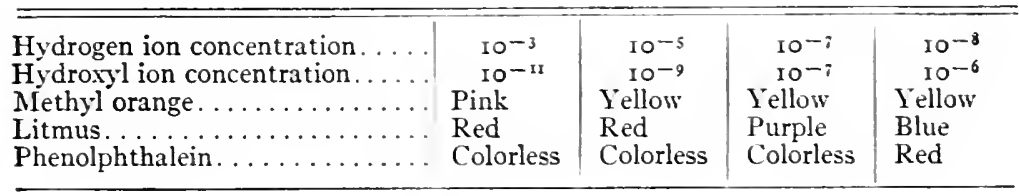

of hydrogen and hydroxyl ion concentrations near those at which the color change occurs. In this table the concentrations are given in gram molecular weights per liter. If the $\mathrm{H}^{+}$concentration is $10^{-3}, \mathrm{I}, \infty$ liters contain $\mathrm{I} g$. of $\mathrm{H}^{+}$ion.

441. Summary on Equilibrium between Soluble Electrolytes.-If we mix solutions of two electrolytes, $A B$ and $C D$, having no ion in common, a double decomposition reaction,

$$
A B+C D \leftrightharpoons A D+C B,
$$

takes place to a greater or less extent, because of the tendency of each positive ion to combine with each negative ion present. 
If all four substances of the preceding equation are highly ionized (Class I, 4r5), the mixed solution will contain largely the four sorts of free ions, $A^{+}, B^{-}, C^{+}$, and $D^{-}$. Only a small percentage of the dissolved material will be present as molecules. Figure 56 represents equilibrium conditions in an example in which equivalent amounts of initial materials were used.

If one of the four substances (say $A D$ ) is little ionized (Class II, 419), then the large numbers of $A^{+}$and $D^{-}$ions shown in Fig. 56 cannot exist side by side in the mixed solution, since they will very largely combine to form $A D$ molecules. The disappearance of $A^{+}$and $D^{-}$ions allows $A B$ and $C D$ molecules more or less completely to dissociate. The final result, shown in Figs. 59, 6r, and 63 , is a nearly complete reaction, $A D$ being present almost wholly in un-ionized form and $C D$ to a small extent as molecules, but largely as $C^{+}$and $D^{-}$ions.

A generalization of much importance is found in the Common Ion Law: suppression of the ionization of a little ionize substance occurs when we add to its solution a highly ionized substance having a common ion.

Since in pure water the number of $\mathrm{H}^{+}$ions is equal to the number of $\mathrm{OH}^{-}$ions, and since we may consider pure water a perfectly neutral substance, we define a neutral solution as one in which the number of $\mathrm{H}^{+}$ions is exactly equal to the number of $\mathrm{OH}^{-}$ions.

Class III $(434,437)$ comprises reactions in which one little ionized substance reacts with a highly ionized substance to form products one of which is slightly, the other highly, ionized. Examples are found in the neutralization of a weak acid by a strong base; or of a weak base by a strong acid. In such cases the reaction is more or less incomplete. The weaker the acid or base taken, the less complete is the neutralization. Conversely, salts of weak acids or of weak bases are hydrolyzed by water. The former give solutions which are alkaline, the latter those which are acid, in reaction. This kind of action is called hydrolytic dissociation.

Under Class IV (438) it was pointed out that weak acids and weak bases always react incompletely, and that when either 
or both are extremely weak, salt formation may not occur in solution (r77).

We have seen that indicators change color according to the concentration of $\mathrm{H}^{+}$and $\mathrm{OH}^{-}$ions present. Litmus shows its neutral tint when the numbers of $\mathrm{H}^{+}$and $\mathrm{OH}^{-}$ions are nearly equal. Phenolphthalein requires an excess of $\mathrm{OH}^{-}$ ions to change color, while methyl orange requires an excess of $\mathrm{H}^{+}$ions. 


\section{CHAPTER XIX}

\section{APPLICATIONS OF THE IONIC HYPOTHESIS. REACTIONS INVOLVING CHANGES OF STATE}

442. Introduction.- - In the present chapter we shall study precipitation from the standpoint of the ionic hypothesis in order to understand the underlying principles of this most important means of separating substances. In equations for precipitation reactions, the substance precipitated will be indicated by a downward-pointing arrow.

If we consider the familiar examples of precipitation represented by the following equations,

$$
\begin{aligned}
& \mathrm{AgNO}_{3}+\mathrm{HCl} \leftrightharpoons \mathrm{AgCl} \downarrow+\mathrm{HNO}_{3}, \\
& \mathrm{BaCl}_{2}+\mathrm{H}_{2} \mathrm{SO}_{4} \leftrightharpoons \mathrm{BaSO}_{4} \downarrow+2 \mathrm{HCl},
\end{aligned}
$$

we might conclude that $\mathrm{AgCl}$ and $\mathrm{BaSO}_{4}$ are precipitated because they are insoluble in water. We might even be tempted to say that in the reaction

$$
A B+C D \leftrightharpoons A D+C B,
$$

if either $A D$ or $C B$ is an insoluble substance it will be precipitated. This statement contains something of the truth, but it is far from the whole truth, as the following examples will prove. Calcium carbonate, $\mathrm{CaCO}_{3}$ (marble), is an almost insoluble substance. If we mix solutions of calcium chloride and carbonic acid we might expect to get a precipitate of calcium carbonate, thus,

$$
\mathrm{CaCl}_{2}+\mathrm{H}_{2} \mathrm{CO}_{3} \div \mathrm{CaCO}_{3} \downarrow+2 \mathrm{HCl} .
$$

Not a trace of precipitate is formed. On the other hand potassium chlorate, $\mathrm{KClO}_{3}$, is easily soluble in water; but if we add a saturated solution of potassium bromide, $\mathrm{KBr}$, to a saturated solution of sodium chlorate, $\mathrm{NaClO}_{3}$, a precipitate of $\mathrm{KClO}_{3}$ forms. Evidently the matter is not as simple as at first thought it appears to be. The separation of a solid from a solution is 
obviously the reverse of the passage of a solid into solution. Accordingly, in beginning the study of precipitation, it will be advisable for the student to read again sections 120-23. In section $\mathbf{I 2 2}$ it is stated, "A solution which at a fixed temperature will dissolve no more of a given substance is called a saturated solution. When we speak of the solubility of a substance we mean the amount of substance dissolved in a given amount of water in the case of the saturated solution."

443. The Kinetic Theory of Solution.-When a soluble salt is brought into water its molecules begin to leave the surface of the solid and pass into the water. Immediately thereafter dissolved salt molecules will occasionally strike the surface of the solid and in some cases remain attached thereto. Finally, when the solution has become saturated we may imagine that the equilibrium between dissolved and solid salt is the result of the passage of molecules into and out of solution at exactly equal rates, thus:

$$
A B \leftrightarrows A B
$$

Solid Dissolved

This picture is, however, incomplete, since the salt is partly ionized. The dissolved molecules are therefore in equilibrium with their ions as well as with the solid salt, thus:

$$
\begin{gathered}
A B \leftrightarrows A B \leftrightarrows A^{+}+B^{-} \\
\text {Solid Dissolved }
\end{gathered}
$$

444. Graphic Representation of a Solid Electrolyte in Equilibrium with Its Saturated Solution.-We shall represent

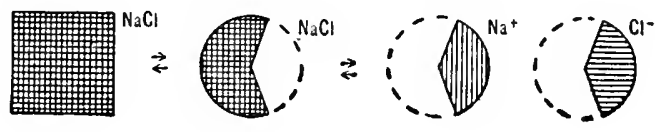

FIG. 70

a solid electrolyte (acid, base, or salt) graphically by a crosshatched square. The condition of a saturated solution of a soluble salt $(\mathrm{NaCl}$, for example) in contact with an excess of the solid salt may then be represented as in Fig. 70. 


\section{The Solubility of Molecules. Molecular Solubility.-} If we except a small number of electrolytes like sulfuric and nitric acids, which mix with water in all proportions, all other acids, bases, and salts have limited solubilities in water. Since all electrolytes are more or less ionized in solution, the dissolved substance is present partly as molecules and partly as ions. Therefore the total solubility of a substance in a solution saturated at a given temperature is the sum of the solubility of its molecules and the solubility of its ions. It seems reasonable to assume that the limited solubility of an electrolyte as a whole is the result of the limited solubility of its molecules rather than of its ions. Two reasons may be given for this assumption which will be amply confirmed by additional evidence to be considered later.

In the first place the solid salt passes into and out of solution as molecules (see Fig. 70). If the molecules have a limited solubility, this would limit the solubility of the ions as well, since the latter and the former are directly in equilibrium. Therefore it is sufficient to assume limited solubility of the molecules in order to explain limited total solubility. Secondly, the small solubility of a difficultly soluble salt like $\mathrm{CaSO}_{4}$ ( 100 c.c. of water dissolve $0.25 \mathrm{~g}$. of $\mathrm{CaSO}_{4}$ ) cannot be due to a correspondingly small solubility of $\mathrm{Ca}^{++}$or $\mathrm{SO}_{4}^{--}$ions, since solutions of $\mathrm{CaCl}_{2}$ and many other easily soluble and highly ionized calcium salts contain an abundance of $\mathrm{Ca}^{++}$ions, and solutions of $\mathrm{H}_{2} \mathrm{SO}_{4}$ and many easily soluble and highly ionized sulfates contain large concentrations of $\mathrm{SO}_{4}^{--}$ions. We shall assume, therefore, that at a given temperature the solubility of an acid, base, or salt is limited by the solubility of its molecules; and we shall call the solubility of the molecules (in the saturated solution) the molecular solubility (abbreviated M.S.) of the substance. Summarizing, we may say that when a solid electrolyte is mixed with water at a fixed temperature the substance dissolves and the concentration of the solution increases until the M.S. is reached; the solution is then saturated (at that temperature), and the molecules are in equilibrium with the ions and with the solid substance. 
446. The Cause of Precipitation.-We are now ready to apply the foregoing principles, together with those learned in chapter xviii, to the process of precipitation. We have learned (414) that in the reaction

$$
A B+C D \leftrightarrows A D+C B,
$$

if all four substances are easily soluble and highly ionized the resulting solution contains largely the four sorts of ions, together

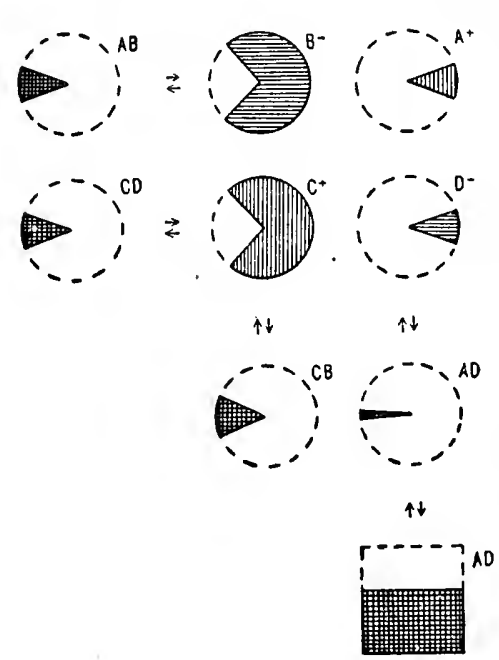

FIG. 7 I with small proportions of the four kinds of molecules. The conditions before and after the reaction are shown in Figs. 55 and 56 . Now let us suppose that one of the products $A D$ is not very soluble, so that its molecular solubility (M.S.) is less than that corresponding to the concentration of the molecules of $A D$ formed in reaction (Fig. 56). In this case the amount of molecules of $A D$ formed in excess of the M.S. will separate out of solution as a precipitate. Fig. 7 I shows the resulting condition for the case where the M.S. of $A D$ is rather small but not extremely small. By comparison of Figs. 56 and $7 \mathrm{I}$ we see that an appreciable shift in equilibrium of the dissolved substances accompanies the partial precipitation of $A D$.

447. The Precipitation of $\mathrm{KClO}_{3}$. - An actual example conforming perfectly to the conditions set forth in the preceding paragraph is found in the reaction

$$
\mathrm{KBr}+\mathrm{NaClO}_{3} \leftrightharpoons \mathrm{KClO}_{3} \downarrow+\mathrm{NaBr} .
$$

Of the four salts, all are very soluble except $\mathrm{KClO}_{3}$, which dissolves only to the extent of $7 \mathrm{~g}$. in roo c.c. of water at $18^{\circ}$. All four salts are highly and about equally ionized in solutions of equal concentration. The conditions of the solutions of $\mathrm{KBr}$ 
and $\mathrm{NaClO}_{3}$ before mixing are shown with sufficient accuracy by Fig. 55, while Fig. 56 shows the condition which the mixed solution would reach if $\mathrm{KClO}_{3}$ were also very soluble. It happens, however, that the amount of molecular $\mathrm{KClO}_{3}$ which tends to be formed exceeds the rather small M.S. of this substance, and in consequence the excess above the M.S. separates as a precipitate. Precipitation continues until the amount of molecular $\mathrm{KClO}_{3}$ left in solution is equal to the M.S. of this substance. The mixture is then in the condition of equilibrium shown in Fig. 7I. Comparison of Figs. 56 and 7 I shows that the removal (by precipitation) of $\mathrm{KClO}_{3}$ from the solution causes a marked shift in the equilibrium. We may trace the stages as follows: Fig. 56 shows the condition that would exist if no precipitation occurred. The removal of $\mathrm{KClO}_{3}$, results in the further union of $\mathrm{K}^{+}$and $\mathrm{ClO}_{3}{ }^{-}$ions to form more $\mathrm{KClO}_{3}$. The resulting loss of $\mathrm{K}^{+}$and $\mathrm{ClO}_{3}{ }^{-}$ions promotes the further ionization of $\mathrm{KBr}$ and $\mathrm{NaClO}_{3}$ respectively and thus increases the numbers of $\mathrm{Br}^{-}$and $\mathrm{Na}^{+}$ions. The latter ions unite in part to form additional molecular $\mathrm{NaBr}$. The final result is the change from the condition of Fig. 56 to that of Fig. 71. The principles here exemplified apply to all double decomposition precipitations.

448. The Precipitation of $\mathrm{CaCO}_{3}$. - Let us now consider a case in which one of the products is precipitated almost completely. The reaction

$$
\mathrm{CaCl}_{2}+\mathrm{Na}_{2} \mathrm{CO}_{3} \Rightarrow \mathrm{CaCO}_{3} \downarrow+2 \mathrm{NaCl},
$$

in which $\mathrm{CaCO}_{3}$ is the precipitate, will serve as a typical illustration. Although $\mathrm{CaCO}_{3}$ appears to be insoluble in water, it is in fact slightly soluble, and has therefore a definite but very small M.S. The other three salts, $\mathrm{CaCl}_{2}, \mathrm{Na}_{2} \mathrm{CO}_{3}$, and $\mathrm{NaCl}$, are easily soluble and highly ionized, and in consequence the reaction between solutions of $\mathrm{CaCl}_{2}$ and $\mathrm{Na}_{2} \mathrm{CO}_{3}$ tends to reach the condition shown in Fig. 56 illustrating a Class I reaction (414). In this respect it completely resembles the reaction

$$
\mathrm{KBr}+\mathrm{NaClO}_{3} \leftrightharpoons \mathrm{KClO}_{3} \downarrow+\mathrm{NaBr} .
$$


It differs however from this reaction in that the M.S. of $\mathrm{CaCO}_{3}$ is extremely small compared with the M.S. of $\mathrm{KClO}_{3}$. In con-

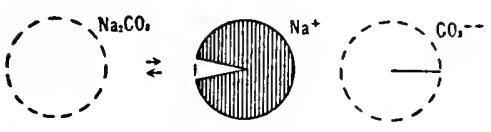
sequence the $\mathrm{CaCO}_{3}$ formed precipitates almost completely, as illustrated in Fig. 72. In all double decomposition reactions

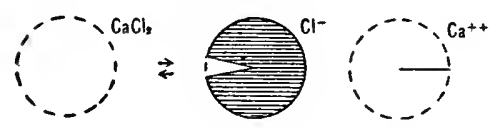
of the above-mentioned types (all involved substances highly ionized) the precipitation is the

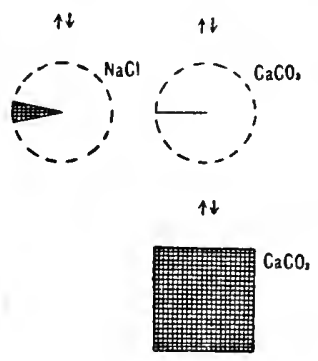
more complete the less the M.S. of the precipitate.

449. The Action of $\mathrm{H}_{2} \mathrm{CO}_{3}$ on $\mathrm{CaCl}_{2}$. - In section 442 it was pointed out that $\mathrm{H}_{2} \mathrm{CO}_{3}$ does not precipitate $\mathrm{CaCO}_{3}$, as we might expect according to the following hypothetical FIG. 72 equation:

$$
\mathrm{CaCl}_{2}+\mathrm{H}_{2} \mathrm{CO}_{3} \leftrightarrows \mathrm{CaCO}_{3} \downarrow+2 \mathrm{HCl} \text {. }
$$

The reason is as follows: carbonic acid $\mathrm{H}_{2} \mathrm{CO}_{3}$ is a very weak acid and in consequence yields but very few $\mathrm{CO}_{3}{ }^{--}$ions; and although $\mathrm{CaCl}_{2}$ gives an abundance of $\mathrm{Ca}^{++}$ions, the concentration of $\mathrm{CO}_{3}^{--}$ions is so small that the concentration of $\mathrm{CaCO}_{3}$ molecules formed is less than the M.S. of this substance. Therefore no precipitation of $\mathrm{CaCO}_{3}$ takes place. The difference in behavior of $\mathrm{H}_{2} \mathrm{CO}_{3}$ and $\mathrm{Na}_{2} \mathrm{CO}_{3}$ toward a solution of $\mathrm{CaCl}_{2}$ is wholly due to the difference in their tendencies to ionize, in consequence of which a solution of $\mathrm{H}_{2} \mathrm{CO}_{3}$ contains exceedingly few $\mathrm{CO}_{3}^{--}$ions as compared with a solution of $\mathrm{Na}_{2} \mathrm{CO}_{3}$.

The behavior of $\mathrm{H}_{2} \mathrm{CO}_{3}$ is typical of that of all weak (little ionized) electrolytes. In the precipitation of salts, weak acids and bases are, in general, less efficient precipitants than their salts, since the latter are highly ionized.

450. The Precipitation of Magnesium Hydroxide, $\mathrm{Mg}(\mathrm{OH})_{2}$. -We shall next discuss in detail the precipitation of $\mathrm{Mg}(\mathrm{OH})_{2}$, 
not so much because of the chemical importance of this substance, but because the reactions illustrate in a striking way some of the most important principles of ionic equilibrium.

If we add $\mathrm{NaOH}$ to a solution of magnesium chloride, $\mathrm{MgCl}_{2}$, we obtain an abundant white precipitate of $\mathrm{Mg}(\mathrm{OH})_{2}$, formed as follows:

$$
\mathrm{MgCl}_{2}+{ }_{2} \mathrm{NaOH} \leftrightharpoons \mathrm{Mg}(\mathrm{OH})_{2} \downarrow+2 \mathrm{NaCl}
$$

If we use $\mathrm{NH}_{4} \mathrm{OH}$ instead of $\mathrm{NaOH}$ the reaction is similar but reaches a state of equilibrium when only a part of the magnesium is precipitated:

$$
\mathrm{MgCl}_{2}+2 \mathrm{NH}_{4} \mathrm{OH} \leftrightharpoons \mathrm{Mg}(\mathrm{OH})_{2} \downarrow+2 \mathrm{NH}_{4} \mathrm{Cl} \text {. }
$$

If we add to a $\mathrm{MgCl}_{2}$ solution a solution of $\mathrm{NH}_{4} \mathrm{OH}$ mixed with sufficient $\mathrm{NH}_{4} \mathrm{Cl}$, no precipitation occurs. We shall now explain these facts. In the first place we may say that the action of $\mathrm{NaOH}$ on $\mathrm{MgCl}_{2}$ is analogous to the action of $\mathrm{Na}_{2} \mathrm{CO}_{3}$ on $\mathrm{CaCl}_{2}$, the two equations being

$$
\begin{array}{rl}
\mathrm{MgCl}_{2}+{ }_{2} \mathrm{NaOH} & \mathrm{Mg}(\mathrm{OH})_{2} \downarrow+2 \mathrm{NaCl}, \\
\mathrm{CaCl}_{2}+\mathrm{Na}_{2} \mathrm{CO}_{3} \leftrightarrows \mathrm{CaCO}_{3} \downarrow+2 \mathrm{NaCl} .
\end{array}
$$

Both $\mathrm{MgCl}_{2}$ and $\mathrm{NaOH}$, like $\mathrm{CaCl}_{2}$ and $\mathrm{Na}_{2} \mathrm{CO}_{3}$, are easily soluble and highly ionized; sodium chloride, one of the products in both reactions, is also highly ionized. The other product in the first reaction, $\mathrm{Mg}(\mathrm{OH})_{2}$, is but slightly soluble, and like $\mathrm{CaCO}_{3}$ it is therefore precipitated almost completely.

If, however, we use $\mathrm{NH}_{4} \mathrm{OH}$ instead of $\mathrm{NaOH}$, the reaction is far from complete. 'The reason can best be seen by the aid of Figs. 73 and 74. Figure 73 shows the condition of the solutions before they are mixed; Fig. 74 represents the condition of the mixture.

It will be recalled, as shown in Fig. 73 , that $\mathrm{NH}_{4} \mathrm{OH}$ is but little ionized. Still its solution yields sufficient $\mathrm{OH}^{-}$ions to produce in reaction with magnesium chloride solution more $\mathrm{Mg}(\mathrm{OH})_{2}$ than the M.S. of the latter difficultly soluble substance. The excess of $\mathrm{Mg}(\mathrm{OH})_{2}$ above its M.S. precipitates, Fig. 74. As these changes go on, molecules of $\mathrm{NH}_{4} \mathrm{OH}$ continue to ionize, thus 
bringing into the solution far more $\mathrm{NH}_{4}^{+}$ions than were originally present (cf. Figs. 73 and 74). The presence of the large excess of $\mathrm{NH}_{4}{ }^{+}$ions restricts the number of $\mathrm{OH}^{-}$ions to such an extent that a state of equilibrium is reached in reaction,

$$
\mathrm{Mg}(\mathrm{OH})_{2}(\text { dissolved }) \leftrightarrows \mathrm{Mg}^{++}+{ }_{2} \mathrm{OH}^{-},
$$

while there is still a considerable amount of magnesium in the form of $\mathrm{Mg}^{++}$ions and $\mathrm{MgCl}_{2}$ molecules left in the solution. After this condition is reached no more $\mathrm{Mg}(\mathrm{OH})_{2}$ precipitates. We therefore conclude that $\mathrm{NH}_{4} \mathrm{OH}$ precipitates $\mathrm{Mg}(\mathrm{OH})_{2}$ only partially, (I) because $\mathrm{NH}_{4} \mathrm{OH}$ is a weak or little ionized base, and (2)
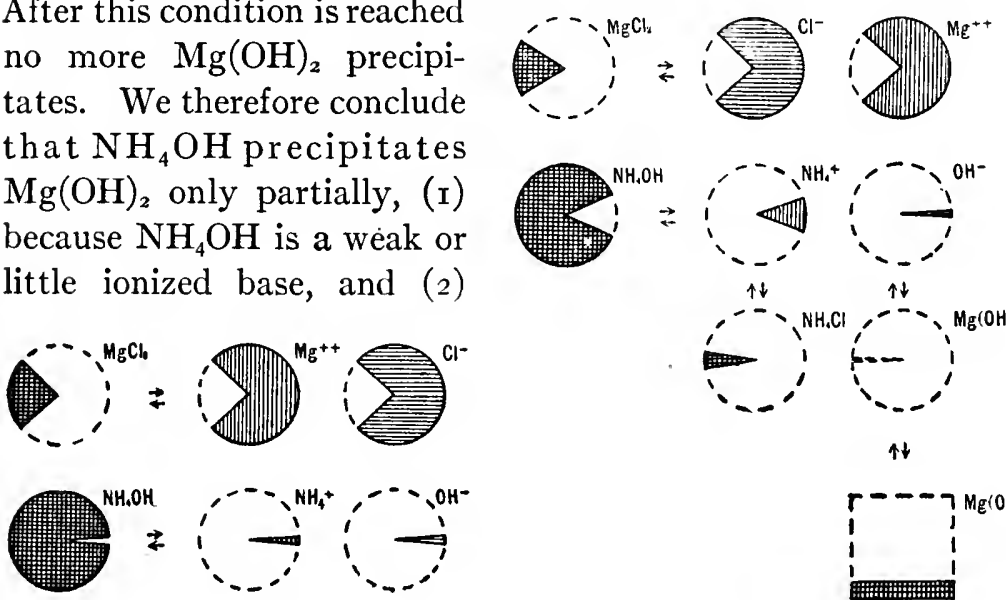

Fig. 73

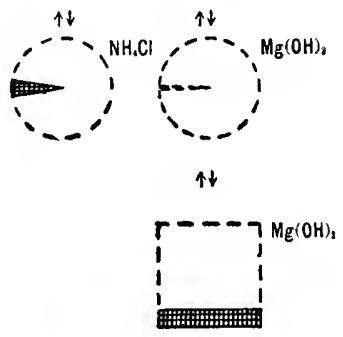

FIG. 74

because the accumulation of $\mathrm{NH}_{4}^{+}$ions (Fig. 74) finally restricts the $\mathrm{OH}^{-}$concentration to so small a value that the $\mathrm{Mg}^{++}$ and $\mathrm{OH}^{-}$ions are just in equilibrium with the amount of $\mathrm{Mg}(\mathrm{OH})_{2}$ corresponding to its M.S. It will now be easy to understand why no $\mathrm{Mg}(\mathrm{OH})_{2}$ is precipitated when $\mathrm{NH}_{4} \mathrm{OH}$, mixed with considerable $\mathrm{NH}_{4} \mathrm{Cl}$, is added to a $\mathrm{MgCl}_{2}$ solution.

The $\mathrm{NH}_{4} \mathrm{Cl}$ furnishes at once such an excess of $\mathrm{NH}_{4}{ }^{+}$ions that the $\mathrm{OH}^{-}$concentration is decreased to so small a value that less $\mathrm{Mg}(\mathrm{OH})_{2}$ is formed in the reaction

$$
\mathrm{Mg}^{++}+{ }_{2} \mathrm{OH}^{-} \leq \mathrm{Mg}(\mathrm{OH})_{2}
$$

than corresponds to its M.S. Therefore no precipitation occurs.

45I. The Precipitation of Ferric Hydroxide, $\mathrm{Fe}(\mathrm{OH})_{3}$. -The reaction

$$
\mathrm{FeCl}_{3}+{ }_{3} \mathrm{NH}_{4} \mathrm{OH} \leftrightarrows \mathrm{Fe}(\mathrm{OH})_{3} \downarrow+{ }_{3} \mathrm{NH}_{4} \mathrm{Cl}
$$


takes place with the practically complete precipitation of brown $\mathrm{Fe}(\mathrm{OH})_{3}$, which is almost insoluble in water. The completeness of precipitation is not noticeably affected by the addition of much $\mathrm{NH}_{4} \mathrm{Cl}$. There are two reasons for the difference in behavior of $\mathrm{Fe}(\mathrm{OH})_{3}$ and $\mathrm{Mg}(\mathrm{OH})_{2}$ : (I) the former is much more insoluble in water than the latter, so that the M.S. of the latter (although small) is perhaps $\mathrm{I}, 000$ times as large as that of the former; (2) $\mathrm{Mg}(\mathrm{OH})_{2}$ is a rather strong (highly ionized) base, while $\mathrm{Fe}(\mathrm{OH})_{3}$ is a very weak base. Therefore in the reactions

$$
\begin{aligned}
& \mathrm{Mg}^{++}+{ }_{2} \mathrm{OH}^{-} \leftrightharpoons \mathrm{Mg}(\mathrm{OH})_{2} \text { (dissolved), } \\
& \mathrm{Fe}^{+++}+{ }_{3} \mathrm{OH}^{-} \leftrightharpoons \mathrm{Fe}(\mathrm{OH})_{3} \text { (dissolved), }
\end{aligned}
$$

for equal concentrations of $\mathrm{Mg}^{++}, \mathrm{Fe}^{+++}$, and $\mathrm{OH}^{-}$ions far less $\mathrm{Mg}(\mathrm{OH})_{2}$ is formed than $\mathrm{Fe}(\mathrm{OH})_{3}$. The presence of $\mathrm{NH}_{4} \mathrm{Cl}$ decreases the $\mathrm{OH}^{-}$concentration of $\mathrm{NH}_{4} \mathrm{OH}$, but not sufficiently to prevent the practically complete precipitation of $\mathrm{Fe}(\mathrm{OH})_{3}$ because of the weakness of the latter and its exceedingly small M.S. The weaker a base and the smaller its M.S., the more completely is il precipitated by $\mathrm{N} \mathrm{H}_{4} \mathrm{OH}$, and the less its precipitation is hindered by the presence of ammonium salts.

452. Classification of Precipitations.- The various examples of precipitation just studied cover the important principles con. cerned We may now cite a few additional examples of each of these classes of precipitation. In the reaction

$$
\mathrm{KBr}+\mathrm{NaClO}_{3}=\mathrm{KClO}_{3} \downarrow+\mathrm{NaBr},
$$

all four substances are highly ionized, and all but $\mathrm{KClO}_{3}$ are very soluble. The latter is partially precipitated because it is formed in excess of its not very large M.S. The following reactions are of this class:

$$
\begin{aligned}
\mathrm{CaCl}_{2}+2 \mathrm{NaClO}_{3} & =2 \mathrm{NaCl} \downarrow+\mathrm{Ca}\left(\mathrm{ClO}_{3}\right)_{2}, \\
\mathrm{~Pb}\left(\mathrm{NO}_{3}\right)_{2}+2 \mathrm{NaCl}_{2} & =\mathrm{PbCl}_{2} \downarrow+2 \mathrm{NaNO}_{3}, \\
\mathrm{CaCl}_{2}+\mathrm{Na}_{2} \mathrm{SO}_{4} & =\mathrm{CaSO}_{4} \downarrow+2 \mathrm{NaCl} .
\end{aligned}
$$

In the first reaction saturated solutions are required to give a precipitate of $\mathrm{NaCl}$. 
In the second example studied,

$$
\mathrm{CaCl}_{2}+\mathrm{Na}_{2} \mathrm{CO}_{3} \leftrightarrows \mathrm{CaCO}_{3} \downarrow+2 \mathrm{NaCl}
$$

the precipitate $\mathrm{CaCO}_{3}$ has an extremely small M.S. Its precipitation by $\mathrm{Na}_{2} \mathrm{CO}_{3}$ is almost complete. Other reactions of this class are:

$$
\begin{aligned}
\mathrm{AgNO}_{3}+\mathrm{NaCl} & =\mathrm{AgCl} \downarrow+\mathrm{NaNO}_{3}, \\
\mathrm{AgNO}_{3}+\mathrm{KBr} & =\mathrm{AgBr} \downarrow+\mathrm{KNO}_{3}, \\
\mathrm{~Pb}\left(\mathrm{NO}_{3}\right)_{2}+\mathrm{CuSO}_{4} & =\mathrm{PbSO}_{4} \downarrow+\mathrm{Cu}\left(\mathrm{NO}_{3}\right)_{2}, \\
\mathrm{BaCl}_{2}+\mathrm{Na}_{2} \mathrm{SO}_{4} & =\mathrm{BaSO}_{4} \downarrow+2 \mathrm{NaCl}, \\
\mathrm{MgCl}_{2}+2 \mathrm{NaOH} & =\mathrm{Mg}(\mathrm{OH})_{2} \downarrow+2 \mathrm{NaCl} .
\end{aligned}
$$

In the third example (449) it was shown that $\mathrm{H}_{2} \mathrm{CO}_{3}$ did not give with $\mathrm{CaCl}_{2}$ a precipitate of $\mathrm{CaCO}_{3}$, because the former is very little ionized and therefore yields very few $\mathrm{CO}_{3}^{--}$ions. The following pairs of substances also fail to give precipitates, because in each case of the weakness of the acid coupled with the moderate solubility of the salt, that might be precipitated:

$$
\begin{aligned}
& \mathrm{CaCl}_{2} \text { and } \mathrm{H}_{3} \mathrm{PO}_{4}, \\
& \mathrm{FeCl}_{2} \text { and } \mathrm{H}_{2} \mathrm{~S}, \\
& \mathrm{AgNO}_{3} \text { and } \mathrm{HC}_{2} \mathrm{H}_{3} \mathrm{O}_{2} \text {. }
\end{aligned}
$$

On the other hand the reactions

$$
\begin{aligned}
& 3 \mathrm{CaCl}_{2}+2 \mathrm{Na}_{3} \mathrm{PO}_{4}=\mathrm{Ca}_{3}\left(\mathrm{PO}_{4}\right)_{2} \downarrow+6 \mathrm{NaCl}, \\
& \mathrm{FeCl}_{2}+\left(\mathrm{NH}_{4}\right)_{2} \mathrm{~S}=\mathrm{FeS} \downarrow+2 \mathrm{NH}_{4} \mathrm{Cl}, \\
& \mathrm{AgNO}_{3}+\mathrm{NaC}_{2} \mathrm{H}_{3} \mathrm{O}_{2}=\mathrm{AgC} \mathrm{H}_{3} \mathrm{O}_{2} \downarrow+\mathrm{NaNO}_{3},
\end{aligned}
$$

give abundant precipitates, because, instead of the weak acids, we use their salts, which are highly ionized.

The fourth example, which belongs to Class II, was taken up in contrast to the fifth example, which is typical of a fourth class of precipitation reactions. The fourth and fifth examples were:

$$
\begin{gathered}
\mathrm{MgCl}_{2}+2 \mathrm{NaOH} \leftrightarrows \mathrm{Mg}(\mathrm{OH})_{2} \downarrow+2 \mathrm{NaCl}, \\
\mathrm{MgCl}_{2}+2 \mathrm{NH}_{4} \mathrm{OH} \leftrightarrows \mathrm{Mg}(\mathrm{OH})_{2} \downarrow+2 \mathrm{NH}_{4} \mathrm{Cl} .
\end{gathered}
$$

The precipitation of $\mathrm{Mg}(\mathrm{OH})_{2}$ is nearly complete in the first reaction but only partial in the second, owing to the moderate solubility of $\mathrm{Mg}(\mathrm{OH})_{2}$ and the little ionization of $\mathrm{NH}_{4} \mathrm{OH}$, especially in the presence of its salts. 
The following reaction of a weak electrolyte $\left(\mathrm{H}_{2} \mathrm{~S}\right)$ also results in partial precipitation:

$$
\mathrm{ZnCl}_{2}+\mathrm{H}_{2} \mathrm{~S} \leftrightarrows \mathrm{ZnS} \downarrow+{ }_{2} \mathrm{HCl} .
$$

In this case the precipitation is prevented by the presence of an excess of $\mathrm{HCl}$ or other strong acid, because of the suppression of the ionization of the $\mathrm{H}_{2} \mathrm{~S}$ by the $\mathrm{H}^{+}$ions of the strong acid.

The sixth example dealt with the precipitation of $\mathrm{Fe}(\mathrm{OH})_{3}$ :

$$
\mathrm{FeCl}_{3}+{ }_{3} \mathrm{NH}_{4} \mathrm{OH} \leftrightarrows \mathrm{Fe}(\mathrm{OH})_{3} \downarrow+3 \mathrm{NH}_{4} \mathrm{Cl} \text {. }
$$

In this case the precipitate is so insoluble (M.S. so small) and so weak (little ionized) that it is practically completely precipitated by $\mathrm{NH}_{4} \mathrm{OH}$ even in the presence oi $\mathrm{NH}_{4} \mathrm{Cl}$. Although we call $\mathrm{NH}_{4} \mathrm{OH}$ a weak base, it is enormously stronger than $\mathrm{Fe}(\mathrm{OH})_{3}$, even when mixed with much $\mathrm{NH}_{4} \mathrm{Cl}$. Other reactions which fall into this class are:

$$
\begin{aligned}
\mathrm{AlCl}_{3}+{ }_{3} \mathrm{NH}_{4} \mathrm{OH} & \leftrightharpoons \mathrm{Al}(\mathrm{OH})_{3} \downarrow+3 \mathrm{NH}_{4} \mathrm{Cl}, \\
\mathrm{CrCl}_{3}+3 \mathrm{NH}_{4} \mathrm{OH} & \leftrightharpoons \mathrm{Cr}(\mathrm{OH})_{3} \downarrow+3 \mathrm{NH}_{4} \mathrm{Cl}, \\
\mathrm{CuSO}_{4}+\mathrm{H}_{2} \mathrm{~S} & \leftrightharpoons \mathrm{CuS} \downarrow+\mathrm{H}_{2} \mathrm{SO}_{4}, \\
\mathrm{Ag}_{2} \mathrm{SO}_{4}+\mathrm{H}_{2} \mathrm{~S} & \leftrightharpoons \mathrm{Ag}_{2} \mathrm{~S} \downarrow+\mathrm{H}_{2} \mathrm{SO}_{4} .
\end{aligned}
$$

Excess of $\mathrm{NH}_{4} \mathrm{Cl}$ in the first two cases, and of $\mathrm{HCl}$ or $\mathrm{H}_{2} \mathrm{SO}_{4}$ in the last two cases, fails to prevent practically complete precipitation.

\section{Precipitation by Adding a Substance Having a Common} Ion.-We have learned in the foregoing chapter (432) that if we add to the solution of an electrolyte, $A B$, enough of another highly ionized electrolyte, $A C$, having a common ion, $A$, to increase the concentration of the common ion, the degree of ionization of the first substance will be suppressed. If now the substance $A B$ is not very soluble, the suppression of its ionization caused by adding $A C$ may increase the concentration of the $A B$ molecules to such an extent as to exceed the M.S. of $A B$. In consequence part of $A B$ will separate out as a precipitate. For example, if a few bubbles of $\mathrm{HCl}$ gas are passed into a saturated solution of $\mathrm{NaCl}$, a precipitate of $\mathrm{NaCl}$ is formed. A similar result is also produced by adding a little concentrated $\mathrm{HCl}$ to a saturated salt solution. In each case the ionization 
of the salt is suppressed by reason of the increase in concentration of the $\mathrm{Cl}^{-}$ions, and the concentration of the molecular $\mathrm{NaCl}$ increased beyond the M.S. of this substance. Salt precipitates until the concentration of molecular $\mathrm{NaCl}$ falls to the value corresponding to its M.S. Another example illustrating the same principle is found in the precipitation of $\mathrm{KClO}_{3}$ from its saturated solution by the addition of a saturated solution of either $\mathrm{KBr}$ or $\mathrm{NaClO}_{3}$. We may say that as a general rule the total solubility of a salt (molecular and ionic) is diminished by the presence in the solution of another electrolyte having a common ion.

454. Conditions Favoring Precipitation.-In the reaction

$$
A B+C D=A D+C B
$$

precipitation will occur if one of the products, say $A D$, is formed as molecules in greater concentration than its molecular solubility. In brief, if the M.S. is exceeded, precipitation will occur. Now the smaller the M.S. of $A D$, the more probably will it be exceeded.

On the other hand the M.S. is the more likely to be exceeded the greater the concentration of $A D$ which tends to be produced in the reaction. The various factors which determine the amount of $A D$ produced (when $A D$ is soluble) have been discussed at length in chapter xviii (Summary, 44I).

These applications of the ionic hypothesis have the following bearing on the practice of precipitation. In the first place, if we are to precipitate from solution an "insoluble" salt of a weak acid we use as the precipitant a soluble salt of the weak acid instead of the acid itself, since the former is highly ionized, while the latter is not. (The term precipitant means the reagent added to cause precipitation.) If, however, we are to precipitate an insoluble chloride we may use either a soluble chloride or hydrochloric acid, since this strong acid is as highly ionized as its salt. When the precipitant is added to a given solution, a precipitate may not appear until considerable reagent has been added. When it is no longer possible to see if more precipitate is forming with further additions of the reagent, a small portion of the mixture is filtered, or, better, the precipitate is allowed 
to settle, and the clear solution is tested with more of the precipitant. Only moderate excesses of the precipitant are used as a rule, since in many cases the precipitant reacts farther with the precipitate to form new and soluble compounds, with the result that the precipitate dissolves in an excess of the reagent added.

455. Dissolving Solid Substances.-Substances which are not readily soluble in water often dissolve easily in solutions of other electrolytes. In such cases we may imagine that chemical reaction gives rise to new products which are soluble in water. Here is a case in point: Calcium hydroxide, $\mathrm{Ca}(\mathrm{OH})_{2}$, is but slightly soluble in water ( $0.12 \mathrm{~g}$. in 100 c.c.), giving a very dilute solution known as limewater. If we mix a few grams of $\mathrm{Ca}(\mathrm{OH})_{2}$ with roo c.c. of water, most of the solid remains undissolved. If now we add dilute $\mathrm{HCl}$ to the mixture, the solid finally passes completely into solution. The explanation is as follows: The small amount of dissolved $\mathrm{Ca}(\mathrm{OH})_{2}$ (which is a strong base) is neutralized by the added $\mathrm{HCl}$ to form very soluble $\mathrm{CaCl}_{2}$,

$$
\mathrm{Ca}(\mathrm{OH})_{2}+{ }_{2} \mathrm{HCl} \leftrightharpoons \mathrm{CaCl}_{2}+2 \mathrm{H}_{2} \mathrm{O} .
$$

More $\mathrm{Ca}(\mathrm{OH})_{2}$ then dissolves in the water in the tendency to keep the concentration of the dissolved $\mathrm{Ca}(\mathrm{OH})_{2}$ up to its M.S.:

$$
\begin{aligned}
& \mathrm{Ca}(\mathrm{OH})_{2} \leftrightarrows \mathrm{Ca}(\mathrm{OH})_{2}=\mathrm{Ca}^{++}+{ }_{2} \mathrm{OH}^{-} \\
& \text {Solid Dissolved }
\end{aligned}
$$

As fast as $\mathrm{Ca}(\mathrm{OH})_{2}$ passes into solution it reacts with the $\mathrm{HCl}$ present. If the chemically equivalent amount of $\mathrm{HCl}$ is added, all $\mathrm{Ca}(\mathrm{OH})_{2}$ will finally dissolve, and the solution will consist simply of $\mathrm{CaCl}_{2}$ dissolved in water.

A perfectly analogous reaction is found in the dissolving of the difficultly soluble, strong base $\mathrm{Mg}(\mathrm{OH})_{2}$ in dilute $\mathrm{HCl}$ :

$$
\mathrm{Mg}(\mathrm{OH})_{2}+{ }_{2} \mathrm{HCl} \leftrightharpoons \mathrm{MgCl}_{2}+{ }_{2} \mathrm{H}_{2} \mathrm{O} \text {. }
$$

Even if the base is weak and much less soluble than either $\mathrm{Ca}(\mathrm{OH})_{2}$ or $\mathrm{Mg}(\mathrm{OH})_{2}$, it will usually dissolve in water upon the addition of a strong acid. For example, $\mathrm{Fe}(\mathrm{OH})_{3}$ is a very weak 
base almost insoluble in water; it dissolves readily in dilute $\mathrm{HCl}$, forming a solution of ferric chloride,

$$
\mathrm{Fe}(\mathrm{OH})_{3}+{ }_{3} \mathrm{HCl} \leftrightharpoons \mathrm{FeCl}_{3}+{ }_{3} \mathrm{H}_{2} \mathrm{O} .
$$

The stages in the process of dissolving may be considered to be comparable to those in the case of the dissolving of $\mathrm{Ca}(\mathrm{OH})_{2}$ in dilute $\mathrm{HCl}$.

Most bases, with the exception of the hydroxides of sodium, potassium, ammonium, and barium, are very little soluble in water. All such so-called insoluble bases dissolve in dilute $\mathrm{HCl}, \mathrm{HNO}_{3}$, and $\mathrm{H}_{2} \mathrm{SO}_{4}$ to form clear solutions, if their corresponding salts with these acids are soluble in water.

456. Dissolving Little Soluble Salts of Weak Acids by Solutions of Strong Acids.- Silver acetate is a rather difficultly soluble salt ( $\mathrm{I} . \circ \mathrm{g}$. dissolves in $\mathrm{I} 00$ c.c. $\mathrm{H}_{2} \mathrm{O}$ at $\mathrm{I} 8^{\circ}$ ) which is easily made by precipitating $\mathrm{AgNO}_{3}$ with $\mathrm{NaC}_{2} \mathrm{H}_{3} \mathrm{O}_{2}$,

$$
\mathrm{AgNO}_{3}+\mathrm{NaC}_{2} \mathrm{H}_{3} \mathrm{O}_{2} \leftrightharpoons \mathrm{AgC}_{2} \mathrm{H}_{3} \mathrm{O}_{2}+\mathrm{NaNO}_{3} \text {. }
$$

If we mix 3 or 4 g. of $\mathrm{AgC}_{2} \mathrm{H}_{3} \mathrm{O}_{2}$ with roo c.c. of $\mathrm{H}_{2} \mathrm{O}$, only a small portion dissolves; but upon addition of dilute $\mathrm{HNO}_{3}$ the whole of the solid passes into solution. Silver acetate is the salt of the weak acid $\mathrm{HC}_{2} \mathrm{H}_{3} \mathrm{O}_{2}$, and, as we have already learned (428), a strong acid reacts more or less completely with the (soluble) salt of a weak acid to form the weak acid and the salt of the strong acid. This was shown earlier in the case of the reaction

$$
\mathrm{HCl}+\mathrm{NaC}_{2} \mathrm{H}_{3} \mathrm{O}_{2} \leftrightarrows \mathrm{HC}_{2} \mathrm{H}_{3} \mathrm{O}_{2}+\mathrm{NaCl} \text {. }
$$

Nitric acid reacts similarly with the dissolved portion of the $\mathrm{AgC}_{2} \mathrm{H}_{3} \mathrm{O}_{2}$,

$$
\mathrm{HNO}_{3}+\mathrm{AgC}_{2} \mathrm{H}_{3} \mathrm{O}_{2} \leftrightarrows \mathrm{HC}_{2} \mathrm{H}_{3} \mathrm{O}_{2}+\mathrm{AgNO}_{3} \text {. }
$$

The reaction is nearly complete, and both products are easily soluble. The dissolved molecular $\mathrm{AgC}_{2} \mathrm{H}_{3} \mathrm{O}_{2}$ being thus removed from the solution, more of the solid passes into solution in the tendency to keep the concentration of molecular $\mathrm{AgC}_{2} \mathrm{H}_{3} \mathrm{O}_{2}$ up to its M.S. But as this salt reacts with the $\mathrm{HNO}_{3}$ present as 
soon as it comes into solution, its M.S. is never reached, so that finally all of the solid passes into solution. The solution consists largely of $\mathrm{AgNO}_{3}$ and its ions, together with molecular acetic acid.

In many other cases so-called "insoluble" salts of weak acids dissolve in solutions of strong acids like $\mathrm{HCl}, \mathrm{HNO}_{3}$, and $\mathrm{H}_{2} \mathrm{SO}_{4}$. 'The following reactions are of this type:

$$
\begin{aligned}
\mathrm{FeS}+2 \mathrm{HCl} & =\mathrm{H}_{2} \mathrm{~S}+\mathrm{FeCl}_{2}, \\
\mathrm{CaCO}_{3}+{ }_{2} \mathrm{HCl} & =\mathrm{H}_{2} \mathrm{CO}_{3}+\mathrm{CaCl}_{2}, \\
\mathrm{Ca}_{3}\left(\mathrm{PO}_{4}\right)_{2}+6 \mathrm{HCl} & ={ }_{2} \mathrm{H}_{3} \mathrm{PO}_{4}+{ }_{3} \mathrm{CaCl}_{2} .
\end{aligned}
$$

However, not all "insoluble" salts of weak acids dissolve in strong acids. For example, CuS, which comes down as a black precipitate when $\mathrm{H}_{2} \mathrm{~S}$ is passed into a solution of a copper salt, and is therefore a salt of the very weak acid $\mathrm{H}_{2} \mathrm{~S}$, does not dissolve appreciably in cold $\mathrm{HCl}$. The reason for this is directly traceable to the extremely small M.S. of CuS. In general the smaller the M.S. of a salt of a weak acid the less soluble it is in a strong acid. Other examples of this sort are found in $\mathrm{Ag}_{2} \mathrm{~S}$ and $\mathrm{HgS}$, neither of which is dissolved appreciably by dilute $\mathrm{HCl}$ or $\mathrm{H}_{2} \mathrm{SO}_{4}$.

457. Weak Acids and Salts of Strong Acids.-We have already learned $(\mathbf{2 8 2})$ that the equilibrium mixture has the same composition whether we start with one pair of substances of a reaction or the equivalent amounts of the other pair. In accord with this principle we always find that if a reaction takes place practically completely in one direction, the reverse of the reaction does not succeed under the same conditions of temperature and concentration. In sections 449 and 452 it was stated that mixtures of the following pairs of substances fail to give precipitates, although little soluble salts would be formed by double decomposition:

$$
\begin{gathered}
\mathrm{H}_{2} \mathrm{CO}_{3} \text { and } \mathrm{CaCl}_{2}, \\
\mathrm{H}_{3} \mathrm{PO}_{4} \text { and } \mathrm{CaCl}_{2}, \\
\mathrm{H}_{2} \mathrm{~S} \text { and } \mathrm{FeCl}_{2}, \\
\mathrm{HC}_{2} \mathrm{H}_{3} \mathrm{O}_{2} \text { and } \mathrm{AgNO}_{3} .
\end{gathered}
$$


Therefore we may be certain that calcium carbonate, calcium phosphate, and ferrous sulphide are soluble in hydrochloric acid, and that silver acetate is soluble in nitric acid. Since the determining factor in dissolving each of these salts is the formation of the weak acid, we may go farther and predict that any strong acid will dissolve these salts. Sometimes a new insoluble salt is formed by the strong acid, as when hydrochloric acid acts on silver acetate; but such reactions are secondary to the solution of the original salts.

458. "Insoluble" Salts of Strong Acids.-The "insoluble" salts of strong acids are not as a rule dissolved to an appreciable extent by solutions of other strong acids. For example, $\mathrm{AgCl}$ is not appreciably dissolved by $\mathrm{HNO}_{3}$, although the products $\mathrm{HCl}$ and $\mathrm{AgNO}_{3}$ of the hypothetical reaction

$$
\mathrm{AgCl}+\mathrm{HNO}_{3} \leftrightarrows \mathrm{HCl}+\mathrm{AgNO}_{3}
$$

are both easily soluble substances. A reaction in which both of the products are highly ionized, as in this case, falls in Class I (4I4). In all such reactions very little chemical change occurs, and this is more strikingly true the more dilute the solution. As we are now considering the case where one of the substances taken is nearly insoluble in water, the solution of this substance must be exceedingly dilute. Comparing the action of $\mathrm{HNO}_{3}$ on $\mathrm{AgC}_{2} \mathrm{H}_{3} \mathrm{O}_{2}$ and $\mathrm{AgCl}$, we may say that the first reaction takes place readily because of the tendency of $\mathrm{H}^{+}$and $\mathrm{C}_{2} \mathrm{H}_{3} \mathrm{O}_{2}^{-}$to unite nearly completely to form little ionized $\mathrm{HC}_{2} \mathrm{H}_{3} \mathrm{O}_{2}$; and that the second reaction does not progress far because of the slight tendency for $\mathrm{H}^{+}$and $\mathrm{Cl}^{-}$ions to unite, since $\mathrm{HCl}$ is nearly completely ionized in very dilute solution.

459. Evolution of a Gas.-Substances may separate from solutions in two ways: (I) as solid precipitates and (2) as gases. We have considered the first case and shall now take up the second, and we shall see that if a substance separates from a solution as a gas the effect on the ionic equilibrium is the same as if the substance separated as a solid. The principles that apply to precipitation apply also, with slight obvious modifications, to gas evolution. Gases have limited solubilities, and 
instead of the M.S. of the precipitate we have the molecular solubility (M.S.) of the gas. Let us now consider a few wellknown reactions as illustrations.

460. The Action of $\mathrm{H}_{2} \mathrm{SO}_{4}$ on $\mathrm{NaCl}$. - If we mix dilute solutions of $\mathrm{H}_{2} \mathrm{SO}_{4}$ and $\mathrm{NaCl}$ no visible effect is produced, although in the solution the reaction

$$
\mathrm{H}_{2} \mathrm{SO}_{4}+\mathrm{NaCl} \leftrightarrows \mathrm{HCl}+\mathrm{NaHSO}_{4}
$$

takes place partially. This is a Class I reaction (4I4) since all four substances are easily soluble and highly ionized. Therefore the dilute solution contains chiefly the ions and relatively few molecules. Nevertheless some $\mathrm{HCl}$ molecules are formed even in dilute solution, but, as $\mathrm{HCl}$ is a very soluble gas, little of it escapes from the solution (25I).

On the other hand the results are quite different if but little water is present. In making hydrochloric acid (103) $58 \mathrm{~g}$. of $\mathrm{NaCl}$, $100 \mathrm{~g}$. of concentrated $\mathrm{H}_{2} \mathrm{SO}_{4}$, and $30 \mathrm{~g}$. of water were mixed in a flask and gently heated.

The proportions of $\mathrm{NaCl}$ and $\mathrm{H}_{2} \mathrm{SO}_{4}$ taken were those indicated by the foregoing equation, since the $100 \mathrm{~g}$. of concentrated acid taken consists of $98 \mathrm{~g}$. of actual $\mathrm{H}_{2} \mathrm{SO}_{4}$ and $2 \mathrm{~g}$. of $\mathrm{H}_{2} \mathrm{O}$. If the reaction should take place completely, $36.5 \mathrm{~g}$. of $\mathrm{HCl}$ would be formed. 'This is far more $\mathrm{HCl}$ than the $32 \mathrm{~g}$. of water present can hold in solution, especially when the mixture is heated. Therefore $\mathrm{HCl}$ gas escapes from the solution. The loss of $\mathrm{HCl}$ by the solution impedes the reverse action on the $\mathrm{NaHSO}_{4}$ present and so causes a great shift to the right of the equilibrium that would otherwise be reached in the reaction

$$
\mathrm{H}_{2} \mathrm{SO}_{4}+\mathrm{NaCl} \Leftrightarrow \mathrm{HCl} \uparrow+\mathrm{NaHSO}_{4} \text {. }
$$

As a consequence this reaction goes nearly completely from left to right under the conditions described (103), the $\mathrm{HCl}$ being given off as gas. In equations for reactions involving gas evolution the gas will be indicated by an upward-pointing arrow.

46r. The Action of $\mathrm{HCl}$ on $\mathrm{CaCO}_{3}$. - We have already seen that carbonic acid, $\mathrm{H}_{2} \mathrm{CO}_{3}$, does not precipitate $\mathrm{CaCO}_{3}$ from a $\mathrm{CaCl}_{2}$ solution, and have learned that this is because $\mathrm{H}_{2} \mathrm{CO}_{3}$ is 
so little ionized that insufficient molecular $\mathrm{CaCO}_{3}$ is formed to exceed its M.S. This fact indicates that the reactions

$$
\begin{aligned}
{ }_{2} \mathrm{HCl}+\mathrm{CaCO}_{3} & \leftrightarrow \mathrm{CaCl}_{2}+\mathrm{H}_{2} \mathrm{CO}_{3}, \\
\mathrm{H}_{2} \mathrm{CO}_{3} & \leftrightarrows \mathrm{H}_{2} \mathrm{O}+\mathrm{CO}_{2} \uparrow,
\end{aligned}
$$

will take place practically completely, since in all reactions between electrolytes exactly the same proportions of the same products result, whether we start with one pair of substances or the chemically equivalent amounts of the other pair (282). The dissolving of $\mathrm{CaCO}_{3}$ in dilute $\mathrm{HCl}$ takes place as follows: $\mathrm{CaCO}_{3}$ first dissolves to the limit of its (very small) M.S. in the water present; the dissolved molecules then ionize rather highly:

$$
\mathrm{CaCO}_{3} \leftrightarrows \mathrm{Ca}+++\mathrm{CO}_{3}--
$$

The $\mathrm{CO}_{3}^{--}$ions unite nearly completely with the $\mathrm{H}^{+}$ions of the highly ionized $\mathrm{HCl}$ present,

$$
{ }_{2} \mathrm{H}^{+}+\mathrm{CO}_{3}^{--} \leftrightarrows \mathrm{H}_{2} \mathrm{CO}_{3},
$$

and to a small extent $\mathrm{Ca}^{++}$and $\mathrm{Cl}^{-}$ions unite to form (easily ionized) $\mathrm{CaCl}_{2}$. The nearly complete removal of $\mathrm{CO}_{3}^{--}$ions allows the further ionization of $\mathrm{CaCO}_{3}$, and this change permits the passage of more solid $\mathrm{CaCO}_{3}$ into solution. The quantitative relations are such that these changes continue until all $\mathrm{CaCO}_{3}$ has dissolved. Incidentally the $\mathrm{H}_{2} \mathrm{CO}_{3}$, which is unstable, dissociates, to a large extent, into water and $\mathrm{CO}_{2}$,

$$
\mathrm{H}_{2} \mathrm{CO}_{3} \leftrightarrows \mathrm{H}_{2} \mathrm{O}+\mathrm{CO}_{2} \uparrow
$$

and, as the latter is not very soluble, much of it passes off as a gas.

The several reactions are shown in the following diagram:

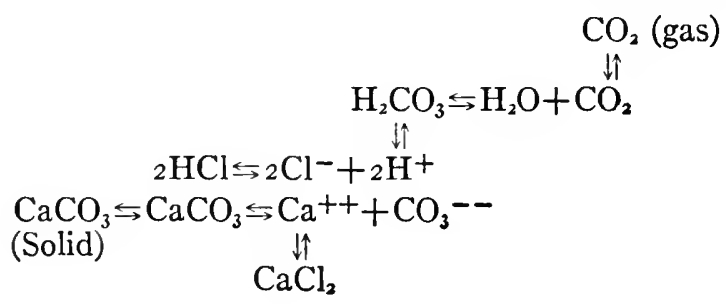


462. The Action of $\mathrm{NaOH}$ on $\mathrm{NH}_{4} \mathrm{Cl}$. - Another example of gas evolution, which, however, does not introduce any new principle, is found in the reaction

$$
\mathrm{NaOH}+\mathrm{NH}_{4} \mathrm{Cl}=\mathrm{NaCl}+\mathrm{NH}_{4} \mathrm{OH},
$$

which takes place more or less completely when solutions of the two initial substances are mixed. This reaction was studied under Class II (426), where it was pointed out that it takes place nearly completely because $\mathrm{NH}_{4} \mathrm{OH}$ is a weak base. This base is also unstable, readily dissociating, thus,

$$
\mathrm{NH}_{4} \mathrm{OH} \leftrightharpoons \mathrm{H}_{2} \mathrm{O}+\mathrm{NH}_{3} \uparrow \text {, }
$$

and, since $\mathrm{NH}_{3}$ is a gas, it will in part escape from the solution. The more concentrated the solution and the higher the temperature the more completely will the $\mathrm{NH}_{3}$ be evolved as gas. The loss of $\mathrm{NH}_{3}$ from the solution promotes the dissociation of $\mathrm{NH}_{4} \mathrm{OH}$, and this in turn favors a further shift in equilibrium from left to right in the main reaction. The various reactions and their relations are fully shown in the following diagram:

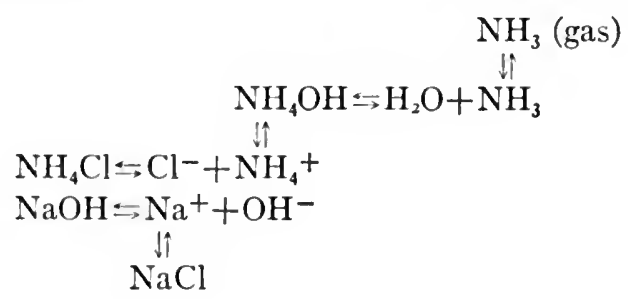

463. The Factors Governing Gas Evolution.--The various factors which are favorable to gas evolution are very similar to those which were found to favor precipitation, although there are some differences aside from the fact that in the one case we are dealing with a gas and in the other with a solid product. If one of the products of a reaction is gaseous it will be given off from the solution, the more completely, the larger the proportion of it formed in the reaction and the less soluble it is. In these respects gas evolution is completely analogous to precipitation. Since all gases are less soluble at high than at low temperatures, gas evolution is always more complete the higher the 
temperature. Gas evolution and precipitation differ in one very important additional respect: at a given temperature the M.S. of a precipitate has a fixed value, while that of a gas depends upon the pressure of the gas above its solution. In most cases the total solubility and therefore also M.S. of a gas is directly proportional to its (partial) pressure (Henry's Law, 126). If during gas evolution the partial pressure of the gas given off is kept down by removing the gas (as by blowing it away with a stream of air) as fast as it is liberated, its M.S. will be reduced to a vanishingly small value. In consequence the dissolved gas will be practically or even completely removed from the solution. Thus, in the reaction between $\mathrm{NaOH}$ and $\mathrm{NH}_{4} \mathrm{Cl}$, if a current of air is blown through the solution every trace of $\mathrm{NH}_{3}$ will finally be removed, so that the reaction will be absolutely complete. The remaining solution will contain only common salt. The same result is attained if the solution is boiled, in which case the evolved steam takes the place of the air current. The high temperature also hastens the removal of the $\mathrm{NH}_{3}$. All reactions giving gases which follow Henry's Law may be driven to completion by the continuous removal of the gas by means of a current of an inert gas or by steam produced when the solution is boiled. We have seen $(455,46 r)$ that the reason why a little soluble salt dissolves is the efficient removal from solution of one or both of its ions to form some new substance, which of course must be soluble or volatile if the resulting mixture is to be a solution. In the reactions studied so far the removal of ions has been accomplished by the formation of little ionized or little soluble substances. 'There are other ways of removing ions. These we shall take up later. We shall find that the dissolving of little soluble substances in question depend upon the same fundamental principle, and that these new cases differ from those studied in this chapter only in secondary ways, the means by which the ions in question are removed from the solution $(532,560,626)$.

464. The Value of the Ionic Hypothesis.-In chapters xviii and xix, we have applied the ionic hypothesis to the interpretation of reactions between acids, bases, and salts and have seen 
that this hypothesis leads to fairly simple explanations of a great variety of facts. Furthermore we have seen that if we know the degrees of ionization and the solubilities of the substances concerned in any reaction we are able to predict what the result of the reaction will be. Herein lies the enormous practical ialue of the ionic hypothesis.

In chapter xvii (4I2) we called attention to some of the glaring inconsistencies of the hypothesis; but we have also pointed out that the practical value of any hypothesis is not its truth but its usefulness. Having now, we hope, shown its usefulness, we shall in later chapters consider whether it is true (chaps. $\mathrm{xx}, \mathrm{xxvii}$ ). 


\section{CHAPTER XX}

\section{ELECTROCHEMISTRY}

465. Introduction.-The present chapter will deal first with some of the marvelous developments of our knowledge of electricity and matter during the last two decades. We now have good reason for believing that electricity like matter is of a granular or "atomic" nature. The grains or "atoms" of free electricity are all exactly alike and of the variety known as negative electricity. These grains are called electrons. Positive electricity is not known in a free state; that is, it is only known as a positive charge on ordinary chemical atoms or larger masses of matter.

466. The Granular Nature of Electricity; Electrons.-In chapter xvii (403) it was shown that Faraday's Law of Electrochemical Equivalents leads directly to the conclusion that all univalent ions, in solution, carry equal charges of electricity (404). The charge on a single univalent ion may be called a unit charge. Each bivalent ion has two unit charges, each trivalent ion three unit charges, etc. As early as 1874 Stoney pointed out that these facts indicate that electricity is gramular in nature, that each univalent atom is associated with one such granule to form a univalent ion, that a bivalent ion is an atom with two granules of electricity, etc. Furthermore a little later he proposed to call the quantity of electricity of a single granule an electron. This quantity is exceedingly minute. The common unit of quantity, one coulomb, is equal to more than a billion billion electrons. According to present-day usage the term electron means a single electronegative granule of electricity.

467. Proof of the Existence of Electrons.-Although evidence was gradually accumulating during the last quarter of the nineteenth century, it was not until more recently that positive evidence was obtained that electricity is granular and is made up of electrons. The crowning work was that of Professor 
Rohert Millikan, an American physicist who showed that when a very small sphere is charged with more and more electricity the quantity of electricity increases by small, equal additions, and not continuously. This is exactly what we should expect if the charge is made up of a small number of electrons.

The spheres used were oil drops of microscopic size, not much larger, in fact, than the particles of dust that can be seen floating in the air when a beam of sunlight penetrates a darkened room. A drop was made visible by a beam of bright light and was viewed through a short-focus telescope. In still, dustfree air the drop fell, under the action of gravity, at a constant velocity that could be accurately measured. It is interesting

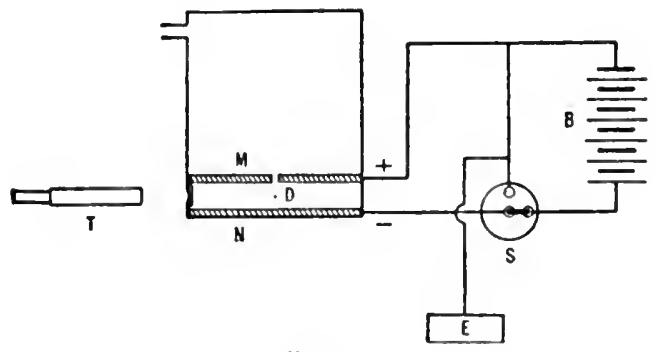

FIG. 75

to note that although every body, however small, will fall with steadily increasing velocity in a vacuum, a very small body falls with constant velocity in air, owing to the viscosity of the latter. The drops used fell with a velocity of about one millimeter per second. The principal parts of Millikan's apparatus are shown in Fig. 75: $M$ and $N$ are parallel metal plates insulated from one another and connected through a switch to the terminals of a high-potential battery, $B$. The upper plate, $M$, can be charged positively and the lower one, $N$, negatively. A minute oil drop, $D$, is caused to fall into the space between $M$ and $N$ through a pinhole in the cənter of $M$, and its rate of fall is measured while $M$ and $N$ are uncharged, observations being made with a telescope, $T$. A minute negative charge is then given to the drop (in a way that need not be considered at present), and the plates $M$ and $N$ are charged. The drop is 
now attracted by $M$ and repelled by $N$, so that it moves upward. When it is close to $M$ the electric field is switched off ( $S$, switch; $E$, earth), and the drop is again allowed to fall, and its speed (time of fall) is again measured. With uncharged plates (field off) the drop falls at exactly the same rate, whether it is charged or uncharged. Next its speed upward is measured with the field on. This speed is always the same as long as the charges on $M$ and $N$ remain constant (constant field), and the charge on the drop is unchanged. But increase of negative charge on the drop increases the upward speed, and decrease of negative charge decreases the upward speed, the field remaining constant. The speed upward is a measure of the force of electrical attraction by $M$ and repulsion by $N$ of the charged drop and is therefore a measure of the charge on the drop. A drop could be made to make hundreds of trips up and down. The downward velocity (field off) was always the same; the upward velocity (field on) varied with the charge. The charge for each upward speed was found by a simple calculation. ${ }^{\text {I }}$ It turned out that the charge on the drop was in every case a multiple by a whole number of the smallest possible charge on the drop. Thousands of observations were made in these experiments, but not an exception was found to the foregoing statement. This proves conclusively that electricity is granular in nature. It has been shown in other ways that the granules of electricity composing the charge on an oil drop are of the same magnitude as the unit charges of ions of electrolytes in solution. We may therefore call them electrons and say that all electric charges are made up of one or more electrons. In Millikan's experiments the oil drops used were observed to carry all possible charges from a single electron to over a hundred electrons; in no single case was a fraction of an electron found. The elcctron is therefore the smallest indivisible particle of electricity.

468. The Nature of an Electric Current.-The relation of an electric charge to an electric current was first clearly established in 1876 by the American physicist Rowland, who showed

'A popular account of Professor Millikan's work is given in his book, The Electron. Chicago: The University of Chicago Press, I9I 7. 
that when an electrically charged gilt disk was very rapidly rotated it produced the same sort of deflection on a magnetic needle as that due to a current of electricity flowing through a wire having the same position with respect to the needle as that occupied by the disk, Fig. 76. This experiment proved that a current of electricity is nothing but an electric charge in motion, just as a current of water is nothing but water in motion.

469. The Electron Theory of Electric Currents.-If we accept the view that an electric current is an electric charge in motion, and also take into account the fact that an electric charge is merely an assemblage of electrons, we are at once led to the supposition that a current in a wire is only a stream of electrons passing through the wire.

470. The Structure of an Atom.-If we think of a metal wire as made up of "solid," impenetrable atoms, it is not very reasonable to imagine that particles of electricity (electrons) could pass through it. However, physicists and chemists have in recent times come to

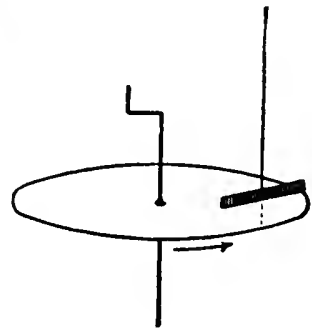

FIG. 76 the conclusion that an atom is by no means a homogeneous, solid lump, but that it is a rather complex structure, consisting largely or even wholly of negative electrons rotating in more or less circular orbits about a positively charged nucleus. The sum of the negative charges of the electrons is exactly equal to the positive charge of the nucleus, so that, as a whole, an atom has no excess of either kind of electricity. The structure of an atom may be likened to that of the solar system, in which the sun corresponds to the nucleus and the planets to the surrounding electrons. The distances between the electrons composing an atom are probably large compared with the size of an electron, so that a stray electron might pass through an atom with the same ease that a comet passes through the solar system, or a bullet may pass through a flock of birds without striking any one of them. 
47r. How a Wire "Carries a Current."-If we think of a wire as made up of atoms of the sort here pictured, it is easy to see how a stream of electrons might pass through it. In a wire (not connected with any electrical source) some electrons are continuously becoming detached from their original atoms; these probably move through and among the atoms, occasionally replacing, for the time being, those that have been lost by other atoms. A metal always contains more or less of these free, wandering electrons, as well as an equal number of atoms which are deficient in electrons. When the terminals of a battery are joined by a wire, the positive pole of the battery attracts and the negative pole repels the free electrons of the wire. This causes a drift of electrons along the wire, and this drift of electrons constitutes the current in the wire. The progress of electrons in the direction of the drift is slow, a matter of a few centimeters per minute. The well-known fact that the effect of closing an electric circuit is felt almost instantaneously at a great distance (as illustrated, for example, by our everyday telephone experience) is explained by the assumption that all the mobile electrons in the wires of the circuit move forward at the instant the circuit is closed. The case is just like that of drawing water from a supply pipe; water flows out the instant the faucet is opened, being pushed forward along the whole pipe by the water forced into the pipe by the pump.

472. The Direction of an Electric Current.-Before the nature of an electric current had been discovered it was customary to consider that the current in the wire flowed from the positive to the negative pole. Since the drift or flow of electrons is in the opposite direction, there is danger of misunderstanding in speaking of the direction of the current. It is perhaps best to speak of the direction of the negative current, which is then the direction of drift of the electrons.

473. Nonconductors of Electricity.-All metals are good conductors, but the non-metals are practically nonconductors or insulators. To account for this difference we have only to suppose that a non-metal, like sulfur, contains but very few free or mobile electrons and therefore has very little ability to carry a current 
474. Production of Electric Charges by Friction.-If a glass rod is rubbed with a piece of silk, the former takes on a positive, the latter a negative, charge. 'This is explained by assuming that a few stray electrons of the glass have been "wiped off" by the silk. The rubbing of the glass by the silk is of importance only in insuring intimate contact between the two. Another example of similar nature is found in the familiar electrification of the hair when combed with a hard-rubber comb in dry weather. Here the comb acquires a negative charge and the hair a positive one. In general, when any two different substances are brought logether they become electrified with opposite charges. This may be taken to mean that electrons accumulate in excess more easily on some kinds of matter than on others.

\section{Cathode Rays.-} When a high-voltage electric current is passed through a Crookes tubc, Fig. 77, which is an evacuated glass bulb having a metallic cathode, $C$, and an anode, $A$, rays,

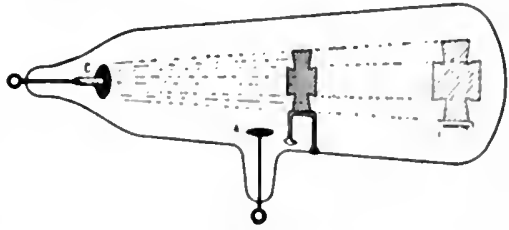

Fic. 77 known as cathode rays, are given off by the cathode and cause a yellowish-green fluorescence of the opposite end of the tube. These rays are readily stopped by a sheet of metal, as shown by the fact that a screen (in the form of a Maltese cross) casts its shadow on the glass. Even transparent substances like glass do not transmit the cathode rays any better than do metal sheets of comparable thickness. Extremely thin sheets of material like aluminum or gold leaf permit partial transmission of the cathode rays.

476. X-Rays. - Cathole ray's produce X-rays, also known as Roentgen rays, which radiate from any object struck by the former. A modern $\mathbf{X}$-ray tube is shown in Fig. 78. This is a modified Crookes tube, intended for the use of large currents and the production of powerful X-rays. 'The cathode rays come from the specially constructed cathode, $C$, and strike a target, $T$, made of metallic tungsten, which metal is chosen because of its very high melting-point $\left(3000^{\circ}\right)$. When the cathode rays are 
stopped by the target, part of their energy is transformed into $\mathrm{X}$-rays, and the balance appears as heat, so that the target becomes red, or even white, hot. Recent work has proved that the $\mathrm{X}$-rays, which are very different from the cathode rays, are, like visible light, vibrations of the so-called luminous ether and differ from visible light in having wave-lengths about onethousand th as great as the latter.

477. The Nature of Cathode Rays. - The extensive investigations of Sir William Crookes on cathode rays, during the seventies of the last century, led this famous English physicist to conclude that these rays were matter in a highly rarefied or

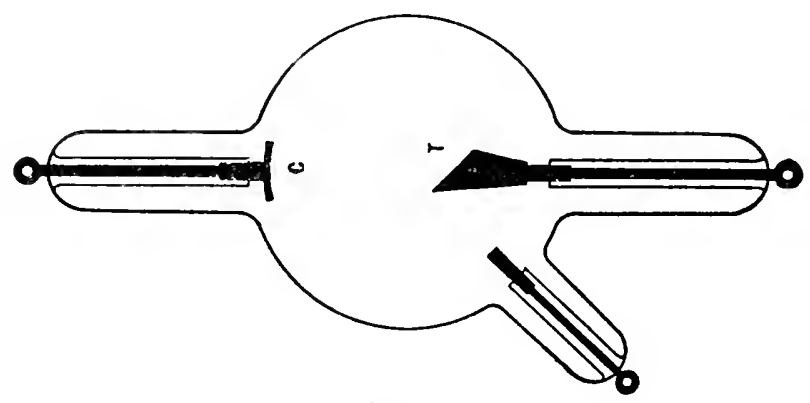

FIG. 78

ultra-gaseous state, which he called a fourth state of matter (the other three states being solid, liquid, and gaseous). And in the light of our present knowledge of the real nature of these remarkable rays we must admit that Crookes's conclusion was substantially correct, although it was by no means the last word on the subject.

It has long been known that cathode rays travel in a straight line in a vacuum, but that they may be deflected in an arc of a circle by a transverse magnetic field. The apparatus shown in Fig. 79 serves for lecture demonstration of this interesting phenomenon. A narrow beam of rays coming from the cathode and passing through a slit in a mica plate strikes along a screen covered with a specially prepared form of zinc sulfide, which becomes luminous in the line where it is struck by the rays. If now a horseshoe magnet is presented so that the $N$ pole is above 
the plane of the paper and the $S$ pole below it, the beam is deflected to the position of the curved line.

It is a well-known fact that a wire, free to move, is deflected by a magnetic field when a current is passed through it. The direction of deflection of the wire is determined by the direction of the current in the wire. The deflection of the cathode rays by a magnetic field indicates that the rays are electricity in motion, the direction of deflection corresponding to that of a stream of negative electricity coming from the cathode, which is, of course, the negative electrode. If we grant that the current in the wire leading to the cathode is, in reality, only a stream of negative electrons in the wire, we have only to suppose that these

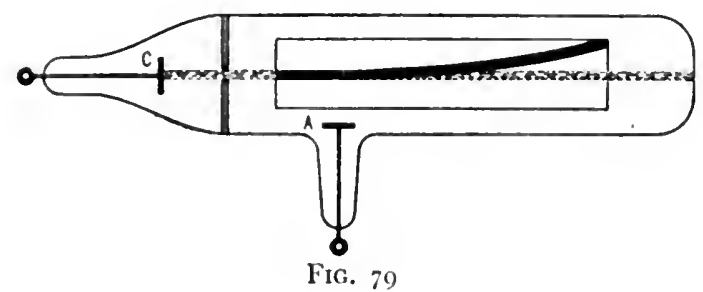

electrons do not stop on reaching the cathode, but shoot out from the surface of the latter and thus constitute the cathode rays.

478. Proof that the Cathode Rays Are a Stream of Electrons. - The conclusive proof that the cathode rays are a stream of negative electricity (presumably electrons, since all negative charges consist of electrons) was furnished by the work of Perrin, a French physicist. Perrin's apparatus is shown in Fig. 80. It was a special form of Crookes tube having the cathode at $C$, the anode at $A$, and at $B$ an insulated metal receiver, into which the cathode ray's could be deflected by means of a magnet. This receiver was connected by a wire to an electroscope, capable of detecting any electric charge given to the box and determining its sign, whether positive or negative. When the cathode rays were started no charge passed into the receiver until the rays were magnetically deflected so as to fall into the receiver; then the latter acquired a large negative 
charge. To guard against stray electric charges the receiver was surrounded by a metal shield connected to the earth, $E$. The experiments above described, together with many other facts, have led to the conclusion that the cathode rays are composed of negative electrons shot out from the cathode with high velocity.

479. The Mass of an Electron.-An electron behaves as thought it had mass. In the first place we know that moving electrons have energy, since the cathode rays can produce light, heat, and X-rays, all of which are forms of energy. Since the kinetic energy of a moving body is proportional to the product

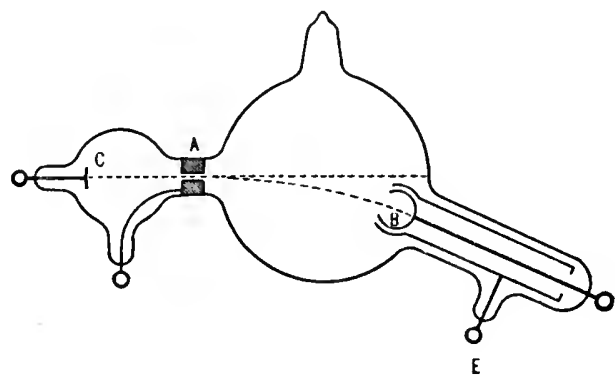

Fic. 80

of its mass and the square of its velocity, we can account for the energy of the cathode rays by assuming the electrons to have mass. Furthermore the fact that it requires an appreciable magnetic force to deflect the cathode rays and that the extent of the deflection (for rays of a given velocity) is proportional to the strength of the magnetic force is also evidence that electrons have mass. One of Newton's laws is to the effect that a moving mass continues in a straight line unless acted upon by a transverse force. Conversely, if a force is required to deflect a moving electron, we are warranted in assuming that the latter has mass. By methods that we cannot explain here it has been shown that the mass of an electron is about one eighteen-hundredth that of an atom of hydrogen.

480. The Beta Rays of Radium.-The spectacular properties of radium have been brought to the attention of nearly everyone, 
whether he is a student of chemistry or not. Radium gives out three kinds of rays, the alpha, beta, and gamma rays. Of these the beta rays very closely resemble the cathode rays. Like cathode rays they are deflected by a magnetic field in a direction which indicates that they too are a stream of electrons shot out with high velocity from the radium. Radium is, by all ordinary tests, an element. It resembles barium as closely as potassium resembles sodium. Here then is an clement that spontaneously gives off negative electricity in the form of electrons shot out with great velocity.

The alpha rays have been proved to be atoms of the element helium, He (atomic weight $=4$ ), each of which carries a double positive charge. These ray's are also shot out with high velocity. The gamma rays are identical with $\mathrm{X}$-rays.

481. The Disintegration Hypothesis. - The extraordinary behavior of radium has been satisfactorily explained by the disintegration hypothesis of Rutherford and Soddy. These scientists assumed that a radium atom is not a homogeneous solid particle but a very complex structure made up of electrons revolving rapidly in more or less circular orbits about a nucleus of positive clectricity in the manner alrearly described. It is further assumed that an alom of radium may become unstable and throw off a single clectron (bcla ray) or a larger mass (an atom of helium, which is an alpha ray), leaing behind an alomic residue of smaller mass and therefore smaller atomic weight. This hypothesis is in complete accord with all known facts concerning radium and radioactive phenomena.

482. The Electrical Nature of Matter. - The sturly of radioactive substances, of which, in addition to radium, about thirty are known, has led to the conclusion that the atoms of all elements, whether radioactive or not, are constructed on about the same plan as that of radium. According to this hypothesis the atom of one element differs from that of another only in the number and arrangement of the electrons composing it. The mass of an atom is, at least in part, accounted for by the mass of the electrons composing it. All matler is considered to be of clectrical origin. 
483. The Nature of an Ion.-A single sodium ion is an atom of sodium having a single positive charge of electricity equal in quantity but opposite in sign to that of an electron. The simplest explanation of the difference between an ion and an atom of sodium is found in the assumption that the ion is an atom which has lost one elcctron. The atom was originally electrically neutral, because the positive charge of its nucleus was just equal to the sum of the negative charges of its surrounding electrons. If one electron is lost, the atom will have an excess positive charge just equal in magnitude to that of one electron. Since metallic atoms all form positive ions we conclude that all such atoms are able to lose electrons. Moreover, an atom of a univalent metal can lose but one electron and its ion will have a single unit charge, thus,

$$
\mathrm{Na}(\text { atom }) \rightarrow \mathrm{Na}^{+}+\text {one electron. }
$$

A bivalent atom can lose two electrons,

$$
\mathrm{Ca}(\text { atom }) \rightarrow \mathrm{Ca}^{++}+\text {two electrons. }
$$

A trivalent atom, such as that of aluminum, can lose three electrons, etc.

Later work has shown that the ions are undoubtedly hydrated to some extent. The actual formula of sodium ion might be represented thus:

$$
\mathrm{Na}\left(\mathrm{H}_{2} \mathrm{O}\right)_{x}{ }^{+} \text {. }
$$

The subscript $x$ represents a small integer, probably 2 or 3 . In practice we do not include the water in the formula, since in the first place the exact data necessary are wanting, and in the second place the relationships in our reactions seem to be satisfactorily represented without it.

484. Valence.-The idea just presented leads to a simple explanation of valence (147, 183). The metals which form only positive ions do so by the loss of one or more electrons from each atom. The valence of an atom of a metallic element is determined by the number of electrons it has lost. 
A negative ion, such as $\mathrm{Cl}^{-}$, is an atom which has taken up an extra electron. Atoms of metals do not take up extra electrons. Only the atoms of non-metallic elements behave in this way. The valence of a negative ion consisling of one atom corresponds to the number of electrons the atom has acquired.

485. Theory of the Union of Sodium and Chlorine.- It is well known that sodium and chlorine unite very energetically to form $\mathrm{NaCl}$. The simplest explanation of the cause of union is found in the assumption that an atom of sodium has a great tendency to lose an electron, and that an atom of chlorine has a great tendency to take up an extra electron. The violent reaction that we observe when we bring these two elements together is only the outward manifestation of the passage of electrons from the atoms of sodium into the atoms of chlorine. The residue of the sodium atom now has an excess of positive electricity, while the chlorine atom with its extra electron is charged negatively. Since unlike electric charges attract each other, we may well assume that the two parts of the $\mathrm{NaCl}$ molecule are held together by electrical attraction.

486. The Cause of Ionization. - If two insulated bodies are oppositely charged, Fig. $8 \mathrm{r}$, the force with which they attract

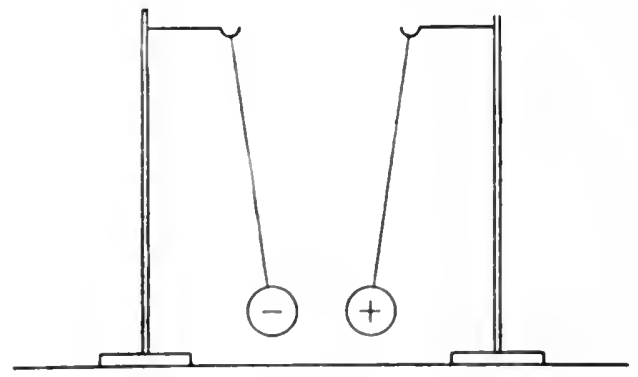

FIC. 8I

each other is proportional to the product of their charges and inversely proportional to the square of the distance between them. There is, however, one additional factor that determines the strength of the attraction, and that is the nature of the surrounding medium. Usually this is air. If the medium were 
glass instead of air the attraction would be only about one-third as great, other things remaining the same; but if the medium were water the attraction would be only one-eightieth as great as for air. If then we dissolve $\mathrm{NaCl}$ in water, the molecules are surrounded by a medium which lessens enormously the attractive force which holds their parts together; as the result, molecules will tend to fall apart, thus,$$
\mathrm{NaCl} \rightarrow \mathrm{Na}^{+}+\mathrm{Cl}^{-} \text {. }
$$

The positive part is the sodium ion, the negative part the chlorine ion. According to this explanation the molecule of salt before it ionizes is made up of two oppositely charged parts. These are not ordinary atoms, since the one has lost an electron which the other has gained. We ought to say that the $\mathrm{NaCl}$ molecule is made up of a sodium ion $\left(\mathrm{Na}^{+}\right)$electrically bound to an ion of chlorine $\left(\mathrm{Cl}^{-}\right)$. The act of ionization, which takes place when the salt is dissolved, is only the falling apart of the ions already present, on account of the great decrease in attractive force caused by the surrounding water. In other words, molecules are composed of bound ions, while in solution part of the ions are free. The ionization of all acids, bases, and salts is explained in precisely analogous fashion to that in the case of $\mathrm{NaCl}$.

487. The Electronic Description of Electrolysis.-According to the electronic description of electrolysis, when an ion reaches an electrode it either gains electrons or loses them. Thus the positive ions $\mathrm{Cu}^{++}, \mathrm{Pb}^{++}, \mathrm{H}^{+}$, etc., each gain enough electrons to make them electrically neutral; while $\mathrm{Cl}^{-}, \mathrm{I}^{-}, \mathrm{S}^{--}$, and $\mathrm{O}^{--}$each lose electrons and become free elements.

488. The Displacement of Non-metals by One Another.-It will be recalled that chlorine acts on a solution of hydrobromic acid or any bromide, setting free bromine, thus:

$$
\mathrm{Cl}_{2}+2 \mathrm{HBr} \rightarrow \mathrm{Br}_{2}+2 \mathrm{HCl} \text {. }
$$

Similarly bromine acts on iodides, as, for example:

$$
\mathrm{Br}_{2}+2 \mathrm{KI} \rightarrow \mathrm{I}_{2}+2 \mathrm{KBr} \text {. }
$$


Iodine acts on $\mathrm{H}_{2} \mathrm{~S}$, in solution, setting free sulfur:

$$
\mathrm{I}_{2}+\mathrm{H}_{2} \mathrm{~S} \rightarrow \mathrm{S}+2 \mathrm{Hl} \text {. }
$$

The order in which the four above-mentioned elements displace one another is therefore as follows:

$$
\mathrm{Cl}, \mathrm{Br}, \mathrm{I}, \mathrm{S} \text {. }
$$

Each will set free from its compounds any one following it. We may also include fluorine and oxygen in the series, and, since fluorine will displace any of the other elements mentioned, it will head the series. The position of oxygen is determined by the fact that a $\mathrm{H}_{2} \mathrm{~S}$ solution reacts with atmospheric oxygen to form free sulfur and water,

$$
\mathrm{O}_{2}+2 \mathrm{H}_{2} \mathrm{~S} \rightarrow 2{ }_{2} \mathrm{~S}+2 \mathrm{H}_{2} \mathrm{O},
$$

and that a solution of $\mathrm{HI}$ also reacts with oxygen of the air (slowly) to form watter and free iodine,

$$
\mathrm{O}_{2}+{ }_{4} \mathrm{HI} \leftrightharpoons 2 \mathrm{I}_{2}+{ }_{2} \mathrm{H}_{2} \mathrm{O} \text {. }
$$

On the other hand, $\mathrm{HBr}$ solution is scarcely affected by oxygen gas, and $\mathrm{HCl}$ solution not at all. Oxygen will therefore precede iodine and sulfur and follow bromine in the list. The whole displacement series is then as follows:

$$
\mathrm{F}, \mathrm{Cl}, \mathrm{Br}, \mathrm{O}, \mathrm{I}, \mathrm{S} \text {. }
$$

489. Electronic Interpretation of Displacement.-If the reaction

$$
\mathrm{Cl}_{2}+2 \mathrm{HBr}=\mathrm{Br}_{2}+2 \mathrm{HCl}
$$

takes place in very dilute solution, the two acids are nearly completely ionized, and we may leave the $\mathrm{H}^{+}$ion out of considerittion. The reaction in its simplest aspect is as follows:

$$
\mathrm{Cl}_{2}+{ }_{2} \mathrm{Br}^{-} \leftrightarrows \mathrm{Br}_{2}+{ }_{2} \mathrm{Cl}^{-} \text {. }
$$

This means that each $\mathrm{Br}^{-}$ion loses an electron, which, passing into a chlorine atom, changes the latter into a $\mathrm{Cl}^{-}$ion. We conclude that chlorine atoms lake up electrons more readily than 
do atoms of bromine. Considering next the six elements of the displacement series, we may say that fluorine has the greatest tendency to take up electrons, and sulfur the least, and that the tendencies of th other elements come in the order indicated in the list as given. Summarizing, we may say that of two elements of the above-mentioned series the one whose atoms have the greater tendency to take up electrons will set the other free from its compounds with positive ions.

490. The Displacement of Metals by One Another.--Strips of metallic zinc placed in solutions of salts of lead, copper, mercury, and silver will react as indicated by the following equations:

$$
\begin{gathered}
\mathrm{Zn}+\mathrm{Pb}\left(\mathrm{NO}_{3}\right)_{2} \rightarrow \mathrm{Pb}+\mathrm{Zn}\left(\mathrm{NO}_{3}\right)_{2}, \\
\mathrm{Zn}+\mathrm{CuSO}_{4} \rightarrow \mathrm{Cu}+\mathrm{ZnSO}_{4}, \\
\mathrm{Zn}+\mathrm{HgCl}_{2} \rightarrow \mathrm{Hg}+\mathrm{ZnCl}_{2}, \\
\mathrm{Zn}+2 \mathrm{AgNO}_{3} \rightarrow 2 \mathrm{Ag}+\mathrm{Zn}\left(\mathrm{NO}_{3}\right)_{2} .
\end{gathered}
$$

In other words, zinc displaces each of the above-mentioned metals from its salts.

If strips of metallic lead are placed in solutions of salts of zinc, copper, mercury, and silver, no reaction takes place with the zinc salt; but the other three metals are set free, while the lead atoms pass into solution as positive ions. In similar fashion metallic copper sets free mercury and silver from their salt solutions, but it does not affect solutions of zinc or lead salts. Mercury displaces silver from its salts but has no action on salts of zinc, lead, or copper. Metallic silver will not displace from their salts any of the other metals just considered. The order of displacement of the five metals is therefore as follows:

$$
\mathrm{Zn}, \mathrm{Pb}, \mathrm{Cu}, \mathrm{Hg}, \mathrm{Ag} \text {. }
$$

491. Electronic Interpretation of Metallic Displacement.The action of zinc on solutions of copper salts may be represented in simplified form thus:

$$
\mathrm{Zn}+\mathrm{Cu}^{++} \rightarrow \mathrm{Cu}+\mathrm{Zn}++
$$

This means that an atom of zinc gives up two electrons to an atom of copper. Since zinc displaces copper equally well from 
solutions of all its simple soluble salts, we conclude that an atom of zinc has a greater tendency to lose electrons than has an atom of copper, but, since metallic copper displaces silver from any of its salts, thus,

$$
\mathrm{Cu}+2 \mathrm{Ag}^{+} \rightarrow 2 \mathrm{Ag}+\mathrm{Cu}^{++},
$$

we conclude that an atom of copper hats a greater tendency to lose electrons than has an atom of silver.

The order of the metals in the displacement series

$$
\mathrm{Zn}, \mathrm{Pb}, \mathrm{Cu}, \mathrm{Hg}, \mathrm{Ag}
$$

is therefore the order in which they fall according to the decreasing ease with which their aloms tend to lose clectrons. In the case of any two metals of the preceding list the one whose atoms have the greater tendency to lose electrons will set the other free from its compounds with negative ions.

492. A More Complete Displacement Series of Metals. Most of the familiar metals may be included in a single displacement series, which shows at the same time the tendencies of the atoms of the metals to lose electrons and so change into positive ions. The list is given in 'Table XIX. In this

TABLE XIX

Displacement Series of tur Metals

$\begin{array}{ll}\text { Potassium } & \text { Nickel } \\ \text { Sodium } & \text { Tin } \\ \text { Barium } & \text { Lead } \\ \text { Calcium } & \text { Hydrogen } \\ \text { Magnesium } & \text { Copper } \\ \text { Aluminum } & \text { Mercury } \\ \text { Zinc } & \text { Silver } \\ \text { Iron } & \text { Ilatinum } \\ \text { Cobalt } & \text { Gold }\end{array}$

list (in which the second column follows the first) each metal tends to displace, or set free from its combination with negative ions, any elcment which follows it.

Hydrogen has been placed in the list between lead and copper. Any metal above hydrogen in the series will react with a nomal 
solution of hydrochloric acid to set free hydrogen (at atmospheric pressure) and pass into solution as chloricle. The metals following hydrogen in the list do not react readily, if at all, with hydrochloric acid. The first four elements of the series react with water to set free hydrogen. Therefore metallic potassium placed in a solution of $\mathrm{NaCl}$ does not set free metallic sodium but causes the evolution of hydrogen. The order shown for the first four elements of the table is in fact that of their tendencies to lose electrons as determined by means other than direct displacement.

493. The Production of an Electric Current.-In the reaction between zinc and copper sulfate the essential change, as we have seen, is that represented by the equation

$$
\mathrm{Zn}+\mathrm{Cu}^{++} \rightarrow \mathrm{Zn}+++\mathrm{Cu} \text {. }
$$

We have said that this change is the result of the passage of two electrons from each atom of zinc into an atom of copper. Now if this is true we ought to be able to get an available elec-

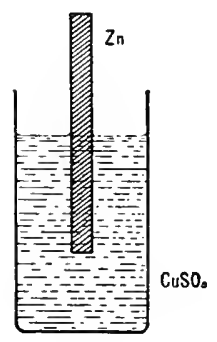

Fig. 82 tric current from the reaction; but if a piece of zinc is dipped into a solution of a copper salt, Fig. 82, no evidence of the production of such a current is to be observed. How indeed could we expect to detect the production of an electric current under the conditions pictured in Fig. 82? If a passage of electrons occurred, it would be between the zinc rod and the layer of copper ions in the solution surrounding the rod, and we could not readily detect such a current, much less make any use of it. If we wish to make this supposed current available for detection and use, we must so arrange the reacting substances that the $\mathrm{Cu}^{++}$ions are not directly in contact with the zinc rod, and then provide a wire for the transfer of electrons from the zinc rod to the copper ions. This can be done by arranging the four substances of the reaction

$$
\mathrm{Zn}+\mathrm{CuSO}_{4} \rightarrow \mathrm{ZnSO}_{4}+\mathrm{Cu}
$$


in the manner shown in Fig. $8_{3}$. Here we have a zinc rod dipping into a solution of $\mathrm{ZnSO}_{4}$ in one beaker, and a copper rod dipping into a solution of $\mathrm{CuSO}_{4}$ in the other beaker. A glass tube filled with $\mathrm{ZnSO}_{4}$ solution and loosely stoppered with cotton plugs forms a so-ca!led salt bridge between the two beakers. If

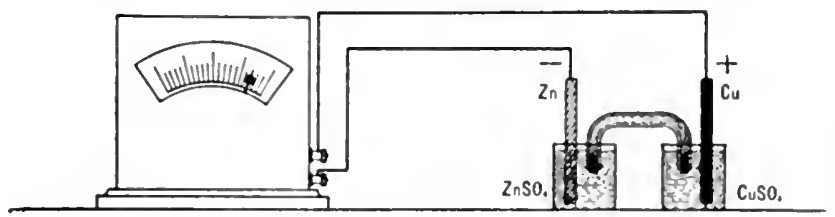

Ií. 83

now the two rods are connected by wires to a galvanometer, a current is found to flow in a direction indicating the passage of electrons from the zinc rod through the wire (and galvanometer) to the copper rod. At the same time metallic copper begins to deposit on the copper plate, and metallic zinc begins to pass into solution. In fact, the reaction

$$
\mathrm{Zn}+\mathrm{CuSO}_{4} \rightarrow \% \mathrm{nSO}_{4}+\mathrm{Cu}
$$

begins to take place just as soon as the metallic circuit is closed between the upper ends of the zinc and copper rods. No action occurs before the circuit is closed, and all action stops when the circuit is broken.

494. The Mechanism of Current Production.-In detail the actions that occur with closed circuit are as follows: zinc atoms pass from the rod into the solution, each atom of zinc leaving behind two electrons and changing thereby into a $\mathrm{Zn}++$ jon. The electrons thus liberated flow through the wire to the copper rod in the $\mathrm{CuSO}_{4}$ solution. Copper ions in contact with the copper rod take up two electrons each, being thereby changed into ordinary copper atoms. These latter adhere to the copper rod as a metallic coating. Fresh $\mathrm{Cu}^{++}$ions move up to the copper rod by diffusion, so that, as the ions in contact with the rod take on electrons and change into copper atoms, others move up by reason of their kinetic motion to take their places. On the other hand $\mathrm{Zn}^{++}$ions, newly formed at the zinc rod, diffuse 
out into the solution. These changes tend to cause a deficiency of $\mathrm{SO}_{4}^{--}$ions about the zinc rod, and an excess of the same ions about the copper rod. The attraction between the excess of $\mathrm{SO}_{4}^{--}$ions, on the one hand, and the excess of $\mathrm{Zn}^{++}$ions, on the other, causes a migration of these ions in opposite directions through the solution and the salt bridge, and thus serves to maintain in each cubic centimeter of the whole solution as many negative as positive ions and thereby to keep the solution, as a whole, electrically neutral.

495. The Function of the Salt Bridge.-The necessity of some sort of connection between the solutions of $\mathrm{ZnSO}_{4}$ and $\mathrm{CuSO}_{4}$ in the two beakers, Fig. $8_{3}$, is obvious. If we remove the salt bridge, which in this case is a $\mathrm{ZnSO}_{4}$ solution, the circuit is broken, and all action comes to a stop. By the use of the bridge we are able, by placing the $\mathrm{CuSO}_{4}$ in a separate beaker, to keep it from coming in contact with the $\mathrm{Zn}$ rod. The use of a metal wire in place of the salt bridge would apparently be a simpler plan, but it would not serve, because new products would be set free by electrolysis at each end of the wire. We could, however, use in the bridge, instead of the $\mathrm{ZnSO}_{4}$, a solution of $\mathrm{CuSO}_{4}$ or, in fact, of $\mathrm{NaCl}$ or almost any other salt. In case the bridge contains $\mathrm{NaCl}$, the $\mathrm{Na}^{+}$ions serve in place of $\mathrm{Zn}^{++}$to carry the positive charge from the $\mathrm{ZnSO}_{4}$ solution to the $\mathrm{CuSO}_{4}$ solution, and the $\mathrm{Cl}^{-}$ions to carry the negative charge in the opposite direction.

496. Galvanic Cells. Electric Batteries.-A galvanic cell, or, as it is more popularly known, an electric battery, is any kind of apparatus by means of which an electric current is produced by chemical reactions. Dry batteries and storage batteries are, at present, the most familiar types. The first practical form of the zinc-copper cell just studied was known as the Daniell cell; a later modification is known as the gravity battery. Properly speaking, the term battery means a group of cells, but this term is frequently used at present to mean a single cell.

497. The Gravity Battery.-A gravity cell is shown in Fig. 84. It consists of thin sheets of copper surrounded by a 
solution of copper sulfate in the lower half of the glass jar and a heavy zinc "crowfout" surrounded by a zinc sulfate solution in the upper half. Attached to the copper sheets is an insulated copper wire. A new cell is set up by filling the jar with water, placing the copper and zinc in position, and adding more than enough solid $\mathrm{CuSO}_{4}$ (blue vitriol or bluestone) to saturate the lower layer. No $\mathrm{ZnSO}_{4}$ need be added; instead, twenty or thirty g. of $\mathrm{NaCl}$ are sprinkled into the water. The solution is not stirred. The $\mathrm{CuSO}_{4}$ gradually dissolves, giving a saturated solution which soon fills the lower part of the cell. If now the insulated wire leading from the copper is connected to the zinc, a current flows through the wire, and the changes already described take place. The $\mathrm{NaCl}$ is used to make the water conduct the current prior

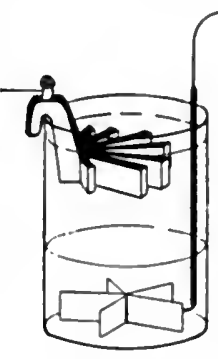

Fig. $S_{4}$ to the formation of sufficient $\mathrm{ZnSO}_{4}$ for this purpose. Until recently the gravity battery was used to operate all telegraph lines.

498. Other Kinds of Galvanic Cells.-It is possible to make a cell that will give a current by the use of any pair of metals (not acted upon by water), each surrounded by a solution of one of its salts. In each cell the experimental arrangement may be that shown in Fig. 83 .

499. Electromotive Force and Voltage. - A body at rest can be set in motion only by the action of a force (Newton's law). In a similar manner we assume that the current (stream of electrons) produced by a battery is the result of an electrical force called the electromotive force, E.M.F. The unit of E.M.F. is the volt (named after the pioneer electrical experimenter Volta). The gravity cell has an E.M.F. of I. I volts.

The farther the two metals forming the electrodes of a cell of any kind are removed from each other in the displacement series (492) the greater the E.M.F. of the cell. The reason for this is found in the fact that the metals heading the list give off electrons most readily (with greatest force). 'The order in the list represents, in fact, the relative force with which the 
element loses electrons. The difference of such forces for the two metals of a cell is, for practical purposes, the chief determining factor of the E.M.F. (voltage) of the cell. This difference of forces between the electrodes is also often called the potential difference of the electrodes.

There is another important factor to be considered besides the nature of the reactions at the electrodes, and that is the concentration of the ions in solution. For example, the more concentrated the copper ions at the copper electrode the faster is the reaction carried on by these ions at a given temperature. Now the difference of potential at the terminals of a cell is a measure of the rate of the reaction in progress; hence it will be increased or decreased by concentration changes in the solutions. To make careful comparison of the electromotive forces of cells the concentrations of the ions must therefore be taken strictly into account. However, in the series we are considering, no moderate variation of the concentrations of the ions from those found in the ordinary laboratory reagents (o:or to $6 \mathrm{~N}$ approximately) will produce results different from those described here, in the cases under consideration. The effect of the concentration of ions on cell potentials should be considered in an exact study of the latter subject.

500. Electrical Energy.-Electrical energy always depends on two factors, voltage and quantity of electricity. The unit of electrical energy is the joule, named after J. P. Joule, the celebrated English scientist, whose work on the mechanical equivalent of heat was discussed earlier (370). One joule is the amount of energy produced when a quantity of one coulomb of electricity flow's through a conductor, the ends of which have a potential difference of one volt. In general, joules $=$ volts $\times$ coulombs. For example, if a gravity cell of $\mathbf{I}$. I volts E.M.F. delivers ro coulombs, the electrical energy produced is $\mathrm{I} . \mathrm{I} \times \mathrm{IO}=\mathrm{I} \mathbf{I}$ joules. Since the joule is an energy unit, its value is expressible in other energy units. Careful experiment has shown that

$$
\begin{aligned}
\text { I joule } & =\text { I0,200 g.cm., } \\
\text { I joule } & =0.24 \text { calorie, } \\
\text { I calorie } & =4.18 \text { joules. }
\end{aligned}
$$


It is electrical energy which a consumer pays for and uses. The same number of electrons go back to the positive pole of a battery as leave the negative pole, but they lose energy in so going. 'The energy which the electrons give up may be liberated as heat or may be converted into work by means of devices like the motor.

501. Electronic Explanation of Oxidation-Reduction Reactions. - The action of chlorine on ferrous chloride in solution $(173,332)$ is a simple, typical example of an oxidation-reduction reaction,

$$
2 \mathrm{FeCl}_{2}+\mathrm{Cl}_{2} \rightarrow 2 \mathrm{FeCl}_{3} \text {. }
$$

This reaction in dilute solution may be represented by the simplified equation

$$
2 \mathrm{Fe}^{++}+\mathrm{Cl}_{2} \rightarrow 2 \mathrm{Fe}^{+++}+2 \mathrm{Cl}^{-} .
$$

The ferrous ion, $\mathrm{Fe}^{++}$, which is the reducing agent, is oxidized to $\mathrm{Fe}^{+++}$by the chlorine atom, which is the oxidizing agent. This is explained by assuming that the $\mathrm{Fe}^{++}$ion (which is an iron atom that has already lost two electrons) gives up a third electron, which, passing into the $\mathrm{Cl}$ atom, changes the latter into a $\mathrm{Cl}^{-}$ion. 'Thus we see that the oxidation of the Fe+t ion

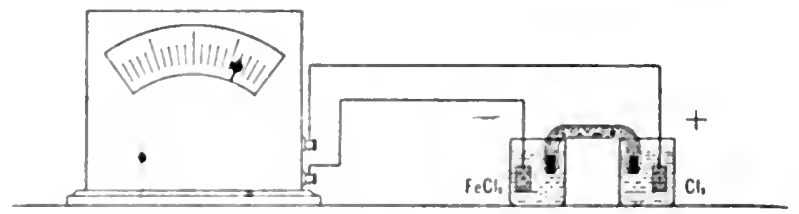

Fic. $8_{5}$

consists in its loss of an electron; and the reduction of the $\mathrm{Cl}$ atom consists in its guin of an clectron.

502. Oxidation-Reduction Cells. - The transfer of electrons which occurs in the reaction just studied can be made to yield an avialable electric current quite as readily as that which takes place in the reaction between metallic zinc and copper sulfate (493). We may demonstrate this fact by means of the arrangement shown in Fig. 85. Platinum electrodes are placed 
in each of two beakers, one of which contains the $\mathrm{FeCl}_{2}$ solution, the other the $\mathrm{Cl}_{2}$ solution (together with some $\mathrm{FeCl}_{3}$ or $\mathrm{NaCl}$ to make the solution conduct). A salt bridge joins the two solutions. Wires from the electrodes are connected with a galvanonometer, which shows the passage of a current in a direction indicating a flow of electrons in the wire from the electrode in the $\mathrm{FeCl}_{2}$ solution to that in the $\mathrm{Cl}_{2}$ solution. The platinum electrodes serve as carriers of electrons into and out of the solutions. Platinum is superior to any other metal except gold for this purpose, because of its very slight tendency to pass into solution as ions.

503. Further Examples of Oxidation-Reduction Reactions.Oxidation-reduction reactions are very common. They may all be interpreted in terms of electron transfers, as the following additional examples will illustrate. Ferric sulfate is reduced by zinc according to the equation

$$
\mathrm{Fe}_{2}\left(\mathrm{SO}_{4}\right)_{3}+\mathrm{Zn} \rightarrow 2 \mathrm{FeSO}_{4}+\mathrm{ZnSO}_{4} \text {. }
$$

The simplified ionic equation is

$$
{ }_{2} \mathrm{Fe}^{+++}+\mathrm{Zn} \rightarrow 2 \mathrm{Fe}^{++}+\mathrm{Zn}^{++} .
$$

Each atom of zinc loses two electrons and changes into a $\mathrm{Zn}{ }^{++}$ ion; these two electrons are taken up, one by each $\mathrm{Fe}^{+++}$ion, which is thereby changed to a $\mathrm{Fe}^{++}$ion. The zinc, which loses electrons, is the reducing agent and is oxidized by ferric ions, which gain electrons and are thereby reduced to ferrous ions.

The action of ferric salts and soluble iodides is illustrated by the following reaction:

$$
{ }_{2} \mathrm{FeCl}_{3}+2 \mathrm{KI} \rightarrow 2 \mathrm{FeCl}_{2}+2 \mathrm{KCl}+\mathrm{I}_{2},
$$

or in simplified form by

$$
{ }_{2} \mathrm{Fe}^{+++}+{ }_{2} \mathrm{I}^{-} \rightarrow 2 \mathrm{Fe}^{++}+\mathrm{I}_{2} .
$$

A closely analogous reaction takes place in the reduction of ferric salts by hydrogen sulfide:

$$
{ }_{2} \mathrm{FeCl}_{3}+\mathrm{H}_{2} \mathrm{~S} \rightarrow 2 \mathrm{FeCl}_{2}+{ }_{2} \mathrm{HCl}+\mathrm{S} \text {. }
$$


The simplified equation is

$$
{ }_{2} \mathrm{Fe}^{+++}+\mathrm{S}^{--} \rightarrow 2 \mathrm{Fe}^{++}+\mathrm{S} .
$$

The electronic explanation of each of the foregoing reactions can easily be made by the student.

Other more intricate oxidation-reduction reactions, which will require a somewhat more extended discussion, will be taken up in subsequent chapters.

In all oxidation-reduction reactions transfers of electrons occur; and in all cases the atom or ion which is oxidized loses one or more electrons, and the atom or ion which is reduced gains one or more electrons. If an ion does not change its charge or its composition in the course of a reaction it is neither oxidized nor reduced.

504. The Oxidation and Reduction of Metals. -When a metal passes into solution its atoms take on positive charges. This means that each atom of a metal loses one or more electrons when it changes into an ion. Since we have defined oxidation as the loss of electrons (50r) we can therefore say that when a metal changes into its ions it is oxidized. For example, in the reaction

$$
\mathrm{Fc}+\mathrm{CuSO}_{4} \rightarrow \mathrm{FeSO}_{4}+\mathrm{Cu}
$$

which we may write in simplified form thus,

$$
\mathrm{Fc}+\mathrm{Cu}^{++} \rightarrow \mathrm{Fe}^{++}+\mathrm{Cu}
$$

we say that the metallic iron is oxidized to ferrous ions, and the copper ions are reduced to metallic copper. We have already seen that the further oxidation of $\mathrm{Fe}^{++}$to $\mathrm{Fe}^{+++}$involves a loss of one additional electron.

505. The Oxidation and Reduction of Non-metals. - When a non-metal (chlorine for example) passes into solution, its atoms take on electrons. We say, therefore, that in such a case the element is reduced. Conversely we say that its ions are oxidized when by loss of electrons they are changed to atoms of the element. 
506. Oxidation-Reduction Potentials.-Every oxidationreduction reaction can by suitable arrangement be made to yield an electric current. The E.M.F. (voltage) of an oxidationreduction cell is the measure of the force with which the reaction tends to take place. The stronger the oxidizing tendency of the oxidizing agent and the stronger the reducing tendency of the reducing reagent the greater the E.M.F. of the cell. A systematic study of such cells has shown that all oxidizing and reducing agents may be arranged in a series in the order of their decreasing oxidizing tendencies and increasing reducing tendencies.

507. Oxidation and Reduction by Means of the Electric Current.-We have shown that oxidation and reduction are capable of producing electric currents. There now remains to show that an electric current can accomplish oxidation and reduction. Two beakers, Fig. 86, are fitted with platinum

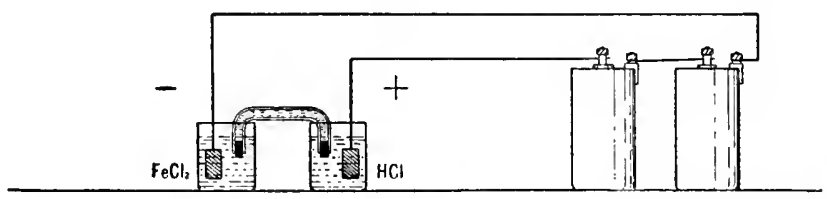

FIG. 86

electrodes and joined with a salt bridge, and in one is placed a solution of $\mathrm{FeCl}_{3}$, and in the other $\mathrm{HCl}$. Upon passing a current from a battery of two dry cells so connected that the electrode in the $\mathrm{FeCl}_{3}$ will be the cathode, it will be found that the $\mathrm{FeCl}_{3}$ is reduced to $\mathrm{FeCl}_{2}$, while at the same time $\mathrm{HCl}$ is oxidized to free chlorine at the anode. The explanation is as follows: The battery sends a steady stream of electrons through the wire to the cathode; one electron passes from the latter into each $\mathrm{Fe}^{+++}$ion coming in contact with it, changing the $\mathrm{Fe}^{+++}$ into $\mathrm{Fe}^{++}$. At the anode $\mathrm{Cl}^{-}$ions coming in contact with this electrode give up to it their electrons and change thereby into ordinary $\mathrm{Cl}$ atoms. The latter then unite in pairs to form molecules, aggregates of which soon form bubbles that escape into the air. In the solution $\mathrm{Fe}^{+++}$ions are attracted by and migrate toward the cathode, while $\mathrm{Cl}^{-}$ions are attracted by and 
migrate toward the anode. Thus the transfer of electricity from one clectrode to the other in the solution is accomplished by means of the moing ions, while in the wire we have a stream of clectrons set in motion by the ballery. A great variety of other oxidations and reductions in solution can be accomplished by means of the electric current. In fact, since we may consider the change of a metal into its ions as an oxidation of the former, and the reverse change a reduction of the ions, we may go farther and say that all processes of electrolysis result in oxidution and reduction. The anode is the seat of oxidation, since it takes up electrons; the cathode is the seat of reduction, because it furnishes electrons. These statements apply to all electrolyses irrespective of whether the substances formed or liberated at the electrodes are elements or compounds.

508. The Conversion of Chemical Energy into Electrical Energy. - The production of heat by a chemical reaction has been explained (373) as being due to the conversion of chemical energy of the reacting'substances into heat energy. If metallic zinc is placed in a solution of copper sulfate so that the reaction

$$
\mathrm{Zn}+\mathrm{CuSO}_{4} \rightarrow \mathrm{ZnSO}_{4}+\mathrm{Cu}
$$

takes place without the production of an available electric current, the quantity of heat liberated is 50,100 calories for one symbol weight of zinc. If the sime amount of zinc reacts with copper sulfite in a gravity cell, $2 \times 96,500$ coulombs of electricity are delivered into the circuit at an E.M.F. of 1.09 volts. The electrical energy produced is $2 \times 96,500 \times 1.09=210,400$ joules. Since I calorie $=4$. I 8 joules, 2 I 0,400 joules $=210,400$ $\div 4$. $18=50,300$ calories. 'Thus we see that electrical energy' equivalent to 50,300 calories is produced in a cell, instead of 50,100 caluries of heat produced when the same amounts of the substances react directly, without the production of a current. 'The small excess of energy produced in a cell is accounted for by the fact, established by experiment, that this amount of energy is absorbed as heat from the surroundings as the cell operates. Similar results are observed in the energy production of all other galvanic cells. The electrical energy produced by any cell is 
equal to the chemical energy liberated or lost, plus or minus an additional amount of energy-plus if heat is taken up from the surroundings and minus if it is given out to the surroundings. We may consider a galvanic cell or battery merely as a device for converting chemical energy into electrical energy.

509. Conversion of Electrical Energy into Chemical Energy.In all processes of electrolysis electrical energy is used up in the production of new chemical substances, and we may conclude at once that the electrical energy used is changed to chemical energy. 


\section{CHAPTER XXI \\ NITROGEN AND AMMONIA}

5ro. Introduction.- Many facts about nitrogen and two of its important compounds, ammonia and nitric acid, have already been discussed. We may recall that about four-fifths by volume of air is uncombined nitrogen, which is left in a nearly pure state as a colorless, odorless gas when air is freed from oxygen (15). Pure nitrogen is obtained by passing ammonia over red-hot copper oxide (84):

$$
{ }_{2} \mathrm{NH}_{3}+{ }_{3} \mathrm{CuO} \rightarrow{ }_{3} \mathrm{Cu}+{ }_{3} \mathrm{H}_{2} \mathrm{O}+\mathrm{N}_{2} .
$$

The symbol weight of nitrogen is 14 , and since 22.4 liters of the gas at $0^{\circ}$ and $76 \mathrm{~cm}$. weigh $2 S \mathrm{~g}$., the formula is $\mathrm{N}_{2}(75)$. This means that a molecule of nitrogen consists of two atoms (2r5).

Ammonia, $\mathrm{NH}_{3}$, is a colorless gas which can be made by the union of nitrogen and hydrogen, by the action of electric sparks,

$$
\mathrm{N}_{2}+3 \mathrm{H}_{2} \rightarrow 2 \mathrm{NH}_{3} \text {. }
$$

It has a very penetrating odor and dissolves easily in water, forming at the same time some ammonium hydroxide:

$$
\mathrm{NH}_{3}+\mathrm{H}_{2} \mathrm{O} \rightarrow \mathrm{NH}_{4} \mathrm{OH} \text {. }
$$

Ammonia and ammonium hydroxide may be completely eliminated from a solution by boiling the latter.

Ammonium hydroxide is a base which neutralizes acids to form salts, among which we have studied the chloride $\mathrm{NH}_{4} \mathrm{Cl}$ (92), the nitrate $\mathrm{NH}_{4} \mathrm{NO}_{3}$ (105), the sulfate $\left(\mathrm{NH}_{4}\right)_{2} \mathrm{SO}_{4}$ (10r), and the acid sulfate $\mathrm{NH}_{4} \mathrm{HSO}_{4}$ (ror).

Ammonium hydroxide is a very much weaker base than sodium hydroxide $(409,429)$; the latter substance reacts with ammonium salts to set free $\mathrm{NH}_{3}$, as illustrated by the following equation:

$$
\mathrm{NH}_{4} \mathrm{Cl}+\mathrm{NaOH} \rightarrow \mathrm{NaCl}+\mathrm{H}_{2} \mathrm{O}+\mathrm{NH}_{3} \text {. }
$$


511. The Occurrence of Nitrogen.-Besides the occurrence of free nitrogen in air, this element is also found in nature as a constituent of many compounds, among which are the familiar substances ammonia and the nitrates of sodium and potassium. Nitrogen is also an essential constituent of the proteins, which constitute the bulk of all kinds of flesh. Proteins are also present in plants, particularly in their seeds. Cereals are especially rich in nitrogenous matter. In the course of the decay of animal and vegetable matter the nitrogen is changed first into $\mathrm{NH}_{3}$ and finally into nitrates.

512. The Element Nitrogen.-Nitrogen was discovered by Rutherford, professor of chemistry in Edinburgh, in 1772. Lavoisier, who was the first to recognize it as an element, called it azote. Its English name, nitrogen, suggested by Chaptal, indicates that the element can be made from saltpeter, $\mathrm{KNO}_{3}$, for which the Greek name was nitron.

513. The Preparation of Nitrogen.--So-called atmospheric nitrogen, obtained by removing oxygen, carbon dioxide, and water from air, is not pure and cannot easily be freed from its residual impurities, amounting to about I per cent, which consist of chemically inactive gases, chiefly argon. Atmospheric nitrogen is prepared on a technical scale by the Linde process, in which air is first liquefied and the more volatile nitrogen distilled from the less volatile liquid oxygen. Pure nitrogen can be made not only from $\mathrm{NH}_{3}$ and $\mathrm{CuO}$ but also in several other ways. One of the best of these is the following: $20 \mathrm{~g}$. of $\mathrm{NH}_{4} \mathrm{Cl}$ and $25 \mathrm{~g}$. of sodium nitrite, a salt having the formula $\mathrm{NaNO}_{2}$, and 50 c.c. of water are placed in a flask (Fig. 87) and heated gently, whereupon the following reaction occurs:

$$
\mathrm{NH}_{4} \mathrm{Cl}+\mathrm{NaNO}_{2} \rightarrow \mathrm{NaCl}+\mathrm{N}_{2}+{ }_{2} \mathrm{H}_{2} \mathrm{O} \text {. }
$$

This reaction takes place in two stages, of which the first is

$$
\mathrm{NH}_{4} \mathrm{Cl}+\mathrm{NaNO}_{2} \rightarrow \mathrm{NaCl}+\mathrm{NH}_{4} \mathrm{NO}_{2} \text {. }
$$

The salt $\mathrm{NH}_{4} \mathrm{NO}_{2}$, ammonium nitrite, is unstable and decomposes at once into $\mathrm{N}_{2}$ and $\mathrm{H}_{2} \mathrm{O}$, thus:

$$
\mathrm{NH}_{4} \mathrm{NO}_{2} \rightarrow \mathrm{N}_{2}+{ }_{2} \mathrm{H}_{2} \mathrm{O} \text {. }
$$


514. The Properties of Nitrogen.-Nitrogen is a colorless, odorless gas, as might be inferred from the fact that four-fifths by bulk of the air is free nitrogen. 'The gas does not support combustion, nor does it burn in the ordinary sense of the term. However, nitrogen unites with oxygen, under certain conditions, to form the oxides $\mathrm{NO}$ and $\mathrm{NO}_{2}$. These very important reactions will be considered in detail in chapter xxii, as by their means nitric acid can be made from the nitrogen of the air.

Nitrogen unites directly with several elements, mostly metals, and especially at high temperatures, to form nitrides, among which calcium nitride, $\mathrm{Ca}_{3} \mathrm{~N}_{2}$; magnesium nitride, $\mathrm{Mg}_{3} \mathrm{~N}_{2}$; and aluminum nitride, AlN, may be

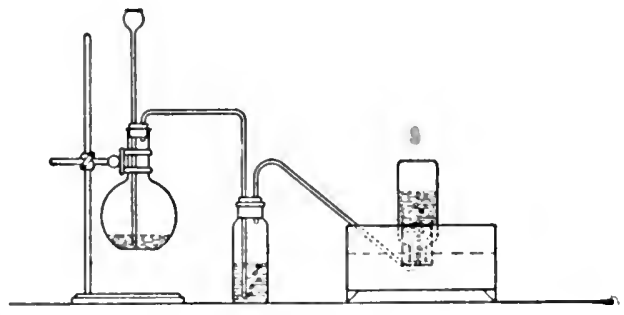

Fig. 87 mentioned. Nitrides usually react readily with water to form ammonia and the hydroxide of the metal:

$$
\mathrm{AlN}+{ }_{3} \mathrm{H}_{2} \mathrm{O} \rightarrow \mathrm{Al}(\mathrm{OH})_{3}+\mathrm{NH}_{3} .
$$

A reaction of great technical importance takes place when nitrogen gas is passed over calcium carbide, $\mathrm{CaC}_{2}$ (49), at a white heat:

$$
\mathrm{CaC}_{2}+\mathrm{N}_{2} \rightarrow \mathrm{CaCN}_{2}+\mathrm{C} \text {. }
$$

The product, $\mathrm{CaCN}_{2}$, is calcium cyanamid; it is also called nitrolime. It is a valuable fertilizer and is also used in the manufacture of ammonia and cyanides by methods which will be described later.

515. The Assimilation of Nitrogen by Plants.-All plants require for their growth nitrogen in some form, in addition to other things. Fertile soils contain considerable nitrogen in the form of compounds, among which are ammonium salts, nitrates, and decaying vegetable and animal matter. Such compounds supply plants with their required nitrogen, since in general plants can assimilate only combined and not free nitrogen. A few 
plants, however, among which are clover, alfalfa, and other legumes, have the power of utilizing uncombined nitrogen from air which has permeated the soil. This they do through the medium of root nodules, which are bacterial growths, whitish in color and often as large as peas. Free nitrogen is taken up by the bacteria in the nodules and changed into compounds, which then serve as a source of nitrogen for the plant. A large part of the nitrogen taken up by plants is concentrated in their seeds as complex compounds called proteins. Wheat, for example, contains io to I 5 per cent of proteins.

516. The Sources of Ammonia.-Although ammonia occurs in very minute amounts in the air, in natural waters, and in the soil, being formed by the decay of animal and vegetable matter, such occurrences are not a practical source of supply. In earlier times ammonia, then called spirits of hartshorn, was made by heating bones, hoofs, horns, and other animal matter with lime (calcium oxide). At present ammonia is produced in large amount as a by-product in the distillation of coal for the production of fuel and illuminating gas and coke. Coal usually contains I or 2 per cent of combined nitrogen, and when heated to a high temperature with exclusion of air, the nitrogen compounds present are decomposed, with the formation of ammonia, which passes off with the gas. The ammonia is absorbed in dilute sulfuric acid, giving a solution which upon evaporation yields crystals of ammonium sulfate; and this salt, when heated with lime, yields free ammonia:

$$
\left(\mathrm{NH}_{4}\right)_{2} \mathrm{SO}_{4}+\mathrm{CaO} \rightarrow \mathrm{CaSO}_{4}+\mathrm{H}_{2} \mathrm{O}+2 \mathrm{NH}_{3} \text {. }
$$

517. The Physical Properties of Ammonia.-For the sake of ready reference we may summarize here the more important data regarding ammonia. It is a colorless gas of penetrating odor; one liter at $0^{\circ}$ and $76 \mathrm{~cm}$. weighs $0.772 \mathrm{~g}$.; and 22.4 liters weigh I 7 grams. Upon being strongly compressed ammonia condenses to a colorless liquid. Liquid ammonia is an important article of commerce; it is marketed in steel cylinders of about two hundred pounds capacity. At $20^{\circ}$ the liquid has a vapor pressure of 8.4 atmospheres. If this pressure is released the 
liquid boils; that is, it changes into gaseous $\mathrm{NH}_{3}$, and its temperature falls to $-33^{\circ}$ (519), which is its boiling-point under one atmosphere pressure.

At $20^{\circ}$ water dissolves about seven hundred times its volume of $\mathrm{NH}_{3}$ gas, part of it going to form the hydroxide $\mathrm{NH}_{4} \mathrm{OH}$, which is a weak or slightly ionized base. Pure ammonium hydroxide of commerce has a specific gravity of 0.90 and contains $2 S$ per cent by weight of $\mathrm{NH}_{3}$.

518. The Uses of Ammonia.-Ammonia is a substance of fundamental importance. It is made and consumed in immense quantities, and its uses are numerous. In the household it is added to water used in washing. In laboratories and chemical works it is indispensable. Ammonium salts, particularly the sulfate, are used in enormous quantities in fertilizers. The manufacture of nitric acid from ammonia has become since the beginning of the war a matter of vital importance; this subject will be taken up in the next chapter (570). The use of ammonia in making ice may now be considered in some detail, as it is not only of commercial importance but also of much scientific interest.

519. The Manufacture of Artificial Ice.-It will be recalled that the change of water into steam at $100^{\circ}$ takes place with the absorption of 540 calories of heat per gram (II5); and further that "every pure liquid has a latent heat of evaporation." The latent heat of evaporation of liquid ammonia at its boilingpoint, $-33^{\circ}$, is $25^{8}$ calories. This means that liquid $\mathrm{NH}_{3}$ released from pressure passes into gas with an absorption of 258 calories of heat per gram. 'This heat is taken from the ammonia and the vessel containing it, and if this vessel is immersed in water the latter is quickly frozen. Since the conversion of $\mathrm{I} g$. of water at $0^{\circ}$ into ice requires an absorption of 79 calories, enough heat is absorbed by the evalporation of $\mathrm{I} g$. of $\mathrm{NH}_{3}$ to freeze about $3 \mathrm{~g}$. of water.

The practical application of these principles to the manufacture of artificial ice is illustrated diagrammatically by Fig. 88.

The pump, $A$, draws ammonia gas from the pipes immersed in the $\operatorname{tank}, O$, and compresses the gas sufficiently so that when 
the latter is cooled by running water in the condenser, $B$, liquid ammonia is formed, and collects in receiver, $C$. From $C$ liquid ammonia flows into the pipes that run through the tank, $O$. In the pipes the ammonia evaporates, thus cooling the brine (freezing-point $-20^{\circ}$ ) with which $O$ is filled. The water to be frozen is contained in cans of 200 pounds or more capacity, immersed in the brine.

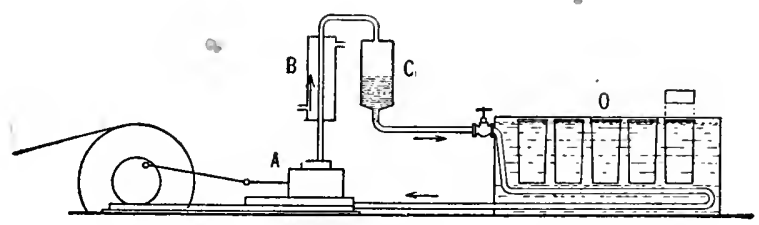

FIG. 88

520. The Synthesis of Ammonia.-We have already learned that $\mathrm{NH}_{3}$ can be made from nitrogen and hydrogen by passing an electric spark through the mixed gases (298). The electric spark is effective mainly as a source of heat:

$$
3 \mathrm{H}_{2}+\mathrm{N}_{2} \rightarrow 2 \mathrm{NH}_{3} \text {. }
$$

This reaction reaches a state of equilibrium when but very little $\mathrm{NH}_{3}$ has been formed, for the reason that the reverse action, the decomposition of $\mathrm{NH}_{3}$ into $\mathrm{H}_{2}$ and $\mathrm{N}_{2}$, takes place very easily. At a temperature of $800^{\circ}$ equilibrium is reached when only one molecule of $\mathrm{NH}_{3}$ remains undecomposed out of every ro, 000 taken. At first thought it would seem to be quite hopeless to try to make use of this reaction as a means of manufacturing $\mathrm{NH}_{3}$ from $\mathrm{H}_{2}$ and $\mathrm{N}_{2}$, because of the very minute proportion of these gases that unite before the reaction reaches equilibrium. In order that a process based on this reaction shall be a practical success, substantially all of the $\mathrm{H}_{2}$ and $\mathrm{N}_{2}$ taken must be converted into $\mathrm{NH}_{3}$. The big problem then is to obtain from the $\mathrm{H}_{2}$ and $\mathrm{N}_{2}$ taken the other 9,999 molecules of $\mathrm{NH}_{3}$. On account of the great technical importance of the subject and as a beautiful illustration of the way in which 
fundamental chemical principles (laws) are used in working toward desired results, we shall consider this problem in detail.

521. The Effect of Temperature on the Equilibrium.-We have already learned (288) that for any reaction the proportions of the substances present when equilibrium is reached are different at different temperatures. With change of temperature the equilibrium of any reaction is shifted in a way that can be definitely predicted from its heat of reaction. If heat is given out when a substance is formed, heat will be absorbed when the same substance is decomposed. When we raise the temperature of a system in equilibrium, the state of equilibrium shifts in such a way that heat is absorbed $(288,367)$. The heat of formation $(36 \mathrm{r})$ of $\mathrm{NH}_{3}$ is $\mathrm{r} 2,200$ calories:

$$
{ }_{3} \mathrm{H}_{2}+\mathrm{N}_{2} \rightarrow 2 \mathrm{NH}_{3}+2 \times \text { I } 2,200 \text { cal. }
$$

Therefore the higher the temperature the smaller the proportion of $\mathrm{NH}_{3}$ present in the equilibrium mixture, and vice versa, as shown by the following table, in which is given the percentage of $\mathrm{NH}_{3}$ at various temperatures and one atmosphere pressure:

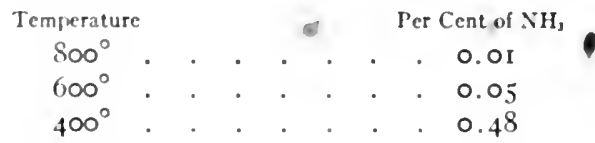

It is evident that the lower the temperature the more favorable the result; but a serious practical difficulty is encountered if one tries to make $\mathrm{NH}_{3}$ at low temperatures; the union of $\mathrm{H}_{2}$ and $\mathrm{N}_{2}$ proceeds the more slowly the lower the temperature, so that at $400^{\circ}$ it would require days for equilibrium to be reached. In this respect this reaction is like all others; the speed of reaction, other things being equal, is slower the lower the temperature. The experimenter then faces this dilemma: at a high temperature the reaction gives very little $\mathrm{NH}_{3}$; at a low temperature it goes too slowly. Is there no way to surmount this difficulty? Is there no way to hasten a reaction except by increasing the temperature? Yes, there is, and this by means of a catalytic agent! 
522. Catalytic Agents for the Ammonia Reaction.-As illustrations of cases of catalysis we may recall that $\mathrm{CuCl}_{2}$ catalyzes the reaction

$$
{ }_{4} \mathrm{HCl}+\mathrm{O}_{2} \rightarrow{ }_{2} \mathrm{H}_{2} \mathrm{O}+{ }_{2} \mathrm{Cl}_{2} \text {; }
$$

that $\mathrm{MnO}_{2}$ promotes the decomposition of $\mathrm{KClO}_{3}(306)$; and that platinum black causes $\mathrm{H}_{2}$ and $\mathrm{O}_{2}$ to unite rapidly at room temperature (303). Therefore it will not be surprising to learn that several catalytic agents were found which hasten the ammonia reaction enormously; among such were the metals iron, manganese, and uranium. By the use of these and probably other substances, the nature of which has not yet been disclosed by the discoverers, the ammonia reaction is caused to reach equilibrium rapidly at $400^{\circ}$ to $500^{\circ}$; but even at $400^{\circ}$ only one-half of I per cent of ammonia is formed. In the next paragraph we shall see how the equilibrium can by another means be shifted still farther in the desired direction.

TABLE XX

Percentages of Ammonia at Various

Temperatures and Pressures

\begin{tabular}{|c|c|c|}
\hline Temperature & $\begin{array}{l}\text { Percentage at } \\
\text { I Atmosphere }\end{array}$ & $\begin{array}{l}\text { Percentage at } \\
\text { Ioo Atmospheres }\end{array}$ \\
\hline $\begin{array}{l}800^{\circ} \ldots \ldots \ldots \\
700^{\circ} \ldots \ldots \ldots \\
600^{\circ} \ldots \ldots \ldots \ldots \\
500^{\circ} \ldots \ldots \ldots\end{array}$ & $\begin{array}{l}0.01 \\
0.02 \\
0.05 \\
0.13\end{array}$ & $\begin{array}{r}\text { I.I } \\
2 . I \\
4 \cdot 5 \\
10.8\end{array}$ \\
\hline
\end{tabular}

523. The Effect of Pressure on the Equilibrium.-In chapter xiii $(\mathbf{2 8 7})$ we learned that "the effect of increase of pressure on any system in equilibrium is in all cases to shift the equilibrium so as to favor the formation of substances occupying a smaller volume." In the reaction

$$
{ }_{3} \mathrm{H}_{2}+\mathrm{N}_{2} \rightarrow{ }_{2} \mathrm{NH}_{3}
$$

three volumes of hydrogen and one volume of nitrogen, if completely united, will yield two volumes of ammonia (77), from which we conclude that an increase of pressure will shift the equilibrium so as to favor the formation of more $\mathrm{NH}_{3}$. Table $\mathrm{XX}$ gives the results. 
It has been found practicable to work at pressures of roo atmospheres ( $\mathrm{I}, 400 \mathrm{lb}$. per square inch) and even higher, and thus greatly to increase the proportion of $\mathrm{NH}_{3}$ formed. It now remains to show how all of the $\mathrm{H}_{2}$ and $\mathrm{N}_{2}$ taken may be converted into $\mathrm{NH}_{3}$.

524. The Effect of Removing One Product of a Reaction.In the discussion of the reaction

$$
\mathrm{NaCl}+\mathrm{H}_{2} \mathrm{SO}_{4} \rightarrow \mathrm{NaHSO}_{4}+\mathrm{HCl}
$$

it was shown that if the $\mathrm{HCl}$ continuously escapes as a gas the reaction will go to completion from left to right. In the reaction between hydrogen and nitrogen equilibrium results from two opposing changes, each of which continues in the equilibrium mixture: these are the union of $\mathrm{H}_{2}$ and $\mathrm{N}_{2}$ on the one hand and the dissociation of $\mathrm{NH}_{3}$ on the other. If the $\mathrm{NH}_{3}$ formed can be removed from the mixture in some systematic way, the $\mathrm{H}_{2}$ and $\mathrm{N}_{2}$ will finally be completely united. We shall now briefly describe the process as actually carried out.

525. The Manufacture of Synthetic Ammonia.-The nitrogen used in making synthetic ammonia might be obtained from the air by the Linde process ( 513 ), and the hydrogen could be made by the electrolysis of water, if these methods were not too expensive. A practical method of obtaining the required mixture of $\mathrm{N}_{2}$ and $\mathrm{H}_{2}$ is said to have been worked out, in which air and steam are passed over heated coke. The carbon comprising the coke unites with the oxygen of both the air and the steam. The $\mathrm{CO}_{2}$ and $\mathrm{CO}$ formed are then removed, leaving $\mathrm{N}_{2}$ and $\mathrm{H}_{2}$.

The apparatus used in the production of $\mathrm{NH}_{3}$ is represented diagrammatically in Fig. 89. The mixture of $\mathrm{N}_{2}$ and $\mathrm{H}_{2}$ under high pressure, say 100 atmospheres, enters at $A$ and passes into the chamber $B$, which holds the catalytic agent and is surrounded by an electric heater $C$. The reaction

$$
\text { a. } 3 \mathrm{H}_{2}+\mathrm{N}_{2} \rightarrow 2 \mathrm{NH}_{3}
$$

takes place to the extent of perhaps 8 to ro per cent in $B$, and the mixture of the three gases passes on to the vessel $D$, surrounded by the refrigerating vessel $E$. 'The high pressure and 
low temperature cause nearly all of the $\mathrm{NH}_{3}$ to condense in $D$ to the liquid state, while the $\mathrm{H}_{2}$ and $\mathrm{N}_{2}$, being much less readily liquefied, pass out and by means of the pump, $F$, are conveyed by a pipe back to the reaction vessel, $B$. Liquid $\mathrm{NH}_{3}$ is removed from $D$ through the valve at the bottom of $D$.

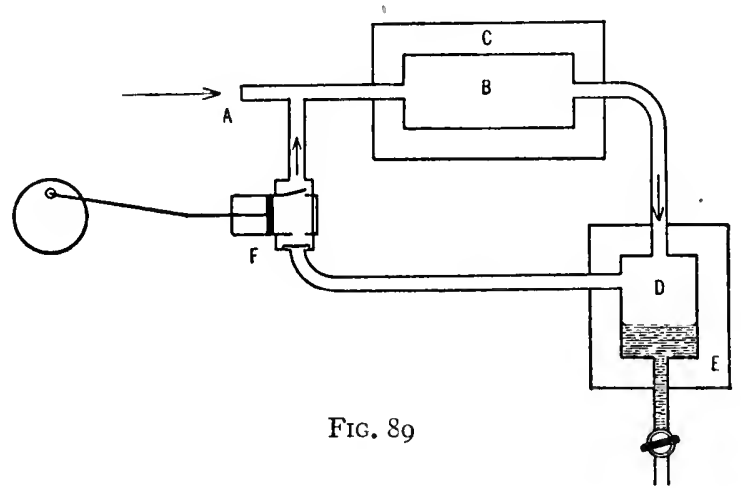

526. Ammonia from Cyanamid--Cyanamid, $\mathrm{CaCN}_{2}$, is made by the action of nitrogen on calcium carbide at a red heat (5I4):

$$
\mathrm{CaC}_{2}+\mathrm{N}_{2} \rightarrow \mathrm{CaCN}_{2}+\mathrm{C} \text {. }
$$

When cyanamid is treated with steam under high pressure the following reaction takes place:

$$
\mathrm{CaCN}_{2}+{ }_{3} \mathrm{H}_{2} \mathrm{O} \rightarrow 2 \mathrm{NH}_{3}+\mathrm{CaCO}_{3} \text {. }
$$

Cyanamid has been manufactured at Niagara Falls, Canada, since I909; and since I9I 5 considerable quantities of ammonia have been made at this place by the reaction described above. Recently the U.S. government has built an immense cyanide ammonia plant at Muscle Shoals, Alabama. It now appears possible that this process will be economically superior to that by which ammonia is made directly from nitrogen and hydrogen.

527. The Chemistry of Ammonia.-One of the most familiar reactions of ammonia is that by which white fumes are formed 
when ammonia gas comes in contact with a volatilized acid. The fumes are, of course, fine particles of the resulting salt:

$$
\begin{gathered}
\mathrm{NH}_{3}+\mathrm{HCl} \rightarrow \mathrm{NH}_{4} \mathrm{Cl}, \\
\mathrm{NH}_{3}+\mathrm{HNO}_{3} \rightarrow \mathrm{NH}_{4} \mathrm{NO}_{3} .
\end{gathered}
$$

In water solution we have the same type of reaction with acids, resulting in the formation of ammonium salts. A consideration of the equilibrium equations

$$
\mathrm{NH}_{3}+\mathrm{H}_{2} \mathrm{O} \leftrightharpoons \mathrm{NH}_{4} \mathrm{OH} \leq \mathrm{NH}_{4}{ }^{+}+\mathrm{OH}^{-}
$$

will show that we may represent the reaction of aqueous ammonia solution with acids as the result of the union of the hydrogen ion of the acid with the hydroxyl ion of the base to form water, and the consequent displacement of the equilibrium to the right; or we may represent the reaction as the union of $\mathrm{NH}_{3}$ with $\mathrm{H}^{+}$ of the acid to form $\mathrm{NH}_{4}{ }^{+}$, and the consequent displacement of the equilibrium to the left. The net result of either reaction is the same, and very likely the truth is that both routes are actually followed. The final solution contains water and the highly ionized ammonium salt.

In these reactions of ammonia, either as a gas or in solution, the nitrogen atom suddenly and very easily takes on one positive and one negative atom or radical. So striking is the ease with which these reactions occur that we think that the nitrogen atom was ready to take on the new groups and so must carry a positive and a negative charge, balanced against each other, in addition to the three negative charges by which it holds the three hydrogen atoms, thus, $\pm \mathrm{NH}_{3}$. From what we know of the complicated structure of an atom it seems plausible to assume the existence of a positive and a negative charge on the same atom. The reaction of aqueous ammonia with an acid may then be represented as follows:

$$
\pm \mathrm{NH}_{3}+\mathrm{H}^{+}+\mathrm{Cl}^{-}=-\mathrm{NH}_{4}^{+}+\mathrm{Cl}^{-} \cong \mathrm{NH}_{4} \mathrm{Cl} \text {. }
$$

A compound which, like ammonia, contains an atom which is not exerting its full valence is said to be unsaturated. There are many other unsaturated compounds. 
Though the above-mentioned reactions are the most characteristic ones shown by ammonia, we have seen one case of a different kind, namely the reducing action of atmmonia on copper oxide $(\mathbf{5 2}, \mathbf{8 4})$ :

$$
{ }_{2} \mathrm{NH}_{3}+{ }_{3} \mathrm{CuO} \rightarrow \mathrm{N}_{2}+{ }_{3} \mathrm{H}_{2} \mathrm{O}+{ }_{3} \mathrm{Cu} \text {. }
$$

An even more vigorous oxidation of ammonia occurs when a mixture of ammonia and air is passed over red-hot platinum. Under the catalytic influence of platinum, nitric acid is formed:

$$
\mathrm{NH}_{3}+{ }_{2} \mathrm{O}_{2} \rightarrow \mathrm{HNO}_{3}+\mathrm{H}_{2} \mathrm{O} \text {. }
$$

This reaction is the basis of one of the great processes for manufacturing nitric acid from the air (570).

When ammonia gas is passed over heated sodium the following reaction takes place:

$$
2 \mathrm{Na}+2 \mathrm{NH}_{3} \rightarrow 2 \mathrm{NaNH}_{2}+\mathrm{H}_{2} \text {. }
$$

The products are hydrogen and sodium amide. 'The latter substance is completely decomposed by water, thus:

$$
\mathrm{NaNH}_{2}+\mathrm{H}_{2} \mathrm{O} \rightarrow \mathrm{NaOH}+\mathrm{NH}_{3} \text {. }
$$

Ammonia reacts with other metals, such as magnesium, forming nitrides (5I4), compounds which are also hydrolyzed by water to form ammonia and the hydroxide of the metal.

528. Ammonium Salts.-Ammonium salts closely resemble the corresponding salts of potassium. They are easily soluble in water and crystallize well from solutions. None of the salts are colored excepting those with colored acid ions.

529. Dissociation of Ammonium Chloride.-Ammonium chloride may be completely volatilized at temperatures much below red heat. When the molecular weight of the salt was calculated on the basis of determinations of the density of the vapor formed at about $300^{\circ}$, the value found was half a formula weight. Apparently a full formula weight of ammonium chloride, if still a gas, at $0^{\circ}$ and $76 \mathrm{~cm}$. pressure would occupy not 22.4 but 44.8 liters. To some chemists sixty to eighty years ago it seemed impossible to co-ordinate these facts with 
Avogadro's hypothesis, and they accordingly abandoned the latter.

A simple experiment gives the clue, however, to the explanation of the enigma. If a bit of $\mathrm{dry}^{r} \mathrm{NH}_{4} \mathrm{Cl}$ is placed in a dry test tube and cautiously heated, a piece of moist red litmus paper held in the mouth of the tube turns blue, thus indicating the formation of free $\mathrm{NH}_{3}$. Elaborate experiments have shown that the vapor of $\mathrm{NH}_{4} \mathrm{Cl}$ dissociates at $300^{\circ}$ almost completely into $\mathrm{NH}_{3}$ and $\mathrm{HCl}$ :

$$
\mathrm{NH}_{4} \mathrm{Cl} \leftrightharpoons \mathrm{NH}_{3}+\mathrm{HCl} \text {. }
$$

Since the molecules of ammonia are much lighter than those of hydrogen chloride they travel (diffuse) more rapidly at the same temperature (197). Hence, although the two gases are released at the same instant, the ammonia molecules are the first to reach the litmus paper in the mouth of the test tube. Upon cooling, the constituents unite again to form solid $\mathrm{NH}_{4} \mathrm{Cl}$. Therefore $\mathrm{NH}_{4} \mathrm{Cl}$ vapor has half the density expected, for the reason that the number of molecules present is practically double the original, because each $\mathrm{NH}_{4} \mathrm{Cl}$ molecule gives one molecule of $\mathrm{NH}_{3}$ and one of $\mathrm{HCl}$. Thus Avogadro's law covers the case perfectly. Many other apparent exceptions to Avogadro's law are known, but in every case a satisfactory explanation has been found in complete harmony with the law.

530. Dissociation of Other Ammonium Salts.-Several other anmonium salts also dissociate into the acid and ammonia. Thus solid ammonium bicarbonate smells strongly of ammonia, at ordinary temperatures, by reason of the following dissociation:

$$
\mathrm{NH}_{4} \mathrm{HCO}_{3}:=\mathrm{NH}_{3}+\mathrm{H}_{2} \mathrm{O}+\mathrm{CO}_{2} .
$$

Ammonium sulfide, formed by the union of ammonia with hydrogen sulfide, dissociates at room temperatures to form ammonia and hydrogen su!fide:

$$
\left(\mathrm{NH}_{4}\right) \mathrm{HS} \leftrightharpoons \mathrm{NH}_{3}+\mathrm{H}_{2} \mathrm{~S} .
$$

Ammonium hydroxide itself, which may be considered as the product of the union of ammonia and the very weak acid water, 
is stable in crystalline form only at a very low temperature (9I). In general we find that the weaker the acid the more unstable is the ammonium salt. It is perhaps well to point out that dissociations of the kind just considered are entirely distinct from the splitting up of salts, acids, and bases into ions in solution, in that the new molecules formed by the dissociation of a gas or vapor are not electrically charged. Furthermore the dissociation products are of different composition in the two cases, as is illustrated by Table XXI.

TABLE XXI

\begin{tabular}{|c|c|c|c|c|c|}
\hline \multirow{2}{*}{$\begin{array}{l}\text { Substance } \\
\mathrm{NH}_{4} \mathrm{Cl} \ldots \ldots \\
\mathrm{NH}_{4} \mathrm{HCO} \\
\mathrm{NH}_{4} \mathrm{HS} \ldots \\
\mathrm{NH}_{4} \mathrm{OH} \ldots\end{array}$} & \multicolumn{2}{|c|}{$\begin{array}{l}\text { Electroly tic } \\
\text { Dissociation }\end{array}$} & \multicolumn{3}{|c|}{$\begin{array}{c}\text { Gaseous } \\
\text { Dissociation }\end{array}$} \\
\hline & $\begin{array}{l}\mathrm{NH}_{4}+ \\
\mathrm{NH}_{4}+ \\
\mathrm{NH}_{4}+ \\
\mathrm{NH}_{4}+\end{array}$ & $\begin{array}{l}\mathrm{Cl}^{-} \\
\mathrm{HCO}_{3}- \\
\mathrm{HS}- \\
\mathrm{OH}-\end{array}$ & $\begin{array}{l}\mathrm{NH}_{3} \\
\mathrm{NH}_{3} \\
\mathrm{NH}_{3} \\
\mathrm{NH}_{3}\end{array}$ & $\begin{array}{l}\mathrm{HCl} \\
\mathrm{H}_{2} \mathrm{O} \\
\mathrm{H}_{2} \mathrm{~S} \\
\mathrm{H}_{2} \mathrm{O}\end{array}$ & $\mathrm{CO}_{2}$ \\
\hline
\end{tabular}

531. Other Compounds of Nitrogen and Hydrogen.-Nitrogen and hydrogen form several other compounds besides $\mathrm{NH}_{3}$; among such hydrazine, $\mathrm{N}_{2} \mathrm{H}_{4}$, and its hydrate, $\mathrm{N}_{2} \mathrm{H}_{5} \mathrm{OH}$, a basic substance, may be mentioned. Hydroxylamine, $\mathrm{NH}_{2} \mathrm{OH}$, is also a base. Hydrazoic acid, $\mathrm{HN}_{3}$, is a violently explosive liquid. It is an acid and forms salts like $\mathrm{NaN}_{3}$, sodium azide, and $\mathrm{AgN}_{3}$, silver azide; the latter is extremely explosive. The ammonium salt $\mathrm{NH}_{4} \mathrm{~N}_{3}$ and the hydrazine salt $\mathrm{N}_{2} \mathrm{H}_{5} \mathrm{~N}_{3}$ may be considered as having the formulae $\mathrm{N}_{4} \mathrm{H}_{4}$ and $\mathrm{N}_{5} \mathrm{H}_{5}$ respectively.

532. The Solubility of Silver Chloride in Ammonia Solution.--Silver chloride, $\mathrm{AgCl}$, as we have seen ( 169 ), is an almost insoluble white salt which is easily obtained by adding any chloride solution to a silver salt solution; for example,

$$
\mathrm{NaCl}+\mathrm{AgNO}_{3} \rightarrow \mathrm{AgCl}+\mathrm{NaNO}_{3} .
$$

This reaction goes nearly to completion by reason of the very small solubility of silver chloride, so that precipitation continues until the concentrations of both $\mathrm{Ag}^{+}$and $\mathrm{Cl}^{-}$ions are very small (452). 
If pure silver chloride is stirred with water, only very little dissolves; in fact, about I. 3 mg. per liter. We have, then,

$$
\begin{aligned}
& \text { Solid } \quad \text { Dissolved } \\
& \mathrm{AgCl} \because \mathrm{AgCl} \because \mathrm{Ag}^{+}+\mathrm{Cl}^{-} \text {. }
\end{aligned}
$$

By far the greater part of the dissolved substance is present as $\mathrm{Ag}^{+}$and $\mathrm{Cl}^{-}$ions.

If a solution of ammonia is added to the $\mathrm{AgCl}$ and water the solid dissolves very easily, giving a clear, colorless solution. This solution is a good electrical conductor, while, as we have already learned, ammonium hydroxide conducts very poorly. These facts may be demonstrated by the use of the apparatus described in sec. 384. If the cell is first filled with ammonia solution, it is found that the current which passes is very weak. As soon as $\mathrm{AgCl}$ is dissolved in the ammonia solution the galvanometer needle is strongly deflected. As a matter of fact the new solution is as good a conductor as solutions of equal concentrations of most salts.

533. The Silver Ammonium Ion, $\mathrm{Ag}\left(\mathrm{NH}_{3}\right)_{2}{ }^{+}$. - It has long been known that dry $\mathrm{AgCl}$ unites with dry $\mathrm{NH}_{3}$ gas to form solid compounds. It is possible that one or more of these is present in the solution formed when $\mathrm{AgCl}$ dissolves in a solution of ammonia; but if so, what are the ions, if any, of the new silver compounds? We have already learned (397) that the composition of the ions of a solution is best investigated by means of migration experiments. If we electrolyze a solution of $\mathrm{AgCl}$ dissolved in ammonia, using a $U$-tube like that shown in Fig. 47 (397), we find that both $\mathrm{Ag}^{+}$and $\mathrm{NH}_{3}$ migrate toward the cathorle or negative electrode, while the chloride ion migrates in the opposite direction. Moreover, we find that the $\mathrm{Ag}^{+}$and $\mathrm{NH}_{3}$ migrating toward the cathode are in the proportion of one atom of $\mathrm{Ag}$ to two molecules of $\mathrm{NH}_{3}$ as represented in the formula $\mathrm{Ag}\left(\mathrm{NH}_{3}\right)_{2}$, so that it would appear that this last formula represents the composition of the positive ion. We are therefore led to conclude that the following reaction first.takes place,

$$
\mathrm{AgCl}+2 \mathrm{NH}_{3}=\mathrm{Ag}\left(\mathrm{NH}_{3}\right)_{2} \mathrm{Cl}
$$


and that the compound so formed ionizes very readily thus:

$$
\mathrm{Ag}\left(\mathrm{NH}_{3}\right)_{2} \mathrm{Cl} \leftrightarrows \mathrm{Ag}\left(\mathrm{NH}_{3}\right)_{2}++\mathrm{Cl}^{-} \text {。 }
$$

The silver ammonium ion, $\mathrm{Ag}\left(\mathrm{NH}_{3}\right)_{2}{ }^{+}$, is called a complex ion.

534. The Stability of the $\mathrm{Ag}\left(\mathrm{NH}_{3}\right)_{2}{ }^{+}$Ion.-It has been proved, by an electrical method which need not be considered here, that in a solution of silver chloride in ammonia only an exceedingly small number of $\mathrm{Ag}^{+}$ions are present. This means that the complex ion, $\mathrm{Ag}\left(\mathrm{NH}_{3}\right)_{2}{ }^{+}$, is very stable and is dissociated only very slightly into $\mathrm{Ag}^{+}$and $2 \mathrm{NH}_{3}$, thus:

$$
\mathrm{Ag}\left(\mathrm{NH}_{3}\right)_{2}+\leftrightarrows \mathrm{Ag}^{+}+2 \mathrm{NH}_{3} \text {. }
$$

535. Why Silver Chloride Dissolves in Ammonia.-We can now explain why $\mathrm{AgCl}$ dissolves in ammonia solution. Let us consider the following formulation of the reaction:

$$
\begin{aligned}
& \text { Solid Dissolved } \\
& \mathrm{AgCl} \leftrightarrows \mathrm{AgCl} \leftrightarrows \mathrm{Ag}^{+}+\mathrm{Cl}^{-} \\
& { }_{2} \mathrm{NH}_{3} \\
& \mathrm{Ag}\left(\mathrm{NH}_{3}\right)_{2}+\text {. }
\end{aligned}
$$

The larger part of the silver chloride dissolved by pure water is present as $\mathrm{Ag}^{+}$and $\mathrm{Cl}^{-}$ions. If $\mathrm{NH}_{3}$ is added, the $\mathrm{Ag}^{+}$ions unite with it nearly completely to form $\mathrm{Ag}\left(\mathrm{NH}_{3}\right)_{2}{ }^{+}$. This reaction greatly reduces the concentration of $\mathrm{Ag}^{+}$and therefore causes more solid $\mathrm{AgCl}$ to pass into solution. If sufficient $\mathrm{NH}_{3}$ is present these changes go on until all the solid $\mathrm{AgCl}$ has dissolved. In the solution we have the salt silver ammonium chloride.

Consideration of the equation

$$
\mathrm{Ag}^{+}+2 \mathrm{NH}_{3} \leftrightarrows \mathrm{Ag}\left(\mathrm{NH}_{3}\right)_{2}{ }^{+}
$$

shows that by adding an excess of $\mathrm{NH}_{3}$ the concentration of $\mathrm{Ag}^{+}$ will be still further decreased. If we wish to dissolve $\mathrm{AgCl}$ completely it is necessary to add a little more than two formula weights of $\mathrm{NH}_{3}$ for one of $\mathrm{AgCl}$ in order to depress the concentration of $\mathrm{Ag}^{+}$sufficiently. The dissolving of silver chloride 
in ammonia is exactly similar to the dissolving of silver acetate in nitric acid, except that in the latter case the acetate ion was suppressed, while in the former the $\mathrm{Ag}^{+}$ion was suppressed.

536. The Effect of Acids on Silver Ammonium Ion.-If a strong acid, nitric acid for example, is added to silver ammonium chloride solution, silver chloride appears as a precipitate. Evidently the nitric acid has caused an increase in the concentration of $\mathrm{Ag}^{+}$ion, and union of the latter with the abundant chloride ion has followed. This reaction is explained by saying that $\mathrm{NH}_{3}$ is converted into $\mathrm{NH}_{4} \mathrm{NO}_{3}$ by the $\mathrm{HNO}_{3}$. This the acid does by virtue of the hydrogen ion which it furnishes. The latter unites with the free ammonia as follows:

$$
\mathrm{NH}_{3}+\mathrm{H}^{+}=\mathrm{NH}_{4}^{+} \text {. }
$$

Some union of the latter ion with the nitrate ion to form $\mathrm{NH}_{4} \mathrm{NO}_{3}$ also takes place, but the critical change is that of ammonia to ammonium ion. 'The removal of $\mathrm{NH}_{3}$ allows the dissociation of the silver ammonium ion to proceed to completion:

$$
\mathrm{Ag}\left(\mathrm{NH}_{3}\right)_{2}+\rightarrow \mathrm{Ag}^{+}+2 \mathrm{NH}_{3} \text {. }
$$

537. Other Silver Ammonium Compounds. - If ammonium hydroxide is added to silver nitrate solution a little at a time, brown silver hydroxide is seen to precipitate but eventually to redissolve, just as the ammonia added exceeds the proportion of two molecules for each molecule of silver nitrate present:

$$
\begin{gathered}
\mathrm{Ag} \mathrm{NO}_{3}+\mathrm{NH}_{4} \mathrm{OH} \rightarrow \mathrm{NH}_{4} \mathrm{NO}_{3}+\mathrm{AgOH} ; \\
\mathrm{AgOH}+2 \mathrm{NH}_{3} \rightarrow \mathrm{Ag}\left(\mathrm{NH}_{3}\right)_{2} \mathrm{OH} .
\end{gathered}
$$

But silver ammonium hydroxide is a strong base, and as fast as it forms it displaces ammonium hydroxide from its salt, $\mathrm{NH}_{4} \mathrm{NO}_{3}$,

$$
\mathrm{NH}_{4} \mathrm{NO}_{3}+\mathrm{Ag}\left(\mathrm{NH}_{3}\right)_{2} \mathrm{OH} \rightarrow \mathrm{NH}_{4} \mathrm{OH}+\mathrm{Ag}\left(\mathrm{NH}_{3}\right)_{2} \mathrm{NO}_{3} \text {, }
$$

so that the final equation for the formation of silver ammonium nitrate from ammonia and silver nitrate is

$$
\mathrm{AgNO}_{3}+2 \mathrm{NH}_{3} \rightarrow \mathrm{Ag}\left(\mathrm{NH}_{3}\right)_{2} \mathrm{NO}_{3} \text {. }
$$


In the same way a solution of silver ammonium sulfate can be made by the addition of aqueous ammonia to silver sulfate. All the silver ammonium salts are found to be highly ionized, just as are potassium and ammonium salts.

538. Other Complex Ions.-Ammonia unites with many other metal ions to form complex ions; for instance, with cupric ion it forms the brilliant blue copper ammonium ion, and with nickel ion it forms the less highly colored blue nickel ammonium ion.

$$
\begin{aligned}
& \mathrm{Cu}+++4 \mathrm{NH}_{3} \rightarrow \mathrm{Cu}\left(\mathrm{NH}_{3}\right)_{4}++ \\
& \mathrm{Ni}^{++}+4 \mathrm{NH}_{3} \rightarrow \mathrm{Ni}\left(\mathrm{NH}_{3}\right)_{4}^{++} .
\end{aligned}
$$

These complex ions form salts such as $\mathrm{Cu}\left(\mathrm{NH}_{3}\right)_{4} \mathrm{SO}_{4}$ and $\mathrm{Ni}\left(\mathrm{NH}_{3}\right)_{4}\left(\mathrm{NO}_{3}\right)_{2}$. The chemistry of these ions parallels that of the silver ammonium ion. The ammonium ions are the first which we have called complex ions. But as a matter of fact we have been dealing with a great number, for example, nitrate, carbonate, phosphate, sulfate, and hydroxyl ions. In the double decomposition reactions studied, these ions have shown no sign of any dissociation into smaller parts corresponding to the dissociation of the silver ammonium ion into silver ion and ammonia. However, there is evidence that these secondary dissociations do exist, but to a very much smaller degree than in the case of a complex silver ion. In other words, these ions are very much more stable than the latter. We shall find later other examples of moderately stable complex ions like those of ammonia. 


\section{CHAPTER XXII}

\section{NITRIC ACID AND THE OXIDES OF NITROGEN}

539. Nitric Acid, $\mathrm{HNO}_{3}$. - That nitric acid is a substance of great importance will be apparent at once when it is known that it is an indispensable agent in the manufacture of explosives and dyestuffs and in addition is used extensively in a great many other ways. We have already learned (IO4) that nitric acid can be made from sodium nitrate, Chile saltpeter, and sulfuric acid:

$$
\mathrm{NaNO}_{3}+\mathrm{H}_{2} \mathrm{SO}_{4} \rightarrow \mathrm{NaHSO}_{4}+\mathrm{HNO}_{3} \text {. }
$$

If a larger proportion of $\mathrm{NaNO}_{3}$ is used, the following reaction can also occur if the temperature is high enough:

$$
2 \mathrm{NaNO}_{3}+\mathrm{H}_{2} \mathrm{SO}_{4} \rightarrow \mathrm{Na}_{2} \mathrm{SO}_{4}+2 \mathrm{HNO}_{3} \text {. }
$$

Until recently the only commercial method of making nitric acid was by means of these two reactions. In practice the action takes place in large cast-iron stills, which are but slightly attacked by the two acids as long as water is not present. The nitric acid distils and is usually condensed in vessels made of fused quartz, which is not acted upon by $\mathrm{HNO}_{3}$, or in Duriron (an alloy of iron and silicon), which is almost unaffected by the acid.

540. Chile Saltpeter, $\mathrm{NaNO}_{3}$.- It is a remarkable fact that there is but one known source of sodium nitrate of sufficient magnitude to be of practical importance: this consists of enormous deposits found in a desert region in the mountains of Chile. These deposits form a stratum averaging five feet in thickness and covering about six hundred square miles. 'The value of the saltpeter exported amounts to three-fourths of the total exports of Chile, and the export duty on it is the chief source of revenue of the country:

Potassium nitrate is found in limited quantities in India, but the deposits are far too small to be of importance for the manufacture of nitric acid. 
54I. Properties of Nitric Acid.-Pure anhydrous (waterfree) nitric acid is a colorless liquid of density $\mathrm{I} .52$; it boils at $78^{\circ}$, undergoing some decomposition into water and oxides of nitrogen. The pure acid of commerce contains only 68 per cent of $\mathrm{HNO}_{3}$, the balance being water. This acid has a density of I.42. When dilute nitric acid is boiled the residual portion grows more concentrated, until it reaches a density of $\mathrm{r} .42$ and 68 per cent purity. Acid of this concentration then distils at the constant temperature of $120^{\circ}$. Acid more concentrated than 68 per cent boils at lower temperatures, and as the boiling continues the temperature rises, while the residual acid becomes less concentrated, until finally, at $120^{\circ}, 68$ per cent acid is left.

Nitric acid unites with water to form two crystalline hydrates, $\mathrm{HNO}_{3} \cdot \mathrm{H}_{2} \mathrm{O}$ and $\mathrm{HNO}_{3} \cdot{ }_{3} \mathrm{H}_{2} \mathrm{O}$, which melt at $-38^{\circ}$ and $-{ }_{\mathrm{I}}^{\circ}$ respectively.

Nitric acid is one of the most highly ionized acids; it therefore has all the properties characteristic of a strong acid. Its salts are all soluble in water-most of them very soluble. Nitrates of strong bases ( $\mathrm{Na}, \mathrm{K}, \mathrm{Ca}, \mathrm{Ba}, \mathrm{Mg}$, and $\mathrm{Ag}$ ) give neutral solutions, while solutions of nitrates of weak bases are acid in reaction, owing to hydrolysis (436). Other important properties demand treatment in separate paragraphs.

542. Nitric Acid as an Oxidizing Agent.-In addition to its action as an acid, nitric acid also acts as a powerful oxidizing agent. It will be recalled that oxidation and reduction always go hand in hand (327); the oxidizing agent is reduced, and the reducing agent is oxidized. Most reducing agents react readily with nitric acid, being themselves oxidized as the result of the reaction. The reduction of $\mathrm{HNO}_{3}$ gives the compounds $\mathrm{NO}_{2}$, $\mathrm{NO}, \mathrm{N}_{2} \mathrm{O}, \mathrm{N}_{2}$, or $\mathrm{NH}_{3}$, according to the reducing agent used. The subject is best approached after one has become familiar with the properties of the oxides. We shall therefore first take up the study of the latter and then return to the discussion of the action of nitric acid as an oxidizing agent (557).

543. Nitric Oxide, NO.-Nitric oxide is a colorless gas almost insoluble in water; it is most easily made by the 
action of dilute $\mathrm{HNO}_{3}$ on copper. We might expect the reaction to be

$$
\mathrm{Cu}+{ }_{2} \mathrm{HNO}_{3} \rightarrow \mathrm{Cu}\left(\mathrm{NO}_{3}\right)_{2}+\mathrm{H}_{2} \text {, }
$$

but copper has virtually no tendency to displace hydrogen from solutions of acids. We find that the reaction actually takes place according to the equation

$$
{ }_{3} \mathrm{Cu}+8 \mathrm{HNO}_{3} \rightarrow{ }_{3} \mathrm{Cu}\left(\mathrm{NO}_{3}\right)_{2}+{ }_{4} \mathrm{H}_{2} \mathrm{O}+2 \mathrm{NO} .
$$

The apparatus shown in Fig. $87(5 \mathrm{r} 3)$ is used. About $20 \mathrm{~g}$. of copper in the form of wire, clippings, turnings, etc., are placed in a 250 c.c. flask, and 60 c.c. of concentrated nitric acid mixed with 60 c.c. of water are added. The wash bottle contains dilute $\mathrm{NaOH}$.

The reaction in the flask starts at once, and the vessel is soon filled with a brown-colored gas. After a few minutes this disappears, and in its place is a faintly colored gas, nitric oxicle, which is impure. When purified by passing through the wash bottle it proves to be colorless. It may now be collected over water in the cylinder set in the pneumatic trough.

544. Direct Union of Nitrogen and Oxygen.-The student's general chemical experience and observations will have already led him to the conclusion that nitrogen does not readily combine directly with oxygen; for if the reaction

$$
\mathrm{N}_{2}+\mathrm{O}_{2} \rightarrow 2 \mathrm{NO}
$$

occurred very casily it would be impossible for the mixture of $\mathrm{N}_{2}$ and $\mathrm{O}_{2}$ composing air to exist without union taking place. Nevertheless the passage of electric sparks through air leads to the formation of a small amount of nitric oxide; but the reaction soon reaches equilibrium because of the decomposition of nitric oxide into its constituents under the action of electric sparks. 'The passage of a flash of lightning through air produces a little nitric oxide.

Although the union of nitrogen and oxygen under the influence of electric sparks was discovered by Cavendish in 1766 , it had only a scientific interest for chemists up to very recent times. 
The modern application of this reaction to the manufacture of nitric acid will be taken up later (566).

545. The Properties of Nitric Oxide, NO.-Nitric oxide is a colorless gas nearly insoluble in water and slightly heavier than air. One liter weighs I. 34 g. and 22.4 liters 30 g., agreeing with that calculated from the formula NO. Nitric oxide is very stable, not being decomposed into its elements except at high temperatures.

When nitric oxide comes in contact with air it turns brown. The same change takes place when nitric oxide is mixed with half its volume of pure oxygen. The reaction takes place thus:

$$
2 \mathrm{NO}+\mathrm{O}_{2} \rightarrow 2 \mathrm{NO}_{2} \text {. }
$$

The brown gas, $\mathrm{NO}_{2}$, is called nitrogen tetroxide. This is the brown gas that first appears when nitric oxide is being made, since the latter unites with the $\mathrm{O}_{2}$ present in the flask to form brown $\mathrm{NO}_{2}$. It is interesting to note that a little heat is given out when $\mathrm{NO}$ and $\mathrm{O}_{2}$ unite.

546. Combustion in Nitric Oxide.-The proportion of oxygen in nitric oxide is about two and one-half times as great as in air, and we might expect that this gas would support combustion very readily; but this is not the case. Burning sulfur or a burning candle is extinguished if brought into a jar of nitric oxide. A mixture of hydrogen and nitric oxide does not explode with an electric spark. On the other hand, a few substances will burn in nitric oxide if strongly heated; thus an iron wire heated to incandescence by an electric current takes fire in this gas and burns, forming an oxide and setting nitrogen free. Briskly burning phosphorus continues to burn when plunged into a jar of nitric oxide, forming phosphorous pentoxide and nitrogen,

$$
{ }_{4} \mathrm{P}+{ }_{\mathrm{I}} \mathrm{NO} \rightarrow{ }_{2} \mathrm{P}_{2} \mathrm{O}_{5}+{ }_{5} \mathrm{~N}_{2} \text {. }
$$

The vapor of carbon bisulfide, $\mathrm{CS}_{2}$, an easily combustible and very volatile liquid, gives with nitric oxide a mixture that readily burns with a bright light. By way of caution it may be pointed out that carbon bisulfide is a very dangerous liquid, 
because of the readiness with which it catches fire. It must be kept aw'ay from all flumes and heated objects.

547. Other Ways of Making Nitric Oxide.-Nitric oxide is formed by the action of dilute nitric acid on many other metals besides copper. It is also formed when various oxidizable substances react with dilute $\mathrm{HNO}_{3}$ as illustrated by the following equation:

$$
{ }_{2} \mathrm{HNO}_{3}+6 \mathrm{FeSO}_{4}+{ }_{3} \mathrm{H}_{2} \mathrm{SO}_{4} \rightarrow{ }_{3} \mathrm{Fe}_{2}\left(\mathrm{SO}_{4}\right)_{3}+2 \mathrm{NO}+{ }_{4} \mathrm{H}_{2} \mathrm{O} .
$$

By means of this reaction very pure nitric oxide can be made.

548. Nitric Oxide and Ferrous Sulfate.-When nitric oxide is passed into a solution of $\mathrm{FeSO}_{4}$ a dark-colored solution is formed containing the compound $\mathrm{FeSO}_{4} \cdot \mathrm{NO}$. When this solution is heated the compound is decomposed, and very pure nitric oxide is given off. The formation of a nearly black solution with $\mathrm{FeSO}_{4}$ serves as a good test for nitric oxide, and in consequence also for nitric acid, by reason of the fact that nitric acid is reduced to nitric oxide by $\mathrm{FeSO}_{4}$ in the presence of $\mathrm{H}_{2} \mathrm{SO}_{4}$, the ferrous sulfate being oxidized to ferric sulfate, $\mathrm{Fe}_{2}\left(\mathrm{SO}_{4}\right)_{3}$, at the same time.

549. A Test for Nitric Acid and Nitrates.-If 2 or 3 c.c. of a mixture of nitric acid or any nitrate and concentrated sulfuric acid are placed in the bottom of a test tube, and a solution of ferrous sulfate is cautiously added in such a way that the two solutions do not mix, a dark-brown ring will be formed at the junction of the two layers. The brown layer contains the eompound $\mathrm{FeSO}_{4} \cdot \mathrm{NO}$.

550. Nitrogen Tetroxide, $\mathrm{NO}_{2}$.-We have learned that the brown gas nitrogen tetroxide is formed by the direct union of nitric oxide and oxygen, thus:

$$
2 \mathrm{NO}+\mathrm{O}_{2}:=2 \mathrm{NO}_{2} .
$$

It is also formed in several other ways, such as by the action of concentrated $\mathrm{HNO}_{3}$ on many metals. For example, with concentrated $\mathrm{HNO}_{3}$ and copper we have

$$
\mathrm{Cu}+{ }_{4} \mathrm{HNO}_{3} \rightarrow \mathrm{Cu}\left(\mathrm{NO}_{3}\right)_{2}+2 \mathrm{NO}_{2}+{ }_{2} \mathrm{H}_{2} \mathrm{O} \text {. }
$$


Dilute $\mathrm{HNO}_{3}$ and copper give $\mathrm{NO}$ instead of $\mathrm{NO}_{2}(543)$. The cause of this difference is found in the behavior of the two oxides of nitrogen toward $\mathrm{H}_{2} \mathrm{O}$ and $\mathrm{HNO}_{3}$, as represented in the equation

$$
{ }_{3} \mathrm{NO}_{2}+\mathrm{H}_{2} \mathrm{O} \leftrightarrows 2 \mathrm{HNO}_{3}+\mathrm{NO}
$$

With much warm water this reaction takes place nearly completely from left to right; but the reaction is reversible, since nitric oxide acts almost completely on an excess of concentrated nitric acid to form nitrogen tetroxide and water. Therefore, in the action of nitric acid on copper, nitric oxide will be given off if the acid is very dilute, and nitrogen tetroxide if it is very concentrated; with an acid of intermediate concentration a mixture of the two oxides will result.

Nitrogen tetroxide is also prepared by heating the nitrates of heavy metals such as copper, lead, mercury, silver, etc. $\left(\mathbf{5 6 5}_{\mathbf{5}}\right)$.

551. The Physical Properties of Nitrogen Tetroxide.Nitrogen tetroxide is a reddish-brown gas having a peculiar, disagreeable odor. It is dangerously poisonous and may produce fatal results after a day or two when inhaled in quantities which seem inconsequential at the time. It is easily condensed to liquid form by cooling it with ice; at $-10^{\circ}$ liquid nitrogen tetroxide solidifies to a mass of nearly colorless crystals. The liquid formed by the melting of these crystals is also nearly colorless at temperatures near the melting-point; as the temperature is raised the liquid becomes first yellow, then orange, and finally boils at $22^{\circ}$, giving a gas or vapor of light reddish-brown color. At higher temperatures the gas becomes much darker in color.

552. The Density of Nitrogen Tetroxide.-The formula $\mathrm{NO}_{2}$ corresponds to a formula or molecular weight of 46 and a gas density I.59 times that of air. The actual density which at room temperature is very much greater than this decreases with rise of temperature and reaches the value I. 59 only at $140^{\circ}$. The exact results are shown in Table XXII.

The very remarkable changes in density and color with change of temperature are thought to be due to the formation 
at low temperatures of double molecules having the formula $\mathrm{N}_{2} \mathrm{O}_{4}$, the equation being

$$
2 \mathrm{NO}_{2} \leftrightharpoons \mathrm{N}_{2} \mathrm{O}_{4} \text {. }
$$

If we accept this view and also suppose $\mathrm{N}_{2} \mathrm{O}_{4}$ to be colorless and $\mathrm{NO}_{2}$ dark reddish-brown we get a very satisfactory explanation of all the known facts. The form $\mathrm{N}_{2} \mathrm{O}_{4}$ exists almost pure in the crystals at $-10^{\circ}$; in the liquid state some dissociation to $\mathrm{NO}_{2}$ has taken place, giving a yellow or at higher temperature an orange-colored liquid.

\section{TABLE XXII}

\begin{tabular}{|c|c|c|c|c|}
\hline Temperature & $\begin{array}{l}\text { Density } \\
\text { Air }=1\end{array}$ & $\begin{array}{c}\text { Calculated } \\
\text { Molecular Weight }\end{array}$ & Per Cent of $\mathrm{NO}_{2}$ & Per Cent of $\mathrm{N}_{2} \mathrm{O}$, \\
\hline $\begin{array}{r}25^{\circ} \ldots \ldots \ldots \\
97^{\circ} \ldots \ldots \ldots \\
140^{\circ} \ldots \ldots \ldots\end{array}$ & $\begin{array}{l}2.50 \\
1.78 \\
1.59\end{array}$ & $\begin{array}{l}72 \\
52 \\
46\end{array}$ & $\begin{array}{r}43 \\
87 \\
100\end{array}$ & $\begin{array}{r}57 \\
13 \\
0\end{array}$ \\
\hline
\end{tabular}

In Table XXII the percentages of $\mathrm{NO}_{2}$ and $\mathrm{N}_{2} \mathrm{O}_{4}$ at various temperatures are given, as calculated from the densities. At $140^{\circ}$ the dissociation into single molecules of $\mathrm{NO}_{2}$ is practically complete. In accord with this explanation it is actually found that the density (corrected of course for thermal expansion) remains constant above $140^{\circ}$.

In chemical equations we shall continue to write the formula $\mathrm{NO}_{2}$ for nitrogen tetroxide, but it will be understood that much of the gas, at room temperature, is in the form of $\mathrm{N}_{2} \mathrm{O}_{4}$.

553. Nitrites and Nitrous Acid.-A very interesting reaction takes place when $\mathrm{NO}_{2}$ is passed into a solution of $\mathrm{NaOH}$. The gas is completely absorbed, and the solution upon evaporation yields crystals of two salts - sodium nitrate, $\mathrm{NaNO}_{3}$, and sodium nitrite, $\mathrm{NaNO}_{2}$. The reaction occurs according to the equation

$$
2 \mathrm{NO}_{2}+2 \mathrm{NaOH} \rightarrow \mathrm{NaNO}_{3}+\mathrm{NaNO}_{2}+\mathrm{H}_{2} \mathrm{O} .
$$

Sodium nitrite is a salt of technical importance, particularly in connection with the manufacture of certain dyestuffs and medicinal chemicals. It is the salt of an acid, $\mathrm{HNO}_{2}$, called 
nitrous acid. The free acid itself is very unstable and its dilute solution easily decomposes, thus:

$$
3 \mathrm{HNO}_{2} \rightarrow \mathrm{HNO}_{3}+2 \mathrm{NO}+\mathrm{H}_{2} \mathrm{O} \text {. }
$$

On the other hand the nitrites of sodium and potassium are stable in water solution. They are also resistant to heat. As a matter of fact they are usually made by the decomposition of the corresponding nitrates under heat (565). Ammonium nitrite, however, decomposes in hot-water solution, forming nitrogen and water $\left(5 \mathbf{5}_{3}\right)$.

All nitrites are easily oxidized to nitrates. Solutions of these reagents undergo this change simply on exposure to air and consequently are never free from nitrates.

Nitrous acid is an oxidizer strong enough to change hydriodic acid into iodine and water, but not strong enough to act similarly with hydrobromic acid:

$$
{ }_{2} \mathrm{HI}+2 \mathrm{HNO}_{2} \rightarrow \mathrm{I}_{2}+2 \mathrm{NO}+2 \mathrm{H}_{2} \mathrm{O} \text {. }
$$

Since nitrous acid is easily reduced to nitric oxide it will give the ring test (549), as the latter depends on the formation of this substance.

554. Nitrogen Trioxide, or Nitrous Acid Anhydride.-An oxide having the composition $\mathrm{N}_{2} \mathrm{O}_{3}$ is found to form white crystals at $-103^{\circ}$. At room temperatures it can exist only in minimal amounts in equilibrium with its decomposition products $\mathrm{NO}$ and $\mathrm{NO}_{2}$ :

$$
\mathrm{N}_{2} \mathrm{O}_{3} \rightarrow \mathrm{NO}+\mathrm{NO}_{2} \text {. }
$$

The decomposition of nitrous acid yields products which apparently come from the dissociation of this gas, so that the latter may be called the anhydride of nitrous acid (313):

$$
{ }_{2} \mathrm{HNO}_{2} \leftrightarrows \mathrm{H}_{2} \mathrm{O}+\mathrm{N}_{2} \mathrm{O}_{3} \text {. }
$$

555. Nitrogen Pentoxide, the Anhydride of Nitric Acid.Nitrogen pentoxide, $\mathrm{N}_{2} \mathrm{O}_{3}$, is a rather unstable, colorless crystalline substance which is formed from pure anhydrous (water-free) $\mathrm{HNO}_{3}$ by the action of $\mathrm{P}_{2} \mathrm{O}_{5}$, thus:

$$
2 \mathrm{HNO}_{3}+\mathrm{P}_{2} \mathrm{O}_{5} \rightarrow 2 \mathrm{HPO}_{3}+\mathrm{N}_{2} \mathrm{O}_{5} \text {. }
$$


The $\mathrm{N}_{2} \mathrm{O}_{5}$ formed is separated from the involatile metaphosphoric acid, $\mathrm{HPO}_{3}$, by careful distillation and condensation in a vessel cooled with ice. Nitrogen pentoxide decomposes readily into $\mathrm{NO}_{2}$ and $\mathrm{O}_{2}$, thus:

$$
2 \mathrm{~N}_{2} \mathrm{O}_{5} \rightarrow 4 \mathrm{NO}_{2}+\mathrm{O}_{2} \text {. }
$$

It also unites vigorously with $\mathrm{H}_{2} \mathrm{O}$ to form $\mathrm{HNO}_{3}$,

$$
\mathrm{N}_{2} \mathrm{O}_{5}+\mathrm{H}_{2} \mathrm{O} \rightarrow 2 \mathrm{HNO}_{3} \text {. }
$$

556. Nitrous Oxide, $\mathrm{N}_{2} \mathrm{O}$, Laughing Gas. - Of the five oxides of nitrogen, nitrous oxide, $\mathrm{N}_{2} \mathrm{O}$, is the only one met with outside of chemical works and laboratories. It is the well-known substance laughing gas, used by all dentists as a mild anaesthetic. This gas is made practically by heating ammonium nitrate, which decomposes easily according to the following equation:

$$
\mathrm{NH}_{4} \mathrm{NO}_{3} \rightarrow \mathrm{N}_{2} \mathrm{O}+2 \mathrm{H}_{2} \mathrm{O} \text {. }
$$

If the substance is heated too strongly the reaction takes place explosively. In fact, $\mathrm{NH}_{4} \mathrm{NO}_{3}$ is being used extensively as an explosive. Therefore great care must be laken to heat the substance cautiously.

Nitrous oxide is a colorless gas, having a faint, not unpleasant odor. It is somewhat soluble in water but is usually collected over warm water. The gas is condensed to liquid form by sufficient pressure. Liquid $\mathrm{N}_{2} \mathrm{O}$ boils at $-90^{\circ}$. This liquid, contained in steel cylinders, is an article of commerce.

Although $\mathrm{N}_{2} \mathrm{O}$ contains a smaller proportion of oxygen than any other oxide of nitrogen, it supports combustion almost as well as oxygen itself. A glowing splint bursts into flame when brought into $\mathrm{N}_{2} \mathrm{O}$, just as it would do in $\mathrm{O}_{2}$.

557. Oxidation by Nitric Acid.-We are now ready to discuss oxidation by nitric acid. Since these reactions involve changes in electric charges on the nitrogen atom (501), let us go over the common reduction products of nitric acid with a view to assigning the electric charges to the nitrogen atoms present in them. In doing this two rules are followed: (I) oxygen in combination is double negatively charged except in the case of a peroxide; 
and (2) hydrogen in combination is always single positively charged. Since the sum of the charges carried by the atoms in an electrically neutral substance must be zero, we may calculate the charge on one of the atoms of a compound if its charge is the only one not known. Table XXIII shows the probable charges on the nitrogen atom in the compounds we have studied. For convenience, charges greater than three in number are written with a superscript to show the number of charges. Thus $\mathrm{N}^{5+}$ is equivalent to $\mathrm{N}^{+++++}$. When nitric acid is reduced to $\mathrm{NO}_{2}$ the original atom of nitrogen has gained one electron $\left(\mathrm{N}^{5+} \rightarrow \mathrm{N}^{4+}\right)$. To form $\mathrm{N}_{2}$ it must gain five electrons, and to form $\mathrm{NH}_{3}$ it must gain eight. Therefore it is a greater feat to

\section{TABLE XXIII}

\begin{tabular}{|c|c|c|c|c|c|c|c|c|c|c|}
\hline \multirow{3}{*}{\multicolumn{6}{|c|}{$\begin{array}{l}\text { Compounds } \\
\mathrm{HNO}_{3}, \mathrm{~N}_{2} \mathrm{O}_{5} \text { and nitrates } \\
\mathrm{NO}_{2}, \mathrm{~N}_{2} \mathrm{O}_{4} \cdot \cdot \cdot \cdot \cdot \cdot \\
\mathrm{HNO}_{2}, \mathrm{~N}_{2} \mathrm{O}_{3} \text { and nitrites }\end{array}$}} & & & & & \multirow{2}{*}{$\begin{array}{l}\text { Nitrogen Atoms } \\
\cdot \mathrm{N}^{5+}\end{array}$} \\
\hline & & & & & & & & & & \\
\hline & & & & & & . & & & & $\begin{array}{ll} & \mathrm{N}^{4+} \\
. & \mathrm{N}^{+++}\end{array}$ \\
\hline NO & . & . . & . & . & . & . & . & & • & . $\mathrm{N}++$ \\
\hline $\mathrm{N}_{2} \mathrm{O}$ & . & . & . & . & . & • & $\cdot$ & • & • & $\mathrm{N}^{+}$ \\
\hline $\mathrm{N}_{2}$ & . & . & . & . & . & • & • & • & . & $\cdot \mathrm{N}^{0}$ \\
\hline $\mathrm{NH}_{3}$ & . & . & . & . & . & • & & $\cdot$ & & . $\quad \mathrm{N}---$ \\
\hline
\end{tabular}

reduce nitric acid to ammonia than to nitric oxide. And in general we find that the more active the reducing agent the more we get of products like $\mathrm{N}_{2} \mathrm{O}, \mathrm{N}_{2}$, and $\mathrm{NH}_{3}$. Thus the main products of the reaction of copper and nitric acid are $\mathrm{NO}$ and $\mathrm{NO}_{2}$ (550); but when zinc reacts with nitric acid, $\mathrm{N}_{2} \mathrm{O}, \mathrm{N}_{2}$, and $\mathrm{NH}_{3}$ may be formed. The latter is found as $\mathrm{NH}_{4} \mathrm{NO}_{3}$, of course, if there is excess nitric acid available for combination. These results agree with our previous experience with these metals, since we have already found in the study of the electromotive series that zinc gives up electrons far more easily than does copper (4วI).

The concentration of the solution, however, as well as the nature of the reducing agent, determines the final products of these reactions by reason of the reversible reaction

$$
{ }_{3} \mathrm{NO}_{2}+\mathrm{H}_{2} \mathrm{O} \rightarrow 2 \mathrm{HNO}_{3}+\mathrm{NO} \text {. }
$$


We find, for example, that NO is the main product of the action of copper on dilute nitric acid, while with concentrated nitric acid $\mathrm{NO}_{2}$ is chiefly formed.

558. Reactions of Nitric Acid with Metals.-All the common metals except platinum and gold dissolve in nitric acid to form nitrates, though the latter are sometimes much hydrolyzed. It might be thought that zinc would displace hydrogen from nitric acid, but as a matter of fact only metals in the electromotive series down to and including aluminum can generate hydrogen fast enough for it to escape the oxidizing action of nitric acid. The cases of copper and zinc already discussed may be taken as illustrative of these reactions.

559. Reactions of Nitric Acid with Non-Metals.-Finely divided sulfur is oxidized to sulfuric acid by hot nitric acid. Iodine is oxidized to iodic acid, $\mathrm{HIO}_{3}$. Red phosphorus is oxidized to phosphoric acid (Care! see 59I). Powdered charcoal (carbon) is oxidized to carbon dioxide. In all of these actions brown fumes are given off, showing the release of $\mathrm{NO}$ or $\mathrm{NO}_{2}$.

560. Nitric Acid as a Chemical Solvent for Salts.-The fact that nitric acid is an oxidizing agent accounts for its ability to dissolve many salts on which hydrochloric acid fails to act. Thus the insoluble copper sulfide does not dissolve appreciably in $\mathrm{HCl}$ (456) but does dissolve in dilute nitric acid, forming copper nitrate and sulfur, or sulfuric acid if the action with the acid is continued. So also mercurous chloride, insoluble in $\mathrm{HCl}$, is dissolved easily by dilute $\mathrm{HNO}_{3}$ to form mercuric nitrate. If neither of the ions of a salt can be oxidized nitric acid is no better chemical solvent than $\mathrm{HCl}$. Thus neither acid can dissolve barium sulfate appreciably.

56r. Balancing of Oxidation and Reduction Equations.Before leaving the subject of oxidation by nitric acid it is worth while to take up a systematic method for balancing oxidation and reduction equations, to be used when the proper coefficients cannot be easily arrived at by inspection. The following illustration of a useful method is given in detail. As has been said, copper reacts with concentrated nitric acid to give copper nitrate, nitrogen tetroxide, and water. We may first write down the 
formulae of the substances taken and of the products in the form of an equation without the coefficients:

$$
\mathrm{Cu}+\mathrm{HNO}_{3} \rightarrow \mathrm{Cu}\left(\mathrm{NO}_{3}\right)_{2}+\mathrm{NO}_{2}+\mathrm{H}_{2} \mathrm{O} .
$$

Inspection of the formulae shows that the only atoms which change their valence are those of copper and those of nitrogen which go to form $\mathrm{NO}_{2}$ (not those which go to form nitrate). The equation for the exchange of charges by these atoms is as follows:

$$
\mathrm{Cu}+2 \mathrm{~N}^{5+} \rightarrow \mathrm{Cu}^{++}+2 \mathrm{~N}^{4+} \text {. }
$$

The equation is balanced so that the number of electrons given up by the copper atoms equal those taken by the nitrogen. We may next write the equation to represent the change between the molecules which contain these atoms:

$$
\mathrm{Cu}+{ }_{2} \mathrm{HNO}_{3} \rightarrow \mathrm{Cu}^{++}+2 \mathrm{NO}_{2}+\mathrm{H}_{2} \mathrm{O}+\mathrm{O}^{--} \text {. }
$$

On the right-hand side we need not trouble to assign positive ions to negative ions to form molecules unless so doing accounts for some of the actual products. $\mathrm{NO}_{2}$ and $\mathrm{H}_{2} \mathrm{O}$ are examples. We may next write equations to show the transformation of the $\mathrm{Cu}^{++}$and $\mathrm{O}^{--}$into the products in which they finally appear,

$$
\mathrm{Cu}^{++}+2 \mathrm{HNO}_{3} \rightarrow \mathrm{Cu}\left(\mathrm{NO}_{3}\right)_{2}+2 \mathrm{H}^{+} \text {. }
$$

Gathering up the $\mathrm{O}^{--}$and $\mathrm{H}^{+}$from (I) and (2), we may combine them to form water,

$$
\mathrm{O}^{--}+{ }_{2} \mathrm{H}^{+} \rightarrow \mathrm{H}_{2} \mathrm{O} \text {. }
$$

Since all the products are accounted for, we may now secure the final equation by adding equations ( 1 ), (2), and (3) and canceling the terms which appear on both sides:

$$
\mathrm{Cu}+{ }_{4} \mathrm{HNO}_{3} \rightarrow \mathrm{Cu}\left(\mathrm{NO}_{3}\right)_{2}+2 \mathrm{NO}_{2}+2 \mathrm{H}_{2} \mathrm{O} \text {. }
$$

A little experience will show that there is a great similarity in oxidation and reduction equations, so that the detail which we have written out so laboriously will soon become so familiar that the intermediate equations need not be written out. How- 
ever, until this facility is gained it is better to write out each step, as was done above.

One more example, which is a difficult one, will now be taken up, namely the reaction between zinc and nitric acid to form zinc nitrate, ammonium nitrate, and water. We shall follow exactly the same scheme as before. The first step is to arrange the initial materials and end products in the form of an equation without the coefficients:

$$
\mathrm{Zn}+\mathrm{HNO}_{3} \rightarrow \mathrm{Zn}\left(\mathrm{NO}_{3}\right)_{2}+\mathrm{NII}_{4} \mathrm{NO}_{3}+\mathrm{H}_{2} \mathrm{O} .
$$

Next we write and balance an equation to show the exchange in charges between the atoms which undergo oxidation and reduction:

$$
{ }_{4} \mathrm{Zn}+\mathrm{N}^{5+} \rightarrow{ }_{4} \mathrm{Zn}^{++}+\mathrm{N}^{---} .
$$

The equation for the reaction of the molecules which contain these atoms is

$$
{ }_{4} \mathrm{Zn}+\mathrm{HNO}_{3} \rightarrow \mathrm{Zn}^{++}+\mathrm{N}^{-\cdots-+}+\mathrm{O}^{--}+\mathrm{H}^{+} .
$$

On the right-hand side of the equation we have not troubled to assign any of the positive ions to the negative ions, since in so doing we would not account for any of the actual products. Next we must account for the actual products one by one. These result from the union of the ions represented in (I) with the ions of the excess acid present:

$$
\begin{aligned}
4 \mathrm{Zn}^{++}+8 \mathrm{HNO}_{3} \rightarrow 4 \mathrm{Zn}\left(\mathrm{NO}_{3}\right)_{2}+8 \mathrm{H}^{+}, \\
\mathrm{N}^{--}+{ }_{3} \mathrm{H}^{+} \rightarrow \mathrm{NH}_{3}, \\
\mathrm{NH}_{3}+\mathrm{HNO}_{3} \rightarrow \mathrm{NH}_{4} \mathrm{NO}_{3} .
\end{aligned}
$$

Gathering up the excess $\mathrm{H}^{+}$and $\mathrm{O}^{--}$from equations (I), (2), (3), and (4) we may write

$$
{ }_{3} \mathrm{O}^{--}+6 \mathrm{H}^{+} \rightarrow{ }_{3} \mathrm{H}_{2} \mathrm{O} \text {. }
$$

All products are now accounted for. We may add equations (I), (2), (3), (4), and (5) and cancel the terms which appear on both sides to secure the final equation

$$
{ }_{4} \mathrm{Zn}+\mathrm{IO} \mathrm{HNO}_{3} \rightarrow 4 \mathrm{Zn}\left(\mathrm{NO}_{3}\right)_{2}+\mathrm{NH}_{4} \mathrm{NO}_{3}+{ }_{3} \mathrm{H}_{2} \mathrm{O} .
$$


562. Aqua Regia.-As has already been stated, gold is not attacked by nitric acid; but this royal metal dissolves readily in the liquid made by mixing nitric acid with three times its volume of concentrated hydrochloric acid. Therefore the liquid solvent for the royal metal was called aqua regia ("royal water") by the alchemists of a thousand years ago. When aqua regia is gently warmed the liquid becomes yellow and gives off gases as the result of a reaction probably best represented by the equation

$$
{ }_{3} \mathrm{HCl}+\mathrm{HNO}_{3} \rightarrow{ }_{2} \mathrm{H}_{2} \mathrm{O}+\mathrm{NOCl}+\mathrm{Cl}_{2} \text {. }
$$

The substance $\mathrm{NOCl}$ is called nitrosyl chloride. The action of aqua regia on gold converts the latter into auric chloride, $\mathrm{AuCl}_{3}$, a yellow salt easily soluble in water. Platinum, which like gold is also insoluble in either nitric or hydrochloric acid singly, dissolves in aqua regia to form platinic chloride, $\mathrm{PtCl}_{4}$.

563. Nitrosyl Chloride, NOCl.- This substance is a brownishcolored gas of very disagreeable odor. It is used extensively for bleaching flour. Less than one gram of $\mathrm{NOCl}$ is required to bleach a barrel of flour. Nitrosyl chloride is readily acted on by water, thus:

$$
\mathrm{NOCl}+\mathrm{H}_{2} \mathrm{O} \rightarrow \mathrm{HCl}+\mathrm{HNO}_{2} .
$$

The nitrous acid formed is unstable and soon decomposes:

$$
{ }_{3} \mathrm{HNO}_{2} \rightarrow \mathrm{HNO}_{3}+2 \mathrm{NO}+\mathrm{H}_{2} \mathrm{O} .
$$

564. Nitrates. - Nearly every basic ion can form a nitrate. The nitrates are all easily, some extremely, soluble in water. A few nitrates derived from weak bases are hydrolyzed by water, forming difficultly soluble basic nitrates. Thus mercuric nitrate and water give $\mathrm{Hg}\left(\mathrm{NO}_{3}\right)_{2} \cdot 2 \mathrm{HgO} \cdot \mathrm{H}_{2} \mathrm{O}$, called basic mercuric nitrate. Bismuth nitrate and water give the basic nitrate $\mathrm{BiONO}_{3}$, called bismuth subnitrate, used extensively in medicine.

The nitrate ion, $\mathrm{NO}_{3}{ }^{-}$, is colorless, and so also are all nitrates of colorless basic ions.

565. Decomposition of Nitrates by Heat.-All nitrates are decomposed when they are heated to sufficiently high temperatures. They fall into three classes with respect to their behavior when heated. 
I. When sodium nitrate, $\mathrm{NaNO}_{3}$, is heated, it first melts without decomposition to give a colorless liquid. At a higher temperature the liquid appears to boil. The gas given off is oxygen, formed as follows:

$$
2 \mathrm{NaNO}_{3} \rightarrow 2 \mathrm{NaNO}_{2}+\mathrm{O}_{2} .
$$

The other product, $\mathrm{NaNO}_{2}$, is sodium nitrite, the formation of which by the action of $\mathrm{NO}_{2}$ on $\mathrm{NaOH}$ has already been mentioned (553). Potassium nitrate, $\mathrm{KNO}_{3}$, when heated gives potassium nitrite, $\mathrm{KNO}_{2}$, and oxygen.

2. The effect of heat on lead nitrate results in the following decomposition:

$$
{ }_{2} \mathrm{~Pb}\left(\mathrm{NO}_{3}\right)_{2} \rightarrow 2 \mathrm{PbO}+{ }_{4} \mathrm{NO}_{2}+\mathrm{O}_{2} .
$$

This reaction is typical of the behavior of the nitrates of most metals excepting those of the alkali group ( $\mathrm{Na}, \mathrm{K}$, etc.). In some cases the oxide of this metal is also decomposed by heat, so that the free metal is formed. This is the case with the nitrates of mercury and silver.

3. The action of gentle heat on ammonium nitrate, which takes place thus,

$$
\mathrm{NH}_{4} \mathrm{NO}_{3} \rightarrow \mathrm{N}_{2} \mathrm{O}+2 \mathrm{H}_{2} \mathrm{O}
$$

has already been described (556).

566. The Equilibrium between Nitrogen and Oxygen.-We are now ready to take up in detail the consideration of the problem of the manufacture of nitric acid from atmospheric nitrogen. We shall begin by the study of the reversible reaction

$$
\mathrm{N}_{2}+\mathrm{O}_{2} \leftrightharpoons 2 \mathrm{NO} \text {. }
$$

This reaction takes place so slowly below $800^{\circ}$ (a bright-red heat) that ordinary observation would lead one to conclude that nitrogen and oxygen have no tendency to unite, and that nitric oxide has no tendency to dissociate into its constituents. At very high temperatures each reaction takes place more rapidly, so that at the very high temperature of $3000^{\circ}$ equilibrium is reached in a small fraction of a second. At $3000^{\circ}$ only about 5 per cent of the $\mathrm{N}_{2}$ and $\mathrm{O}_{2}$ are combined as $\mathrm{NO}$ in the equilibrium 
mixture. At lower temperatures the equilibrium mixture contains still smaller proportions of NO. Table XXIV shows the percentage by volume of $\mathrm{NO}$ in the equilibrium mixture at each of several temperatures, and also the length of time required to reach practically complete equilibrium (that is, within one-tenth of I per cent complete). Inspection of the table shows that the higher the temperature the greater the proportion of $\mathrm{NO}$ formed and the quicker the attainment of equilibrium.

TABLE XXIV

\begin{tabular}{|c|c|c|}
\hline Temperature & Per Cent of NO & Time \\
\hline $\begin{array}{l}\mathrm{I}_{500^{\circ}} \ldots \ldots \ldots \ldots \\
2000^{\circ} \ldots \ldots \ldots \ldots \\
2500^{\circ} \ldots \ldots \ldots \\
3000^{\circ} \ldots \ldots \ldots \ldots\end{array}$ & $\begin{array}{l}0 . I \\
\text { I. } \\
2.6 \\
5.3\end{array}$ & $\begin{array}{l}\text { I } 2 \text { days } \\
30 \text { sec. } \\
0 . \text { I sec. } \\
0.0004 \text { sec. }\end{array}$ \\
\hline
\end{tabular}

567. The Manufacture of Nitric Acid from Air.-It is a simple matter to make nitric acid from nitric oxide, NO. We have learned that NO unites readily at ordinary temperatures with oxygen of the air to form nitrogen tetroxide,

$$
2 \mathrm{NO} \rightarrow \mathrm{O}_{2}+2 \mathrm{NO}_{2} \text {. }
$$

The latter gas reacts with water to give nitric acid and nitric oxide,

$$
{ }_{3} \mathrm{NO}_{2}+\mathrm{H}_{2} \mathrm{O} \rightarrow{ }_{2} \mathrm{HNO}_{3}+\mathrm{NO} ;
$$

and since the NO by direct union with oxygen of the air passes readily into $\mathrm{NO}_{2}$, the whole of the $\mathrm{NO}$ can finally be converted into nitric acid. The reactions discussed in this and the foregoing paragraphs are now carried out on a very large scale for the manufacture of nitric acid from atmospheric nitrogen. The difficult part of the process is the preparation of NO. We see from the table that the higher the temperature to which the mixture of $\mathrm{N}_{2}$ and $\mathrm{O}_{2}$ is heated the greater the proportion of NO present in the equilibrium mixture. Now it is not at all difficult to heat air, which is simply a mixture of $\mathrm{N}_{2}$ and $\mathrm{O}_{2}$, to $3000^{\circ}$ or even higher by means of an electric arc. The air at this temperature then contains 5 per cent or more of NO, which 
is a very satisfactory proportion. But a most serious difficulty is now encountered: this gas mixture is at a dazzling white heat, $3000^{\circ}$ ! It must be cooled almost to ordinary temperatures before it will unite with more oxygen to form $\mathrm{NO}_{2}$; and the latter must be nearly cold before it is combined with $\mathrm{H}_{2} \mathrm{O}$ to produce $\mathrm{HNO}_{3}$. Since the cooling cannot be accomplished instantaneously, the temperature for an appreciable time will be between $2500^{\circ}$ and $2000^{\circ}$. Therefore the reaction

$$
\mathrm{N}_{2}+\mathrm{O}_{2} \leftrightharpoons 2 \mathrm{NO}
$$

will reverse to a greater or less extent, since at $2500^{\circ}$ only 2.6 per cent of $\mathrm{NO}$ is stable and at $2000^{\circ}$ only I. 2 per cent. If the mixture which contained 5 per cent of $\mathrm{NO}$ at $3000^{\circ}$ remains at $2000^{\circ}$ for 30 seconds only I. 2 per cent of NO will be left! This makes it

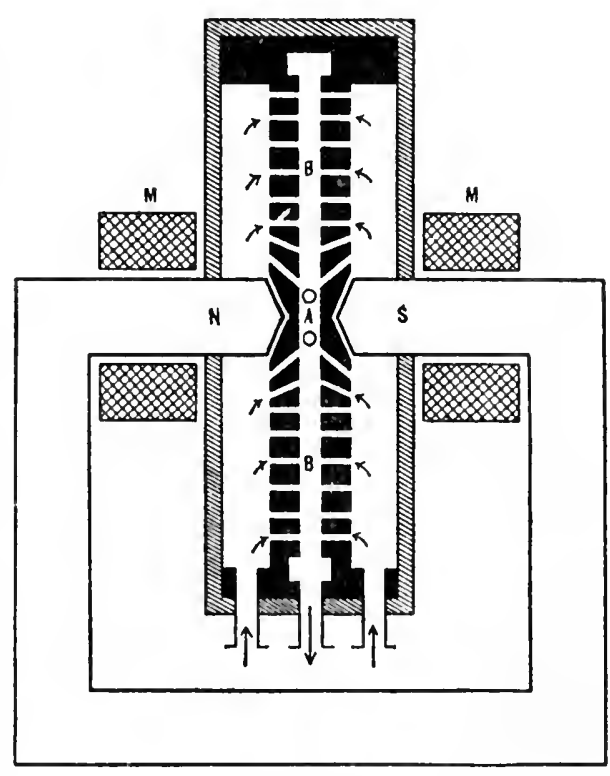

Fig. 90 imperative to cool the gas mixture very rapidly to a temperature below $1000^{\circ}$, where the speed of decomposition of $\mathrm{NO}$ becomes negligible.

One of the most successful practical methods of making NO will now be described.

568. The Birkland and Eyde Process.-This process was invented in 1903 by two Norwegians, Birkland and Eyde. Air is passed through an immense electric arc and then cooled as rapidly as possible. The mixture of unchanged air and nitric oxide is then used to produce nitric acid by means of the reactions already discussed (567). 'The Birkland-Eyde electric furnace is shown in cross-section in Fig. 90. An arc formed at $A$ between 
the electrodes which enter from the front and back is spread out by the action of a powerful magnet $N-S$ into a great electric flame which fills the space $B-B$. The wires of the magnet are shown at $M-M$. The arc is inclosed by refractory walls, pierced by numerous holes. (A refractory substance is one which is incombustible and may be heated to a high temperature.) Air passes through the furnace in the manner shown by the arrows. The temperature of the perforated walls inclosing the arc does not exceed $800^{\circ}$, owing to the cooling effect of the inflowing air. In the arc the gases reach a temperature of $3500^{\circ}$, but are subsequently quickly cooled by the walls, so that they escape at

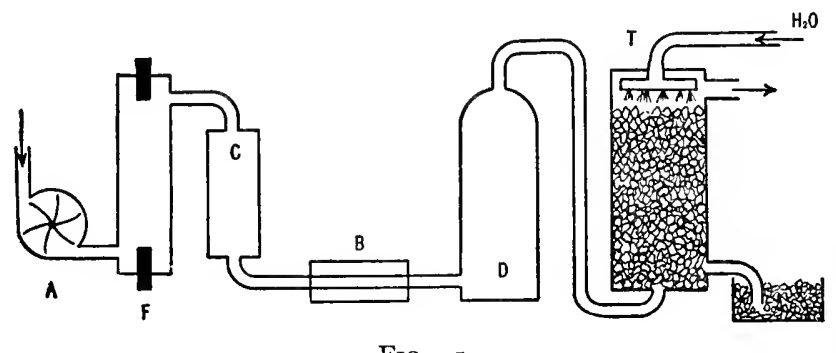

FIG. 9 I

about $1000^{\circ}$. The NO content is about 2 per cent. In addition to the furnace just described, several others accomplishing similar results are in practical use.

569. Conversion of Nitric Oxide into Nitric Acid.-Figure 9r illustrates diagrammatically a typical plant for the manufacture of nitric acid from air.

A pump, $A$ (air compressor), delivers air to the electric firnace, $F$, from which the escaping mixture of air and $\mathrm{NO}$ at I $000^{\circ}$ passes into a cooler, $C$, where the temperature is lowered to $500^{\circ}$. The gases are now passed under steam boilers, $B$, to generate steam for use in the plant, and are thereby still further cooled. The reaction

$$
2 \mathrm{NO}+\mathrm{O}_{2} \rightarrow 2 \mathrm{NO}_{2}
$$

now takes place at about $50^{\circ}$ in the vessel, $D$, there being a large excess of $\mathrm{O}_{2}$ still present. The absorption tower, $T$, into the 
base of which the gases now pass, is filled with pieces of quartz and is supplied with a stream of water at the top. Here the reaction

$$
3 \mathrm{NO}_{2}+\mathrm{H}_{2} \mathrm{O} \rightarrow 2 \mathrm{HNO}_{3}+\mathrm{NO}
$$

takes place. Nitric acid is drawn off at the base of the tower, while the NO and air pass out at the top. The escaping $\mathrm{NO}$ is not lost, since it can combine with more $\mathrm{O}_{2}$ to form $\mathrm{NO}_{2}$, which is also converted into nitric acid, so that finally all $\mathrm{NO}$ is changed into $\mathrm{HNO}_{3}$. Large amounts of nitric acid are now manufactured in this way.

570. The Manufacture of Nitric Acid from Ammonia.-The conversion of $\mathrm{NH}_{3}$ into $\mathrm{HNO}_{3}$ was mentioned in the foregoing chapter. When a mixture of one volume of $\mathrm{NH}_{3}$ and two volumes of oxygen or ten volumes of air is passed over a gauze of fine platinum wire at about $700^{\circ}$ the following reaction takes place:

$$
\mathrm{NH}_{3}+{ }_{2} \mathrm{O}_{2} \rightarrow \mathrm{HNO}_{3}+\mathrm{H}_{2} \mathrm{O} \text {. }
$$

The platinum acts catalytically, and the speed of the reaction is so great that contact with the gauze for 0 . or second is sufficient. Considerable heat is given out, so that by regulation of the rate of flow of the gases the most favorable temperature is maintained. Although this reaction has been known since $18_{30}$, it did not assume commercial importance until after cheap, synthetic ammonia became available. Since the beginning of the war a large proportion of the nitric acid required by Germany has been made from $\mathrm{NH}_{3}$ by this process. As stated in the foregoing chapter (526), an immense amount of nitric acid will be made at the new American works at Muscle Shoals, Alabama, from $\mathrm{NH}_{3}$ made by the cyanamid process.

57r. Uses of Nitric Acid. Explosives. - With very few exceptions all practical explosives are made by the use of nitrates, or nitric acid. The oldest explosive is gunpowder (now better known as black powder). This is a mechanical mixture of charcoal, sulfur, and potassium nitrate. Modern explosives are made by the action of nitric acid on various substances, such as glycerine, cotton, phenol, toluene, etc. The chemistry of these 
nitrated substances, all of which are carbon compounds, will be considered in chapter xxvi. Suffice it to say here that without nitric acid we should have no modern explosives, and without modern explosives present forms of warfare would not exist. We should also remember, however, that the arts of peace are quite as dependent upon explosives as are those of war. Modern methods of mining and quarrying would be impossible without explosives; and without the aid of these marvelous chemical products the Panama Canal could not have been dug.

Nitric acid is an indispensable reagent in the manufacture of most artificial dyes. It also has many other important uses.

572. Gunpowder, Black Powder.-The history of the discovery of gunpowder, usually ascribed to Roger Bacon, is rather obscure. Gunpowder seems first to have been used in cannon toward the end of the thirteenth century. This explosive is a black, granular substance, composed of about 74 per cent potassium nitrate, I 6 per cent charcoal, and so per cent sulfur. In making gunpowder the finely powdered components are moistened with water, and the mass is thoroughly mixed. The product is pressed into cakes; these are dried and broken into grains of suitable size. The explosion of gunpowder results in the change of $\mathrm{KNO}_{3}, \mathrm{~S}$, and $\mathrm{C}$ into $\mathrm{CO}_{2}, \mathrm{SO}_{2}, \mathrm{~N}_{2}$, and $\mathrm{K}_{2} \mathrm{~S}$. Of the products all but the potassium sulfide are gases. The very large volume of gases formed in a fraction of a second is the cause of the result which we call an explosion. These gases, at the instant of their liberation, are at a very high temperature and occupy only the same space as that of the powder. They therefore exert an enormous pressure on all sides. In a gun the projectile is shot out, while the gun itself recoils.

573. Ammonium Nitrate as an Explosive.-Ammonium nitrate, $\mathrm{NH}_{4} \mathrm{NO}_{3}$, is a very important cxplosive. We have seen that when gently heated it decomposes, thus:

$$
\mathrm{NH}_{4} \mathrm{NO}_{3} \rightarrow \mathrm{N}_{2} \mathrm{O}+{ }_{2} \mathrm{H}_{2} \mathrm{O}
$$

but when heated to a very high temperature or when exploded by a powerful detonator the decomposition is more complete:

$$
{ }_{2} \mathrm{NH}_{4} \mathrm{NO}_{3} \rightarrow 2 \mathrm{~N}_{2}+{ }_{4} \mathrm{H}_{2} \mathrm{O}+\mathrm{O}_{2} \text {. }
$$


At the high temperature of the explosion $\mathrm{H}_{2} \mathrm{O}$ is of course gaseous. A detonator is a substance that explodes readily and produces a sharp shock that starts the explosion of a large quantity of another less easily exploded substance. Ammonium nitrate is not easily exploded. It may therefore safely be used in shells fired from cannon without risk of its premature explosion. A time or impact detonator is required for such shells. Enormous quantities of $\mathrm{NH}_{4} \mathrm{NO}_{3}$ were used in the war.

574. The Cycle of Nitrogen in Nature.-We have already learned (5I) that the flesh of all animals is made up of compounds of nitrogen (with carbon, hydrogen, and oxygen chiefly). Animals cannot derive their required nitrogen from the air or from any of the simple nitrogen compounds so far studied. Much more complex nitrogen compounds (proteins) are required for the food of animals (including man). These nitrogenous animal foods are obtained from the flesh of other animals and from plants, particularly cereals. Plants have the power of building up proteins from simple nitrogen compounds, such as ammonium salts and nitrates, which are present in the soil. A small group of plants (especially the legumes: clover, alfalfa, etc.) can, by virtue of bacteria that infest their roots, take up free nitrogen and convert it into proteins. All other plants thrive only on soils containing compounds of nitrogen. Soils deficient in combined nitrogen are greatly improved in fertility by the application of ammonium salts or of nitrates as fertilizers. In normal times about three-fourths of all ammonium salts and nitrates used in Ameriça are employed as fertilizers. A large part of the nitrogen of animal food is eliminated in the form of a rather simple substance, urea, $\mathrm{CO}\left(\mathrm{NH}_{2}\right)_{2}$. This substance changes slowly into $\mathrm{CO}_{2}$ and $\mathrm{NH}_{3}$ by the action of water,

$$
\mathrm{CO}\left(\mathrm{NH}_{2}\right)_{2}+\mathrm{H}_{2} \mathrm{O} \rightarrow \mathrm{CO}_{2}+2 \mathrm{NH}_{3} \text {. }
$$

Urea itself, as well as $\mathrm{NH}_{3}$, can serve as plant food.

The decay of animal remains and refuse results in the change of their proteins into simpler substances. Ammonium salts are first formed and later are oxidized to nitrates. Thus nitrogen in nature passes through an endless cycle of changes. 
575. The Problem of the Fixation of Nitrogen.-In this chapter and the foregoing chapters we have tried to point out the practical importance of the compounds of nitrogen. The abundance of food is determined by the fertility of the soil. Fertility may be conserved and increased by means of nitrogenous fertilizers. Aside from animal refuse and manures and the ammonia obtained as a by-product of the manufacture of gas and coke, the great deposits of sodium nitrate in Chile were until recently the only important source of nitrogenous fertilizers. These deposits are by no means inexhaustible. They may last one hundred years at the present rate of consumption. Fortunately, within the last twenty-five years several methods have been developed for making nitrogen compounds directly from the free nitrogen of the air. All such methods are now referred to as processes for the fixation of nitrogen. We have at present three successful methods, each of which is in extensive use. These are: (I) the making of nitric acid from air (566), (2) the making of cyanamid from calcium carbide and atmospheric nitrogen (526), and (3) the synthesis of ammonia from hydrogen and atmospheric nitrogen (520). We have also seen that ammonia is readily made from cyanamid, and that nitric acid is easily produced by the oxidation of ammonia by air. Since the nitrogen contained in the air over each square mile of the earth's surface amounts to nearly 20,000,000 tons, the supply may be said to be inexhaustible.

The final question to be settled before deciding upon the practicability of any technical process is whether it is economically sound; in other words, will it pay? With regard to the three general methods of fixing nitrogen above mentioned, it may be said that the direct manufacture of nitric acid from air requires exceedingly cheap electric power. The other two general methods can be used where power is more expensive. At present all three processes are profitable. Only time will decide which will prove most economical. In any case we may feel satisfied that the problem of the fixation of nitrogen has been solved. 


\section{CHAPTER XXIII}

\section{PHOSPHORUS}

576. Review.-We have already had some experience with the chemistry of phosphorus and its compounds. The burning of the free element was used to show the presence of oxygen in air (10). In chapter ix phosphoric acid and some of its salts were described (158, 159). The importance of phosphates for the life of plants and animals was pointed out ( 160 ), and their widespread distribution in nature was mentioned. One method of preparing free phosphoric acid was also given. In chapter xii (247) the chemistry of phosphorus was continued in the description of the union of phosphorus with the halogens to form trivalent and pentavalent phosphorous compounds, and the reactions of these compounds with water to set free the hydrogen halides and an oxygen acid of phosphorus, as illustrated by the following typical equations:

$$
\begin{aligned}
& \mathrm{PCl}_{3}+{ }_{3} \mathrm{H}_{2} \mathrm{O} \rightarrow \mathrm{H}_{3} \mathrm{PO}_{3}+{ }_{3} \mathrm{HCl}, \\
& \mathrm{PBr}_{5}+{ }_{4} \mathrm{H}_{2} \mathrm{O} \rightarrow \mathrm{H}_{3} \mathrm{PO}_{4}+{ }_{5} \mathrm{HBr} .
\end{aligned}
$$

In chapter xxii the powerful dehydrating property of the pentoxide of phosphorus was mentioned in the formation of the anhydride of nitric acid:

$$
2 \mathrm{HNO}_{3}+\mathrm{P}_{2} \mathrm{O}_{5} \rightarrow 2 \mathrm{HPO}_{3}+\mathrm{N}_{2} \mathrm{O}_{5} .
$$

We shall now take up a systematic study of the element phosphorus and its compounds.

577. The Discovery of the Element.-The free element, which does not occur in nature, was discovered by Brandt, of Hamburg, sometime before I669. In searching for a suitable solvent for turning silver into gold he was led to ignite a mixture of dried urine and charcoal in a clay retort. Since the urine contained phosphates he was really carrying out one of the 
methods now in use for preparing free phosphorus. When we have studied some of the peculiarities of the free element we shall realize what a stir this new substance must have made in the thinking world of that day. Historians record with unction that Kraft, who purchased the secret of its preparation from Brandt, exhibited it before many of the crowned heads of Europe.

578. The Physical Properties of White Phosphorus.-At ordinary temperatures the free element in pure form is a white, waxy, translucent solid of specific giavity I.8. It does not conduct electricity. It is usually kept under water, since when it is exposed to air it soon takes fire. At ordinary temperatures the solid is slightly volatile. Calculations made from the density of the vapor show that it has a formula of $\mathrm{P}_{4}$ at temperatures up to $I, 500^{\circ}$. It melts at $45^{\circ}$ and boils at $287^{\circ}$. It is not soluble in water but is readily soluble in many solvents, such as carbon disulfide, turpentine, olive oil, and many of its own compounds. The so-called yellow phosphorus of commerce is white phosphorus made yellow by the presence of impurities.

579. The Conversion of White Phosphorus into Red Phosphorus.-When white phosphorus is exposed to light it darkens in color, owing to the formation of a new form called from its color red phosphorus. Samples of white phosphorus which have been kept for some time show a coating of this substance. At higher temperatures the change from white to red goes on more rapidly. It can be hastened also by the presence of a trace of iodine, which acts as a catalyzer.

580. The Manufacture of Phosphorus.-Though some phosphorus is manufactured from bone ash, most of it is prepared from phosphate minerals. The principal materials are phosphate rock and apatite. The first varies in composition from $\mathrm{Ca}_{3}\left(\mathrm{PO}_{4}\right)_{2}$ to the composition of apatite, $\mathrm{Ca}_{3}\left(\mathrm{PO}_{4}\right)_{2} \mathrm{CaF}_{2}$, or $\mathrm{Ca}_{3}\left(\mathrm{PO}_{4}\right)_{2} \mathrm{CaCl}_{2}$.

The most widely used method of making phosphorus consists in heating a mixture of sand, phosphate rock, and charcoal to high temperatures in an electric furnace, so designed that the phosphorous vapor distils off and condenses under water. The residual materials of the reaction are withdrawn from time to 
time, and new mixtures are put in in a continuous process. The equation of the reaction follows:

$$
{ }_{2} \mathrm{Ca}_{3}\left(\mathrm{PO}_{4}\right)_{2}+6 \mathrm{SiO}_{2}+\mathrm{IOC} \rightarrow 6 \mathrm{CaSiO}_{3}+\mathrm{IOCO}+\mathrm{P}_{4} .
$$

Red phosphorus is made on a commercial scale by heating yellow phosphorus in the absence of air.

581. The Properties of Red Phosphorus.-Although red phosphorus is often given the title amorphous phosphorus, which means that it is without crystalline form, it is really a crystalline powder. Its specific gravity is 2.I. Commercial red phosphorus often contains among other impurities small traces of yellow phosphorus and some phosphoric acid. It is on this account sticky and hygroscopic (water-absorbing), although if free from these impurities it is a dry powder quite insoluble in water and also in most other solvents. It has far greater stability than white phosphorus, as is evidenced by the fact that it must be heated to $240^{\circ}$ before it will take fire in air, and that it may be kept in contact with the latter at ordinary temperatures for years without alteration. If pure it is non-poisonous. It is for these reasons to be preferred to white phosphorus in all laboratory experiments in which the substitution can be marle. The chemistry of red phosphorus parallels that of yellow phosphorus, but in every case the reactions are less violent.

582. Allotropic Forms. - White and red phosphorus are called allotropic forms of phosphorus. The noun allotropy translated means simply "other modes." We shall find many examples of elements which exist in more than one form. We have already met ordinary oxygen and ozone (316), allotropic forms of the same element. We found that these two forms differed from each other in the number of atoms and in the energy content of the molecule. The same causes undoubtedly account for the differences in the two forms of phosphorus.

583. The Slow Oxidation of Phosphorus.-If white phosphorus is exprosed to moist air the heat given out by its oxidation will slowly raise its temperature to the melting-point, and it then takes fire. It is this fact that makes the storage of white phosphorus under water necessary. 
If a stick of moist phosphorus is viewed in the dark it is seen to give out a soft, yellow light. It is from this property that the element received its name, which means "bearer of light." We can imagine what an interest the discovery of this substance aroused in a superstitious age! The appearance of this light is apparently due to the slow oxidation which is in progress; for if conditions are such that oxidation cannot occur, no light is given out by the phosphorus. The light is simply a part of the energy of the reaction, which appears in this unusual form instead of as heat.

584. Phosphorescence. - When light is given off by bodies at ordinary temperatures they are said to phosphoresce, and the process is called phosphorescence. But the same phenomenon is shown by substances other than phosphorus. Moist, decaying wood often glows in the dark as the result of the liberation of energy in the form of light. The so-called fox fire seen in forests after night is an example. We have another case in the process by which the firefly gives out light from parts of the surface of its body. All our processes of making light are very wasteful, since high temperatures must be maintained, and so most of the energy used must go into heat, and only a small portion is changed into light. Hence the process by which the firefly gives out light at the temperature of its body is indeed marvelous, and its imitation has been the despair of scientists for generations.

585. Danger in the Use of White Phosphorus. - In conclusion it should be especially emphasized that white phosphorus is a very dangerous reagent. It should never be touched by the hands directly, since it sticks to the skin and the heat of the hand is sufficient to ignite it.

586. Matches.-On account of the serious harm to workers with yellow phosphorus, by reason of poisonous vapor, the making of yellow-phosphorus matches has been made illegal in all the great countries of the world. These matches were at one time very popular because of their easy ignition. Matches are now made with red phosphorus, phosphorus sulfide, $\mathrm{P}_{4} \mathrm{~S}_{3}$, or other non-poisonous compounds of phosphorus. The ordinary 
match is a stick of non-resinous wood dipped first in paraftin and then in a preparation to make the ignition head. The latter is usually a mixture of a good oxidizing material like lead dioxide or potassium chlorate and a reducing agent such as red phosphorus or phosphorus sulfide. With these is mixed some powdered glass to increase the friction when the match is struck. The material of the head is kept in place by glue or varnish. Friction starts the action between the oxidizer and the reducer, and the heat of this reaction kindles the paraffin, which in turn kindles the wood. The heads of safety matches are made of less easily reacting mixtures, usually sulfur and potassium chlorate. They are ignited by striking on a prepared surface coated with red phosphorus, antimony trisulfide, and glue. Match sticks are often treated with phosphoric acid or sodium phosphate to prevent "afterglow," the formation of glowing coals of charcoal after the flame of the match has been blown out.

587. Smoke Screens. - A new use for phosphorus was developed during the war. It was discovered that the most effective smoke screens for concealing troop movements were produced by means of burning phosphorus. The explosion of shells filled with phosphorus ignited the latter, which burned to give dense white clouds of the pentoxide, $\mathrm{P}_{2} \mathrm{O}_{5}$. Large quantities of phosphorus were used for this purpose.

588. Compounds of Phosphorus.-Phosphorus forms compounds with many elements. With hydrogen it unites to form several hydrides. Of these, phosphine, $\mathrm{PH}_{3}$, has been of particular interest, since it apparently resembles ammonia. It is a poisonous, ill-smelling gas, little soluble in water, but like ammonia it unites with acids to form salts, though far less readily. For instance, phosphine and hydrogen chloride unite at low temperature to form a cloud of solid phosphonium chloride:

$$
\mathrm{PH}_{3}+\mathrm{HCl} \leftrightharpoons \mathrm{PH}_{4} \mathrm{Cl} \text {. }
$$

Some of the halogen compounds of phosphorus have been already studied. These are very important substances used to synthesize many valuable halogen compounds. 'The formation of hydrogen bromide or hydrogen iodide by the action of water 
on the tribromide or the tri-iodide is typical of these important reactions and should be reviewed $(256,264)$.

With oxygen, phosphorus unites to form $\mathrm{P}_{2} \mathrm{O}_{3}, \mathrm{P}_{2} \mathrm{O}_{4}$, and $\mathrm{P}_{2} \mathrm{O}_{5}$, respectively. The first two are formed when phosphorus burns in a poor supply of oxygen; the last is formed when the burning takes place in an excess of oxygen. All three are white hygroscopic solids.

Phosphorus trioxide unites with cold water to form phosphorous acid:

$$
{ }_{3} \mathrm{H}_{2} \mathrm{O}+\mathrm{P}_{2} \mathrm{O}_{3} \leftrightharpoons 2 \mathrm{H}_{3} \mathrm{PO}_{3} .
$$

The latter is a very unstable acid. When the solution is heated some of the molecules of the acid are oxidized to phosphoric acid at the expense of others, which are reduced, forming phosphine.

Phosphorus pentoxide unites with water with great vigor, forming first metaphosphoric acid, $\mathrm{HPO}_{3}$. The stability of this acid and the completeness of the reaction make phosphorus pentoxide our most effective drying agent. The last traces of moisture are taken from air exposed to this oxide.

589. The Phosphoric Acids. - When phosphorus pentoxide is thrown into water spattering is likely to occur because of the heat given out in the formation of metaphosphoric acid:

$$
\mathrm{H}_{2} \mathrm{O}+\mathrm{P}_{2} \mathrm{O}_{5} \leftrightharpoons 2 \mathrm{HPO}_{3}
$$

The excess water may be evaporated, leaving the pure acid, a colorless, glassy solid. It changes to orthophosphoric acid, $\mathrm{H}_{3} \mathrm{PO}_{4}$, if it is kept a long time in water solution. The change may be carried out in the course of an hour if the metaphosphoric acid solution is mixed with a strong acid and heated on the water bath:

$$
\mathrm{HPO}_{3}+\mathrm{H}_{2} \mathrm{O} \leftrightharpoons \mathrm{H}_{3} \mathrm{PO}_{4} \text {. }
$$

The action is hastened by the catalytic action of the hydrogen ion of the strong acid.

590. The Properties of Orthophosphoric Acid.-Orthophosphoric acid, usually called simply phosphoric acid, forms colorless, odorless crystals, which are deliquescent and very soluble 
in water. The acid is usually sold in the form of a concentrated solution of sirupy consistency. Its dilute solution has an agrecable sour taste and is not poisonous. This acid cannot be oxidized and is very stable toward most reducing agents. It is a moderately strong acid (409); but since it is tribasic (159) we shall consider, in some detail, in a separate section, its interesting mode of ionization.

Orthophosphoric acid is not volatile. For this reason it reacts at elevated temperatures with salts of volatile acids like chlorides, bromides, and nitrates to form phosphates and to set free the volatile acids. For example,

$$
\mathrm{H}_{3} \mathrm{PO}_{4}+\mathrm{NaBr} \rightarrow \mathrm{NaH}_{2} \mathrm{PO}_{4}+\mathrm{HBr} \text {. }
$$

When phosphoric acid is heated to $210^{\circ}-215^{\circ} \mathrm{in}$ an open vessel for about an hour it is converted, by slow loss of water, into pyrophosphoric acid, $\mathrm{H}_{4} \mathrm{P}_{2} \mathrm{O}_{7}$ :

$$
{ }_{2} \mathrm{H}_{3} \mathrm{PO}_{4} \rightarrow \mathrm{H}_{4} \mathrm{P}_{2} \mathrm{O}_{7}+\mathrm{H}_{2} \mathrm{O} \text {. }
$$

If the heating is continued longer and the temperature is raised to $400^{\circ}$ or higher, the proluct slowly loses more water, giving finally metaphosphoric acid, $\mathrm{HPO}_{3}$ :

$$
\mathrm{H}_{4} \mathrm{P}_{2} \mathrm{O}_{7} \rightarrow 2 \mathrm{HPO}_{3}+\mathrm{H}_{2} \mathrm{O} \text {. }
$$

Metaphosphoric acid volatilizes slowly at a red heat $\left(700^{\circ}-800^{\circ}\right)$.

591. The Preparation of Phosphoric Acid.-Phosphoric acid is casily made in the laboratory by boiling red phosphorus with concentrated nitric acid. (Caution! White phosphorus must not be used.) The ensuing reaction produces red fumes of nitrogen tetroxide, $\mathrm{NO}_{2}$, and gives finally a colorless, sirupy solution, from which the exeess of $\mathrm{HNO}_{3}$ can be removed by heating, since $\mathrm{HNO}_{3}$ is eatsily volatile, while $\mathrm{H}_{3} \mathrm{PO}_{4}$ is not. Incomplete oxidation gives more or less phosphorous acid, $\mathrm{H}_{3} \mathrm{PO}_{3}$. 'The latter is readily oxidized to $\mathrm{H}_{3} \mathrm{PO}_{4}$ by sufficient boiling nitric acid.

Phosphoric acid is made commercially by the double decomposition of calcium phosphate and sulfuric acid ( 158 ) and subsequent purification of the product. The crude acid is very 
likely to contain magnesium and calcium salts and also some arsenic acid, $\mathrm{H}_{3} \mathrm{AsO}_{4}$, the first coming from the rock used and the second mostly from the acid employed, though sometimes from the rock as well.

592. Ionization of Phosphoric Acid.-According to the ionic theory phosphoric acid dissociates as follows:

$$
\begin{aligned}
\mathrm{H}_{3} \mathrm{PO}_{4} & \leftrightarrows \mathrm{H}^{+}+\mathrm{H}_{2} \mathrm{PO}_{4}^{-}, \\
\mathrm{H}_{2} \mathrm{PO}_{4}^{-} & \leftrightarrows \mathrm{H}^{+}+\mathrm{HPO}_{4}^{--}, \\
\mathrm{HPO}_{4}^{--} & \leftrightarrows \mathrm{H}^{+}+\mathrm{PO}_{4}^{---} .
\end{aligned}
$$

In a water solution equilibrium is established between the three pairs of opposed reactions, and each equilibrium is dependent on the other two. In all cases of this kind the first dissociation goes on to a greater degree than the second, the second to a greater degree than the third, etc. In a solution of phosphoric acid containing one-tenth formula weight per liter at $18^{\circ}$ the total concentration of hydrogen ion is 0.0282 and that of the primary phosphate ion, $\mathrm{H}_{2} \mathrm{PO}_{4}{ }^{-}$, virtually the same; the concentration of the secondary phosphate ion, $\mathrm{H}_{2} \mathrm{PO}_{4}^{--}$, is calculated to be 0.0000002 , while that of the phosphate ion, $\mathrm{PO}_{4}^{---}$, is very much smaller.

It is important that we understand that, inasmuch as $\mathrm{H}_{2} \mathrm{PO}_{4}^{-}$ and $\mathrm{HPO}_{4}^{--}$both dissociate to give hydrogen ion, they are acids, just as is phosphoric acid itself. Since, however, their degrees of dissociation are far less than that of phosphoric acid, even at greater dilutions they are far weaker acids. Obviously $\mathrm{HPO}_{4}^{--}$ is much weaker than $\mathrm{H}_{2} \mathrm{PO}_{4}{ }^{-}$. These acids are furnished by the soluble and highly ionized salts $\mathrm{NaH}_{2} \mathrm{PO}_{4}$ and $\mathrm{Na}_{2} \mathrm{HPO}_{4}$ as a result of their ionization:

$$
\begin{aligned}
& \mathrm{NaH}_{2} \mathrm{PO}_{4} \leftrightharpoons \mathrm{Na}^{+}+\mathrm{H}_{2} \mathrm{PO}_{4}^{-}, \\
& \mathrm{Na}_{2} \mathrm{HPO}_{4} \leftrightharpoons 2 \mathrm{Na}^{+}+\mathrm{HPO}_{4}{ }^{--} .
\end{aligned}
$$

593. Phosphate Baking Powder.-The ion $\mathrm{H}_{2} \mathrm{PO}_{4}^{-}$is a weak acid of about the same strength as carbonic acid, but it has an advantage over the latter in being more stable in water solution. As a result, when water is added to a mixture of sodium hydrogen carbonate, $\mathrm{NaHCO}_{3}$, and sodium acid phosphate, $\mathrm{NaH}_{2} \mathrm{PO}_{4}$, we 
get, as we expect, an effervescence due to the escape of carbon dioxide from the solution of carbonic acid formed. Thanks to the escape of the gas the action becomes complete:

$$
\mathrm{NaHCO}_{3}+\mathrm{NaH}_{2} \mathrm{PO}_{4} \leftrightharpoons \mathrm{Na}_{2} \mathrm{HPO}_{4}+\mathrm{H}_{2} \mathrm{O}+\mathrm{CO}_{2} \text {. }
$$

This is the reaction made use of in phosphate baking powders The essential ingredients are sodium bicarbonate and an acid phosphate which may be the sodium, calcium, or magnesium salt, or mixtures of them, together with some inert material, such as starch, which serves as a dilutent. When water is added to the mixture of the dry salts and flour the tiny bubbles of carbon dioxide given off make the dough spongy.

594. The Phosphates. The Sodium Salts.-Primary sodium phosphate, or sodium acid phosphate, $\mathrm{NaH}_{2} \mathrm{PO}_{4}$, is prepared in solution by adding one molecular weight of sodium hydroxide for every molecular weight of phosphoric acid:

$$
\mathrm{H}_{3} \mathrm{PO}_{4}+\mathrm{NaOH} \rightarrow \mathrm{NaH}_{2} \mathrm{PO}_{4}+\mathrm{H}_{2} \mathrm{O} \text {. }
$$

That $\mathrm{NaH}_{2} \mathrm{PO}_{4}$ alone forms, and not a mixture of the three possible phosphates, we can understand if we remember that the secondary and tertiary salts are salts of weaker acids than $\mathrm{H}_{3} \mathrm{PO}_{4}$; and so if momentarily formed they would undergo double decomposition with unused phosphoric acid to form $\mathrm{NaH}_{2} \mathrm{PO}_{4}$. A solution of $\mathrm{NaH}_{2} \mathrm{PO}_{4}$ is acid to litmus, as we would expect from the presence of the acid $\mathrm{H}_{2} \mathrm{PO}_{4}{ }^{-}$.

Common sodium phosphate, $\mathrm{Na}_{2} \mathrm{HPO}_{4}$, the secondary salt, is prepared by adding two molecular weights of sodium hydroxide for every molecular weight of phosphoric acid in solution. Solutions of secondary sodium phosphate are alkaline to litmus, owing to a slight hydrolysis (436).

$\mathrm{HPO}_{4}^{--}$is so weak an acid that when three molecules of sodium hydroxide are furnished for every molecule of phosphoric acid in solution the action is far from complete, and we have only a small part of the possible $\mathrm{Na}_{3} \mathrm{PO}_{4}$ formed when equilibrium is established. However, we can force the reaction to yield the desired salt by evaporating off the water, or better by furnishing 
a very large excess of sodium hydroxide and then evaporating the water. In either case the tertiary or normal sodium phosphate will separate from solution. As we would expect, if we redissolve this salt we get back the same solution made originally, and we find that as a result of the hydrolysis involved the solution is strongly alkaline.

As solids, all three salts are white crystalline substances and are hydrated as indicated by the following formula:

$$
\mathrm{NaH}_{2} \mathrm{PO}_{4} \cdot{ }_{4} \mathrm{H}_{2} \mathrm{O} ; \mathrm{Na}_{2} \mathrm{HPO}_{4} \cdot \mathrm{I}_{2} \mathrm{H}_{2} \mathrm{O} ; \mathrm{Na}_{3} \mathrm{PO}_{4} \cdot \mathrm{I}_{2} \mathrm{H}_{2} \mathrm{O} \text {. }
$$

When heated the primary and secondary salts both give off water and form sodium metaphosphate and pyrophosphate respectively. The tertiary phosphate remains unchangerl:

$$
\begin{gathered}
\mathrm{NaH}_{2} \mathrm{PO}_{4} \rightarrow \mathrm{NaPO}_{3}+\mathrm{H}_{2} \mathrm{O}, \\
2 \mathrm{Na}_{2} \mathrm{HPO}_{4} \rightarrow \mathrm{Na}_{4} \mathrm{P}_{2} \mathrm{O}_{7}+\mathrm{H}_{2} \mathrm{O} .
\end{gathered}
$$

595. The Titration of Phosphoric Acid, Normal Solution.If phenolphthalein is used as the indicator and phosphoric acid is titrated with sodium hydroxide ( 137 ), the color change of the indicator will be observed when two formula weights of sodium hydroxide have been added for every formula weight of the acid. This is the point at which the acid has been changed to the secondary salt, $\mathrm{Na}_{2} \mathrm{HPO}_{4}$. Therefore a liter of a solution of phosphoric acid which contains one-half formula weight of the acid will neutralize one liter of a normal sodium hydroxide solution and has the same neutralizing power as have solutions of hydrochloric acid or nitric acid which contain one formula weight of the acid per liter respectively, or of sulfuric acid which contain one-half formula weight of the acid per liter. A half molar solution of phosphoric acid is therefore a normal solution of the latter.

596. The Bead Test.- Sodium metaphosphate is much used in analytical chemistry for the so-called metaphosphate bead tests. The bead tests are carried out by first fusing microcosmic salt, sodium ammonium hydrogen phosphate, on the end of a platinum wire. This salt, which is used because it 
is easily prepared in pure form, decomposes, giving sodium metaphosphate as follows:

$$
\mathrm{NaNH}_{4} \mathrm{HPO}_{4} \rightarrow \mathrm{NaPO}_{3}+\mathrm{H}_{2} \mathrm{O}+\mathrm{NH}_{3} \text {. }
$$

Then a tiny speck of the material to be tested is picked up on the bead and fused into it. Salts of metals fuse into the bead, furming phosphates, which often are characteristically colored and thus indicate the metal present in the original substance. Presumably the salt of the metal first forms an oxide and then unites with the metaphosphate to form an orthophosphate, a reaction which is really a reverse of the formation of the metaphosphate from the orthophosphate described above. For instance, copper oxide would be clissolved, forming sodium copper phosphate, thus:

$$
\mathrm{CuO}+\mathrm{NaPO}_{3} \rightarrow \mathrm{NaCuPO}_{4} .
$$

597. Qualitative Tests for Phosphates.-Phosphates are usually tested for by the formation of yellow ammonium phosphomolybdate, $\left(\mathrm{NH}_{4}\right)_{3} \mathrm{PO}_{4} \mathrm{I} \mathrm{IMOO}_{3} 6 \mathrm{H}_{2} \mathrm{O}$, which is insoluble in nitric acid solution. As the formula indicates, the successful operation of this test necessitates an enormous excess of the reagent ammonium molybdate.

Owing to the fact that solutions of the metaphosphates and pyrophosphates are stable and only form the orthophosphate after the lapse of considerable time, unless they are heated with strong acids, it is desirable to be able to distinguish between the three kinds of phosphates. This is done on the basis of the fact that silver orthophosphate, $\mathrm{Ag}_{3} \mathrm{PO}_{4}$, is insoluble in water and yellow, whereas the other silver phosphates, $\mathrm{AgPO}_{3}$ and $\mathrm{Ag}_{4} \mathrm{P}_{2} \mathrm{O}_{7}$, though also insoluble, are white. The metaphosphate is distinguished from the pyrophosphate by the fact that the free acid which can be made from the salt will coagulate albumen, commonly known in the form of white of egg, while pyrophosphoric acid will not. As a matter of fact this test distinguishes the metaphosphoric acid from the orthophosphoric acid as well.

598. Use and Production of Phosphates.-Phosphates and phosphoric acid are used extensively in medicine, but the great 
bulk of the phosphate production supplies the match industry and the still greater demands of agriculture. The meeting of the latter need is of incalculable importance, since the phosphate taken from the land by the crops must be put back or the soil will become sterile. The natural phosphates are mined on an enormous scale either for direct use as fertilizer or for the production of superphosphate (I60), to be used for the same purpose. 


\section{CHAPTER XXIV \\ SULFUR AND ITS COMPOUNDS}

599. Introduction.-Many isolated cases of the use of sulfur compounds have already been encountered. Some of the properties of sulfuric acid were given (93), and in subsequent work its use was described in the preparation of hydrochloric (103), nitric (104), and phosphoric (158) acids, and also in the making of many sulfates and acid salts. Hydrogen sulfide was used as a reducing agent (339), the most important instance being the preparation of hydrogen iodide by the action of aqueous hydrogen sulfide upon iodine. Sulfurous acid was also shown to be a reducing agent because of its easy oxidation to sulfuric acid (340). As a matter of fact, so commonly are these and other sulfur reagents used by chemists, because of their cheapness and wide range of adaptability, that they may be counted among the most important tools of the trade. It is therefore from the point of view of possessing ourselves of a working knowledge of these important reagents that we are next to undertake the further study of sulfur and its compounds.

600. The Physical Properties of Sulfur.-The commonest form of free sulfur is called rhombic sulfur from the shape of its crystals. It is light yellow in color, not soluble in water, slightly soluble in alcohol or ether, but very soluble in some of its own compounds, such as carbon disulfide, $\mathrm{CS}_{2}$ (546). Another form of sulfur, monoclinic sulfur, which is also named from the shape of its crystals, has the same color as rhombic sulfur and is soluble in the same solvents but to a different degree. At $95^{\circ}$, the so-called transition point, these two forms of sulfur can exist in contact with each other, just as ice and water can at $0^{\circ}$. If the mixture of the two forms is heated above $95^{\circ}$, all the rhombic sulfur becomes monoclinic sulfur. On the other hand, if the mixture is cooled below $95^{\circ}$ all the monoclinic sulfur changes to 
rhombic sulfur. If the monoclinic sulfur is cooled quickly to room temperature the change to rhombic sulfur goes on very slowly, just as the change of white to red phosphorus goes on slowly at ordinary temperatures, though it is rapid at higher temperatures. It is not possible to heat a solid substance above its melting-point without melting it, but it is possible to heat a solid above its transition point to another solid form and to determine the melting-point of the first form, provided the operation is carried on very rapidly. In this way it has been found that rhombic sulfur melts at $114^{\circ}$. Monoclinic sulfur melts at $119^{\circ}$.

Still another form of sulfur, plastic sulfur, is prepared by heating sulfur to its boiling-point and then pouring it into cold water. Sulfur so treated is a plastic solid, dark amber in color, and when free from rhombic sulfur, which may be formed with it, it is insoluble even in carbon disulfide. In time it changes to rhombic sulfur.

With rising temperature molten sulfur is at first a mobile, straw-colored liquid, but at about $160^{\circ}$, curiously enough, it becomes dark brown and extraordinarily viscous. But when its temperature has reached $260^{\circ}$, its color becomes a yellow red, and its viscosity again decreases. These changes are due to the formation of allotropic liquid forms. This is the first case of the kind which we have encountered.

6or. Chemical Properties of Sulfur.-Sulfur unites chemically with most metals and non-metals. Thus it not only forms sulfides of metals, but it also forms oxides, chlorides, bromides, phosphides, etc. For example, when it is rubbed together with mercury, black mercuric sulfide forms:

$$
\mathrm{Hg}+\mathrm{S} \rightarrow \mathrm{HgS} \text {. }
$$

If sulfur is heated with iron, the reaction to form ferrous sulfide begins and soon gives out enough heat to raise the whole mass to incandescence (339). The oxides of sulfur are of such importance that their chemistry will be described later in detail. Chlorine passed over melted sulfur forms sulfur monochloride, 
$\mathrm{S}_{2} \mathrm{Cl}_{2}$. The latter substance, which is a liquid at ordinary temperatures, is an important solvent for sulfur. It has been produced in enormous quantities for the preparation of the terrible mustard gas (695).

602. The Sources of Sulfur.-Sulfur is found in large deposits, supposedly of volcanic origin, in Sicily, Japan, and elsewhere. Of these deposits, those of Sicily supplied most of the world's trade up to I904. At that time two mines in Louisiana and one in Texas became of tremendous importance, owing to the development of the Frasch process of getting out the sulfur. The deposits now being mined there are several hundred feet below the surface. Iron pipes are driven down into the beds, and superheated steam is forced down to melt the sulfur, which collects in a pool at the end of the pipe. From this pool it is forced to the surface by compressed air. Single wells deliver per day as much as four hundred to five hundred tons of sulfur, 99. 5 per cent pure, and keep it up for months at a time. The sulfur issuing at the surface is piped into bins, where it is spread out in thin layers, which immediately solidify. In this way great blocks of sulfur are built, sometimes as much as 150 feet wide. 250 feet long, and 60 feet high. The greater part of the sulfur carried to the coast from the mines is transported in dumpbottom cars, much as coal is transported.

A very considerable quantity of sulfur is produced as a by-product of other industries.

603. Rock or Roll Sulfur, Flowers of Sulfur.-Sulfur from the mines or industry is called roll or rock sulfur. Sometimes the vapor of sulfur is quickly cooled to produce a powder called flowers of sulfur. It is usually contaminated with traces of sulfurous and sulfuric acid.

604. Commercial Importance of Sulfur.-One of the very. strikingly important uses of sulfur is found in the process of vulcanizing rubber. Experts tell us that without sulfur there would be no rubber industry worthy of the name. It seems that crude rubber is stiff and hard at the temperature of ordinary winter weather, and at moderate summer heat it becomes very sticky. But after vulcanizing, a treatment with sulfur, 
commonly as vapor or with antimony sulfide (red rubber), it gains greatly in strength and elasticity and becomes remarkably indifferent to heat.

Free sulfur is an important constituent of many germicide and of many fungicide mixtures. Finely divided sulfur is dusted on vines and hops. Before the war Europe used more than one hundred thousand tons per annum for this purpose alone. Some sulfur is used in making gunpowder, the so-called "black powder." Enormous quantities of sulfur are used to make sulfur compounds such as sulfur dioxide, sulfites, sulfuric acid, carbon disulfide, and a host of other important substances. Before the war the annual consumption of sulfur in the United States was about three hundred thousand tons, but during the war it was reported to be as high as nine hundred thousand tons.

605. Hydrogen Sulfide.-The important reagent hydrogen sulfide will have been used in the laboratory by the time the work has been carried to this point. A brief description of its physical properties and chemistry has already been given (339). Usually generators of this gas are available in every laboratory. Many special types have been designed, but in principle they are much like the hydrogen generators already described in 294. Ferrous sulfide and hydrochloric acid are almost universally used to charge them.

Hydrogen sulfide burns in air to form water and sulfur dioxide. If, however, the flame is allowed to play on a cool surface it deposits sulfur. Either as a gas or dissolved in water it is a very powerful poison, small doses causing insensibility or even death. Fortunately its very bad odor warns of its presence, so that it can usually be avoided.

606. Water Solution of Hydrogen Sulfide. Hydrosulfurous Acid.- One volume of water at $0^{\circ}$ dissolves eighteen volumes of the gas, but only about three volumes at room temperature. At the latter temperature and atmospheric pressure the saturated solution is approximately one-tenth molar in concentration. All the hydrogen sulfide may be removed from a solution by boiling it. 
The solution will turn neutral litmus faintly pink. Hydrogen sulfide, or hydrosulfurous acid, ionizes in stages as follows:

$$
\begin{gathered}
\mathrm{H}_{2} \mathrm{~S} \rightarrow \mathrm{H}^{+}+\mathrm{HS}^{-}, \\
\mathrm{HS}^{-} \rightarrow \mathrm{H}^{+}+\mathrm{S}^{--} .
\end{gathered}
$$

The first or primary dissociation is weaker than that of carbonic acid or of dihydrogen phosphate ion, $\mathrm{H}_{2} \mathrm{PO}_{4}^{-}$(592). The secondary dissociation is extremely small. $\mathrm{H}_{2} \mathrm{~S}$ reacts with sodium hydroxide to form two products, sodium hydrogen sulfide and sodium sulfide:

$$
\begin{aligned}
\mathrm{H}_{2} \mathrm{~S} & +\mathrm{NaOH} \rightarrow \mathrm{NaHS}+\mathrm{H}_{2} \mathrm{O}, \\
\mathrm{NaHS} & +\mathrm{NaOH} \rightarrow \mathrm{Na}_{2} \mathrm{~S}+\mathrm{H}_{2} \mathrm{O} .
\end{aligned}
$$

607. Sodium and Ammonium Sulfides, Yellow Ammonium Sulfide.-The first of these reactions is nearly complete, but the second is far from being so. Crystalline sodium sulfide, $\mathrm{Na}_{2} \mathrm{~S}$, can, however, be prepared. A water solution of sodium hydrogen sulfide, NaHS, is slightly alkaline, but a solution of the other salt is strongly alkaline. These are the results which we would expect from the weakness of the acids $\mathrm{H}_{2} \mathrm{~S}$ and $\mathrm{HS}^{-}(436)$. Solutions of sodium sulfide find important use as a depilatory for the removal of hair from the skins of slaughtered animals and for other similar purposes.

The ammonium salts, $\mathrm{NH}_{4} \mathrm{HS}$ and $\left(\mathrm{NH}_{4}\right)_{2} \mathrm{~S}$, are prepared by passing hydrogen sulfide into ammonium hydroxide solution. The resulting solution, which is of course alkaline in reaction, is extensively used in chemical analysis.

Although pure ammonium sulfide solutions are colorless, the ordinary reagent is usually yellow, owing to the presence of dissolved sulfur formed because of exposure to the oxidizing influence of the air. Sulfur dissolves in ammonium sulfide forming a series of complex ions which, because of their similarity to peroxide ion, are called persulfide ions. Ions of the formula $\mathrm{S}_{2}^{--}, \mathrm{S}_{3}^{--}$, etc., are known. The laboratory reagent, yellow ammonium sulfide, which is prepared by dissolving sulfur in ammonium sulfide, probably contains a mixture of persulfides. Its formula is usually given $\left(\mathrm{NH}_{4}\right)_{2} \mathrm{~S}_{x}$, the subscript $x$ denoting 
an undetermined proportion of sulfur. Upon acidification of this reagent, sulfur is precipitated and hydrogen sulfide given off :

$$
\begin{aligned}
\left(\mathrm{NH}_{4}\right)_{2} \mathrm{~S}_{x}+2 \mathrm{HCl} & \rightarrow 2 \mathrm{NH}_{4} \mathrm{Cl}+\mathrm{H}_{2} \mathrm{~S}_{x}, \\
\mathrm{H}_{2} \mathrm{~S}_{x} & \rightarrow \mathrm{H}_{2} \mathrm{~S}+(x-\mathrm{I}) \mathrm{S} .
\end{aligned}
$$

608. The Precipitation of Sulfides.-The precipitation of sulfides from solution is a very important procedure in making an analysis of a substance of unknown composition. The process of a general metal analysis depends on the separation of metals from the mixture in order that they may be recognized by their characteristic reactions. Thus if we have in solution copper sulfate and zinc sulfate, we may separate the metals as follows: First, the solution is made acid $(0.25$ to $0.5 \mathrm{~N})$ with hydrochloric acid, and hydrogen sulfide is passed in. Copper sulfide alone is precipitated, because the excess of acid makes the concentration of sulfur ion so small (432) that only the extremely insoluble copper sulfide forms in sufficient amount to exceed its molecular solubility (445). Next, the filtrate, which is practically free from copper, is made alkaline with ammonium hydroxide. Immediately the separation of the white zinc sulfide, from solution begins and is carried to completion by the further addition of ammonium sulfide. In practice the extremely insoluble sulfides of mercury, lead, bismuth, cadmium, arsenic, antimony, tin, etc., if present, are brought down with the copper sulfide, and then further separation of these metals from each other follows. With zinc sulfide appear the sulfides of iron, cobalt, nickel, and manganese, together with certain insoluble hydroxides. The separation by means of hydrogen sulfide is therefore one of the fundamental processes in the analysis of metals.

609. Aqueous Hydrogen Sulfide as a Reducing Agent.Aqueous hydrogen sulfide is a good reducing agent by virtue of the easily oxidizable sulfur ion, $\mathrm{S}^{--}$, which it furnishes (503). Thus when it is oxidized by the air (339) the fundamental change may be represented as follows:

$$
{ }_{2} \mathrm{~S}^{--}+\mathrm{O}_{2} \rightarrow 2 \mathrm{~S}+{ }_{2} \mathrm{O}^{--} \text {. }
$$


The hydrogen ions which had been associated with the sulfur ion before the change unite with the oxygen ion produced, forming water.

Since hydrogen sulfide is acted upon in water solution by such mild oxidizing agents as the oxygen of the air, it is not surprising to find that it is attacked by very powerful agents such as acid permanganate (343) or acid dichromate solutions (346). If dilute sulfuric acid is added to potassium permanganate solution, and hydrogen sulfide is passed into the mixture, the intense color of the permanganate soon disappears, and in its place we have the nearly colorless solution of manganous sulfate. The sulfur ion may be oxidized to free sulfur or to sulfite ion or sulfate ion according to the experimental conditions. If free sulfur is the main product, as shown by a fine, almost white suspension in solution, the fundamental change $(56 \mathrm{r})$ in charges of the atoms undergoing oxidation and reduction is as follows:

$$
2 \mathrm{Mn}^{7+}+{ }_{5} \mathrm{~S}^{--} \rightarrow 2 \mathrm{Mn}+++{ }_{5} \mathrm{~S},
$$

and the final equation is

$$
{ }_{2} \mathrm{KMnO}_{4}+{ }_{5} \mathrm{H}_{2} \mathrm{~S}+{ }_{3} \mathrm{H}_{2} \mathrm{SO}_{4} \rightarrow 2 \mathrm{MnSO}_{4}+\mathrm{K}_{2} \mathrm{SO}_{4}+{ }_{5} \mathrm{~S}+8 \mathrm{H}_{2} \mathrm{O} .
$$

When hydrogen sulfide is led into potassium dichromate solution mixed with a strong acid, sulfuric acid for instance, the characteristic color change from orange to green or violet of the latter reagent on reduction is soon observed. In this case also free sulfur, sulfurous acid, or sulfuric acid may be produced by the reaction. If the fine, almost white, precipitate of sulfur is observed the fundamental equation is

$$
{ }_{2} \mathrm{Cr}^{6+}+{ }_{3} \mathrm{~S}^{--} \rightarrow 2 \mathrm{Cr}^{+++}+{ }_{3} \mathrm{~S},
$$

and the final equation is

$$
\mathrm{K}_{2} \mathrm{Cr}_{2} \mathrm{O}_{7}+{ }_{4} \mathrm{H}_{2} \mathrm{SO}_{4}+{ }_{3} \mathrm{H}_{2} \mathrm{~S} \rightarrow \mathrm{K}_{2} \mathrm{SO}_{4}+\mathrm{Cr}_{2}\left(\mathrm{SO}_{4}\right)_{3}+{ }_{3} \mathrm{~S}+{ }_{7} \mathrm{H}_{2} \mathrm{O} \text {. }
$$

6ro. Sulfur Dioxide.-Sulfur dioxide is a sharp-odored, colorless gas formed when sulfur or sulfides are burned in air. It is often found in volcanic gases and is to be noticed in the air 
about towns, since much of the coal burned contains traces of sulfur. A 99 per cent pure gas is made on a huge scale by burning sulfur, but an enormous supply is also obtained by the burning of pyrite, $\mathrm{FeS}_{2}$, one of the richest of the sulfur ores:

$$
{ }_{4} \mathrm{FeS}_{2}+\mathrm{IIO}_{2} \rightarrow{ }_{2} \mathrm{Fe}_{2} \mathrm{O}_{3}+8 \mathrm{SO}_{2} \text {. }
$$

Since the gas is very easily liquefied it is generally marketed in that form and stored in iron tanks, the material of which it does not attack. At ordinary temperatures the pressure in these tanks is about 2.5 atmospheres. The gas dissolves easily

water, about 70 volumes to one of water at $0^{\circ}$ and about 40 volumes at room temperature. A saturated solution at $20^{\circ}$ and one atmosphere pressure is about a I. 6 molar solution. The sulfurous acid of commerce contains about 6 per cent of sulfur dioxide.

6ri. Water Solution of Sulfur Dioxide, Sulfurous Acid.-In water solution sulfur dioxide forms sulfurous acid (340) just as carbon dioxide forms carbonic acid (I52). Sulfurous acid is a moderately weak dibasic acid. Its reducing power has already been described (340). On this account it is used on an enormous scale commercially as "antichlor," that is, to remove the residual hypochlorous acid left after the bleaching action of chlorine. Sulfurous acid is also used to bleach substances which chlorine rots, such as wool, silk, etc. Sulfurous acid and sulfites are used in breaking up the fibers of wood pulp preliminary to papermaking. Sulfur dioxide is also used in the preservation of food, particularly dried fruits.

6r2. Salts of Sulfurous Acid, the Sulfites.-Sulfurous acid forms both acid and normal salts. Most of the acid salts are soluble, but of the normal salts only the sodium, potassium, and ammonium sulfites are soluble in water. Like carbonates (46I) sulfites interact with strong acids to form the correspondingly strong acid salt and the weak and unstable acid, which decomposes, giving off the acid anhydride:

$$
\begin{aligned}
\mathrm{BaSO}_{3}+{ }_{2} \mathrm{HCl} & \rightarrow \mathrm{BaCl}_{2}+\mathrm{H}_{2} \mathrm{SO}_{3}, \\
\mathrm{H}_{2} \mathrm{SO}_{3} & \rightarrow \mathrm{H}_{2} \mathrm{O}+\mathrm{SO}_{2} .
\end{aligned}
$$


This, of course, means that sulfites, which are only moderately insoluble, are dissolved extensively by acids, particularly if the sulfur dioxide is removed from solution by boiling (463).

613. Sulfur Trioxide. Fuming Sulfuric Acid.-Sulfur trioxide, $\mathrm{SO}_{3}$, the anhydride of sulfuric acid, is a choking gas. It dissociates into sulfur dioxide and oxygen more and more with increasing temperature; at $400^{\circ}$ this dissociation is only about 2 per cent of the whole; at $500^{\circ}$ it is about 9 per cent; at $1000^{\circ}$ it is virtually complete. Sulfur trioxide can be condensed into needle-shaped crystals which mclt at $18.8^{\circ}$ and form a liquid which may be distilled undecomposed at $44.8^{\circ}$. In the presence of a trace of moisture which, of course, forms sulfuric acid, sulfur trioxide changes to a white, asbestos-like mass which can be melted at $50^{\circ}$. This substance probably consists of molecules, each one of which contains sevcral sulfur trioxide molecules. Substances the molecules of which are made up of small molecules of a simpler substance are called polymers. Sulfur trioxide is very hygroscopic. When thrown into water it reacts violently to form sulfuric acid.

Sulfur trioxide dissolves in sulfuric acid to form a product called fuming sulfuric acid or oleum.

6r4. Sulfuric Acid.-Sulfuric acid has a clear title to being the most important chemical used in industry. Very few chemical industries exist which do not employ this acid in some process. During normal times the United States produced about three and a half million tons annually, but during the Great War the yearly output is reported to have exceeded six million tons (see 6r8). This statement does not include enormous amounts made and used without being put on the market.

6r5. The Manufacture of Sulfuric Acid.-The problems of the manufacture of sulfuric acid are much like those of the synthesis of ammonia (520). At low temperatures the union of sulfur dioxide with oxygen to form sulfur trioxide, the anhydride of sulfuric acid, will go to completion, but too slowly for the process to be profitable. If higher temperatures are used the rate of the reaction is increased, but equilibrium is established 
with less of sulfur trioxide formed the higher the temperature used. The manufacture of the acid has been made successful by the use of catalysts, which hasten the reaction at low temperatures. Two general methods are used. The one called the chamber process relies on a gas catalyst and allows the reaction to come to completion in huge reaction chambers of lead. The other, called the contact process, employs a solid catalyst, on the surface of which the reaction proceeds.

6r6. The Chamber Process.-In the first method, which is the older, the catalyst is a mixture of nitric oxide and nitrogen tetroxide. Sulfur dioxide, supplied usually from the burning of sulfur or pyrite, is mixed with the right amount of air and is then swept over pots which contain niter and sulfuric acid. After receiving the nitric acid fumes from the latter the mixed gases are sent to the so-called Glover tower to receive more of the catalyst, which has been recovered from previous charges in a manner which will be described. The reaction begins in the Glover tower, but from this tower the gases are forced into the great lead chambers in which the reaction is completed. Jets of cold water or dilute sulfuric acid in the chambers dissolve the sulfur trioxide, forming a moderately concentrated sulfuric acid, called chamber acid:

$$
\mathrm{SO}_{3}+\mathrm{H}_{2} \mathrm{O} \rightarrow \mathrm{H}_{2} \mathrm{SO}_{4} \text {. }
$$

The actual mechanism by which the catalyst acts is not completely understood. The mixture of $\mathrm{NO}$ and $\mathrm{NO}_{2}$ undoubtedly contains a little $\mathrm{N}_{2} \mathrm{O}_{3}(554)$ in equilibrium with the former. If nitrous acid anhydride is regarded as the active agent, the reaction may be represented by the following equation:

$$
\mathrm{N}_{2} \mathrm{O}_{3}+\mathrm{SO}_{2} \rightarrow \mathrm{SO}_{3}+2 \mathrm{NO} \text {. }
$$

The nitric oxide left combines with more oxygen, and the regenerated oxides are again able to oxidize more sulfur dioxide.

The gases left in the chambers are next forced through the Gay Lussac tower. The latter is filled with broken brick, over which concentrated sulfuric acid constantly trickles. In this both sulfur trioxide and nitric oxide dissolve, the first to form 
fuming sulfuric acid $\left(6 \mathrm{r}_{3}\right)$ and the second to form nitrosyl sulfuric acid:

$$
{ }_{4} \mathrm{NO}+\mathrm{O}_{2}+{ }_{4} \mathrm{H}_{2} \mathrm{SO}_{4} \rightarrow{ }_{4} \mathrm{NO} \cdot \mathrm{HSO}_{4}+{ }_{2} \mathrm{H}_{2} \mathrm{O} \text {. }
$$

The mixture is drawn off and carried to the top of the Glover tower. From this point it is allowed to run down over the brick with which the tower is filled. As it does so it meets a stream of dilute acid. The dilution of the nitrosyl sulfuric acid effects its decomposition, and oxides of nitrogen are given off to the gases entering the tower from the niter pots:

$$
2 \mathrm{NO} \cdot \mathrm{HSO}_{4}+\mathrm{H}_{2} \mathrm{O} \rightarrow 2 \mathrm{H}_{2} \mathrm{SO}_{4}+\mathrm{NO}_{2}+\mathrm{NO} .
$$

The nitric oxide is of course changed into higher oxides by the oxygen of the air, so that the catalyst is being continually re-formed. The mixed gases then go on the next trip through the lead chambers. A little fresh catalyst is furnished continually from the niter pots to make up a small unavoidable loss in the process. The object of the broken brick in the two towers is to afford a large surface of contact for the gases. The yield of sulfuric acid is about 95 per cent of the amount theoretically possible. The chamber acid is of a concentration of about 78 per cent $\mathrm{H}_{2} \mathrm{SO}_{4}$ and 22 per cent water. More concentrated acid is made by subsequent evaporation.

The following diagram, a so-called flow sheet, shows by means of arrows how the various materials entering into and formed in the chamber process are related to one another:

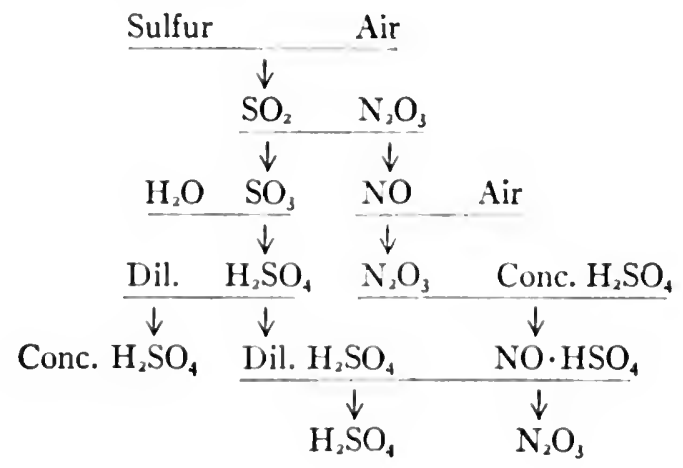


Sulfur, air, water, and the oxides of nitrogen are the starting materials. Of these the oxides of nitrogen, which act catalytically, are largely recovered in the process and used over and over again. The small deficiency caused by the failure to recover them completely is made up by gases from concentrated nitric acid.

6r7. The Contact Process.-The more recent method of making sulfuric acid is the so-called contact process. Sulfur dioxide is made to unite with oxygen on the surface of a catalyst which can be iron oxide, nickel oxide, brick, quartz, etc. Platinum is an especially good agent for this purpose, since it allows the reaction to be carried on at temperatures as low as $45^{\circ}-500^{\circ}$. Other catalysts are reported to be in use. If platinum is employed the gases must be carefully freed from arsenic, since

TABLE XXV

\begin{tabular}{|c|c|c|c|}
\hline $\begin{array}{c}\text { Bé } \\
\text { (Degrees Baumé) }\end{array}$ & $\begin{array}{c}\mathrm{Tw} \\
\text { (Degrees Twadell) }\end{array}$ & Specific Gravity & $\begin{array}{l}\text { Percentage of } \\
\text { Sulfuric Acid }\end{array}$ \\
\hline $\begin{array}{l}50^{\circ} \\
60 \\
66\end{array}$ & $\begin{array}{l}105^{\circ} \\
141 \\
167\end{array}$ & $\begin{array}{l}\text { I. } 53 \\
\text { I. } 7 \text { I } \\
\text { I. } 84\end{array}$ & $\begin{array}{l}62 \\
78 \\
93\end{array}$ \\
\hline
\end{tabular}

this substance, which is commonly present in pyrite, renders the platinum ineffective. The sulfur trioxide formed is dissolved in concentrated sulfuric acid, and water is added to the product to make the more dilute acids. The contact method has the advantage that much more concentrated solutions of sulfuric acid may be prepared directly by it than by the chamber process; but in spite of this advantage and the greater simplicity of the method, the chamber process is still the more commonly used, owing to many skilful improvements in the details of manufacture.

618. The Baumé and Twadell Scales of Specific Gravity.-In the market reports, etc., the concentration of sulfuric acid is usually given in terms of either of two arbitrary scales of specific gravity: in England and the United States the Baumé (abbreviation Bé) and on the Continent the Twadell (abbreviation Tw). Table XXV gives some data for the temperature of $15^{\circ}$. 
The "concentrated sulfuric acid" of the laboratory is usually understood to be $66^{\circ} \mathrm{Bé}$. The annual production of sulfuric acid is given in terms of $66^{\circ}$ acid (6r4). The so-called chamber acid $(6 \mathrm{I} 6)$ is usually $60^{\circ} \mathrm{Be}$. The specific gravity of other liquids heavier than water is also given on these scales. Another Baume scale is used for liquids lighter than water. Complete tables showing the relationship between the specific-gravity scales will be found in most collections of chemical tables.

6rg. Physical Properties of Sulfuric Acid.-Pure sulfuric acid is a heavy, oily liquid often called oil of vitriol. Like hydrochloric acid it forms with water a mixture of constant boilingpoint (25I). At one atmosphere pressure the composition of this mixture is $98.4^{-6}$ per cent acid. It boils at $33^{\circ}$. Pure sulfuric acid boils at $290^{\circ}$. The vapor of sulfuric acid consists almost entirely of sulfur trioxide and water.

Sulfuric acid is very hygroscopic and therefore a good drying agent. Since the 98.4 per cent acid does not give off fumes at ordinary temperatures, a little is often kept in storage vessels, such as desiccators, to keep the air dry. If a moist substance is put in such a vessel it will continuously lose water to the air, and as the water vapor is constantly being taken up by the sulfuric acid the substance is soon dry. The great heat which is given out as sulfuric acid is diluted with water has already been mentioned. Students have been warned (93) that there is danger from spattering whenever sulfuric acid is mixed with water. On this account the acid is always poured very slowly into water, with gentle stirring in order that the heavy layer may be well mixed into the lighter layer. The process of pouring water into sulfuric acid is very likely to result in a violent spattering of the hot and corrosive acid.

620. Chemical Properties of Sulfuric Acid.-Dilute sulfuric acid is a dibasic acid a little weaker than the strongest acids:

$$
\begin{aligned}
\mathrm{H}_{2} \mathrm{SO}_{4} & =\mathrm{H}^{+}+\mathrm{HSO}_{4}^{-}, \\
\mathrm{HSO}_{4}{ }^{-} & =\mathrm{H}^{+}+\mathrm{SO}_{4}^{--} .
\end{aligned}
$$

Even its secondary dissociation is that of a moderately strong acid. Like other acids, dilute sulfuric acid reacts with metals 
above hydrogen in the electromotive series (492) to give off hydrogen, but the sulfate ion is usually very slowly affected by reducing agents.

On account of its high boiling-point concentrated sulfuric acid, contrary to the usual rule, can be made to displace stronger acids from their salts, since the acid formed by the partial double decomposition of sulfuric acid with the salt is distilled off at temperatures at which the sulfuric acid is but little volatile. In this way even the insoluble silver chloride may be dissolved, though the action is very slow:

$$
\begin{aligned}
& \mathrm{AgCl} \text { (solid) } \leftrightharpoons \mathrm{AgCl} \text { (dissolved) } \leftrightharpoons \mathrm{Ag}^{+}+\mathrm{Cl}^{-} \\
& \mathrm{H}_{2} \mathrm{SO}_{4} \leftrightarrows \mathrm{HSO}_{4}{ }^{-}+\mathrm{H}^{+} \\
& \Downarrow \\
& \mathrm{AgHSO}_{4} \quad \mathrm{HCl}
\end{aligned}
$$

Concentrated sulfuric acid, unlike the dilute, is an oxidizing agent as well as an acid, though its power to oxidize is not as strong as is that of nitric acid. Some of the important and characteristic reactions will be discussed shortly (622).

If concentrated sulfuric acid is put on filter paper the latter will be found to blacken. This is a typical behavior of sulfuric acid with any carbon compound from which it can take water from combination. Thus sugar, $\mathrm{C}_{12} \mathrm{H}_{22} \mathrm{O}_{11}$, is charred by sulfuric acid. For the same reason sulfuric acid burns the skin. These reactions are in reality more complex than simple dehydrations.

621. Concentrated Sulfuric Acid and Metals.-Sulfuric acid does not attack silver or gold, but the impure acid does attack platinum. In the cold it does not react with the less active metals like copper, but does do so when hot, forming the sulfate of the metal and sulfur dioxide. The more active metals like zinc are attacked by the warm acid with the liberation of $\mathrm{SO}_{2}$; but sulfur and hydrogen sulfide may be formed according to experimental conditions. That sulfuric acid in the absence of air does not attack iron is of great technical importance, since the latter material is widely used for containers of this acid.

622. Oxidation by Concentrated Sulfuric Acid.-The solution of metals in sulfuric acid is of course the result of the oxidizing 
action of the acid. Table XXVI states briefly the charges which the sulfur atom carries in sulfuric acid and its reduction products, as well as in the other common compounds which we have studied. Apparently a sulfur atom in sulfuric acid must gain two electrons to become sulfite, six to become free sulfur, and eight to become hydrogen sulfide.

\section{TABLE XXVI}

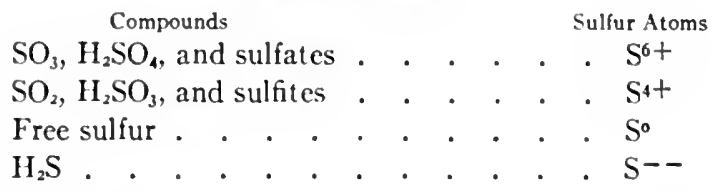

Of the possible reduction products of sulfuric acid, $\mathrm{S}$ and $\mathrm{H}_{2} \mathrm{~S}$ are capable of further reaction with excess acid. Thus $\mathrm{H}_{2} \mathrm{~S}$ bubbled through concentrated $\mathrm{H}_{2} \mathrm{SO}_{4}$ forms sulfur and the unstable sulfurous acid:

$$
\mathrm{H}_{2} \mathrm{~S}+\mathrm{H}_{2} \mathrm{SO}_{4} \rightarrow \mathrm{S}+\mathrm{H}_{2} \mathrm{O}+\mathrm{H}_{2} \mathrm{SO}_{3} \text {. }
$$

Sulfur warmed with concentrated $\mathrm{H}_{2} \mathrm{SO}_{4}$ reduces the acid, forming $\mathrm{SO}_{2}$ :

$$
\mathrm{S}+{ }_{2} \mathrm{H}_{2} \mathrm{SO}_{4} \rightarrow \mathrm{SO}_{2}+2 \mathrm{H}_{2} \mathrm{SO}_{3} \text {. }
$$

As in the case of nitric acid (557), so with sulfuric acid, the concentration of the acid, the temperature of the reaction, the fineness of division of the reducing agent if it is a solid, and whether or not the acid is present in excess, all affect the nature of the products.

In the case of zinc the fundamental reactions by which each of the possible products is formed are as follows:

$$
\begin{aligned}
& \text { for } \mathrm{SO}_{2}, \quad \mathrm{Zn}+\mathrm{S}^{6+} \rightarrow \mathrm{Zn}+++\mathrm{S}^{4+}, \\
& \text { for } \mathrm{S}, \quad 3 \mathrm{Zn}+\mathrm{S}^{6+} \rightarrow 33 \mathrm{Zn}^{++}+\mathrm{S}^{\circ}, \\
& \text { for } \mathrm{H}_{2} \mathrm{~S}, \quad 4 \mathrm{Zn}+\mathrm{S}^{6+} \rightarrow 4 \mathrm{Zn}^{++}+\mathrm{S}^{--},
\end{aligned}
$$

and the final equations arc

$$
\begin{gathered}
\mathrm{Zn}+{ }_{2} \mathrm{H}_{2} \mathrm{SO}_{4} \rightarrow \mathrm{ZnSO}_{4}+\mathrm{SO}_{2}+{ }_{2} \mathrm{H}_{2} \mathrm{O} \\
3 \mathrm{Zn}+{ }_{4} \mathrm{H}_{2} \mathrm{SO}_{4} \rightarrow{ }_{3} \mathrm{ZnSO}_{4}+\mathrm{S}+{ }_{4} \mathrm{H}_{2} \mathrm{O} \\
{ }_{4} \mathrm{Zn}+{ }_{5} \mathrm{H}_{2} \mathrm{SO}_{4} \rightarrow{ }_{4} \mathrm{ZnSO}_{4}+\mathrm{H}_{2} \mathrm{~S}+{ }_{4} \mathrm{H}_{2} \mathrm{O} .
\end{gathered}
$$


623. Action of Non-Metallic Reducing Agents on Concentrated Sulfuric Acid.-The non-metallic reducing agents show the same differences in behavior with concentrated sulfuric acid as do the metals. Thus hydrogen bromide reduces sulfuric acid to form sulfurous acid and bromine, while hydriodic acid reduces sulfuric acid to sulfur and hydrogen sulfide, iodine and water being formed at the same time (34I). The action of sulfur and hydrogen sulfide respectivcly has just been discussed (622). Charcoal (carbon) reduces the acid to sulfurous acid and is itself oxidized to carbon dioxide:

$$
\mathrm{C}+2 \mathrm{H}_{2} \mathrm{SO}_{4} \rightarrow \mathrm{CO}_{2}+2 \mathrm{H}_{2} \mathrm{SO}_{3} \text {. }
$$

624. Sulfates.-Solutions of the acid sulfates are acid to litmus, owing to the fact that the $\mathrm{HSO}_{4}{ }^{-}$ions are very largely dissociated in solutions of even moderate dilution. Sulfates as a rule are easily soluble in water, but those of barium, strontium, calcium, and lead are little soluble. Under dry heat the acid sulfates of sodium and potassium first lose water, forming the so-called pyrosulfates. These on further heating change into the normal sulfates with the loss of sulfur trioxide:

$$
\begin{aligned}
2 \mathrm{Na}\left(\mathrm{HSO}_{4}\right) & \rightarrow \mathrm{Na}_{2} \mathrm{~S}_{2} \mathrm{O}_{7}+\mathrm{H}_{2} \mathrm{O}, \\
\mathrm{Na}_{2} \mathrm{~S}_{2} \mathrm{O}_{7} & \rightarrow \mathrm{Na}_{2} \mathrm{SO}_{4}+\mathrm{SO}_{3} .
\end{aligned}
$$

Normal sulfates of the alkalies are very resistant to heat. The others break down, forming the corresponding oxides and sulfur trioxide:

$$
\mathrm{Fe}_{2}\left(\mathrm{SO}_{4}\right)_{3} \rightarrow \mathrm{Fe}_{2} \mathrm{O}_{3}+{ }_{3} \mathrm{SO}_{3} \text {. }
$$

625. Sodium Thiosulfate.-One other sulfur compound should be mentioned. This is sodium thiosulfate, the "hypo" used in photography to "fix" negatives. If sulfur is boiled in a solution of sodium sulfite it will soon be seen to dissolve. Upon evaporation the solution gives clear, colorless crystals of sodium thiosulfate:

$$
\mathrm{Na}_{2} \mathrm{SO}_{3}+\mathrm{S} \rightarrow \mathrm{Na}_{2} \mathrm{~S}_{2} \mathrm{O}_{3} \text {. }
$$

This salt is extremely soluble in water. At $0^{\circ}, 217$ parts dissolve in 100 parts by weight of water. It will be remembered as one 
of the substances used to show the phenomenon of supersaturation of a solution (123). When solutions of hypo are made, acid sulfur is deposited and sulfurous acid forms:

$$
\mathrm{Na}_{2} \mathrm{~S}_{2} \mathrm{O}_{3}+2 \mathrm{HCl} \rightarrow 2 \mathrm{NaCl}+\mathrm{H}_{2} \mathrm{SO}_{3}+\mathrm{S} .
$$

Sodium thiosulfate is manufactured in immense quantities for purposes sinilar to those for which sodium sulite is used. The reaction with chlorine is as follows:

$$
\mathrm{Na}_{2} \mathrm{~S}_{2} \mathrm{O}_{3}+{ }_{4} \mathrm{Cl}_{2}+{ }_{5} \mathrm{H}_{2} \mathrm{O} \rightarrow{ }_{2} \mathrm{NaCl}+{ }_{2} \mathrm{H}_{2} \mathrm{SO}_{4}+6 \mathrm{HCl} \text {. }
$$

Sodium thiosulfate reacts differently with iodine, forming sodium tetrathionate and sodium iodide:

$$
2 \mathrm{Na}_{2} \mathrm{~S}_{2} \mathrm{O}_{3}+\mathrm{I}_{2} \rightarrow 2 \mathrm{NaI}+\mathrm{Na}_{2} \mathrm{~S}_{4} \mathrm{O}_{6} .
$$

626. The Silver Thiosulfate Complex Ion.-If a solution of sodium thiosulfate is added to a solution of silver nitrate a white precipitate of silver thiosulfate appears:

$$
2 \mathrm{AgNO}_{3}+\mathrm{Na}_{2} \mathrm{~S}_{2} \mathrm{O}_{3} \rightarrow \mathrm{Ag}_{2} \mathrm{~S}_{2} \mathrm{O}_{3}+2 \mathrm{NaNO}_{3} \text {. }
$$

The precipitate dissolves very easily in excess of the hypo solution, forming complex silver thiosulfate ions, $\mathrm{Ag}_{2} \mathrm{~S}_{4} \mathrm{O}_{6}^{--}$, and secondarily some of the sodium silver thiosulfate molecules:

$$
\mathrm{Ag}_{2} \mathrm{~S}_{2} \mathrm{O}_{3}+\mathrm{Na}_{2} \mathrm{~S}_{2} \mathrm{O}_{3} \rightarrow \mathrm{Na}_{2} \mathrm{Ag}_{2} \mathrm{~S}_{4} \mathrm{O}_{6} \text {. }
$$

If we adc sodium chloride to the resulting solution no precipitate of silver chloride will appear. Or if we treat silver chloride with sodium thiosulfate solution the former will dissolve. So little dissociated is the complex silver thiosulfate ion that even silver bromide, which is less soluble than silver chloride, is dissolved by the hypo solution.

627. The Action of Hypo as a Fixing Solution.-The coating of a photographic plate or film which is sensitive to light is ordinarily made of gelatine and silver bromide. After the plate has been "exposed" and "developed" the parts acted upon by light are black coatings of finely divided silver. The unchanged part of the "negative" is still coated with the light-yellow silver bromide, which must be removed before the former is again 
exposed to light, or the whole plate will become evenly black and the picture be obliterated. Accordingly the unchanged silver bromide is dissolved in the hypo bath, and the soluble sodium silver thiosulfate thus formed is washed away.

628. Other Sulfur Compounds.--The chemistry of sulfur and its compounds has not been exhausted by any means by the discussions of this chapter. The treatment here has been limited to the more important properties of the commonest sulfur compounds; but there are numerous others, some of which are of technical importance. Of the latter class are persulfuric acid, $\mathrm{H}_{2} \mathrm{~S}_{2} \mathrm{O}_{8}$ permonosulfuric or Caro's acid, $\mathrm{H}_{2} \mathrm{SO}_{5}$; and hyposulfurous acid, $\mathrm{H}_{2} \mathrm{~S}_{2} \mathrm{O}_{4}$, together with their salts. The first two of these acids are closely related to hydrogen peroxide. Their graphic formulae (323), together with that of sulfuric acid, are thought to be as follows:<smiles>O=S(=O)(O)OOOS(=O)(=O)O</smiles>

Persulfuric acid<smiles>O=S(=O)(O)OO</smiles>

Permonosulfuric acid<smiles>O=S(=O)(O)O</smiles>

Sulfuric acid

They and their salts are powerful oxidizing agents. The $\mathrm{Zn}$ salt of hyposulfurous acid is readily formed by dissolving zinc dust in sulfurous acid:

$$
\mathrm{Zn}+2 \mathrm{H}_{2} \mathrm{SO}_{3} \rightarrow \mathrm{ZnS}_{2} \mathrm{O}_{4}+2 \mathrm{H}_{2} \mathrm{O} \text {. }
$$

The sodium salt $\mathrm{Na}_{2} \mathrm{~S}_{2} \mathrm{O}_{4}$, known in commerce as sodium hydrosulfite, is a powerful reducing agent and has strong bleaching action on vegetable colors. It is used for bleaching soap, etc. Five other acids of theoretical but at present little practical importance are the following: dithionic acid, $\mathrm{H}_{2} \mathrm{~S}_{2} \mathrm{O}_{6}$; trithionic acid, $\mathrm{H}_{2} \mathrm{~S}_{3} \mathrm{O}_{6}$; tetrathionic acid, $\mathrm{H}_{2} \mathrm{~S}_{4} \mathrm{O}_{6}$; pentathionic acid, $\mathrm{H}_{2} \mathrm{~S}_{5} \mathrm{O}_{6}$; hexathionic acid, $\mathrm{H}_{2} \mathrm{~S}_{6} \mathrm{O}_{6}$. 


\section{CHAPTER XXV}

CARBON AND CARBON COMPOUNDS: ORGANIC COMPOUNDS. I

629. Review.-Some relatively simple reactions of carbon and carbon compounds have already been studied. When carbon is burned in oxygen, the gas carbon dioxide is formed and may be identified by its reaction with limewater to form a white precipitate of calcium carbonate (18, I5I). The burning of other carbon compounds in excess of air is found to produce the same gas (20). Charcoal heated with copper oxide reduces the latter, forming free copper and carbon dioxide (328). The commercial production of phosphorus depends on the reducing action of carbon on calcium phosphate mixed with sand (580). Carbon monoxide is also a good reducing agent at high temperatures (329). When carbon dioxide dissolves in water the weak and unstable carbonic acid is formed ( $152,285,449)$. The bicarbonate and carbonate of sodium and of potassium are common reagents ( $161,162,448$ ). Limestone and marble, different forms of calcium carbonate, are used on a huge scale for the preparation of both lime ( 150 ) and carbon dioxide ( $16_{3}$ ). The precipitation of carbonates is governed by the usual conditions which control the precipitation of little soluble salts of weak acids (448). All carbonates are soluble in strong acids (46r). Another common reagent, acetic acid, is also a carbon compound (157). It is a colorless liquid, of sour odor, miscible with water in all proportions. It is used mainly as a weak and stable acid (424).

630. Physical Properties of Carbon.-The free element carbon appears in several allotropic forms. An impure form of great industrial importance is the non-volatile portion of coal left after the latter has been subjected to processes which free it of volatile matter. This material is called coke. It is employed as a fuel directly but is also used for making different forms of fuel gas, which are usually mixtures of carbon monoxide 
with other gases. Coke is also used for the reduction of oxide ores such as the important iron ore hematite, $\mathrm{Fe}_{2} \mathrm{O}_{3}$. Thus coke is a necessary material for many industries.

Graphite is another form of carbon. It occurs to some extent in nature but is now produced in enormous quantities by heating coke in the electric furnace (Acheson process). It is formed in slippery black scales which serve as an excellent lubricant for parts exposed to high temperatures and for wood surfaces which must rub together. Since graphite conducts electricity it is an important material for electrodes, particularly for the preparation of substances like chlorine. Mixed with clay, graphite is the "lead" of lead pencils.

The diamond is pure crystalline carbon. The natural crystals are cut into many-faced shapes in order to bring out the power of this material to reflect light. Diamonds were first produced artificially by Moissan. He took advantage of the fact that carbon, though insoluble in all ordinary solvents, is soluble in molten iron, and dissolved graphite in the latter substance. He then chilled the mass quickly by plunging it into molten lead. The outside layer of iron was first to cool, and as it shrank in so doing it put the inner part under great pressure. When the entire mass was cooled tiny diamonds, about $0.5 \mathrm{~mm}$. in diameter at the most, were found in the interior. The diamond has the distinction of being the hardest substance known and is consequently an important abrasive and cutting material. Black diamonds, which have no ornamental value, are set in the cutting surfaces of rock drills, while small diamond chips are used to cut glass.

631. Chemical Properties of Carbon.-Any form of carbon when burned gives either carbon monoxide or carbon dioxide, according to the supply of oxygen available.

If sulfur vapor is led over hot carbon, carbon disulfide, $\mathrm{CS}_{2}$, is formed. Carbon disulfide is a colorless liquid. It is an important solvent for a variety of substances. An instance of interest is its use to dissolve rubber. As has already been pointed out, it is very easily inflammable and therefore dangerous (546). 
Carbon does not unite with hydrogen (except to a very small extent at high temperatures) without the assistance of a catalyst; but if hydrogen is led over a mixture of carbon and very finely divided nickel at $250^{\circ}$ a very good yield of the gas methane, $\mathrm{CH}_{4}$, is obtained.

Carbon can be made to unite with metals, forming carbides. Thus when coke is heated with lime in the electric furnace calcium carbide is formed:

$$
\mathrm{CaO}+{ }_{3} \mathrm{C} \rightarrow \mathrm{CaC}_{2}+\mathrm{CO} .
$$

This substance is of interest because of the part it plays in the cyanamide process for fixing atmospheric nitrogen (526). It is also of importance because of its ready action with water to form acetylene:

$$
\mathrm{CaC}_{2}+{ }_{2} \mathrm{H}_{2} \mathrm{O} \rightarrow \mathrm{Ca}(\mathrm{OH})_{2}+\mathrm{C}_{2} \mathrm{H}_{2} .
$$

When sand is heated with coke under special conditions carborundum, $\mathrm{SiC}$, is formed. This important abrasive is ground, mixed with a binder, and molded into grinding wheels, knife sharpeners, etc.

632. Carbon Monoxide.-Wherever combustion of carbon or carbon compounds goes on with a deficient supply of air, the odorless, colorless, and very poisonous gas carbon monoxide is likely to be formed. It is often seen burning as a pale-blue flame on the top of coal fires. Care must be taken that furnaces are sufficiently ventilated to prevent this dangerous gas from getting into living quarters. Carbon monoxide is made in large quantities for industrial fuel gas. If made from air and coke it is mixed with nitrogen. Steam and coke give carbon monoxide and hydrogen:

$$
\mathrm{H}_{2} \mathrm{O}+\mathrm{C} \rightarrow \mathrm{CO}+\mathrm{H}_{2} \text {. }
$$

633. Carbon Dioxide.-Liquid carbon dioxide is sold in steel cylinders in which it is under about 60 atmospheres' pressure. When it is allowed to flow rapidly from these into a cloth bag it is cooled so strongly by its own evaporation that tiny particles of the solid carbon dioxide are formed and collect in the bag as carbon dioxide snow. This substance has a vapor pressure of 
$76 \mathrm{~cm}$. at $-79^{\circ}$, a temperature which is $23^{\circ}$ below the meltingpoint of the solid. Thus the latter evaporates at $-79^{\circ}$ without melting. Carbon dioxide snow has a limited but important use as a refrigerant.

The gas is very stable and will not support ordinary combustion. It is so much heavier than air that it can be poured from one vessel to another much as we would pour a liquid. These properties make it very serviceable as a fire extinguisher. Usually the apparatus contains concentrated carbonate solution and a bottle of sulfuric acid. When the handle of the device is turned the bottle is broken, and a concentrated solution of carbon dioxide is formed under considerable pressure of the gas. A stream of the gas and supersaturated solution may then be directed against the fire.

The so-called charged water (soda water) is, of course, a solution of carbon dioxide under pressure. When the pressure is released the solubility of the gas decreases, and the liquid is seen to froth with the escaping bubbles.

Carbon dioxide plays an important part in plant life, as will be shown later $(690,69 \mathrm{r})$.

634. Illuminating Gas.-The distillation of coal yields illuminating gas, together with other important products. These vary in composition according to the kind of coal used and the temperature to which the latter is heated. The following data are representative of the nature and the approximate amounts of products gained from one ton of coal:

Illuminating gas, i $\mathrm{I}, 000 \mathrm{cu}$. $\mathrm{ft}$.

Ammonia, $6 \mathrm{lb}$.

Coal tar, $120 \mathrm{lb}$.

Coke, I, $500 \mathrm{lb}$.

The coal is heated in retorts, $A$, to about $1300^{\circ}$ (Fig. 93). The vapors given off are sent through water in the so-called hydraulic main, $B$, in which some of the ammonia and tar are collected. The gas is then passed first through condensers, $C$, to take out the rest of the coal tar, and next through trays of broken brick, $D$, "scrubbers," over which water is trickling, 
to dissolve the last of the ammonia. Next, objectionable impurities such as carbon dioxide and hydrogen sulfide are removed. Finally the gas is stored in huge holders preliminary to its passage into the city main. Illuminating gas consists largely of hydrogen and methane, $\mathrm{CH}_{4}$, together with a small proportion of ethylene, $\mathrm{C}_{2} \mathrm{H}_{4}(660)$.

635. Organic Chemistry. - The number of definite compounds containing the element carbon far exceeds that of all other chemical substances. It was once thought that all except the very simplest carbon compounds were products of vegetable and animal organisms, and for this reason they were called

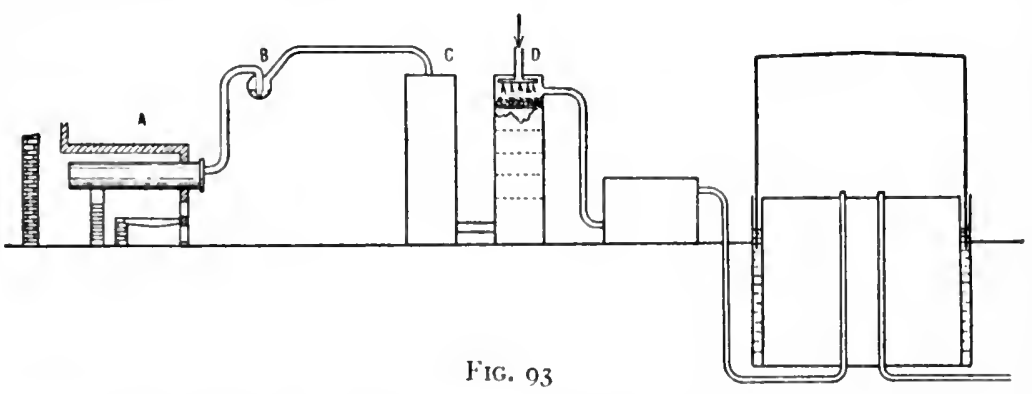

organic substances. We now know that this view is erroneous, because the great majority of organic substances can at present be made by purely chemical methods (synthesized) from the elements composing them. Although the term "organic" is a misnomer, it is retained and serves to distinguish the great class of compounds of carbon from all others, which by contrast are known as inorganic substances. A few of the simplest compounds of carbon are classed as inorganic; these include- such substances as the oxides of carbon, carbonates, carbides (e.g., $\mathrm{CaC}_{2}$ ), etc.

It will be the purpose of the rest of this chapter to give the student a brief glimpse of the fundamental principles of organic chemistry, and the object of the next chapter to show some of the great successes of this branch of the science. No attempt should be made on the part of the reader to secure an intimate knowledge of the detail presented, since such effort is best 
expended under circumstances in which more time is given to laboratory work than is possible in a general chemistry course. But the student should aim to understand the fundamental principles pointed out.

We shall begin by the study of one of the commonest and best known of organic substances, starch. We shall then show how from this plant product a variety of other substances can be derived by chemical processes. This procedure will lead us to a knowledge of the properties and interrelations of several of the most important organic compounds and thus pave the way for the further study of the compounds of carbon.

636. Starch.--Starch in nearly pure form is a well-known article of merchandise, used extensively as food and also for

\section{TABLE XXVII}

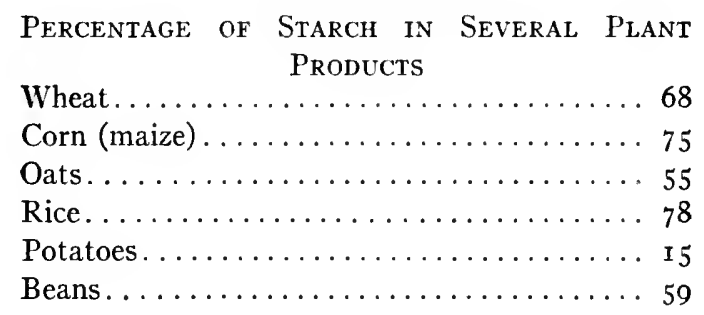

the stiffening (starching) of laundered clothes, etc. Most starch is made from corn (maize), of which it constitutes about 75 per cent. Starch forms a large part of the mass of most grains and seeds and is present in large amount in tubers, bulbs, and other parts of plants. Table XXVII shows the starch contents of a variety of plant products.

Starch may be easily prepared by grating a potato and stirring the pulp with water. If the mixture is poured through a coffee strainer, the starch goes through with the water, while the fiber is left behind. The (insoluble) starch soon settles out of the water, which can then be decanted. After being dried at room temperature the starch is obtained as a white powder.

It is well known that starch is one of the indispensable food constituents for man and all herbivorous animals. It constitutes 
the most valuable ingredient of vegetables and fruits and forms a very considerable part of all breadstuffs.

The simplest formula calculated from the analysis of starch is $\mathrm{C}_{6} \mathrm{H}_{\mathrm{ro}} \mathrm{O}_{5}$; but since it is not possible to volatilize this substance its molecular weight cannot be found by the vapordensity method. The physical and chemical properties of starch lead us to believe that its formula is less simple than that given, and that it is better represented by $\left(\mathrm{C}_{6} \mathrm{H}_{10} \mathrm{O}_{5}\right)_{n}$, where $n$ may be a rather large number.

637. The Properties of Starch.-Starch occurs in plants in minute grains easily visible under the microscope. Fig. 94 shows the appearance of wheat starch. It scarcely need be said that the visible grains are not the molecules of starch, for each grain contains an enormous number of molecules.

Natural starch is nearly insoluble in cold water, but when boiled with water the grains burst, producing so-called starch paste. A solution of starch does not conduct an electric current much

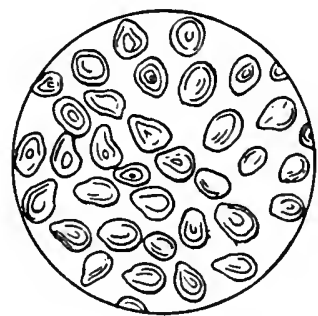

FIG. 94 better than water; it is not an acid, base, or salt. 'The action of iodine on starch (263), giving a blue color, forms the simplest and best test for this substance.

638. The Conversion of Starch into Glucose.-The most important reaction of starch is that in which it unites with water to form glucose, or grape sugar:

$$
\mathrm{C}_{6} \mathrm{H}_{60} \mathrm{O}_{5}+\mathrm{H}_{2} \mathrm{O} \rightarrow \mathrm{C}_{6} \mathrm{H}_{12} \mathrm{O}_{6} \text {. }
$$

This reaction requires the aid of a catalytic agent. Acids of all kinds act as catalytic agents for the conversion of starch into glucose. The latter substance is made commercially by heating starch with very dilute hydrochloric acid. The acid rapidly promotes the union of water with the starch but is not itself changed. At the end of the reaction the $\mathrm{HCl}$ is neutralized with soda and thus converted into $\mathrm{NaCl}$, the presence of which in minute amounts is not objectionable. The union of starch 
with water is called hydrolysis, and we say that starch is hydrolyzed to glucose.

639. Glucose, $\mathrm{C}_{6} \mathrm{H}_{\mathrm{r} 2} \mathrm{O}_{6}$.- - The sugar of ripe grapes and of many other fruits is largely glucose, which is also called dextrose and grape sugar. In pure form glucose is a white crystalline substance looking much like ordinary "granulated sugar." It is very soluble in water and is about half as sweet as ordinary sugar. Very little pure glucose is made, but glucose sirup is produced in enormous quantities.

Glucose sirup is used directly as a table sirup and is commonly known as corn sirup. It is frequently colored and flavored to imitate maple sirup. It is a good and cheap foodstuff. A large part of the production of glucose sirup is used in the manufacture of candy. The sirup is now shipped largely in tank cars.

640. The Fermentation of Glucose and the Formation of Alcohol.- The fermentation of fruit juices is due to the decomposition of their glucose content into alcohol, $\mathrm{C}_{2} \mathrm{H}_{6} \mathrm{O}$, and $\mathrm{CO}_{2}$, according to the equation

$$
\mathrm{C}_{6} \mathrm{H}_{\mathrm{I} 2} \mathrm{O}_{6} \rightarrow{ }_{2} \mathrm{C}_{2} \mathrm{H}_{6} \mathrm{O}+{ }_{2} \mathrm{CO}_{2} \text {. }
$$

This change requires the aid of a catalytic agent produced by growing yeast, which is a simple form of vegetable organism (Fig. 95). It was once thought that the

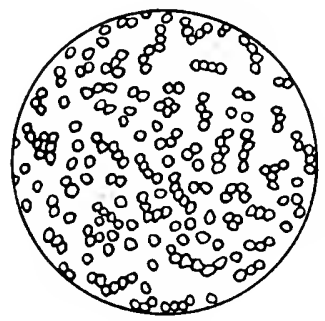

FIG. 95 yeast consumed the glucose as food and produced alcohol and $\mathrm{CO}_{2}$ as products. The falsity of this idea was shown by grinding up growing yeast so as to destroy every plant cell, then pressing out the plant sap and mixing it with glucose. The sap or extract so obtained fermented glucose and therefore contained the active catalytic agent of fermentation. The change of glucose into alcohol and carbon dioxide is therefore strictly a chemical decomposition.

64I. Alcohol, $\mathrm{C}_{2} \mathrm{H}_{6} \mathrm{O}$. - There are many kinds of alcohols, but the term alcohol is commonly and popularly applied to the 
fermentation product of glucose, $\mathrm{C}_{2} \mathrm{H}_{6} \mathrm{O}$. This is also known as grain alcohol (because it is usually made from corn, barley, or rye as starting materials), and by chemists as ethyl alcohol. Alcohol is a colorless liquid which burns with a non-luminous, sootless flame. It is miscible with water in all proportions.

In the manufacture of alcohol, corn from which the germ has been removed is ground up coarsely and boiled with water. The product is cooled to $60^{\circ}$ or $65^{\circ}$ and mixed with the malt, which furnishes the diastase necessary to convert the starch into maltose, $\mathrm{C}_{12} \mathrm{H}_{22} \mathrm{O}_{11}$, which is an intermediate product between starch and glucose. Maltose is easily changed into glucose by heating with dilute $\mathrm{HCl}$ or $\mathrm{H}_{2} \mathrm{SO}_{4}$ :

$$
\mathrm{C}_{12} \mathrm{H}_{22} \mathrm{O}_{11}+\mathrm{H}_{2} \mathrm{O} \rightarrow{ }_{2} \mathrm{C}_{6} \mathrm{H}_{12} \mathrm{O}_{6},
$$

and like glucose is easily fermented to give alcohol and $\mathrm{CO}_{2}$ :

$$
\mathrm{C}_{12} \mathrm{H}_{22} \mathrm{O}_{11}+\mathrm{H}_{2} \mathrm{O} \rightarrow{ }_{4} \mathrm{C}_{2} \mathrm{H}_{6} \mathrm{O}+{ }_{4} \mathrm{CO}_{2} \text {. }
$$

After all starch has changed into maltose, yeast is added to start fermentation, which is finished in three days. The product, which contains io to I 2 per cent of alcohol, is then distilled. The alcohol (boiling-point $78^{\circ}$ ) distils off more readily than the water and is thus easily separated from most of the water as well as from the other soluble and insoluble materials present. Repeated distillation finally yields a product containing 95 per cent of alcohol. Proof spirit contains about 50 per cent of alcohol. Pure alcohol, free from water, is called absolute alcohol. Denatured alcohol is alcohol to which has been added methyl alcohol, benzene, pyridine, etc., to render it unfit for drinking. It is poisonous.

642. Ether, $\mathrm{C}_{4} \mathrm{H}_{10} \mathrm{O}$. - Ether is made by allowing alcohol to drop slowly into a mixture of alcohol and two parts of concentrated $\mathrm{H}_{2} \mathrm{SO}_{4}$ at $\mathrm{I}_{4} 0^{\circ}$. Crude ether distils off and is condensed by cooling its vapor (Fig. 96). The reaction in its simplest form may be represented thus:

$$
{ }_{2} \mathrm{C}_{2} \mathrm{H}_{6} \mathrm{O} \rightarrow \mathrm{C}_{4} \mathrm{H}_{20} \mathrm{O}+\mathrm{H}_{2} \mathrm{O} \text {. }
$$


The $\mathrm{H}_{2} \mathrm{SO}_{4}$ acts as a powerful dehydrating (water-absorbing) agent. It is very probable that the reaction is not as simple as indicated, but that an intermediate compound of $\mathrm{C}_{2} \mathrm{H}_{6} \mathrm{O}$ and $\mathrm{H}_{2} \mathrm{SO}_{4}$ is first formed and later decomposed.

Ether is a colorless, mobile, and very volatile liquid boiling at $35^{\circ}$. It is our most important anaesthetic, being almost universally used in surgical operations. It is also of enormous importance chemically. It is an excellent solvent for numerous organic substances. It mixes with alcohol in all proportions but is only slightly soluble in water.

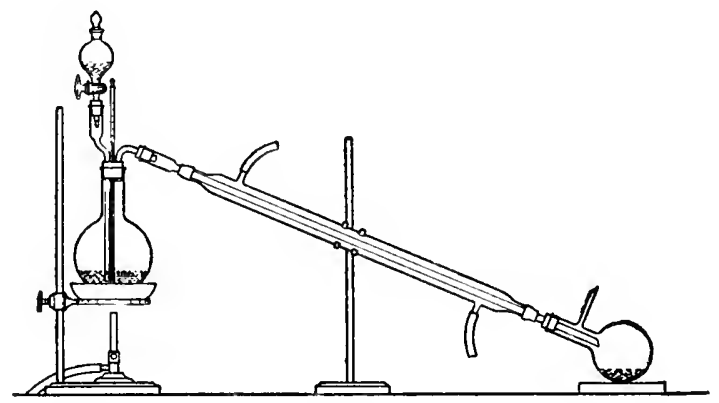

FIG. 96

643. The Paraffine Series.--From crude petroleum and the accompanying gases a very remarkable series of carbon and hydrogen compounds, called hydrocarbons, can be obtained. The simplest member of the series is methane, $\mathrm{CH}_{4}(\mathbf{5 4})$. It was pointed out that this gas is the chief component of natural gas and of marsh gas. Methane may be made in the laboratory by heating a mixture of sodium acetate, sodium hydroxide, and lime to a high temperature in an iron retort. The reaction is substantially as follows:

$$
\mathrm{NaC}_{2} \mathrm{H}_{3} \mathrm{O}_{2}+\mathrm{NaOH} \rightarrow \mathrm{CH}_{4}+\mathrm{Na}_{2} \mathrm{CO}_{3} \text {. }
$$

The second member of the series is ethane, $\mathrm{C}_{2} \mathrm{H}_{6}$, a gas closely resembling methane. The third member is propane, $\mathrm{C}_{3} \mathrm{H}_{8}$. The formula, name, physical state, boiling-point (B.P.), and melting-point (M.P.) of a number of these compounds are given in Table IXXVIII. It will be seen that the number of 
$\mathrm{H}$ atoms is two more than twice the number of carbon atoms in any molecule. Therefore the general formula for a compound having $n$ carbon atoms is $\mathrm{C}_{n} \mathrm{H}_{2 n+2}$. All the members of this series of compounds up to $\mathrm{C}_{20}$ are known, and also several with more carbon atoms in the molecule.

TABLE XXVIII

\begin{tabular}{|c|c|c|c|c|}
\hline & B.P. & & B.P. & M.P. \\
\hline 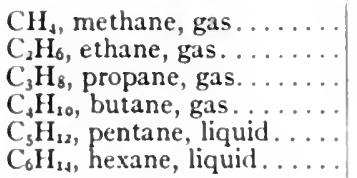 & $\begin{array}{r}-164^{\circ} \\
-\quad 93^{\circ} \\
-17^{\circ} \\
+\quad 1^{\circ} \\
38^{\circ} \\
71^{\circ}\end{array}$ & 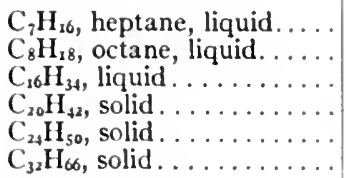 & $\begin{array}{r}98^{\circ} \\
124^{\circ} \\
288^{\circ}\end{array}$ & $\begin{array}{l}18^{\circ} \\
37^{\circ} \\
51^{\circ} \\
68^{\circ}\end{array}$ \\
\hline
\end{tabular}

The petroleum products in common use are mixtures of hydrocarbons. The principal components of some of these are given in Table XXIX.

TABLE XXIX

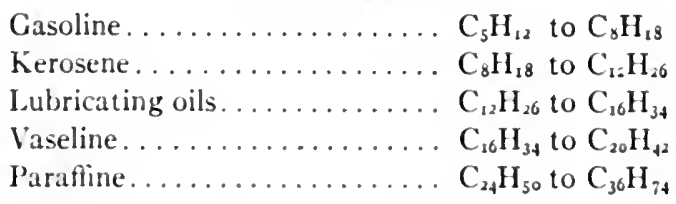

644. The Action of Chlorine on Methane.-Chlorine acts on methane to form a series of four compounds. The first reaction yields methyl chloride, $\mathrm{CH}_{3} \mathrm{Cl}$ :

$$
\mathrm{CH}_{4}+\mathrm{Cl}_{2} \rightarrow \mathrm{CH}_{3} \mathrm{Cl}+\mathrm{HCl} \text {. }
$$

Methyl chloride is a colorless gas which can react still further with chlorine:

$$
\mathrm{CH}_{3} \mathrm{Cl}+\mathrm{Cl}_{2} \rightarrow \mathrm{CH}_{2} \mathrm{Cl}_{2}+\mathrm{HCl} \text {. }
$$

The product, $\mathrm{CH}_{2} \mathrm{Cl}_{2}$, methylene chloride, is a colorless liquid, which gives, with more chlorine, chloroform, $\mathrm{CHCl}_{3}$ :

$$
\mathrm{CH}_{2} \mathrm{Cl}_{2}+\mathrm{Cl}_{2} \rightarrow \mathrm{CHCl}_{3}+\mathrm{HCl} \text {. }
$$

Chloroform is a sweet-smelling, colorless liquid boiling at $6 \mathrm{I}^{\circ}$ and insoluble in water, but miscible with alcohol or ether in all 
proportions. It is a good anaesthetic but is not as safe as ether for patients with weak hearts. Finally the action of more $\mathrm{Cl}_{2}$ on $\mathrm{CHCl}_{3}$ gives carbon tetrachloride:

$$
\mathrm{CHCl}_{3}+\mathrm{Cl}_{2} \rightarrow \mathrm{CCl}_{4}+\mathrm{HCl} \text {. }
$$

This is a colorless liquid which is not only incombustible but an excellent fire extinguisher. It is the principal component used in Pyrene and similar fire extinguishers. Devices of this type should be at hand in every laboratory for the speedy control of fires that might otherwise prove dangerous.

Carbon tetrachloride and closely related substances are used as cleaning fluids. They are perfectly safe, since they are not combustible.

645. Methyl Alcohol, $\mathrm{CH}_{4} \mathrm{O}$. - If methyl chloride, $\mathrm{CH}_{3} \mathrm{Cl}$, is heated with water at a high temperature under pressure it reacts as follows:

$$
\mathrm{CH}_{3} \mathrm{Cl}+\mathrm{H}_{2} \mathrm{O} \rightarrow \mathrm{CH}_{4} \mathrm{O}+\mathrm{HCl} \text {. }
$$

The substance, $\mathrm{CH}_{4} \mathrm{O}$, is methyl alcohol. This method of making methyl alcohol is only of scientific interest. The substance is obtained in large quantity as one of the volatile products of the distillation of wood (7or). The common name of methyl alcohol is wood alcohol. It is a colorless liquid, boiling at $66^{\circ}$ and miscible with water in all proportions It resembles common (ethyl) alcohol in its physical and chemical properties. It is a dangerous poison, often accidentally causing permanent blindness or death. It is a valuable solvent and is manufactured on a large scale.

646. Methyl Ether, $\mathrm{C}_{2} \mathrm{H}_{6} \mathrm{O}$.- - Methyl ether is made from methyl alcohol in practically the same way that ethyl ether, $\mathrm{C}_{4} \mathrm{H}_{\mathrm{ro}} \mathrm{O}$, is made from ethyl alcohol:

$$
{ }_{2} \mathrm{CH}_{4} \mathrm{O} \rightarrow \mathrm{C}_{2} \mathrm{H}_{6} \mathrm{O}+\mathrm{H}_{2} \mathrm{O} \text {. }
$$

Methyl ether, $\mathrm{C}_{2} \mathrm{H}_{6} \mathrm{O}$, is a colorless gas having chemical properties resembling those of ethyl ether.

647. Isomerism.-The formula given for methyl ether is that based upon analysis and gas density. It will be recalled 
that exactly the same formula, $\mathrm{C}_{2} \mathrm{H}_{6} \mathrm{O}$, was also ascribed to ethyl alcohol. This formula was also fixed by analysis and vapor density. Bolh substances, allhough cntirely distinct in physical and chemical properties, have exactly the same percentage composition, the same zafor density, and therefore the same molecular weight! This is a most remarkable fact but by no means the only known case of the kind. Indeed, among organic compounds there are hundreds, yes thousands, of cases in which two or even several totally different substances have the same percentage composition and the same molecular weight and in consequence the same formula. Such pairs or groups of substances are called isomers. In consequence we speak of the isomerism of methyl ether and ethyl alcohol. We shall next consider some theoretical matters with the object of developing an explanation of the remarkable phenomenon of isomerism.

648. The Valence of Carbon.-If we consider the six compounds of carbon, $\mathrm{CH}_{4}, \mathrm{C}_{2} \mathrm{H}_{2}, \mathrm{C}_{2} \mathrm{H}_{4}, \mathrm{C}_{2} \mathrm{H}_{6}, \mathrm{C}_{3} \mathrm{H}_{6}$, and $\mathrm{C}_{3} \mathrm{H}_{8}$ (see Table IV, 63), we shall have much difficulty in deciding upon the valence of carbon. In $\mathrm{CH}_{4}$, assuming the valence of hydrogen to be one, carbon evidently has a valence of four, but this value does not seem to harmonize with the formulae of the other substances. Consistent conclusions are to be reached only by considering the question of the graphic formulae of the substances (323). If we start with the plausible assumption that carbon has a valence of four, while hydrogen and chlorine each have unit valence, we may write, as the graphic formulae of methane and the substances formed from it by the action of chlorine, the following:<smiles>C</smiles><smiles>CCl</smiles><smiles>ClCCl</smiles><smiles>ClC(Cl)Cl</smiles><smiles>ClC(Cl)(Cl)Cl</smiles>

Since we write for water the graphic formula

$$
\mathrm{H}-\mathrm{O}-\mathrm{H} \text {, }
$$


thereby assuming the valence of oxygen to be two, we may write for methyl alcohol, $\mathrm{CH}_{4} \mathrm{O}(645)$, the graphic fornula<smiles>CO</smiles>

It seems probable that these graphic formulae show the actual relations of the atoms to one another in the molecule. If so, they show the structure of the molecule, and in consequence they may be called structural formulae. As stated earlier (323), the lines joining the symbols in such formulae are called bonds.

Only one possible arrangement of two atoms of carbon and six atoms of hydrogen $\left(\mathrm{C}_{2} \mathrm{H}_{6}\right)$ satisfies the condition that the valence of carbon is four and that of hydrogen one, namely the following:<smiles>CC</smiles>

This is therefore the accepted formula of ethane (643).

649. The Structural Formulae of Ethyl Alcohol and Methyl Ether.-Ethyl alcohol and methyl ether are both represented by the simple formula $\mathrm{C}_{2} \mathrm{H}_{6} \mathrm{O}$. There are two structural possibilities:<smiles>CCO</smiles>

and<smiles>COC</smiles>

Since methyl alcohol is<smiles>CO</smiles>

and since methyl and ethyl alcohol show similar chemical behavior, we may assume provisionally that the first of the 
preceding formulae is that of ethyl alcohol and the second that of methyl ether.

The formation of ethyl ether from ethyl alcohol could then be considered to take place by the removal of a molecule of water from each pair of alcohol molecules, thus:<smiles>CCOCCO</smiles>

which leads to the following as the formula for ethyl ether, $\mathrm{C}_{4} \mathrm{H}_{10} \mathrm{O}$ :<smiles>CCOC</smiles>

This conclusion is supported by the fact that this formula is entirely analogous to that assigned to methyl ether. In general, the structure of the molecules of any substance is discovered by a study of its reactions and mode of synthesis.

650. The Cause of Isomerism.-Isomerism finds a simple explanation in the assumption that the atoms composing molecules of isomers are arranged in two (or more) ways, each in harmony with simple valence laws. Hundreds of cases of isomerism are all adequately explained in this fashion.

65I. Discussion. - We have now seen how a number of the sinpler organic compounds can be obtained from natural sourcés. We have also learned a little regarding the reactions of organic substances.

The disclosure of the isomerism of ethyl alcohol and methyl ether demanded an explanation which was found in the assumption that the atoms of a molecule are joined to one another in definite fashion and always in accord with simple fixed rules of valence.

The importance of structural formulae to the chemist can well be illustrated with the examples of methyl ether and ethyl 
alcohol. The $\mathrm{OH}$ group attached to a carbon atom which carrics no other oxygen atom is called an alcohol group. When an organic chemist sees this group in a structure he knows that the substance must have the properties of an àlcohol modified to some degree, according to what other groups are near it in the molecule in question. An oxygen atom joined on either hand to a carbon atom which carries no other oxygen atoms is the group characteristic of the oxygen ethers. The latter are very different substances from alcohols and have their own characteristics. Thus at a glance the trained chemist can read the characteristics of a substance by observing the arrangements of the atoms in the structure formula of the molecule and recognizing groups which have pronounced characteristics.

Most of the substances encountered in preceding chapters belong to one or another of five principal classes: elements, oxides, acids, bases, or salts. Among organic compounds we find a large number of new and important classes. The paraffine hydrocarbons, $\mathrm{C}_{n} \mathrm{H}_{2 n+2}$, starting with methane form the simplest class of organic substances. The halogen derivatives like methyl chloride, $\mathrm{CH}_{3} \mathrm{Cl}$, and chloroform, $\mathrm{CHCl}_{3}$, form another class. 'The alcohols and the ethers are also important classes. The balance of this chapter will be devoted to the brief description of several other classes of carbon compounds. It is necessary that the student should have at least a slight acquaintance with the more important classes of organic substances if he wishes to get an insight into the nature of foodstuffs, as well as of those organic substances which play so important a rôle in our modern daily life. Among the latter are found medicinals, dyes, explosives, photographic developers, perfumes, poisons, etc.

It is not to be expected that the student who reads, however carefully, the balance of this chapter will get very definite ideas of the methods of preparation and properties of the various classes of substances there described. Familiarity with organic substances can be gained only by prolonged and detailed study in both text and laboratory. Our object in presenting the topics about to be discussed will be largely attained if we impress upon the student that carbon forms an enormous variety of com- 
pounds, that these compounds are of known molecular structure, that they fall into definite classes according to the presence in them of certain active groups of atoms, and that the members of each class show similar behavior, namely, that of the active groups, the characteristics of each of which may be modified somewhat according to the nature of other groups present in the same molecule.

In what follows, bonds between characteristic groups will frequently be represented by dots or entirely omitted. Thus $\mathrm{CH}_{3}-\mathrm{OH}$ will be written $\mathrm{CH}_{3} \cdot \mathrm{OH}$, or for still greater simplicity $\mathrm{CH}_{3} \mathrm{OH}$.

652. Aldehydes. - The mild oxidation of ethyl alcohol gives acetaldehyde. The reaction is best carried out with a mixture of a dichromate and sulfuric acid. Omitting details, the equation is

$$
\mathrm{CH}_{3} \cdot \mathrm{CH}_{2} \cdot \mathrm{OH}+\mathrm{O} \rightarrow \mathrm{CH}_{3} \cdot \mathrm{CO} \cdot \mathrm{H}+\mathrm{H}_{2} \mathrm{O} .
$$

Acetaldehyde is a very volatile liquid.

The oxidation of methyl alcohol, $\mathrm{CH}_{3} \mathrm{OH}$, gives the closely related substance formaldehyde, $\mathrm{H} \cdot \mathrm{CO} \cdot \mathrm{H}$. This is a gas of pungent odor which comes on the market as a 40 per cent solution in water called formalin. It is extensively used as a germicide and antiseptic and also in the manufacture of other important organic substances. In general, the oxidation of any alcohol of the formula $\mathrm{R} \cdot \mathrm{CH}_{2} \cdot \mathrm{OH}$, where $\mathrm{R}$ is $\mathrm{H}$ or a hydrocarbon radical, as for example $\mathrm{C}_{2} \mathrm{H}_{5}$ or $\mathrm{C}_{3} \mathrm{H}_{7}$, gives an aldehyde, $\mathrm{R} \cdot \mathrm{CO} \cdot \mathrm{H}$. The aldehydes are good reducing agents because they are readily oxidized to the corresponding acids. For example, acetaldehyde may be oxidized by suitable agents to acetic acid:

$$
\mathrm{CH}_{3} \cdot \mathrm{CO} \cdot \mathrm{H}+\mathrm{O} \rightarrow \mathrm{CH}_{3} \cdot \mathrm{CO} \cdot \mathrm{OH} .
$$

653. Acetic Acid, $\mathrm{HC}_{2} \mathrm{H}_{3} \mathrm{O}_{2}$. - As stated earlier (157), acetic acid is the most important component of vinegar, of which it forms about 4 per cent. Cider vinegar is made from cider, the juice of apples. The sweetness of fresh cider is due to glucose. Upon standing several days the glucose gradually ferments, owing to the growth of yeast, the germs of which are always 
present in the dust of the air, the glucose changing to alcohol and $\mathrm{CO}_{2}$. The fermented product is popularly known as hard cider. If hard cider is allowed to stand in an open or loosely stoppered vessel it changes in a few weeks into vinegar. This change is the result of the oxidation of alcohol to acetic acid:

$$
\mathrm{C}_{2} \mathrm{H}_{6} \mathrm{O}+\mathrm{O}_{2} \rightarrow \mathrm{HC}_{2} \mathrm{H}_{3} \mathrm{O}_{2}+\mathrm{H}_{2} \mathrm{O} \text {. }
$$

The oxidation requires the assistance of a catalytic agent produced by the so-called vinegar plant, or mother of vinegar (micoderma aceti). "White distilled vinegar" is made from dilute alcohol produced from corn, substantially in the manner already described (640). The dilute alcohol is allowed to trickle slowly through large casks filled with beechwood shavings, coated with the slimy mother of vinegar, while oxygen is furnished by a countercurrent of air that enters near the bottom and passes out at the top of the cask. This so-called "quick process" produces finished vinegar in eight to ten days.

Pure acetic acid is a colorless liquid having a sharp, characteristic odor. When free from water it solidifies at $I 7^{\circ}$ to glassy crystals called glacial acetic acid. We have already studied the reactions of solutions of acetic acid and its salts $(157,424,456)$.

654. The Graphic Formula of Acetic Acid.-Expressed graphically, we have as the equation for the oxidation of alcohol,<smiles>CCOC(=O)C=O</smiles>

The group $-\underset{\mathrm{O}}{\mathrm{C}}-\mathrm{OH}$, or briefly $-\mathrm{CO} \cdot \mathrm{OH}$, is the carboxyl

radical. One of the oxygen atoms of acetic acid is said to be attached to one of the carbon atoms by a double bond (324). Of the four $\mathrm{H}$ atoms of acetic acid, one occupies a unique position in that it is attached to an atom of $\mathrm{O}$, while the others are attached to one of the carbon atoms. Since only one of the $\mathrm{H}$ 
atoms of the molecule is ionizable we may safely conclude that it is the one attached to oxygen.

655. The Fatty Acids. - There is a series of acids closely related to acetic acid, all having a carboxyl radical attached to a hydrocarbon radical. The general formula of such acids is $\mathrm{R} \cdot \mathrm{CO} \cdot \mathrm{OH}$, where $\mathrm{R}$ stands for hydrogen or any hydrocarbon radical such as $\mathrm{C}_{2} \mathrm{H}_{5}, \mathrm{C}_{3} \mathrm{H}_{7}, \mathrm{C}_{4} \mathrm{H}_{9}$, etc. The names and formulae of a few of the more important are given in Table XXX.

\section{TABLE XXX}

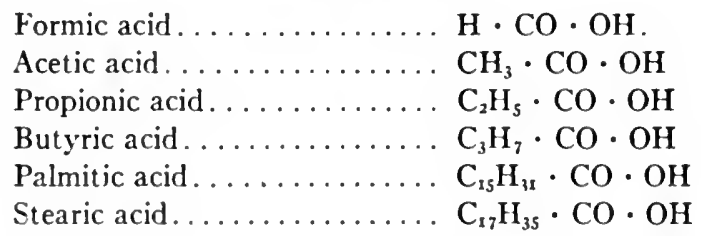

'These acids are known as fatty acids, because some of them are obtained from fats (680).

656. Ketones.-Calcium acetate, $\mathrm{Ca}\left(\mathrm{C}_{2} \mathrm{H}_{3} \mathrm{O}_{2}\right)_{2}$, is an important article of commerce made by the action of acetic acid on limestone $\left(\mathrm{CaCO}_{3}\right)$. When calcium acetate is strongly heated it decomposes into $\mathrm{CaCO}_{3}$ and acetone, $\mathrm{C}_{3} \mathrm{H}_{6} \mathrm{O}$ :

$$
\mathrm{Ca}\left(\mathrm{C}_{2} \mathrm{H}_{3} \mathrm{O}_{2}\right)_{2} \rightarrow \mathrm{CaCO}_{3}+\mathrm{C}_{3} \mathrm{H}_{6} \mathrm{O} \text {. }
$$

The acetone distils off and is condensed to a liquid. Purified acetone is a colorless liquid of mild but peculiar odor. It boils at $56^{\circ}$. It mixes with water or alcohol in all proportions. It is an excellent solvent for many organic substances, and it is also used in the preparation of several important organic compounds, of which chloroform is one. The structural formula of acetone is indicated by its formation from calcium acetate:

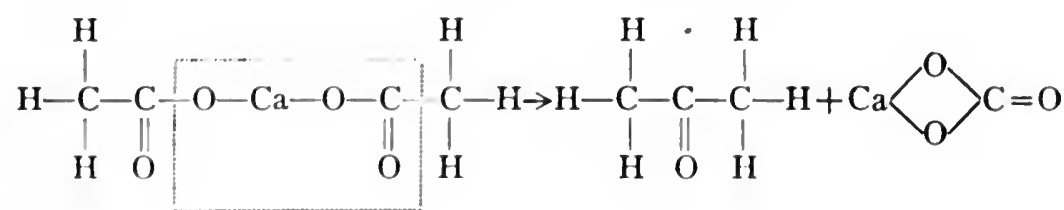


Acetone is the simplest member of a class of substances called ketones. The general formula for a ketone is $\mathrm{R}_{\mathrm{I}} \cdot \mathrm{CO} \cdot \mathrm{R}_{3}$, where $R_{1}$ and $R_{2}$ represent the formulae of hydrocarbon radicals. In acetone $R_{1}$ and $R_{2}$ are both methyl, $\mathrm{CH}_{3}$; but if, say, $\mathrm{R}_{1}$ is ethyl, $\mathrm{C}_{2} \mathrm{H}_{5}$, and $\mathrm{R}_{2}$ is propyl, $\mathrm{C}_{3} \mathrm{H}_{7}$, the formula of the ketone would be $\mathrm{C}_{2} \mathrm{H}_{5} \cdot \mathrm{CO} \cdot \mathrm{C}_{3} \mathrm{H}_{7}$.

Ketones are closely related to aldehydes, since if $R_{2}$ is $H$ we have $\mathrm{R}_{\mathrm{I}} \cdot \mathrm{CO} \cdot \mathrm{H}$, the formula of an aldehyde. They are reducing agents but are not as active as aldehydes.

657. Esters. - The esters are an important class of compounds, inasmuch as all animal and vegetable fats and oils are included therein. One of the simplest esters, ethyl acetate, $\mathrm{CH}_{3} \mathrm{COOC}_{2} \mathrm{H}_{5}$, is obtained by the action of ethyl alcohol on acetic acid:

$$
\mathrm{CH}_{3} \cdot \mathrm{CO} \cdot \mathrm{OH}+\mathrm{C}_{2} \mathrm{H}_{5} \cdot \mathrm{OH} \rightarrow \mathrm{CH}_{3} \cdot \mathrm{CO} \cdot \mathrm{OC}_{2} \mathrm{H}_{5}+\mathrm{H}_{2} \mathrm{O} .
$$

Ethyl acetate is a colorless liquid, boiling at $75^{\circ}$. It has a rather pleasant odor and is somewhat soluble in water.

Just as most acids can form salts with most bases, so most acids can form esters with most alcohols. However, alcohols are not bases, since they do not yield $\mathrm{OH}^{-}$ions, and esters are not salts. Their water solutions are not ionized, and they do not give the ionic reactions shown by solutions of the acids from which they are derived. The formation of esters requires in general the stimulus of $\mathrm{H}^{+}$ions as a catalytic agent. In making ethyl acetate we add to the mixture of acetic acid and alcohol some $\mathrm{HCl}$ or $\mathrm{H}_{2} \mathrm{SO}_{4}$. The union of acetic acid and alcohol does not take place completely but reaches equilibrium when about two-thirds of the possible amount of ester has been formed. This is because the reaction is reversible.

The change of ester and water into acid and alcohol is promoted by the presence of much water and also by the catalytic influence of acids. The speed of ester formation and also the speed of reaction of ester and water are greatly increased with increase of temperature.

Methyl acetate, $\mathrm{CH}_{3} \mathrm{CO} \cdot \mathrm{OCH}_{3}$, closely resembles ethyl acetate. It is miscible with water in all proportions. Other 
esters, including fats and oils, will be considered in the next chapter.

658. Amines. - The amines are derivatives of ammonia and, like the latter, are base-forming substances capable of yielding salts with acids. The simplest member of the class is methyl amine, $\mathrm{CH}_{3} \mathrm{NH}_{2}$. If methyl iodide (660) and ammonia are mixed they unite to form methyl ammonium iodide:

$$
\mathrm{CH}_{3} \mathrm{I}+\mathrm{NH}_{3} \rightarrow \mathrm{CH}_{3} \mathrm{NH}_{3} \mathrm{I} \text {. }
$$

The product is a soluble salt of the base $\mathrm{CH}_{3} \mathrm{NH}_{3} \mathrm{OH}$. This unstable base, which is set free by the action of sodium hydroxide on the salt, easily dissociates into methyl amine and water. Methyl amine is a colorless gas with an odor resembling ammonia. It is abundantly soluble in water, with which it partially unites, thus:

$$
\mathrm{CH}_{3} \cdot \mathrm{NH}_{2}+\mathrm{H}_{2} \mathrm{O}=\mathrm{CH}_{3} \cdot \mathrm{NH}_{3} \cdot \mathrm{OH} \text {. }
$$

Ethyl iodide, $\mathrm{C}_{2} \mathrm{H}_{5} \mathrm{I}$, and ammonia give ethyl ammonium iodide, $\mathrm{C}_{2} \mathrm{H}_{5} \cdot \mathrm{NH}_{3} \cdot \mathrm{I}$, from which we readily obtain ethyl amine, $\mathrm{C}_{2} \mathrm{H}_{5} \cdot \mathrm{NH}_{2}$, a substance closely resembling methyl amine.

Methyl amine acts on methyl iodide as follows:

$$
\mathrm{CH}_{3} \cdot \mathrm{NH}_{2}+\mathrm{CH}_{3} \mathrm{I} \rightarrow\left(\mathrm{CH}_{3}\right)_{2} \mathrm{NH}_{2} \mathrm{I} \text {, }
$$

from which dimethyl amine, $\left(\mathrm{CH}_{3}\right)_{2} \mathrm{NH}$, is obtained by the action of alkali. Dimethyl amine, by further action of methyl iodide, yields $\left(\mathrm{CH}_{3}\right)_{3} \mathrm{NHI}$, from which by the acion of alkali we get trimethyl amine, $\left(\mathrm{CH}_{3}\right)_{3} \mathrm{~N}(59)$. Amines of various kinds are usually found among the products of decomposition of proteins (685). Trimethyl amine, for example, is contained in herring brine.

659. Amides. - The interaction of ethyl acetate and ammonia gives acetamide and alcohol:

$$
\mathrm{CH}_{3} \mathrm{COOC}_{2} \mathrm{H}_{5}+\mathrm{NH}_{3} \rightarrow \mathrm{CH}_{3} \mathrm{CONH}_{2}+\mathrm{C}_{2} \mathrm{H}_{5} \mathrm{OH} \text {. }
$$

Acetamide is also easily made by distilling ammonium acetate:

$$
\mathrm{CH}_{3} \mathrm{COONH}_{4} \rightarrow \mathrm{CH}_{3} \mathrm{CONH}_{2}+\mathrm{H}_{2} \mathrm{O} \text {. }
$$


The substance is a white crystalline solid easily soluble in water. It unites with hydrochloric acid to form a saltlike compound, $\mathrm{CH}_{3} \mathrm{CONH}_{3} \mathrm{Cl}$. This fact shows that the $\mathrm{NH}_{2}$ radical in an amide has still some basic properties.

Most organic acids and some mineral acids are able to form amides. Carbonic acid, for example, forms the amide $\mathrm{CO}\left(\mathrm{NH}_{2}\right)_{2}$. This important substance is commonly known as urea. By far the larger part of the nitrogen content of the food of all animals is excreted as urea. Urea reacts slowly with water to form ammonia and carbon dioxide:

$$
\mathrm{CO}\left(\mathrm{NH}_{2}\right)_{2}+\mathrm{H}_{2} \mathrm{O} \rightarrow{ }_{2} \mathrm{NH}_{3}+\mathrm{CO}_{2} \text {. }
$$

660. Ethylene, $\mathbf{C}_{2} \mathbf{H}_{4}$. $-\mathrm{A}$ modification of the process of making ether (642) yields ethylene. To make ethylene, a mixture of alcohol with six parts by weight of concentrated sulfuric acid is heated to $I 65^{\circ}$, and a mixture of one part of alcohol to two parts of sulfuric acid is dropped in slowly. The gas $\mathrm{C}_{2} \mathrm{H}_{4}$ is given off. The reactions in this case are probably also complex, but the net result is the decomposition of alcohol into water and ethylene:

$$
\mathrm{C}_{2} \mathrm{H}_{6} \mathrm{O} \rightarrow \mathrm{H}_{2} \mathrm{O}+\mathrm{C}_{2} \mathrm{H}_{4} \text {. }
$$

Ethylene is a colorless gas nearly insoluble in water. It burns with a luminous flame. It gives several interesting and important reactions. With $\mathrm{Cl}_{2}$ it unites to form ethylene chloride:

$$
\mathrm{C}_{2} \mathrm{H}_{4}+\mathrm{Cl}_{2} \rightarrow \mathrm{C}_{2} \mathrm{H}_{4} \mathrm{Cl}_{2} \text {, }
$$

a colorless, oil-like liquid, boiling at $84^{\circ}$. Ethylene chloride is insoluble in water. It has none of the properties of a salt. Ethylene also unites with bromine, thus:

$$
\mathrm{C}_{2} \mathrm{H}_{4}+\mathrm{Br}_{2} \rightarrow \mathrm{C}_{2} \mathrm{H}_{4} \mathrm{Br}_{2} \text {. }
$$

The product, ethylene bromide, resembles the chloride. Ethylene and $\mathrm{HBr}$ unite readily to form $\mathrm{C}_{2} \mathrm{H}_{5} \mathrm{Br}$, ethyl bromide, a liquid boiling at $38^{\circ}$ :

$$
\mathrm{C}_{2} \mathrm{H}_{4}+\mathrm{HBr} \rightarrow \mathrm{C}_{2} \mathrm{H}_{5} \mathrm{Br} \text {. }
$$


Ethylene and HI give ethyl iodide, boiling-point $72^{\circ}$ :

$$
\mathrm{C}_{3} \mathrm{H}_{4}+\mathrm{HI} \rightarrow \mathrm{C}_{2} \mathrm{H}_{5} \mathrm{I} \text {. }
$$

Neither ethyl bromide or ethyl iodide has any of the properties of a salt. Methyl iodide, $\mathrm{CH}_{3} \mathrm{I}$, closely resembles ethyl iodide. It is used in the preparation of other organic compounds (658).

66r. The Structural Formula of Ethylene.-The fact that ethylene unites with chlorine, hydrobromic acid, etc., leads to the conclusion that two of the valence bonds of the carbon atoms of the $\mathrm{C}_{2} \mathrm{H}_{4}$ molecule are either free,<smiles>CCC(C)C</smiles>

or, more probably, are attached to, or satisfied by, one another so as to form a double bond (324) between the two carbon atoms:<smiles>C=C</smiles>

In the reaction with chlorine, for example, the extra bonds unite with chlorine to give<smiles>ClCCl</smiles>

662. The Ethylene Series, $\mathrm{C}_{n} \mathrm{H}_{2 n}$. $-\mathrm{A}$ long series of hydrocarbons which have twice as many $\mathrm{H}$ as $\mathrm{C}$ atoms per molecule and of which the first member is $\mathrm{C}_{2} \mathrm{H}_{4}$ is known. The second member is propylene, $\mathrm{CH}_{3}-\mathrm{CH}=\mathrm{CH}_{2}$. There are theoretically just. three butylenes, $\mathrm{C}_{4} \mathrm{H}_{8}$ :

(I) $\mathrm{CH}_{3} \cdot \mathrm{CH}_{2} \cdot \mathrm{CH}=\mathrm{CH}_{2}$

(2) $\mathrm{CH}_{3} \cdot \mathrm{CH}=\mathrm{CH} \cdot \mathrm{CH}_{3}$

(3) $\stackrel{\mathrm{CH}_{3}}{\mathrm{CH}_{3}}>\mathrm{C}=\mathrm{CH}_{2}$ 
These are all known. All of the members of this series unite with $\mathrm{Cl}_{2}, \mathrm{Br}_{2}, \mathrm{HI}$, etc. For example,

$$
\mathrm{CH}_{3}-\mathrm{CH}_{2}-\mathrm{CH}=\mathrm{CH}_{2}+\mathrm{Cl}_{2} \rightarrow \mathrm{CH}_{3}-\mathrm{CH}_{2}-\mathrm{CHCl}-\mathrm{CH}_{2} \mathrm{Cl} \text {. }
$$

663. The Acetylene Series, $\mathrm{C}_{n} \mathrm{H}_{2 n-2}$. -The acetylene series of hydrocarbons has the general formula $\mathrm{C}_{n} \mathrm{H}_{2 n-2}$. It is headed by acetylene, $\mathrm{C}_{2} \mathrm{H}_{2}(49,83)$. It is probable that the four extra valence bonds of acetylene,<smiles>CC(C)C(C)C</smiles>

are united in pairs, so that the carbon atoms are joined by a triple bond,

$$
\mathrm{H}-\mathrm{C} \equiv \mathrm{C}-\mathrm{H} \text {. }
$$

In accord with this view we should expect acetylene to unite with chlorine, thus:<smiles>C#C[14CH2][CH]C(Cl)C(Cl)Cl</smiles>

and, in fact, it actually behaves in this way. Because of the fact that $\mathrm{C}_{2} \mathrm{H}_{4}$ and $\mathrm{C}_{2} \mathrm{H}_{2}$ have unsatisfied valences, as shown by their union with $\mathrm{Cl}_{2}, \mathrm{HBr}$, etc., these hydrocarbons are said to be unsaturated.

664. Isomerism of Hydrocarbons.-All of the compounds $\mathrm{C}_{n} \mathrm{H}_{2 n+2}$, where $n$ is four or more, can exist in two or more isomeric forms. The simplest case is that of the butanes, $\mathrm{C}_{4} \mathrm{H}_{\mathbf{x}}$, of which two are known: normal butane,

$$
\mathrm{CH}_{3}-\mathrm{CH}_{2}-\mathrm{CH}_{2}-\mathrm{CH}_{3} \text {, }
$$

and isobutane,<smiles>CC(C)C</smiles> 
There are three isomeric pentanes:

(I) $\mathrm{CH}_{3} \cdot \mathrm{CH}_{2} \cdot \mathrm{CH}_{2} \cdot \mathrm{CH}_{2} \cdot \mathrm{CH}_{3}$

(2)<smiles>CCC(C)C</smiles>

(3)<smiles>CC(C)(C)C</smiles>

The first of these is said to have a straight carbon chain; the second and third have branched chains. The B.P. and M.P. columns of Table XXVIII (643) refer to the normal hydrocarbons, with straight chains.

665. Some Common Organic Acids. - We shall now briefly describe several of the commoner organic acids. The simplest fatty acid is formic acid, $\mathrm{HCOOH}$. It is present in the bodies of ants and constitutes the poison of bees' stings. It can be made artificially in several ways. It is a colorless liquid dissolving easily in water to form a moderately well-ionized acid solution.

Oxalic acid, $\mathrm{H}_{2} \mathrm{C}_{2} \mathrm{O}_{4}$, is a white crystalline substance. Its structural formula is

\section{$\mathrm{COOH}$ $\mathrm{COOH}$.}

It is a dibasic acid (102), forming both acid and neutral salts, as for example $\mathrm{NaHC}_{2} \mathrm{O}_{4}$ and $\mathrm{Na}_{2} \mathrm{C}_{2} \mathrm{O}_{4}$. The free acid, which is easily soluble in water and rather highly ionized, crystallizes from water as a hydrate of the formula $\mathrm{H}_{2} \mathrm{C}_{2} \mathrm{O}_{4} \cdot{ }_{2} \mathrm{H}_{2} \mathrm{O}$. The acid is decidedly poisonous, probably because of the ease with which it decomposes into the powerful poison carbon monoxide (632) and water:

$$
\mathrm{H}_{2} \mathrm{C}_{2} \mathrm{O}_{4} \rightarrow \mathrm{H}_{2} \mathrm{O}+{ }_{2} \mathrm{CO} \text {. }
$$

This decomposition takes place rapidly when the acid is heated with concentrated sulfuric acid, and so affords a good way of making carbon monoxicle. Oxalic acid occurs in some plants. 
The chief acid present in sour milk is lactic acid, the graphic formula of which is $\mathrm{CH}_{3} \cdot \mathrm{CHOH} \cdot \mathrm{COOH}$. It gives a colorless water solution of a pleasant sour taste. Tartaric acid, a dibasic acid having the formula $\mathrm{H}_{2} \mathrm{C}_{4} \mathrm{H}_{4} \mathrm{O}_{6}$, is abundant in grapes in the form of its acid potassium salt, $\mathrm{KHC}_{4} \mathrm{H}_{4} \mathrm{O}_{6}$. This salt is obtained as argol in large amount in the manufacture of wine. The refined salt is known as cream of tartar. High-grade baking powder is a mixture of cream of tartar and sodium bicarbonate. The dry mixture is fairly stable. In the presence of water the substances react thus:

$$
\mathrm{KHC}_{4} \mathrm{H}_{4} \mathrm{O}_{6}+\mathrm{NaHCO}_{3} \rightarrow \mathrm{KNaC}_{4} \mathrm{H}_{4} \mathrm{O}_{6}+\mathrm{CO}_{2}+\mathrm{H}_{2} \mathrm{O} \text {. }
$$

The leavening power of baking powder is due to the $\mathrm{CO}_{2}$ given off (cf. 593). Tartaric acid forms colorless crystals easily soluble in water. Its graphic formula is
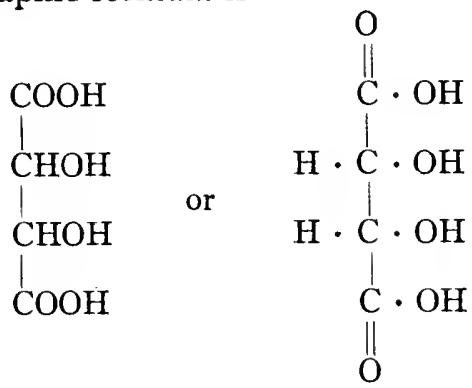

Citric acid, $\mathrm{H}_{3} \cdot \mathrm{C}_{6} \mathrm{H}_{5} \mathrm{O}_{7}$, is the acid of lemon and other citrus fruits. It is a tribasic acid (I59) rather closely resembling tartaric acid but having a more complex formula. Prussic or hydrocyanic acid, $\mathrm{HNC}$, is an extremely poisonous and very weak acid. Its salts, sodium cyanide, $\mathrm{NaNC}$, and potassium cyanide, KNC, are made from calcium cyanamid (526). Both salts react readily with either oxygen or sulfur, forming the corresponding cyanate or sulfocyanate respectively:

$$
\begin{gathered}
2 \mathrm{KNC}+\mathrm{O}_{2} \rightarrow 2 \mathrm{KNCO}, \\
\mathrm{KNC}+\mathrm{S} \rightarrow \mathrm{KNCS} .
\end{gathered}
$$

The cyanide ion unites with many metal ions to form complex cyanide ions. Salts containing these ions, such as potas- 
sium ferrocyanide, $\mathrm{K}_{4} \mathrm{Fe}(\mathrm{CN})_{6}$, and potassium ferricyanide, $\mathrm{K}_{3} \mathrm{Fe}(\mathrm{CN})_{6}$, are important analytical reagents.

666. The Aliphatic and Aromatic Series of Organic Compounds.-Broadly speaking, organic compounds constitute two great series, the aliphatic and the aromatic. The substances thus far mentioned are all members of the aliphatic series. We may consider that the paraffin hydrocarbons, $\mathrm{C}_{n} \mathrm{H}_{2 n+2}$, are the fundamental substances from which all aliphatic compounds are derived. Thus paraffin hydrocarbons by loss of two hydrogen atoms per molecule give members of the ethylene series, $\mathrm{C}_{n} \mathrm{H}_{2 n}$, or by loss of four hydrogen atoms per molecule

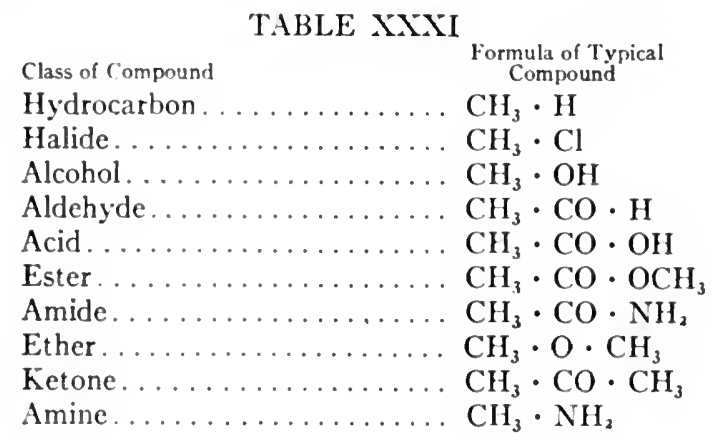

give members of the acetylene series. Substitution of hydrogen by halogens or by various radicals such as methyl, $\mathrm{CH}_{3}$; hydroxyl, $\mathrm{OH}$; carboxyl, $\mathrm{COOH}$; amid, $\mathrm{NH}_{2}$; etc., gives rise to the various classes of compounds already briefly studied.

Table XXXI shows the formulae of typical members of the most important classes of aliphatic compounds.

Instead of the methyl radical, $\mathrm{CH}_{3}$ (Table $\mathrm{XXXI}$ ), we may have ethyl, $\mathrm{C}_{2} \mathrm{H}_{5}$, propyl, $\mathrm{C}_{3} \mathrm{H}_{7}$, or any radical derived from any hydrocarbon in each case and thus obtain a very great variety of substances. All of these substances belong to the aliphatic series.

The aromatic compounds differ from the aliphatic in that they are derived from hydrocarbons entirely different from the paraffins. The most fundamental and characteristic aromatic hydrocarbon is benzene. 
667. Benzene, $\mathrm{C}_{6} \mathrm{H}_{6}$. - Benzene is a colorless liquid, boiling at $79^{\circ}$ and practically insoluble in water. It is a by-product of the manufacture of coke and coal gas $\left(6_{34}\right)$. When coal is heated in the absence of air it yields, in addition to coke and gas, a large amount of black liquid tar. By the distillation of tar a number of very important aromatic hydrocarbons are obtained. One of the most useful of these is benzene. We shall give the structural formula of benzene without attempting to justify our reasons therefor, since that story (although a most interesting one) is entirely too long for this text. This formula is

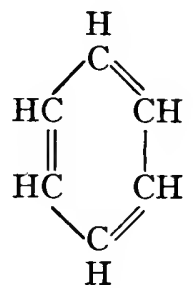

It represents a ring of six carbon atoms, with three single and three double bonds, and six hydrogen atoms, one attached to each atom of carbon.

Benzene differs from the paraffine hydrocarbons (which are very inactive) in being remarkably active chemically. This activity is shown in two ways: (I) by substitution of various radicals for one or more hydrogen atoms of each molecule, and (2) by addition after the manner of unsaturated compounds (660) but much less readily.

668. Other Aromatic Hydrocarbons.-Next to benzene the simplest aromatic hydrocarbon is toluene, or methyl benzene, $\mathrm{C}_{6} \mathrm{H}_{5} \mathrm{CH}_{3}$, the structural formula of which is

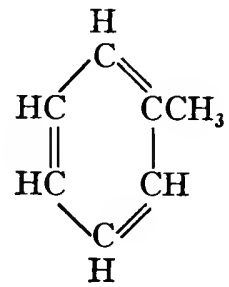


In order to save labor the $\mathrm{C}_{6} \mathrm{H}_{5}$ radical is frequently represented graphically by a hexagon called the benzene ring, so that the formula of toluene is written thus:

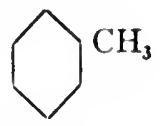

Toluene is a liquid, boiling at $110^{\circ}$ and closely resembling benzene. It is obtained from coal tar. Xylene, also obtained from the same source, is dimethyl benzene, $\mathrm{C}_{6} \mathrm{H}_{4}\left(\mathrm{CH}_{3}\right)_{2}$. In writing the structural formula of xylene we note that there are three possible arrangements:
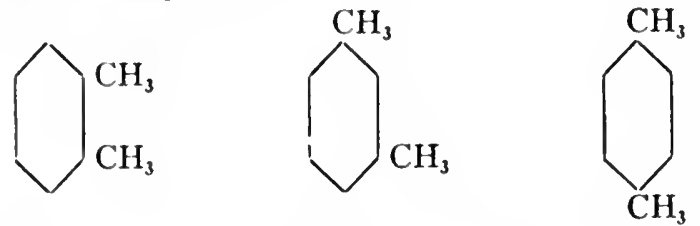

As a matter of fact three different xylenes are known. 'These are called ortho, meta, and para xylene, respectively written 0 -xylene, m-xylene, and p-xylene.

669. Naph thalene, $\mathrm{C}_{10} \mathrm{H}_{8}$. - Naphthalene is a white crystalline substance obtained from the high-boiling portion of coal tar. It is extensively used in the household under the name of moth balls. Its structure is represented thus:<smiles>c1ccc2ccccc2c1</smiles>

As great a variety of organic substances are derived from naphthalene as from benzene. For example, we have two methyl naphthalenes, $\mathrm{C}_{{ }_{10}} \mathrm{H}_{7} \cdot \mathrm{CH}_{3}$ :
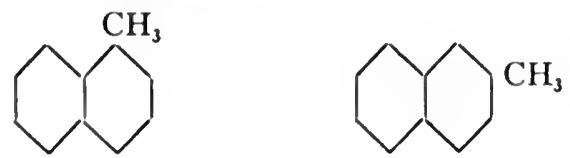
The first is called alpha and the second beta methyl naphthalene. Naphthalene and its derivatives are important starting materials for the manufacture of dyestuffs.

670. Aromatic Alcohols and Aldehydes.-Toluene, $\mathrm{C}_{6} \mathrm{H}_{5}$. $\mathrm{CH}_{3}$, can be considered as derived from methane, $\mathrm{CH}_{4}$, by the substitution of the phenyl radical, $\mathrm{C}_{6} \mathrm{H}_{5}$, for one hydrogen atom of $\mathrm{CH}_{4}$. The substitution of $\mathrm{C}_{6} \mathrm{H}_{5}$ for $\mathrm{H}$ in methyl alcohol, $\mathrm{CH}_{3} \cdot \mathrm{OH}$, would give $\mathrm{C}_{6} \mathrm{H}_{5} \cdot \mathrm{CH}_{2} \cdot \mathrm{OH}$. The substance actually exists and is known as benzyl alcohol. Like other alcohols, it can be oxidized to an aldehyde called benzaldehyde:

$$
\mathrm{C}_{6} \mathrm{H}_{5} \cdot \mathrm{CH}_{2} \cdot \mathrm{OH}+\mathrm{O}_{2} \rightarrow \mathrm{C}_{6} \mathrm{H}_{5} \cdot \mathrm{CO} \cdot \mathrm{H}+\mathrm{H}_{2} \mathrm{O} \text {. }
$$

This aldehyde is identical with the principal constituent of the oil of bitter almonds. Benzaldehyde can be made from toluene by converting the latter into a chlorine compound and then treating this product with water:

$$
\begin{aligned}
\mathrm{C}_{6} \mathrm{H}_{5} \cdot \mathrm{CH}_{3}+{ }_{2} \mathrm{Cl}_{2} & \rightarrow \mathrm{C}_{6} \mathrm{H}_{5} \cdot \mathrm{CHCl}_{2}+{ }_{2} \mathrm{HCl}, \\
\mathrm{C}_{6} \mathrm{H}_{5} \cdot \mathrm{CHCl}_{2}+\mathrm{H}_{2} \mathrm{O} & \rightarrow \mathrm{C}_{6} \mathrm{H}_{5} \cdot \mathrm{CO} \cdot \mathrm{H}+2 \mathrm{HCl} .
\end{aligned}
$$

Benzaldehyde is slowly oxidized by contact with air to form benzoic acid, $\mathrm{C}_{6} \mathrm{H}_{5} \cdot \mathrm{CO} \cdot \mathrm{OH}$ :

$$
{ }_{2} \mathrm{C}_{6} \mathrm{H}_{5} \cdot \mathrm{CO} \cdot \mathrm{H}+\mathrm{O}_{2} \rightarrow{ }_{2} \mathrm{C}_{6} \mathrm{H}_{5} \cdot \mathrm{CO} \cdot \mathrm{OH} \text {. }
$$

671. Benzoic Acid, $\mathrm{C}_{6} \mathrm{H}_{5} \cdot \mathrm{CO} \cdot \mathrm{OH}$.- This important acid is a white crystalline solid which can be made in several ways in addition to the one just mentioned. It is used extensively in the form of its sodium salt, sodium benzoate, $\mathrm{C}_{6} \mathrm{H}_{5} \mathrm{CO} \cdot \mathrm{ONa}$, as a preservative for catsup and other articles of food. It can be used legally in the United States as a food preservative if its presence is indicated on the label. It occurs naturally as a constituent of cranberries.

Benzoic acid forms salts with bases of all kinds. With alcohols it forms esters. The latter are fragrant liquids. Ethyl benzoate, $\mathrm{C}_{6} \mathrm{H}_{5} \cdot \mathrm{CO} \cdot \mathrm{OC}_{2} \mathrm{H}_{5}$, is a colorless liquid, boiling at $2 \mathrm{II}^{\circ}$.

672. Phenol, $\mathrm{C}_{6} \mathrm{H}_{5} \cdot \mathrm{OH}$.- Phenol (popularly known as carbolic acid) is contained in coal tar, from which it is separated in crude form by distillation. Phenol is also made on a large 
scale from benzene. The latter substance reacts slowly with sulfuric acid, forming benzene sulfonic acid and water, thus:

$$
\mathrm{C}_{6} \mathrm{H}_{6}+\mathrm{H}_{2} \mathrm{SO}_{4} \rightarrow \mathrm{C}_{6} \mathrm{H}_{5} \mathrm{SO}_{2} \mathrm{OH}+\mathrm{H}_{2} \mathrm{O} \text {. }
$$

The product is an acid the sodium salt of which when fused with sodium hydroxide gives phenol:

$$
\mathrm{C}_{6} \mathrm{H}_{5} \mathrm{SO}_{2} \mathrm{ONa}+\mathrm{NaOH} \rightarrow \mathrm{C}_{6} \mathrm{H}_{5} \mathrm{OH}+\mathrm{Na}_{2} \mathrm{SO}_{3} \text {. }
$$

Phenol is a white crystalline substance having a peculiar, characteristic odor. It is moderately soluble in water. It is a violent poison and is extensively used as a germicide. Phenol is a very weak acid and forms salts with strong bases:

$$
\mathrm{C}_{6} \mathrm{H}_{5} \mathrm{OH}+\mathrm{NaOH} \rightarrow \mathrm{C}_{6} \mathrm{H}_{5} \mathrm{ONa}+\mathrm{H}_{2} \mathrm{O} \text {. }
$$

It is interesting to contrast phenol and ethyl alcohol. The formulae of the two substances<smiles>CCO</smiles><smiles>Oc1ccccc1</smiles>

show that both contain the hydroxyl radical united to a hydrocarbon radical. In consequence we might expect similar properties, but we find quite the contrary. Phenol shows but few of the characteristic chemical properties of an alcohol.

673. Aromatic Nitro Compounds.-Aromatic hydrocarbons, like benzene, react very readily with concentrated nitric acid in a peculiar way, as illustrated by the following equation:

$$
\mathrm{C}_{6} \mathrm{H}_{6}+\mathrm{HNO}_{3} \rightarrow \mathrm{C}_{6} \mathrm{H}_{5} \mathrm{NO}_{2}+\mathrm{H}_{2} \mathrm{O} \text {. }
$$

The new product is nitro benzene, a light-yellow colored liquid of aromatic odor. It is not soluble in water and is not a salt. The structural formula is<smiles>O=S1(=O)CCCCC1</smiles> 
Toluene and nitric acid give two isomeric nitro compounds, $\mathrm{CH}_{3} \cdot \mathrm{C}_{6} \mathrm{H}_{4} \cdot \mathrm{NO}_{2}$. These have the following formulae:<smiles>CC1CCCCC1=O</smiles><smiles>CC1CCN([O+])CC1</smiles>

The left-hand formula is that of ortho nitro toluene; the righthand one that of para nitro toluene. A third nitro toluene can be made by indirect methods. This substance, called meta nitro toluene, has the formula<smiles>CC1CCCC([N+](=O)[O-])C1</smiles>

The further action of nitric acid on either ortho or para nitro toluene gives finally tri nitro toluene, $\mathrm{CH}_{3} \cdot \mathrm{C}_{6} \mathrm{H}_{2} \cdot\left(\mathrm{NO}_{2}\right)_{3}$. This substance is the violent explosive so extensively used in the war and known popularly as T.N.T. Its formula is

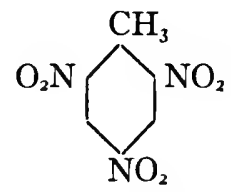

The action of nitric acid on phenol gives first a mixture of ortho and para nitro phenol,<smiles>O=[N+]([O-])C1CCCCC1</smiles><smiles>O=[N+]1CCC(O)CC1</smiles>

Further action of nitric acid finally yields tri nitro phenol, or picric acid, $\mathrm{C}_{6} \mathrm{H}_{2}\left(\mathrm{NO}_{3}\right)_{3} \mathrm{OH}$. The nitro phenols are much stronger acids than phenol itself. In fact, picric acid is nearly as strong (highly ionized) an acid as hydrochloric.

Ammonium picrate, $\mathrm{C}_{6} \mathrm{H}_{2}\left(\mathrm{NO}_{3}\right)_{3} \mathrm{ONH}_{4}$, is a powerful explosive. It has been extensively used in the war. 
674. Aromatic Amines.-Nitro compounds are easily acted on by reducing agents, as illustrated by the case of nitro benzene:

$$
\mathrm{C}_{6} \mathrm{H}_{5} \cdot \mathrm{NO}_{2}+{ }_{3} \mathrm{H}_{2} \rightarrow \mathrm{C}_{6} \mathrm{H}_{5} \cdot \mathrm{NH}_{2}+{ }_{2} \mathrm{H}_{2} \mathrm{O} \text {. }
$$

The new product is called aniline. It is the simplest aromatic amine. The aromatic amines resemble the aliphatic amines (658). They are base-forming substances and are to be considered as ammonia in which hydrogen has been replaced by an aromatic radical. Aniline unites with hydrochloric acid to form a true salt, a chloride:

$$
\mathrm{C}_{6} \mathrm{H}_{5} \mathrm{NH}_{2}+\mathrm{HCl} \rightarrow \mathrm{C}_{6} \mathrm{H}_{5} \mathrm{NH}_{3} \mathrm{Cl} \text {. }
$$

This salt corresponds to $\mathrm{NH}_{4} \mathrm{Cl}$. A great variety of aromatic amines can be made by the reduction of nitro compounds. Usually the reduction is carried out by mixing the nitro compound with zinc or iron and hydrochloric acid. The hydrogen liberated by the action of the metal and acid then reacts with the nitro compound in the way above indicated. Aniline and other aromatic amines are made in immense quantities to be used as intermediates in the manufacture of so-called aniline or coal-tar dyes. Further reference to this subject will be found in the next chapter. 


\section{CHAPTER XXVI}

ORGANIC COMPOUNDS. II

675. Introduction.-We have promised to show the reader some of the successes achieved in organic chemistry as a result of the systematic study of the science. First we shall take up the chemistry of foods and next the chemistry of explosives and the related substances, such as celluloid, artificial silk, etc. After this we shall treat briefly the poison gases used in warfare, then the synthesis of essential oils, perfumes, spices, dyes, medicinals, and rubber. This may seem a bewildering list, but organic chemists ca? say without fear of contradiction that they have accomplish.: a great deal more than is even casually mentioned in this chapter. Finally we shall discuss briefly the chief sources of materials for the manufacture of organic chemicals.

676. The Three Classes of Foods.-All foods fall into three great classes: the fats, including also edible oils; the carbohydrates, comprising starches and sugars; and the proteins, in which class are included eggs, lean meat, and certain nitrogenous vegetable products. A well-balanced diet for man should be made up of foods of all three of these classes. Fats and carbohydrates are compounds of carbon, hydrogen, and oxygen only; while all proteins, in addition to these three elements, contain also nitrogen. Of the three classes the fats are from the chemical point of view the simplest, and their chemistry was worked out long before that of the other two classes. The chemistry of the carbohydrates was well cleared up during the last two decades of the nineteenth century. The chemistry of the proteins is far more complex and is even today far from completely solved.

677. Fats.-The term fats includes liquid as well as solid animal and vegetable products. Liquid fats like olive oil, cottonseed oil, peanut oil, castor oil, and linseed oil are chemically very different from the paraffin or mineral oils described in the 
foregoing chapter. Most natural fats (butter fat for example) are mixtures of several chemical compounds all of which belong to a single group of organic substances, the esters (657). The chemical nature of these esters is most readily shown by the conversion of fats into soap.

678. Soap.-Any fat is changed into soap when it is boiled with a solution of sodium hydroxide. If a good grade of white soap is dissolved in water and the solution acidified with hydrochloric acid a dense white precipitate forms. The evaporated filtrate yields only common salt. The white precipitate is a mixture of three or four fatty acids (655). Among the commonest fatty acids obtained in this way are palmitic acid, $\mathrm{C}_{15} \mathrm{H}_{35} \cdot \mathrm{CO} \cdot \mathrm{OH}$, and stearic acid, $\mathrm{C}_{17} \mathrm{H}_{35} \cdot \mathrm{CO} \cdot \mathrm{OH}$.

The radicals $\mathrm{C}_{55} \mathrm{H}_{31}$ and $\mathrm{C}_{17} \mathrm{H}_{35}$ form straight or unbranched carbon chains, as illustrated in the following formula for palmitic acid:

\author{
$\mathrm{H} \mathrm{H} \mathrm{H} \mathrm{H} \mathrm{H} \mathrm{H} \mathrm{H} \mathrm{H} \mathrm{H} \mathrm{H} \mathrm{H} \mathrm{H} \mathrm{H}$ \\ $\mathrm{HC} \cdot \mathrm{C} \cdot \mathrm{C} \cdot \mathrm{C} \cdot \mathrm{C} \cdot \mathrm{C} \cdot \mathrm{C} \cdot \mathrm{C} \cdot \mathrm{C} \cdot \mathrm{C} \cdot \mathrm{C} \cdot \mathrm{C} \cdot \mathrm{C} \cdot \mathrm{C} \cdot \mathrm{C} \cdot \mathrm{CO} \cdot \mathrm{OH}$ \\ $\mathrm{H} \mathrm{H} \mathrm{H} \mathrm{H} \mathrm{H} \mathrm{H} \mathrm{H} \mathrm{H} \mathrm{H} \mathrm{H} \mathrm{H} \mathrm{H} \mathrm{H}$
}

Stearic acid contains one more $\mathrm{CH}_{2}$ group per molecule than palmitic acid.

Soaps are the sodium (or potassium) salts of fatty acids. 'The action of $\mathrm{HCl}$ on sodium palmitate takes place thus:

$$
\mathrm{C}_{15} \mathrm{H}_{31} \mathrm{COONa}+\mathrm{HCl} \rightarrow \mathrm{C}_{15} \mathrm{H}_{31} \mathrm{COOH}+\mathrm{NaCl} \text {. }
$$

679. Glycerine and Its Esters. - The action of sodium hydroxide on a fat always gives in addition to a soap one other product, glycerine. Glycerine is a sweet, sirupy, colorless liquid, the structural formula of which is

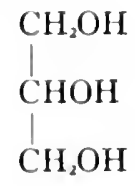

Glycerine is an alcohol, but it differs from simple (monatomic) alcohols like methyl alcohol, $\mathrm{CH}_{3} \mathrm{OH}$, and ethyl alcohol, 
$\mathrm{CH}_{3} \cdot \mathrm{CH}_{2} \mathrm{OH}$, in having three hydroxyl groups in a molecule. It is called a triatomic alcohol. It will be recalled (657) that alcohols are not bases, in spite of the presence of hydroxyl groups. They do not yield $\mathrm{OH}^{-}$ions.

Just as acetic acid and ethyl alcohol unite to form ethyl acetate (an ester) and water (657), so a fatty acid and glycerine can unite to form an ester in which three molecules of the acid are combined with one of glycerine. Thus palmitic glycerine ester, or palmitin, is

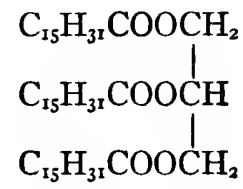

This substance is one of the principal constituents of beef fat. Fats in general are the glycerine esters of various fatty acids. Just as ethyl acetate gives, with sodium hydroxide, sodium acetate and ethyl alcohol,

$$
\mathrm{CH}_{3} \cdot \mathrm{CO} \cdot \mathrm{OC}_{2} \mathrm{H}_{5}+\mathrm{NaOH} \rightarrow \mathrm{CH}_{3} \cdot \mathrm{CO} \cdot \mathrm{ONa}+\mathrm{C}_{2} \mathrm{H}_{5} \mathrm{OH},
$$

so a fat and sodium hydroxide yield a soap and glycerine. On account of the close chemical relation between these two reactions the first as well as the second is spoken of as a saponification of the ester, although of course sodium acetate is not a soap in the ordinary sense of the term.

680. The Composition of Fats.-We are now in a position to understand the cause of the differences between fats from various sources. In general, a given sort of fat is a mixture of the glycerine esters of several fatty acids. Among such, in addition to palmitic and stearic acids, already mentioned, we have oleic acid, $\mathrm{C}_{17} \mathrm{H}_{33} \mathrm{COOH}$; lauric acid, $\mathrm{C}_{1 \mathrm{I}} \mathrm{H}_{21} \mathrm{COOH}$; caprylic acid, $\mathrm{C}_{7} \mathrm{H}_{15} \mathrm{COOH}$; caproic acid, $\mathrm{C}_{5} \mathrm{H}_{15} \mathrm{COOH}$; valeric acid, $\mathrm{C}_{4} \mathrm{H}_{3} \mathrm{COOH}$; and butyric acid, $\mathrm{C}_{3} \mathrm{H}_{7} \mathrm{COOH}$.

Beef fat is composed largely of the esters of palmitic, stearic, and oleic acids. These esters are known respectively as palmitin, stearin, and olein. The first two are solids, while the last is an oil at room temperature. Mutton fat resembles 
beef fat in composition but contains a smaller proportion of olein, while hog fat in the form of lard contains appreciably more olein than beef fat. Butter fat contains, in addition to palmitin, stearin, and olein, a considerable proportion of butyrin, the glycerine ester of butyric acid. The chief constituent of olive oil is olein. The same ester, together with others, comprises cottonseed oil, an edible oil of cnormous economic importance.

68I. The Hardening of Oils.-Oleic acid, $\mathrm{C}_{17} \mathrm{H}_{33} \mathrm{COOH}$, differs from stearic acid, $\mathrm{C}_{17} \mathrm{H}_{35} \mathrm{COOH}$, by two atoms of hydrogen per moleculc. This difference is the result of one double bond $(66 \mathrm{I}, 662)$ between two of the carbon atoms of the $\mathrm{C}_{17} \mathrm{H}_{33}$ radical, which is therefore an unsaturated (663) compound. By the addition of hydrogen to the double bond, oleic acid can be converted into stearic acid:

$$
\mathrm{C}_{77} \mathrm{H}_{33} \mathrm{COOH}+\mathrm{H}_{2} \rightarrow \mathrm{C}_{37} \mathrm{H}_{35} \mathrm{COOH}
$$

By a similar addition of hydrogen, olein is changed into stearin. 'This change is accomplished by the aid of a catalytic agent, fincly divided metallic nickel. By means of this process of hydrogenation, liquid fats like cottonseed oil are readily changed into solid or partially solid fats. During the last twenty years this so-called hardening of fats has developed into an immense industry. The product made from cottonseed oil has about the consistency of lard and finds extensive use as a substitute for the latter.

682. The Carbohydrates.-In the preceding chapter starch $(636)$ and glucose, or grape sugar (639), were briefly described. These substances belong to an important class of organic compounds known as carbohydrates. 'This name was chosen because these substances are composed of carbon, together with hydrogen and oxygen, the two latter in the proportion corresponding to water. Thus glucose is $\mathrm{C}_{6} \mathrm{H}_{12} \mathrm{O}_{6}$, which is equivalent to $\mathrm{C}_{6}\left(\mathrm{H}_{2} \mathrm{O}\right)_{6}$. However, the carbohydrates are not simply carbon with water of hydration in the sense that $\mathrm{Na}_{2} \mathrm{SO}_{4} \cdot \mathrm{IoH}_{2} \mathrm{O}$ is the hydrate of $\mathrm{Na}_{2} \mathrm{SO}_{4}$. 
The simplest formula which would represent the composition of starch is $\mathrm{C}_{6} \mathrm{H}_{10} \mathrm{O}_{5}\left(6_{3} 6\right)$; but it is certain that the molecule of starch is much larger than that represented by this formula. The formula is more correctly written $\left(\mathrm{C}_{6} \mathrm{H}_{10} \mathrm{O}_{5}\right)_{n}$, where $n$ is an integer probably as large as 30 or 40 . Ordinarily the simpler formula is employed.

The hydrolysis of starch to form glucose $(638)$,

$$
\left(\mathrm{C}_{6} \mathrm{H}_{10} \mathrm{O}_{5}\right)_{n}+n \mathrm{H}_{2} \mathrm{O} \rightarrow n \mathrm{C}_{6} \mathrm{H}_{12} \mathrm{O}_{6},
$$

is a very important reaction. It takes place readily in acid solution by reason of the catalytic action of $\mathrm{H}^{+}$ions. The higher the temperature of the solution the more rapid the hydration proceeds.

Glucose has a large number of isomers (650), all having, of course, the same formula. These sugars, called hexoses, all have properties more or less like those of glucose. Levulose, or fruit sugar, is one of the commonest of the hexoses. It is the sugar most abundant in many fruits.

Ordinary table sugar, commonly known as cane sugar and called by chemists sucrose, has the formula $\mathrm{C}_{12} \mathrm{H}_{22} \mathrm{O}_{11}$. It is made from two principal sources, sugar cane and sugar beets. It has the same composition in each case. Numerous other plants also produce sucrose. Maple sugar is largely sucrose.

Milk sugar, or lactose, $\mathrm{C}_{12} \mathrm{H}_{22} \mathrm{O}_{11}$, is an isomer of cane sugar. It is present in cow's milk to the extent of 4 per cent. It is much less sweet than cane sugar. Maltose, $\mathrm{C}_{\mathrm{I} 2} \mathrm{H}_{22} \mathrm{O}_{\mathrm{II}}$, is another isomer of cane sugar. It is formed by the hydrolysis of starch in the presence of a catalytic agent occurring in germinating seeds. The reaction may be written

or better,

$$
{ }_{2} \mathrm{C}_{6} \mathrm{H}_{10} \mathrm{O}_{5}+\mathrm{H}_{2} \mathrm{O} \rightarrow \mathrm{C}_{12} \mathrm{H}_{22} \mathrm{O}_{\mathrm{II}},
$$

$$
{ }_{2}\left(\mathrm{C}_{6} \mathrm{H}_{10} \mathrm{O}_{5}\right)_{n}+n \mathrm{H}_{2} \mathrm{O} \rightarrow n \mathrm{C}_{12} \mathrm{H}_{22} \mathrm{O}_{11} \text {. }
$$

The catalytic agent is called diastase. A similar substance, ptyalin, is present in saliva. It promotes the digestion of starch by hydrolyzing it to maltose. The catalytic agents diastase and ptyalin are classed as enzymes. 
683. The Structure of the Sugars. - The structure of the simpler sugars, like glucose, was worked out at the end of the nineteenth century. The following structure of glucose was discovered after long experimentation, which established the presence of the groups indicated.

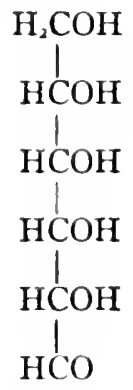

The five hydroxyl groups behave like those of an alcohol. In this respect glucose is an alcohol somewhat resembling glycerine (679). One end carbon atom of the glucose molecule forms an aldehyde radical $(652)$. The reactions of glucose are those of an alcohol and of an aldehyde. Like all aldehydes glucose is a good reducing agent. It reduces an alkaline solution of copper to cuprous oxide. This reaction, which serves as the best test for glucose, is carried out by warming glucose with Fehling's solution. This solution is made by mixing copper sulfate solution with a solution of sodium tartrate (665) containing an excess of sodium hydroxide. In the presence of glucose the deep-blue Fehling's solution gives a red precipitate of cuprous oxide, $\mathrm{Cu}_{2} \mathrm{O}$.

The structure of levulose is represented thus:

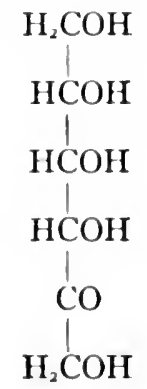


It is an alcohol ketone (656). The behavior of cane sugar with dilute acids throws much light on its structure, since in this reaction it unites with water and forms equal amounts of glucose and levulose:

$$
\mathrm{C}_{12} \mathrm{H}_{22} \mathrm{O}_{11}+\mathrm{H}_{2} \mathrm{O} \rightarrow \mathrm{C}_{6} \mathrm{H}_{12} \mathrm{O}_{6}+\mathrm{C}_{6} \mathrm{H}_{12} \mathrm{O}_{6} \text {. }
$$

From this it follows that in the molecule of cane sugar a molecule of glucose is joined with one of levulose, with the elimination of a molecule of water.

Since maltose gives by hydrolysis two molecules of glucose its molecule may be considered to be made up of two glucose radicals.

Cane sugar and maltose do not reduce Fehling's solution. If their solutions are first hydrolyzed the resulting solution reduces Fehling's solution readily.

684. Cellulose.-Cellulose, which occurs nearly pure in cotton, is an isomer of starch $\left(6_{3} 6\right)$. Its simplest formula is $\mathrm{C}_{6} \mathrm{H}_{10} \mathrm{O}_{5}$, but its true formula should be written $\left(\mathrm{C}_{6} \mathrm{H}_{\mathrm{I0}} \mathrm{O}_{5}\right)_{m}$, where $m$ is an integer probably even larger than $n$ in the starch formula. Cellulose is far less active chemically than starch and is practically indigestible by man. It is possible to hydrolyze cellulose to glucose, but the reaction takes place slowly. Wood and vegetable fiber in general contain a large proportion of cellulose. The latter is classed with starch and sugars as a carbohydrate.

685. The Proteins. - We shall use the term protein to include the various complex nitrogenous substances forming the characteristic constituents of lean meat, white of eggs, etc. Most plant seeds also contain more or less proteins. Wheat is particularly rich in this respect.

Proteins are complex substances containing in addition to carbon, hydrogen, and oxygen a rather large percentage of nitrogen and a much smaller percentage of sulfur; a few of the proteins also contain phosphorus. Neither the exact formula nor the structure of any typical protein is definitely known. The composition of albumin (white of egg) is approximately expressed by the formula $\mathrm{C}_{720} \mathrm{H}_{1134} \mathrm{~N}_{218} \mathrm{~S}_{5} \mathrm{O}_{248}$. Although considerable 
progress has been made in recent years toward the elucidation of the structure of the proteins, much remains to be done. When the proteins are heated with acids or alkalies they are split up into simpler substances which are found to be nitrogen derivatives of fatty acids (655). We have seen that acetic acid, $\mathrm{CH}_{3} \mathrm{COOH}$, for example, forms an amide, $\mathrm{CH}_{3} \mathrm{CONH}_{2}$, acetamide (659). We have also learned something of the amines, of which methyl amine, $\mathrm{CH}_{3} \mathrm{NH}_{2}$, is the simplest representative, and it will therefore not be surprising to learn that a substance having the formula

\section{$\mathrm{H}_{2} \mathrm{NCH}_{2} \mathrm{COOH}$,}

which we may call amino acetic acid, or glycocoll, exists. This acid can form an amide,

\section{$\mathrm{H}_{2} \mathrm{NCH}_{2} \mathrm{CONH}_{2}$,}

which can unite with one or more molecules of glycocoll to form such products as

$$
\mathrm{H}_{2} \mathrm{NCH}_{2} \mathrm{CONHCH}_{2} \mathrm{CONH}_{2}
$$

and

\section{$\mathrm{H}_{2} \mathrm{NCH}_{2} \mathrm{CONHCH}_{2} \mathrm{CONHCH}_{2} \mathrm{CONH}_{2}$.}

Still more complex substances, amino acids, have been built up artificially in the laboratory. The fact that these substances are identical with, or related to, the decomposition products of the proteins leads us to think that the molecules of the latter are made up of amino acid radicals, among which are those of fatty acids other than acetic. Proteins from different sources differ markedly from one another by reason of the kinds and relative amounts of the amino acids which compose them.

Many proteins are of vegetable origin. The cereals like wheat, oats, rye, barley, and corn are comparatively rich in these nitrogenous substances (5II). Beans, peas, and other legumes also contain large percentages of proteins, whilc vegetables contain but small amounts.

686. Why the Body Needs Food.- The body needs food for growth, repair, and the sunply of energy. For the adult only 
the last two are of importance. The average amount of carbon dioxide exhaled per day by an adult is about 1,100 grams; but the amount varies, greatly increasing with the amount of work done. Nitrogen is excreted largely as urea (659) but also in smaller amounts in the form of other compounds. The average daily loss calculated as nitrogen amounts to about 20 grams for an adult. Hydrogen and oxygen are also lost in large quantities, principally in the form of water and to a smaller extent in compounds with carbon, nitrogen, etc. These losses must be compensated by food and water in order to maintain bodily weight; for of course the law of the conservation of matter (2I) applies rigorously to all bodily processes.

687. The Law of the Conservation of Energy for Bodily Processes.-The body expends energy in two ways: in doing work and in giving off heat to the surroundings. The source of this energy is found in the chemical changes of the food eaten and the oxygen inhaled. The amount of energy produced when a known amount of a given foodstuff, together with sufficient oxygen, is changed to the same products as those formed in the body can be determined by means similar to that described earlier (357). The energy per gram of a food may be expressed in calories and called its fuel value.

Until recent years it was a question whether the amount of energy supplied by the food eaten was exactly equal to that expended in the form of work plus that given off as heat when the body neither gained nor lost in weight. This important problem was solved by the very elaborate experiments of the American chemists Atwater and Benedict. These scientists constructed a huge calorimeter (357) in which a man could live and perform work for hours at a time. The amount of work done and heat given off was accurately measured, and the energy or calorific value of all food eaten was determined. After several years of the most careful work, in which animals as well as men were experimented upon, it was conclusively proved that the energy given out by the body is exactly equal to that produced when the food and oxygen taken are changed to the same forms as those excreted by the body. In other 
words, it was found that the law of the conservation of energ: applies rigidly to all bodily processes.

688. The Science of Dietetics. - The facts set forth in the two preceding sections serve as the basis for a scientific treatment of the subject of nutrition. This branch of science is called dietetics. A satisfactory, well-balanced ration must supply sufficient amounts of each class of food to compensate for the known or determinable body losses of material and at the same time yield sufficient energy to enable the performance of the required amount of physical work and also keep up bodily temperature, all without the loss of body weight.

The energy requirement of a man doing moderate work is about 3,200 kilogram calories. ${ }^{\mathrm{I}}$ To get this he must consume food more than sufficient to satisfy his requirements for carbon; but this will not necessarily also supply his requirements for nitrogen. Therefore a sufficient ration will result from eating enough protein to compensate the nitrogen loss, and in addition enough fats or carbohydrates, or better both, to bring the fuel value up to his requirement. The ratio of carbohydrates to fat in the diet is not of fundamental importance; furthermore the protein consumption may, for some individuals or even for whole races, be safely increased far above the necessary minimum. For adult male Americans, professional, business men, and students, $100 \mathrm{~g}$. of protein, $125 \mathrm{~g}$. of fat, and $400 \mathrm{~g}$. of carbohydrates, with a total fuel value of 3,200 kilo calories, constitutes an average daily ration.

Table XXXII gives the data for sample meals for one day for one man doing moderately heavy work. It shows the weight in fractions of a pound and in grams of each article, and also its protein content and fuel value.

689. Vitamines. - It would naturally be inferred from the discussion of the preceding sections that in providing for a dietary sufficient to maintain health and weight it is necessary to consider only the protein content and fuel value of the food

s On account of the fact that the calorie (III) is so small a unit of heat, a unit one thousand fold larger is in common use. This larger unit is the heat required to raise the temperature of one kilogram of water one degree. It niay be called a kilogram calorie, or kilo calorie. 
supplied. Yet it has long been known that in earlier times sailors who lived for long periods on an abundance of food of

\section{TABLE XXXII}

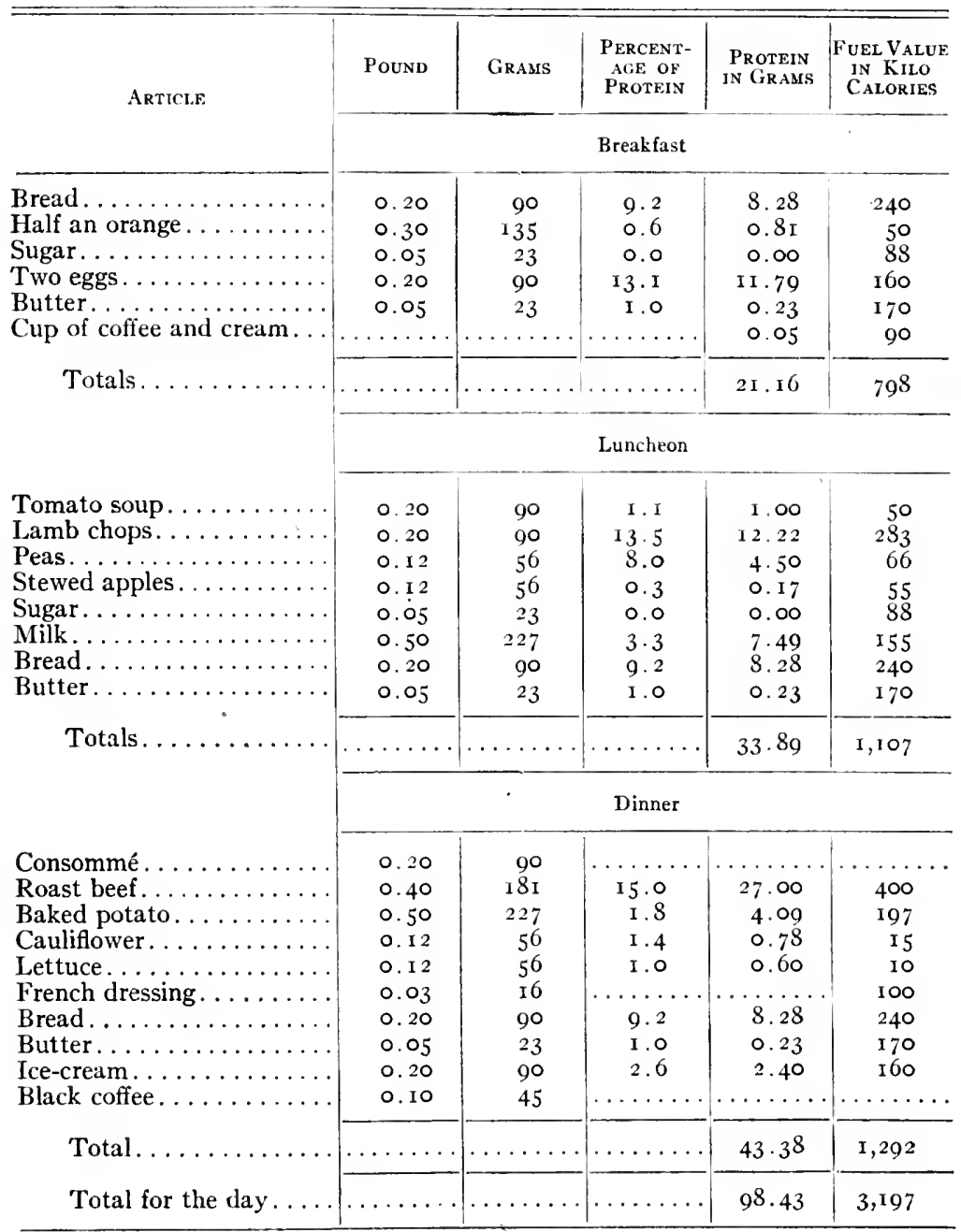

very limited variety, such as salt pork and "hard-tack," were liable to be afflicted with a peculiar and often fatal disease called scurvy. Afflicted persons rapidly recovered when supplied with 
fresh vegetables or even with the juice of oranges, lemons, or limes. The British navy and mercantile marine have for fifty years or more been required to provide sailors regularly with lime juice. Scurvy is now of rare occurrence.

A still more remarkable case is found in the cause of and remedy for the peculiar oriental disease beri-beri. This affects people who live chiefly on a diet of rice and fish. Recent experiments, particularly with pigeons, has shown that these birds thrive on natural rice, while if fed on the polished grains they quickly suffer from the disease and soon die. Dangerously sick pigeons make marielous recoiery when given small amounts of the malerial remoied from the grains in the process of polish. ing. Further experimentation has shown conclusively that beri-beri is the result of a diet deficient in a substance known as water soluble $\mathbf{B}$ found in the germ of the rice grain. This same substance is also found in the germ of wheat and other grains.

Very extensive experiments have shown conclusively that rats cannot grow nor even live long on diets containing adequate amounts of proteins, fats, and carbohydrates if every article of food has been highly purified by chemical processes. This is because certain essential substances contained in the natural foods have been removed in the processes of purification. Milk is especially rich in these essential substances. By adding small amounts of milk to the diet of rats living on purified food a wholly satisfactory ration is obtained. The results of such experiments are shown in Fig. 97. The lower curve shows the change in average weight of

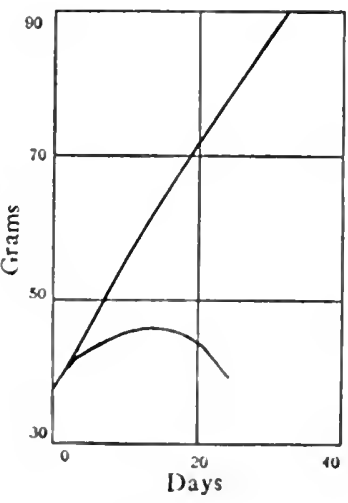

FIG. 97 six young rats fed on a diet of purified foods alone. The upper curve shows the weights of the same number of young rats which received the same fool ration as the first six but had in addition 2 c.c. each of milk per day. Work of F. G. Hopkins. 
In addition to water soluble $B$, milk contains also another peculiar substance called fat soluble A. The latter occurs also in the leafy parts of most vegetables. The term vitamines is usually used to designate the important substances, fat soluble A and water soluble B. It is now definitely established that animals, including man (especially children), must have for growth and health a constant supply of both these vitamines. A diet (for man) of roots, tubers, seeds, and meat may furnish sufficient proteins and have adequate fuel value and still be markedly deficient in vitamines, particularly fat soluble A. The deficiency is best avoided by the liberal use of milk and leafy vegetables; of these, milk is the more important.

The chemical nature of the vitamines still remains to be discovered, but their importance as food constituents is no longer in question. There is little doubt that vitamines act catalytically.

69o. Dependence of Animals upon Plants.-All animals, including man, depend for their food upon plants or upon other animals which in turn feed upon plants. Animals cannot subsist upon the free elements that compose their food, nor even upon the simpler compounds of these elements, such as carbon dioxide, hydrocarbons (643), ammonia, amines (658), etc., but must have the far more elaborate compounds, the carbohydrates (682), fats (677), and proteins (685), and in addition mineral salts and vitamines (689).

Plants, on the other hand, require for their sustenance far simpler materials, principally carbon dioxide, water, and simple nitrogen compounds, such as ammonia or nitrates, and also small amounts of mineral matter. Some of the simplest organisms contain species of bacteria, by aid of which they assimilate free nitrogen from the air (515).

691. Photosynthesis.--The plant products formed from these simple inorganic substances have far more energy than the latter. What then is the source of this energy? Plainly the light and heat necessary for the growth of all plants (excepting fungi and other parasitic plants which live on decaying animal or vegetable matter). The energy of sunlight is transformed 
in the growing plant into the chemical energy of the plant products.

Cellulose and starch are the most abundant plant products. For the formation of these only carbon dioxide and water are theoretically required:

$$
6 \mathrm{CO}_{2}+{ }_{5} \mathrm{H}_{2} \mathrm{O} \rightarrow \mathrm{C}_{6} \mathrm{H}_{10} \mathrm{O}_{5}+6 \mathrm{O}_{2} \text {. }
$$

However, this reaction does not take place in the simple fashion indicated by this equation. It takes place only in plants and then only in such parts as contain the characteristic green substance chlorophyl. Probably several intermediate stages exist in the change of carbon dioxide into starch or cellulose; in any case oxygen is always a product of plant growth. Since this building up of complex products occurs by the aid of light the process is termed photosynthesis.

692. Some Common Organic Explosives.-We have referred earlier (57I) to the great practical importance of explosives. The most useful explosives are made by the nitration of organic substances such as glycerine (679), cotton (684), phenol (672), and toluene (668). The products thus obtained will now be briefly described.

Nitroglycerine is formed when glycerine is added drop by drop to a cooled mixture of concentrated nitric and sulfuric acids. 'The nitroglycerine separates as an insoluble heavy oil when the product is poured into water. It is a violent explosive which finds cxtensive use in blasting. Nitroglycerine is the nitric acid ester $(657)$ of the alcohol glycerine (679). Its formula is

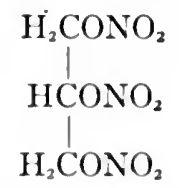

Its explosive nature arises from the fact that it contains more than sufficient oxygen to change all its hydrogen and carbon into water and carbon dioxirle respectively. At the high temperature reached in the explosion water is, of course, gaseous, as are likewise the other products, $\mathrm{CO}_{2}$ and $\mathrm{N}_{2}$. 'Therefore the products 
of the explosion occupy many times the volume of the original substance. This explains the enormous force produced by the explosion. Dynamite, which is a solid mixture of infusorial earth and nitroglycerine, is much safer to handle than the latter substance.

693. Nitrocellulose, or guncotton, is made from cotton by a process similar to that used in making nitroglycerine. The product is a white solid scarcely differing in appearance from the cotton from which it is made. It is far less sensitive to shock than nitroglycerine, especially when in a moist state, and since it can be perfectly exploded while moist by the use of a detonator (573) it is one of the safest and most useful of explosives. Guncotton can be physically compounded with nitroglycerine to form cordite, a transparent solid resembling amber in appearance. This is one of the most important propellants for large projectiles. Modern smokeless powders are products closely related to guncotton.

The complete nitration of phenol, $\mathrm{C}_{6} \mathrm{H}_{5} \mathrm{OH}\left(\mathbf{6 7 2}_{2}\right)$, yields tri nitro phenol, or picric acid, $\mathrm{C}_{6} \mathrm{H}_{2}\left(\mathrm{NO}_{3}\right)_{3} \mathrm{OH}$. This substance and its ammonium salt $(673)$ are powerful explosives. Their most extensive use is in shrapnel.

The explosive commonly designated as T.N.T. is tri nitro toluene. It has also been mentioned earlier (57I). During the war it was used in enormous amounts in shrapnel and other explosive shells.

A few words may be added at this point regarding the differences in explosives, since the student will naturally wonder why so many different explosives are used. The most important properties which determine the character of an explosive are (I) its sensitiveness, (2) its force of explosion, determined by the volume and temperature of the gaseous products, and (3) its velocity of explosion. Of these three properties the last is very important; for although ordinary observation would indicate that every explosion is instantaneous, this is far from being the case. Every explosion requires time for its completion. A satisfactory propellant must not explode too rapidly. It must allow time for the projectile to get under way, otherwise 
it would burst the gun. For exploding shrapnel shells and for blasting rocks rapidly exploding substances are used.

694. Other Products of Nitrocellulose. - When the nitration of cotton is not carried so far as in the preparation of guncotton the product is known as pyroxyline or soluble cotton. This substance resembles guncotton closely but is less explosive. It dissolves readily in many organic solvents, such as acetone (656) and esters (657), and in a mixture of alcohol (64I) and ether (642). The solution so obtained is called collodion; it is used as an adhesive, as a liquid court-plaster, as a coating for incandescent gas mantles, and for many other purposes.

Artificial silk, a product resembling silk in appearance, but totally different chemically, is made in one way from collodion. The process consists in "spinning" a concentrated collodion solution from a fine glass capillary by means of high pressure. Upon coming into the air the solvents evaporate at once, leaving a filament of the fineness of a natural silk fiber. These filaments are made in to threads; but this material is extremely combustible and must be denitrated by a chemical process which changes it back into cellulose without altering its beautiful silky luster. Artificial silk is made in large quantities.

A rough way of telling the difference between artificial and natural silk is to set fire to a small piece of the fabric. Natural silk burns the way hair does, melting back of the flame and giving off a characteristic orlor. Artificial silk burns as does a thin piece of cotton. It does not melt and gives virtually no odor.

Celluloid is made by thoroughly kneading and rolling a warm mixture of pyroxyline and camphor, $\mathrm{C}_{30} \mathrm{H}_{56} \mathrm{O}$. The latter is a product of the camphor tree. 'The many forms and uses of celluloid are too well known to require description. Photographic films made from this material are highly inflammable. On this account celluloid is combined with less inflammable materials like cellulose acetate (acetic acid ester of cellulose) in the preparation of photographic films for moving pictures.

Leather substitute, or artificial leather, is male by coating heavy cotton cloth with a preparation in which pyroxyline is the principal ingredient. 
695. The Materials of Chemical Warfare.-The introduction by the Germans of chlorine in warfare was rapidly followed by the use of several other poisonous and irritating substances, practically all of which were carbon compounds. One of the most important of these was phosgene, or carbon oxychloride, $\mathrm{COCl}_{2}$. This gas is made by the union of carbon monoxide (632) and chlorine:

$$
\mathrm{CO}+\mathrm{Cl}_{2} \rightarrow \mathrm{COCl}_{2}
$$

It has a powerful, choking odor and is very poisonous. It is readily liquefied (boiling-point $8^{\circ}$ ). It was frequently mixed with liquid chlorine in gas attacks.

The so-called mustard gas is not a gas at all but a colorless liquid known to chemists as dichlor diethyl sulfide $\left(\mathrm{ClC}_{2} \mathrm{H}_{4}\right)_{2} \mathrm{~S}$. It has a rather faint odor and at first appears harmless enough; but inhalation of the vapor or mist produced by explosion of a shell containing some of it causes terrific inflammation of the lungs, frequently proving fatal. Almost inconceivably minute amounts on the skin cause in the course of a few days deep and dangerous wounds. The substance is made from ethylene, $\mathrm{C}_{2} \mathrm{H}_{4}$, and sulfur chloride, $\mathrm{S}_{2} \mathrm{Cl}_{2}$ (6or), and has the following structure:<smiles>CSCCCCl</smiles>

It seems to act by being absorbed by the skin and then slowiy hydrolyzing within the tissues to form hydrochloric acid and other products.

Chloropicrin, $\mathrm{CCl}_{3} \mathrm{NO}_{2}$, made by the action of bleaching powder (35I) on picric acid (673), is a high-boiling liquid. It is extremely irritating to the eyes, is poisonous, and causes vomiting. It passes through clothing and the fabric of a gas mask rather readily, and it is difficultly absorbed in the canister of chemicals used with a mask. This substance was used in large amounts toward the end of the war. Chloropicrin had no use before the war; it is now proposed to employ it as an insecticide. 
696. Organic Synthesis.-The term organic synthesis means the artificial building up of organic compounds from simpler substances, ultimately from the elements. A century ago it was generally believed that the complex carbon-containing products of plants and animal organisms (organic compounds) were formed as the result of vital forces, and that it was impossible for chemists to make them artificially. In I $\$_{2} 8$ Wöhler proved the fallacy of this idea by the synthesis of one of the most characteristic of animal products, urea, $\mathrm{CO}\left(\mathrm{NH}_{2}\right)_{2}(659)$. His method consisted in oxidizing potassium cyanide, $\mathrm{KNC}(665)$, to the cyanate $\mathrm{KNCO}$ by heating it with litharge, $\mathrm{PbO}$ :

$$
\mathrm{KNC}+\mathrm{PbO} \rightarrow \mathrm{KNCO}+\mathrm{Pb} \text {. }
$$

From $\mathrm{KNCO}$ and $\left(\mathrm{NH}_{4}\right)_{2} \mathrm{SO}_{4}$ he obtained by double decomposition ammonium cyanate, $\mathrm{NH}_{4} \mathrm{NCO}$. This salt, when warmed in solution gradually changes into urea,

$$
\mathrm{NH}_{4} \mathrm{NCO} \rightarrow \mathrm{CO}\left(\mathrm{NH}_{2}\right)_{2} \text {. }
$$

The announcement of Wöhler's discovery created a profound sensation and led to the expectation that other vital products could also be synthesized. This expectation has in the past ninety years been realized far more completely than the most enthusiastic chemist of Wöhler's day could have predicted; for not only have the greatest variety of vital products been synthesized, but thousands of related and new and unrelated organic substances have been made in the laboratory, so that today their number is legion. True, however, much remains to be done, for as we have seen in one instance the proteins have as yet not been synthesized. By way of illustrating the nature and scope of organic synthesis we shall in the following paragraphs recount briefly a few typical cases.

697. Essential Oils and Perfumes.-Spices, fruits, and flowers owe their characteristic odors or perfumes to small amounts of substances which can in many cases be isolated and purified. These fragrant substances are volatile oils or solids known generally as essential oils. Frequently the oil from a given source is a mixture of several definite chemical substances, although in other cases but a single substance is present. 
The first essential oil made synthetically was that contained in bitter almonds and well known as the oil of bitter almonds. Examination of the natural oil showed it to be benzaldehyde, a substance having the formula

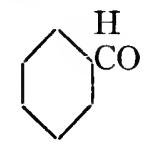

When toluene (668) is treated with chlorine it gives benzal chloride, $\mathrm{C}_{6} \mathrm{H}_{5} \mathrm{CHCl}_{2}$. The latter, by treatment with water, gives benzaldehyde (670). The product so obtained has the same agreeable odor as that made from bitter almonds and is extensively used as a substitute for the natural oil in flavoring extracts.

The chief constituent of oil of cinnamon, known as cinnamic aldehyde, has the formula

$$
\mathrm{C}_{6} \mathrm{H}_{5} \mathrm{CH}=\mathrm{CHCHO} \text {. }
$$

It can also be made synthetically from toluene, and when so prepared has the same fragrant odor as cinnamon.

The characteristic constituent of vanilla is also an aldehyde, called vanillin, somewhat related to the two foregoing substances. Its formula is

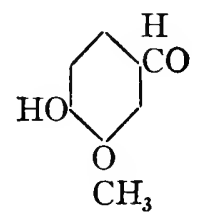

It is a white crystalline solid, which is present in vanilla beans to the extent of about I per cent. After the discovery of its formula as just given it became possible to make the substance synthetically. It was soon found that other essential oils were closely related to vanillin. Thus oil of cloves consists largely of<smiles>C=CCC1CCCO1</smiles> 
This oil is easily and cheaply made from cloves. It can be converted into vanillin by a process of oxidation, which thus affords a good practical method of making the latter valuable substance.

It is a well-known fact that organic substances with closely related formulae have similar properties, and this is beautifully illustrated by the near relatives of the two substances just considered. The fragrant oil of sassafras consists largely of safrole,

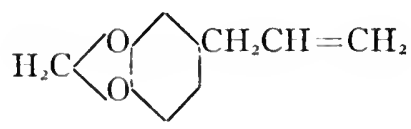

This substance can be oxidized to an aldehyde piperonal,<smiles>O=C1CC2OCC1O2</smiles>

a substance having the delightful odor of heliotrope. Thus an exquisite and costly perfume is made in the laboratory from the cheap and abundant oil of sassafras.

Many essential oils belong to the class of substances known as esters (657). For example, oil of wintergreen is the methyl ester of salicylic acid, readily made by following reaction:

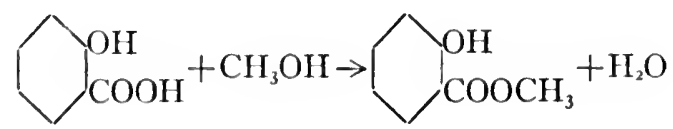

The ester, which is made technically on a large scale, is a colorless oil having exactly the odor of wintergreen. It will be of interest to trace the manufacture of this substance from its beginning. To get salicylic acid we start with benzene (667), obtained from coal tar. This by treatment with sulfuric acid gives benzene sulfonic acid $(672)$,

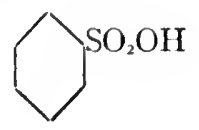


When the sodium salt of this acid is fused with an excess of sodium hydroxide it yields phenol or carbolic acid (672),

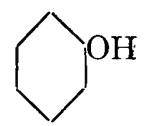

The sodium salt of this substance reacts with carbon dioxide to form the salt of salicylic acid,

$$
\mathrm{C}_{6} \mathrm{H}_{5} \mathrm{ONa}+\mathrm{CO}_{2} \rightarrow \mathrm{C}_{6} \mathrm{H}_{4} \mathrm{OHCOONa},
$$

from which the free acid is readily obtained by the action of sulfuric acid.

The subjects discussed in this section are of importance chiefly as a means of illustrating the ways in which some natural products are made in the laboratory. It is not worth while for the student to attempt to memorize the formulae or the reactions involved. The examples given are chosen from hundreds equally important and interesting.

698. Dyes. The Synthesis of Indigo.-In earlier times dyes were usually plant or animal products. A few coloring substances, not dyes in the strict sense of the word, were of mineral origin. At present all but a small proportion of dyes are made artificially from substances obtained from coal tar. In a few cases dyes originally obtained from plants are now made in the laboratory. The most interesting case in point is that of indigo, which has long been one of the most important blue dyes. It is the product of a plant grown extensively in India, Java, and elsewhere. After the development of synthetic chemistry it was apparent that the artificial production of any natural chemical substance was possible, though doubtless in many cases difficult of realization. This possibility led chemists to hope that indigo could be made in the laboratory. The first step was to discover its structural formula. The purification of indigo proved easy, and the analysis of the pure crystalline substance was a matter of routine. The simplest formula possible according to the analysis was $\mathrm{C}_{8} \mathrm{H}_{5} \mathrm{NO}$; but the vapor density $(7 \mathrm{I}, 2 \mathrm{I} 7)$ indicated a molecular weight corresponding to twice this formula, namely, $\mathrm{C}_{16} \mathrm{H}_{10} \mathrm{~N}_{2} \mathrm{O}_{2}$. Now it would be 
possible to think of hundreds of molecular arrangements of 16 atoms of carbon, ro atoms of hydrogen, and 2 each of nitrogen and oxygen, all in accord with accepted laws of valence (648); only experiment could decide which if any of these formulae represented the structure of indigo. Let us try to explain how the organic chemist attacks this kind of a problem, for he must solve it beyond doubt if he hopes to synthesize the substance. By distillation of indigo with $\mathrm{KOH}$ one obtains aniline (674), the structure of which is known to be<smiles>NC1CCCCC1</smiles>

The oxidation of indigo yielded a related substance, isatine, for which the structure had been shown to be<smiles>O=C1NC2CCCCC2C1=O</smiles>

These facts indicate that the indigo molecule contains the atomic group<smiles>C=C1C(=C)N(C)C2CCCCC12</smiles>

This group contains 8 atoms of carbon and I of nitrogen; since indigo is $\mathrm{C}_{16} \mathrm{H}_{10} \mathrm{~N}_{2} \mathrm{O}_{2}$, the latter probably contains two isatine groups. Finally, after many years of research the structure of indigo was shown to be the following:

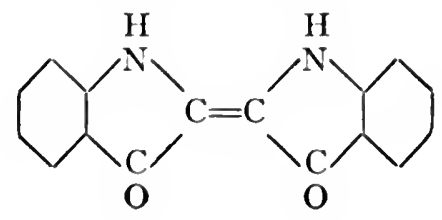


After the structure of indigo had been found it was not long until several methods were devised by which it could be made synthetically; for once he knows its structure the organic chemist can attempt the building up of a molecule with nearly as great certainty of ultimate success as an architect can construct a building from prepared plans. A successful technical synthetic process must employ starting materials that are sufficiently abundant and cheap. For the artificial production of indigo we start with naphthalene (moth balls), $\mathrm{C}_{\mathrm{ro}} \mathrm{H}_{8}$ (669), the structure of which is

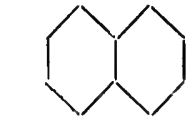

Oxidation of this substance yields phthalic acid,

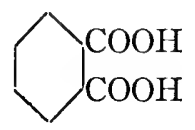

which in turn yields, by the aid of ammonia, a substance having the formula

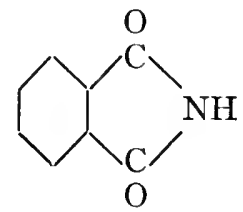

This by the action of sodium hypochlorite (350) gives an acid of the following composition:

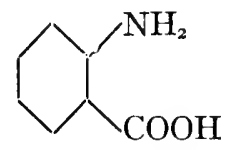

The next step is the union of this acid with chloracetic acid,

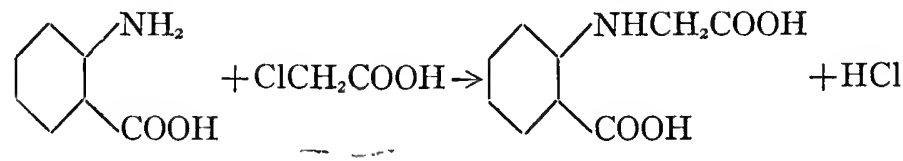


When the product is heated with sodium hydroxide it is changed into

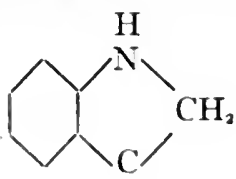

$\mathrm{O}$

Upon oxidation with air, two molecules of this substance give water and a molecule of indigo,

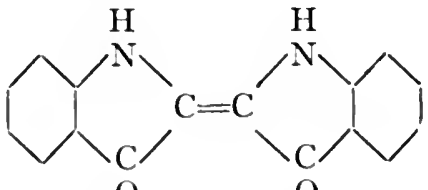

$\mathrm{O}$

$\mathrm{O}$

Artificial indigo can now be made cheaper than the natural dye and has nearly driven the latter from the market.

The story of indigo is closely paralleled by that of madder, an extract from the root of which dyes turkey red. The same dye is now made synthetically from anthracene, $\mathrm{C}_{\mathrm{r} 4} \mathrm{H}_{\mathrm{ro}}$, a coal-tar product resembling naphthalene.

The story of indigo has been told in some detail largely to illustrate the method by which a natural substance is reproduced synthetically. The steps in the process, which in all cases are the same, are the following: first, the isolation and purification of the substance; second, the analysis; third, the molecularweight determination to discover whether a multiple of the simplest possible formula is the true formula; fourth, the discovery of the structure; fifth and last, the synthesis from simple substances or from the elements. Of these steps the fourth is usually the most difficult, although both this step and the last demand the highest skill of the chemist. It would require the space of a chapter to give even a brief account of the coal-tar dyes. Suffice it to say here that about nine hundred different coal-tar dyes are made, and of these about three hundred are in active denand. Each is a definite chemical substance, the preparation of which is on the average as complicated a process as that of making indigo. At the beginning of the war 
90 per cent of all dyes used in America were imported, largely from Germany. At the close of the war we were making in the United States a greater tonnage of dyes than we required, although many less important kinds of dyes were not yet made in this country.

699. Synthetic Medicinals and Photographic Chemicals.At the present time a large number of valuable medicinal substances are produced synthetically from coal-tar products. Many of these substances are obtained from the same intermediate products, so-called intermediates, that are used in making dyes. Chemicals used as photographic developers are also derived from similar intermediates. These facts give an added significance to the development of an American dye industry, since it means that we shall also be able to manufacture in this country our required medicinals and photochemicals. A long step in this direction was taken during the period of the war.

700. Rubber.- It has long been known that the composition of natural rubber is represented by the very simple formula $\mathrm{C}_{5} \mathrm{H}_{8}$, but that the actual formula is probably $\mathrm{C}_{10} \mathrm{H}_{16}$ or perhaps $\left(\mathrm{C}_{\mathbf{r} 0} \mathrm{H}_{\mathrm{r} \sigma}\right)_{n}$. Thus rubber has the same percentage composition as the comparatively cheap and abundant substance turpentine, $\mathrm{C}_{10} \mathrm{H}_{16}$ (248). It is therefore not surprising that enormous efforts have been made to produce synthetic rubber. On account of the great practical importance of this subject a brief discussion may prove of interest. One of the products of the distillation of rubber is a colorless volatile liquid called isoprene. This has the simple formula $\mathrm{C}_{5} \mathrm{H}_{8}$ and has been shown to have the structure

\section{$\mathrm{CH}_{2}: \mathrm{C}\left(\mathrm{CH}_{3}\right) \mathrm{CH}: \mathrm{CH}_{2}$}

Isoprene has been made synthetically by several methods. It is a colorless liquid, which by treatment with certain catalytic agents changes into synthetic rubber:

$$
{ }_{2} \mathrm{C}_{5} \mathrm{H}_{8} \rightarrow \mathrm{C}_{10} \mathrm{H}_{16} \text {. }
$$

As long ago as I9I2 a synthetic-rubber automobile tire that had run 5,000 miles and was still in good condition was exhibited at the International Congress of Applied Chemistry. 
The practical problem of the synthesis of rubber is then resolved inte the sufficiently cheap production of isoprene. The starting material must be a compound of carbon and hydrogen with or without other elements, which is cheap and abundant and convertible with a good yield into isoprene. No wholly satisfactory technical method has yet been found, for the simple reason that the cost of artificial rubber by any of the processes so far devised is greater than that of natural rubber. Here then is a most interesting and important problem for the future. To the chemist who solves it will come both fame and wealth.

70r. Sources of Organic Material.-The possibilities of synthetic organic chemistry are very alluring to one possessed of an active imagination; but it is necessary to keep in mind that all synthetic substances must be made from other carbon compounds, and that if a substance is to be made on a practical scale it can be made only from something sufficiently abundant and cheap. These facts make it worth while to consider the natural sources of organic materials.

The principal sources are (I) plants, (2) animals, (3) coal, and $(4)$ petroleum and natural gas. It is well known that coal is of regetable origin; and it seems probable that petrolcum comes from both vegetable and animal matter. Going back a step farther we recall that all animals are dependent on plants for nourishment, so that we find that plants are the ultimate source of all other organic material. We have also seen that plants obtain their carbon from the carbon dioxide of the air. Although the $\mathrm{CO}_{2}$ content of the air is but three parts in 10,000 by volume, the weight of this gas above every square mile of the earth's surface amounts to about 10,000 tons. Therefore there is in the air an immense reserve of the element carbon.

We have already referred to the many important constituents of coal tar $(634,667)$. These serve as the more immediate starting materials for numerous organic syntheses. The distillation of wood also yields, in addition to charcoal, several very useful products, of which methyl or wood alcohol (645), acetone $(656)$, and acetic acid $(653)$ are the most important. 


\section{CHAPTER XXVII}

\section{THEORY OF DILUTE SOLUTIONS}

Our purpose in this chapter is to develop in greater detail than before the picture of the condition of a dissolved substance. This will be accomplished to best advantage by going over at the same time parallel work done on gases.

702. The Reality of Molecules.-According to the kinetic theory gases are composed of tiny particles moving at random with velocities of rifle bullets and spaced from each other distances which on the average are large compared with the diameter of the particles (196). This picture has given us an understanding of why all gases diffuse through and occupy any space in which they are released. It has also explained why all gases follow the same laws, namely those of Charles and Boyle $(4,5)$. Further than this, however, the kinetic theory has explained why under great pressures and low temperature gases do not follow these laws closely (225). So great has been the assistance given by the theory in understanding the behavior of gases that the actual existence of molecules has seemed to many beyond the possibility of doubt, even though the molecules themselves have never been seen. More recently, however, the crowning evidence in proof of the reality of molecules has been brought out by work with the ultra-microscope.

703. Perception of Molecules.-The celebrated French physicist Perrin, to some of whose work on the cathode rays we have referred earlier (478), says, "Direct perception of the molecules in agitation is not possible for the same reason that the motion of the waves is not noticed by an observer at too great distance from them. But if a ship comes in sight, he is able to see that it is rocking," and this "will enable him to infer the existence of a possibly unsuspected motion of the sea's surface." While we cannot see molecules themselves, we can 
by devices now to be described see the phenomenon which is the counterpart of the boat rocking on the invisible waves.

In place of a ship buffeted about by waves we shall observe particles of smoke floating in the air and exposed to the bombardment of air molecules. Although these smoke particles are far larger than the largest molecules (each consisting of many molecules), they are still too small to be seen even under a microscope unless they are brilliantly illuminated. The smoke (from a cigarette or the like) is contained in a small glass box bencath the objective of the microscope and is illuminated by a beam of light from a projection lantern focused at a point in

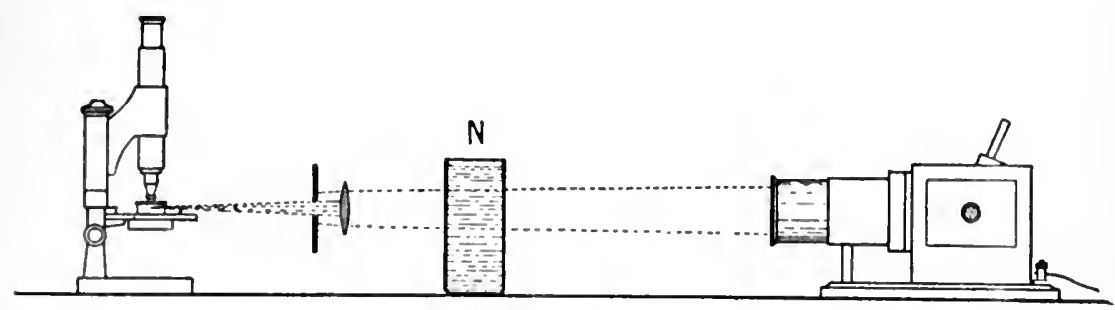

FIC. 98

the smoke just under the objective. 'The arrangement is illustrated in Fig. 98. 'The observer looks down through the microscope upon tiny smoke particles which are illuminated against the dark background by the horizontal rays from the lantern. The field of the microscope seems covered with a "milky way" of bright stars which are the flashes of light reflected to the observer's eye by the particles. 'Thanks to the dark background these can be easily distinguished.

The flat-sided jar of water, $N$, shown in the figure is interposed in the beam from the lantern in order to cool it as much as possible before allowing it to strike the box; but even so convection currents are set up within the box. As a consequence of these currents all the particles drift along in one direction, but if the observer watches a single particle it will be seen to be dancing about in a most erratic way. This jiggling motion keeps up endlessly, as long as the particles themselves are in 
suspension. The smaller the particles the more violent is the motion. In more refined apparatus still smaller particles can be seen; these dart over distances many hundreds of times their own diameter. The fact that the motion is absolutely erratic, and that any suspension of any substance, the individual particles of which are very small, exhibits this same motion, shows that the latter is due to the irregular bombardment of the particles by the molecules of the gases in which they are suspended. The particles must be small enough to be moved appreciably by a few more hits by molecules on one side than on the other. This motion, called from its discoverer Brown the Brownian movement, is the phenomenon which is the counterpart of the rocking of the boat upon the invisible waves.

704. The Kinetic Theory of the Liquid State.-In comparing liquids with gases we note at once the far greater density of the former. The kinetic theory (198) describes molecules in the liquid state as moving about, much as they do in the gaseous state, but as greatly hampered in their motion on account of the crowding of the molecules. In liquids the average path between collisions is much shorter than in gases, so that diffusion through the former is very much slower than through the latter. The smaller distance between the molecules means that the attraction between them is of greater moment than in the gaseous state. This attraction falls off rapidly with increasing distance, so that each molecule is affected by its immediate neighbors only. As a result, within the liquid this effect is balanced, since the pull exerted by the neighbors comes equally from all sides. Only at the surface of the liquid does this force become onesided and so hampers the free motion of the molecules. As a result only a few of the faster-moving molecules can escape from the surface (I99). According to the kinetic theory the average kinetic energy of molecules in solution is the same as that of molecules in the gaseous state at the same temperature, so that the light molecules in solution are to be pictured as moving very much faster than the heavy ones. In many ways the success of the kinetic theory in helping us to understand the liquid state has been so marked that its validity was accepted 
even before the development of the more direct evidence of the ultra-microscope.

705. Brownian Movements in Liquids.-The existence of molecular motion in liquids can be demonstrated by the Brownian movements of very tiny particles in suspension in liquids, just as it can be in the case of smoke particles. Suspended particles of suitable size, whether of clay, lampblack, starch, or anything else, all exhibit these movements irrespective of their composition.

The pigment gamboge, when rubbed in a little water or dissolved in alcohol and added to water, gives a yellowish turbid liquid which consists of suspended droplets of suitable size to show the Brownian movements. A microscope with oil-immersion objective is best used. If a drop of the gamboge suspension is mounted for observation under the objective and viewed with the ordinary illumination reflected from the mirror below the stage of the instrument many tiny particles will be seen in the field. Careful observation will show that the smaller particles are in a constant tremor, moving erratically over paths approximately one to several times their own diameters in length. It seems difficult for the observer to believe that these moving particles are not alive, so lifelike is their motion!

706. The Revelations of the Ultra-Microscope.-Suspensions of far smaller particles may be observed if instead of the ordinary microscope we use the ultra-microscope. To build a crude form of the latter we may employ the same set-up as was used for the examination of the smoke particles, except that we must use an oil-immersion lens and a so-called condenser. In this case the light from the lantern is again made to pass through water, but this time, instead of being directed next against the object, it is made to strike the mirror below the stage of the microscope and is reflected into the condenser, which directs it obliquely against the droplet on the slide, as shown by the path of the arrows in Fig. 99. A drop of pure water placed on the slide appears only as a dark field to the observer; but if there are tiny particles present in it flashes of light are reflected from them to the eye. Zsigmondy, who with Siedentopf perfected the ultra-microscope, describes his first view of a fine suspension 
of gold particles in water thus: "The small gold particles move and that with astonishing rapidity. A swarm of gnats in a sunbeam will give one an idea of the motion of the gold particles. ... . They hop, dance, jump, dash together and fly away from each other so that it is difficult to get one's bearings." The very fine particles seen under the ultra-microscope are observed to

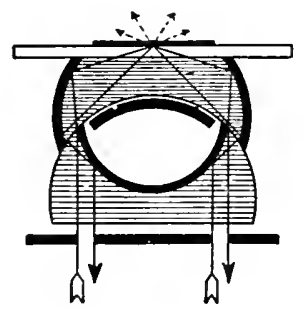

FIG. 99 travel much more rapidly and to cover greater distances than those previously described. It should be realized that any suspension in any liquid shows this same erratic motion. Specimens have been under observation for years, but no abatement of the Brownian movement has been observed. Suspensions in liquids taken from the interior of quartz crystals show these same motions, and yet they are thousands of years old! We have, therefore, the same type of evidence of the reality of the existence of swift-moving molecules in liquids as we have in gases.

707. Visible Atmospheres.-Perrin was able to show that fine suspensions may be considered as visible atmospheres. The pressure due to the impacts of the ultra-microscopically visible particles is proportional to the concentration and to the absolute temperature, just as the pressure due to gas molecules is proportional to their concentration and to the absolute temperature. The actual number (194) of molecules in a gram molecular weight of a substance in the gaseous state has been estimated as $6 \times 10^{23}$. Perrin estimated the number of suspension particles in what would be a gram molecular weight of a suspended substance, namely the weight of substance in 22.4 liters of a suspension the particles of which at $0^{\circ}$ exert a pressure of $760 \mathrm{~mm}$. The numbers found for a great variety of suspensions were close to $6 \times 1 \mathrm{I}^{23}$. The viscosity of the suspension medium seemed to make no difference in the result. It did not matter whether the suspension medium was pure water or the very viscous glycerine. The average weight of the particles was varied 70,000 fold, and yet the results were the same. Thus the gas 
laws and Avogadro's hypothesis apply to these suspensions. I'e are then to think of fine suspensions as miniature atmospheres of colossal molccules which are actually visible.

708. Particles in Solution vs. Particles in Suspension.Particles visible in the ultra-molecular microscope are called suspensions, but particles just too small to be seen in this way must be counted as in solution. The change from solution to suspension should be thought of as gradual rather than abrupt, for the distinction secms to be fundamentally one of the size of the particle.

709. Substances in Solution vs. Gases. - The knowledge that particles in suspension in a liquid act like huge gas molecules inclosed in the same volume as that of the solution leads at once to the conception that substances in solution may also be considered in the same light. To test this theory the pressure of the dissolved molecules must be measured separately from that of the molecules of the solvent. This is obviously not a simple undertaking, since the full impacts of molecules in solution are not felt by a surface in contact with the latter because of the attraction inward of the molecules which come to the surface layer. The same force which prevents the free migration of molecules from the surface of the liquid prevents the measurement of the force of their impacts by such devices as are used to measure gas pressure. However, devices to suit the purpose have been found, and these will now be studied. Since they depend on the operation of a semipermeable membrane, a simple example of the latter will first be described.

710. Semipermeable Membranes. $A$ socalled semipermeable membrane is a membrane which is permeable to one of two substances in a mixture but not to the other. A layer of

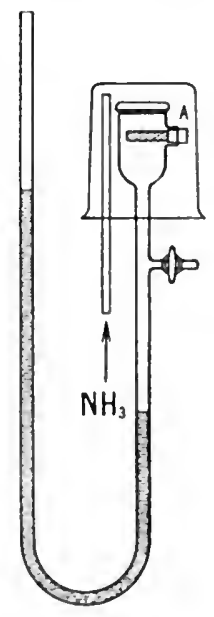

Fir. 100 thin cloth (muslin) stretched over the tubulated end of the oil manometer, as shown in Fig. 100, and wet with water will serve as a membrane permeable to ammonia but not to air. The 
stopper at $A$ permits the insertion of red litmus paper into the inner chamber, as shown in the figure. When ammonia is collected in a beaker inverted over the membrane, the oil in the manometer moves, showing an increase of pressure in the inner chamber. Meantime the litmus paper is seen to turn blue, thus showing that ammonia has entered. This it has done by dissolving in the upper surface of the water film and diffusing through it. As a result some ammonia molecules soon appear at the inner surface of the film and then

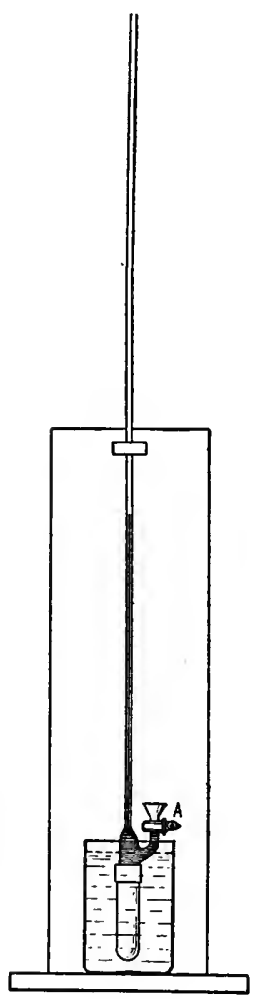

FIG, IOI escape into the air within the apparatus, just as in the case of the diffusion through a gas (rgr) the migration of ammonia molecules through the water and air layers proceeds spontaneously from regions of higher concentration to those of lower concentration until there is no longer any difference between the two. Meantime the air inclosed in the apparatus is not able to escape, since it is not appreciably soluble in water, and therefore the total pressure increases.

7rr. The Solution Cell.-A similar apparatus for use with solutions may be easily prepared for demonstration purposes by attaching a parchment-paper thimble to a glass tube, as shown in Fig. ror. The thimble is filled with concentrated sugar solution colored with cochineal and immersed in a beaker of pure water. Water can pass through the parchment wall in either direction, but the sugar and the cochineal cannot do so appreciably. Since the color can only accompany the sugar, it serves as a convenient marker for the presence of the latter. Soon after the cell is filled and the stopcock of the filling tube, $A$, is closed the level of the sugar solution begins to rise in the vertical tube. In a few hours the surface of the sugar solution will be three or four feet above the level of the water in the outside 
beaker. There is obviously a close analogy between this experiment and the preceding one. The water in the former corresponds to the ammonia in the latter, since each can pass through the membrane. The sugar, like the air, cannot pass through the membrane. Whatever the mechanism by which the cell operates, it is plain that the weight of the column of solution is borne by the membrane, except for the slight assistance given by the pressure exerted against it by the water in the outside beaker. If the column is allowed to get too high the thimble will be broken. For accurate measurements stronger membranes must be used, and they must also be of substances more truly impermeable to sugar. The best membranes have been made by precipitating copper ferrocyanide in the pores of unglazed porcelain cups.

A cell of this type is shown in Fig. 102. The porcelain cup which holds the sugar solution is fitted with a mercury manometer, the closed end of which contains nitrogen gas. As water enters the cell through the copper ferrocyanide membrane deposited in the pores of the cup, the nitrogen in the manometer is compressed. Since the manometer is very narrow, only a small increase of volume on the part of the solution in the cell results in a very great compression of the nitrogen. The change in volume of the latter (together with the very small difference in levels of the mercury in the manometer arms) gives the necessary data for calculating the pressure within the cup. When no further

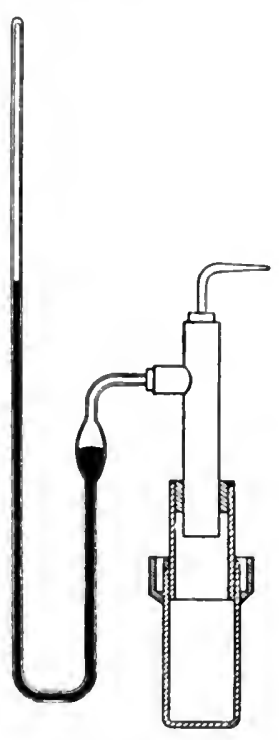

FIG. 102 change in volume of the nitrogen is seen, the pressure of the latter is just sufficient to check the inflow of water through the membrane. The pouring of the water into the sugar solution through the membrane is called osmosis, and the pressure which checks this flow is called the osmotic pressure of the solution.

712. Results of Osmotic-Pressure Measurement.-Table XXXIII gives the osmotic pressures of cane-sugar solutions 
at $60^{\circ}$ at the concentrations indicated. From indirect evidence chemists long ago made up their minds that the molecular weight of sugar is 342 , corresponding to the formula $\mathrm{C}_{\mathrm{I} 2} \mathrm{H}_{22} \mathrm{O}_{1 \mathrm{I}}$ (682). Hence we may give the concentration in percentage by weight of sugar in solution and also in terms of gram molecular weights of sugar to I,000 grams of water, i.e., weight-molar concentration. The osmotic pressure is given first in lbs. per square inch, in order that the magnitude of these pressures may be visualized, and then in terms of atmospheres. From the table it is evident that even for a 3 per cent solution the membrane

\section{TABLE XXXIII}

Osmotic Pressures of Sugar Solutions

\begin{tabular}{|c|c|c|c|c|c|}
\hline \multicolumn{2}{|c|}{ CONCENTRATION } & \multicolumn{2}{|c|}{ OsMotic Pressure } & \multirow{2}{*}{$\begin{array}{l}\text { Gas Pressure } \\
\text { IN } \\
\text { AtMospheres }\end{array}$} & \multirow{2}{*}{$\begin{array}{c}\text { Ratio } \\
\text { O.P. to G.P. }\end{array}$} \\
\hline Percentage & Weight-Molar & Lbs. Per Sq. In. & Atmospheres & & \\
\hline $3 \cdot 3$ & O.I & 40 & 2.72 & 2.72 & I. .00 \\
\hline 6.4 & .2 & 80 & $5 \cdot 44$ & $5 \cdot 43$ & I . $\infty$ \\
\hline $9 \cdot 3$ & $\cdot 3$ & I 20 & 8.14 & 8.15 & 1.00 \\
\hline 12.0 & .4 & 160 & I0.87 & I0. 87 & 1.00 \\
\hline 14.6 & .5 & $2 \dot{v} 2$ & I 3.67 & I $3 \cdot 5^{8}$ & I.OI \\
\hline I $7 . I$ & .6 & 244 & I6. 54 & 16.30 & I.OI \\
\hline $\pm 9 \cdot 3$ & .7 & 285 & 19.40 & 19.02 & I. .02 \\
\hline $21 \cdot 5$ & 0.8 & 329 & 22.33 & $21 \cdot 73$ & 1.03 \\
\hline
\end{tabular}

must stand a pressure of $40 \mathrm{lb}$. to the square inch. For a 20 per cent solution the membrane must stand a pressure of over $300 \mathrm{lb}$. per square inch. Students will readily appreciate the experimental skill necessary to make these measurements, when they think of their own difficulties in securing an apparatus tight enough to stand less than a pound of pressure without leaking! In the fifth column is given the pressure which a like molecular concentration of hydrogen would exert at the same temperature. The close agreement between osmotic pressure and gas pressure is at once apparent from a comparison of the fourth and fifth columns. This is brought out in the sixth column, which represents the ratio of osmotic pressure to gas pressure. Where the ratio is equal to $\mathbf{I . 0 0}$, the two may be regarded as equal. As has been said, this data is for solutions 
at $60^{\circ}$. How osmotic pressure compares with gas pressure at other temperatures is shown in Table XXXIV, which represents the ratio of osmotic pressure to gas pressure for solutions at other temperatures as well. The data for both tables have been adapted from the very fine work of Morse and Frazer, of Johns Hopkins University.

In both Table XXXIII and Table XXXIV it is apparent that the osmotic pressures are indeed close to the corresponding gas pressures. If we look at the deviations from the gas laws by noting how much greater than $\mathrm{I} .00$ the ratios are, we find

\section{TABLE XXXIV}

Ratio of Osmotic Pressure to Gas Pressure at Various Temperatures

\begin{tabular}{|c|c|c|c|c|}
\hline Concentration & $0^{\circ}$ & $20^{\circ}$ & $40^{\circ}$ & $60^{\circ}$ \\
\hline $0.1 \ldots \ldots \ldots$ & (I.II) & I.OS & I.,$\infty$ & 1.00 \\
\hline $0.3 \ldots \ldots \ldots \ldots$ & 1.06 & I. .06 & 1.02 & I., \\
\hline $0.5 \ldots \ldots \ldots \ldots$ & 1.07 & 1.07 & 1.05 & I.OI \\
\hline $0.8 \ldots \ldots \ldots \ldots$ & I. OO & I. 09 & 1.07 & 1.03 \\
\hline $1.0 \ldots \ldots \ldots \ldots$ & I. I 2 & I. 12 & 1.09 & 1.04 \\
\hline
\end{tabular}

that they are larger the lower the temperature and the more concentrated the solution. This reminds one of the deviations of the behavior of the gases themselves from strict agreement with the gas laws (225).

713. Interpretation of Osmotic-Pressure Measurements.How are we to explain the striking results of osmotic-pressure measurements? The fact that the measured osmotic pressure of a solution equals the pressure due to a gas at like temperature and of like molecular concentration as that of the dissolved substance in question is a coincidence so remarkable that we cannot call the agreement one of chance. Apparently the cell operates because water is at a different concentration on the two sides of the membrane just as the air-ammonia cell operated because ammonia which could pass through the water membrane was at first more concentrated on one side than on the other. In the sugar-water cell presumably the pressure due to the impacts of the molecules against the membrane is at first 
the same from both sides; hence the pressure of water molecules inside the cell is short of that outside by just the pressure due to the impacts of the sugar molecules. This pressure must be put upon the solution in order that the water may be made to pass back from solution to pure water at the same rate that it is coming into the cell and equilibrium thus be established. In this way we may explain the operation of the cell and the peculiar values found.

In our previous work we have always given concentrations of solutions in terms of weight of dissolved substance per liter of solution. In the above-mentioned work concentration is given in terms of weight of dissolved substance per liter of solvent, since the values so obtained are a little nearer to the corresponding gas pressures, presumably because the amount of solvent is a measure of the actual free space through which the dissolved particles move.

In measuring the pressure which must be put upon a solution to check the inflow of solvent into it when separated from the solvent by a semipermeable membrane, we are measuring a force which is equal to the pressure exerted by the impacts of the molecules of the dissolved substances. From such measurements we learn that substances in dilute solution act much as though they were in the gaseous state, at the same temperature and inclosed in volumes equal to the volume of the pure solvent in the solution.

714. Other Theories of Osmosis.--The theory just presented of the mechanism by which the semipermeable membrane cell operates is not the only possible explanation of the facts. For instance, some prefer to regard the motion of the solvent through the membrane as due to an attraction by the solution. Osmotic pressure would be the result of a sort of suction. As yet the theory presented above has been by far the more useful.

7r5. Van't Hoff's Theory of Solutions.-The great Dutch chemist van't Hoff was the first to point out that the gas laws should hold for substances in very dilute solution. He drew his conclusions from considerations of a purely theoretical nature at a time ( 1887 ) when the data at hand for the experi- 
mental confirmation of his theory were far less accurate than those given in Tables XXXIII and XXXIV and showed far less clearly the truth of his statements. Van't Hoff pointed out that the laws of gases should be followed by substances in very dilute solution only, since in the more concentrated solutions the formation of complexes between the solvent and the dissolved substance and between the molecules of the latter make conditions which are not parallel to those found in gases which follow the simple gas laws.

716. The Avogadro-van't Hoff Hypothesis.-Van't Hoff also asserted the so-called Avogadro-van't Hoff hypothesis, a generalization of tremendous importance in the growth of modern chemistry. This hypothesis is simply the application of Avogadro's hypothesis to substances in solution. Equal volumes of solutions of all substances which have equal osmotic pressures at the same temperature contain the same number of dissolved molecules.

717. Molecular Weights from Osmotic-Pressure Data.As a first result of the establishment of this principle we have learned how to find the relative weights of molecules in solution (molecular weights) by finding what weight of the substance in question will exert $76 \mathrm{~cm}$. of osmotic pressure at $0^{\circ}$ when dissolved in 22.4 liters of solvent.

718. Raoult's Work. Shorter Methods of Determining Molecular Weights.-Before van't Hoff's work was published, Raoult, a French chemist, working with a great variety of substances the molecular weights of which were known, had shown that a gram molecular weight of each dissolved in a given weight of solvent produced about the same lowering of the vapor pressure of the latter, provided the dissolved substance was not volatile. Van't Hoff pointed out (on the basis of reasoning which we need not now consider) that this is a necessary consequence of the fact that Avogadro's hypothesis applied to dilute solutions. Therefore the molecular weight of an unknown substance in solution may be determined by finding what weight of this substance is required to give a solution of vapor pressure equal to that of a half-molar solution of a substance the moleccular weight of which is known. Twice the value found would 
be the molecular weight of the new substance. Raoult's observations are for dilute solutions, so that the work should not be done with concentrations greater than half-molar.

We have already seen that one result of the lowering of the vapor pressure of a liquid by dissolving a non-volatile foreign substance in it is that the solution boils at a higher temperature than the pure solvent (128). Raoult showed that the elevation of the boiling-point is proportional to the number of gram molecular weights of substance dissolved in unit weight of solvent. If one gram molecular weight of a non-volatile substance is dissolved in $1,000 \mathrm{~g}$. of water the boiling-point is raised $0.50^{\circ}$. This is the so-called molar elevation for water. A gram molecular weight of a substance dissolved in $\mathrm{r}, 000 \mathrm{~g}$. of ether elevates the boiling-point of the latter $2.20^{\circ}$. Here then is another way of determining molecular weights of substances in solution. We have only to find how much of the new substance must be dissolved in $1,000 \mathrm{~g}$. of solvent to produce the molar elevation of the boiling-point, in order to find the molecular weight of the substance in question.

The freezing-point of a solution affords still another method. The depression of the freezing-point of the solvent, like the elevation of the boiling-point, is proportional to the number of gram molecular weights present per unit weight of solvent, provided the latter is the only substance which separates from solution during the freezing. Water solutions which contain one gram molecular weight of dissolved substance per $\mathrm{I}, 000 \mathrm{~g}$. of water freeze at $-\mathrm{r} .85^{\circ}$. Hence the molar depression of the freezing-point of water may be taken as I $85^{\circ}$. That of the solvent benzene $(667)$ is $5 \cdot 3^{\circ}$. Accordingly we need only find what weight of substance is necessary to produce the molar depression of the freezing-point of a suitable solvent to find the molecular weight of the substance in question in tine solvent used.

719. The Importance of the Work of van't Hoff and of Raoult.-The general acceptance of Avogadro's hypothesis (about I860) initiated a new era in chemistry. A second great development of chemistry began in 1887 , with the announcement of the Avogadro van't Hoff hypothesis. 'The latter furnished 
the basis for the understanding of reactions in dilute solution, just as the original hypothesis had done for gas reactions. Much of the rapidity of the development of the work on dilute solutions is due to the relatively simple experimental methods of Raoult for determining molecular weights of dissolved substances. 'The immediate result of the work of these two men was the presentation of new and independent evidence concerning the ionic hypothesis which was just being developed by Arrhenius (in I $88_{7}$ ), mainly from work on conductivity measurements.

720. Molecular Weights of Acids, Bases, and Salts.-When the molecular weights of acids, bases, and salts were determined in water solution abnormal results were found. Thus in the case of hydrogen chloride, which as a gas has the molecular weight 36.5 , the molecular weight in water solution was found to be much less than this, and furthermore, when measurements were made on solutions of greater and greater dilution, the results found grew smaller, reaching about i 8.3 as a limit.

The significance of abnormally low molecular weight in the case of ammonium chloride vapor (529) was shown to be due to dissociation into ammonia and hydrogen chloride. Arrhenius was quick to point out that the abnormally low molecular weights of electrolytes must also be due to the fact that the original molecules must have dissociated into ions in water solution. The fact that these molecular weights get smaller with increasing dilution is evidence of increased dissociation with dilution; but finally, when the degrees of dissociation were calculated from freezing-point data and compared with the values found for the same concentrations by the conductivity method (408), the two were found to agree so closely that little doubt remained of the correctness of the ionic hypothesis.

\section{The Degree of Dissociation from Molecular-Weight} Determinations. - The method of calculation of the degree of dissociation from molecular-weight determinations is best illustrated by an example. In the case of potassium chloride the simplest formula is $\mathrm{KCl}$, indicating a molecular weight of 74.6 . If $\mathrm{KCl}$ ionizes thus,

$$
\mathrm{KCl}=\mathrm{K}^{+}+\mathrm{Cl}^{-}
$$


it is plain that if roo molecules were originally present and 80 per cent of them broke down into ions, the total number of free particles present would be 180 instead of 100 . If we start with $74.6 \mathrm{~g}$. of $\mathrm{KCl}$ in $1,000 \mathrm{~g}$. of water and 80 per cent is dissociated, we shall have present 0.2 gram molecular weight of $\mathrm{KCl}, 0.8$ gram molecular weight of potassium ion, and 0.8 gram molecular weight of chlorine ion, making a total of I. 8 gram molecular weights from the $74.6 \mathrm{~g}$. of salt dissolved instead of one gram molecular weight. The observed depression of the freezing-point of water will be 1.8 times as great as is expected for one gram molecular weight of total substance dissolved in the same volume. Hence the apparent molecular weight of $\mathrm{KCl}$ calculated from the freezing-point of a solution 80 per cent ionized will be $74.6 \div \mathrm{I} .8=4 \mathrm{I} \cdot 4$. Conversely, if the molecular weight of a $\mathrm{KCl}$ solution found in the usual way by the freezing-point method is $4 \mathrm{I} .4$, the degree of dissociation of the solution is found thus: $74.6 \div 4 \mathrm{I} \cdot 4=\mathrm{I} .80$. From this we conclude that the dissociation is 80 per cent.

722. Degree of Dissociation of Electrolytes by Two Methods.-The degrees of dissociation of a number of electrolytes at different concentrations as calculated from molecularweight determinations by the method just illustrated are shown in Table XXXV (line I for each substance) in comparison with like data for the same substance as found from electrical conductivities (line II). (In the more concentrated solutions the latter data have been corrected for changes in the viscosity of the solution with concentration. Obviously, if the viscosity of the solution increases, the rapidity with which the same ions will move under the same attractive force will be diminished so that a correction is necessary.) Concentrations are given in terms of molecular weights per liter except in the cases of salts having bivalent ions. In these cases the fraction $\frac{1}{2}$ indicates that the concentration is one-half the concentration indicated at the head of the column. 'The solutions represented in the same column contain equivalent weights of materials (403).

Apparently the agreement between the results of the two methods is within a few per cent for concentrations up to 
0.5 molar for salts consisting of two univalent ions in combination, and for concentrations up to 0.25 molar jor salts consisting of two univalent ions united to one bivalent ion. With increasing concentrations for both ypes the divergences become larger. Data for salts made up of higher valent ions show even greater disagrements in the two methods. However,

T.ABLE XXXT

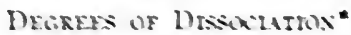

\begin{tabular}{|c|c|c|c|c|c|}
\hline Concatrations & o or & 0.5 & o is & 020 & $0 \leqslant 0$ \\
\hline $\begin{array}{l}\mathbf{K C I} \mathbf{I} \\
\mathbf{K C I} \mathrm{II} \ldots \ldots \ldots \ldots\end{array}$ & $04 \%$ & sor & $50 \%$ & $s \%$ & $\begin{array}{l}30 \\
-S\end{array}$ \\
\hline $\mathrm{XaCl}_{\mathrm{XaCI}} \mathrm{II}_{\ldots} \ldots \ldots \ldots$ & $\begin{array}{l}04 \\
04\end{array}$ & $\begin{array}{l}S o \\
\ldots\end{array}$ & $\begin{array}{ll}3 \\
\vdots 5\end{array}$ & $\begin{array}{ll}s: \\
\vdots:\end{array}$ & $\begin{array}{l}s: \\
\because:\end{array}$ \\
\hline $\begin{array}{l}\text { KBrI } \ldots \ldots \ldots \\
\text { KBr II . . . . }\end{array}$ & $\mathrm{OH}_{4}$ & io & $\begin{array}{l}50 \\
s 0\end{array}$ & $\begin{array}{l}S \\
s\end{array}$ & $\begin{array}{l}s i \\
\because ;\end{array}$ \\
\hline $\begin{array}{l}H C I \\
H C\end{array}$ & $0 ;$ & $0 ;$ & & & \\
\hline 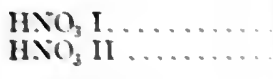 & on & $\begin{array}{l}0: \\
\text { at }\end{array}$ & & & \\
\hline $\begin{array}{l}\mathrm{RaCl}_{3}\left(\frac{1}{2}\right) \\
\mathrm{KaCl}_{2}\left(\frac{1}{2}\right)\end{array}$ & ss & $\begin{array}{l}s: \\
\vdots 0\end{array}$ & is & $\vdots$ & \\
\hline $\begin{array}{l}\mathrm{CaCl}_{2}(1) \text { I } \ldots . . . \\
\mathrm{CaCl}_{2}(1) \text { II } \ldots . .\end{array}$ & & St & $\begin{array}{l}5: \\
; 0\end{array}$ & $\therefore$ & \\
\hline $\begin{array}{l}\mathrm{Pb}\left(\mathrm{NO}_{3}\right)_{2}\left(\frac{1}{2}\right) \\
\mathrm{Ib}\left(\mathrm{NO}_{3}\right)_{2}\left(\frac{1}{2}\right)\end{array}$ & $s$ & $\because:$ & 3 & si & 43 \\
\hline $\begin{array}{llll}\mathrm{K}_{5} \mathrm{SO}_{4}\left(\frac{1}{1}\right) & \mathrm{I} & \ldots & \ldots \\
\mathrm{K}_{6} \mathrm{SO}_{4}(1) & 11 & \ldots & \ldots\end{array}$ & $\therefore$ & $\because$ & $\because:$ & $\because ;$ & $\begin{array}{ll}5: \\
12\end{array}$ \\
\hline
\end{tabular}

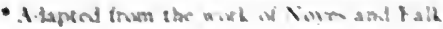

the complications which arise under both these circumstances make these discrepuncies appear to be in kerping with the iundamental theors:

723. Summary. - The material developed in this chapter may be summarized as follows: The existence of fremoving molecules in liquids as describer by the kinetic theory has been put beyond the posibility of doubt by the diswery that all very tine suspensions are in constant motion. Perrin has shown 
that these fine suspensions behave like atmospheres of huge molecules. The kinetic energy of a suspension particle is equal to that of a gas molecule at the same temperature. In fact, the gas laws may be applied to these suspensions. Even when the average mass of the molecules is varied 70,000 fold, the same results are found. Since the distinction between an extremely fine suspension and a solution is fundamentally one of the size of the particles, it seems plausible that a substance in dilute solution might also be found to behave as though in the gaseous state.

The pressure exerted by the molecules of a dissolved substance is determined by measuring an equal and opposite pressure, namely that of the inflow of solvent into the solution through a membrane permeable to the solvent but not to the dissolved substance. The pressures so measured are found to be very nearly equal to the pressure which a gas would exert if of the same molecular concentration and at the same temperature as the dissolved substance. Thus the analogy between substances in dilute solution and in the gaseous state is completely borne out. Van't Hoff, who was the first to develop the theory of dilute solutions, pointed out that Avogadro's Law should hold for substances in dilute solution. On the basis of this assumption the molecular weight of a substance in solution can be obtained by finding what weight of the latter dissolved in 22.4 liters will exert $760 \mathrm{~mm}$. osmotic pressure at $0^{\circ}$. Raoult, working with a great variety of substances, the molecular weights of which were known, showed that a gram molecular weight of each produced about the same change in the vapor pressure of the solvent. The corresponding changes in the boiling-point and freezing-point can be easily measured. The theoretical deductions of Van't Hoff showed that the experimental methods of Raoult were simply other methods for determining the relative weights of the molecules as they existed in the solutions.

On the basis of the work of these two men the existence of particles smaller in weight than the original molecules of electrolyte dissolved in water solutions was established. Furthermore the amounts of these smaller particles were found to be 
in agreement with the requirements of the ionic theory. As a consequence the discovery of these facts put the ionic theory on a firm foundation.

724. Osmosis in Nature.-Osmosis is one of the important processes operative in nature. This is illustrated by the effect of water on wilted plants. The latter have lost water in the air, but upon being put into water they "freshen" because the water pours through the cell walls and thus puts a pressure against the latter which stiffens them. This pressure could not accumulate if the cell walls were not only membranes, permeable to water, but also impermeable to much of the other contents of the cell. If a fresh flower is put into concentrated sugar solution, in a very few minutes it will be found to be badly wilted. This occurs because the concentration of water is less in the sugar solution than it is in the plant cells $\left(71_{3}\right)$. The effect of sugar in withdrawing the juice from fruit is another wellknown phenomenon of the same type. The foregoing are easily observed examples. However, many less easily observed but important phy'siological processes both of plants and animals depend on osmosis 


\section{CHAPTER XXVIII}

\section{DISPERSE SYSTEMS}

725. Introduction.-The surface layer of a massive solid or of a liquid, as it is contained in an ordinary vessel, is of negligible importance chemically compared to its total mass. But if the solid is made into dust or the liquid is transformed into fine droplets, the surface layer is enormously increased and new phenomena become prominent. Dusts, mists, and other finely divided materials may be characterized as disperse systems. They are to be the subject of our next study.

726. Cohesion and Adhesion.-The structure of gases and solids need not be discussed further here. But some points about the structure and behavior of liquids may well be taken up in preparation for the subsequent discussion. We are familiar with the sight of a liquid falling in drops from the tip of a pipette or burette. As soon as a given volume of liquid is free from outside influence, such as gravity, attraction from the walls of the containing vessel, etc., it tends to form a sphere. If the drop is very small, the sphere will be nearly perfect even when pulled down against a supporting surface by gravity. This is well illustrated in the case of small drops of mercury resting on paper. The attraction between molecules falls off very rapidly as the distance between them increases. As a consequence only the immediate neighbors of a given molecule in a liquid exert any appreciable influence upon it, and, except in the surface layer, this attraction is balanced, since it comes equally from all sides. But in the surface layer the molecules are pulled inward and there is no compensating pull outward from beyond the surface. The net result is that the surface layer acts as a compressing membrane upon the rest of the drop. Work must be done against this inward pressure if a liquid is to be subdivided. This fact may be easily observed if the finger is used to divide a large drop of mercury resting on a flat glass surface. 
The floating of a needle on the surface of water is another wellknown experiment, used to illustrate this resistance of a liquid to deformation. When two drops touch each other they. promptly merge into a single drop. This occurs because at the instant of contact the surface layer no longer exists where the drops touch. The tension on the remaining surface layer forces the drops together into a new sphere, since the latter has the smallest surface of any shape which a given volume of substance may take.

The attraction of like particles for each other is called cohesion, in contrast to adhesion, the attraction between unlike particles.

If a clean glass plate (free from grease) is allowed to touch the surface of water and is then gently raised, the water will be lifted with it several millimeters before the surface breaks and the glass is released. The water is said to adhere to the glass. As a matter of fact the water molecules hold tighter to the glass than to each other, for when the separation comes water parts from water and not from glass. The surface of water in a small, clean glass ressel is concave upward because of the spreading of the water upon the surface of the latter. If a glass capillary tube is placed in water the latter rises in the tube high above its level outside (Fig. I03A). The rising of the water in the tube against the force

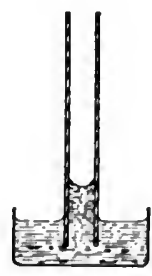

Fic. 103.1 of gravity occurs because of the attraction of the glass for the water molecules, and stops when this force is just balanced by the weight of water.

On the same principle, when blotting paper or filter paper is put into water the latter rises in the paper even against the force of gravity. Before blotting paper was introduced, ink was dried by dusting sand over it. 'The excess ink was taken up (adsorbed) on the surface of the sand grains and was removed with the latter. The adsorption of liquids by fine powders has become of great importance in industry. Nitroglycerine is adsorbed by a very fincly divided silica (silicon dioxide) called infusorial earth, an important substance which will be spoken 
of again (732). In this form, called dynamite (692), nitroglycerine is much more safely handled than as a pure liquid.

All liquids do not wet (adhere to) all solids. For example, mercury does not wet glass. When mercury is contained in a glass vessel the shape of its surface is convex upward

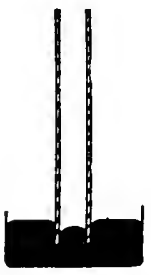
(Fig. I०3 $B$ ). This curvature is due to the usual pulling inward of the molecules of the surface layer of the liquid. If a glass capillary tube is thrust into mercury, the level of the latter inside the tube is lower than outside simply because the pull inward of the surface layer resists the deformation made FIG. 1о $_{3} B$ by the tube. Mercury is not adsorbed by blotting paper. Water, of course, behaves the same way toward surfaces which it does not wet. Apparently the force of adhesion depends on the nature of the substances in question.

727. Surface Areas.- The foregoing examples are sufficient to illustrate well-known surface phenomena. Table XXXVI, which gives the surface area of a cube with continuous subdivision, illustrates how greatly the surface of a given mass of substance may vary, and consequently how effects which are ordinarily slight may become of great importance.

TABLE XXXVI

\begin{tabular}{|c|c|c|}
\hline Length of Edge of Cube & $\begin{array}{c}\text { Number of } \\
\text { Cubes }\end{array}$ & Total Surface \\
\hline $\begin{array}{l}\text { I } \mathrm{cm} \ldots \ldots \ldots \ldots \\
\text { I } \mathrm{mm} \ldots \ldots \ldots \\
0.00 \mathrm{I} \mathrm{mm} \ldots \ldots \ldots \\
0.000,00 \mathrm{Imm} \cdot \ldots \ldots\end{array}$ & $\begin{array}{l}1 \\
1 O^{3} \\
1 O^{12} \\
1 O^{21}\end{array}$ & $\begin{array}{rl}6 & \mathrm{sq} . \mathrm{cm} \\
60 \mathrm{sq} . \mathrm{cm} \\
6 \text { sq. } \mathrm{m} . \\
6000 \mathrm{sq} . \mathrm{m} .\end{array}$ \\
\hline
\end{tabular}

If these areas are changed into the system to which we are accustomed, we find that if a cube one centimeter on one side is divided into little cubes, each $0.00 \mathrm{Im}$. on one side, the total surface is about 65 square feet. If, however, the original cube is subdivided into smaller cubes, each $0.000,00 \mathrm{Imm}$. on a side, the total surface is almost an acre and a half!

728. Adsorption of Gases by Charcoal.-If charcoal is freshly heated and thrust into ammonia gas confined over 
mercury, the mercury will be seen to rise in the tube as the charcoal cools and finally to remain high above its old level (Fig. I04), showing that the ammonia has been taken up by the charcoal.

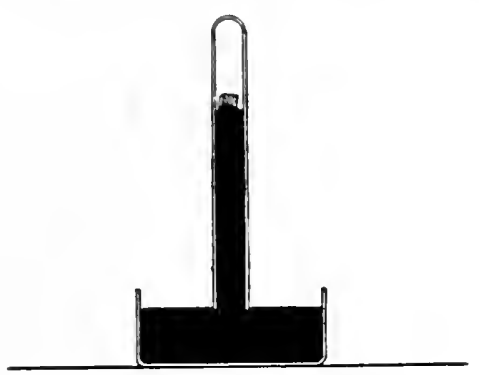

FIG. IO4 There remains in the latter the cellular structure of the substance from which it was made, so that it is composed of innumerable tiny pores and therefore presents an enormous surface. The fast-moving ammonia molecules encounter this and are held upon it. A few of the molecules are able to escape the carbon, apparently, for all are not taken up, and finally there is an equilibrium between the free ammonia and that adhering to the charcoal. The ammonia is said to be adsorbed by the charcoal. The higher the temperature, the more rapidly is equilibrium reached, but the smaller is the amount adsorbed. It was on this account that the charcoal was freshly heated before it was used in the foregoing experiment, in order that its surface should be free from other gases. Charcoal is a very good adsorbent for gases, but it does not adsorb all gases to an equal degree. Thus a given sample was found to adsorb $9 \circ$ times its own volume of ammonia, 35 times its volume of carbon dioxicle, but only I.7 times its volume of hydrogen. In the recent war, filters of specially prepared cocoanut-shell charcoal, mixed with other chemicals, were used in gas masks to remove poison gases from air inhaled through them.

729. Air and Glass.--So strongly is air adsorbed on the surface of glass that great difficulty is found in preparing barometer. tubes which shall have a perfect vacuum in the space over the mercury. It is not sufficient to fill a glass tube with mercury and then invert it with the open end under mercury. 'The tube is filled, put under vacuum, and heated for some time before the air is removed and the tube is ready to be put in place.

730. Water Vapor and Glass.-Accurate workers have long known that glass surfaces are covered with a thin water film 
which is with difficulty removed. Water molecules encounter the glass surface and are held there owing to the very strong attraction of water to glass, to which attention has already been called.

These and other experiences show that on the surface of every solid exposed to a gas we may expect to find an adsorbed layer of the latter. If the solids are finely divided, so that the surface is large, the result may become of great importance.

73I. Adsorption and Catalysis.-We have many times noticed the catalytic effect of finely divided metals on gas reactions. For example, the union of hydrogen and oxygen (303) is greatly accelerated by the presence of finely divided platinum. We find that platinum takes up both hydrogen and oxygen in considerable quantity. As early as 1844 Faraday pointed out that one result of the presence of a metallic catalyst is that on its surface the gases undergoing reaction are at far greater concentration than in the gas mixture itself. Such a layer would react far more rapidly than the main mixture. Undoubtedly adsorption is the first stage of the reaction on these contact agents. But some absorption, or penetration of the adsorbed gas beneath the surface on which it is at first held, follows, and in some cases compounds are formed.

732. Adsorption from Solution.-If a little of the dyestuff methyl violet, often used as the purple tint of indelible ink pencils, is added to water, a beautiful purple solution results which may be filtered unchanged. But if charcoal (from sugar, for instance) is shaken in this solution and the mixture is then poured on the filter, the filtrate is found to be colorless. The methyl violet has been adsorbed by the charcoal. That the dye has not been destroyed may be shown by pouring alcohol through the filter. The filtrate again shows the brilliant color of the dye. An enormous number of substances are adsorbed from water by charcoal. It is because of this property that it is an effective filter for purifying water. However, there are limits to the amount that a given column of charcoal can adsorb, so that it soon loses this power. Bone black, charcoal made from bones, is extensively used to take out objectionable impurities 
from sugar solution in the process of refining sugar. Fusel oil, a poisonous by-product present in crude whiskey, is also removed from the latter by filtration through charcoal.

Besides charcoal there are other good adsorbents. Fullers earth, a very fine clay (mainly aluminum silicate) which varies in composition, is an important industrial adsorbent for the purification of edible oils. The particles of this earth are usually between 0.007 of a millimeter and 0.0002 of a millimeter in diameter. Another important adsorbent is infusorial earth or kieselguhr, a deposit of the skeletons of diatoms, which are tiny aquatic organisms. Beds of this substance of as much as a thousand feet in thickness are found in the United States. Other adsorbents are finely divided metals, plant and animal fibers such as cotton, silk, wool, etc. As has been pointed out in the case of metals (73I), absorption may of course follow adsorption in the action of these substances. The fundamental requirement for a good adsorbent seems to be an enormous surface of contact with the solution.

733. Suspensions Produced by Grinding of Solids under Liquids. - If clay is stirred up in water a turbid mixture results. First the coarser particles settle to the bottom, and then gradually finer and finer particles follow as time goes on. Pulverized emery (174), used for grinding, is graded according to the number of minutes required for it to settle after being stirred in water. That which will settle in one-half minute is a coarse grade. Ten-minute emery is for very delicate work. Careful grinding of any substance will produce powders which are still slower in settling than any of these, but we have other ways of producing them.

734. Arsenious Sulfide Suspension.-Arsenious sulfide, $\mathrm{As}_{2} \mathrm{~S}_{3}$, is very insoluble in water. If this solid is shaken up in pure water only a minute trace will be found in a liter of the latter after filtration. When arsenious acid, $\mathrm{H}_{3} \mathrm{AsO}_{3}$, mixed with a little hydrochloric acid, is treated with hydrogen sulfide, a precipitate of arsenious sulfide appears. But if the hydrochloric acid is omitted, only a yellow, opalescent liquid results. When the latter is poured through filter paper, merely a trace of precipitate 
is held back and the liquid passes through unchanged. That the latter is not a supersaturated solution may be easily established by adding to it a little of the solid arsenious sulfide. No settling out of the arsenious sulfide follows, as would be the case if the solution were supersaturated (123).

In whatever form the arsenious sulfide exists in the yellow liquid, its presence in water seems to have only a slight effect upon the boiling-point and freezing-point of the latter. The osmotic pressure ( $7 \mathrm{I} I)$ of such a solution is very small. If we calculate from the latter the amount of arsenious sulfide necessary to give one gram molecular weight, we obtain enormous numbers; for instance, in one case six thousand grams was the result, a number which is more than twenty-four times that indicated by the formula $\mathrm{As}_{2} \mathrm{~S}_{3}$.

The examination of the liquid under the ordinary microscope shows nothing. But if it is examined even with a crude form of the ultra-microscope described in 706, it is found to be full of dancing particles which must be arsenious sulfide. All the evidence shows that the arsenious sulfide exists in the form of particles which are very large compared to the simple arsenious sulfide molecules, but small compared to particles which settle from solution.

735. Colloids.- Such a non-settling suspension is referred to as a colloidal solution, or as a colloidal suspension. Usually matter is said to be in a colloidal state if it is too finely divided to be held back by a good filter paper and still coarse enough to be seen in the ultra-microscope. This means that the average diameter of the particles is between $0.000, \mathrm{I}$ and $0.000,00 \mathrm{Imm}$. The setting aside of these systems as a special class is obviously entirely a matter of convenience. Colloidal suspensions of substances which are solids when in massive form are often called suspensoids, while colloidal suspensions of liquids are called emulsoids. The name colloid, which means glue-like, was originated by Thomas Graham, the first important investigator of this subject. He worked mainly with gum arabic, starch, glue, and glue-like substances which belong to a more complex type than we have yet studied. 
736. Properties of Colloidal Arsenious Sulfide, Diffusion.The most striking thing about the colloidal arsenious sulfide suspension is its stability. If it is kept in good-grade glass bottles, months may pass without its settling.

If two test tubes are half filled with 5 per cent hot gelatin solution which is allowed to cool and set to a gel, and to one tube is added a layer of copper sulfate solution, while to the other the yellow arsenious sulfide suspension is added, after about twentyfour hours the blue solution will have penetrated well into the gel layer, but the yellow solution will not have entered the layer below it. The effect can best be seen by corking the tubes and then inverting them. The gel in the copper sulfate tube will be found to be partly colored, while the other gelatin layer will be found to be unchanged. Apparently the rate of diffusion of the arsenious sulfide particles is very slow. This is explained by the work of Perrin (707). Each arsenious sulfide particle is very much larger than a copper sulfate molecule or a copper ion, and since it is at the same temperature its velocity must be much smaller.

737. Effect of an Electrical Current on Colloidal Arsenious Sulfide.-Colloidal arsenious sulfide solution is a very poor conductor of electricity, but if we fill a U-tube with this material and pass a i Io-volt current through it, in from ten to twenty minutes a colorless layer will be plainly visible at the negative electrode, and the region near the positive electrode will be deepened in color.

The effect is better shown by using the device described by Professor A. A. Noyes and shown in Fig. 105. The ends of the inner tube are covered with thin parchment paper, or better with goldbeater's skin. Over the ends are fitted extension tubes, which are joined to the $U$-tube by rubber. The device is

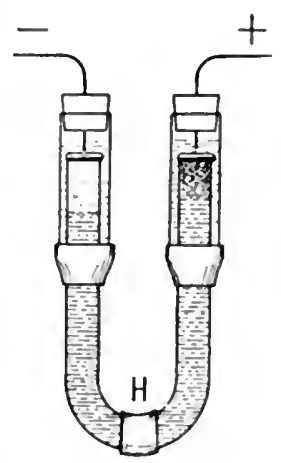

FIG. 105 inverted for filling through the hole, which is finally closed by sliding a rubber tube, $H$, over it. The spaces around the electrodes are filled with ordinary distilled water. The shaded 
area of the figure shows the region of yellow color after the current has been passing for some time.

Since the suspension drifts away from the negative and toward the positive electrode, its particles are negatively charged. We have already noticed that when unlike substances are in contact, a loss of electrons by one and a gain of electrons by the other are very likely to occur (474). It would not be surprising if this were the cause of the existence of this difference of potential between the solid and the liquid. But we shall soon see that other factors may also account for this phenomenon.

738. Effect of Electrolytes upon Colloidal Arsenious Sulfide.-We have already seen that in the presence of hydrochloric acid the colloid does not form, and we find that if a little hydrochloric acid is added to the suspension the liquid becomes very much more turbid, and with further addition of the acid a precipitate soon appears and settles to the bottom of the container. The curious thing is that any other good electrolyte will accomplish the same thing.

The positive ion seems to be the active part of the electrolyte; for about the same concentrations of salts which have univalent positive ions are necessary to cause complete coagulation of the precipitate from a given volume of solution. Smaller concentrations of salts which have bivalent positive ions are needed, and very much smaller concentrations of salts which have trivalent positive ions. Relatively little difference is made by varying the negative ion of the salt.

739. Adsorption of the Precipitating Agent.-Careful experiments have shown that the active ion is carried down by the precipitate, leaving in solution an equivalent amount of the corresponding ion of water; thus arsenious sulfide coagulated with calcium chloride contained calcium, and the liquid left behind contained an equivalent concentration of hydrogen chloride.

Apparently the positive ion neutralizes the charge on the suspension and is carried down by the precipitate. Since the proportion of ion carried down by the precipitate bears no simple and constant relation to the weight of the precipitate, as would 
be the case if a chemical compound of the type we know had been formed, it is apparent that the product is of a different class. For the sake of convenience we may call the former an adsorption compound. If the adsorption compound is washed with pure water, the calcium is not removed, but it can be taken away by washing the substance with some other electrolyte, ammonium chloride, for example. In this case ammonium takes the place of calcium with the arsenious sulfide, and the calcium is found as calcium chloride in solution.

The proportion of the adsorbed ion to the precipitate is usually very small, though of course different with different preparations according to the valence of the ion, the charge on the suspensions, and other conditions. For example, in the case of certain colloidal arsenious sulfide suspensions coagulated with calcium chloride the proportion was found to be one equivalent of calcium to fifty equivalents of the sulfide. If very pure substances are desired, as is the case in exact analysis, even this small proportion may be of moment, especially if the adsorbed ion has a high molecular weight.

Experience shows that the adsorption of ions from solution by a precipitate in the processes of its formation is the rule and always must be taken into consideration in the preparation of pure substances.

740. Influence of the Charge on the Stability of a Suspensoid.- If in the experiment on the migration of the suspensoid (737) the particles of the latter had been allowed to reach the positive electrode, they would have been precipitated. Apparently the existence of the charge on the suspension is necessary for its stability. We can understand that the presence of like charges on the arsenious sulfide would have a tendency to keep them from coming together, but undoubtedly the effect of the charge is more complex than this.

74r. Preparation of Colloidal Ferric Hydroxide.-Another method of preparing colloidal solutions is illustrated in the following preparation of colloidal ferric hydroxide. Ferric chloride solution, which is of course acid in reaction (436), is treated with ammonium carbonate as long as the precipitate which first 
forms redissolves. The dark-red solution thus made contains mainly ferric chloride, colloidal ferric hydroxide, and ammonium chloride. The ferric hydroxide and ammonium chloride are

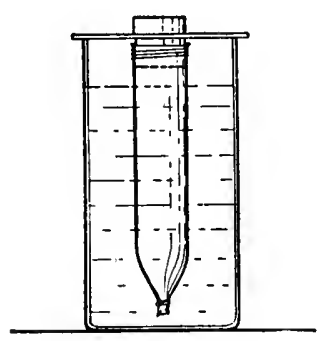

FIG. 106 produced as the ammonium carbonate reacts with the hydrochloric acid formed by the hydrolysis of ferric chloride:

$$
\mathrm{FeCl}_{3}+{ }_{3} \mathrm{H}_{2} \mathrm{O} \leftrightarrows \mathrm{Fe}(\mathrm{OH})_{3}+{ }_{3} \mathrm{HCl}
$$

This mixture is next placed in a parchmentpaper bag (Fig. 106), and the whole is suspended in a water bath through which a current of fresh water is constantly flowing. If this arrangement is left for about four days the contents of the bag will be found to give only a very small test for chloride, showing that virtually all of the ferric chloride has been transformed into ferric hydroxide. The preparation is in fact colloidal ferric hydroxide.

742. Properties of Colloidal Ferric Hydroxide.-When the liquid is poured through filter paper no precipitate is left on the latter. The freezing-point, boiling-point, and osmotic-pressure determinations all show that the substance is present in the form of particles which are large in comparison with simple molecules. The dark-red liquid is stable if carefully kept. If it is placed in the apparatus, as shown in Fig. 104, and an electric current is applied, the red substance is found to migrate toward the negative electrode, proving that this suspension is positive to its solution.

Electrolytes precipitate the red hydroxide, leaving the liquid colorless. This time it is the negative ion of the electrolyte which is active and goes to form the adsorption compound. Again the valence of the precipitating ion is found to be an important factor. In a given instance about one-fortieth as great a concentration of sulfate as chloride ion was needed to coagulate a given amount of ferric hydroxide.

When the latter suspension is added a little at a time to arsenious sulfide suspensions, the mixture becomes turbid, and finally a precipitate of the two substances settles out. This is 
the result of mutual adsorption and precipitation. Curiously enough the addition of a large excess of cither colloid does not give a precipitate, but gives a complex colloid.

743. Explanation of the Preparation of Colloidal Ferric Hydroxide.-If a layer of the colloidal ferric hydroxide is put over a layer of gel, as was described in the case of arsenious sulfide (736), the ferric hydroxide will be found to diffuse very slowly indeed. This fact was made use of in the original preparation of the colloidal. 'The ferric hydroxide made by the action of ammonium carbonate did not diffuse through the parchment paper with appreciable speed, and at the same time it adsorbed the ferric chloride so that the latter remained in the bag. The hydrochloric acid and the ammonium chloride, however, passed readily through the paper and were washed away on the other side. The continuous loss of hydrochloric acid allowed the hydrolysis of the ferric chloride to go to completion. The separation of colloids from dissolved substances by a process of diffusion through a membrane is called dialysis and the apparatus is called a dialyzer.

744. Colloidal Silver.-If an electric arc is passed between two silver wires submerged in water, a dark cloud will form around the electrodes. 'This is, in fact, a colloidal suspension of silver. A little alkali added to the liquid will increase the stability of the suspension. The dark liquid has the same general properties as those of the other suspensions we have described. It is electro-negative to water. 'This method of producing a colloidal suspension of a metal by volatilizing the latter in the electric arc is named Bredig's method after its liscoverer, the German chemist Bredig.

745. Protecting Agents.-The silver suspension can be made of considerable concentration if about I per cent of gelatin is added to the solution. 'The effect of the gelatin may be shown by mixing silver nitrate $(\mathrm{N} / 10)$ and hydrochloric acid $(\mathrm{N} / \mathrm{ro})$ solutions, to each of which about I per cent of gelatin has been freshly added. Instead of the copious white precipitate which we have so often seen result from mixing these reagents, only a slight white turbity appears. The gelatin has prevented large 
particles from forming. Many other agents besides gelatin stabilize suspensions. Their function is not understood, but it is thought that they surround the particles, thus preventing further union between them. Hence they are called protecting agents.

746. Red Gold Suspensions.-A very useful reagent in making suspensions of metals is tannin, a complex organic substance which is both a good reducing agent and a protecting agent. A very finely divided gold suspension may be made with a neutralized (about I per cent) gold chloride solution and dilute (o.I per cent) solution of tannin, according to a method described by Dr. Wolfgang Ostwald. First a few drops of the gold solution are mixed with roo c.c. of water, then a few drops of the tannin solution are added, and the mixture is heated for a few minutes, with constant shaking. Meantime the red color of the gold suspensoid appears. More gold chloride and tannin may be added alternately a little at a time.

The particles of the red gold suspension are very tiny, usually about one or two one-hundred-thousandths of a millimeter in diameter. These were the particles described by Zsigmondy (706). They are negative to the water in which they are suspended and are precipitated by positively charged ions. Coarser suspensions of gold may be violet or blue in color. Brown gold suspensions settle in a very short time.

747. Summary of Work with Suspensoids.-The general methods of preparing suspensoids are illustrated in the foregoing. Either the material is subdivided by grinding or volatilization in the electric arc, or it is formed in solution and the particles are not permitted to grow to a size large enough to settle from the liquid. The absence of electrolytes and the presence of protecting agents assist in making concentrated suspensions. Colloidal suspensions of an enormous number of different substances have been prepared. Apparently any substance can exist in the colloidal state in liquids in which it is not soluble. Thus colloidal sodium suspension has been prepared in ether, phosphorus in water, sodium chloride in benzene, etc.

Apparently many inorganic substances stabilize colloidal suspensions to a moderate degree, for we often see opalescent 
liquids form when precipitation in dilute solutions is in progress. As the last of the precipitating agent is added, the solution becomes clear and the precipitate settles. Thus if 5 c.c. of $N / 20$ silver nitrate is added to 20 c.c. of $\mathrm{N} / \mathrm{I} O$ potassium chloride solution, a part of the silver chloride appears as a non-settling suspension. If more and more silver nitrate is added, with constant shaking of the mixture, the liquid finally becomes transparent and the precipitate settles just as the amount of silver nitrate added becomes nearly equivalent to the potassium chloride. The excess potassium chloride present at first stabilizes the silver chloride suspension.

The origin of the charges on these suspensions is not understood. As has been said, it would not be surprising to find a frictional charge on them, but we must also consider that an unequal adsorption of positive and negative ions from solution might also account for the existence of these charges. In addition, the loss of ions from the suspended particles to the solution might occur. The fact that all suspensions of bases are positive to the liquid in which they exist would seem to favor the idea of the ionization of the solid particles in these cases. Thus the hydroxide particle might lose one or more hydroxyl ions to the solution, leaving a residue which would be a very large positive ion. But in explaining the presence of these charges we must also consider that most other suspensions in water are negative. Thus, finally, suspended clay, lamp black, metals, sulfur, salts, etc., are negative to their solutions as a rule, though not always. Very probably all three possible causes cited for the existence of the charges come into play at different times.

748. Tyndall Effect. - When a pencil of light from a lantern is brought to bear on water, we see only a faint glow over its path through the latter; but as soon as a colloidal suspension is added, the path of the light ray appears as a cone of bright cloud. This effect is called the Tyndall effect in honor of J. Tyndall, who was the first to make extensive use of this phenomenon. 'The 'Tyundall effect is, of course, best observed in a darkened room. It depends on the difference in refracting power for light of the particles of the suspension and of the pure 
liquid. In some cases this difference is not great, so that no cone appears. Hence the absence of the Tyndall cone does not always mean that the liquid in question contains no colloidal matter. The ultra-microscope is of course simply a refined apparatus for examining the Tyndall effect in detail. It is interesting to note that concentrated solutions of sugar, sodium acetate, and many other salts show the Tyndall effect.

749. Test for the Charge on a Suspension.-A crude distinction may be made between positive and negative colloids as follows: Strips of filter paper are suspended so that one end of each strip is wet by a colloid. If a strip dips into an arsenious sulfide or gold solution, the liquid which rises in the paper will show the color of the colloid. The colloid is spreading through the paper, though usually not so rapidly as the water. But if the strip dips into a ferric hydroxide solution, the ferric hydroxide will be found to diffuse but a little way into the paper, although water from the suspension rises through the latter as easily as in the other cases. The test is reliable only under average conditions, which are those of dilute negative suspensions and relatively more concentrated positive suspensions. If turbid suspensions are to be tested, they should be filtered before the trial is made, otherwise coarse particles will impede the rise of the colloid through the paper.

750. Important Suspensoids.- - Suspensoids have become of considerable practical importance. Colloidal silver is an important antiseptic. It is prepared with a protecting agent so that it may be sold as a dry powder (argyrol), which forms a dark-brown colloid upon the addition of water. Colloidal copper, mercury, and sulfur have also come in to use in medicine. India ink is mainly colloidal carbon. The important lubricants aquadag and oildag are colloidal graphite in water and oil, respectively, each protected by tannin. The lubricating power of graphite is much improved when it is in the colloidal state.

We shall next take up emulsoids. These, as we shall see, are of tremendous importance in biochemistry.

75I. Oil and Water.-If oil, benzene for example, is shaken up in water, for a few seconds there is a general mixture of oil 
and water. Then two cloudy layers separate. The lower is an emulsion of benzene in water, and the upper an enulsion of water in benzene. Very soon, however, the two layers become transparent. Momentarily the oil and water are in the colloidal state, but this is not permanent. If a drop of water touches another drop of water, the two promptly coalesce $(\mathbf{7 2 6})$. The oil has very little attraction for the water, and its drops continue uniting just as the water drops do until two layers are made from all the small drops. If, however, soap $\left(6_{7} 8\right)$ is added and the oil and water layers are shaken together, a stable suspension of oil in water will form. The suspension may be termed an emulsoid if the droplets are small enough (735).

752. Cleansing Action of Soap.-Apparently the emulsifying power of soap is an important factor in its cleansing or detergent action. Thus a little fine dirt shaken with water settles out, but shaken with soap and water it remains in suspension. Dirt usually sticks to the soap solution more than it does to the fabric being washed, and hence rinsing carries off the dirt. Undoubtedly some hydrolysis of soap in water does occur, since soaps are all salts of the very weak fatty acids (678), but the alkali formed is not an important factor in the detergent action of soap, since alkali alone has no such power to emulsify mineral oils, etc. Vegetable oils can be emulsified to some extent with dilute alkalies. But it must be remembered that these are esters $\left(6_{77}\right)$ and that some soap is formed by the interaction of esters with the alkali. If alkali, washing soda, or ammonia is added to soap solution, its detergent power is increased. Of course the hydrolysis of soap is decreased at the same time.

753. Nature of Soap Solution.-Ultra-microscopic examination of soap solution is unsatisfactory, presumably because there is very little difference in the power of soap and water to refract light. The fact that soap has little effect on the boiling-point of water seems to indicate a very high molecular weight. All evidence points to the conclusion that the soap molecules in solution are groups of large numbers of simple molecules.

754. Work of Harkins and of Langmuir. - It has long been known that a soap solution is more concentrated in the surface 
layer than in the inner region. Professor W. D. Harkins and co-workers have found that this is true of solutions of sodium oleate $(678,68 \mathrm{I})$ as dilute as 0.002 normal. The surface layer of a 0.002 normal solution is saturated. Stronger solutions up to 0.1 normal have the same concentration in the surface layer as have these very dilute solutions. In each case there is equilibrium between molecules of soap in the surface layer and in the body of the solution. Evidently the surface layer allows the escape of relatively few molecules to the solution, or the inequality of concentration could not be kept up. Further, Professor Harkins and Dr. Langmuir, of the General Electric Company, working independently and by different methods, have shown that the soap molecules of the surface layer are not simply jumbled together but are arranged in a definite order, The long sodium oleate molecules

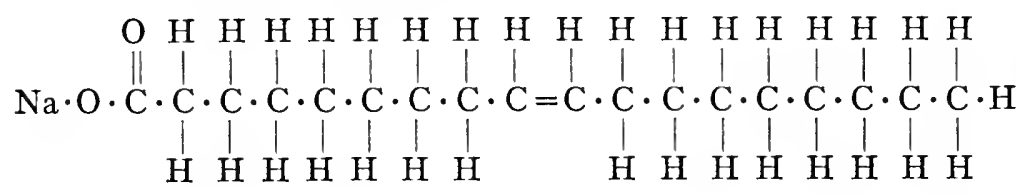

O

are all placed with the $(\mathrm{Na} \cdot \mathrm{O} \cdot \mathrm{C} \cdot)$ group toward the body of the solution and the long hydrocarbon chain (664) outward.

It is an old and well-known rule that "like dissolves like." Thus oils are good solvents for fats and greases but poor solvents for sugar, while water is a good solvent for the latter but a poor

solvent for the former. Apparently water attracts the $\mathrm{Na} \cdot \mathrm{O} \cdot \mathrm{C}$ group of the soap molecule strongly but has no attraction for the long hydrocarbon chain. As a result of this attraction on one end of the long molecule, this end is pulled in toward the liquid, and the hydrocarbon chain is left on the surface. The surface of the soap solution is therefore that of an oil, and it is on this account that soap solutions can wet oils while water cannot.

755. Other Emulsifying Agents.-Other emulsifying agents are in common use, but their function is not so well understood. 
Thus in mayonnaise dressing the yolk of an egg is used to keep the oil and vinegar together. Many substances are capable of forming emulsoids without the assistance of special agents. Examples of these are gelatin, gum arabic, egg white, etc. These are all substances which are themselves wet by water.

756. General Properties of Emulsoids.-Emulsoids are sharply differentiated from suspensoids by their relatively high viscosity. The tiny particles of both show Brownian movements (705). Unlike suspensoids, many emulsoids seem to be without electrical charges. Salts in small amounts have little effect on these systems. In large amounts they may cause precipitation. Thus soaps are precipitated ("salted out") from solution upon the addition of considerable quantities of sodium chloride. Although we have discussed emulsions in water only, it is obvious that they may exist in other media.

757. Important Emulsoids.-Virtually all fluids of plant and animal bodies are emulsoids. Thus the sap of plants and the blood and milk of animals are complex emulsoids.

758. Gels. - If the solvent is evaporated from a suspensoid, the latter is left as a powder. But if the solvent is evaporated from an emulsoid, or if a concentrated emulsoid is cooled, a gel usually forms, though not always. Soap melted in hot water sets as a gel on cooling. Solutions of gelatin of greater concentration than 0.25 per cent will set at temperatures above $0^{\circ}$. These gels are stiffer the higher the concentration of the original emulsion.

Unfortunately they refract light little differently from water, so that an examination of their structure under the ultra-microscope is not satisfactory. Gels have been precipitated by the addition of alcohol, etc., and then examined. 'Their structure then seems to be that of a delicate latticework or honeycomb, but it is more likely that this form is acquired in the process of precipitation.

We have already seen that substances in solution diffuse through gels very readily. In most cases they do so as easily as through water, but colloidal substances, either emulsoid or suspensoid, diffuse through gels only very slowly. Some gels 
are elastic and others are not. If the former are stretched they become warm, and cool again on contraction. Another very striking property of gels is their ability to absorb (73I) water. If a warm, concentrated gelatin solution is poured out on a glass plate and allowed to set, it may be cut into pieces of equal volume. The latter should then be dried. If a piece is allowed to soak in water, it will be found to swell. If at the same time other pieces of the same weight are allowed to soak about twenty-four hours in dilute acid or dilute alkali of equivalent concentration, the rate of swelling of the gel will be found to be most in the acid solution, next in the alkali, and least in the pure water. Salts added to pure water may increase or decrease the rate of swelling. Still another important property of gels is their separation into two layers, one liquid and the other gel. If evaporation is prevented, all gels act in this way in the course of time.

759. Plant and Animal Tissue.-Virtually all of the firmer parts of animals and plants are gels of great complexity, but the properties given above for gelatin belong to most of these substances. For example, the experiment with the gelatin squares may be repeated with animal tissue (frogs' legs, for example) with the same type of result. The development of a watery fluid by a gel is repeated in the formation of many animal and plant secretions.

760. Rubber.--If rubber, which is a typical colloid, is allowed to soak in benzene or carbon disulfide, it swells enormously, at

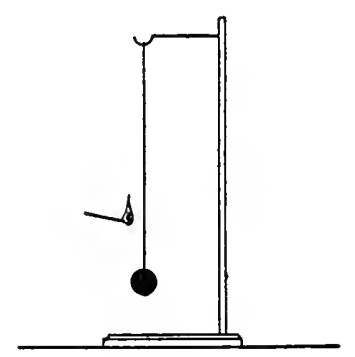

FIG. IO7 the same time taking up the liquid. Upon being stretched rubber becomes warm, and cools when contracting, just as do other gels. An interesting application of the general law governing all changes, applications of which we have observed so many times $(\mathbf{3 6 7})$, may be shown in the following experiment: A stout rubber band is attached by one end to a support and is stretched by a weight, as in Fig. I07. If a lighted match is held near the rubber, so that it is heated quickly but not melted, the weight 
will be seen to rise. The application of heat has favored the change which absorbs heat, namely, contraction.

76r. Inorganic Gels.-Many inorganic substances form gels casily; for example, silicic acid, ferric hydroxide, and aluminium hydroxide. These are all extremely insoluble substances. Von Weimarn has shown that in general, if a very high degree of supcrsaturation is attained preliminary to precipitation, the precipitate will be a gel. Thus barium sulfate is usually secn to precipitate as a powder. But if saturated solutions of the very soluble salts sodium sulfate and barium sulfocyanate are mixed, a gel of barium sulfate forms. Concentrated solutions of sodium carbonate and calcium chloride give a gel of calcium carbonate instead of the usual powder.

762. Relative Stability of Precipitates.-Apparently the crystalline form is more stable than either the gel or the powder precipitate. On long standing in contact with the solution most non-crystalline precipitates become crystalline. The rate of change is accelerated by a rise of temperature which increases the rate of molecular agitation and usually also increases the solubility of the precipitate. Even such substances as gelatin can be prepared in crystalline form. A substance can separate from solution in the crystalline form only if there is time and opportunity for the orderly arrangement of molecules in crystals. Hence only the more soluble substances usually appear in this form.

763. Explanation of Adsorption from Solution.-The fact that soap is more concentrated in the surface layer than in the solution is a phenomenon of a type which is very common among substances of complex molecular structure. Most emulsoids and suspensoids show this to a marked degree, though substances in solution do also to some extent. Under ordinary conditions this is not noticeable. But when the surface of the solution in question is enormously increased by being mixed with a porous or finely divided solicl like charcoal or infusorial earth, the net result is that a great amount of the colloid goes to the surface layer and adheres to the solid with the latter, while the rest of the liquid is drained away. The very large extent of the surface 
means a very great loss of material from the original liquid. The latter may be virtually freed from the colloidal matter if passed through a thick layer of filter.

764. Importance of Colloid Chemistry.--The many complex systems discussed in this chapter have one thing in commonan enormous surface of contact between different simpler systems. The porous charcoal and gas, arsenious sulfide and water, oil, soap and water, etc., may all be called disperse systems. They are also called colloids and their study colloid chemistry. Even this brief treatment should be sufficient to show the reader the enormous importance of the subject. As a matter of fact the majority of practical applications of chemistry involve disperse systems. Students will find the five lectures written on this subject for the general pubic by Wolfgang Ostwald ${ }^{\mathrm{r}}$ well worth reading.

IOstwald and Fisher, Theoretical and Applied Chemistry. Published by John Wiley \& Sons, New York. 


\section{CHAPTER XXIX}

\section{THE ATMOSPHERE AND RELATED TOPICS}

765. The Composition of the Air. - We have already learned that the most important components of air are nitrogen, oxygen, water vapor, and carbon dioxide. In addition to these four, there is but one other component the proportion of which exceeds 0.01 per cent. This is the element argon (5I3), discovered in the air in 1894 . The percentage by volume of the five named components is given in Table XXXVII.

\section{TABLE XXXVII}

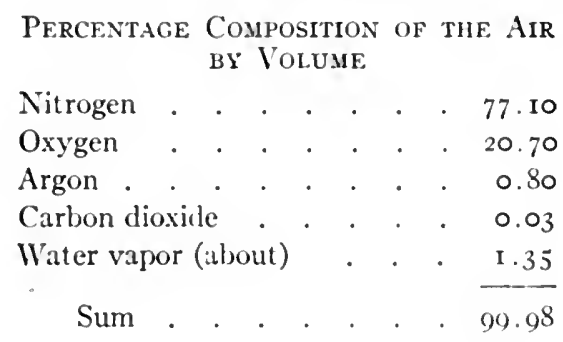

It is self-evident that the air must also contain minute amounts of several other gases and vapors, since these are being poured into the air from numerous industrial sources and are also being formed by natural chemical changes. Among the minor components of the air there may be mentioned hydrogen, methane, sulfur dioxide, hydrogen sulfide, ammonia, and nitrogen tetroxide. These chemically active gases are continuously being removed from the air by various means, so that they never accumulate in appreciable proportions. Other minor gaseous components of the air will be considered later (79I-799).

\section{Why the Composition of the Air Remains Constant.-} It is plain that oxygen is removed from the air on every hand by the burning of substances and by the respiration of all animals. If oxygen were not being constantly renewed the percentage of 
this element would in time steadily decrease. But as we have learned (69I), all growing plants take in carbon dioxide and water, from which they form, in addition to other products, cellulose, or starch, and oxygen, thus:

$$
6 \mathrm{CO}_{2}+{ }_{5} \mathrm{H}_{2} \mathrm{O} \rightarrow \mathrm{C}_{6} \mathrm{H}_{10} \mathrm{O}_{5}+6 \mathrm{O}_{2}
$$

The oxygen supplied to the air in this way serves to keep the percentage of this element fairly constant.

Closely connected with the oxygen balance in the air is that of carbon dioxide. The removal of this gas by growing plants is compensated by its formation in the oxidation of wood, coal, fuel gas, and other carbon compounds $\left(35^{6-365}\right)$. One other agency has been of much importance in past geological ages in diminishing the carbon dioxide content of the air. This is the formation of the shells of aquatic animals, consisting largely of calcium carbonate. Immense deposits of limestone (I50) have been built up from the shells of marine animals.

The water-vapor content of the air varies greatly from place to place and from time to time in a given place. Over the ocean the lower layers of the air are nearly saturated with water vapor, so that the vapor pressure tends to approach, at each prevailing temperature, the value shown in Table VII (II2). In desert regions the relative degree of saturation (the humidity) is very small.

The percentages of nitrogen, oxygen, and argon in air freed from water vapor and carbon dioxide are practically constant at all times the world over. The carbon dioxide content rarely falls below 0.03 per cent but may reach or exceed 0.04 per cent in congested parts of cities. Indoors it may at times run much higher.

767. Dew and Frost.-After sundown the temperature of the air falls, on clear nights in particular, because of the more rapid radiation of heat in the absence of clouds. If the humidity has been rather high by day the air may become supersaturated with water vapor at night. For every temperature the vapor of liquid water exerts a definite pressure, its saturation vapor pressure (I 2 , Table VII). If air containing a fixed proportion 
of vapor falls in temperature below the point at which it becomes saturated, the excess water separates out on all exposed objects as dew. 'The dew point is defined as the temperature to which a given sample of moist air must be cooled just to reach a condition of saturation. If cooled further it forms dew.

When the dew point lies below zero, if the temperature falls sufficiently low, the water vapor deposits in the form of frost (ice).

768. Dust in the Air and Cloud Formation.-The presence of dust in the air is, in general, considered nothing less than a nuisance; but, as we shall point out shortly, it has a very important function in connection with the formation of clouds and consequently of rain. For this reason especially we shall consider the dust content of the air.
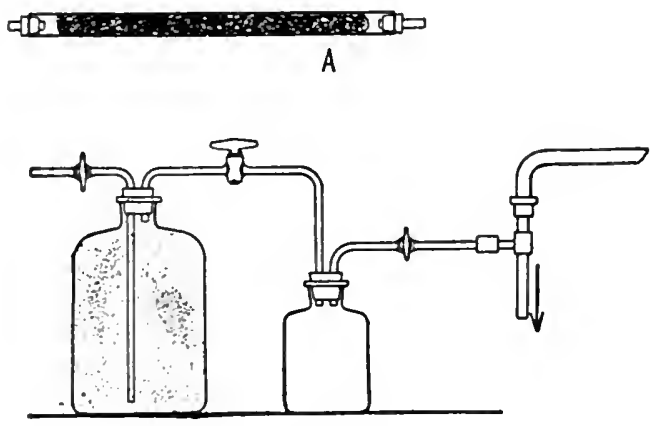

FIG. IOS

A cloud, or a fog which is a cloud at the earth's surface, is made up of countless drops of water, each so minute that it does not fall with appreciable speed (compare Millikan's experiment, 467). A cloud may form when a mass of moist air is cooled below its dew point. But this is true only under certain conditions, as may be demonstrated by a lecture-table experiment. If air not specially freed from dust, and saturated with water vapor, is suddenly cooled several degrees, a cloud or fog forms at once; but if the air is entircly free from dust no cloud is formed. To show these phenomena experimentally we may fill a large bottle (Fig. 108), with air that has been bubbled 
slowly through water in order to insure its saturation with vapor. The bottle should be closed with a stopper through which passes a glass tube attached by a rubber tube to a second bottle half the size of the first. The second bottle should have a twohole stopper and a second tube leading to a vacuum pump. The rubber tube connecting the two bottles is now closed by means of a clamp and the smaller bottle evacuated. If now the clamp is released, the air in the large bottle expands rapidly, partly passing over into the smaller bottle, and at the same time a dense cloud develops in the larger bottle. The expanding air does work in the process, and the energy to do the work is taken from the air as heat; in consequence the temperature falls so much that the air becomes supersaturated at the lower temperature, and the excess moisture separates as a cloud. If we repeat this experiment with the single change that the air used in filling the large bottle is freed from dust before it is bubbled through water, by causing it to filter through a cotton plug ( $A$, Fig. IOS), no cloud forms upon expansion. If now a small amount of dust (for example a little cigarette smoke) is admitted to the partially evacuated large bottle a cloud is formed. (The smoke must be introduced quickly, before the temperature has time to rise.)

769. Counting Dust Particles.-Anyone who has noticed the particles of dust floating in air, which may be seen when a bright beam of light enters a darkened room, would be inclined to say that counting the stars or the grains of sand by the seaside would be a simple task in comparison with the enumeration of the dust particles in a given volume of air. But thanks to the facts related in the preceding section the undertaking proved rather easy. Without dust, cloud formation does not take place. In the presence of dust each dust particle acts as a nucleus for the formation of a water drop. Therefore there are as many water drops in the cloud as there were dust particles present! A practical instrument has been devised by Aitken to count, by the aid of a microscope, the drops formed in a known volume of air and thus to ascertain the dust content of the air. The number of dust particles per c.c. of air varies greatly with circumstances, as would be expected. The smallest values, a 
few hundred particles per c.c., are found in air above mid-ocean. Clear mountain air contains a few thousand particles per c.c., while the air of large cities often contains over 100,000 per c.c. In his interesting article in the Encyclopaedia Brilannica on "Dust," Aitken writes:

Without atmospheric dust not only would we not have the glorious cloud scenery we at present enjoy, but we should have no haze in the atmosphere, none of the atmospheric effects that delight the artist. The white haze, the blue haze, the tender sunset glows of red, orange and yellow, would all be absent, and the moment the sun dipped below the horizon the earth would be in darkness; no twilight, no after glows; none of the poetry of eventide. Why it may be asked is this so? Simply because all these are due to matter suspended in the air, to dust.

770. The Ionization of Gases. - The topic about to be discussed is related to cloud formation in an important way, and for this reason we shall have to digress somewhat before going on with our subject. It is well known that air under ordinary conditions is an almost perfect electrical insulator. However, it may be made appreciably conducting in several ways. The phenomena may be readily shown by the use of a gold- or aluminum-leaf electroscope, Fig. Io9. If the electroscope has good insulation (sulfur or amber, A, Fig. rog) it will retain its charge for a long time. If a flame of any kind is brought near the electrode, $B$, the gold leaf, $C$, drops almost as rapidly as if

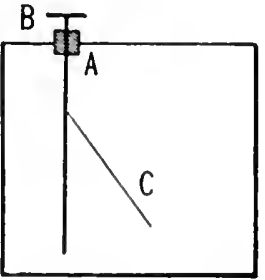

FIG. 109 one had touched $B$ with the finger. The gases in and about a flame conduct electricity thousands of times better than ordinary air. Elaborate investigations, which cannot profitably be discussed here, have proved that part of the gas molecules of a flame are electrically charged, half positively, half negatively. Consequently the gas is said to be ionized; and just as in the case of an ionized solution the gas conducts electricity.

Every gas can be ionized by a variety of means, among which are intense heat, $\mathrm{X}$-ray's $(476)$, and radium rays $(480)$. Even the most energetic means do not convert into ions more than a very small fraction of the total number of gas molecules. 
771. Gaseous Ions and Cloud Formation.-Gaseous ions behave like dust particles in being able to serve as nuclei for water drops in cloud formation. If dust-free air saturated with water vapor, is ionized by X-rays, by radium, or in any other way and is then suddenly cooled (as by expansion in the manner described in 768) a cloud will form, each ion acting as a nucleus for a single drop of water.

772. The Formation of Rain.-There are always present in the air a small number per c.c. of gaseous ions formed in part at least by radioactive matter in the air. These ions, together with a much larger number of dust particles, serve as the nuclei of the drops forming ordinary clouds. Cloud formation occurs when a current of warm, moist air meets a current of cold air. If the water drops are large enough they tend to coalesce and thus grow so large that they fall to earth as rain. Rain is frequently accompanied by lightning, the cause of which we may now consider.

773. The Cause of Lightning.-If air is only slightly supersaturated with water vapor the negative ions present are much more effective in the condensation of moisture than are the positive ions, so that it frequently happens that only the negative ions are at first removed from the upper layers of air and carried to the earth with the falling rain. This results in the accumulation of opposite electrical charges in the air and on the earth beneath. The lightning that frequently accompanies rain is the electric discharge between air and earth, tending to neutralize the unlike charges.

774. The Atmosphere, a Disperse System.-The behavior of tiny water and dust particles in the air suggests the behavior of small particles in liquids. We have already noted (703) the Brownian movement of smoke particles. The accumulation of an electrical charge by clouds in the process of formation and the formation of rain with the discharge of the former have their counterparts in the behavior of suspensoids. The atmosphere with its dust and water particles is, of course, a disperse system.

775. The Liquefaction of Gases.-Faraday's experiments on the liquefaction of chlorine have already been described (242). 
We have also seen that ammonia can be liquefied when strongly compressed (5I7). Carbon dioxide gas can be liquefied (633) at a very high pressure if at the same time the temperature is below $3 \mathrm{I}^{\circ}$. Above this temperature no pressure, however great, causes liquefaction. At $3 \mathrm{I}^{\circ}$ the pressure required is 72 atmospheres. At lower temperatures less pressure is required. Experiment has shown that for every gas there is a definite temperature above which no pressure (however great) will cause liquefaction. This temperature is called the critical temperature of the gas. The vapor pressure exerted at its critical temperature by a liquefied gas is called its critical pressure. Any gas may be liquefied if cooled below ils critical temperaturc

T.LBLE XXXVIII

\begin{tabular}{|c|c|c|c|}
\hline Gas & $\begin{array}{c}\text { Critical Temperature } \\
\text { in Degrees } E\end{array}$ & $\begin{array}{l}\text { Critical Pressure } \\
\text { in Atmospheres }\end{array}$ & $\begin{array}{l}\text { Boiling-Point in } \\
\text { Degrees C. }\end{array}$ \\
\hline 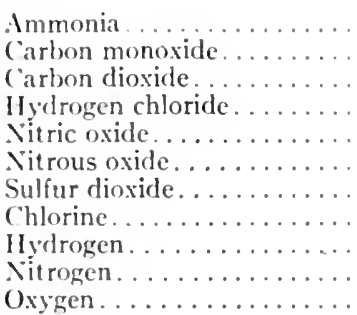 & $\begin{array}{r}131^{\circ} \\
-140 \\
31 \\
52 \\
-\quad 94 \\
37 \\
155 \\
145 \\
-243 \\
-145 \\
-119\end{array}$ & $\begin{array}{r}113 \\
36 \\
72 \\
84 \\
71 \\
72 \\
79 \\
84 \\
11 \\
34 \\
51\end{array}$ & $\begin{array}{l}-39^{\circ} \\
-190 \\
-\quad 76 \\
-\quad 35 \\
-154 \\
-\quad 88 \\
-10 \\
-\quad 34 \\
-252 \\
-194 \\
-182\end{array}$ \\
\hline
\end{tabular}

and subjected to a pressure which need not cxcecd its crilical pressure. All known gases except five (hydrogen, nitrogen, oxygen, nitric oxide, and carbon monoxide) were liquefied by Faraday by the year 1845 . The critical temperatures, critical pressures, and boiling-points at one atmosphere pressure of a number of gases are given in Table XXXVIII.

Solid carbon dioxide $\left(6_{33}\right)$ mixed with ether $\left(6_{42}\right)$ to make it a better heat conductor was used by Faraday as a cooling agent. By means of this mixture a temperature of $-80^{\circ}$ is easily obtained. Even at $-80^{\circ}$ and at very high pressures Faraday could not liquefy the five named gases. These he called permanent gases. We now know that these gases differ from others only in having critical temperatures considerably 
lower than $-80^{\circ}$. They have all been liquefied in more recent times.

776. Liquefaction of Air.--Practical methods for the liquefaction of air have been devised by Hampson, by Linde, and by Claude. The principle of the apparatus of the first two of these inventors is the same and will be understood by reference to the diagrammatic Fig. I Io and the following description. Air is compressed to 200 atmospheres pressure by a powerful pump, $A$. The work of compression is largely changed to heat so that the air becomes very hot. It is next cooled by means of running water in a cooler, $B$, and afterwards passes through a thickwalled steel cylinder, $C$, containing solid caustic potash to free it from water vapor and carbon dioxide. The compressed air

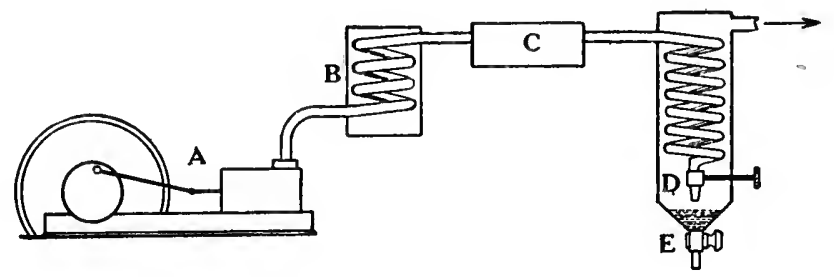

FIG. IIO

next passes through a metal pipe ending in a needle valve, $D$, from which it escapes and expands to normal atmospheric pressure. Upon expansion at the needle valve the air falls greatly in temperature (compare the preparation of carbon dioxide snow, 633), but not sufficiently to cool it to its temperature of liquefaction. But the very cold expanded air now flows upward through the larger pipe surrounding the pipe leading to the needle valve and thus cools the compressed air greatly before it escapes. The result is that the air reaching the valve becomes colder and colder until finally it reaches its liquefaction point; that is, its boiling-temperature at atmospheric pressure. From this time on liquid air collects in the lower part of $E$ and can be drawn off through a second valve.

777. The Properties of Liquid Air.-Liquid air is a faintly blue mobile liquid. It can be kept in the liquid state at atmos- 
pheric pressure only so long as its temperature remains at or below its boiling-point, about $-190^{\circ}$. If it is contained in an open vessel, it boils away more or less rapidly, and the absorption of heat during its evaporation (II5) keeps its temperature down to its boiling-point at atmospheric pressure. The more slowly external heat flows into the liquid air the more slowly it boils away and therefore the longer it may be kept. Sir James Dewar has invented ingenious containers for liquid air that provide the best attainable heat insulation. These are glass ressels with double walls, having the space between the walls evacuated (Fig. III). Often the walls are silvered. Glass is a poor conductor of heat, and as there is no air between the walls to transfer heat by convection, heat can reach the interior only by conduction, by way of a long path through glass. Radiant heat is kept out by the silvered surface, which reflects both heat and light. Heat reaches the interior so slowly that a five-liter flask of liquid

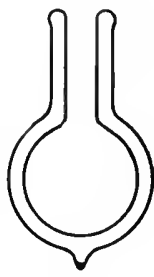

FIG. III air will lose by evaporation only 20 to 25 per cent of its contents in twenty-four hours. In recent years Dewar vessels have come into extensive general use (as "Thermos" bottles, "Icy-Hot" bottles, etc.).

Liquid air, being a mixture of liquid nitrogen and liquid oxygen, does not have a constant boiling-point. Liquid nitrogen boils at $-194^{\circ}$ and liquid oxygen at $-182^{\circ}$. The former, being the more volatile, boils away more rapidly, so that the partially' evaporated liquid is largely oxygen. This is decidedly blue in color. Liquid nitrogen is colorless. By a process of systematic fractional distillation the component gases of liquid air can be nearly completely separated. 'These separate gases are made commercially on a large scale in this way $(309,513)$.

778. Liquefaction of Other So-called Permanent Gases.With the liquefaction of air it became apparent that there were no permanent gases in the sense that these could not be liquefied. It was plain that any gas could be liquefied either at atmospheric or higher pressure, provided it could be cooled below its critical temperature. Faradiay had failed to liquefy five gases because 
he had no means of cooling them to sufficiently low temperatures. With liquid air, boiling at atmospheric pressure, a temperature of $-190^{\circ}$ is available. Since carbon monoxide boils at $-190^{\circ}$ and nitric oxide at $-\mathrm{I} 54^{\circ}$ both these gases are readily liquefied by cooling them with liquid air. In the case of the former a little pressure above atmospheric is required. Hydrogen, however, cannot be liquefied at $-190^{\circ}$ even when strongly compressed. This fact indicates that the critical temperature of this gas is lower than $-190^{\circ}$.

779. Liquid Hydrogen.-Hydrogen was condensed to a liquid (in appreciable amounts) by Dewar in 1898 . The method employed was similar to that used to make liquid air (776); but the compressed hydrogen before entering the liquefier, $D$, Fig. I Io (776), was cooled to $-I 85^{\circ}$ by liquid air. Liquid hydrogen is a colorless liquid, about one-seventh as dense as water. Its critical temperature is $-243^{\circ}$, its critical pressure I I atmospheres. It boils at $-252^{\circ}$ at atmospheric pressure, equal to $2 \mathrm{I}^{\circ}$ absolute.

780. Flames.-That a flame is a burning gas is of course well known to the reader; but it may not have occurred to him that the flame of a candle or kerosene lamp is a gas flame. In the case of a burning candle the wax melted by the heat forms a small pool of liquid; this the wick takes up by capillary action and brings to the center of the flame, where the

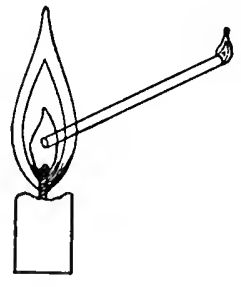

FIG. I I 2 intense heat decomposes the wax into volatile (gaseous) products. By holding a narrow glass tube 3 inches long in a candle flame so that the lower end of the tube is at the tip of the wick, unburned gas may be drawn from the center of the flame and burned at the upper end of the tube, Fig. I 2 . This experiment also shows that in the center of the candle flame the gas is as yet unburned. It unites with oxygen in the outer layer of the flame; this is therefore the hottest part of the flame, while the interior is much cooler. If a piece of writing paper is held for a few seconds in a candle flame at the tip of the wick and perpendicularly to the latter, it will be scorched in a ring, the center of which is unburned. 
The flame of burning wood or coal is formed similarly to the candle flame by reason of the preliminary conversion of the fuel into gaseous products.

781. The Bunsen Burner.-If we close the air vents at the base of a Bunsen burner the ignited gas burns with a flame resembling the candle flame in structure. The flame is luminous and is likely to be smoky. If we open the air vents sufficient oxygen becomes mixed with the gas to cause much more rapid burning, since now it is not necessary for gas and air to mix by the rather slow process of diffusion after the gas has left the burner tube. With the ideal adjustment of the air vents somewhat less oxygen is supplied than the total necessary, so that some oxygen is taken from the air around the flame. If too much air mixes with the gas at the vents, the mixture burns with such great rapidity that the speed of ignition exceeds the speed with which the mixed gas and air travel upward in the burner tube, with the result that the flame "strikes back" and "burns at the base." In an improved form of burner known as the Meker burner (Fig. I I3) the design of the tube and vents is such that a larger proportion of air is taken in than in the ordinary Bunsen burner, and air and gas are more intimately mixed before reaching the top of the burner. To prevent striking back, the wide upper end of the burner tube is provided with a metal grid, in the narrow passages of which the hot gases are

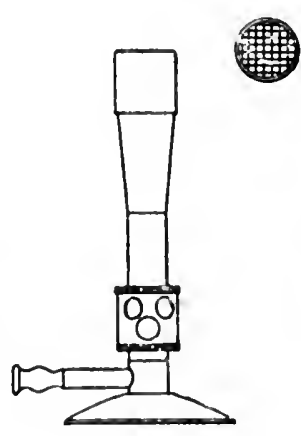

FIG. II3 so greatly cooled that the lownward speed of the ignition wave no longer exceeds that of the upwarl-moving gas stream.

The Bunsen flame with open vents consists of two distinct parts: the inner cone of a greenish color and the outer cone, bluish in color and less luminous. The hottest part of the flame is found in the center of the outer cone, just above the apex of the inner cone.

782. Luminous and Non-luminous Flames.-The cause of luminosity of a gas flame, such as that from a "fish tail" burner, has been the subject of extensive investigation. It is probable 
that certain compounds in the gas, notably acetylene, $\mathrm{C}_{2} \mathrm{H}_{2}$ (663), are decomposed by heat giving free carbon; and that the particles of the latter, being intensely heated, give out light. Indeed we have only to hold a cold object in a luminous flame to collect on it a deposit of soot (carbon). Furthermore it is well known that if acetylene is strongly heated in the absence of air it dissociates into hydrogen and carbon. The particles of carbon in a luminous flame are finally more or less completely burned.

The action of air in rendering a Bunsen flame non-luminous is said by some chemists to be due to more perfect combustion. But this explanation is not quite sufficient, since a gas flame is rendered non-luminous by admixture with nitrogen or carbon dioxide instead of with air. Probably these inert gases so act because the temperature of the flame is reduced by the dilution to a point below that at which the acetylene, etc., is decomposed before it burns. Air, being four-fifths nitrogen, must also act as a diluent in the Bunsen flame.

We always use the non-luminous Bunsen flame when we wish to heat anything most efficiently; and we might be led to conclude that a given volume of gas produces more heat as a non-luminous than as a luminous flame. This, however, is not true; the heat production is exactly the same in the two cases, that is, if the combustion is complete (363). Nevertheless a beaker of water will be heated more quickly and a piece of glass will be heated hotter in the non-luminous flame. One reason is this: the luminous flame radiates heat, as well as light, in much greater amount than does the non-luminous flame, as is easily proved by holding the hand at a distance of a few inches on one side of a Bunsen flame and opening and closing the air vent. Most of the radiated heat is lost for practical heating purposes. A second reason why the non-luminous flame is more effective is that it is more concentrated (compact), and that the gases are in more rapid motion. For both these reasons hot molecules strike the object to be heated more frequently than is the case in the luminous flame and so raise its temperature more rapidly.

783. Reactions in the Flame. Bead Tests.-It is perhaps a new point of view to consider the flame as a reagent, but that 
it is a valuable one may be illustrated by some well-known bead tests. If a metaphosphate bead is made in the usual way and a tiny speck of a copper salt is melted into it by heating the two in the non-luminous flame of the burner, after the resulting bead is cool it will be found to be blue, the usual color of dilute solutions of copper salts. But if the air holes of the burner are partly closed, so that a small, luminous sheath appears on the tip of the inner cone of the flame and the bead is first melted in this sheath and then lowered into the inner cone of unburned gas until it is cool, upon removal from the flame the bead will be seen to be opaque and reddish, owing to the presence of fincly divided copper. If the red bead is reheated in the outer flame the blue color reappears. As a consequence of such reactions the inner cone of the flame is known as the reducing region and the outer as the oxidizing region. The reducing region of the non-luminous flame is relatively thin, but its depth is increased by shutting off a little of the air as indicated above. Many other bead tests can be made in a similar way.

784. Colored Flames. - A number of elements if brought into a non-luminous Bunsen flame in the form of volatile compounds

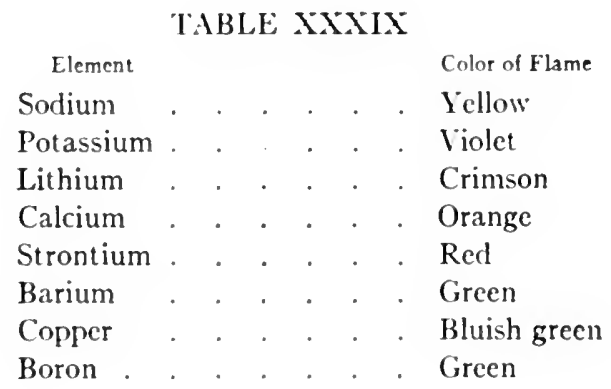

(usually salts) give to the flame characteristic colors. 'Thus, common salt and other compounds of sodium give intensely yellow flames. The flame color is, in general, dependent on the elements present irrespective of whether they are free or combined. Table XXXIX gives a list of the commoner elements forming colored flames. 
Very interesting and important facts about colored flames are brought out by the use of the spectroscope, to the description of which we shall now turn.

785. The Spectroscope.-When a narrow beam, $A$, of white light, with parallel rays, strikes a prism, $B$, in the manner illus-

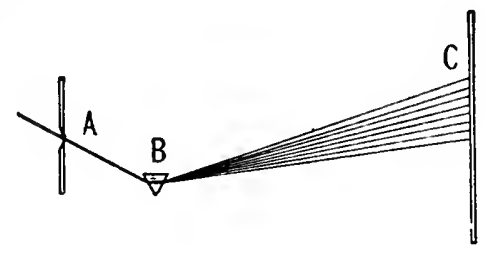

FIG. II 4 trated in Fig. II4, its colors are refracted to different degrees so that the emerging light is spread out as a spectrum at $C$, with its.red rays least and its violet rays most changed in direction. A spectroscope (Fig. I I5) consists of a prism, $A$, with one tube, $B$, carrying lenses so arranged as to throw a narrow beam of light on the prism, and a second tube, $C$, carrying a set of lenses forming a microscope through which the spectrum is viewed. In most cases there is in addition a third tube, $D$, carrying a scale which can be illuminated, by means of which the different colors of the spectrum can be located. If one examines with the spectroscope the light from an incandescent electric bulb he sees the entire spectrum from red on the one hand to violet on the other, with no single color noticeably brighter than any other. The same kind of spectrum is given by any white-hot body, as for example an incandescent gas mantle.

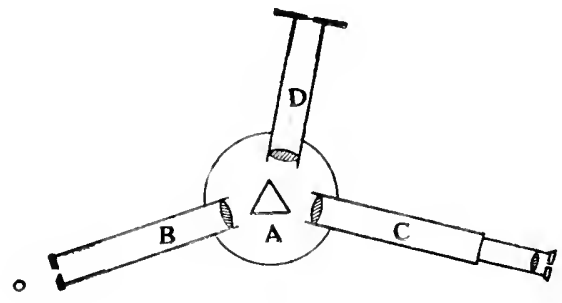

FIG. II5

Such a spectrum is called a continuous spectrum. A luminous gas flame also gives a continuous spectrum.

786. Bright-Line Spectra.-An entirely different picture is presented when one looks at a non-luminous Bunsen flame made yellow by a sodium salt. Instead of the entire spectrum only a bright-yellow line appears. If the instrument is a good one 
and a very narrow beam of light is used, the yellow line is found to consist of two parallel lines very close together in the position on the scale occupied by the yellow part of the spectrum when the entire spectrum is present. The spectrum of the element lithium is still more striking, in that it appears as a single bright line of the purest red. The spectrum of potassium shows two lines, one in the red but not in the same position as that of lithium or of the same shade of red, and another in the violet. Some elements like calcium give a rather complex spectrum made of several lines, some of which are rather broad. Each element gives its own characteristic spectrum, so that if one is familiar with the various line spectra of the elements it becomes a very simple matter to identify at once any element which gives a colored flame. This identification is made easy because each line of the spectrum alway's appears at a fixed position on the scale of the instrument, so that the observer has only to note the scale position of the lines without considering critically their colors. It is not difficult to detect spectroscopically the presence of two or more elements in a mixture. Thus for example if a little lithium chloride, $\mathrm{LiCl}$, is mixed with some common salt the presence of the former can easily be detected with the spectroscope. To do this the end of a platinum wire is dipped in the solution to be tested and then held in a non-luminous flame toward which the slit of the spectroscope is directed. One sees the lines of both lithium and sodium.

787. Other Means of Examining Spectra.-Only a small number of elements give colored flames suitable for spectroscopic study. By suitable means every element can be made to show a bright line spectrum. In general it is necessary for this purpose to heat the element to a higher temperature than that of the Bunsen flame. 'To do this we may make use of the electric arc; or we may cause sparks to pass between a platinum wire and a solution of the substance. In either case bright-line spectra are seen; but there is usually more or less difference between the flame, arc, and spark spectra of a given element. A still different method applicable to gases and volatile substances consists in rendering them luminous by the discharge of an 
induction coil while they are contained in a glass tube under low pressure. The spectroscope is of great importance in the analysis of substances, and in several cases its use has led to the discovery of new elements.

788. The Ether Wave Hypothesis.-Light is a vibratory disturbance in the so-called luminous ether, the waves being set up (according to hypothesis) by atomic vibrations. Light of a definite color is due to waves all of equal length. In so far as the visible spectrum is concerned, red light has the longest waves and violet light the shortest. Waves of intermediate lengths give all the intervening colors of the spectrum. An incandescent solid sends out waves of all lengths and gives therefore a continuous spectrum. On the other hand, a glowing gas emits waves of but very few wave-lengths, corresponding to the bright lines of its spectrum. The reason for this is thought to be that each distinct length of wave is caused by a single sort of vibrator. Very likely it is the electrons composing an atom (482) which act as the vibrators which set up the ether waves appearing to the eye as light.

789. Dark-line Spectra.--If a colored flame (say of sodium light) is interposed between a highly luminous solid and a spectroscope one might expect to see a continuous spectrum with its yellow portion crossed by a still brighter yellow line. The effect, however, is quite the contrary: where we should expect the bright line of sodium a dark line appears instead. Under similar conditions the colored flame of any other element would in like fashion show a dark-line spectrum of that element, having a dark line corresponding to every bright line of its ordinary spectrum. The explanation of this curious fact seems to be the following: vibrators (atoms or electrons) which can emit light of a certain wave-length are also able to absorb light of this same wave-length. Therefore those wave-lengths of the light of the bright continuous spectrum which correspond to the bright lines of the glowing gas are absorbed in passing through the latter, so that in their places dark lines appear in the spectrum.

790. The Composition of the Sun and the Stars.-The spectrum of daylight (sunlight) shows a great number of dark lines. 
These were discovered by Wollaston in I SO2 and first mapped by Fraunhofer in 1814 ; they are commonly called Fraunhofer lines. These lines correspond to the elements present in the sun's glowing atmosphere. Among the elements so indicated are many common on earth such as calcium, iron, hydrogen, and sodium. A few lines are present, however, which do not correspond to any known elements. Some of these are thought to be due to elements not as yet discovered on earth.

We may even learn much of the chemical composition of the stars, which are in fact very distant suns, by a study of their dark-line spectra.

791. The Discovery of Argon.-In the year 1 890 there was probably not a single reputable chemist in the world who would have conceded the possibility of the existence in the air, to the extent of nearly I per cent, of a hitherto unknown gaseous element. The discovery of argon in 1894 by Rayleigh and Ramsay forms, therefore, one of the most interesting and suggestive chapters of modern chemistry. The whole story as related by Lord Rayleigh himself in the article on argon in the eleventh edition of the Encyclopaedia Britannica is well worth reading. Briefly stated the circumstances leading to the discovery are these: Lord Rayleigh, one of the world's greatest physicists, and Professor Ramsey (later Sir William), a brilliant chemist, both Englishmen, were engaged in a research on the determination of gas densities, in which results of the utmost accuracy were desired. Nitrogen was one of the gases studied. Now, as the student well knows, nitrogen can be prepared from various nitrogen compounds (5r3) as well as also (so it was thought) from the air by the removal of oxygen, carbon dioxide, and water vapor, and the minute amounts of other known gases. Rayleigh and Ramsey found that nitrogen extracted in this way from air had a density half of I per cent greater than nitrogen made from ammonia or other pure compounds of nitrogen. This difference in density was about fifty times as great as could be accounted for by experimental error! The only logical conclusion was that "atmospheric nitrogen" contained an appreciable proportion of a new gas denser than nitrogen and equally or 
more inert chemically than the latter. A thoroughgoing search of the literature of nitrogen brought to light a most valuable clue. Nearly a century earlier Cavendish (292), the discoverer of hydrogen, had carried out an experiment in which atmospheric nitrogen mixed with an excess of oxygen was subjected to the prolonged action of clectric sparks (566). The oxides of nitrogen so formed were absorbed in alkali, and the volume of the nitrogen was thus reduced to I/ 20 of that taken. But further diminution of volume could not be made to take place.

Rayleigh and Ramsay's repetition of Cavendish's experiment with much more refined and larger apparatus enabled these scientists to obtain considerable amounts of the chemically inert residual gas. When this was freed from nitrogen and other known gases it was found to be a colorless, odorless gas I . 25 times as dense as oxygen ( $\mathrm{r}$ liter weighs $\mathrm{I} .78 \mathrm{~g}$.). It was named argon and given the symbol $\mathrm{A}$.

Every effort to cause argon to combine with other elements or react in any way with any other substance proved completely futile. Furthermore argon could not be decomposed by any physical means. It was therefore classed as an element, but one without chemical affinity and therefore without chemical properties.

792. The Molecular and Atomic Weights of Argon.-At standard conditions, 22.4 liters of argon weigh 39.9 g.; therefore the molecular weight of the element is 39.9 . Since argon does not combine with other elements it is impossible, by methods so far discussed in this text, to determine its atomic weight. If, however, we can by some independent method find the number of atoms in a molecule of argon we have only to divide its molecular weight by this number to get its atomic weight.

By means of calculations based on the kinetic-molecular hypothesis it has been found that if no expansion is permitted the amount of heat required to raise the temperature of one gram molecular weight of a gas one degree (the so-called molecular heat) should be three calories, if each molecule consists of a single atom. In this case the heat applied is wholly used 
to increase the velocities of the molecules. If however the molecules are made up of two or more atoms each, then part of the heat is used up in increasing the internal energy of each molecule (that is, increasing the vibrational or rotational velocities of the atoms within each molecule with respect to one another): Therefore the heat required to raise the temperature of one gram molecular weight of a diatomic gas should theoretically be more than three calories.

Now it has been found by experiment, in strict accord with theory, that the vapors of sodium and mercury, elements having single atom molecules (75), have molecular heats of three calories. No known gas or vapor has a smaller molecular heat than this value. On the other hand, just as theory predicts, oxygen, hydrogen, and nitrogen, all forming diatomic molecules, have molecular heats of about 4.9 calories. Gaseous elements and compounds with still more complex molecules show still larger molecular heats. When the molecular heat of argon was found to be three calories the conclusion was at once drawn that its molecules consist of single atoms and therefore that its atomic weight is identical with its molecular weight, namely 39.9. Indeed this conclusion is quite in harmony with the fact that argon does not unite with other elements, for it would be very strange if its atoms united with one another when they show no inclination to unite with other atoms.

793. The Ratio of Molecular Heats of Gases.-The molecular heat referred to in the previous section is the so-called molecular heat at constant volume, $C_{\xi}$. It represents the heat necessary to raise one gram molecular weight of the gas through one degree centigrade when the volume of the gas is kept constant. If the gas is allowed to expand at constant pressure while it is being heated, two more calories per molar weight are needed to raise the temperature one degree. These two calories are used up in the work of overcoming the external pressure during the expansion of the gas. The value so determined is the molecular heat at constant pressure, $C_{p}$. Now it appears that the ratio of the two molecular heats $C_{b} / C_{v}$ for a given gas may be calculated from the velocity of sound in the 
gas in question, and that the ratio is a relatively easy value to determine. As a result it is usual to find $C_{p} / C_{v}$ instead of the separate values. For if $C_{v}$ for monatomic gases is equal to three, then the ratio of the molecular heats may be represented thus:

$$
\frac{C_{p}}{C_{v}}=\frac{3+2}{3}=1.66
$$

The ratio of the molecular heats of a diatomic gas is in no case more than $\mathbf{I} .40$. The ratio of the molecular heats is at its maximum in the case of monatomic gases.

794. The Story of the Discovery of Helium.-Of all the elements known in 1894 argon stood alone in refusing to unite chemically with any other element. Naturally this proved a matter of surpassing interest to chemists at the time. After the extensive laboratory experiments in which Rayleigh and Ramsay hoped to make compounds of argon had all failed, it occurred to these scientists that such compounds might possibly exist in nature. But if this were true, where should they seek these strange substances? Once more a search of old chemical literature furnished a clue. In a paper by the American chemist Hillebrand there appeared a statement that the mineral cleveite when heated gave off nitrogen. Before the discovery of argon it was considered sufficient identification of a gas as nitrogen to show that it was chemically inert and not one of the other known gases. Perhaps the "nitrogen" from cleveite might be argon! Ramsay repeated Hillebrand's experiment and obtained very readily an inert gas, but it was not nitrogen, since it would not unite with oxygen under the action of electric sparks (566). Moreover, it was not argon, since its density was but one-tenth as great as that of argon. It was plainly a new element, with a density only twice that of hydrogen; but like argon, entirely devoid of chemical affinity. The spectrum of the new gas showed a yellow line not far from that of sodium but not identical with that of any hitherto known element. Search of the literature of spectroscopy, however, revealed a most interesting fact. In 1868 the astronomer Lockyer studied the photographs of spectra of the sun's corona (the incandescent atmosphere of the sun) 
taken during a total eclipse and had noted the presence of a Fraunhofer dark line in the yellow of the spectrum which could not be identified as due to any known element. In explanation Lockyer suggested that this line was doubtless due to a new element to which he gave the name helium (Gr. helios, the sun). Careful comparison of the position of the dark line of the Lockyer photograph with the yellow line of the new gas showed beyond any doubt that the two were produced by the same element. Therefore the new gas was Lockyer's helium! 'Thus Rayleigh and Ramsay's search for a possible compound of argon led to the discovery on earth of an element whose existence in the sun had been announced thirty years earlier on the evidence of a photograph taken of an object 92 million miles away.

795. Helium and the Alpha Rays of Radioactive Substances. - We have already stated (480) that the alpha rays of radioactive substances are helium atoms. The further discussion of this scientifically important topic will be taken up in chapter xxxi. At this point we shall only add that minerals like cleveite, in which helium is contained, all contain radioactive substances, and that the helium present is beyond doubt the product of radioactive changes (867). Helium is present in the atmosphere in minute amount, about four parts in a million.

796. The Properties of Helium.-As we have stated, helium, like argon, is devoid of all chemical properties. It is a colorless, odorless gas of twice the density of hydrogen. One liter weighs $0.18 \mathrm{~g}$. and 22.4 liters weigh $4.0 \mathrm{~g}$. Therefore its molecular weight is 4 . Since the ratio of its two molecular heats (793) is I.66, it is a monatomic gas and its atomic weight is therefore 4 . Of all gases helium is the most difficult to liquefy. It has the lowest critical temperature, $-268^{\circ}$ or $5^{\circ}$ absolute. By cooling compressed helium by boiling liquid hydrogen and allowing it to expand in a liquefier similar in principle to that used for liquid air $(\mathbf{7 7 6})$ it has been condensed to a liquid which boils at $4.3^{\circ}$ absolute. The boiling-point of helium under reduced pressure showed a temperature of about $3^{\circ}$ absolute; this is up to the present time the lowest temperature ever produced experimentally. The liquefaction of helium was accomplished by 
Dr. Kammerlingh Onnes, of the University of Leiden, in the year 1908 .

797. Helium Balloons.--The use of balloons in warfare is extremely precarious on account of the inflammability of the hydrogen with which they are filled. Helium being incombustible would be an ideal substitute for hydrogen if it could only be had in sufficient quantity. The lifting power of a helium balloon is eleven-twelfths of that of the same balloon filled with hydrogen. For a number of years it had been known that helium was often found in small proportions in natural gas, and when the matter of helium balloons began seriously to be considered in the last year of the war (I918) it was found that a certain gas well in Texas produced gas containing nearly I per cent of helium. Calculation showed that this well also produced a sufficient total amount of helium to be of practical importance. The United States government appropriated $\$ 500,000$ for a plant to produce pure helium, and at the end of the war production had already begun. It is not unlikely that immense dirigibles filled with helium will in the near future furnish a safe means of aërial travel. If this turns out to be the case it will be because two English scientists were keen to discover the cause of a seemingly trivial matter-the small discrepancy in the density of nitrogen as prepared in different ways! But this is the usual course of great practical discoveries, the scrupulous following up of every outstanding scientific inconsistency.

798. Three Other Inactive Gases.-Shortly after the disrovery of helium three other inert gases were discovered. in

TABLE XL

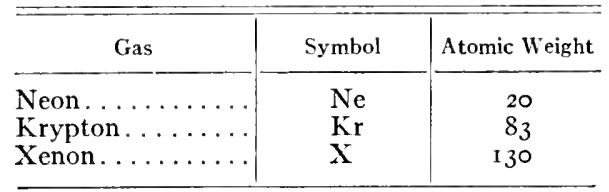

minute amount in the atmosphere. Like helium and argon these new gases do not form any chemical compounds; they are also monatomic. These gases with their symbols and atomic weights are given in Table XL. 
799. Summary and Conclusions. - The air may be considered as a great gaseous ocean into which flow many gases of terrestrial origin and from which are drawn the gases entering into the earth's chemical activity. It is evident that in addition to the several well-known components of the air there must also be present numerous other gases and vapors in minute proportions. The composition of the air remains nearly constant because of the approximate equality of formation and removal of the several components. In addition to the gases present, water vapor and dust are components of prime importance. Without dust (or gaseous ions) we should have no clouds, and without clouds no rain, although we should have high humidity and tremendous dews. Viewed in the light of the matters considered in chapter xxviii we must see that the atmosphere is a disperse system consisting of a complex gaseous mixture filled with a suspension of minute solid particles (dust) and liquid droplets (water). The gaseous ions of the air become in part attached to these suspended particles giving to them plus and minus charges, corresponding to the charges on particles of colloidal solutions.

The work on the liquefaction of gases so ably started by Faraday in ${ }^{8} 823$ was brilliantly completed when Kammerlingh Onnes liquefied helium in I908. Every known gas has now been liquefied, and all save helium have been solidified. In the liquefaction of gases two principles are most noteworthy: first, the gas can exist as a liquid only below its critical temperature; second, a highly compressed and sufficiently cold gas will, upon being allowed to expand, fall greatly in temperature.

The spectra of elements volatilized at high temperatures, as in a flame or by means of an electric spark or arc, appear as bright lines. Each element gives definite and characteristic lines. If white light from a glowing solid passes through an incandescent gas, the continuous spectrum of the former is seen to be crossed by dark lines which are the Fraunhofer spectrum of the gas. The spectroscope is of great importance for the detection of elements. By its aid the composition of the atmosphere of the sun and of the stars has been definitely revealed. 


\section{CHAP'TER XXX}

\section{SOME ADDITIONAL ELEMENTS AND THEIR COMPOUNDS}

8oo. Introduction.- - The total number of elements known at the present time is 83 (if we exclude the products of radioactive change, chap. xxxii). These with their symbols and atomic weights are given in Table XLI. In the foregoing chapters we have become acquainted with but little more than one-third of the elements. In the present chapter we shall study more or less briefly the chemistry of a number of additional elements. These, with those studied earlier, include all of the elements of practical importance. This list does not embrace several elements which are fairly abundant but for which no technical uses have as yet been discovered. In chapter xxxi these remaining elements will also be considered.

Our study of part of the elements has enabled us to develop and illustrate many principles and laws; but the student will naturally wonder whether the study of the remaining elements will bring forth an entirely new set of generalizations. We can hasten to assure him that this is not the case, for in the study of new elements and compounds he will encounter very few facts and phenomena that do not have their counterparts among those of more familiar substances.

8or. Boron.-Borax and boric acid (also known as boracic acid) are doubtless known to everyone. These are compounds of the non-metallic element boron. This element occurs plentifully as boric acid, $\mathrm{H}_{3} \mathrm{BO}_{3}$, and also as borates of sodium, magnesium, and calcium, but never as free boron. It is found chiefly in Italy, California, and Thibet. Boric acid, which is volatile with steam, is present in the steam escaping from the earth in certain volcanic regions, especially in Italy. Sodium borate, or borax, which is easily soluble, occurs abundantly in the waters of some California and Nevada lakes. It is also found in the deposits left by the drying up of certain lakes of this same region. Borax 
is also made technically from the borates of magnesium and calcium. Boric acid and borax are the only compounds of this

TABLE XLI

INternational Atomic Weights, 19 I7

\begin{tabular}{|c|c|c|c|c|c|}
\hline Element & Symbol & $\begin{array}{l}\text { Atomic } \\
\text { il' eight }\end{array}$ & Element & Symbol & $\begin{array}{l}\text { Atomic } \\
\text { Weight }\end{array}$ \\
\hline Aluminum...... & .11 & 27.1 & Molybdenum. & Mo & $9^{6.0}$ \\
\hline Antimony..... & $\mathrm{Sb}$ & $120 \quad 2$ & Neodymium & $\mathrm{Nd}$ & $144 \cdot 3$ \\
\hline Argon........... & A & 3988 & Neon....... & $\mathrm{Ne}$ & 20.2 \\
\hline Arsenic......... & As & 7496 & Nickel....... & $\mathrm{Ni}$ & 58.68 \\
\hline Barium........ & $\mathrm{Ba}$ & $137 \cdot 37$ & Niton (radium & & \\
\hline Berỵllium..... & $\mathrm{Bc}$ & 91 & emanation). & $\mathrm{Nt}$ & 222.4 \\
\hline Bismuth..... & $\mathrm{Bi}$ & 208.0 & Nitrogen ..... & $N$ & 14.01 \\
\hline Boron............ & $B$ & 110 & Osmium... & Os & 190.9 \\
\hline Bromine...... & $\mathrm{Br}$ & 70.92 & Oxygen....... & $\mathrm{O}$ & 16.00 \\
\hline Carlmiun ..... & $\mathrm{Cd}$ & 112.40 & Pailladium... & $\mathrm{Pd}$ & 106.7 \\
\hline Caesium........ & Cs & I 32.81 & Phosphorus... & $\mathbf{P}$ & 31.04 \\
\hline Calcium... & $\mathrm{Ca}$ & 40.07 & Platinum... & $\mathrm{Pt}$ & 195.2 \\
\hline Carbon... & C & 12.005 & Potassium. & $\mathrm{K}$ & 39.10 \\
\hline Cerium... & $\mathrm{Ce}$ & $140 \quad 25$ & Praseodymium & Pr & 1409 \\
\hline Chlorine..... & $\mathrm{Cl}$ & 3546 & Radium ..... & Ra & 226.0 \\
\hline Chromium...... & $\mathrm{Cr}$ & 520 & Rhodium . . & $\mathrm{Rh}$ & 102.9 \\
\hline Cobalt. ... & Co & 5807 & Rubidium.... & $\mathrm{Rb}$ & 85.45 \\
\hline Columbium..... & $\mathrm{Cb}$ & 931 & Ruthenium... & $\mathrm{Ru}$ & 101.7 \\
\hline Copper.......... & $\mathrm{Cu}$ & 6357 & Samarium... & $\mathrm{Sa}$ & 150.4 \\
\hline Dysprosium..... & Dy & 162.5 & Scandium.... & $\mathrm{Sc}$ & 441 \\
\hline Erbium........ & $\mathrm{Fir}$ & 1677 & Selenium..... & Se & 79.2 \\
\hline Europium ....... & $\mathrm{Eu}$ & 1520 & Silicon.... & $\mathrm{Si}$ & 28.3 \\
\hline Fluorine. ... & F & 190 & Silver. . . . . & $\mathrm{Ag}$ & 107.88 \\
\hline Gadolinium. . & Gid & 1573 & Sodium.... & $\mathrm{Na}$ & 23.00 \\
\hline Gallium... & Gia & 699 & Strontium. . & $\mathrm{Sr}$ & 87.63 \\
\hline Germanium. . & Ge & 72.5 & Sulfur...... & $\mathrm{S}$ & 32.00 \\
\hline Gold ........ & Au & 1972 & Tantalum. . & $\mathrm{Ta}$ & 181.5 \\
\hline IJelium... . & $\mathrm{He}$ & 4.00 & Tellurium. . & $\mathrm{Te}$ & 127.5 \\
\hline Holmium....... & Ho & 163.5 & Terbium.. & $\mathrm{Tb}$ & 159.2 \\
\hline Hydrogen... & II & 1.008 & Thallium... & $\mathrm{Tl}$ & 204.0 \\
\hline Indium.... & In & 114.8 & Thorium... & Th & 232.4 \\
\hline Iodine.... & I & 12692 & Thulium.... & ' $\mathrm{m}$ & I 68.5 \\
\hline Iridium. . & ir & 1031 & Tin........ & $S n$ & 118.7 \\
\hline Iron ..... & Fe & 55.84 & Titanium... & $\mathrm{Ti}$ & 48.1 \\
\hline Krypton..... & $\mathrm{Kr}$ & 8202 & Tungsten... & $11{ }^{\circ}-(-a+a)$ & $184 \circ$ \\
\hline Irinthanum. & Lat & 1390 & Uranium... & U & 238.2 \\
\hline Lead. ... & $\mathrm{Ph}$ & 207.20 & lanadium. & 1 & 51.0 \\
\hline Lithium.... & $1, \mathrm{i}$ & 604 & Xenon.... & $\mathrm{Xc}$ & 130.2 \\
\hline Latecium... & I.u & 1750 & Viterbium. . & ib & 173.5 \\
\hline Magnesium & $1 / g$ & 2432 & Yitrium... & It & 88.7 \\
\hline Manganese.. & $\operatorname{Mn}$ & $54 \quad 93$ & Kinc . . . . . & $\mathrm{Zn}$ & $65 \cdot 37$ \\
\hline Mercury..... & $\mathrm{HIg}$ & 2006 & Zirconium. . & $\mathrm{Z} / \mathrm{r}$ & 90.6 \\
\hline
\end{tabular}

element that are of any commercial importance; these are cheap substances and are produced in large quantities. 
802. Boric Acid, $\mathrm{H}_{3} \mathrm{BO}_{3}$.--Boric acid forms white crystals which dissolve in 25 parts of cold or 3 parts of boiling water. It is a very weak acid, weaker even than carbonic acid. Its solution is almost tasteless; it certainly has no sour taste. The solution is used extensively in medicine as an antiseptic lotion. A cold, saturated solution is an excellent eye wash. If alkali has by accident got into the eye, after the former has been washed out at once with water, boric acid solution should be copiously applied. In the past boric acid was rather extensively used as a preservative for foods, especially milk. Its use in this way is now prohibited, although it is not poisonous if taken internally in small amount. It is sometimes prescribed in medicine for internal use (dose 0.3 to $\mathrm{I}$ g.).

Boric acid is readily decomposed by heat into water and metaboric acid, $\mathrm{HBO}_{2}$, tetraboric acid, $\mathrm{H}_{2} \mathrm{~B}_{4} \mathrm{O}_{7}$, and finally boron trioxide, $\mathrm{B}_{2} \mathrm{O}_{3}$ :

$$
\begin{aligned}
& \mathrm{H}_{3} \mathrm{BO}_{3} \rightarrow \mathrm{HBO}_{2}+\mathrm{H}_{2} \mathrm{O}, \\
& { }_{4} \mathrm{HBO}_{2} \rightarrow \mathrm{H}_{2} \mathrm{~B}_{4} \mathrm{O}_{7}+\mathrm{H}_{2} \mathrm{O}, \\
& \mathrm{H}_{2} \mathrm{~B}_{4} \mathrm{O}_{7} \rightarrow{ }_{2} \mathrm{~B}_{2} \mathrm{O}_{3}+\mathrm{H}_{2} \mathrm{O} .
\end{aligned}
$$

The first two reactions take place at rather low temperatures, the last at a red heat. These reactions are analogous to those by which pyrophosphoric and metaphosphoric acids are formed from orthophosphoric acid (590).

The ordinary salts of boric acid are those derived from metaboric, or more commonly tetraboric, acid. Borax, sodium tetraborate, $\mathrm{Na}_{2} \mathrm{~B}_{4} \mathrm{O}_{7} \cdot \mathrm{IoH}_{2} \mathrm{O}$, forms colorless, glassy crystals. Boric acid is easily made from a solution of borax by adding hydrochloric or sulfuric acid to a hot solution. On cooling, crystals of boric acid separate out:

$$
\mathrm{Na}_{2} \mathrm{~B}_{4} \mathrm{O}_{7}+{ }_{2} \mathrm{HCl}+{ }_{5} \mathrm{H}_{2} \mathrm{O} \rightarrow 2 \mathrm{NaCl}+4 \mathrm{H}_{3} \mathrm{BO}_{3} \text {. }
$$

803. Borax.-Borax, or sodium tetraborate, $\mathrm{Na}_{2} \mathrm{~B}_{4} \mathrm{O}_{7} \cdot \mathrm{I}^{\circ} \mathrm{H}_{2} \mathrm{O}$, is the only salt of boric acid of commercial importance. It is easily soluble in water, and its solution is alkaline by reason of hydrolysis (436). Borax is used extensively in the laundry for softening hard water (156). Hardness of water is due to the 
presence of the bicarbonates and sulphates of calcium principally and magnesium to a smaller extent. The bicarbonates cause temporary hardness, so called because they are decomposed and the carbonates precipitated when the water is boiled. The two sulphates cause what is called permanent hardness. Borax softens water by precipitating all the calcium and most of the magnesium present in hard water, as illustrated for the calcium salts by the following equations:

$$
\begin{gathered}
\mathrm{Na}_{2} \mathrm{~B}_{4} \mathrm{O}_{7}+\mathrm{Ca}\left(\mathrm{HCO}_{3}\right)_{2}+{ }_{5} \mathrm{H}_{2} \mathrm{O} \rightarrow \mathrm{Na}_{2} \mathrm{CO}_{3}+\mathrm{CaCO}_{3}+{ }_{4} \mathrm{H}_{3} \mathrm{BO}_{3} \\
\mathrm{CaSO}_{4}+\mathrm{Na}_{2} \mathrm{CO}_{3} \rightarrow \mathrm{CaCO}_{3}+\mathrm{Na}_{2} \mathrm{SO}_{4} .
\end{gathered}
$$

Upon being heated borax partially melts and at the same time gives off its water of hydration and in so doing swells to a spongy, viscous mass (intumesces); this finally melts completely when all the water has been driven off, and it then resembles melted glass. The cold product, which is anhydrous borax, $\mathrm{Na}_{2} \mathrm{~B}_{4} \mathrm{O}_{7}$, is a clear, colorless, brittle solid called borax glass. Beads made of borax glass are used in the same way as metaphosphate beads (596), which they closely resemble in

T.IBLE XLII

Colors of Borax Beads

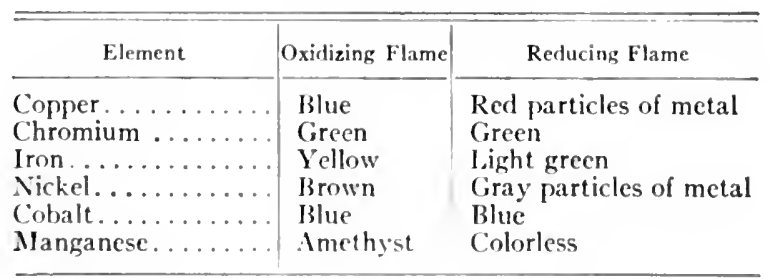

appearance. Various metallic oxides readily dissolve in fused borax glass, and in many cases the beads have colors characteristic of the added metallic element. If the element is readily oxidized or reduced the color of the bead will depend on whether it has been heated in an oxidizing or a reducing flame (783). Table XLII shows the colors of borax beads of several elements. 
In explanation of the action of melted borax on oxides it may be supposed that reactions of the type illustrated by the following equation occur:

$$
\mathrm{Na}_{2} \mathrm{~B}_{4} \mathrm{O}_{7}+\mathrm{CuO} \rightarrow 2 \mathrm{NaBO}_{2}+\mathrm{Cu}\left(\mathrm{BO}_{2}\right)_{2} \text {. }
$$

Elements which give differently colored beads in the oxidizing and reducing flames do so because they undergo oxidation and reduction. Thus the yellow iron bead obtained in the oxidizing flame contains ferric metaborate, while the green one from the reducing flame contains a ferrous salt. The borates of copper and nickel are reduced to the corresponding metals in the reducing flame. It may be added that boron trioxide also forms a glass after being fused, and that it also readily unites with metallic oxides at high temperatures to form borates. Some useful optical glasses contain borates as essential ingredients. Borates are also used in making some kinds of enamels and glazes. In the welding of iron a little borax sprinkled on the hot metal dissolves the iron oxide always present and thus gives a clean surface for the weld.

804. Silicon.-The element silicon is the second most abundant of the components of the earth's crust. It is estimated that about 50 per cent of the known part of the earth is oxygen and 25 per cent is silicon. This is not surprising when we realize that common quartz sand is essentially silicon dioxide, $\mathrm{SiO}_{2}$, and that numerous minerals composing the bulk of the earth are compounds of silicon. Among such minerals, besides quartz, we may mention clay, granite, agate, and opal as being well known. The element silicon does not occur free in nature, although it can be made by reduction of the oxide with carbon at the high temperature of the electric-arc furnace:

$$
\mathrm{SiO}_{2}+{ }_{2} \mathrm{C} \rightarrow \mathrm{Si}+{ }_{2} \mathrm{CO} \text {. }
$$

Free silicon made in this way is only go to $9 \delta$ per cent pure. It is a semimetallic solid somewhat resembling cast-iron in appearance and having a density of 2.35 and a melting-point of about $1400^{\circ}$. It conducts electricity fairly well. It is not attacked by any of the common acids, with the exception of hydrofluoric, 
with which it reacts to form silicon tetrafluoride, $\mathrm{SiF}_{4}(\mathbf{2 7 0})$. Metallic silicon, as it is called by technical men, is too brittle to be of much importance for the manufacture of vessels or machines; but an alloy with iron containing about 14 per cent of silicon finds important use in chemical industry for the construction of vessels, pumps, etc., for the handling of strongly acid solutions. These alloy's, known under the trade names of Duriron, Tantiron, etc., are extremely resistant to the action of acids. Ferro-silicon is an alloy of iron and silicon containing 50 to 75 per cent of the latter and is used in making other silicon iron alloys containing less silicon.

805. Compounds of Silicon with Hydrogen Carbon and Chlorine.-Silicon forms a great many compounds with hydrogen, carbon, and chlorine, of much theoretical but little practical importance. Many of these compounds are analogous to certain compounds of carbon. Thus we have silico-methane, $\mathrm{SiH}_{4}$, a combustible gas, and silico-ethane, $\mathrm{Si}_{2} \mathrm{H}_{6}$, a spontaneously combustible liquid, corresponding to methane and ethane $\left(6_{43}\right)$ respectively. Silicon tetrachloride, $\mathrm{SiCl}_{4}$, is a colorless liquid formed by the union of silicon and chlorine and also by the action of chlorine on a red-hot mixture of silica and carbon (charcoal):

$$
\mathrm{SiO}_{2}+{ }_{2} \mathrm{C}+{ }_{2} \mathrm{Cl}_{2} \rightarrow \mathrm{SiCl}_{4}+2 \mathrm{CO} \text {. }
$$

Silicon tetrachloride boils at $58^{\circ}$ and resembles in many respects carbon tetrachloride, $\mathrm{CCl}_{4}(644)$, of boiling-point $76^{\circ}$. Unlike the latter, the former reacts readily with water. Silicic acid, $\mathrm{H}_{2} \mathrm{SiO}_{3}$, and $\mathrm{HCl}$ are formed:

$$
\mathrm{SiCl}_{4}+{ }_{3} \mathrm{H}_{2} \mathrm{O} \rightarrow \mathrm{H}_{2} \mathrm{SiO}_{3}+{ }_{4} \mathrm{HCl} \text {. }
$$

The formation and properties of silicon tetrafluoride, $\mathrm{SiF}_{4}$, and of hydrofluosilicic acid and its salts have already been discussed (270-272).

By the action of $\mathrm{HCl}$ gas on heated silicon, silico-chloroform, $\mathrm{SiHCl}_{3}$, is obtained as a low-boiling colorless liquid. This is the analogue of chloroform, $\mathrm{CHCl}_{3}(644)$, but unlike the latter it is readily decomposed by water. Silicon tetrachloride vapor reacts with heated silicon as follows:

$$
{ }_{3} \mathrm{SiCl}_{4}+\mathrm{Si} \rightarrow{ }_{2} \mathrm{Si}_{2} \mathrm{Cl}_{6} \text {. }
$$


The product, silicon hexachloride, is a liquid boiling at $148^{\circ}$, analogous to carbon hexachloride, $\mathrm{C}_{2} \mathrm{Cl}_{6}$, boiling at $187^{\circ}$. There is little doubt that the structure of these substances is that represented by the following formulae:<smiles>ClC(Cl)(Cl)C(Cl)(Cl)Cl</smiles><smiles>Cl[Si](Cl)(Cl)[Si](Cl)(Cl)Cl</smiles>

Much more complicated silicon compounds are known, in some of which long chains (664) of silicon atoms occur. The resemblance between compounds of silicon and carbon extends to a great variety of compounds. By way of further illustration the following pairs of formulae of related substances are shown:<smiles>CC(C)(C)C</smiles>

Tetramethyl-Methane<smiles>O=C(O)C(=O)O</smiles>

Oxalic Acid (665)<smiles>O=C(O)OC(=O)C(=O)O</smiles>

Mesoxalic Acid<smiles>C[Si](C)(C)C</smiles>

Tetramethyl-Silico-Methane

$\mathrm{OSiOH}$ $\mathrm{OSiOH}$

Silico-Oxalic Acid<smiles>O=S(=O)(O)O[Sb](O)(O)O</smiles>

Silico-Mesoxalic Acid

806. Silica or Silicon Dioxide, $\mathrm{SiO}_{2}$. - - Silica is one of the most abundant of all minerals. It occurs in many forms both crystalline and amorphous. In a pure state it forms colorless, transparent, hexagonal crystals called quartz or rock crystal. Amethyst is a variety of quartz colored by manganese. The commonest kinds of sand are usually largely or wholly quartz. Sandstone is essentially quartz, red varieties being colored 
with ferric oxide. A massive form of silica is called quartzite. Amorphous (58I) varieties constitute agate and onyx. Flint and opal are hydrated varieties of silica.

The various forms of silica are extremely resistant to chemical and physical changes by reason of the great hardness, high melting-point, and chemical inertness of this substance. These properties, together with the abundance and cheapness of various forms of silica, cause them to be used in enormous amounts for a great variety of purposes. As an abrasive silica finds use in grindstones, whetstones, sandpaper, and polishing material, the latter containing silica powder, tripoli, or infusorial earth (732). Mortar and concrete always contain quartz sand. Fire bricks for furnace linings are often made of silica. Various kinds of glass, including that made from pure quartz, will be considered later (808).

807. Silicic Acid and Silicates.-Silica reacts with sodium carbonate (soda ash) at a red heat to form a viscous fluid consisting of sodium silicate, $\mathrm{Na}_{2} \mathrm{SiO}_{3}$. During the fusion carbon dioxide is evolved:

$$
\mathrm{SiO}_{2}+\mathrm{Na}_{2} \mathrm{CO}_{3} \rightarrow \mathrm{Na}_{2} \mathrm{SiO}_{3}+\mathrm{CO}_{2} .
$$

On cooling, sodium silicate solidifies to a glassy solid easily soluble in water. By adding hydrochloric acid to a solution of sodium silicate we get silicic acid, which under certain conditions appears as a gelatinous precipitate (a gel, 76r). 'The composition of this silicic acid is approximately represented by the formula $\mathrm{H}_{4} \mathrm{SiO}_{4}$. Its formation may be represented thus:

$$
\mathrm{Na}_{2} \mathrm{SiO}_{3}+\mathrm{H}_{2} \mathrm{O}+2 \mathrm{HCl} \rightarrow \mathrm{H}_{4} \mathrm{SiO}_{4}+2 \mathrm{NaCl} \text {. }
$$

Silicic acid is an extremely weak acid, but it will dissolve in sodium hydroxide solution to form a solution of sodium silicate. The solution is strongly alkaline by reason of hydrolysis.

Silicic acid gel gradually loses water when heated, and in so doing it doubtless gives a series of derived acids and finally silica. In this respect it resembles phosphoric (590) and boric acids (802). Theoretically we have the following possibilities 
as one or two molecules of $\mathrm{H}_{4} \mathrm{SiO}_{4}$ lose water. (In the following expressions the water lost is omitted for the sake of brevity.)

$$
\begin{aligned}
\mathrm{H}_{4} \mathrm{SiO}_{4} & \rightarrow \mathrm{H}_{2} \mathrm{SiO}_{3} \rightarrow \mathrm{SiO}_{2} \\
2 \mathrm{H}_{4} \mathrm{SiO}_{4} & \rightarrow \mathrm{H}_{6} \mathrm{Si}_{2} \mathrm{O}_{7} \rightarrow \mathrm{H}_{4} \mathrm{Si}_{2} \mathrm{O}_{6} \rightarrow \mathrm{H}_{2} \mathrm{Si}_{2} \mathrm{O}_{5} \rightarrow 2 \mathrm{SiO}_{2} .
\end{aligned}
$$

Still more complex acids would result from the dehydration of three or more molecules of silicic acid. Although none of these acids has been made in pure form, salts of several of them are found as minerals. Silicon dioxide is of course the anhydride (313) of all the possible derivatives of silicic acid, and the formulae of all the latter may be written in the general form $\left(\mathrm{H}_{2} \mathrm{O}\right)_{n}\left(\mathrm{SiO}_{2}\right)_{m}$, where $n$ and $m$ are integers. The formulae of the salts may also be written similarly, as illustrated in Table XLIII, which shows the composition of several minerals and the acids from which they are derived.

\section{TABLE XLIII}

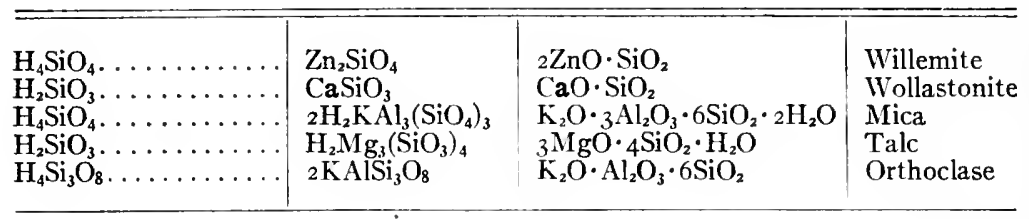

808. Glass.-There are a great many kinds of glass, nearly all of which are silicates, although borates are also common ingredients. The basic constituent elements include sodium potassium, calcium, aluminum, zinc, lead, and other metals less commonly used. Water glass having approximately the composition $\mathrm{Na}_{2} \mathrm{Si}_{4} \mathrm{O}_{9}$ is made by dissolving infusorial earth (732) in hot sodium hydroxide solution. 'It comes on the market as a heavy syrup which gives on evaporation a glasslike solid easily soluble in water. It is used as a component of laundry soaps, as a cement, as an oil-proof glaze for lard barrels, and for a number of other purposes.

Glass made from the silicates of sodium and calcium is used for window panes, bottles, etc. The ordinary soft glass tubing of the laboratory is also made of this kind of glass. Hard glass, which contains potassium in place of the sodium in soft glass, 
fuses at a higher temperature than the latter. Flint glass, a potassium-lead silicate, has a high index of refraction and on this account possesses great brilliancy. It is used for cut-glass ware and lenses.

Glassware for chemical use, beakers, flasks, etc., should possess several special properties. It must be practically insoluble in water, acids, and alkalies, must not crack when heated, and must be thick enough to withstand ordinary handling without breaking. The cracking of glass when heated is the result of rapid local expansion. The thinner the glass the less liable it is to crack when heated; but if ordinary glass is made thin enough to withstand heating in the usual way it is very fragile. Before the war nearly all chemical glassware came from Germany and Bohemia. There were two principal kinds, typically represented by Kavalier glass and Jena glass, the latter being much better in quality than the former. The composition of each of these is shown in Table XLIV.

TABLE XLIV

Percentage Couposition of Glass for Laboratory Ware*

\begin{tabular}{|c|c|c|c|c|}
\hline & Kavalier & Jena & Pyrex & Nonsol \\
\hline 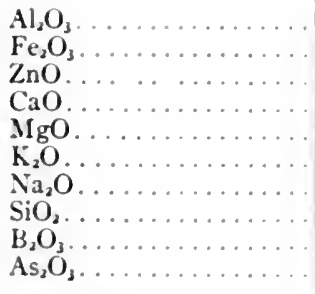 & $\begin{array}{cc}0 & 14 \\
\ldots & \ldots \\
3 & 70 \\
7 & 90 \\
7 & 10 \\
75 & 90\end{array}$ & $\begin{array}{rr}4 & 20 \\
0 & 25 \\
10 & 90 \\
0 & 63 \\
0 & 21 \\
0 & 37 \\
7 & 50 \\
64 & 70 \\
10 & 90 \\
0 & 14\end{array}$ & $\begin{array}{rr}2 & \infty \\
0 & 25 \\
\cdots & \cdots \\
0 & 29 \\
0 & 00 \\
0 & 20 \\
4 & 40 \\
80 & 50 \\
11 & 30 \\
0 & 70\end{array}$ & $\begin{array}{r}250 \\
0.23 \\
7.80 \\
0.79 \\
3.40 \\
0.30 \\
10.90 \\
6730 \\
6.20 \\
\ldots\end{array}$ \\
\hline
\end{tabular}

- Walker \& Smith, U.S. Bureau of Standards, Bullclin 107, 1918.

During the war several American manufacturers succeeded in producing first-class chemical glass. 'The composition of two sorts, Pyrex and Nonsol, is shown in 'Table XLIV. Pyrex glass has so small a coefficient of expansion that beakers and flasks can be made with thick walls and still be far less liable to crack with change of temperature than the best German glass. Several 
other sorts of American chemical glass are also of excellent quality, so that today we have better domestic ware than was heretofore imported.

Quartz glass is made from pure silicon dioxide (806) melted in an electric furnace. This glass resembles ordinary glass in appearance but differs from the latter in having an extremely high melting-point and an extremely small coefficient of expansion. White-hot quartz will not crack when plunged into water. This glass finds important uses in both scientific and technical chemistry.

809. Tin and Its Compounds. - The metal tin ( $\mathrm{Sn}=\mathrm{II} 9)$ was known from very early times, its Latin name being stannum. It is widely used in making a number of alloys, such as bronze and bell metal (tin and copper), solder (tin and lead), and tin amalgam (tin and mercury used in making mirror backs). Sheet iron coated with tin and known as tin plate is made in immense quantities for the manufacture of common tinware. Tin in the form of thin sheets is tin foil, but the common grade of this article is usually largely lead.

Tin is a rather soft metal of low melting-point, $232^{\circ}$. It is permanent in air and in water, and, as shown by its position in the displacement series (Table XIX, 492), it is a rather inactive element. It dissolves somewhat slowly in hydrochloric acid, forming stannous chloride, $\mathrm{SnCl}_{2}$,

$$
\mathrm{Sn}+{ }_{2} \mathrm{HCl} \rightarrow \mathrm{SnCl}_{2}+\mathrm{H}_{2} \text {. }
$$

The hydrate $\mathrm{SnCl}_{2} \cdot 2 \mathrm{H}_{2} \mathrm{O}$, easily soluble in water, is known in commerce as tin salt and is used in dyeing. It is frequently used in the laboratory as a reducing agent. Stannous sulfate, $\mathrm{SnSO}_{4}$, and nitrate, $\mathrm{Sn}\left(\mathrm{NO}_{3}\right)_{2}$, can also be made. The action of chlorine gas on melted tin gives stannic chloride, $\mathrm{SnCl}_{4}$,

$$
\mathrm{Sn}+{ }_{2} \mathrm{Cl}_{2} \rightarrow \mathrm{SnCl}_{4} \text {. }
$$

The product is a colorless liquid boiling at $\mathrm{II}_{4}^{\circ}$, which forms with water the crystalline hydrates $\mathrm{SnCl}_{4} \cdot 5 \mathrm{H}_{2} \mathrm{O}$ and $\mathrm{SnCl}_{4} \cdot 8 \mathrm{H}_{2} \mathrm{O}$. These salts are also used as mordants in dyeing, a mordant being a substance which by combining with the fiber fixes the dye upon the latter. Numerous other stannic salts are known. 
Thus it will be seen that tin forms two series of salts: the stannous, in which the metal is bivalent, and the stannic, in which it is quadrivalent. Stannous salts form stannous ions, $\mathrm{Sn}^{++}$, which by oxidation give stannic ions, $\mathrm{Sn}^{4+}$. The latter by reduction give the former. Stannous solutions are strong reducing agents.

Tin forms two oxides, stannous, $\mathrm{SnO}$, and stannic, $\mathrm{SnO}_{2}$. The corresponding hydroxides are precipitated when a stannous or a stannic solution is treated with the equivalent amount of sodium hydroxide. Each precipitate is soluble in an excess of the alkali, thus showing that the hydroxides of tin have acidic as well as basic properties. Like aluminum hydroxide they are amphoteric (177). Stannous hydroxide, $\mathrm{Sn}(\mathrm{OH})_{2}$, yiclds sodium stannite, $\mathrm{Na}_{2} \mathrm{SnO}_{2}$; while stannic hydroxide, also called stannic acid, gives sodium stannate, $\mathrm{Na}_{2} \mathrm{SnO}_{3}$. $\Lambda$ solution of sodium stannite is a very powerful reducing agent, since it is readily oxidized to sodium stannate. An isomer (647) of stannic acid, called metastannic acid, is formed by the action of hot nitric acid on tin. This is a white solid, insoluble in water and nitric acid, and differs greatly from the other form. Metastannic acid forms complex salts such as $\mathrm{Na}_{2} \mathrm{Sn}_{5} \mathrm{O}_{1 \mathrm{r}} \cdot 4 \mathrm{H}_{2} \mathrm{O}$. Hydrogen sulfide precipitates brown stannous sulfide from solutions of stannous chloride and yellow stannic sulfide from solutions of stannic chloride. Both precipitates are soluble in concentrated hydrochloric acid. Stannic sulfide is dissolved by ammonium sulfide, owing to the formation of ammonium sulfo-stannate. Stannous sulfide does not dissolve appreciably in pure ammonium sulficle but does so in yellow ammonium sulfide (607), forming also ammonium sulfo-stannate:

$$
\begin{aligned}
& \mathrm{SnS}_{2}+\left(\mathrm{NH}_{4}\right)_{2} \mathrm{~S} \rightarrow\left(\mathrm{NH}_{4}\right)_{2} \mathrm{SnS}_{3}, \\
& \mathrm{SnS}+\left(\mathrm{NH}_{4}\right)_{2} \mathrm{~S}_{2} \rightarrow\left(\mathrm{NH}_{4}\right)_{2} \mathrm{SnS}_{3} .
\end{aligned}
$$

8ro. Arsenic.-In its chemistry arsenic $(\Lambda s=75)$ is much more like the non-metallic than the metallic elements. In many of its compounds it bears a close resemblance to phosphorus. Arsenic is an abundant element, occurring as a mineral both free and in combination with oxygen and sulfur, and with 
iron, copper, and many other metals. The trioxide $\mathrm{As}_{2} \mathrm{O}_{3}$, commonly called white arsenic, is obtained in large amounts as a by-product of the smelting of ores of copper and some other metals.

The free element is easily prepared by heating the trioxide with carbon; the vapor of arsenic so formed condenses on cooling to nearly black crystals. The latter are semimetallic in appearance and, like metals, conduct electricity well. Arsenic is not oxidized by air at ordinary temperatures but burns when strongly heated, forming the trioxide. When heated in a stream of chlorine, arsenic forms a trichloride, $\mathrm{AsCl}_{3}$, a colorless liquid boiling at $130^{\circ}$. This hydrolyzes almost completely when treated with much water, thus showing that the corresponding hydroxide, $\mathrm{As}(\mathrm{OH})_{3}$, is a very weak base.

On the other hand the hydroxide and also the trioxide dissolve readily in dilute alkalies to form salts in which $\mathrm{As}(\mathrm{OH})_{3}$ plays the part of an acid, called arsenious acid. Two series of salts are known: one derived from $\mathrm{H}_{3} \mathrm{AsO}_{3}$, the other from $\mathrm{HAsO}_{2}$. Arsenious acid, like phosphorous acid, $\mathrm{H}_{3} \mathrm{PO}_{3}$ (588), is a good reducing agent. It is oxidized by halogens or nitric acid to form arsenic acid, $\mathrm{H}_{3} \mathrm{AsO}_{4}$ :

$$
\mathrm{H}_{3} \mathrm{AsO}_{3}+\mathrm{H}_{2} \mathrm{O}+\mathrm{Cl}_{2} \rightarrow \mathrm{H}_{3} \mathrm{AsO}_{4}+2 \mathrm{HCl} \text {. }
$$

Arsenic acid, which is derived from the pentoxide $\mathrm{As}_{2} \mathrm{O}_{5}$, is a very soluble, moderately strong acid closely resembling phosphoric acid (590). Like the latter, it loses water when heated, giving first pyroarsenic acid, $\mathrm{H}_{4} \mathrm{As}_{2} \mathrm{O}_{7}$, and then metarsenic acid, $\mathrm{HAsO}_{3}$. Arsenic acid resembles phosphoric acid in giving with ammonium molybdate solution a yellow precipitate of ammonium arseno-molybdate, insoluble in nitric acid (597).

The numerous salts of arsenic acid closely resemble the corresponding salts of phosphoric acid. In fact Mitscherlich in the year I8I9 discovered that corresponding salts, e.g., $\mathrm{H}_{2} \mathrm{KPO}_{4}$ and $\mathrm{H}_{2} \mathrm{KAsO}_{4}$, were identical in crystalline form. Such compounds were said to be isomorphous. Later Mitscherlich declared that if two substances are isomorphous they must have the same numbers of atoms similarly arranged in the molecule. 
This principle enabled him to discover the correct formulae of many compounds of little-known elements at a time when much uncertainty prevailed regarding valence and atomic weights.

The action of hydrogen sulfide on a solution of arsenious acid acidified with $\mathrm{HCl}$ gives a precipitate of yellow arsenic trisulfide, $\mathrm{As}_{2} \mathrm{~S}_{3}$ (608). Acidified solutions of arsenic acid or its salts give under similar conditions arsenic pentasulfide, $\mathrm{As}_{2} \mathrm{~S}_{5}$, along with some trisulfide and sulfur, according to experimental conditions. These sulfides are insoluble in water and hydrochloric acid but are easily soluble in concentrated nitric acid, which changes the substances into sulfuric and arsenic acids. The sulfides also dissolve in ammonium sulfide to form soluble sulfarsenite, $\left(\mathrm{NH}_{4}\right)_{3} \mathrm{AsS}_{3}$, and sulfarsenate, $\left(\mathrm{NH}_{4}\right)_{3} \mathrm{AsS}_{4}$.

Arsenic and hydrogen form a gaseous compound called arsine, $\mathrm{AsH}_{3}$. This is produced along with hydrogen when any soluble arsenic compound is added to a mixture of zinc and hydrochloric acid. It is an extremely poisonous gas and must be handled with great caution. Arsine is readily decomposed into its elements by heat, so that if it is passed through a glass tube heated locally a dark mirror-like deposit of arsenic is formed in the tube. Marsh's test for arsenic is based on these reactions.

Most arsenic compounds are poisonous; one-fifth of a gram of the trioxide usually proves fatal. A good antidote consists of freshly prepared ferric hydroxide, which combines readily with arsenious acid to form an insoluble and therefore littlepoisonous compound.

8Ir. Antimony.-Antimony $(\mathrm{Sb}=\mathrm{I} 20)$ is classed as a metallic element because of the decidedly metallic properties of the free element and its ability to form salts with acids such as sulfuric and nitric. Nevertheless its hydroxides are also acidic and give rise to salts with bases, just as do the hydroxides of arsenic. Antimony is a widely distributed and rather common element occurring usually in combination with oxygen, sulfur, or metallic elements. China is the largest single source of the element. The metal forms brittle, silver-white crystals, melting at $630^{\circ}$. It forms useful alloys with many other metals. Type 
metal, an alloy with lead and bismuth, expands at the moment of solidifying in the mold and thus produces type with sharp, clear-cut edges. Britannia, made from tin, antimony, and copper, is used as a cheap substitute for silver plate. Babbitt metal, used for antifriction bearings, consists of antimony and tin with some lead and copper.

Antimony unites readily with chlorine to form the trichloride $\mathrm{SbCl}_{3}$, white crystals melting at $73^{\circ}$ and boiling at $223^{\circ}$. The chloride is to be considered the salt of the weak base $\mathrm{Sb}(\mathrm{OH})_{3}$; it is soluble in aqueous hydrochloric acid but is hydrolyzed by pure water. With a small proportion of water a white precipitate of sparingly soluble antimony oxychloride, $\mathrm{SbOCl}$, is formed,

$$
\mathrm{SbCl}_{3}+\mathrm{H}_{2} \mathrm{O} \rightarrow \mathrm{SbOCl}+2 \mathrm{HCl} \text {. }
$$

This reaction is reversible, $\mathrm{SbOCl}$ dissolving readily in concentrated $\mathrm{HCl}$. The univalent radical $\mathrm{SbO}$ present in several salts is called antimonyl; therefore $\mathrm{SbOCl}$ is also called antimonyl chloride.

Antimony nitrate, $\mathrm{Sb}\left(\mathrm{NO}_{3}\right)_{3}$, and antimony sulfate, $\mathrm{Sb}_{2}\left(\mathrm{SO}_{4}\right)_{3}$, are typical salts as far as their composition is concerned, but they are more or less completely hydrolyzed by water, depending on the proportion and temperature of the latter. The double salts potassium antimonyl tartrate, $\mathrm{K}(\mathrm{SbO}) \mathrm{C}_{4} \mathrm{H}_{4} \mathrm{O}_{6}$ (see 665), and sodium antimony fluoride, $\mathrm{NaSbF}_{4}$, are easily soluble salts which are not hydrolytically decomposed by water. Both of these salts are important mordants (809), and the former, commonly known as tartar emetic, is also used in medicine. In both cases stable complex ions $\left({ }_{538}\right)$ are formed; these are $(\mathrm{SbO}) \mathrm{C}_{4} \mathrm{H}_{4} \mathrm{O}_{6}^{-}$and $\mathrm{SbF}_{4}^{-}$respectively.

Antimony forms three oxides: $\mathrm{Sb}_{2} \mathrm{O}_{3}, \mathrm{Sb}_{2} \mathrm{O}_{4}$, and $\mathrm{Sb}_{2} \mathrm{O}_{5}$. The first and second can be made by direct union of antimony with oxygen, the second being the one stable in air at a red heat. The third, made indirectly, loses oxygen when strongly heated, giving $\mathrm{Sb}_{2} \mathrm{O}_{4}$. The trioxide corresponds to a hydroxide, $\mathrm{Sb}(\mathrm{OH})_{3}$, or $\mathrm{H}_{3} \mathrm{SbO}_{3}$, which by reason of its faintly acidic nature is known as antimonous acid. The latter unites with bases to form salts such as $\mathrm{Na}_{3} \mathrm{SbO}_{3}$ and $\mathrm{NaSbO}_{2} \cdot{ }_{3} \mathrm{H}_{2} \mathrm{O}$. Antimony pentoxide is 
the anhydride of antimonic acid, $\mathrm{H}_{3} \mathrm{SbO}_{4}$, a white powder sparingly soluble in water. This weak acid loses water when heated, giving in turn pyroantimonic acid, $\mathrm{H}_{4} \mathrm{Sb}_{2} \mathrm{O}_{7}$, metantimonic acid, $\mathrm{HSbO}_{3}$, and finally $\mathrm{Sb}_{2} \mathrm{O}_{5}$. Potassium hydrogen pyroantimonate, $\mathrm{K}_{2} \mathrm{H}_{2} \mathrm{Sb}_{2} \mathrm{O}_{7}$, is one of the best-known salts of these acids. Its solution gives with sodium salts a precipitate of sodium hydrogen pyroantimonate, $\mathrm{Na}_{2} \mathrm{H}_{2} \mathrm{Sb}_{2} \mathrm{O}_{7} \cdot 6 \mathrm{H}_{2} \mathrm{O}$.

Acid solutions of antimony salts give with hydrogen sulfide brick-red precipitates of antimony trisulfide, $\mathrm{Sb}_{2} \mathrm{~S}_{3}$, or pentasulfide, $\mathrm{Sb}_{2} \mathrm{~S}_{5}$. These sulfides are almost insoluble in water and cold dilute acids but dissolve easily in potassium hydroxide by reason of reactions like the following:

$$
{ }_{2} \mathrm{Sb}_{2} \mathrm{~S}_{3}+{ }_{4} \mathrm{KOH} \rightarrow{ }_{3} \mathrm{KSbS}_{2}+\mathrm{KSbO}_{2}+{ }_{2} \mathrm{H}_{2} \mathrm{O} .
$$

The soluble salt $\mathrm{KSbS}_{2}$ is called potassium sulfantimonite.

Antimony trisulfide also dissolves in yellow ammonium sulfide to form soluble ammonium sulfantimonate, $\left(\mathrm{NH}_{4}\right)_{3} \mathrm{SbS}_{4}$.

Antimony forms a gaseous hydride, $\mathrm{SbH}_{3}$, called stibine. This gas resembles arsine closely and is also decomposed by heat, with the formation of a black deposit of free antimony.

812. Bismuth.-Bismuth $(\mathrm{Bi}=208)$ is strictly metallic in properties, in distinction from arsenic, which is non-metallic, and antimony, which stands midway between the two in this respect. In its ordinary compounds bismuth is trivalent, but products probably pentavalent also exist. The element is much scarcer than either arsenic or antimony and commands a much higher price. It occurs free (native) and as oxide or sulfide, often admixed with ores of copper or lead. Bolivia is the chief source of bismuth. A considerable amount of it is obtained in the United States as a by-product in the electrolytic refining of lead. The metal forms rather hard, brittle crystals, silverwhite of a reddish tint. The melting-point is $269^{\circ}$. The metal is used in the making of easily fusible alloys. Thus Rose's metal, consisting of tin I, lead I, and bismuth 2 parts, melts at $94^{\circ}$; Wood's metal, tin I, lead 2 , bismuth 4 , and cadmium I, melts at $6 \mathrm{I}^{\circ}$. Fusible alloys are used for fire-sprinkler nozzles, electric fuse wires, etc. 
Bismuth forms with acids salts in which this element acts as a trivalent positive ion. Bismuth trichloride, $\mathrm{BiCl}_{3}$, meltint at $227^{\circ}$ and boiling at $428^{\circ}$, is made by the direct union of chlorine with the metal, or by the action of aqua regia (562) on the latter. The salt is soluble in water, but when diluted the salg hydrolyzes and forms a white precipitate of bismuth oxychloride, $\mathrm{BiOCl}$ (bismuthyl chloride or basic bismuth chloride). Bismuth nitrate, $\mathrm{Bi}\left(\mathrm{NO}_{3}\right)_{3}$, results from the action of nitric acid on the metal. It is soluble in water or dilute nitric acid but is hydrolyzed by much water, forming the basic nitrate $\mathrm{BiONO}_{3}$ (also called bismuth subnitrate). This product is a nearly insoluble white powder used both internally and externally in medicine. Quite large doses are easily tolerated. Bismuth sulfide, $\mathrm{Bi}_{2} \mathrm{~S}_{3}$, is formed as a brown precipitate when acid solutions of bismuth salts are treated with hydrogen sulfide. Sodium hydroxide or ammonia precipitates white bismuth hydroxide, $\mathrm{Bi}(\mathrm{OH})_{3}$, from a bismuth salt solution. Bismuth does not form a hydride.

813. Molybdenum.-Molybdenum $(\mathrm{Mo}=96)$ is found in nature chiefly as molybdenite, $\mathrm{MoS}_{2}$; a mineral closely resembling graphite $\left(6_{30}\right)$ in appearance. When the sulfide is heated in air (roasted) it is oxidized to the trioxide $\mathrm{MoO}_{3}$. The latter heated with hydrogen or carbon gives the metallic element. Molybdenum is a hard but malleable metal of very high meltingpoint (over $2400^{\circ}$ ). It is not oxidized by air at ordinary temperatures. The pure metal is not commercially important, but an alloy with iron, ferro-molybdenum, is made on a rather large scale. This alloy finds use in the making of certain kinds of highgrade tool steel.

The trioxide $\mathrm{MoO}_{3}$ is the most important of the several oxides. It is the anhydride of molybdic acid, $\mathrm{H}_{2} \mathrm{MoO}_{4} \cdot \mathrm{H}_{2} \mathrm{O}$, which consists of yellow crystals difficultly soluble in water. The hydrate loses water when heated gently, giving white $\mathrm{H}_{2} \mathrm{MoO}_{4}$, Sodium molybdate, $\mathrm{Na}_{2} \mathrm{MoO}_{4} \cdot 10 \mathrm{H}_{2} \mathrm{O}$, resembles Glauber's salt. $\mathrm{Na}_{2} \mathrm{SO}_{4} \cdot 10 \mathrm{H}_{2} \mathrm{O}$, in appearance. Molybdic acid resembles silicic acid in forming salts of complex formulae (807). Thus for example a sodium salt, $\mathrm{Na}_{6} \mathrm{Mo}_{7} \mathrm{O}_{24} \cdot{ }_{2} \mathrm{H}_{2} \mathrm{O}$, and an ammonium salt, $\left(\mathrm{NH}_{4}\right)_{6} \mathrm{Mo}_{7} \mathrm{O}_{24} \cdot{ }_{4} \mathrm{H}_{2} \mathrm{O}$, are known. The latter salt dis- 
solved in an excess of nitric acid gives the so-called ammonium molybdate solution, extensively used in the detection and quantitative analysis of phosphates (597). With this reagent a nitric acid solution of a phosphate or phosphoric acid gives quantitatively a yellow precipitate of ammonium phospho-molybdate, $\left(\mathrm{NH}_{4}\right)_{3} \mathrm{PO}_{4} \cdot 11 \mathrm{MoO}_{3} \cdot 6 \mathrm{H}_{2} \mathrm{O}$. Arsenic acid solutions give the corresponding arsenic compound under similar conditions (8Io).

814. Tungsten. - The element tungsten $(W=r 84)$ is of considerable practical importance. In the United ${ }^{\circ}$ States it is found chiefly in Colorado. Its principal ore is Wolframite, a tungstate of iron and manganese. The separate tungstates $\mathrm{FeWO}_{4}$ and $\mathrm{MnWO}_{4}$ also occur as minerals. Metallic tungsten is made in very pure form by the reduction of its oxide, $\mathrm{WO}_{3}$, by hydrogen at a high temperature. By reason of the extremely high melting-point of the metal the product is obtained as a powder. To get this into compact malleable and ductile form the powder is made into bars by powerful compression; these are then again heated electrically and the granules caused to melt together. Pure metallic tungsten, first made by Cooledge in I9I $\mathrm{I}$, is a hard, silver-white, and very ductile metal. Its most important use is for the manufacture of the filaments of incandescent electric lamps. Its great value for this purpose depends on the fact that its melting-point, $3267^{\circ}$, is higher than that of any other metal. It may be added that the efficiency of an electric lamp (ratio of light energy to electrical energy) increases greatly with the temperature to which the filament can be heated.

Ferro-tungsten ( 70 per cent $\mathrm{W}, 30$ per cent $\mathrm{Fe}$ ), made on a large scale by an electric-furnace process, is used in the manufacture of special steels. Tungsten forms two oxides, $\mathrm{WO}_{2}$ and $\mathrm{WO}_{3}$, the latter being far more important. The trioxide is the anhydride of tungstic acid, $\mathrm{H}_{2} \mathrm{WO}_{4}$, a yellow powder insoluble in water. This acid forms simple tungstates such as $\mathrm{Na}_{2} \mathrm{WO}_{4} \cdot 2 \mathrm{H}_{2} \mathrm{O}$, $\mathrm{K}_{2} \mathrm{WO}_{4}, \mathrm{CaWO}_{4}, \mathrm{PbWO}_{4}$, and the tungstates of iron and manganese already mentioned. Calcium tungstate, $\mathrm{CaWO}_{4}$, gives a fluorescent light with $\mathrm{X}$-rays and finds important use for the manufacture of fluoroscopic screens. Sodium tungstate is used to render cotton cloth slow-burning ("fireproof"). 
8i5. Selenium and Tellurium.-The elements selenium $(\mathrm{Se}=79)$ and tellurium $(\mathrm{Te}=\mathrm{I} 27 \cdot 5)$ are more closely related to sulfur than to any others. The first, selenium, is essentially non-metallic in its behavior. The second, tellurium, in free form is a silver-white crystalline metal which, in addition to forming some salts in which it is the basic ion, also forms two well-characterized acids. These elements are not very common. Practically they are obtained as by-products in the refining of copper. No important use has been found for tellurium. Selenium has two uses, one in the manufacture of red glass, the other in the construction of light-sensitive electrical apparatus. The electrical conductivity of selenium varies greatly with the intensity of its illumination, so that by ingenious use of this principle pictures, etc., have been transmitted long distances by wire. Lights on beacons and buoys may be turned on at dark by the use of selenium cells. Selenium and tellurium form compounds analogous to $\mathrm{H}_{2} \mathrm{~S}$ by methods similar to those that yield the latter gas.

Hydrogen selenide, $\mathrm{H}_{2} \mathrm{Se}$, is an ill-smelling gas, dangerously irritating to mucous membranes and temporarily paralyzing the sense of smell. Hydrogen telluride, $\mathrm{H}_{2} \mathrm{Te}$, is a similar gas. Selenium and tellurium both burn in air or oxygen when ignited and form white solid dioxides, $\mathrm{SeO}_{2}$ and $\mathrm{TeO}_{2}$. These oxides both unite with water to form selenous acid and tellurous acid respectively:

$$
\begin{aligned}
& \mathrm{SeO}_{2}+\mathrm{H}_{2} \mathrm{O} \rightarrow \mathrm{H}_{2} \mathrm{SeO}_{3}, \\
& \mathrm{TeO}_{2}+\mathrm{H}_{2} \mathrm{O} \rightarrow \mathrm{H}_{2} \mathrm{TeO}_{3} .
\end{aligned}
$$

These acids are the analogues of sulfurous acid, $\mathrm{H}_{2} \mathrm{SO}_{3}$ (6II), but are more stable than the latter in that both are white crystalline solids. They form typical salts with bases; not only normal salts like $\mathrm{K}_{2} \mathrm{SeO}_{3}$ and $\mathrm{K}_{2} \mathrm{TeO}_{3}$, but also acid salts like $\mathrm{KHSeO}_{3}$ and $\mathrm{KHTeO}_{3}$.

Unlike sulfurous acid, these acids are oxidizing agents, since they are reduced to selenium and tellurium respectively by reducing agents, as illustrated in the following equation:

$$
\mathrm{H}_{2} \mathrm{SeO}_{3}+{ }_{2} \mathrm{SO}_{2}+\mathrm{H}_{2} \mathrm{O} \rightarrow \mathrm{Se}+2 \mathrm{H}_{2} \mathrm{SO}_{4} \text {. }
$$

On the other hand, powerful oxidizing agents convert selenous and tellurous acids into selenic acid, $\mathrm{H}_{2} \mathrm{SeO}_{4}$, and telluric acid, 
$\mathrm{H}_{2} \mathrm{TeO}_{4}$, respectively. These acids form white crystals and are analogous in composition to sulfuric acid. Like the latter, they form both neutral and acid salts, e.g., potassium acid tellurate, $\mathrm{KHTeO}_{4}$. The selenates and tellurates are oxidizing agents tending to pass into selenites and tellurites. Thus, for example, potassium tellurate, when strongly heated, gives the tellurite and oxygen, or when heated with concentrated hydrochloric acid it gives potassium tellurite and chlorine. Telluric acid is decomposed by heat into its anhydride, tellurium trioxide, $\mathrm{TeO}_{3}$, and water.

816. Cobalt and Nickel.-The metals cobalt $\left(\mathrm{Co}=5^{8.97}\right)$ and nickel $(\mathrm{Ni}=58.68)$ resemble iron in both their physical and their chemical properties but are less active chemically, corresponding to positions below iron in the displacement series (492). Nickel is by far the more important of the two commercially, although cobalt commands the higher price. The two metals usually occur together, nickel being the more abundant. They are commonly found with iron, copper, or silver as sulfides or arsenides. Large deposits of nickel and cobalt occur in the province of Ontario, Canada.

A unique method of extracting nickel from its ores is based on the fact that carbon monoxide, $\mathrm{CO}\left(6_{32}\right)$, unites with finely divided metallic nickel at $80^{\circ}$ to form a compound, $\mathrm{Ni}(\mathrm{CO})_{4}$, nickel carbonyl, which is gaseous above $44^{\circ}$. The ores, after they have been roasted to remove sulfur and arsenic and to convert the metals into oxides, are first extracted with sulfuric acid to remove copper and are then reduced to metal by water-gas, a mixture of $\mathrm{H}_{2}$ and $\mathrm{CO}$, at $400^{\circ}$. The residue, cooled to $80^{\circ}$, is exposed to a stream of highly compressed $\mathrm{CO}$, and the volatile $\mathrm{Ni}(\mathrm{CO})_{4}$ which passes off is decomposed at $200^{\circ}$ into pure metallic nickel and carbon monoxide,

$$
\mathrm{Ni}(\mathrm{CO})_{4} \rightarrow \mathrm{Ni}+{ }_{4} \mathrm{CO} \text {. }
$$

This is the Mond process. Other processes of refining nickel are also in use.

Nickel is so extensively used for plating copper, brass, and iron that its appearance and permanence in the air are known to everyone. It is harder than iron and melts at $1450^{\circ}$. The metal 
finds one of its most important uses in the manufacture of nickel steel, of which it may constitute several per cent. Cobalt may also be present to advantage in such steel. Nickel steel is very hard and tough and is largely used for armor plate.

Our five-cent coins, "nickels," are an alloy of 25 per cent nickel and 75 per cent copper. The well-known properties of this alloy need no comment. The alloy called German silver consists of nickel, copper, and zinc. Monel metal is $\mathrm{Ni} 68$ per cent, $\mathrm{Cu} 30.5$ per cent, and $\mathrm{Fe} \mathrm{I} .5$ per cent. It resembles the alloy of our nickel coins but is harder and more resistant to chemicals. It is obtained by direct smelting of copper-nickel-iron ores.

Cobalt resembles nickel in appearance and properties. It is not much used as a metal. Its oxide, $\mathrm{CoO}$, is used in making blue glass, and since no other means is known of making a good blue glass this element is of considerable importance. Blue glazes for porcelain and a blue pigment, smalt, are also made from cobalt oxide.

8I7. Salts of Cobalt and Nickel.-The bivalent oxides and hydroxides of cobalt and nickel are basic and have no acidic properties. They form salts with practically all acids. In its salts nickel is always and cobalt usually bivalent, although a few trivalent salts of the latter element are known. The salts of these elements correspond closely in composition to those of ferrous iron. Of the commoner salts of (bivalent) cobalt and nickel we may mention the fluorides, chlorides, bromides, iodides, nitrates, sulfates, carbonates, and phosphates. These salts, with the exception of the carbonates and phosphates, are readily soluble in water and as a rule form crystals with six molecules of water to one of the salt, e.g., $\mathrm{Co}\left(\mathrm{NO}_{3}\right)_{2} \cdot 6 \mathrm{H}_{2} \mathrm{O}$ and $\mathrm{NiCl}_{2} \cdot 6 \mathrm{H}_{2} \mathrm{O}$. Nickel sulfate forms besides the hexahydrate a heptahydrate, $\mathrm{NiSO}_{4} \cdot 7 \mathrm{H}_{2} \mathrm{O}$, isomorphous (8Io) with $\mathrm{MgSO}_{4} \cdot{ }_{7} \mathrm{H}_{2} \mathrm{O}$ (144). Cobalt sulfate also forms a hexahydrate and a heptahydrate, the latter isomorphous with $\mathrm{FeSO}_{4} \cdot{ }_{7} \mathrm{H}_{2} \mathrm{O}$ (I73). Many double salts (I75) are also known. Ammonium nickel sulfate, $\left(\mathrm{NH}_{4}\right)_{2} \mathrm{SO}_{4} \cdot \mathrm{NiSO}_{4} \cdot 6 \mathrm{H}_{2} \mathrm{O}$, is used in electroplating.

The hydrated salts of cobalt are red in crystalline form and also in solution, in which latter $\mathrm{Co}^{++}$ions are present. Many 
anhydrous cobalt salts are blue. If one uses a dilute cobalt chloride solution, pale red in color, as ink, the writing will be practically invisible when air dry. If the paper is heated the writing appears in easily legible blue characters. The effect of the heating is to drive off the water of hydration. A writingfluid of this kind is known as sympathetic ink. Cobalt forms a large number of compounds called cobalt amines. These are complex substances containing ammonia and trivalent cobalt. Hexamine cobaltic chloride, $\left(\mathrm{NH}_{3}\right)_{6} \mathrm{CoCl}_{3}$, formed by the action of ammonia and oxidizing agents on a solution of cobaltous chloride, $\mathrm{CoCl}_{2}$, is a typical example. Solutions of cobalt amines must contain complex ions (538), since they do not give the ordinary reactions of solutions of simple cobalt salts. Nickel does not form analogous compounds.

Hydrated nickel salts are green and form green solutions in which bivalent $\mathrm{Ni}^{++}$ions are present. Simple cobalt and nickel solutions give precipitates with alkaline hydroxides, carbonates, phosphates, and sulfides. These are all soluble in ammonia solution by reason of the formation of complex ions of various sorts.

818. Platinum.-Platinum $(\mathrm{Pt}=195.2)$ occurs only in the metallic state, often as an alloy with the rarer metals osmium and iridium. It comes chiefly from the Ural Mountains in Russia. Platinum is almost indispensable in chemical laboratories, where it is used as crucibles, dishes, tubes, etc. Its value for such uses arises from the fact that it is very inactive and has a high melting-point. It is not attacked by any of the common acids singly, although it is slowly converted into its chloride, $\mathrm{PtCl}_{4}$, by aqua regia (562), a mixture of hydrochloric and nitric acids. Platinum is an active catalytic agent for many reactions, such as the union of hydrogen and oxygen (303), the union of sulfur dioxide and oxygen (6/7), and the oxidation of ammonia to nitric acid (570). It finds extensive use catalytically in the manufacture of sulfuric and nitric acids. Since platinum has in recent years become much more valuable than gold, it has gained great popularity as a setting for jewels. Its use in jewelry was restricted and later prohibited during the war in order that the metal might be conserved for chemical uses. The use in 
jewelry of this noble metal, so necessary for chemistry and industry, ought permanently to be prohibited. The compounds of platinum are not of much practical importance. Until recently the chloride $\mathrm{PtCl}_{4}$, a very soluble yellow salt, was used in the quantitative determination of potassium. Potassium chloride forms with $\mathrm{PtCl}_{4}$ a very difficultly soluble complex salt, potassium platinic chloride, $\mathrm{K}_{2} \mathrm{PtCl}_{6}$, in to which all the potassium in a given sample could be converted and this form isolated and weighed.

All platinum compounds are easily reduced to the free element. The chloride, for example, gives off chlorine upon being heated to a red heat,

$$
\mathrm{PtCl}_{4} \rightarrow \mathrm{Pt}+{ }_{2} \mathrm{Cl}_{2} \text {. }
$$

8ig. Gold.-Gold (Au= 197.2$)$ has been known from prehistoric times. It usually occurs in free form (native gold) but is also found in compounds such as the telluride (8I5). Several methods are in use for the extraction of gold from its ores. The simplest one is the amalgamation process, in which sand or crushed rock containing free gold is carried by running water over copper plates covered with a film of mercury. The gold forms an alloy (amalgam) with the mercury, from which it can be freed subsequently by distilling off the latter. In the chlorination process ores are extracted with a solution of chlorine, in which gold dissolves as auric chloride, $\mathrm{AuCl}_{3}$. From the chloride solution the gold may be precipitated by a variety of reducing agents, e.g., ferrous sulfate, etc. More important than the preceding is the cyanide process, by which enormous quantities of gold are recovered from low-grade ores. This process is based on the formation of readily soluble sodium auricyanide, $\mathrm{NaAu}(\mathrm{NC})_{2}$, which contains the very stable complex ion $\mathrm{Au}(\mathrm{NC})_{2}{ }^{-}$. The reaction is as follows:

$$
4 \mathrm{Au}+8 \mathrm{NaNC}+{ }_{2} \mathrm{H}_{2} \mathrm{O}+\mathrm{O}_{2} \rightarrow{ }_{4} \mathrm{NaAu}(\mathrm{NC})_{2}+{ }_{4} \mathrm{NaOH} \text {. }
$$

The crushed ore, which must sometimes be roasted to free it from sulfide, etc., is placed in immense tanks and extracted with a solution of sodium cyanide (665). The resulting extract is then run over zinc shavings to precipitate the gold,

$$
2 \mathrm{NaAu}(\mathrm{NC})_{2}+\mathrm{Zn} \rightarrow \mathrm{Na}_{2} \mathrm{Zn}(\mathrm{NC})_{4}+2 \mathrm{Au} \text {. }
$$


Many copper, silver, and lead ores contain gold which accompanies the metals when they are obtained by smelting processes. This gold is recovered when the metals are refined electrolytically.

The principal gold-producing regions in the order of their importance are the Transvaal (South Africa), United States including Alaska, Australia, Russia including Siberia, Mexico, and Canada. The value of the world's production of gold during the year 1915 was 473 million dollars.

Gold is a rather soft metal and is usually alloyed with copper or other metals. Pure gold is designated as $\mathbf{2 4}$ carat. Eighteencarat gold, used in high-grade jewelry, is 75 per cent pure. United States gold coins consist of 90 per cent gold and ro per cent copper. Gold is the most malleable and ductile of all metals.

Metallic gold is not attacked by any of the common acids. It dissolves in aqua regia (562), a mixture of hydrochloric and nitric acids, to form auric chloride, $\mathrm{AuCl}_{3}$. This salt gives with hydrochloric acid, chlorauric acid, $\mathrm{HAuCl}_{4} \cdot{ }_{3} \mathrm{H}_{2} \mathrm{O}$, forming yellow crystals, and with sodium chloride, sodium chloraurate, $\mathrm{NaAuCl}_{4} \cdot{ }_{2} \mathrm{H}_{2} \mathrm{O}$. Aurous chloride, $\mathrm{AuCl}$, is an insoluble salt obtained by gently heating $\mathrm{AuCl}_{3}$.

A solution of auric chloride is used in toning photographs. In the ensuing reaction the silver of the picture is replaced (492) by metallic gold $\mathrm{Au}$,

$$
\mathrm{AuCl}_{3}+3 \mathrm{Ag} \rightarrow \mathrm{Au}+3 \mathrm{AgCl} .
$$

Two oxides of gold are known, aurous oxide, $\mathrm{Au}_{2} \mathrm{O}$, and auric oxide, $\mathrm{Au}_{2} \mathrm{O}_{3}$; these both give oxygen and metallic gold when heated.

820. The Rare Earth Elements. - The term rare earth elements is used to designate a large group of similar trivalent elements. The name indicates that these more or less rare elements bear some resemblance to aluminum, the silicate of which constitutes clay, a typical earthy substance. One of the most abundant sources of these elements is the mineral monazite, which is found in North Carolina, South Carolina, 
Florida, Idaho, Brazil, and India. Monazite ordinarily occurs as a heavy yellow sand. It is a mixed phosphate of cerium, lanthanum, neodymium, and thorium, with smaller proportions of many other rare earth elements.

Monazite sand is worked up primarily for the thorium it contains. Strictly speaking, thorium is not a rare earth element, although in its chemical behavior it is similar to these elements.

Thorium nitrate, $\mathrm{Th}\left(\mathrm{NO}_{3}\right)_{4}$, is extensively used to make incandescent gas mantles; these consist, to the extent of over 99 per cent, of thorium oxide, $\mathrm{ThO}_{2}$, and must contain 0.6 to 0.8 per cent of cerium oxide, $\mathrm{CeO}_{2}$ (301).

A brief description of the chemistry of cerium, lanthanum, and neodymium will serve to illustrate the nature of the rare earth elements, which are all much alike in their properties. In free form the three elements mentioned are metals stable in air and toward water. An alloy of the three metals, called mixed metal, is made technically by the electrolysis of the molten chlorides $\mathrm{CeCl}_{3}, \mathrm{LaCl}_{3}, \mathrm{NdCl}_{3}$. An alloy of 70 per cent mixed metal and 30 per cent iron gives out a shower of sparks of burning metal when scratched with a file. It is for this reason known as pyrophoric alloy and finds use in cigar lighters, gas lighters, etc.

These elements form bases and salts in which they are trivalent. The hydroxides, e.g., $\mathrm{La}(\mathrm{OH})_{3}$, are almost insoluble, moderately strong bases. The nitrates, chlorides, and sulfates are soluble and are not appreciably hydrolyzed in solution. The carbonates, phosphates, and oxalates are insoluble in water. Cerium differs from the other two elements in forming a series of quadrivalent salts derived from the oxide $\mathrm{CeO}_{2}$. In these the ceric ion, $\mathrm{Ce}^{4+}$, is a strong oxidizing agent. The ceric ion, $\mathrm{Ce}^{++}$, is orange in color, while cerous ion, $\mathrm{Ce}^{+++}$, is colorless, as is also lanthanum ion $\mathrm{La}^{+++}$. Neodymium ion, $\mathrm{Nd}^{+++}$, is a beautiful rose color. In addition to these rare earths there are eleven others, all of which are less common than the three here mentioned. 


\section{CHAPTER XXXI}

\section{CLASSIFICATION OF THE ELEMENTS. THE PERIODIC SYSTEM}

821. Introduction.-By reason of the enormous number of known chemical substances the best that a trained chemist can hope or even wish to do is to become thoroughly familiar with the principles and laws of his science and to know a moderate number of facts regarding the commoner substances, together with those with which his own field of work brings him in contact. His mind should be a laboratory and not a warehouse; otherwise he will soon find it so crowded with useless and unrelated data (chemical junk!) that he has no ability to solve the new chemical problems that will constantly confront him. If one knows well the fundamental chemical principles and is familiar with the chemical behavior of the most typical of the elements he will find it easy to understand the chemistry of any unfamiliar element he may have occasion to study. Morcover, by reason of the relationships between the elements to be discussed in this chapter he will be able from a knowledge of a very few facts regarding the element in question to predict with more or less assurance the general behavior of the element and its compounds.

We shall now consider briefly the entire list of the elements with the view of bringing out their relations to one another and of showing that they may be classified into groups (families) and series in such a way as clearly to exhibit these relationships. In fact, every element finds a definite place in this system of classification, which for reasons soon to be shown is called the Periodic System.

822. Chemical Groups or Families. The Halogens.-The similarities in properties and behavior of chlorine, bromine, and iodine and of their corresponding compounds must be well known to the reader at this stage of advancement. Let us review some of the facts already established and consider also 
a few additional ones. These elements are all non-metals which form the colorless gases $\mathrm{HCl}, \mathrm{HBr}$, and $\mathrm{HI}$, all of which dissolve easily in water to form strong acids. In these acids and their salts the halogen is always univalent. Although the free halogens are colored their ions in solution are all colorless. The salts, with the exception of those of silver, lead, and univalent mercury, are easily soluble in water. The three halogens being considered all form oxygen acids and salts of a class represented by $\mathrm{KClO}_{3}$. Thus we have the acids chloric, $\mathrm{HClO}_{3}$, bromic, $\mathrm{HBrO}_{3}$, and iodic, $\mathrm{HIO}_{3}$, and their corresponding salts, the chlorates, bromates, and iodates.

If now we turn to the differences between the corresponding compounds of the three halogens we observe that the properties of bromine and its compounds are in most cases nearly intermediate between those of chlorine and iodine. Let us consider first the matter of atomic weights:

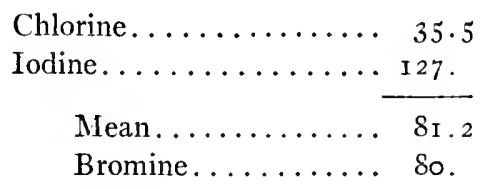

The atomic weight of bromine is seen to be very close to the mean of the atomic weights of the other two elements. The data shown in Table XLV illustrate the fact that bromine and

TABLE XLV

Comparison of the Properties of Chlorine, Bromine, and IOdine AND Their CoMpounds

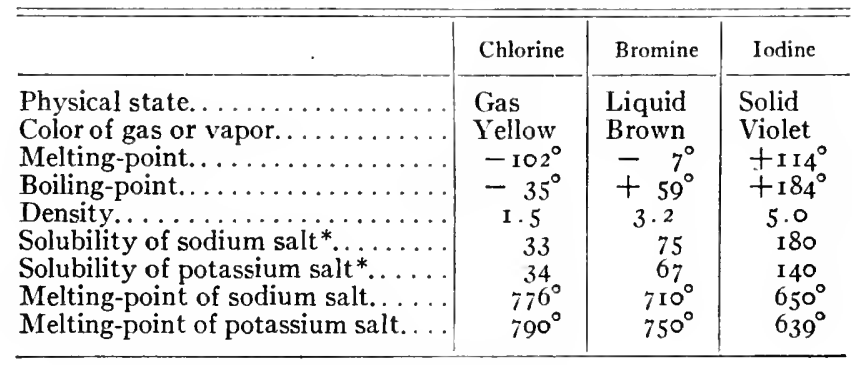

* Grams of salt dissolved by $100 \mathrm{~g}$. of water at $20^{\circ}$. 
its compounds stand nearly midway between chlorine and iodine and their corresponding compounds; so that if we know the properties of compounds of the latter we can predict pretty nearly those of the former.

Fluorine, although classed as a halogen, is less closely related to the three elements just discussed than are the latter to one another.

823. The Alkali Metals.--Sodium and potassium are called the alkali metals. This group or family also includes lithium (786), a much less common element, and in addition two rare elements, rubidium and caesium. These two elements form with sodium and potassium as closely related a group as do chlorine, bromine, and iodine. Lithium, although showing many points of similarity to the other four elements, bears about the same sort of relation to the others that fluorine does to the other halogens. Table XLVI gives some typical data on this

TABLE XLVI

Comparison of the Properties of the Alkali Metals and Their Compounds

\begin{tabular}{|c|c|c|c|c|c|}
\hline & Lithium & Sodium & Potassium & Rubidium & Caesium \\
\hline 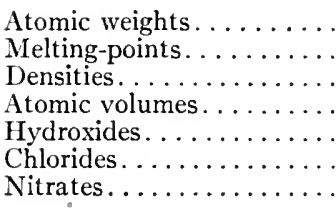 & $\begin{array}{l}6.94 \\
186^{\circ} \\
0.59 \\
\mathrm{rI} .9 \\
\mathrm{LiOH} \\
\mathrm{LiCl} \\
\mathrm{LiNO}_{3}\end{array}$ & $\begin{array}{l}23.00 \\
98^{\circ} \\
0.97 \\
23.7 \\
\mathrm{NaOH} \\
\mathrm{NaCl} \\
\mathrm{NaNO}_{3}\end{array}$ & $\begin{array}{c}39.01 \\
62^{\circ} \\
\circ .87 \\
44.8 \\
\mathrm{KOH} \\
\mathrm{KCl} \\
\mathrm{KNO}_{3}\end{array}$ & $\begin{array}{c}85 \cdot 45 \\
38^{\circ} \\
\mathrm{I} \cdot 5^{2} \\
56 \cdot \mathrm{I} \\
\mathrm{RbOH} \\
\mathrm{RbCl} \\
\mathrm{RbNO}_{3}\end{array}$ & $\begin{array}{l}132.8 \mathrm{I} \\
26^{\circ} \\
\mathrm{I} .88 \\
70.6 \\
\mathrm{CsOH} \\
\mathrm{CsCl} \\
\mathrm{CsNO}_{3}\end{array}$ \\
\hline
\end{tabular}

group. Again we find some simple relations between atomic weights, as in the case of the halogens. Thus the atomic weight of sodium, 23, is practically the mean of the atomic weights of lithium and potassium, while that of rubidium stands almost midway between those of potassium and caesium. Curiously enough, however, the atomic weight of potassium, 39, is much less than the mean of the values for the neighboring elements sodium and rubidium, namely 54 .

All of the five elements of this family are typical metals. As such they are all very active, caesium most so and lithium least. With water they all react readily to form strong, soluble 
bases, resembling sodium hydroxide. This means that they all form positive ions, all of which are univalent. The metals are all soft (easily cut with a knife). They are all silver-white and have low melting-points, rubidium and caesium melting below blood heat. The melting-points decrease in order from lithium to caesium. The densities are all lower than those of any other metals. In general the higher the atomic weight the greater the density; but we find that the value for sodium is exceptional in being a little higher than that of potassium. If, however, we calculate from the density and atomic weight the so-called atomic volume, that is the volume in cubic centimeters occupied by one gram atomic weight of the element, we find that these constants increase steadily with increasing atomic weight, and that the value for sodium is no longer irregular. Since the gram atomic weights (symbol weights) of all elements contain equal numbers of atoms, the atomic volumes are the volumes of equal numbers of atoms. By reference to Table XLVI we see that in this family the heavier an atom is the greater the volume it occupies.

824. The Alkali Earth Group.-The alkali earth group includes the four elements calcium, strontium, barium, and radium. These are all bivalent metals whose hydroxides are moderately strong bases, and whose salts are typically represented by calcium chloride, $\mathrm{CaCl}_{2}$, and sulfate, $\mathrm{CaSO}_{4}$. A detailed examination of this group would show that its members closely resemble one another in general, and also that a systematic and gradual change of properties accompanies change of atomic weight just as in the halogen and alkali metal families.

825. The Inert Gases.-The inert gases helium, neon, argon, krypton, and xenon (79x-797) must obviously be considered as belonging to a separate family. They are all devoid of chemical properties and are therefore of valence zero in all cases. As gases their densities are of course all proportional to their molecular weights (Avogadro's Law, I93). The ratio of the molecular heat at constant pressure to that at constant volume is 1.66 in each case, thus showing that each gas is monatomic (793). The critical temperatures and boiling-points of the liquid gases all increase with increasing atomic weight from the 
lowest values found in the case of helium. No other elements (excepting niton, radium emanation, chap. xxxii) resemble the five members of this family at all closely. Sometimes the inert gases are called the noble gases, because the most striking characteristic of the noble metals, gold and platinum, is the reluctance with which they enter into chemical combination.

826. Series of Elements. - If we arrange the elements in the order of their atomic weights the first of the series is obviously hydrogen. The next is helium. The next seven are shown in Table XLVII. The data presented in this table show that the

TABLE XLVII

The First Series of Elements

\begin{tabular}{|c|c|c|c|c|c|c|c|}
\hline & $\mathrm{Li}$ & $\mathrm{Be}$ & B & C & $\mathrm{N}$ & 0 & $F$ \\
\hline $\begin{array}{l}\text { Serial numbers } \ldots \ldots \ldots \\
\text { Atomic weights . . . . . } \\
\text { Melting-points. . . } \ldots \ldots \\
\text { Chlorine compounds .... } \\
\text { Valence toward chlorine . } \\
\text { Hydrogen compounds. . } \\
\text { Valence toward hydrogen. }\end{array}$ & $\begin{array}{c}3 \\
7 \\
186^{\circ} \\
\mathrm{LiCl} \\
\quad \mathbf{I} \\
\end{array}$ & $\begin{array}{c}4 \\
9 \\
\mathrm{I} 80^{\circ} \\
\mathrm{BeCl}_{2} \\
2 \\
2\end{array}$ & $\begin{array}{c}5 \\
\mathrm{II} \\
2350^{\circ} \\
\mathrm{BCl}_{3} \\
3 \\
3\end{array}$ & $\begin{array}{c}6 \\
\mathrm{I} 2 \\
3600^{\circ} \\
\mathrm{CCl}_{4} \\
4 \\
\mathrm{CH}_{4} \\
4\end{array}$ & $\begin{array}{c}7 \\
14 \\
-210^{\circ} \\
\ldots \ldots \ldots\end{array}$ & $\begin{array}{c}8 \\
16 \\
-218^{\circ} \\
\ldots \ldots \ldots\end{array}$ & 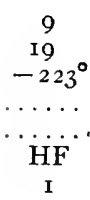 \\
\hline
\end{tabular}

valence toward chlorine increases by one as we pass from lithium, one, to carbon, four, and that the valence toward hydrogen decreases by units from carbon, four, to fluorine, one. There is also a gradual change of both physical and chemical properties as we go from lithium to fluorine. For example, lithium is a light, soft, silver-white metal (density 0.59). It readily decomposes cold water. Lithium hydroxide is a strong base, and its salts are not hydrolyzed in solution. Beryllium is a white malleable metal of density r.64. It does not tarnish in air, but it slowly decomposes hot water. Beryllium hydroxide is a rather weak base. The chloride is appreciably hydrolyzed in water solution. Boron $(80 \mathrm{r}-803)$ is a non-metallic solid of very high melting-point. It is not acted on by water or by oxygen except at high temperatures. Its hydroxide, $\mathrm{B}(\mathrm{OH})_{3}$, or $\mathrm{H}_{3} \mathrm{BO}_{3}$, boric acid, is an extremely weak acid. The chloride $\mathrm{BCl}_{3}$ is completely hydrolyzed by water to form boric and 
hydrochloric acids. Carbon is strictly non-metallic in the form of diamond (the latter is an electrical, insulator-like glass), and only semimetallic as graphite (this has a semimetallic luster and conducts the current fairly well, 630). Carbon tetrachloride, $\mathrm{CCl}_{4}(644)$, is a colorless liquid with none of the characteristics of a salt. The other three members of the series, nitrogen, oxygen, and fluorine, are all gases and all strictly non-metallic in character. Their activity increases very markedly from nitrogen, through oxygen, to fluorine. In their hydrogen compounds the last four elements of the series show a progressive decrease in valence from four for carbon to one for fluorine. Only the hydrogen compounds of nitrogen, oxygen, and fluorine form compounds with metals, e.g., $\mathrm{NaNH}_{2}$ (527), $\mathrm{NaOH}$, and $\mathrm{NaF}$. The first is decomposed completely by water.

827. The Second Series.-The seven elements beginning with sodium taken in increasing order of their atomic weights form a second series as illustrated in Table XLVIII. Again in

\section{TABLE XLVIII}

The Second Series of Elemexts

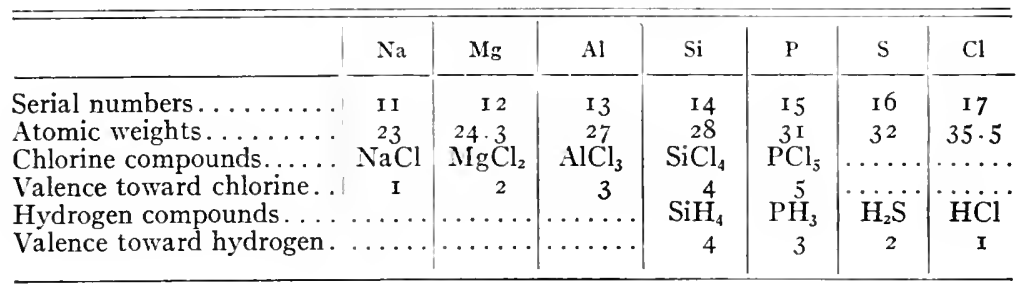

this series metallic properties characterize the first three elements. The fourth, silicon, is a hard, black, difficultly fusible element with fair electrical conductivity; it may well be classed as semimetallic. The following three elements are truly non-metallic. Chemical activity, great in the case of sodium, diminishes toward silicon, which is quite inert, and then increases again to a maximum with chlorine. In this connection it is of importance to note that the activities of the first three metals are represented by their tendencies to take on positive charges (lose electrons, 49I), while those of the last three elements are due to a tendency 
to acquire negative charges (gain electrons, 489). Furthermore the valences change by unity as we pass from one to the next element in the series.

828. Correlation of the First and Second Series.-If we write the two series of elements in parallel columns a very remarkable relationship at once appears, as shown in Table XLIX. We see that $\mathrm{Li}$ and $\mathrm{Na}$, both members of the alkali-

TABLE XLIX

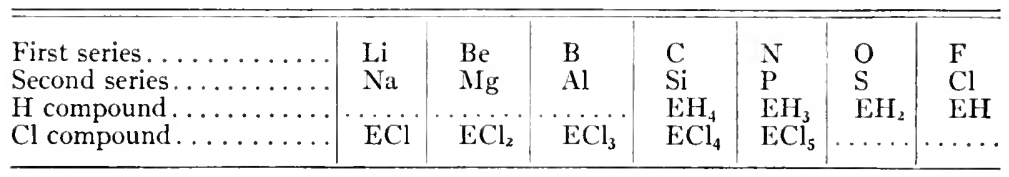

metal group, fall in the same vertical column; likewise the halogens, $\mathrm{F}$ and $\mathrm{Cl}$. Furthermore, if $\mathrm{E}$ is written as the general symbol of any element, the compounds with $\mathrm{H}$ or $\mathrm{Cl}$ for the two elements of any vertical column can be represented by the same formula. In other words, this arrangement brings elements of similar properties into the same vertical columns.

829. The Periodic Table of the Elements.-Table $\mathrm{L}$ shows a systematic arrangement of 70 of the 83 elements. In this table the first two horizontal lines reproduce the two series of the preceding section and in addition include the elements helium, $\mathrm{He}$, and neon, Ne. Following chlorine all known elements to and including cerium, Ce, I4O.2, are given in the order of increasing atomic weights, with the exception that argon, A, 39.9, precedes potassium, K, 39.I; and tellurium, Te, I 27.5, precedes iodine, I, I26.9. Between cerium, Ce, I40.2, and tantalum, Ta, I8I.5, a number of rare earth elements are omitted $(820,846)$.

The first and second series, called also the first and second periods respectively, each includes eight elements. The double series of 18 elements beginning with argon, A, 39.9, and ending with bromine, 79.9, forms the third period. It will be noticed that a group of three elements, iron, Fe, cobalt, $\mathrm{Co}$, and nickel, $\mathrm{Ni}$, follows manganese, $\mathrm{Mn}, 54.9$, in the column headed Group VIII. Copper, the next element after nickel, is placed in Group I and not under argon in Group O. The 


\begin{tabular}{|c|c|c|c|c|c|c|c|c|c|}
\hline & $\begin{array}{l}5 \\
5 \\
00 \\
0.04 \\
0\end{array}$ & 1 & & 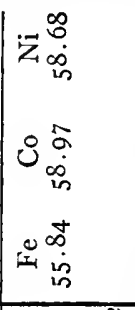 & 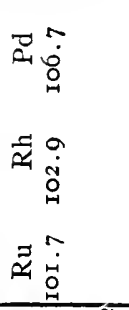 & & 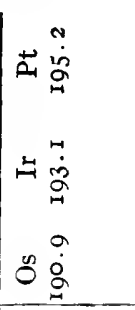 & & 屌 \\
\hline : & 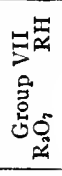 & 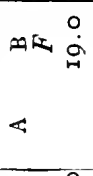 & 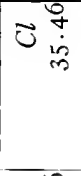 & 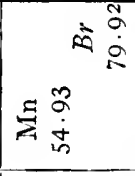 & r. & ^. & a. & & 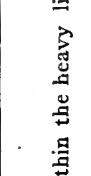 \\
\hline & 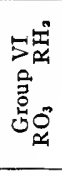 & $\begin{array}{l}0 \\
0 \\
\infty\end{array}$ & v & जั & 先 & : & $\geqslant \stackrel{\circ}{\dot{G}}$ & 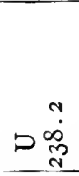 & 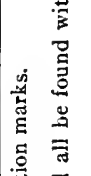 \\
\hline 窇 & 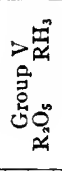 & 飞杵 & $2 \stackrel{\substack{0 \\
\dot{m}}}{\text { D }}$ & 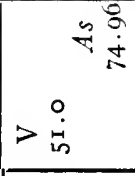 & 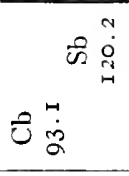 & : & 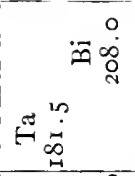 & 2 & 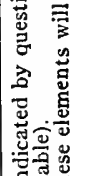 \\
\hline 总 & 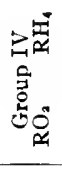 & ৫ & $\ddot{s} \underset{\sim}{m}$ & 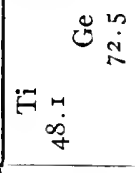 & 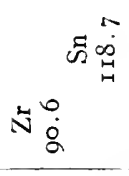 & ن & हि & 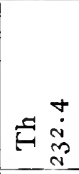 & 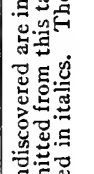 \\
\hline & 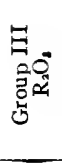 & 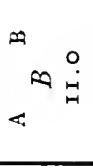 & 落 & 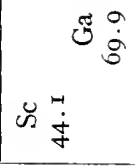 & 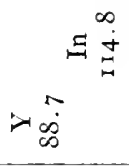 & 今̊요 & E 웡 & n. & 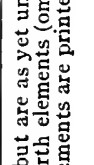 \\
\hline & 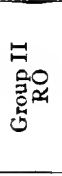 & $\begin{array}{l}\infty \\
\ddot{0} \dot{0}\end{array}$ & 赵 & जี & 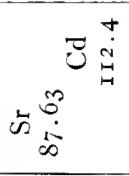 & 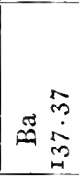 & 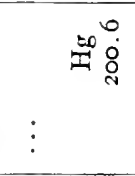 & 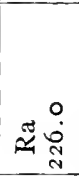 & 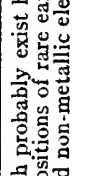 \\
\hline & ت્م0 & 《ت口 & 艺号 & is & 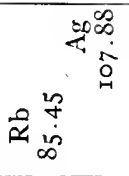 & $\stackrel{\stackrel{H}{\infty}}{\stackrel{\infty}{\sim}}$ & $\underset{殳}{3} \stackrel{2}{9}$ & $n$ & 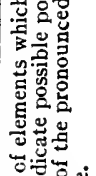 \\
\hline & 足 & $\stackrel{\oplus}{\Delta}+$ & $\ddot{z} \stackrel{\circ}{\circ}$ & $\varangle^{\infty} \stackrel{\infty}{\dot{m}}$ & मू & A & & 艺 & 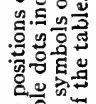 \\
\hline & 表 & $\rightarrow$ & $\sim$ & $m$ & + & in & 0 & $N$ & 胥言 \\
\hline
\end{tabular}


fourth period begins with krypton, $\mathrm{Kr}, 82.9$, and ends with iodine, I26.9. The fifth period includes the elements beginning with xenon, $\mathrm{X}$, I30.2. The sixth period, which is defective, shows tantalum as its first and bismuth as its last member. The seventh period contains only four elements, of which niton, $\mathrm{Nt}, 222.4$, is the first and uranium, U, 238.2, the last. No element of higher atomic weight than uranium is known. The first two periods are called short periods, and the next four long periods. The seventh, and last, period is fragmentary.

830. The Nine Groups of Elements. The Zero Group.-The elements contained in any one of the nine vertical columns headed $\mathrm{O}$ to VIII constitute a group. There are, therefore, nine such groups. The zero group consists of the five inert gases (rare or noble gases studied earlier, 825), together with niton (radium emanation, see chap. xxxii), which is also inert chemically. None of these gases forms any chemical compounds. The members of the zero group never have other than zero valence.

831. The First Group. A and B Families.-Reference to the Periodic Table shows that five of the eight elements of Group I belong to the alkali-metals family. These elements are lithium, sodium, potassium, rubidium, and caesium. The other three elements, copper, silver, and gold, as we already know from their earlier study, are widely different from sodium and potassium, the best-known alkali metals. In many respects copper, silver, and gold are much alike and may be classed together as a family. Let us compare and contrast the two families of Group I, which we may designate as IA and IB respectively. The differences are set forth in Table LI.

TABLE LI

\begin{tabular}{|c|c|c|}
\hline \multirow{2}{*}{ ElesheNts } & IA & IB \\
\hline & $\mathrm{Li}, \mathrm{Na}, \mathrm{K}, \mathrm{Rb}, \mathrm{Cs}$ & $\mathrm{Cu}, \mathrm{Ag}, \mathrm{Au}$ \\
\hline 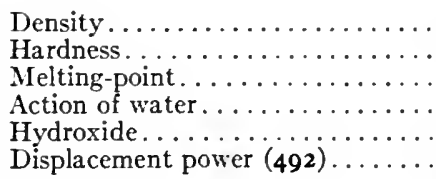 & $\begin{array}{l}0.59 \text { to } 1.88 \\
\text { Very soft } \\
26^{\circ} \text { to } 186^{\circ} \\
\text { Give hydroxides } \\
\text { Soluble strong bases } \\
\text { Very high }\end{array}$ & $\begin{array}{l}8.8 \text { to } 19.2 \\
\text { Hard } \\
960^{\circ} \text { to } 1060^{\circ} \\
\text { No action } \\
\text { Insoluble or unstable } \\
\text { Very low }\end{array}$ \\
\hline
\end{tabular}


The differences are set forth in Table LI. The members of IA are uniformly univalent when in combination and form such compounds as $\mathrm{ECl}, \mathrm{EBr}, \mathrm{ENO}_{3}, \mathrm{E}_{2} \mathrm{SO}_{4}, \mathrm{EOH}$, and $\mathrm{E}_{2} \mathrm{O}$. In sodium peroxide, $\mathrm{Na}_{2} \mathrm{O}_{2}$, we believe that sodium is still univalent and represents the structural formula thus: $\mathrm{Na} \cdot \mathrm{O} \cdot \mathrm{O} \cdot \mathrm{Na}(324)$. In IB copper and gold are variable in their valences; thus copper forms in addition to the commoner compounds in which it is bivalent (165) a series of univalent compounds, of which cuprous chloride, $\mathrm{CuCl}$, is an example. Gold has so little chemical affinity that it does not form many stable compounds, but in these it is usually univalent or trivalent. It forms two oxides, $\mathrm{Au}_{2} \mathrm{O}$ and $\mathrm{Au}_{2} \mathrm{O}_{3}$. If we fix our attention on the resemblances between copper, silver, and gold rather than on the divergences it is easy to see why they should be classed in the same family. The metals themselves are all permanent in air and not very active chemically; none of them sets free hydrogen from acids appreciably. They are moderately hard but are very malleable and ductile. They all melt in the neighborhood of $1000^{\circ}$. They are the best three conductors of heat and of electricity. They all form oxides, $\mathrm{E}_{2} \mathrm{O}$, and their chlorides, $\mathrm{ECl}$, are all white salts insoluble in water and dilute acids. It will be noted that $\mathrm{Cu}$, $\mathrm{Ag}$, and $\mathrm{Au}$ occur in the long periods 3, 4, and 6 respectively, and in each case in the second line of the period. Inspection will show the presence of $A$ and B families in Groups II to VII but not in groups $\mathrm{O}$ and VIII.

832. The Second Group.-Group II is made up of two families, IIA and IIB. The alkali earth elements $\mathrm{Ca}, \mathrm{Sr}, \mathrm{Ba}$, and $\mathrm{Ra}$ are the typical members of IIA (824); while $\mathrm{Zn}, \mathrm{Cd}$ (cadmium), and $\mathrm{Hg}$ constitute IIB. Beryllium, Be, and magnesium, $\mathrm{Mg}$, in the first and second periods are somewhat more closely related in their properties to the family IIA than to the family IIB. The alkali earth family, IIA, is so called because of the resemblance of its members to the alkalies, IA, on the one hand, and to the earths, typified by aluminum in Group III, on the other. Metallic calcium is a moderately soft metal and is acted on rather rapidly by water to form a strongly basic hydroxide and hydrogen, but 
by no means so rapidly as are the alkali metals. The hydroxides of IIA are not quite as strong bases as those of IA; but their salts with strong acids give neutral solutions. The three members of IIB, $\mathrm{Zn}, \mathrm{Cd}$, and $\mathrm{Hg}$, are typical metals and are all unacted upon by water and are all untarnished by air. The hydroxides of IIB are weak, insoluble bases, and solutions of salts with strong acids are acid in reaction by reason of hydrolysis (436). Comparison of IIA and IIB shows less difference in properties than was found in the case of IA and IB. With the exception of mercury all the elements of IIA and IIB form exclusively compoundsin which they are bivalent. Typical formulae are $\mathrm{ECl}_{2}$, $\mathrm{EBr}_{2}, \mathrm{E}\left(\mathrm{NO}_{3}\right)_{2}, \mathrm{ESO}_{4}, \mathrm{ECO}_{3}, \mathrm{EO}$, and $\mathrm{E}(\mathrm{OH})_{2}$. Mercury is exceptional, since in mercurous compounds (333) it is univalent.

833. The Third Group.-Of the nine elements composing the third group, only two, boron (8or) and aluminum (174), are common. The others, with the exception of lanthanum, are rare. In their compounds the third-group elements are always trivalent and give products of the following types: $\mathrm{E}_{2} \mathrm{O}_{3}, \mathrm{E}(\mathrm{OH})_{3}$, $\mathrm{ECl}_{3}, \mathrm{E}\left(\mathrm{NO}_{3}\right)_{3}, \mathrm{E}_{2}\left(\mathrm{SO}_{4}\right)_{3}$.

Boron is the only member of the group that does not form salts with acids; its hydroxide, boric acid (802), has only weak acid properties. Aluminum hydroxide, $\mathrm{Al}(\mathrm{OH})_{3}$, is amphoteric (I77), forming salts with both acids and bases; but these salts are all considerably hydrolyzed in solution, so that, for example, a solution of $\mathrm{AlCl}_{3}$ reacts acid, while one of $\mathrm{NaAlO}_{2}$ reacts basic, to indicators $(436)$.

Lanthanum hydroxide, $\mathrm{La}(\mathrm{OH})_{3}$, a white solid, is a rather strong base forming salts, e.g., $\mathrm{La}\left(\mathrm{NO}_{3}\right)_{3}$, which are not hydrolyzed in solution.

The rare earths (820), which doubtless should be considered as third-group elements, will be treated separately in a later section (846).

834. The Fourth Group.-All the elements of the fourth group, with the exception of germanium $(\mathrm{Ge}=72.5)$, are of use technically. Carbon and silicon are of course the most important, and tin and lead come next. Titanum, zirconium, cerium, and thorium find interesting minor uses. The characteristic 
valence of these elements, in their compounds, is four, so that the typical compounds have formulae like the following: $\mathrm{EO}_{2}$, $\mathrm{E}(\mathrm{OH})_{4}, \mathrm{H}_{2} \mathrm{EO}_{3}, \mathrm{ECl}_{4}, \mathrm{E}\left(\mathrm{NO}_{3}\right)_{4}, \mathrm{E}\left(\mathrm{SO}_{4}\right)_{2}$, etc. Only titanium, zirconium, tin, cerium, and thorium form salts of the last two types. Several of the members of this group form compounds in which they have a valence of two or three. Thus carbon and lead form the oxides $\mathrm{CO}\left(\mathbf{6 3 2}_{32}\right)$ and $\mathrm{PbO}\left(\mathbf{I 6 7}_{7}\right)$ respectively. In all stannous compounds (809) tin is bivalent, while in all its ordinary salts lead also has a valence of two. In the more stable salts of cerium, like cerous nitrate, $\mathrm{Ce}\left(\mathrm{NO}_{3}\right)_{3}$, and cerous sulfate, $\mathrm{Ce}_{2}\left(\mathrm{SO}_{4}\right)_{3}$, this element is trivalent; but moist cerous hydroxide, $\mathrm{Ce}(\mathrm{OH})_{3}$, takes up oxygen from the air to form the more stable ceric hydroxide, $\mathrm{Ce}(\mathrm{OH})_{4}$. All the fourth-group elements form chlorides of the type $\mathrm{ECl}_{4}$. All of these except $\mathrm{CeCl}_{4}$ and $\mathrm{PbCl}_{4}$ are stable toward heat; the chlorides of carbon, silicon, titanium, germanium, and tin are colorless liquids of low boiling-points $\left(59^{\circ}\right.$ to $\left.\mathrm{I} 35^{\circ}\right)$.

The hydroxides of the elements of the fourth group form only weak acids or weak bases. Carbon and silicon give carbonic acid and silicic acid (807) respectively; and titanium, zirconium, cerium, and thorium form hydroxides which are weak bases. Stannous and stannic hydroxides (809) and lead hydroxide, $\mathrm{Pb}(\mathrm{OH})_{2}$, are amphoteric. Titanium, zirconium, and thorium are the typical members of IVA; the position of cerium in this group is a bit uncertain. Germanium, tin, and lead compose IVB. Carbon and silicon bear resemblances to both of these families. Tin and lead are easily obtained as free metals, but it is doubtful whether even moderately pure metallic thorium has ever been obtained.

835. The Fifth Group.-We have studied only five of the eight elements given in Group V, Table L. These are nitrogen, chapter xxi; phosphorus, chapter xxiii; arsenic (810), antimony (8II), and bismuth (8I2). The last three form family VB, to which the first two show points of similarity. Of the members of family VA only vanadium $(\mathrm{V}=5 \mathrm{I})$ is of technical importance. It is a rather scarce element which finds an important use as a minor component of high-grade machine steel. Its common 
oxide is $\mathrm{V}_{2} \mathrm{O}_{5}$, which is weakly basic and rather strongly acidic in character. The metavanadates, e.g., $\mathrm{NH}_{4} \mathrm{VO}_{3}$, correspond to the salts of metaphosphoric acid, $\mathrm{HPO}_{3}$ (589). No technical uses have yet been found for the other two members of this family, the rare elements columbium (also called niobium) and tantalum.

The following discussion will be confined to the members of VB, together with nitrogen and phosphorus. Of the five elements referred to, all but bismuth form gaseous hydrogen compounds of the type $\mathrm{EH}_{3}$. Of these only $\mathrm{NH}_{3}$ forms with water a basic hydroxide. In these hydrides the fifth-group element is trivalent. All five of the elements (including bismuth) form trimethyl derivatives, $\mathrm{E}\left(\mathrm{CH}_{3}\right)_{3}$, corresponding to trimethyl amine (59).

All five elements form trichlorides, $\mathrm{ECl}_{3}$, and $\mathrm{P}, \mathrm{As}$, and $\mathrm{Sb}$ form pentachlorides, $\mathrm{ECl}_{5}$. All of the five elements being considered form trioxides, $\mathrm{E}_{2} \mathrm{O}_{3}$, and all with the possible exception of bismuth form pentoxides, $\mathrm{E}_{2} \mathrm{O}_{5}$. Also, with the exception of bismuth, these elements form acids derived from their pentoxides. These acids exist in various stages of hydration, of which $\mathrm{HEO}_{3}$ is the most common.

The highest valence of the elements of the fifth group is five, although in many cases the valence is only three.

836. The Sixth Group.-The two families VIA and VIB are each made up of four elements. The first, VIA, consists of chromium (344), molybdenum (8I3), tungsten (814), and uranium; the second, VIB, embraces oxygen, sulfur (chap. xxiv), selenium (815), and tellurium (8I5). The members of VIA have a characteristic maximum valence of six, as exemplified by the oxides, $\mathrm{EO}_{3}$. These are all acidic in nature and lead to salts such as $\mathrm{Na}_{2} \mathrm{EO}_{4}$ and $\mathrm{Na}_{2} \mathrm{E}_{2} \mathrm{O}_{7}$ and even more complex formulae, e.g., $\mathrm{Na}_{6} \mathrm{Mo}_{7} \mathrm{O}_{24} \cdot 22 \mathrm{H}_{2} \mathrm{O}$. In all these salts the sixth-group element is hexavalent. The members of family VIB all form hydrogen compounds, $\mathrm{H}_{2} \mathrm{E}$, and these decrease in stability as the atomic weight of the sixth-group element increases. Water is extremely stable: hydrogen telluride decomposes with ease (8I5). We should expect a normal valence of six for these elements; 
but we find that for oxygen the ordinary valence is only two, although in some cases (not discussed in this book) a valence of four is very probable. Sulfur and tellurium form trioxides, $\mathrm{EO}_{3}$, and in these the two former are undoubtedly hexavalent. as they are also in the corresponding acids $\mathrm{H}_{2} \mathrm{EO}_{4}$ and their salts, While selenium trioxide has not been made, the corresponding acid $\mathrm{H}_{2} \mathrm{SeO}_{4}$ and its salts are well known (8I5). In them selenium has a valence of six. Sulfur, selenium, and tellurium also form oxides $\mathrm{EO}_{2}$ and acids $\mathrm{H}_{2} \mathrm{EO}_{3}$ and their salts. These, as we have learned, are converted by oxidation into the corresponding acids $\mathrm{H}_{2} \mathrm{EO}_{4}$ or their salts.

Contrasting VIA with VIB, we may say that while all the elements are acid-forming, only chromium and uranium are base-forming and give salts with acids. Chromium forms hydroxides in which it is trivalent. Thus $\mathrm{Cr}(\mathrm{OH})_{3}$ is a weak base like $\mathrm{Fe}(\mathrm{OH})_{3}$. It also is capable of further reduction and gives a more strongly basic hydroxide, $\mathrm{Cr}(\mathrm{OH})_{2}$. The commonest uranium salts are derived from the trioxide $\mathrm{UO}_{3}$. These salts may be considered as being formed from a compound $\mathrm{UO}_{2}(\mathrm{OH})_{2}$, a diacid base about as strong as $\mathrm{Fe}(\mathrm{OH})_{2}$. Its reaction with nitric acid may be represented thus:

$$
\mathrm{UO}_{2}(\mathrm{OH})_{2}+{ }_{2} \mathrm{HNO}_{3} \rightarrow \mathrm{UO}_{2}\left(\mathrm{NO}_{3}\right)_{2}+{ }_{2} \mathrm{H}_{2} \mathrm{O} \text {. }
$$

This salt is called uranyl nitrate. It forms large yellow crystals, $\mathrm{UO}_{2}\left(\mathrm{NO}_{3}\right)_{2} \cdot 6 \mathrm{H}_{2} \mathrm{O}$. The corresponding uranyl chlorides, sulfates, acetates, etc., are readily prepared. In these salts uranium is undoubtedly hexavalent.

837. The Seventh Group.-Only one member of the A family of the seventh group is known; this is manganese (342). It is a hard metal the color of iron and does not tarnish in air. It does not form a hydride. In its common halogen compounds (true salts) it is bivalent. Its corresponding hydroxide is $\mathrm{Mn}(\mathrm{OH})_{2}$, an insoluble base which forms salts like $\mathrm{MnBr}_{2}$, $\mathrm{Mn}\left(\mathrm{NO}_{3}\right)_{2}, \mathrm{MnSO}_{4}$, etc., and an oxide, $\mathrm{MnO}$. Manganese also gives higher oxides, some of which are acid anhydrides. Potassium manganate, $\mathrm{K}_{2} \mathrm{MnO}_{4}$, soluble green crystals, is a salt of the (unstable) manganic acid $\mathrm{H}_{2} \mathrm{MnO}_{4}$, the anhydride of which would 
be $\mathrm{MnO}_{3}$. By oxidation (with chlorine, for example) solutions of manganates give permanganates,

$$
2 \mathrm{~K}_{2} \mathrm{MnO}_{4}+\mathrm{Cl}_{2} \rightarrow 2 \mathrm{KCl}+2 \mathrm{KMnO}_{4} .
$$

Potassium permanganate (343) by treatment with sulfuric acid gives an explosive liquid (danger!) which is very probably manganese heptoxide, $\mathrm{Mn}_{2} \mathrm{O}_{7}$, the anhydride of permanganic acid, $\mathrm{HMnO}_{4}$. In the latter and its salts and anhydride manganese has a valence of seven. These compounds are all violent oxidizing agents.

The halogen family, VIIB, is so well known and the close resemblances of its members have been so often referred to that further discussion of them would be superfluous. A few lines may be added touching the valence of chlorine, bromine, and iodine in their oxyacids and salts. Fluorine, it will be recalled, does not form such compounds. The other three halogens reach their maximum oxygen valence in the acids perchloric, $\mathrm{HClO}_{4}$, perbromic, $\mathrm{HBrO}_{4}$, and periodic, $\mathrm{HIO}_{4}$. These may be considered as derived from hypothetical heptoxides, $\mathrm{E}_{2} \mathrm{O}_{7}$, in which the halogen has a positive valence of seven. There is thus an analogy between these elements and manganese in the peracids and their salts.

838. The Eighth Group.-An inspection of the Periodic Table (829) will show that the arrangement of the members of the eighth group is different from that in any other group. In this group there are three lines of three elements each in place of the usual $\mathbf{A}$ and $\mathbf{B}$ columns. In first line we find iron, cobalt, and nickel, which are the only common elements of Group VIII. These elements have many similar properties. First of all it will be noted that their atomic weights are all close together. These three elements are all metals and all form salts of the types $\mathrm{ECl}_{2}, \mathrm{E}\left(\mathrm{NO}_{3}\right)_{2}, \mathrm{ESO}_{4}, \mathrm{E}_{3}\left(\mathrm{PO}_{4}\right)_{2}$, and hydroxides and oxides $\mathrm{E}(\mathrm{OH})_{2}$ and $\mathrm{EO}$ respectively. In all these compounds these three elements are bivalent. However, iron and to a lesser extent cobalt form hydroxides, oxides, and salts in which they are trivalent, e.g., $\mathrm{E}(\mathrm{OH})_{3}, \mathrm{E}_{2} \mathrm{O}_{3}$, and $\mathrm{EF}_{3}$. The second line of the eighth group contains the three metals ruthenium, $\mathrm{Ru}$, 
rhodium, Rh, and palladium, $\mathrm{Pd}$, all rare elements which closely resemble one another. Recently an alloy of palladium with gold has come into use as a substitute for platinum in the manufacture of crucibles and dishes for laboratory use. This alloy closely resembles platinum in its physical properties and in its inertness toward chemical reagents. The elements of the third line osmium, Os, iridium, Ir, and platinum, Pt, form another subgroup in which the three members are much alike. All are extremely resistant to attack by most chemical reagents. Metallic osmium is of interest in that it has the greatest density of any known substance, namely 22.5. It is also extremely hard and melts only at the very high temperature of $2500^{\circ}$. It is also unique in forming an easily volatile and extremely poisonous oxide, $\mathrm{OsO}_{4}$, a solution of which, known as osmic acid, is a very important staining material for microscopic preparations. In this oxide osmium has a valence of eight.

839. Valence and the Structure of Inorganic Molecules.The study of organic chemistry has made it very clear that the valence of each element of a compound can be definitely determined only when the structure $(648)$ of the molecule of the substance is known. It is much easier to discover the structural formulae of organic compounds than of inorganic. There are two reasons for this: first, a single element, carbon, forms the backbone, so to speak, of all organic substances; and second, the enormous number of carbon compounds makes it possible to test theory by fact in a multitude of cases. The problem is very different with inorganic compounds, where we must deal with 80 or more elements, any one of which (excepting hydrogen, oxygen, and nitrogen) forms but few compounds in comparison with the host of carbon derivatives. Therefore when we attempt to show the structure of inorganic molecules the element of uncertainty is often great.

In writing graphic formulae for the chlorides, oxides, hydroxides, etc., of the elements of the first three groups we are on pretty safe ground, as these formulae are very simple. For common salt we have only one choice, $\mathrm{Na} \cdot \mathrm{Cl}$; similarly for other univalent elements. If for water $(\mathbf{3 2 3})$ we write $\mathrm{H} \cdot \mathrm{O} \cdot \mathrm{H}$, for 
sodium hydroxide we must write $\mathrm{Na} \cdot \mathrm{O} \cdot \mathrm{H}$. Sodium oxide, $\mathrm{Na}_{2} \mathrm{O}$, must be $\mathrm{Na} \cdot \mathrm{O} \cdot \mathrm{Na}$. In the second and third groups the task is nearly as easy. Calcium chloride is of course $\mathrm{Cl} \cdot \mathrm{Ca} \cdot \mathrm{Cl}$, and the oxide must be simply $\mathrm{Ca}=\mathrm{O}$, calcium and oxygen both being bivalent. Calcium hydroxide then is represented by $\mathrm{H} \cdot \mathrm{O} \cdot \mathrm{Ca} \cdot \mathrm{O} \cdot \mathrm{H}$.

In the third group boron, which forms a trichloride, $\mathrm{BCl}_{3}$, is evidently trivalent. If boric acid, $\mathrm{H}_{3} \mathrm{BO}_{3}$, is written

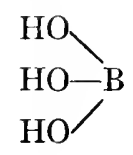

the normal valences of all three elements are correctly represented. Metaboric acid, $\mathrm{HBO}_{2}$, would then be $\mathrm{HO} \cdot \mathrm{B}=\mathrm{O}$ and sodium metaborate $(802) \mathrm{NaO} \cdot \mathrm{B}=\mathrm{O}$. Sodium aluminate, $\mathrm{NaAlO}_{2}$ (I77), would then be written $\mathrm{NaO} \cdot \mathrm{Al}=\mathrm{O}$, which shows aluminum with its correct valence of three.

The compound $\mathrm{C}(\mathrm{OH})_{4}$ is not known, but carbonic acid, which would result from this by loss of water, is doubtless correctly represented by

$$
\mathrm{HO}_{\mathrm{C}=\mathrm{O}}^{\mathrm{HO}}
$$

which shows carbon with a valence of four. The structure of silicic acid is probably analogous.

Nitrogen pentoxide, $\mathrm{N}_{2} \mathrm{O}_{5}$ (555), is the anhydride of nitric acid. We might expect a hydroxide $\mathrm{N}(\mathrm{OH})_{5}$ or $\mathrm{H}_{5} \mathrm{NO}_{5}$. By loss of water this could form first $\mathrm{H}_{3} \mathrm{NO}_{4}$ and then $\mathrm{HNO}_{3}$. As a matter of fact a crystalline substance, $\mathrm{HNO}_{3} \cdot \mathrm{H}_{2} \mathrm{O}$, which is really $\mathrm{H}_{3} \mathrm{NO}_{4}$, is actually known (54I). For this hydrate we may write the formula

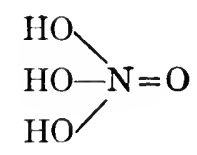

and for nitric acid itself<smiles>O=[W](=O)=O</smiles> 
Both formulae give nitrogen a valence of five. Orthophosphoric metaphosphoric acids probably have analogous formulae.

For the two oxides of sulfur we write

$$
\mathrm{O}=\mathrm{S}=\mathrm{O} \text { and } \mathrm{O}=\mathrm{S} \mathbb{O}_{\mathrm{O}}^{\mathrm{O}}
$$

respectively, thereby assuming the valence of sulfur to be four in the first case and six in the second. The corresponding acids then become<smiles>O=S(=O)(O)[14CH](O)[14CH2]O[Si](O)(O)O</smiles>

The acids of the seventh group of the type $\mathrm{HEO}_{4}$ very probably have the structure<smiles>O=[As](=O)(O)O</smiles>

with a manganese or halogen atom of valence seven.

Osmium, the only element of the eighth group which forms an oxide, $\mathrm{EO}_{4}$, doubtless has a valence here of eight. If so this oxide must be represented thus:<smiles>O=P(=O)([O-])([O-])[O-]</smiles>

840. Positive and Negative Valence.-We have already seen that the valence of an element is represented by the number of electrons each of its atoms loses or gains when it reacts to form a compound (484). Thus when copper and chlorine unite (246) to form $\mathrm{CuCl}_{2}$ each atom of copper loses two electrons and each atom of chlorine gains one. When sulfur burns to form $\mathrm{SO}_{2}$ (340) each atom of sulfur loses four electrons and each atom of oxygen gains two. But when sulfur and hydrogen combine to torm $\mathrm{H}_{2} \mathrm{~S}$ it is the hydrogen which loses electrons, since this element forms positive ions only $\left(\mathrm{H}^{+}\right)$, and therefore each atom 
of sulfur must gain two electrons. The graphic formulae of the two sulfur compounds showing the distribution of charges resulting from the transfers of electrons are as follows:

Sulfur dioxide,

Hydrogen sulfide,

$$
\overline{\mathrm{O}}=+++++--
$$

$$
\stackrel{+}{\mathrm{H}}-\overrightarrow{\mathrm{S}}-\stackrel{+}{\mathrm{H}}
$$

In these formulae each plus sign indicates a loss of one electron. In $\mathrm{SO}_{2}$ the valence of sulfur is four; in $\mathrm{H}_{2} \mathrm{~S}$ it is two. But very plainly these are different kinds of valence, and we should distinguish them by calling the first a positive valence and the second a negative valence.

Other elements also exhibit both positive and negative valence. Thus phosphorus in $\mathrm{PH}_{3}(588)$ has a negative valence of three and in $\mathrm{PCl}_{3}(247,576)$ a positive valence of three, while in $\mathrm{PCl}_{5}$ it has a positive valence of five.

\begin{tabular}{|c|c|c|c|c|c|}
\hline Group & IV & $v$ & IT & VII & VIIII \\
\hline $\begin{array}{l}\text { Compound.......... } \\
\text { Negative valence...... } \\
\text { Compound........... } \\
\text { Positive valence..... } \\
\text { Snm of }+ \text { and }- \text { valence. }\end{array}$ & $\begin{array}{c}\mathrm{CH}_{4} \\
\mathrm{CO}_{2}, \mathrm{CCl}_{4} \\
4 \\
8\end{array}$ & $\begin{array}{c}\mathrm{PH}_{3} \\
\mathrm{P}_{2} \mathrm{O}_{5},{ }_{5} \mathrm{PCl}_{5} \\
8\end{array}$ & $\begin{array}{c}\mathrm{H}_{2} \mathrm{~S} \\
\stackrel{2}{2} \mathrm{SO}_{3}, \underset{2}{\mathrm{SO}_{4}} \\
\underset{8}{8}\end{array}$ & $\begin{array}{c}\mathrm{HCl} \\
\mathbf{I} \\
\mathrm{HClO}_{4} \\
\stackrel{7}{8}\end{array}$ & $\begin{array}{c}\stackrel{\circ}{\mathrm{O}_{5}} \\
8 \\
8 \\
8\end{array}$ \\
\hline
\end{tabular}

TABLE LII

A very remarkable law can be formulated from facts like those brought out by Table LII. For many elements of Groups IV to VIII the sum of the maximum positive and negative valences of an element is eight.

84I. Metals and Non-Metals. - We may now consider the way in which the metals and the non-metals are distributed in the Periodic Table (829). In the table the heavy line starting between beryllium and boron and ending with the boundary between the seventh and eighth groups separates all of the pronounced non-metallic elements from the others, which are all more or less metallic in character. The symbols of the former are printed in italics. It is true, however, that a few elements, 
particularly chromium, molybdenum, and manganese, thus included with the non-metals, are in the free state definitely metallic and form salts with strong acids, e.g., $\mathrm{Cr}_{2}\left(\mathrm{SO}_{4}\right)_{3}$ and $\mathrm{Mn}\left(\mathrm{NO}_{3}\right)_{2}$. But it is also a fact that these elements all form acids that yield with bases typical salts, e.g., $\mathrm{Na}_{2} \mathrm{CrO}_{4}, \mathrm{Na}_{2} \mathrm{MoO}_{4}$, and $\mathrm{KMnO}_{4}$, and in this respect the elements resemble the nonmetals.

It is particularly the non-metallic elements segregated by the heavy line of Table $\mathrm{L}$ that exhibit both positive and negative valence. The rare gases (Group $\mathrm{O}$ ) have little in common with either the metallic or the non-metallic elements.

842. Chemical Activity and the Periodic Table.-For practical purposes we may define the chemical activity of an element as its tendency to enter into combination. If one element displaces another from the solution of one of its compounds (488-492) the first is the more active of the two. For example, in family IA the displacement order is $\mathrm{Cu}, \mathrm{Ag}, \mathrm{Au}$, and this is therefore the order of the elements in respect to decreasing activity. For the halogen family, VIIB, the displacement order is $\mathrm{F}, \mathrm{Cl}, \mathrm{Br}, \mathrm{I}$, and this again is the order of decreasing activity. A thorough study of the displacement tendencies and activities has established the following important generalization: In Groups I to VII, inclusice, in the A families the activity of an element increases with increasing atomic weight; in the B series activity decreases as the atomic weight increases. In accord with this law the greatest activities are found in the elements caesium, $\mathrm{Cs}$, at the bottom of IA and fluorine at the top of VIIB. It is also important to note further that the valence of caesium in its compounds is positive, while that of fluorine is negative.

On the other hand, the least active elements are met with at the bottom of Group VIII in the metals Os, $\mathrm{Ir}$, and $\mathrm{Pt}$, and at the top of Group $\mathrm{O}$, where we find helium an element of no chemical activity and so little physical affinity (cohesion) that in liquid form it has the lowest boiling-point of all the elements and is the only element that has not been solidified.

843. Periodic Properties.-We have now discussed, as far as the limits of this text will permit, the ways in which the 
properties of the elements change as we pass from element to element in each family and from family to family and group to group in the table. It now remains to call attention to the remarkable manner in which the properties of the elements change as we pass through the table from period to period in the order of increasing atomic weights. The periodic fluctuation of properties is most easily exhibited by means of a diagram or graph. Let us take for illustration the variation of atomic volume (823) with atomic weight. In Fig. Ir6 the atomic volumes are plotted on the vertical axis and the atomic weights on the horizontal axis. The result is a very remarkable graph, in which each period is represented by a crude U-shaped curve. We see that there is a periodic repetition of like properties as we pass along the graph. If we should plot in a similar way any one of many other properties of the elements, for instance the melting-points or the compressibilities, we obtain periodic curves (graphs) of more or less similar forms. These facts are summarized by the statement first made by the great Russian chemist Mendelejeff: The properties of the elements are periodic functions ${ }^{1}$ of their atomic weights. This means that the atomic weight of an element determines its properties. This is plain if we note that the place of any element in the Periodic Table is fixed by its atomic weight, and that the properties of the element are indicated by its position in the table. Among the properties of elements which show periodic relations we may mention valence, chemical activity, melting- and boiling-temperatures, conductivity for heat and for electricity, hardness, etc.

844. History of the Periodic System.--The family relationships of the elements began to be discovered long before the Periodic Table as a whole was developed. As early as I829 Döbreiner pointed out the existence of triads of elements, such as $\mathrm{Cl}, \mathrm{Br}, \mathrm{I}$; $\mathrm{Li}, \mathrm{Na}, \mathrm{K}$; and $\mathrm{Ca}, \mathrm{Sr}, \mathrm{Ba}$, in which the atomic weight and other properties of the middle element of the triad were close to the mean of the other two. As time went on other families and their relations were recognized. The first

I The term function is much used in mathematics. If, for example, the value of $x$ is dependent on the value of $y$, then $x$ is said to be a function of $y$. 
attempt to arrange all of the elements in a single table was made by Newlands in I864-66. A little later (1869-70) Mendelejeff

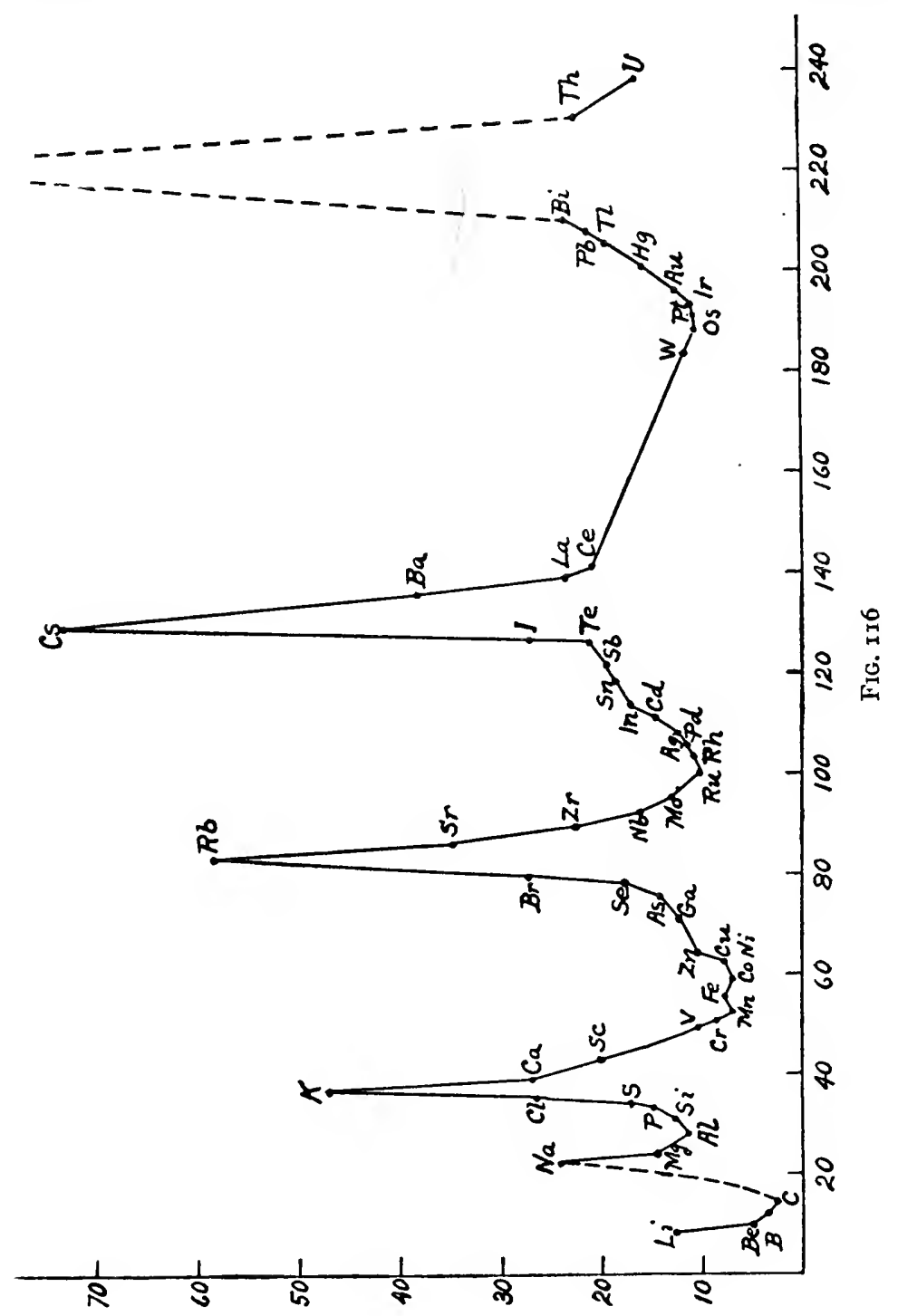

published a table almost in the form of Table L. There were several places left vacant by Mendelejeff for elements as yet 
undiscovered, and the whole zero group was of course absent, as none of these elements was discovered until many years later. It was Mendelejeff who pointed out many of the relations between the properties of the elements in essentially the form discussed in this chapter and drew the far-reaching conclusion that the properties of the elements are periodic functions of their atomic weights. This statement is known as the periodic law. The German chemist Lothar Meyer also discovered the periodic law independently and published a periodic table closely resembling that of Mendelejeff very shortly after the appearance of the latter's table.

845. The Vacant Places of Mendelejeff's Table.-In Mendelejeff's table the spaces now occupied by scandium, gallium, and germanium were left vacant. He very boldly took the stand that these places represented elements still to be discovered. In fact, his description of these undiscovered elements, which he called ekaboron, ekaluminum, and ekasilicon respectively, was so complete and accurate that chemists knew just how these elements would behave if present in mixtures with other elements. In other words, they knew in just what sorts of chemical residues to expect these new elements, and it was not long until all three had been found. In 1875 Lecoq de Boisbaudran discovered ekaluminum and called it gallium (from Gaul, in honor of France); in 1879 Nilson discovered ekaboron and called it scandium (for Scandinavia); and in 1886 Winkler

\section{TABLE LIII}

MendelejefF's Predictions, is7 I

Ekasilicon; Es $=72$

Metal; density $5 \cdot 5$

Oxide, $\mathrm{EsO}_{2}$; density 4.7

Chloride, $\mathrm{EsCl}_{4}$; density I.9

Chloride should boil below $100^{\circ}$

Fluoride, $\mathrm{EsF}_{4}$; not gaseous

Sulfide, $\mathrm{EsS}_{2}$; insoluble in water, but soluble in ammonium sulfide

Tetra ethyl compound, $\mathrm{Es}\left(\mathrm{C}_{2} \mathrm{H}_{5}\right)_{4}$; density 0.96 ; boiling-point $160^{\circ}$
WinKLER'S OBSERVATIONS, I 886

Germanium; $\mathrm{Ge}=72.5$

Metal; density 5.47

Oxide, $\mathrm{GeO}_{2}$; density 4.7

Chloride, $\mathrm{GeCl}_{4}$; density I $.88_{7}$

Chloride boils at $86^{\circ}$

Fluoride, $\mathrm{GeF}_{4}$; solid

Sulfide, $\mathrm{GeS}_{2}$; insoluble in water, but soluble in ammonium sulfide Tetra ethyl compound, $\mathrm{Ge}\left(\mathrm{C}_{2} \mathrm{H}_{5}\right)_{4}$; density a little less than water; boiling-point $160^{\circ}$ 
discovered ekasilicon, which he named germanium (for Germany). In Table LIII some of the properties of the last-named element are set down opposite those predicted by Mendelejeff.

Mendelejeff also predicted the existence of ekamanganese with atomic weight about Ioo, but this element remains as yet unknown.

When radium (480) was discovered by Mme Curie and its atomic weight was determined to be 226 it was at once placed at the bottom of IIA as a member of the alkaline earth family, of which its chemical properties (see chap. xxxii) show it to be a member. The zero family of the Periodic Table was added by Ramsay after the discovery of the inert gases $\left(79 \mathrm{I}^{-798)}\right.$ and served to round out the system in an unexpected but most satisfactory and suggestive fashion.

846. The Rare Earths and the Periodic Table.-The fitting of the rare earths (820) into the Periodic Table presents a difficulty that is not yet very satisfactorily solved. These elements, as we have seen, are all trivalent in their characteristic compounds and all much alike in their properties. They plainly form one large family quite as homogeneous as any other. Only the first member, lanthanum, La, and possibly also the second, cerium, $\mathrm{Ce}$, fall into their proper places when these elements are arranged in the order of their increasing atomic weights. The problem of the position of the rare earths in the table is an interesting one that still awaits solution.

847. The Position of Hydrogen in the Periodic Table.-As the Periodic Table is usually written hydrogen is not included. The chemistry of hydrogen is so unique that this element is in reality in a class by itself. Its positive valence of one suggests a place in the first group; but solid hydrogen (296) is not metallic and bears no physical resemblance to the elements of Group I. A critical discussion of the position of hydrogen in the periodic system would carry us beyond the scope of this text.

848. Two Striking Anomalies.-In Table $\mathrm{L}$ there are two striking anomalies: argon with an atomic weight of 39.9 precedes potassium with a value of 39.10 ; and tellurium, I 27.5 , precedes iodine, I26.9. Before the discovery of argon the case 
of tellurium and iodine was unique, and for a long time chemists were inclined to think that the then accepted values of the atomic weights of these two elements were in error, for it seemed incredible that there should be an exception to a law that held so consistently for all other known elements. It was, of course, out of the question to place iodine before tellurium, for this would bring iodine in VIB with sulfur and selenium (815) and place tellurium among the halogens! It was possible that either or both of the elements used in early atomic-weight determinations were impure, or that methods of analysis were faulty, and that in consequence the atomic weight of iodine should be, in reality, greater than that of tellurium. The scientific importance of the problem thus presented made chemists realize the need of determining atomic weights of all elements with the greatest possible accuracy. As a result some of the world's most skilled chemists have given their best efforts to such work. In the cases of the two elements under discussion the final and universally accepted results are

$$
\begin{aligned}
& \text { Tellurium }=\mathrm{I} 27.5 \\
& \text { Iodine }=\mathrm{I} 26.92
\end{aligned}
$$

and we are therefore forced to conclude that the position of an element in the Periodic Table is not of necessity strictly fixed by its atomic weight. The more recently discovered case of argon and potassium serves to confirm this conclusion.

A discovery nade by Mosely in I9I4 and discussed in the following chapter has thrown a new light on this whole matter. This discovery consists in nothing less than the finding of a property of the elements more fundamental than their atomic weights, namely, their atomic numbers. If the elements are arranged according to these numbers the two anomalies disappear and every other element falls in its correct position. But this is another story, which finds its logical place in the following chapter.

849. The Newer Tables: The Harkins Table.-Since the time of Mendelejeff many other tables have been arranged. We shall have space to present only one of these, namely, that 
of Professor W. D. Harkins. This table should be of particular interest to those who expect to specialize in chemistry, since it shows the relationships between the elements much more clearly than does the Mendelejeff table. Fig. II 7 is a projection of a space model of the table.

The elements are arranged about a continuous helix in such a way that those of the same family are in the same vertical column. In the actual model the path of the helix is indicated by a heavy wire connecting balls which are lettered with the symbols of the elements which they represent. The families (83I) are also connected by heavy vertical rods of brass, which thus form the supporting structure of the model. Each unit of atomic weight is represented by $\mathrm{I} \mathrm{cm}$. vertical distance on the spiral (see scale at the side of Fig. II7). The elements are arranged in the order of their atomic numbers.

The helix begins with hydrogen, which is uniquely placed at the top of the table. Next the helix descends to the position of helium and makes a big loop, coming back to neon, which is of course just under helium. On this loop are found the elements of the first period of the Mendelejeff table, namely lithium, beryllium, boron, carbon, nitrogen, oxygen, and fluorine. After arriving at the position of neon the helix makes another big loop back to argon, and on this are the elements of the second period of the Mendelejeff table. These are sodium, magnesium, aluminum, silicon, phosphorus, sulfur, and chlorine. From argon the helix passes next to potassium, calcium, scandium, and titanium; then it begins the first inner loop, passing successively through the positions of vanadium, chromium, manganese, iron, cobalt, nickel, copper, zinc, and gallium before returning to the outer loop at the position of germanium. Next the helix takes a full outer turn through the positions of arsenic, selenium, bromine, krypton, etc.

If we continue following the helix we find that the first four A-family elements and the last four B-family elements are on the outer loops, together with the zero group. The second four A-family elements, the eighth group, and the first four B-family elements are on the inner loops. This brings corresponding A 
Classification of the Elements. The Periodic System $5_{5}^{6}$

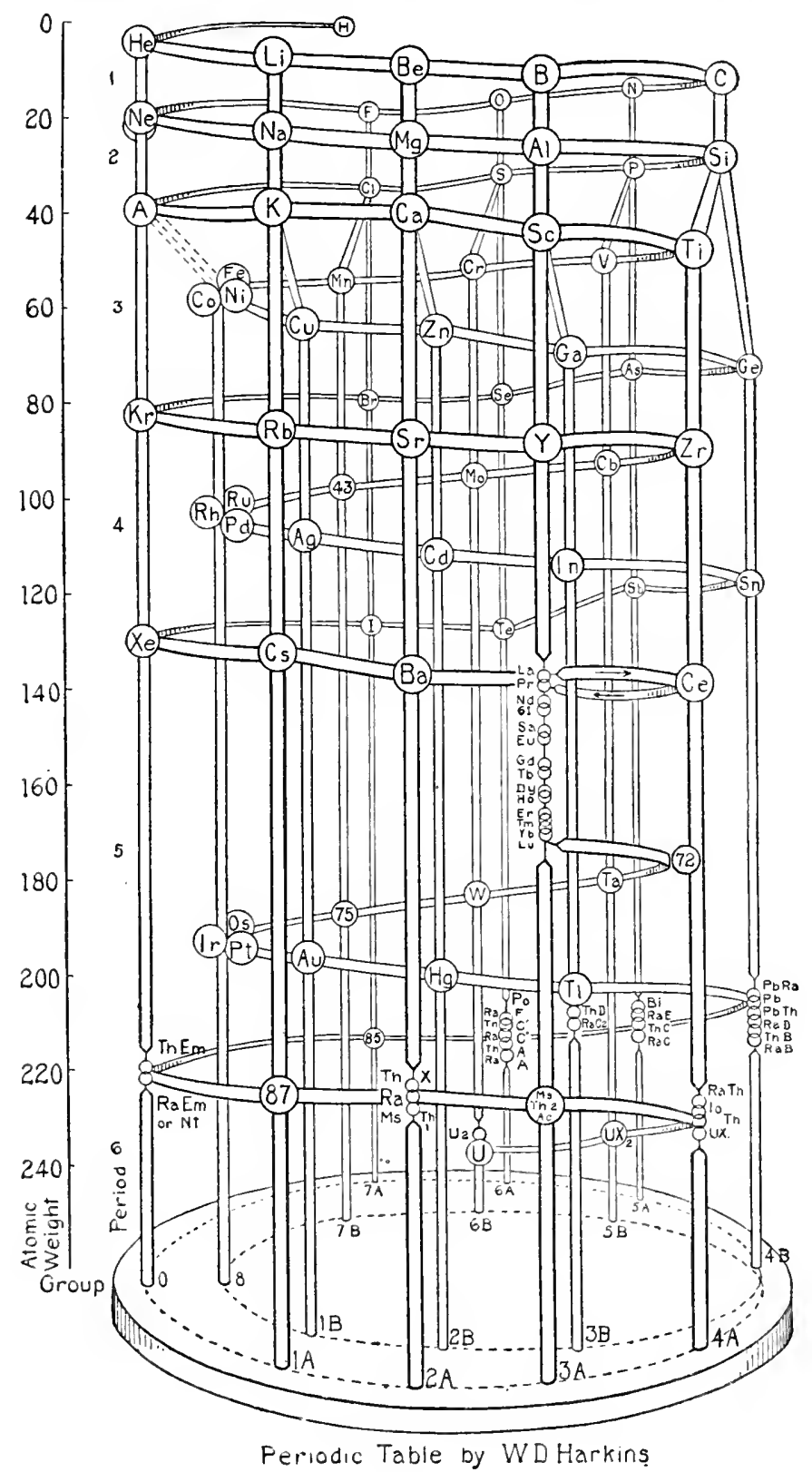

FIG. II? 
and $B$ families together in a very ingenious way. At the bottom of the model a dotted line is traced to show the relative positions of the inner and outer loops. It will be noticed that the inner loops spring from the outer at the fourth group, and that from that point the distance between the two increases, becoming greatest between the zero and the eighth group. The rare earths are placed on the vertical column of IIIA, since their chemical properties show them to be closely related to this family. The last loop of the helix represents the positions of the radioactive elements, which will be discussed in the next chapter.

This table has the advantage of representing the elements in a continuous series. It also shows the relationship of the $\mathrm{A}$ and B families more plainly than does the Mendelejeff table. The vacancies in this table are for atomic numbers as yet unassigned to any element, and there is therefore a very strong probability that elements will be found to occupy these places. This was of course not true of many of the vacant spaces of the Mendelejeff table. Professor Harkins counts hydrogen and helium as the first period and calls it the zero period. The subsequent periods he counts as beginning with the alkalies and ending with the noble gases. Thus, period one would begin with lithium and end with the neon.

850. Summary.-In this chapter we have discussed a system of classification which brings all the elements into a single table (Table L, 829), in which the individuals are arranged in nine groups (vertical columns) and seven periods (horizontal rows). The first two periods are short ones, the others long (829); and in the latter the elements of Group I to VII are further classified into $A$ and $B$ subgroups or families (83I). In the first two (short) periods the elements of Groups I and II belong to the corresponding $\mathrm{A}$ families $\left(8_{3} \mathrm{I}, \mathbf{8 3 2}\right)$, while in the same periods the members of Groups VI and VII are B-family elements $\left(8_{3} 6,837\right)$. The first- and second-period members of Groups III, IV, and V may be classed with either the respective $\mathbf{A}$ or $\mathbf{B}$ families.

In the periodic system of arrangement most of the properties of elements and their compounds vary gradually as we pass 
from one element to its neighbor in the table. This is true whether we pass from top to bottom of the vertical columns, considering each family separately, or pass through any one of the seven periods in the order of increasing atomic weights. Within a given family the propertics change in a systematic manner in such a way that a knowledge of the chemical properties and the behavior of a given member can be deduced from those of each of its nearest relations. This is one of the most important facts about the periodic system. Furthermore in each period we find a recurrence, in like order but of different degree, of the properties that appear in all the periods. In consequence, if we plot the graph of any given property, taking the elements in serial order (in the order of increasing atomic numbers, 848), we get a periodic curve more or less closely resembling in form that for atomic volumes shown in Fig. II6. These facts are epitomized in the periodic law of Mendelejeff: "The properties of the elements are periodic functions of their atomic weights."

The simple and systematic way in which valence (both positive and negative) changes from group to group $(839,840)$ is one of the most striking and useful facts brought out in this chapter, and one that should be firmly fixed in the mind of every chemist.

Finally the Periodic Table is of great value to all students of chemistry in presenting to them a bird's-eye view of all the elements. In so doing it furnishes a mass of evidence for the unity of matter in the sense indicated earlier $\left(47^{\circ}, 482\right)$, as will be discussed more fully in the following chapter. It seems to set a limit to the possible number of elements and also to indicate the nature of the yet undiscovered ones. Of more practical importance than all else the table makes possible a system of classification for an enormous number of chemical facts and thus permits through its mastery an orderly arrangement of the knowledge that constitutes the science of chemistry. 


\section{CHAPTER XXXII}

\section{RADIOACTIVITY AND THE NATURE OF MATTER}

851. Introduction.- The study of the history of chemistry shows very plainly that the rapid growth of chemical knowledge was in no small measure due to the innumerable attempts of the alchemists to convert the base metals into gold. Before the dawn of scientific chemistry belief in the possibility of transmutation of the elements was nearly universal. As time went on and the facts and ideas discussed in the early chapters of this book became known, alchemy merged gradually into chemistry, which soon became a science as well as an art. With the science of chemistry came the conviction that the elements were immutable kinds of matter, and that transmutation was a myth consisting of nothing more substantial than the hopes of the avaricious or the cupidity of charlatans.

After some years of disrepute new life was injected into the old idea in ISr 4 by a suggestion of Prout. This Englishman called attention to the fact that the atomic weights of the gaseous elements were whoie numbers and therefore even multiples of the atomic weight of hydrogen and expressed the opinion that the atoms of other elements were made up of atoms of hydrogen. This idea came to be known as Prout's hypothesis. The first defeat for the hypothesis came when it was proved that the atomic weight of chlorine was 35.5. Prout now claimed a half-atom of hydrogen as the unit. Later work on atomic weights (see Table XLI, 80o) proved even this half-size unit too large if the hypotheses were to be applied to all elements. On the whole Prout had but few supporters. Nevertheless it is an incontestable fact that for many elements the atomic weights are much nearer whole numbers than can be accounted for by chance.

The discovery of the periodic law furnished new and to many the most convincing evidence that all elements are but 
modifications of one primitive form of matter. But no satisfactory theory of the nature of matter and the interrelations of elements was developed until the knowledge of the main facts of radioactivity (480) had led to the disintegration hypothesis as their most plausible explanation. Since the discovery of radium and radioactive phenomena has furnished the key to one of nature's most profound mysteries we shall devote the present chapter to an account of this subject. The story of the discovery of radium is most instructive in illustrating the way in which scientific advances of the greatest significance often result from the thorough investigation of phenomena that seem trivial to persons who call themselves practical. We shall therefore relate the facts in their historical sequence.

852. The X-Rays and Phosphorescence.-The X-ray. apparatus has already been described (476), and it has been mentioned that the $\mathrm{X}$-rays are produced when the cathode rays strike the target. It has also been stated that the cathode rays, non-luminous themselves, cause the glass walls of a cathoderay tube to glow with a greenish-yellow phosphorescence. Thus there seemed to be a causal relation between phosphorescence and X-rays. This supposed relationship was made the subject of an extensive and thorough investigation by Henri Becquerel, professor of physics at the Sorbonne (University of Paris). It had long been known that a number of substances gave out a faint phosphorescent light for some time after exposure to any bright light. All such phosphorescent substances were carefully examined by Becquerel to learn if these glowing bodies gave out $\mathrm{X}$-rays. The latter have the same effect on photographic plates that light has, but they can pass directly through lightproof paper. Accordingly Becquerel tested the effect of these phosphorescent substances on plates wrapped in black paper. None showed any indication of the production of $\mathrm{X}$-rays, with one exception: this was potassium uranyl sulfate, $\mathrm{K}_{2} \mathrm{UO}_{2}\left(\mathrm{SO}_{4}\right)_{2} \cdot{ }_{4} \mathrm{H}_{2} \mathrm{O}$, a salt of the rather uncommon element uranium $(836)$.

853. The Becquerel Rays.-Not only did the abovementioned uranium salt give out rays that, like $\mathrm{X}$-rays, penetrated black, lightproof paper and acted on a photographic 
plate while phosphorescing after it had been exposed to sunlight, but the salt showed equal photoactivity if prepared and also tested wholly in the dark, under which condition it did not phosphoresce. Furthermore all other uranium compounds tested showed similar photoactivity, although they were not phosphorescent even if previously exposed to light. Obviously these new rays, called at first Becquerel rays, were not caused by phosphorescence but were produced by the element uranium in all forms of chemical combination.

854. Ionization of Air by Becquerel Rays.-It was also soon discovered that Becquerel rays ionize air (770), so that by means of the discharge of a gold-leaf electroscope the activity of a substance could be tested much more readily than by the photographic method. A convenient form of electroscope suitable for projection-lantern experiments is shown in Fig. Io9 (770). Two parallel sides are made of glass plates; the rest of the case is of metal. A wire passes through the amber plug insulator and ends in a brass strip 6 to $8 \mathrm{~mm}$. wide and 5 or $6 \mathrm{~cm}$. long. A single leaf of gold or aluminum is attached to the brass strip, as shown in the figure. The electroscope may be charged by means of an ebonite rod, celluloid comb, fountain-pen holder, etc., electrified by being rubbed with a piece of woolen cloth. The uranium compound is held within 2 or $3 \mathrm{~cm}$. of the end of the wire carrying the gold-leaf system. The rate of discharge of the electroscope under fixed conditions is a measure of the intensity of the Becquerel rays.

855. The Discoveries of Mme Curie.-Becquerel's discovery of the rays known by his name was published in 1896 , about two years after Roentgen had discovered X-rays. At about this time Becquerel suggested to Mme Curie, the brilliant wife of Becquerel's colleague, Professor Curie, that the investigation of natural uranium minerals should prove interesting. Mme Curie's experiments soon showed that all uranium minerals were photographically active; they were considerably more active in ionizing air than one would expect from their uranium content. This fact seemed to suggest that the larger part of the activity of the minerals was eliminated during the chemical 
treatment used in making the pure uranium compounds. Search was therefore made for other active substances among the by-products of the preparation of uranium salts; whereupon it was discovered that the small amount of barium extracted from the waste materials was many times as active as any pure uranium salt, notwithstanding the fact that ordinary barium salts were entirely inactive.

856. The Discovery of Radium.-After considerable quantities of active barium chloride had been extracted from uranium mineral residues Mme Curie undertook to separate from the barium the new substance to which she attributed the activity. She found that if a hot, saturated solution of the active barium chloride was allowed to cool about half of the dissolved salt crystallized out, but these crystals contained 70 per cent or more of the active material. Thus the crystals were appreciably richer in the active matter than the original material. By repeated recrystallization of the crystals that separated in this way Mme Curie finally obtained a product that was thousands of times more active than an equal weight of a uranium salt. This product was still largely barium chloride; but that it also contained a new element was shown conclusively by the fact that its spectrum (786) contained in addition to those of barium several lines not belonging to any known element. It was therefore certain that a new element had been discovered, and it seemed probable that it was this element which had produced the powerful Becquerel rays that caused the photoactivity and ionizing power of the material. On account of its ability to send out rays or radiations M.me Curie named the supposed new element radium.

The next problem was to free the radium salt from the accompanying barium. This was finally accomplished by a long series of crystallizations of the chlorides of the barium-radium mixture. Finally pure radium chloride free from barium was obtained. It was a white crystalline salt closely resembling barium chloride in appearance. Its activity, called now radioactivity, was two or three million times as great as that of an equal weight of a uranium salt. 
857. The Atomic Weight of Radium and Its Position in the Periodic Table.-If an element does not form volatile compounds its atomic weight cannot be determined by means of the law of minimum weights (64); and if it is not obtainable in considerable quantity in uncombined solid form the method based on the law of Dulong and Petit (229) is also unavailable. One might make use of a method based on direct or indirect determination of the osmotic pressure of a solution (717-722), but this involves a complication in case an ionized salt is used. As none of the ways just mentioned could be applied in the case of radium, of which but a fraction of a gram was available and that worth thousands of dollars in its cost of preparation, other means of fixing its atomic weight were used.

If we analyze a chloride of a new element and find its percentage composition, one additional fact must be known in order to fix the atomic weight of the element, namely, its valence. But if we can discover to what group of the Periodic Table the new element belongs we can of course infer its valence (839). We have seen that radium resembles barium so closely, in some respects at least, that the separation of the chlorides of the two elements is very difficult. This indicates that radium is a member of the alkali earth group containing calcium, strontium, and barium $(\mathbf{8 2 4}, \mathbf{8 3 2})$. Furthermore it was found that radium also resembles barium in the following respects: its carbonate is insoluble in water, its sulfate is insoluble in water and in strong acids, and its hydroxide is soluble, since radium chloride solution gives no precipitate with either ammonia or sodium hydroxide. Furthermore the free element cannot be deposited on a platinum electrode from a water solution by electrolysis. It therefore seemed very probable that radium was a second-group element and had a valence of two. The analysis of the pure chloride was made by Mme Curie; the result, with an assumed valence of two, led to a calculated atomic weight of 226 . Since the space in the Periodic Table (829) corresponding to this atomic weight was vacant, no doubt remained of the correctness of the atomic weight assigned to the new element. 
858. The Alpha Rays. - It has already been stated that radium emits three kinds of rays, designated as alpha, beta, and gamma, although, strictly speaking, only the last named is a true radiation (that is, a vibration in the luminous ether), since the alpha rays are atoms of helium (795) and the beta rays electrons (480). Entirely reliable experiments, which are too intricate to be described here, have shown that the alpha particles, as we shall now call them, are shot out from radioactive atoms with velocities of 5,000 to I 2,000 miles a second. They travel in straight lines through air for distances (called their ranges) of 3 to $8 \mathrm{~cm}$. before they slow down to the average velocity of air molecules (about one-fourth of a mile a second, 197). The alpha particle of radium has a range of $3.5 \mathrm{~cm}$., and in this distance it collides with many thousands of air molecules; but instead of rebounding as an elastic body and changing its direction after each collision the helium atom constituting an alpha particle passes straight through the air molecule, as a rule, without changing its direction of motion. This fact furnishes the most convincing evidence that molecules are not solid but are made up of a group of widely spaced particles, the electrons (470, 483). It is the alpha particles which cause the major part of the ionization of air exposed to radium rays. Each alpha particle leaves in its wake thousands of ions: each ion is an air molecule from which an electron has been detached, leaving a positive ion; or to which a detached electron has united, forming a negative ion. All air ions carry single electric charges only. Alpha particles are easily stopped by thin sheets of any solid substance, such as paper, glass, or metal.

Proof that the alpha rays are helium atoms was conclusively furnished in the following way: Radium, contained in a sealed glass tube with walls so thin that the alpha particles could barely pass through, was surrounded by a sheet of lead which completely stopped the particles. After several hours the lead was removed and sealed in a larger glass tube having wires for the passage of electric sparks. The lead was then melted by heating the glass tube; this set free the helium atoms that were imbedded in the 
lead, as was proved by the fact that on passing an electric discharge through the tube the spectrum of helium was easily recognized by observation with a spectroscope $(785)$.

859. Luminous Effect of the Alpha Rays.-When alpha rays strike a screen covered with a layer of a specially prepared form of crystalline zinc sulfide, $\mathrm{ZnS}$, a pale phosphorescent light is produced. Luminous watch dials, which have recently become so popular, are produced on this principle. From one to ten parts of radium to ten thousand parts of zinc sulfide are used in making the luminous paint for such purposes. Radium luminous

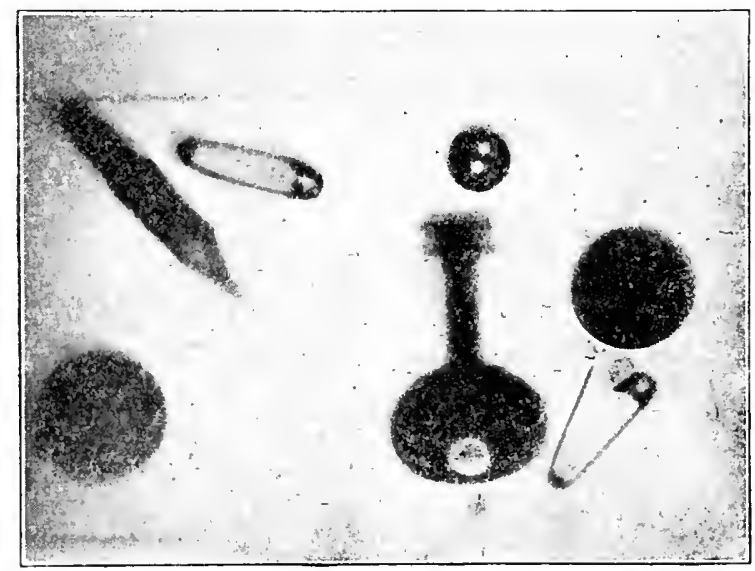

FIG. I I8

paint was extensively used in the war for making luminous dials for aëroplane instruments, submarine instruments, trench compasses, gun sights, etc.

If one views in the dark, by means of a good lens, a zinc sulfide screen exposed to alpha rays from a minute amount of radium a very beautiful effect will be seen. Instead of a uniform glow sparks of light will be seen appearing here and there at random over the screen. It is now certain that each tiny flash of light is the result of the striking of a single helium atom. The simple device consisting of a zinc sulfide screen, a bit of radium, and a lens was called a spinthariscope by Crookes (477), its inventor. The same effect may be seen if the radium paint 
on a luminous watch dial is examined in the dark with a powerful lens.

860. The Beta Rays. - The beta rays are electrons (480) shot out from radioactive atoms with velocities closely approaching the velocity of light. The beta rays are much more penetrating than the alpha rays and can pass through ten to twenty sheets of paper. It is chiefly these rays (and in small measure the gamma rays) that are photographically active. Fig. II 8 shows a photograph taken by radium rays. The photographic plate was wrapped in lightproof paper; the objects shown in the figure were laid on the paper and the whole exposed to the radium at a distance of $10 \mathrm{~cm}$. for a few minutes. The plate was then dereloped in the usual way, and from the negative so obtained the positive print reproduced in the figure was made.

A curious radium clock has been constructed by R. J. Strutt, an English physicist, to show the continuous loss of negative electricity as electrons by radium. The radium is inclosed in a small tube, $A$, Fig. II9, made of glass thick enough to prevent the alpha rays from escaping but not thick enough to stop the beta rays. This small tube is attached at the top to an insulating rod, $B$, of quartz and carries at its lower end a pair of gold leaves, $C$. The tube and gold leaves are fixed in a glass vessel having a

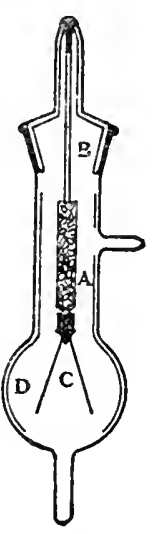

FIG. II9 pair of tin-foil strips, $D$, pasted on the inside opposite the gold leaves. These strips are electrically connected to a wire sealed through the glass and intended to furnish a connection to the earth. The larger glass vessel is highly evacuated. The action of the apparatus is as follows: The beta rays (electrons) from the radium tube escape to earth by way of the tin-foil strips, $D$. This leaves the radium tube with a deficiency of negative electricity and therefore positively charged. The positive charge causes the gold leaves to diverge (by reason of the repulsion of like charges) until they touch the tin-foil strips, when they are at once discharged and fall together. Then the whole cycle begins again. Since the discharge of the gold leares occurs at 
regular intervals Strutt called the device a radium clock. If the vessel were not evacuated ionization of the air would occur and conduct electricity away so fast that the gold leaves would not become charged. The beta rays can also ionize gases but are less effective than the alpha rays.

86I. The Gamma Rays and X-Rays.--The gamma rays closely resemble $\mathrm{X}$-rays. They are far more penetrating than the beta rays and can pass through several millimeters of metal with little absorption and are only completely absorbed by several centimeters of lead, which is more effective in stopping these rays than any other common substance. The gamma rays ionize gases and also affect a photographic plate. Several minerals glow perceptibly when exposed to the gamma rays of a considerable quantity of radium (say roo mg.). Willemite, a silicate of zinc (807), is especially active in this way. Diamonds also glow with a clear white light under the gamma rays, so that these rays may be used practically to distinguish a real from an imitation diamond.

The true physical nature of gamma rays and X-rays was for a long time in doubt. The critical work which cleared up the question will be discussed later (877).

862. Heat from Radium.- Not long after radium had been obtained in considerable quantity it was discovered that a tube of this unique element was somewhat warmer than its surroundings. Subsequent investigation by Rutherford (48I) showed that one gram of radium gives out $\mathrm{I}_{34}$ calories per hour and keeps up this rate of heat production continuously with apparently little change.

863. Radium Emanation.-The ionization of a gas does not occur spontaneously, as does the ionization of an electrolyte. Moreover, very quickly after the ionizing agent (say a tube of radium) is removed the ions of a gas neutralize one another's charges and the gas becomes nonconducting. With this fact in mind let us consider the following curious observation: If a current of air is led over a radium compound, or better through a solution of a radium salt, the air will become strongly ionized and remain so for hours and even days, losing its electrical con- 
ductivity so slowly that only after about four days is half of the effect lost. In fact, it seemed as if the air had taken up radioactive matter from the radium, since this active air also caused a zinc sulfide screen to glow (859), and this glow was found by examination with a lens to be the result of scintillating flashes like those caused by alpha rays. In consequence the radium was said to have given off a radioactive emanation to the air. The material nature of the emanation was first clearly shown by experiments by Rutherford, in which air containing emanation was passed through a metal tube cooled externally by liquid air (776). Every trace of emanation was removed from the air that passed through the cooled tube; but when the latter was removed from the liquid air and allowed to come to room temperature the whole of the emanation was recovered by blowing fresh air through the tube. The explanation of this experiment given by Rutherford is very simple: Radium emanation is a gaseous radioactive substance which condenses practically completely at the temperature of liquid air. Upon becoming warm it again volatilizes. This explanation has proved to be correct in every particular.

864. The Formation and Decay of Radium Emanation.-The whole of the emanation contained in a solution of a radium salt can be removed by blowing a current of air through the solution for a few minutes. If the solution is sealed air-tight, new emanation will gradually accumulate in it. After about 40 days there is present in the sealed vessel a maximum of emanation strictly proportional to the quantity of radium present. The emanation accumulates at a regular rate, as follows: after 3.85 days from the time the solution was sealed (after all emanation has previously been removed) half of the maximum quantity of emanation is present; after $2 \times 3.85$ days, three-fourths of the maximum; after $3 \times 3.85$ days, seven-eighths of the maximum, etc., so that after 40 days over 99.9 per cent of the maximum is present. We have seen that half of the activity of a given portion of emanation is lost in about 4 days (863). Strictly speaking, exactly half is lost in 3.85 days; in $2 \times 3.85$ days half of the remainder is lost, or a total of three-fourths; in $3 \times 3.85$ 
days half of the remainder is again lost, or a total of seveneighths, etc. The emanation is said to decay to half value in 3.85 days. The interval of 3.85 days is called the period of half decay, or briefly the period. The rates of formation and decay of radium emanation are shown by the two graphs of Fig. I 20. The proportion of emanation contained in a sealed sample of radium after an interval of 40 days is a maximum, because from

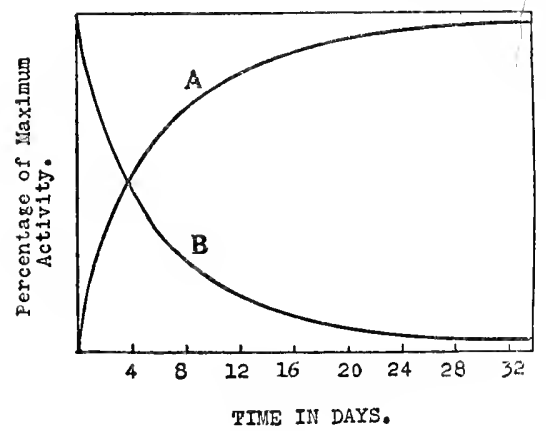

FIG. I 20 this time on the decay of the emanation present exactly compensates the formation of new emanation.

865. Theory of Radioactive Change.-The many facts already cited show that radium has properties that place it in a class by itself. For a number of years after the discovery of radium the cause of its

strange behavior was the most conspicuous of all scientific mysteries. Some faint-hearted scientists declared that the known facts disproved the two most fundamental scientific laws: the law of the conservation of matter and the law of the conservation of energy. It was also said that a serious inconsistency existed in the statement that radium is an element, if it is really true that radium gives rise to helium and radium emanation. The epoch-making hypothesis which fairly met and explained every known fact and withstood the most searching criticism was one of the boldest and at the same time the simplest and most comprehensive ever introduced into our science. This was the distintegration hypothesis of Rutherford and Soddy (48I). These scientists were at the time professors in the department of physics of McGill University, Montreal.

The sketch of this remarkable hypothesis already given (48r) should now be read again. According to this hypothesis radioactive atoms are more or less unstable systems. The first step in the disintegration of a radium atom is the throwing off of an 
atom of helium (an alpha particle) and the leaving behind of a residual atom of smaller mass, an atom of radium emanation $(\mathrm{Em})$,

$$
\mathrm{Ra} \rightarrow \mathrm{He}+\mathrm{Em} .
$$

If we suppose that the heliun atom shot out was in rapid orbital motion while it formed a part of the radium atom we have at once a simple explanation of the ligh velocity of the alpha particle and therefore of the energy given out by radium as heat (862), since the kinetic energy of the alpha rays must be changed into heat when the rays are stopped (368-37I).

866. The Radium Series.-A solid body of any kind exposed to radium emanation for some time becomes itself strongly radioactive, giving out alpha, beta, and gamma rays. This so-called excited activity decays rather rapidly (to half in about thirty minutes). Elaborate experiments have shown that the explanation of these facts is as follows: Just as a radium atom disintegrates, throwing off an alpha ray (helium atom) and leaving an atom of emanation, so an atom of the latter in turn shoots out another helium atom and leaves a new and lighter residual atom of a substance called radium A, having a very short period (864). Radium $A$ also gives out alpha rays and forms radium $B$, and the latter in turn passes into radium $\mathrm{C}$. The series of radioactive products of radium is therefore as follows:

$$
\mathrm{Ra} \rightarrow \mathrm{Em} \rightarrow \mathrm{RaA} \rightarrow \mathrm{RaB} \rightarrow \mathrm{RaC} .
$$

The excited activity obtained from the emanation consists of the products $\mathrm{A}, \mathrm{B}$, and $\mathrm{C}$. These, together with the emanation, are present in radium sealed so as to retain the emanation. Radium itself gives only alpha rays. The beta and gamma rays come largely from radium $C$.

867. The Origin of Radium.- -Soon after the discovery of the nature of radioactive change attempts were made to discover the rate of decay of radium itself. Several methods were devised. The simplest consisted in counting the number of alpha particles given off by a known amount of radium in a measured period of time. It was arranged so that each alpha particle would strike a zinc sulfide screen and produce a flash of 
light (859), the number of which flashes could be counted. Since we know the atomic weight of radium (857) and the actual number of atoms in one gram atomic weight of any element $\left(6.06 \times 10^{23}\right)$ (194), such an experiment furnished a means of calculating the rate of decay of radium. This rate is about 0.04 per cent per year, which corresponds to a period of $I, 85^{\circ}$ years. But even this period, long as it is in terms of the span of human life, is very short compared with the age of the earth as judged by the conclusions of geologists; so that if the earth were at the beginning made of pure radium, after even a million years far less radium would remain than we now find in many radium-bearing minerals. But as the age of the earth is to be reckoned in hundreds of millions of years, and as there is a practical certainty that the proportion of radium never was high, we are forced to the conclusion that the radium now found in the earth is being formed as fast as it decays. When these conclusions had been reached it became a matter of much scientific interest to discover the origin of radium.

The parent of radium, as we may call the hypothetical substance from which we may suppose radium to be formed, would very likely conform to the following specifications: It would be associated with radium in minerals; the ratio of radium to the substance would be constant (for the same sort of reason that sealed radium contains, after a sufficient interval, a constant proportion of emanation); the parent would probably be radioactive, with a very slow rate of decay compared with that of radium; the atomic weight of the parent would exceed that of radium. Only one element conforms to all these specifications, namely uranium (836). This element is beyond doubt the parent of radium. The latter element is found only in uranium minerals and in the fixed ratio of one part of radium to three million parts by weight of uranium.

Uranium-bearing minerals always contain mechanically imprisoned helium which has been formed by radioactive change. In fact it was in such a mineral, cleveite, that helium was discovered (794). 
868. The Uranium Series.-The change of uranium into radium is not direct but takes place by the intermediate formation of a series of radioactive products, as indicated in Table LIV, which also includes the entire radium series.

TABLE LIV

The Uranium-Radium Series (Slightly Condensed)

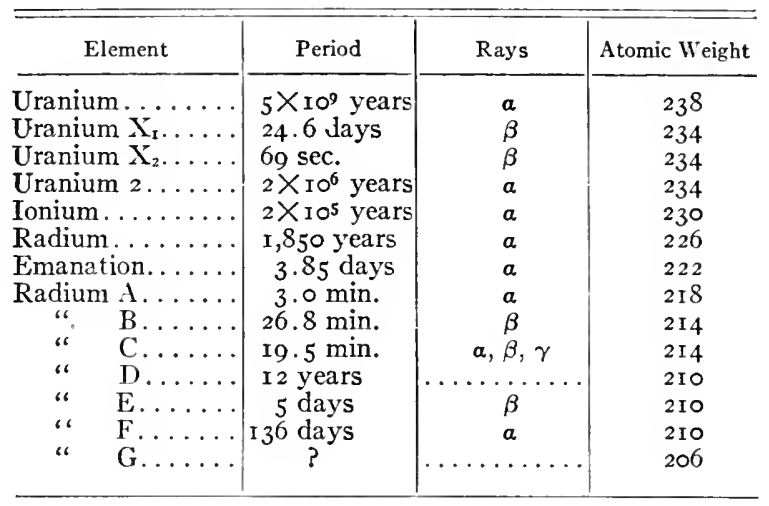

869. The Atomic Weights of Radioactive Substances.-If the disintegration hypothesis is correct the atomic weight of a product formed by an alpha-ray change should be less than that of its parent by the atomic weight of helium, namely 4 units. By reason of the very small mass of an electron (479) the atomic weight is practically unchanged as the result of a beta-ray change. Taking the atomic weight of uranium (836) in round numbers as 238 , the calculated atomic weights of its products are those given in the last column of Table LIV (868). We can easily test these conclusions in one case, that of radium. The calculated value is 226.2 , if $U=232.2$, while Mme Curie found by experiment the nearly identical value 226 . Therefore it appears probable that the calculated values given in the table are essentially correct.

870. Thorium and Its Active Products. - The element thorium $(820,834)$ is radioactive with an intensity of activity about equal to that of uranium. Like the latter, the former gives rise to a 
long series of active products, of which the first, mesothorium, is the only one of technical importance. Mesothorium is identical with radium in its chemical behavior, and no means is known of separating $\dot{a}$ mixture of the two. They are readily distinguished by their periods and by their radioactive products. Mesothorium has a period of $5 \cdot 5$ years as against 1,850 years for radium. Mesothorium itself gives no rays but changes into a series of active products giving alpha, beta, and gamma rays. Each product has its own characteristic chemical properties, rays, and period. One of these products is radiothorium, having a period of two years. This gives out alpha rays and forms thorium $\mathrm{X}$, which in turn forms a gaseous emanation having a period of less than one minute. Mesothorium is produced as a by-product of the manufacture of thorium and is used as a substitute for radium.

871. Isotopes.-A most unlooked-for set of facts was brought to light by the investigation of the chemical behavior of certain radioactive products. We have mentioned that mesothorium and radium are identical in chemical behavior (870). Thorium $\mathrm{X}$ is also identical chemically with these two, and no means is known of separating mixtures of this substance and either radium or mesothorium. Substances identical in chemical behavior are called isotopes.

Several groups of isotopes are known. Thus radiothorium and uranium $\mathrm{X}$ are isotopic with thorium, since in every chemical reaction they both behave qualitatively and quantitatively exactly like thorium. Each of the three can be obtained separately, but if we mix the separate substances it is impossible, by any known means, to separate them. The final product of the radium series, formed by the decay of $\mathrm{RaF}$ and known as $\mathrm{RaG}$ (at. wt. 206), is an inactive substance isotopic with lead (at. wt. 207.2), and curiously enough $\operatorname{RaD}$ (at. wt. 210 ) is also isotopic with lead and therefore also with $\mathrm{RaG}$.

Isotopes are not identical in all their properties; otherwise they would be identical substances and consequently indistinguishable. Thus, for example, radium differs from mesothorium in the following respects: Radium has a period of 
I, $85^{\circ}$ years; mesothorium has a period of $5 \cdot 5$ years. Ra gives alpha rays; Ms gives no rays. Ra changes into a gas, Em, which gives alpha rays and has a period of 3.85 days; Ms changes into a solid which gives beta rays and has a period of 6.2 hours. $\mathrm{Ra}$ has an atomic weight of 226.0 , while Ms has the value 228.4. No difference in the spectra of isotopes has yet been discovered.

872. The Valence of Radioactive Substances.-Although but very few of the radioactive products of the uranium-radium series (Table LIV) or the thorium series have as yet been obtained in weighable amounts in pure form, still we know much about the chemical and physical properties of these substances and are thus able to learn their valence. For example, when we know that mesothorium is isotopic with radium and that the valence of the latter is two, we are safe in concluding that mesothorium has the same valence. Since $\mathrm{UX}_{\mathrm{r}}$ and ionium are isotopic with thorium we conclude that all three have the same valence, namely four; and since $U_{2}$ is isotopic with uranium itself its valence is $\operatorname{six}\left(8_{3} 6\right)$. These examples suffice to illustrate the principle employed. Let us now consider the first part of the uranium-radium series. In Table $\mathrm{LV}$ the first line gives the

TABLE LV

\begin{tabular}{c|c|c|c|c|c|c|c}
\hline \hline & $\mathrm{U}^{*}$ & $\mathrm{UX}_{\mathrm{t}}$ & $\mathrm{UX}_{3}$ & $\mathrm{U}_{2}$ & Io & $\mathrm{Ra}$ & $\mathrm{Em}$ \\
\hline Rays......... & $a$ & $\beta$ & $\beta$ & $\alpha$ & $a$ & $a$ & $a$ \\
Valence...... & 6 & 4 & 5 & 6 & 4 & 2 & 0 \\
\hline
\end{tabular}

symbols of the products of uranium in the order of their formation (868); the next line shows the kind of rays given out by each substance; while the third line shows the valence of each.

Fajans discovered a remarkable relation between the facts set forth in this table, namely, (I) the valence of the product of an alpha-ray change (858) is less by two than that of the parent; (2) the valence of the product of a beta-ray change (860) is greater by one than that of the parent. This statement is called Fajans' Law. With a little amplification the law applies to all radioactive substances and their transformations. 
873. The Meaning of the Term Element.-A direct definition of the term element has not been given in this text (see $3 \mathrm{I}$ ). To say that an element is a substance incapable of further decomposition would exclude uranium, radium, and thorium as well as, of course, all other radioactive substances from the list. of elements; and this would certainly be an unwise classification. Since radioactive changes are always spontaneous and entirely beyond human control, it would seem best to define the term element thus: An element is a substance which cannot be decomposed into simpler substances at the will of the experimenter. We shall then class all radioactive products (even those like thorium emanation, period 53 seconds) as elements. All these "new" elements have been satisfactorily fitted into enlarged periodic tables. For example, see the lower part of the Harkins table (849). It will be seen that several elements occupy the same position. Except for the rare earths these are isotopes.

874. The Technical Production of Radium.-Radium was first made in considerable quantities from pitchblende, a mineral having the composition $\mathrm{U}_{3} \mathrm{O}_{8}$. This mineral is so rare that it is no longer a satisfactory source of radium. The bulk of the radium now produced is made from carnotite, a mineral of somewhat variable composition, but containing uranium, vanadium, and potassium as its usual constituents. This ore is found chiefly in southwestern Colorado and adjoining regions of Utah, for the most part as cementing material in sandstone. The ordinary ore as mined carries less than I per cent of uranium and therefore but one part of radium in 300 million parts by weight of ore! There are several ways of extracting the radium, but all of them are based on the fact that the radium present remains with the barium present when the latter is separated. If insufficient barium is present in the ore, enough barium chloride is added to bring the proportion up to about I per cent. Usually the barium (with the radium) is first obtained as sulfate (164). This salt is converted into chloride and the latter subjected to fractional crystallization in the manner already described (856). A much more rapid separation of barium and radium results from the crystallization of the bromides, since 
in this case above 95 per cent of the radium separates with half of the barium salt. In another process the hydroxides of barium and radium are crystallized, in which case the radium concentrates in the mother-liquors instead of in the crystals.

The world's total production of radium up to the end of I9I8 was between 100 and 125 grams, over half of which was made in the United States of America.

875. How Radium Is Measured and Sold.-Radium usually comes on the market as an isomorphous (8Io) mixture of its bromide with about an equal amount of barium bromide. The selling price of radium is always based on the radium content of material, stated in milligrams of radium element. The radium content of a given sample is not found by the use of a balance but is determined by the ionizing power of the gamma rays (86I) as shown by an electroscope $(770,854)$. Comparative measurements are always made with a tube of radium salt of exactly known radium content (a standard). Every purchaser of radium, before paying for it, can have the material measured by the United States Bureau of Standards and get a certificate for a small fee. During the year I9I8 the price was close to $\$ 120.00$ per milligram of radium element. This is more than 100,000 times the value of gold! But when it is known that a ton of ordinary ore yields only two milligrams of radium and that it requires three to four months' work to extract and refine the radium, the high price is readily understood.

876. The Use of Radium in Therapeutics.-The term therapeutics is defined as being that branch of medical science that treats of the action of remedial agents on the human body. The beta and gamma rays of radium are capable of producing serious "burns" of the skin and underlying tissues. The effect produced is proportional to the quantity of radium and the length of time of the exposure. Healthy tissue is much less affected by these rays than is abnormal and unhealthy tissue. It is by reason of this fact that radium finds an important use as a therapeutic agent. Exposure of certain kinds of abnormal growths on the body to radium rays results in their destruction and subsequent removal. Some forms of cancer respond very favorably to such 
treatment. In cases where large masses of cancerous tissue are removed by surgical operation treatment with radium rays is often subsequently employed to destroy the remaining portions not accessible to the knife. In hopeless cases, where a cure cannot be expected, radium treatment is useful in greatly alleviating pain. In most respects radium rays produce therapeutic effects like those due to $\mathrm{X}$-rays. The great advantage over $\mathrm{X}$-rays in the use of radium arises from the fact that the radium container is so minute that it can be applied exactly where its rays are required to act, a procedure impossible with an $\mathrm{X}$-ray bulb. Radium in considerable quantity (100 mg. or more) may be safely handled if contained in thick-walled lead tubes.

877. X-Ray Spectra.-Ordinary visible light, which is made up of ether vibrations of various wave-lengths (788), can be spread out into a spectrum of its component colors in another way besides that by the use of a prism, namely by means of a diffraction grating. The latter consists of a polished plane surface, as of glass or metal, ruled with an enormous number of fine parallel lines, often as little as one-thousandth of a millimeter apart. The principle of the production of spectra by such gratings is discussed in most textbooks on physics.

For many years after the discovery of X-rays numerous attempts were made to obtain X-ray spectra, but all without any success, by the use of either prisms or gratings. It then occurred to Laue that if $\mathrm{X}$-rays consisted of very much shorter waves than visible light it would be impossible to rule gratings with lines sufficiently fine and close together to show the expected effect. In fact, he calculated that if $\mathrm{X}$-rays consist of waves onethousandth as long as those of visible light, in order to get their spectrum it would require a grating with lines no farther apart than the diameters of ordinary atoms (196). To rule such a grating would be a task beyond human skill. Then came the brilliant idea that nature makes such gratings on every hand, since in every crystal the molecules are arranged in rows and layers like bricks in a wall (204). Several experimenters applied Laue's icleas and soon brought forth a great number of new and interesting facts, a few of which we shall discuss briefly. 
When X-rays from a given source strike the flat surface of a crystal obliquely the reflected rays, brought to focus on a photographic plate, produce a well-defined line spectrum consisting of a small number of lines. Each line corresponds to a definite wave-length (just as in the case of visible light), which length can be easily calculated.

878. Atomic Numbers.-The X-ray spectrum given by one element used as the target of the $X$-ray bulb is characteristic for that element and different from that of any other element. In this connection a remarkably simple relation was discovered in I9I4 by the young English physicist Moseley: the wave-length of the

TABLE LVI

Periodic Table According to Atomic Numbers, H: $\mathrm{r}$

\begin{tabular}{|c|c|c|c|c|c|c|c|c|c|}
\hline$\underset{\text { riod }}{\mathrm{Pe}-}$ & $\mathrm{O}$ & $\begin{array}{lll} & \mathrm{I} & \\
\mathrm{A} & & \mathrm{B}\end{array}$ & $A{ }^{\text {II }}$ B & $\mathrm{A}^{\text {III }} \mathrm{B}$ & $\mathrm{A}^{\mathrm{IV}} \mathrm{B}$ & $\mathrm{A}^{\mathrm{V}}$ B & $A_{A} \begin{array}{lll} & V I & \\
B\end{array}$ & ${ }_{A}{ }_{B}^{\text {VII }}$ & VIII \\
\hline$I$ & $\stackrel{2}{\mathrm{He}}$ & $\mathrm{Li}^{3}$ & $\mathrm{Be}^{4}$ & $\begin{array}{l}5 \\
\mathrm{~B}\end{array}$ & $\stackrel{6}{\mathrm{C}}$ & $\stackrel{7}{N}$ & $\begin{array}{ll}8 & 0\end{array}$ & ${ }^{9} \mathrm{~F}$ & \\
\hline 2 & $\begin{array}{l}\mathrm{IO} \\
\mathrm{Ne}\end{array}$ & $\mathrm{Na}^{\mathrm{xI}}$ & $\mathrm{Mg}^{\mathrm{I} 2}$ & $\begin{array}{l}\mathrm{I}_{3} \\
\mathrm{Al}\end{array}$ & $\begin{array}{l}\mathrm{I} 4 \\
\mathrm{Si}\end{array}$ & $\begin{array}{l}\text { I5 } \\
\text { P }\end{array}$ & I6 $\mathrm{S}$ & ${ }^{1} 7 \mathrm{Cl}$ & \\
\hline \multirow{2}{*}{3} & $\begin{array}{l}\text { I } 8 \\
\mathrm{~A}\end{array}$ & $\mathrm{~K}^{\mathrm{I9}}$ & $\mathrm{Ca}^{20}$ & $\mathrm{Sc}^{2 \mathrm{I}}$ & $\mathrm{Ti}^{22}$ & $V^{23}$ & $\mathrm{Cr}^{24}$ & $\mathrm{Mn}^{25}$ & $\begin{array}{lll}26 & 27 & 28 \\
\mathrm{Fe} & \mathrm{Co} & \mathrm{Ni}\end{array}$ \\
\hline & & ${ }^{29} \mathrm{Cu}$ & $3^{30} \mathrm{Zn}$ & ${ }^{3 \mathrm{I}} \mathrm{Ga}$ & ${ }^{32} \mathrm{Ge}$ & ${ }^{33} \mathrm{As}$ & ${ }^{34} \mathrm{Se}$ & ${ }^{35} \mathrm{Br}$ & \\
\hline \multirow{2}{*}{4} & $\begin{array}{l}36 \\
\mathrm{Kr}\end{array}$ & $\mathrm{Rb}^{37}$ & $\mathrm{Sr}^{38}$ & $Y^{39}$ & $\mathrm{Zr}^{40}$ & $C b^{4 x}$ & $\mathrm{Mo}^{42}$ & $?^{43}$ & $\begin{array}{lll}44 & 45 & 46 \\
\mathrm{Ru} & \mathrm{Rh} & \mathrm{Pd}\end{array}$ \\
\hline & & ${ }^{47} \mathrm{Ag}$ & ${ }^{48} \mathrm{Cd}$ & ${ }^{49}$ In & ${ }^{50} \mathrm{Sn}$ & ${ }^{5 \mathrm{I}} \mathrm{Sb}$ & ${ }^{52} \mathrm{Te}$ & 53 & \\
\hline \multirow{2}{*}{5} & $\begin{array}{l}54 \\
\mathrm{Xe}\end{array}$ & $\mathrm{Cs}^{55}$ & $\mathrm{Ba}^{56}$ & $\begin{array}{l}57 \\
\text { La }\end{array}$ & $\stackrel{58}{\mathrm{Ce}}$ & $\begin{array}{l}59 \\
\operatorname{Pr}\end{array}$ & $\begin{array}{l}60 \\
\mathrm{Nd}\end{array}$ & $\begin{array}{c}6 \mathrm{I} \\
?\end{array}$ & \\
\hline & & $\begin{array}{l}62 \\
\mathrm{Sa}\end{array}$ & $\begin{array}{l}63 \\
\text { Eu }\end{array}$ & $\begin{array}{l}64 \\
\mathrm{Gd}\end{array}$ & $\begin{array}{l}65 \\
\mathrm{~Tb}\end{array}$ & $\begin{array}{l}66 \\
\text { Dy }\end{array}$ & $\begin{array}{l}67 \\
\text { Ho }\end{array}$ & $\begin{array}{l}68 \\
\text { Er }\end{array}$ & \\
\hline \multirow{2}{*}{6} & & $\begin{array}{l}69 \\
\text { Tm }\end{array}$ & $\begin{array}{l}70 \\
?\end{array}$ & $\begin{array}{l}7 \mathrm{I} \\
\mathrm{Yb}\end{array}$ & $\begin{array}{l}72 \\
\text { Lu }\end{array}$ & $\mathrm{Ta}^{73}$ & $w^{74}$ & $?^{75}$ & $\begin{array}{lll}76 & 77 & 78 \\
\text { Os } & \text { Ir } & \mathrm{Pt}\end{array}$ \\
\hline & & ${ }^{79} \mathrm{Au}$ & ${ }^{80} \mathrm{Hg}$ & $\begin{array}{lll}8 \mathrm{I} & \\
& & \mathrm{Tl}\end{array}$ & ${ }^{82} \mathrm{~Pb}$ & ${ }^{83} \mathrm{Bi}$ & 84 & 85 & \\
\hline 7 & $\begin{array}{l}86 \\
\mathrm{Nt}\end{array}$ & $?^{87}$ & $\mathrm{Ra}^{88}$ & $?^{89}$ & $\mathrm{Th}^{90}$ & $?^{91}$ & $U^{92}$ & & \\
\hline
\end{tabular}


most intense line decreases quite uniformly as we pass from one element to the next higher in atomic weight. Moseley also showed that if the elements are assigned numbers, called atomic numbers, in the order of their increasing atomic weights there is a very simple numerical relation between the atomic number of an element and the wave-length of the strongest line of its X-ray spectrum. The atomic numbers calculated from the wave-lengths as found by experiment differed from whole numbers by not more than I or 2 per cent, and this difference could easily be ascribed to error of experiment. The only cases where the atomic numbers so found did not follow exactly the order of increasing atomic weights were met with in those pairs of elements (A and $\mathrm{K}, \mathrm{Te}$ and $\mathrm{I}$ ) where a departure from the usual order is necessary to bring the elements into their proper groups in the Periodic Table (829). In Table LVI the elements are arranged in a periodic table strictly in the order of their atomic numbers. The anomalies (848) now disappear completely. It would therefore appear that the atomic number of an element is its most fundamental constant. In Table LVI the rare earth elements $(820,846)$ are included within the heavy lines. These elements form a single family of Group III (849) and do not belong to the other groups indicated by their positions in this table.

879. The Structure of Crystals.-Professor W. H. Bragg and his son W. L. Bragg, English physicists, have followed Laue's ideas in another direction and obtained a great deal of information regarding the arrangement of molecules and atoms in a great variety of crystals. The simplest way of investigating the structure of a crystal consists in getting the impression made on a photographic plate when a small, round beam of $\mathrm{X}$-rays passes through a thin slice of the crystal and strikes the plate some inches beyond. The reproduction of such a photograph is shown in Fig. I Ir. From the nature of the pattern produced the geometrical arrangement and distances from one another of the atoms of a crystal can be calculated.

880. Atomic Structure and the Nature of Matter.-The modern view of the structure of an atom has been described 
briefly in section 470. The facts epitomized in the periodic law, together with the phenomena of radioactivity, show very convincingly that atoms must be constructed according to very definite plans, and that the arrangement of the parts (electrons, atoms of helium, and the atomic nucleus, 470) in the atom of an element must determine its properties. Some of the world's ablest physicists and chemists have devoted much attention to the problems of atomic structure, and as a result several more or less definite hypotheses have resulted, each aiming to explain and correlate as many facts as possible. As yet none of these suggestions is wholly

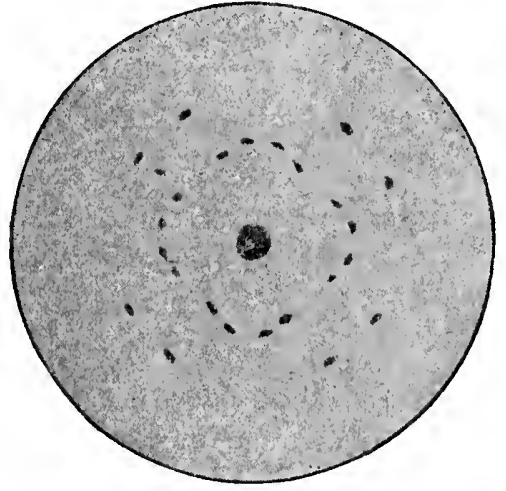

FIG. I 2 I satisfactory, although several of them account very well for many of the facts with which we are familiar.

On one point there is general agreement: namely, that the atomic number of an element represents the number of electrons cncircling the electro-positive nucleus of the atom. Since atoms, as such, are electrically neutral, the positive charge of the nucleus must also be proportional to the atomic member. It seems probable that the nucleus also contains some electrons and that its positive charge represents the excess of its positive over its negative electricity.

In changes of atoms into ions and vice versa (oxidations and reductions, $5^{\circ} \mathrm{I}^{-507}$ ) the valence electrons lost or gained are those of the outer ring, and not of the nucleus. In radioactive changes the alpha and beta rays doubtless come from the nucleus, so that the new residual atom has a nucleus different in positive charge from its parent and therefore surrounded by a different number of electrons in the outer rings. It is on this basis that Fajans' Law (872) receives its explanation. 
It is of interest to note that the chemist's control over the composition of the atom is limited to the removal and replacement of some of the electrons of the outer rings; changes of the nucleus are solely the result of spontaneous radioactive processes entirely beyond human control.

Since the positive charge of the nucleus is the excess of its positive over its negative electricity, it is possible for two or more differently composed nuclei to have equal nuclear charges. Atoms containing nuclei differing thus would have equal numbers of electrons in the outer rings and therefore be identical in chemical but not in radioactive properties. In consequence it would be possible to have two (or more) different elements with identical chemical properties, in other words isotopes (87I).

In conclusion, it may be stated that all chemists and physicists are now agreed that the elements are but modifications of the same primitive forms of material, including in the latter term electrons and whatever else may compose an atom. It may well be that all matter is entirely of an electrical nature. This view has led some persons to declare that "there is no such thing as matter," that "everything is electricity." This statement is scarcely warranted. It is just as if, when we have discovered that a potato is composed of starch, fiber, and water, we should declare "there is no such thing as a potato"! As for matter, it remains what it always has been, only we know more about it. 


\section{CHAPTER XXXIII}

\section{METALLURGY}

881. Introduction.-Metallurgy, or the art of extracting metals from their ores, had its beginning in prehistoric times. In our present-day civilization it is the basis, of one of our greatest industries. The colossal importance of it may be judged from Table LVII, which gives the annual production of metals in the United States, the values of the output, and the percentage of the world's supply furnished by the United States. In the cases of iron, copper, silver, zinc, lead, and aluminum, the United States produces more than any other one country. The total value of its annual production is over two billion dollars.

As will be shown later, this enormous industry may well be called a chemical industry since, in the first place, the science of chemistry has contributed greatly to improvements practised in the general procedures, and since, in the second place, virtually all of the enormous output of metals is prepared under supervision from chemical laboratories.

It will be the purpose of the present chapter to present a brief survey of the metallurgy of the more important metals, namely, iron, copper, zinc, lead, and aluminum, and incidentally certain aspects of the metallurgy of silver and gold.

TABLE LVII

Anvual Production of Metals in the Uxited States

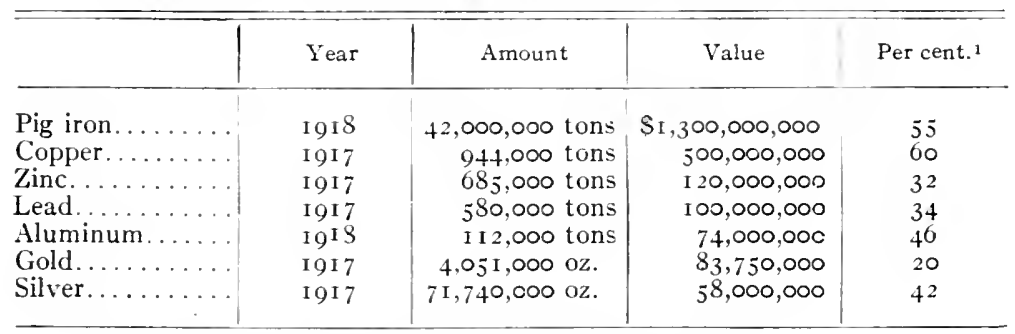

${ }^{1}$ U. S. A. production as percentage of total for the world. 
882. Economic Importance of Iron.--Of all metals, iron is the most important. Not only is this true because it is abundant, but because of the cheapness of its production and its manifold useful properties. To appreciate this we have only to reflect that most of our earthly possessions which do not consist wholly or in part of iron have been made with the aid of iron machinery, which is driven by engines or motors consisting wholly or in part of iron. Iron is the only element which is decidedly magnetic (of high magnetic permeability) and so is the essential material for magnets. Thus dynamos, motors and other electrical machinery which are the basis of the modern generation and use of electricity would not be possible without iron. It is easy to see that the existing material aspect of our present-day civilization is dependent upon this element. It is therefore not strange that iron is produced in far greater quantity than any other metal, and it is interesting to find that the tonnage of iron produced in the United States is about twenty times that of all the other metals combined. (See Table LVII.)

883. Commercial Grades of Iron. Steel.-Broadly speaking, commercial grades of iron belong to one of three classes: wrought iron, cast iron, and steel. All grades contain small amounts of certain other elements which have important bearing on the properties of the product. Of these elements the most important is carbon. Others are silicon, manganese, phosphorus and sulfur.

The terms wrought iron and cast iron designate substances of more definite composition than does the term steel, which applies to products of certain processes. Some kinds of steel are nearly identical in composition and properties with wrought iron. The latter and also low carbon or mild steel contain less than 0.3 per cent of carbon. Half-hard and high carbon steel contains from 0.3 per cent to 2.2 per cent of carbon. Cast iron contains from 2.2. to 5 per cent of carbon.

Wrought iron and mild steel are moderately soft metals. High carbon steel may be either extremely hard or rather soft, 
according to the heat treatment given it. Cast iron is moderately hard and brittle, but can be machined to shape.

884. Iron Ores.-It seems probable that iron constitutes about 5 per cent of the earth's crust (F.W. Clarke). The only iron-bearing minerals of importance are those which contain 40 per cent or more of this element and only small percentages of objectionable impurities, especially phosphorus and sulfur. In the United States the most important ore is hematite, $\mathrm{Fe}_{2} \mathrm{O}_{3}$, (328). Limonite, a hydrated hematite called also brown ore, and magnetite, $\mathrm{Fe}_{3} \mathrm{O}_{4}$ (I73) are also mined in considerable amounts; but altogether they constitute only 6 to 8 per cent of the total iron ore of this country.

No less than 85 per cent of all the iron ore mined in the United States comes from a single region which lies to the south and west of Lake Superior, in adjacent parts of the states of Minnesota, Michigan and Wisconsin. The ore of the Lake Superior region is hematite. The next most important district, located in Alabama, produces 8 per cent of the total ore. New York state ranks third in importance, with 3 per cent. The remaining states produce but 4 per cent of the total.

885. The Chemistry of the Metallurgy of Iron.-The principal reactions involved in the conversion of ferric oxide, $\mathrm{Fe}_{2} \mathrm{O}_{3}$ (hematite), into metallic iron (329) are

$$
\mathrm{Fe}_{2} \mathrm{O}_{3}+{ }_{3} \mathrm{C} \rightarrow 2 \mathrm{Fe}+{ }_{3} \mathrm{CO}
$$

and

$$
\mathrm{Fe}_{2} \mathrm{O}_{3}+{ }_{3} \mathrm{CO} \rightarrow 2 \mathrm{Fe}+{ }_{3} \mathrm{CO}_{2}
$$

Taken together, these may le written:

$$
{ }_{2} \mathrm{Fe}_{2} \mathrm{O}_{3}+{ }_{3} \mathrm{C} \rightarrow 4 \mathrm{Fe}+{ }_{3} \mathrm{CO}_{2}
$$

If iron ores consisted of pure cxides, it would suffice to heat these with carbon and nothing els.. However in practice this is not the case. The average composition of Lake Superior ore for the year I9o9 was that shown in Table LVIII.

It thus appears that components other than iron oxide are present in large amount and must be removed in the metallurgical process. The elimination of silica, alumina, calcium, etc. is accomplished by converting them into a fusible glass- 
TABLE LVIII

Average Percentage Composition of Lake Superior Iron Ore (Ries)

Iron........................ $5^{8.45}$ per cent

Silica........................... 7.67

Alumina...................... 2.23

Calcium, magnesium and manganese....... I.80

Phosphorus.......................... .

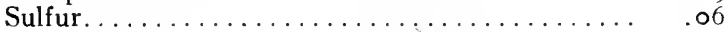

Loss on ignition................... 4.12

like product called slag, which at a white heat forms a fluid that floats on molten iron like oil on water and can be separated mechanically.

In the formation of slag we have a typical reaction in a fused mass. For example, calcium oxide is a base forming oxide. Silica is an acid forming oxide. Heated together they form a salt, calcium silicate, just as acids and bases do in water solution. The salt is molten at the temperature of the furnace and dissolves many other oxides and salts. The following is representative of the percentage composition of slag:

$\begin{array}{cccccc}\mathrm{SiO}_{2} & \mathrm{Al}_{2} \mathrm{O}_{3} & \mathrm{CaO} & \mathrm{MgO} & \mathrm{CaS} & \text { Remainder } \\ 34 & \mathrm{I} 2 & 43 & 6 & 3 & 2\end{array}$

Slag is therefore a silicate of aluminum, calcium, and magnesium with small proportions of other substances. Since but few ores contain the slag forming elements in ideal proportions, it is customary to add to the ore sufficient materials from other sources to make up the deficiency. Usually it is only necessary to add limestone (I5).

We shall now describe the smelting of iron ore as actually carried out in the blast furnace.

886. The Blast Furnace.-A blast furnace is represented in Fig. I22. This is a huge structure, 60 to 90 feet in height, built of iron and lined with fire brick. This furnace gets its name from the fact that a blast of air is supplied near the base at a pressure of about $I_{5}$ pounds per square inch above atmospheric pressure. The air before entering the furnace is heated to about $700^{\circ}$ (corresponding to a red heat) in a way later to be described. The charge or burden, consisting of ore, limestone and coke $(328,630)$ is introduced at the top of the furnace, 
which is closed by a cone or "bell." In a typical case, a charge of 9 tons of ore, 5 tons of coke and 3 tons of limestone is added every quarter of an hour, day and night, week in and week out, for many months at a time. As the charge passes downward it soon becomes hot enough to react so that by the time it has reached the level of the blast pipes (tuyeres) it consists largely of molten iron and slag. The former collects in the lowest part of the furnace as a white hot fluid, on which floats the equally hot but somewhat more viscous liquid slag. From time to time slag is tapped from the furnace through an opening made for the purpose. At intervals of a few hours the iron is also drawn off at a lower level opening and run into troughs made in a bed of sand. The smaller troughs, about 3 feet long and 4 inches wide, branch off from the main channel at uniform distances. The solidified product is called pig iron. A large furnace charged as above indicated will produce 500 tons ( $\mathrm{I}, 000,000$ pounds) of pig iron in 24 hours.

887. The Blast Furnace Plant.-In order that the reduction of ore to metal. may be complete, it is necessary to use

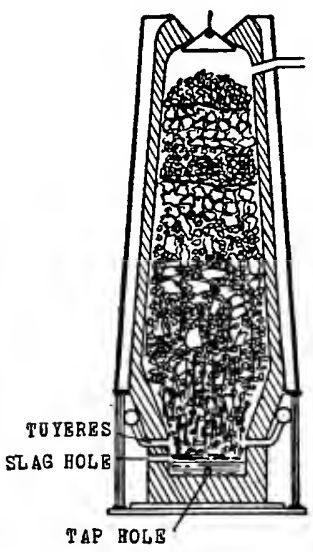

FIG. I 22 more carbon (in the form of coke) than that indicated by the chemical equations (885). As a result, the gases issuing from the top of a furnace contain a large proportion of carbon monoxide. A typical product will contain 25 per cent of carbon monoxide, I 3 per cent of carbon dioxide, 3 per cent of hydrogen, and 57 per cent of nitrogen. This gas is saved and used as fuel to heat the air used for the blast and also to produce the power to run the air blowers. For the latter purpose it is either burned under the boilers of steam engines or, in more modern plants, it is used directly as the fuel for huge gas engines. The blast furnace requires therefore a large accessory equipment, the whole forming the blast furnace plant. The more important items of this plant will now be briefly described. The gases 
escaping from the furnace carry a great deal of dust. This is first separated and collected in a dust catcher consisting of an immense cylindrical vessel with a conical base in which the dust settles out to a large extent. The gases may be further purified in washers.

For heating the blast, so-called stoves are used, of which three or fcur are required for each blast furnace. A stove is a cylindrical furnace nearly half as large as the blast furnace. It consists of a central chamber in which the gas burns surrounded by a honey-comb like arrangement (checker-work) of fire brick, through which the flame and hot gases pass before they escape at the smoke-stack. After a short time the stove becomes very hot. The supply of gas is then turned off and the blast of (cold) compressed air is led through the hot iron stove whereby the air is raised to a temperature of about $700^{\circ}$. This hot air is used to blow the blast furnace. While one stove is being used to heat the blast, others are being heated by burning gas.

888. Pig Iron and Slag.-Pig iron is by no means pure iron. Its composition is variable. It contains about 94 per cent of iron, 3 to 4 per cent of carbon, I to 2 per cent of silicon, I per 'cent of manganese, o.I to 2 per cent of phosphorus, and less than o.I per cent. of sulfur. Pig iron is so brittle that the pigs are easily broken into smaller pieces by means of a sledge hammer. Small pieces can be ground to a powder in a mortar. In appearance, pig iron is metallic gray, and shows a crystalline or granular facture.

Pig iron is an intermediate product which is used to make purer forms of iron, about 78 per cent of the production going into steel, I9 per cent into cast iron, and $2 \frac{1}{2}$ per cent into wrought iron.

Slag is largely waste material which is used for road building and for ballast for railroads. A good quality of cement is made from slag. Mineral wool, used for fire and acid-proof packing, is made by blowing steam through molten slag.

889. Cast Iron.--Cast iron is the form of the metal used in making stoves, steam radiators, street lamp posts, water and gas 
mains, fire hydrants, etc. where strength and rigidity but not flexibility nor elasticity are required. Iron castings are made by pouring the molten metal into moulds made in sand from wooden patterns of the form to be reproduced. The metal is melted in a cupola furnace, Fig. I 23. This furnace consists of a cylindrical sheet steel shell 3 to 6 feet in diameter and ${ }_{5} 5$ to 25 feet high lined with fire-brick. The bottom is covered with a thick layer of sand. A tapping hole for the iron is located at the upper level of the sand layer and on the opposite side of the cupola a little higher up is another tap hole for slag. Several inlets (tuyeres) for the air blast are provided. Thus the cupola furnace resembles a very small blast furnace. Its charge consists of pig iron and coke together with enough limestone to form a slag with the silica of the coke ash and a certain additional amount formed by oxidation of a small part of the silicon of the pig iron used. Usually some old scrap iron, broken and worn out castings, etc. are added to the charge. In the furnace the coke burns in the

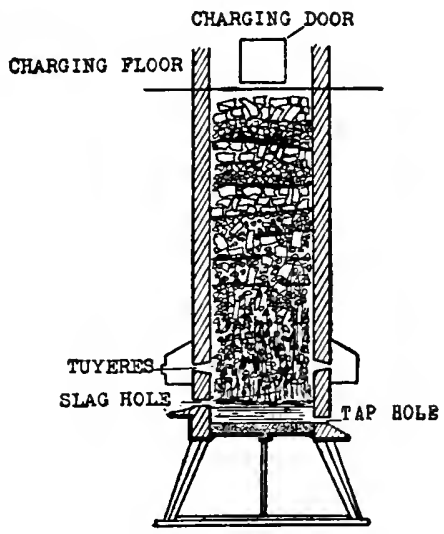

FIG. I 23 strong air blast and raises the temperature above the melting point of the pig iron (about $1100^{\circ}$ ). The molten iron, covered by a layer of molten slag, collects in the bottom of the furnace. The white hot liquid iron is drawn off through the tap hole into a ladle from which it is poured into the mould. An iron casting or moulding establishment is called a foundry.

There are many grades of cast iron which merge imperceptibly into one another. The differences are due to two causes: the chemical composition and the heat treatment the product has undergone. In addition to a little manganese and phosphorus and a minute amount of sulfur, cast iron contains between $3 / 4$ and $2 \frac{1}{2}$ per cent of silicon and $23 / 4$ to $3 \frac{1}{2}$ per cent of carbon. It is the last two elements in particular that determine 
the character of the cast iron. The two principal sorts of cast iron are designated as white and gray respectively. If a molten iron low in a silicon (e.g. 3/4 per cent) and containing about $23 / 4$ per cent of carbon is cooled rapidly, white cast iron results. This is silver white on the fracture and very brittle. It is so hard that it cannot be machined to shape in a lathe. On the other hand, iron high in silicon (e.g. $2 \frac{1}{2}$ per cent) and carbon (31/2 per cent) if cooled slowly forms gray cast iron, gray in color, and soft enough to be easily machined. The reason for these facts will be considered in a later section.

89o. Wrought Iron.-Wrought iron is one of the purest commercial forms of the metal. It contains less than 0.3 per cent of carbon and only traces of silicon, phosphorus and sulfur;

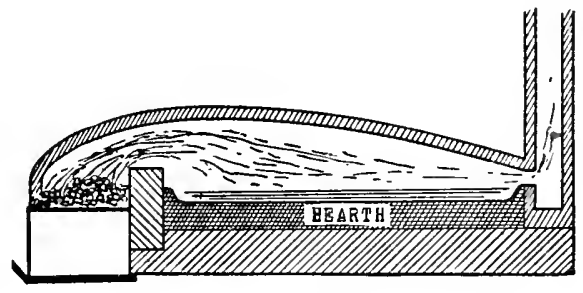

FIG. I 24

although it holds in mechanical admixture a small amount of solid slag. The conversion of molten pig iron into wrought iron is accomplished by oxidizing the elements to be eliminated, thus converting the carbon into gaseous monoxide, $\mathrm{CO}$, and the others into a molten slag consisting largely of ferrous silicate and ferrous phosphate. The oxidation is carried out by mixing the molten pig iron at a white heat with oxides of iron.

This process of making wrought iron, usually known as pig boiling, is carried out in a so-called puddling furnace, Fig. I 24. This is a small, simple form of reverberatory furnace. This kind of furnace is so called because heat from the flame produced in the fire box is reflected or reverberated from the top of the furnace to the material on the hearth beneath. In a reverberatory furnace the material to be heated is not contaminated by contact with the fuel. In the puddling furnace the hearth 
is lined with iron oxides, which may be very pure ore (hematite or magnetite) or so-called hammer scale, which is chiefly $\mathrm{Fe}_{3} \mathrm{O}_{4}$. The process consists in melting about 400 pounds of pig iron on the hearth and adding to the molten metal powdered iron oxides and stirring (rabbling) the mixture thoroughly with an iron bar. A vigorous reaction soon begins. The carbon monoxide formed by the reactions

$$
\begin{aligned}
& \mathrm{Fe}_{3} \mathrm{O}_{4}+{ }_{4} \mathrm{C} \rightarrow{ }_{4} \mathrm{CO}+{ }_{3} \mathrm{Fe} \\
& \mathrm{Fe}_{3} \mathrm{O}_{4}+\mathrm{C} \rightarrow \mathrm{CO}+{ }_{3} \mathrm{FeO}
\end{aligned}
$$

escapes, causing the material to appear to boil, hence the term pig boiling. The ferrous oxide formed in the last reaction unites with silicon dioxide formed by oxidation of the silicon to form a silicate and with phosphorus pentoxide produced by oxidation of the phosphorus to form a phosphate. These fused salts form the bulk of the slag. The sulfur present is partly converted into sulfide that passes into the slag, and partly into sulfur dioxide, which escapes as a gas.

The purified iron that results from these reactions melts at a much higher temperature than does pig iron, with the result that as the process approaches completion semi-solid masses of iron form in the mixture. These are gathered into a white hot ball by the puddler and worked about on the hearth by means of an iron rod (rabble) until the mass is nearly freed from slag. It is next subdivided and the smaller plastic lumps, still white hot, removed and worked by hammers or mechanically operated squeezers to remove as much as possible of the still liquid slag. The product is then rolled into bars, etc.

Wrought iron is exceedingly tough and not at all brittle. When it is heated to a high temperature it is easily welded (803), or it may be wrought (forged) into any desired shape on a blacksmith's anvil.

89x. The Bessemer Process.-The manufacture of wrought iron is both laborious and expensive. This is because it requires most strenuous manual labor and that by a skilled workman to produce in the course of several hours a batch of a few hundred pounds of this desirable form of iron. Imagine, then, 
the importance of a process that produces from pig iron a product equal or superior to wrought iron in quality in batches of I 5 to 20 tons, with little hand labor, in 20 minutes' time, using the cheapes $\imath$ of all oxidizing agents, namely, air!

This process was first carried out by an American, William Kelly, in $\mathbf{1} 846$, and later perfected after many years of persistent experimenting by Sir Henry Bessemer, an Englishman. Theoretically the process is very simple: a powerful blast of air is forced through molten pig iron contained in a vessel lined with silica or silicates. The air oxidizes the carbon, silicon and manganese (889) of the melted iron to oxides. The oxide of carbon, being a gas, passes off, the silicon dioxide

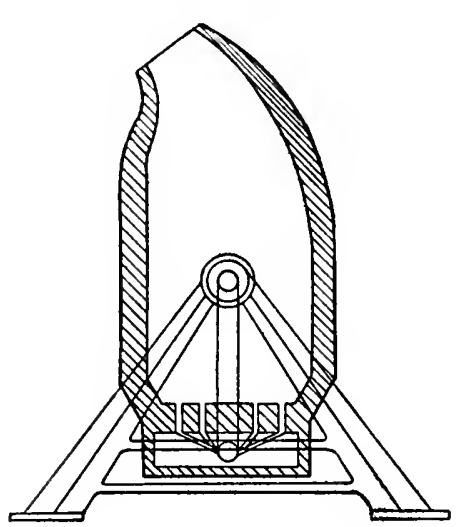

FIG. 125 unites with the oxides of iron and manganese (as in the pig boiling process) to form a fusible slag. The iron is thus freed from other elements excepting sulfur and phosphorus. By a modification of the process, even the latter can be removed. During the process much heat is rapidly produced and the temperature of the materials rises so high that both metal and slag remain molten. Simple as it appears when thus stated, enormous difficulties had to be overcome before the process was perfected. The product is known as Bessemer steel.

The process is carried out in a huge vessel called a converter, Fig. I25. This is built of steel plates and lined with silicious material. It is supported on trunions, so that it can be tilted to pour out its contents. The pig iron used for the charge is not allowed to cool after being drawn from the blast furnace (887) but is swiftly carried in a great ladle mounted on a railroad truck and run into the converter and blown at once. Silicon and manganese burn first; after a few minutes, when the oxidation of the carbon begins, a flame shoots out to a 
height of 20 or 30 feet with a great shower of brilliant sparks. After I $_{5}$ or 20 minutes the flame dies down, all the carbon having been burned out. At this stage a small but definite proportion of an iron-manganese alloy called ferro-manganese is added This, by reason of its manganese content, removes excess oxygen or reduces oxides of iron in the metal and thus prevents brittleness at a red heat (red shortness) that would otherwise exist. The carbon content of the product is brought up to the desired point by adding anthracite coal or other form of carbon, and the metal is then cast in iron molds (ingot molds) about $I_{5}$ inches square and 4 or 5 feet deep.

The whole process, from the time the molten pig iron is drawn from the blast furnace until it has been cast into ingots, requires less than an hour.

892. The Basic Bessemer Process.-The process just described does not lower the phosphorus content of the pig iron appreciably. Since the finished steel must not contain more than a small fraction of one per cent of phosphorus, pig iron, which carries more than the upper limit of phosphorus allowed in steel, is unsuitable for the Bessemer process in its original form. While a large part of the pig iron produced in the United States is sufficiently low in phosphorus, that produced in Germany runs over 2 per cent of this element. Two Englishmen, 'Thomas and Gilchrist, modified Bessemer's process by lining the converter with basic materials, such as burnt magnesite (largely $\mathrm{MgO}$ ) and by adding limestone along with the charge. The strongly basic slag produced takes up the phosphorus of the pig iron, forming calcium phosphate, and gives steel low in phosphorus. In the basic Bessemer process, a large part of the heat is derived from the burning of the phosphorus. To be suitable for this process the pig iron must contain 2 per cent or more of phosphorus. The basic process (Thomas-Gilchrist process) is not much used in America.

893. The Open Hearth Process.-Progress is ever the watchword of the successful chemical technologist. If a given process has some inherent defect or limitation, he tries to invent a new one to overcome the difficulty. Now, the Bessemer 
process is suitable for pig iron with less than o.I per cent of phosphorus (acid lining) or. more than 2 per cent (basic lining) but not for material of intermediate composition. The puddling (pig boiling) process (89r) is handicapped because it is limited to the production of batches of a few hundred pounds and because the temperature is not high enough to keep the charge melted to the end of the purification and thus allow the production of steel. The open hearth process surmounts all these difficulties. It is one that combines the advantages of the puddling and Bessemer processes and produces high grade steel from material having a wide range of composition with respect to phosphorus and other components as well. The development of this process was made possible by the inven-

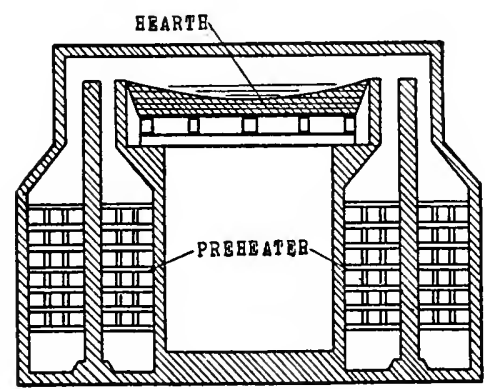

FIG. I $_{2} 6$ tion by Sir William Siemens, an Englishman, of a method of producing an extremely high furnace temperature by the use of gaseous fuel and without recourse to the heat furnished by the oxidation of the silicon, phosphorus, or carbon of the charge. Siemens' invention consists in a method of heating the fuel gas and air separately to a high temperature before the gas is burned. This is accomplished by the use of the waste heat of the very hot gaseous products of combustion that come from the furnace. The process resembles somewhat that by which the air supplied to the blast furnace is heated in stoves (887). In this case, however, both fuel gas and air are preheated. Since both fuel and air are at a high temperature before they combine, the heat of combustion raises the temperature to a point well above the melting point of pure iron, $1530^{\circ}$. This temperature is about $400^{\circ}$ higher than the melting point of pig iron. The essential features of the Siemens open hearth furnace are shown diagrammatically in Fig. I26. The fuel gas burns in the space above the hearth, the waste gases pass out 
and traverse one of the two brick checker work preheaters from which they pass up the chimney. At the same time, air and fuel gas are being heated separately by passage through the other preheater which previously has been heated white hot by waste gases. Every half hour the direction of flow of the waste gases on the one hand and the fuel gas and air on the other is changed so that one preheater is being heated while the other is giving up its heat to the fuel and air.

The open hearth process may be used with either acid (silica) or basic (magnesia) lining just as in the Bessemer process. But it is the basic process that is of far the greater importance, since this allows the treatment of charges with all ranges of phosphorus content. Furthermore, by a procedure introduced by Messrs. Martin of France, scrap steel is usually added to the pig iron charge; hence arises the name, the Siemens-Martin open hearth process. At the present time about three-fourths of all the stecl produced in the United States is made by this process.

The process is carried out by charging the furnace (basic lining) with 20 to 60 tons of pig iron (either cold or molten), scrap steel, and scrap iron, together with several tons of lime or limestone. As the charge melts down, part of the silicon and manganese are oxidized and pass into the slag. The addition of iron ore at this stage accomplishes further oxidation and elimination of all important impurities, excepting sulfur, which is only partly removed. The regulation of the temperature, the composition of the slag and the intensity of oxidation are all subject to easy control. The products, metal and slag, remain molten until a chemical analysis ${ }^{1}$ of the steel shows the correct composition. The metal is then cast into ingots.

894. Other Processes for Making Steel.-The cementation process of making steel antedates the other process already described. It consists in heating wrought iron in contact with powdered charcoal to a high temperature for several days. The iron strips or bars are packed in the charcoal powder and con-

\footnotetext{
1 Every steel works has a chemical laboratory and a staff of chemists. A steel analysis can be completed in about 20 minutes.
} 
tained in closed iron boxes which are then heated in a furnace. Sufficient carbon is taken up by the iron to change it into a product which, with proper subsequent heat treatment, becomes extremely hard and elastic. This steel was used for knives, tools, etc. The product of the cementation process was of variable composition, owing to unequal absorption of carbon even by the different iron bars of a single lot. A greatly inproved and very uniform product is produced by melting cementation steel in crucibles of clay or graphite. The product, called crucible steel, is the highest grade steel made. It is used for razors, dies, fine steel inștruments, etc.

During the last 15 or 20 years electric arc furnaces have come into rather extensive use for the manufacture of steel. These furnaces allow far easier control of conditions such as temperature, oxidizing, or reducing atmosphere, acidity or basicity of flux, etc. than is possible in any other process. Socalled electric steel seems to offer great possibilities.

895. The Working of Iron and Steel.-In our description of the Bessemer and open hearth processes we ended in each case with the casting of ingots, pieces of metal weighing several tons. As a rule these are not allowed to cool but are worked up into merchantable shapes, such as rails, beams, plates, etc., in the rolling mills that form an important part of every steel works. The ingots while still red hot are drawn from the molds and carried at once to a furnace in which they are heated uniformly until the metal is somewhat plastic; since fortunately iron has the property of becoming gradually softer as its temperature is raised. The white hot ingot is then run through a series of power driven rolls having grooved faces, for the production of the desired finished shapes. Each pair of rolls diminishes the cross section of the piece and increases its length. Finally the finished pieces are cut into the desired lengths, while still hot, by steel saws.

Many shapes that cannot be produced by rolling are made by forging the hot metal by the aid of huge power driven hammers. Complex shapes are made by the use of steel dies into which the hot plastic metal is forced by powerful hammer blows. 
The welding of iron is a process of uniting two pieces of iron by hammering or pressing them together at a white heat. In welding, the surfaces to be united should be clean and free from oxide. Borax is usually used as a flux to dissolve the oxide (803). Only wrought iron and mild (low carbon) steel can be welded. The use of the oxyacetylene torch in the cutting and welding of iron has already been mentioned (315). In acetylene welding, a rod of nearly pure iron is used to furnish molten iron to weld together two adjacent pieces of metal.

896. The Effect of Sulfur and Phosphorus in Iron or Steel.Sulfur is always an objectionable impurity in iron and steel and its amount should never exceed 0.05 of one per cent. If present in larger proportions, sulfur causes cracks to form in the metal, especially when the latter is worked at a red heat. The behavior of sulfur seems to be due to the formation of easily fusible ferrous sulfide $\mathrm{FeS}(339,601)$. The presence of manganese tends to counteract the bad effect of sulfur by forming with it a sulfide, MnS, which is less harmful than FeS.

The presence of phosphorus in steel in amounts over 0.I of one per cent causes marked brittleness in the cold steel. The danger is increased by reason of the fact that as the steel solidifies in the ingot mold (8gI), the phosphorus compounds tend to segregate in the last portions to solidify (upper central portion). If this part of the ingot is not rejected, the rails (for example) will be defective in places where the phosphorus content is high. The failure of such high phosphorus rails has led in the past to numerous railroad wrecks. These facts were established in numerous cases by chemical analysis of the rails that had failed.

The presence of phosphorus in cast iron is in some cases desirable, and may be as high as one-half of one per cent, or sometimes higher. Phosphorus increases the fluidity of molten cast iron and makes easier the production of thin castings. In small proportions, it is said to increase the strength of the cast iron.

897. The Tempering of Steel.-The most important difference between steel and iron is the quality of the former by rea- 
son of which it may be rendered hard or soft by certain heat treatments. If steel containing about 0.9 per cent of carbon is suddenly cooled from a bright red heat (about $800^{\circ}$ ) to ordinary temperature it is rendered extremely hard. At the same time its elasticity and brittleness are increased. If the same sample of hardened steel, after being again heated red hot, is cooled very slowly it will be made so soft that it can easily be filed or cut with a lathe tool. Files and lathe tools are made of hardened steel. It is usually desirable to produce steel having less than the maximum hardness and brittleness. This is accomplished by partially drawing the hardness of completely hardened steel by heating it for a short time to 200 to $300^{\circ}$ and then suddenly cooling the piece with water. This procedure is called tempering. In this way, it is possible to produce a product of the desired hardness and toughness. Iron containing little or no carbon cannot be hardened by sudden cooling.

For various classes of steel, the ordinary practical limits of carbon lie between 0.2 and I.5 per cent; since with greater proportions of carbon the hardened steel is too brittle to be serviceable.

The causes of the variations of properties of steel and cast iron with percentage composition and with heat treatment have been the subject of very extensive investigations, for which neither money nor brains have been lacking. A great deal has been accomplished; but much still remains to be discovered. The matter is so complex that it can only be touched upon here. Iron, like sulfur (600), phosphorus (582), carbon (630), and many other elements can exist in more than one allotropic form. The three forms of iron are known as alpha $(\alpha)$ iron, beta $(\beta)$ iron and gamma $(\gamma)$ iron. The first, $\alpha$, is stable at ordinary temperatures and changes to the second, $\beta$, at $750^{\circ}$. Upon being cooled, $\beta$ iron changes to $\alpha$ iron below this temperature. The case is like that of rhombic and monoclinic sulfur, for which the transition temperature is $95^{\circ}(600)$.

Beta iron is stable between $750^{\circ}$ and $860^{\circ}$ at which latter temperature it changes into $\gamma$ iron. The two transition points for $\alpha-\beta$ and $\beta-\gamma$ iron may be found experimentally by appli- 
cation of the same principle that enables one to find the melting point of ice. When ice melts, heat is absorbed (II8). A thermometer surrounded by ice in a warm room remains at $0^{\circ}$ until the ice is all changed to water. Now when $\alpha$ iron changes to $\beta$ iron (at $75^{\circ}$ ) heat is absorbed. In consequence, if a block of iron is placed in a furnace at $1000^{\circ}$, for example, its temperature will rise steadily up to $750^{\circ}$ and then remain stationary at this point until the change of $\alpha$ to $\beta$ is completed. The temperature of the iron block will again rise steadily until it reaches $860^{\circ}$, where it stops once more during the change of $\beta$ to $\gamma$. Upon cooling the reverse occurs: the fall of temperature halts at two places corresponding to the two transition points.

Let us now turn to another topic, the carbon content of the iron. Iron, like many other metals (calcium, silicon, aluminum, 63I) forms a carbide. This has the formula $\mathrm{Fe}_{3} \mathrm{C}$ and contains 6.7 per cent of carbon. This carbide of iron is known as cementite. It decomposes into its constituents above $1000^{\circ}$.

Pure iron melts at $\mathrm{I}_{53} 0^{\circ}$. At this temperature it dissolves several per cent of carbon. The solidification point (freezing point) of iron is lowered by dissolved carbon just as is the freezing point of water containing a dissolved substance (7r8). The maximum lowering is produced by 4.3 per cent of carbon. Iron of this composition (cast iron) solidifies at II $40^{\circ}$. At the same time, more than half of the 4.3 per cent of carbon separates out as graphite, which gives to gray iron its characteristic appearance. The remainder of the carbon, about 2.0 per cent, remains uniformly disscminated through the solid jron (gamma form) and is said to form a solid solution called martensite in the $\gamma$ iron. At temperatures below $1000^{\circ}$ this carbon of the solid solution can unite with iron to form the carbide $\mathrm{Fe}_{3} \mathrm{C}$ (cementite).

Now, it appears that the changes of $\gamma$ into $\beta$ iron and $\beta$ into $\alpha$ iron are greatly hindered by the presence of carbon, so that if steel containing, let us say, r.o per cent of carbon is cooled quickly to ordinary temperature, the change to $\alpha$ iron does not occur to an appreciable extent. The very hard and brittle product is therefore largely the solid solution of carbon in $\beta$ 
(or $\gamma$ ) iron, called martensite. When this is tempered by gentle heating to 200 to $300^{\circ}$, more or less of the martensite changes to $\alpha$ iron (ferrite) and cementite, whereby the product increases in toughness and decreases somewhat in hardness.

The most useful method of studying the physical composition of steel is by means of the microscopic examination of polished and etched samples. Photomicrographs made of such samples always show steel to be of heterogeneous structure. The study of alloys in this fashion is called metallography. The metallographic examination of steel forms an indispensable part of the tests made in practice to determine whether the material comes up to the specifications of the contract on which it is bought.

898. Alloy Steels. - When steel tools are heated and then cooled slowly, the steel loses its "temper" and they become worthless. As a consequence, the invention of special alloy steels which are indifferent to heat treatment has been of tremendous importance for cutting tools used on lathes. These are tungsten steels which have been subjected to a special heat treatment. With the best carbon-steel tool a lathe could be run at about 30 feet a minute without spoiling the tool. With a high speed steel tool it can be run at the rate of 300 feet a minute. The tool may even become red hot without losing its cutting power.

Many other steel alloys are in use for special purposes. For example a I4 per cent manganese steel is used for burglar proof safes, since it is too hard to be cut or broken; silicon-manganese steels are used for springs; nickel-chromium steel for engine shafts, projectile cases, and armor plate. The familiar"stainless steel" used in cutlery is a chromium steel containing small proportions of cobalt, manganese, etc. It is not stained by contact with food stuffs. Vanadium steel is much used for automobile parts because of its great tensile strength and elasticity.

899. Copper and Its Ores.-Copper ranks next to iron in economic importance. Over half of the world's output of copper is produced in the United States. Four states, Arizona, Montana, Utah and Michigan produce over 80 per cent of the 
domestic copper. Much of the ore is converted into metallic copper in the states where it is mined. A great variety of copper bearing minerals are known; but the number of practical importance is small.

Metallic or native copper is the chief ore in Michigan. In the western states, sulfide ores predominate, the most important being chalcopyrite, $\mathrm{CuFeS}_{2}$. Ores of copper containing arsenic, antimony, iron, lead, zinc, silver, gold or other elements also occur. Near the earth's surface, in place of sulfides there are found oxides, carbonates (e.g. malachite, 34 ), silicates, etc., which have formed by the natural oxidation of the sulfides and reaction with water, carbon dioxide, silica, etc.

The minimum copper content that an ore must have to be of commercial value depends on several factors, among which are the locality of the deposit, the cost of extracting the metal, and the prevailing price of metallic copper. At present (19r9) the Michigan mines producing native copper can be run at a profit on ores carrying less than one per cent of this metal. The average copper content of ores being mined at present in the United States is less than 2 per cent.

900. Ore Dressing.-If an ore contains but 2 per cent of copper it will usually consist to a large extent of rock and earthy material, the so-called gangue. The gangue of copper ores is usually high in silica and silicates of aluminum, magnesia, calcium, etc. Sometimes carbonates of the last two elements are present. Ores from the western states usually contain a large percentage of pyrite, $\mathrm{FeS}_{2}$. Most copper ores before being smelted are put through mechanical processes which separate a large part of the gangue from the ore as mined and thus give concentrates much richer in copper than the original ores. These processes are known in general as ore dressing. The possibility of concentrating ores like those here considered rests on several radically different principles. The most important of these, on which all of the older methods of concentration are bases, depends the fact that the valuable minerals are all much denser than the gangue. Thus the density of chalcopyrite is 4.2 while that of quartz (silica) is 
2.6. In concentrating ores the latter are crushed so as to produce small fragments which consist either of fairly pure mineral or of worthless gangue. The fine material is then screened or otherwise treated so as to classify the product with respect to size of grains. If now grains of approximately uniform size are agitated with water, the denser grains of mineral will tend to settle to the bottom of the container so that the gangue can be washed away with the water. Numerous ingenious devices for the practical application of this principle are in extensive use. These comprise jigs, spitzkasten, vanners, Wilfley tables, etc., descriptions of which are to be found in most books on metallurgy. The miner's pan by which gold was freed from gangue by washing with water was the original concentrating device.

The flotation process of concentration consists in violently agitating finely powdered sulfide ores with water and air and a minute quantity of an oil. The oil adheres to sulfide minerals as a thin film and the oil coated particles become attached to minute air bubbles and are thus carried to the surface of the water as a froth while the worthless gangue settles to the bottom. This process has in recent years become of very great importance in the concentration of the ores of copper, zinc, lead, etc.

By means of various methods of dressing it is usually practicable to eliminate two-thirds or more of the worthless gangue.

90r. Principles of the Metallurgy of Copper.-The principal problem confronting the metallurgist of western America is the preparation of copper of 99.95 per cent purity or better from ores containing less than 2 per cent of this metal together with far larger proportions of iron, sulfur and silica, and considerable amounts of many or all of the elements, lead, zinc, aluminum, calcium, magnesium, arsenic, antimony, and small amounts of many others, notably selenium, tellurium, bismuth, silver and gold. In the solution of this problem he must not only make pure copper; but he must get a good recovery of the ore content of this metal and at the same time save nearly the whole of the valuable silver and gold present. It is easy to see that this problem is far more complex than that involved 
in the production of pig iron (888). Naturally the details of the metallurgical processes will vary in different localities by reason of considerable variation in the nature of the ores. Nevertheless the general procedure is tolerably uniform throughout the whole of western America, including Canada and Mexico. The usual process consists of three or four separate steps. In the four-step process the ores are first strongly heated in air (roasted) to oxidize iron, sulfur and arsenic and drive off the last two as oxides. The product (calcine) is then melted down (smelted) in huge reverberatory furnaces (89o) to form two products, slag and matte, both liquid at a white heat. The matte is largely a crude sulfide of copper and iron and contains also the silver and gold of the ore. In the third step the molten matte is oxidized by a blast of air in a vessel resembling a Bessemer converter. Here the sulfur is eliminated as $\mathrm{SO}_{2}$, the iron oxidized and combined with the silica of the lining of the converter to form a slag, and the copper set free as crude metal containing also silver and gold. The fourth and final step in the purification is the electrolytic refining of the crude copper. In the three step process, the first two steps above mentioned are combined. In this case the ore, rich in sulfur (pyrite), is smelted directly in a blast furnace somewhat resembling that used for iron (889). Here a large part of the heat is supplied by the burning of the pyrite $\left(\mathrm{FeS}_{2}\right)$, the remainder being furnished by the burning of coal added to the charge. The non-gaseous products are again slag and matte. The latter is finished as above described.

It must be evident that a great variety of chemical reactions take place in the processes here outlined. We shall consider the more important ones in connection with the detailed description that follows.

902. The Roasting Process.-A diagram of the vertical section of a common type of roasting furnace is shown in Fig. I27. This is a cylindrical structure ${ }_{5} 5$ feet or more in diameter having 6 or 7 hearths, one above the other. The heating furnace is at the base and the ore is fed in at the top. Power driven, water cooled revolving arms stir the ore and on one 
hearth slowly rake it to a center opening where it falls to the next lower hearth. Here the rakes are so set as to carry the ore to the circumference where it again falls to the hearth below. The material, usually finely divided concentrates, is heated to bright redness; but not hot enough to melt it. Much heat is developed by the oxidations that occur in the process, so

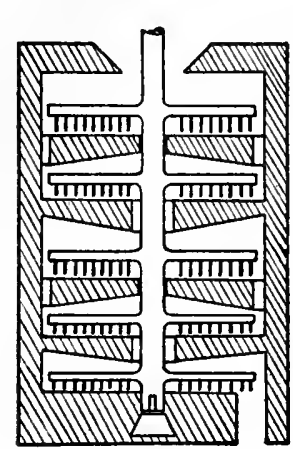

FIG. I 27 that for ores rich in pyrite little or no other fuel is required once the process is started.

In the roasting process the following reactions occur:

$$
\begin{gathered}
\mathrm{FeS}_{2}+\mathrm{O}_{2} \rightarrow \mathrm{FeS}+\mathrm{SO}_{2} \\
{ }_{2} \mathrm{FeS}+3 \mathrm{O}_{2} \rightarrow 2 \mathrm{FeO}+2 \mathrm{SO}_{2} \\
4 \mathrm{FeO}+3 \mathrm{O}_{2} \rightarrow 2 \mathrm{Fe}_{2} \mathrm{O}_{3} \\
4 \mathrm{CuS}+2 \mathrm{O}_{2} \rightarrow 2 \mathrm{Cu}_{2} \mathrm{~S}+{ }_{2} \mathrm{SO}_{2}
\end{gathered}
$$

If ore with about 30 per cent of $\mathrm{S}$ is used, the roasted product (calcine) contains 7 or 8 per cent $S$.

903. The Reverberatory Smelting Process.-Modern copper reverberatories are of enormous size, having hearths $20 \times 140$ feet. These furnaces are heated with powdered coal blown in by an air blast, in this way producing the maximum heating effect of the fuel in the smelting chamber. The great heat energy of the waste combustion gases is utilized to produce steam power for running the plant.

In the reverberatory the ore melts and its silica content unites with the oxides of iron and smaller amounts of aluminum, calcium, magnesium, etc. to form a slag which is nearly free from copper. Further oxidation of sulfides takes place with the formation of $\mathrm{SO}_{2}$. Any oxides of copper now present react with iron sulfide to form cuprous sulfide, $\mathrm{Cu}_{2} \mathrm{~S}$ and oxide of iron, which latter passes into the slag as a silicate. The sulfides of copper and iron and of other metals in much small proportions form at a white heat a fluid matte, insoluble in molten slag. The former, being of about twice the density of the latter, sinks to the bottom of the hearth and is tapped out from time to time. The yield of matte is about one-fourth of the weight 
of the charge. It contains 35 per cent or more of copper and most of the silver and gold.

904. The Converter Process.-A copper converter resembles a Bessemer converter (Fig. I25); but in some instances it is much larger and may have a capacity of more than 60 tons of matte. In the copper conversion process, the sulfur and iron are oxidized and eliminated almost completely, the 'ormer as $\mathrm{SO}_{2}$, the latter as ferrous silicate (slag). The silica is supplied from the clay and quartz sand converter lining or, in more modern practice, by the addition of silica with the charge and the use of a converter lined with calcined magnesite, which is but little attacked. The matte is charged in the white hot molten state immediately after it is tapped from the reverberatory. During the conversion, which lasts several hours, instead of $I_{5}$ to 20 minutes as in the Bessemer process, the temperature is maintained by the great heat of combustion of the sulfur and the iron. In addition to $\mathrm{SO}_{2}$, the products of the conversion are slag (mostly ferrous silicate) and crude metallic copper (blister copper). The formation of metallic copper is largely brought about by the following reactions:

$$
\begin{gathered}
{ }_{2} \mathrm{Cu}_{2} \mathrm{~S}+{ }_{3} \mathrm{O}_{2} \rightarrow 2 \mathrm{Cu}_{2} \mathrm{O}+{ }_{2} \mathrm{SO}_{2} \\
2 \mathrm{Cu}_{2} \mathrm{O}+\mathrm{Cu}_{2} \mathrm{~S} \rightarrow 6 \mathrm{Cu}+\mathrm{SO}_{2} \\
{ }_{3} \mathrm{Cu}_{2} \mathrm{O}+\mathrm{FeS} \rightarrow 6 \mathrm{Cu}+\mathrm{FeO}+\mathrm{SO}_{2}
\end{gathered}
$$

905. The Copper Blast Furnace.-The furnaces recently constructed by the Anaconda (Mont.) Copper Company are the largest blast furnaces ever built for any purpose. They are rectangular in section $4 \frac{1}{2}$ to $6 \times 87$ feet inside and take a charge 15 feet deep. These furnaces have a daily capacity of 2500 tons. The charge consists of lumps of unroasted (raw) ore and a small proportion of coal or coke. Powerful blasts of (unheated air, introduced through numerous tuyeres, supply oxygen for the burning of the coal and also a large part of the sulfur. The reactions that occur are similar to those that take place in the processes of roasting (902) and reverberatory smelting (903) already described. The gaseous products are $\mathrm{SO}_{2}, \mathrm{CO}_{2}$ and water vapor. Much arsenic is 
volatilized as $\mathrm{As}_{2} \mathrm{O}_{3}$. This is largely collected after it condenses upon cooling along with other valuable dust in specially devised dust collectors. The liquid products are slag and matte. The latter goes directly to the converters.

906. Lake Copper.-The native copper ores of Michigan occur in a small region on the south shore of Lake Superior. For many years these deposits were the most productive in America. Michigan now ranks fourth among the copper producing states. The metallurgy of the Michigan ores is simple, since they contain free copper in a high state of purity. The ores are crushed and concentrated, and the concentrate smelted in reverberatory furnaces with enough limestone to yield a good slag. Since the copper is present in the ore in metallic form, it is merely melted in this process, without in the main being altered chemically. Copper from this district is known in trade as Lake copper.

907. The Refining of Copper.-Crude copper (blister copper) obtained by the processes here described must always be refined. Blister copper is melted in a reverberatory furnace in an oxidizing atmosphere. Sometimes air is blown into the melted metal through an iron pipe. A small amount of slag and dross (consisting of oxides of various metals) is skimmed off. To remove cuprous oxide, $\mathrm{Cu}_{2} \mathrm{O}$, which is now present (in solution) in the molten copper, the latter is stirred with poles of green wood. When the oxide has been thus reduced to metal, the latter is tapped from the furnace and cast into anode plates or other forms. The metal is now over 99 per cent pure; but it is not pure enough to be used as wire for electrical transmission or for the construction of electrical apparatus such as generators, motors, transformers, etc., since the electrical conductance of copper is very greatly decreased by the presence of minute amounts of impurities. Furthermore all western copper contains silver and gold in amounts sometimes representing 20 per cent or more of the value of the product. Very complete removal of impurities and recovery of the precious metals is accomplished by a process of electrolytic refining. The process is very simple in principle. If a solu- 
tion of copper sulfate is electrolyzed with copper electrodes, the anode passes into solution as $\mathrm{Cu}^{++}$ions. These migrate toward the cathode while $\mathrm{SO}_{4}^{--}$ions travel in the opposite direction. Cupric ions arriving at the cathode take up electrons (487) and deposit in metallic form. The sulfate ions remain in solution. In practice, the cast anodes (referred to above) are about $2 \frac{1}{2} \times 3$ feet and over an inch thick at the start while the cathodes are extremely thin sheets of very pure copper. Alternate cathodes and anodes are hung in a solution of copper sulfate and free sulfuric acid contained in a lead lined wooden vat. The anodes are all connected to the positive wire from the electric generator, the cathodes to the negative wire. The rate of electrolysis follows Faraday's law (403). About I ounce of copper is deposited in twenty-four hours for each ampere of current. During the electrolysis the impurities either pass into solution and remain dissolved (arsenic, nickel, iron, zinc) or fall to the bottom of the vat as insoluble residue (silver, gold, lead, etc.). The electrolyte is purified from time to time and the mud-like residue worked up for silver and gold. The cathodes are removed when they have reached a thickness of half an inch, metled down and cast into ingots, bars, rods, etc. The metal now contains but a few hundredths of $\mathbf{I}$ per cent impurity.

908. Uses of Copper.-The various electrical industries consume the bulk of the copper produced. Next to silver, copper is the best electrical conductor. Copper is used extensively in making various utensils (kettles, stills, condenser worms, etc.). Much copper is used in the making of brass, an alloy containing about 70 per cent of copper and 30 per cent of zinc. Brass is superior to copper for many purposes. It can be readily cast and easily machined and is much harder and more elastic than copper. Brass is cheaper than copper because zinc is much the cheaper of the two components. Many other alloys of copper are in use. Bronze contains copper, tin, zinc, and sometimes lead. German silver contains copper, zinc and nickel. All United States coins contain copper: one-cent pieces contain 95 per cent copper, 3 per cent of tin 
and 2 per cent of zinc. Nickel five-cent pieces (nickels) contain 75 per cent of copper and 25 per cent of nickel. Gold and silver coins contain ro per cent of copper.

909. The Occurrence and Production of Lead.-In I9I3, the last year prior to the war, the world's production of lead was about I,300,000 tons. The United States was the largest producer, furnishing about one-third of the whole. During the war the United States production increased considerably. In I9I3, 92 per cent of the domestic production came from four states, which, with the percentages produced by each, were: Missouri, 35; Idaho, 3I; Utah, I7; Colorado, Io. The principal ore is galena, $\mathrm{PbS}$, although the carbonate, cerussite, $\mathrm{PbCO}_{3}$ and the sulfate anglesite, $\mathrm{PbSO}_{4}$, are of some importance. Lead ores often contain copper, silver and gold as valuable components and also zinc sulfide, which is as a rule detrimental.

910. Principles of the Metallurgy of Lead.-Lead is even more easily recovered from its ores than is copper. The various oxides are all easily reduced when heated with hydrogen or carbon (coal or coke). Lead oxide, $\mathrm{PbO}$, is formed by heating the carbonate and also by the oxidation of the sulfide by air (roasting).

$$
\begin{gathered}
\mathrm{PbCO}_{3} \rightarrow \mathrm{PbO}+\mathrm{CO}_{2} \\
{ }_{2} \mathrm{PbS}+{ }_{3} \mathrm{O}_{2} \rightarrow 2 \mathrm{PbO}+{ }_{2} \mathrm{SO}_{2}
\end{gathered}
$$

The roasting of galena also gives rise to lead sulfate.

$$
\mathrm{PbS}+{ }_{2} \mathrm{O}_{2} \rightarrow \mathrm{PbSO}_{4}
$$

At a high temperature lead sulfide reacts with the oxide or the sulfate to form the metal and sulfur dioxide.

$$
\begin{gathered}
\mathrm{PbS}+{ }_{2} \mathrm{PbO} \rightarrow{ }_{3} \mathrm{~Pb}+\mathrm{SO}_{2} \\
\mathrm{PbS}+\mathrm{PbSO}_{4} \rightarrow 2 \mathrm{~Pb}+{ }_{2} \mathrm{SO}_{2}
\end{gathered}
$$

Lead sulfide also reacts with iron at a red heat to form lead and ferrous sulfide.

$$
\mathrm{PbS}+\mathrm{Fe} \rightarrow \mathrm{Pb}+\mathrm{FeS}
$$

All of the reactions here mentioned take place in the smelting of galena. 
9II. Lead Smelting.-In the United States nearly all lead smelting is carried out in blast furnaces. These resemble in principle those used for iron (887) and copper (905) but are of smaller size. The charge usually consists of lead ore and coal or coke. The lead ore, which may have an average lead content of 20 per cent, is in part roasted to remove a large part of the sulfur. In the furnace the charge melts down, giving in addition to gases and vapors three products which are liquid at a red heat: lead, slag and matte. The lead is set free by the reactions above given. The slag is formed from the silica gangue of the lead ore and the added limestone and iron ore. It consists largely of silicates of calcium and iron. The matte (903), which consists principally of ferrous sulfide, contains in form of sulfide nearly the whole of the copper content of the ore. The slag and matte are tapped out together in ladles. The matte, being heavier, settles to the bottom and is easily separated from the slag when the two have solidified. The copper content of the matte is recovered as described earlier (904). The lead which accumulates in a well at the bottom of the blast furnace is drawn off through a siphon and cast into pigs, usually called base bullion.

912. The Refining of Lead.-The chief impurities of the base bullion (crude lead) smelted from western United States ores are antimony, arsenic, copper, bismuth, silver and gold. Since the first three of these impurities render the lead hard, the process by which they are eliminated is termed softening. The bullion is melted in a reverberatory furnace in an oxidizing atmosphere. Part of the antimony and arsenic are volatilized and the remainder together with the copper pass into the oxidized dross that is skimmed off. The further purification of the lead, which also accomplishes the recovery of the silver and gold, is usually carried out in either of two ways. The first and older of these is the Parkes process in which a small amount of zinc is added to the molten lead, and the mixture well stirred. Upon being allowed to stand, the zinc, which has dissolved most of the silver and gold, rises to the surface and is skimmed off. This process is in principle precisely like that 
by which ether shaken with a water solution of bromine removes most of the latter and then separates as a layer floating on the denser water. The lead which has thus been freed from silver and gold is again heated in a reverberatory furnace to remove the zinc and small amounts of other metallic impurities that may still be present. It is then cast into pigs.

The Betts process for refining lead consists in the electrolysis of a solution of lead fluosilicate, $\mathrm{PbSiF}_{6}$ (272), using heavy anodes of crude lead and starting with very thin cathodes of pure lead. The process resembles in a general way the electrolytic refining of copper. In this case the impurities fall to the bottom of the vessel as an insoluble mud, while very pure lead deposits on the cathode. Silver, gold and bismuth are recovered from the mud.

Lead from the Missouri district does not contain enough silver (or gold) to make its recovery profitable.

9r3. Uses of Lead.-With the exception of iron, lead is the cheapest metal. Its normal, pre-war price was about $4 \frac{1}{2}$ cents per pound. As metal, alloys and compounds, it serves a great variety of useful purposes. Lead lined tanks are extensively used in chemical works for sulfuric acid solutions. Chambers built of sheet lead are used in making this acid (6r6). Shot and bullets are made of lead. Solder, which consists of lead and tin, melts at a lower temperature than either of the separate metals. Type metal (8II) contains a large percentage of lead. Fusible alloys (8I2) also usually contain lead. Two of the most important uses of lead are found in the manufacture of "white lead" (basic lead carbonate) which is extensively used in making paint, and in the construction of so-called storage batteries.

914. The Storage Battery.-A storage cell has electrodes of lead immersed in 20 per cent sulfuric acid. When the cell is charged, one of the electrodes is coated with a layer of lead dioxide, $\mathrm{PbO}_{2}(\mathbf{1 6 7}, 326)$, the other with a deposit of metallic lead in spongy form. Upon closing the circuit a current passes through the connecting wire in a direction indicating 
a flow of electrons from the lead covered plate to that holding the lead dioxide (47I-2).

At the same time the following reaction takes place in the cell:

$$
\mathrm{PbO}_{2}+{ }_{2} \mathrm{H}_{2} \mathrm{SO}_{4}+\mathrm{Pb} \rightarrow 2 \mathrm{PbSO}_{4}+{ }_{2} \mathrm{H}_{2} \mathrm{O} \text {. }
$$

This cell may most simply be considered an oxidation-reduction cell, in which the lead dioxide is the oxidizing agent, and the spongy lead the reducing agent (502-4). Each atom of the spongy lead passing into solution as a $\mathrm{Pb}^{++}$ion leaves behind on the electrode two electrons. Each molecule of lead dioxide tends to yield $\mathrm{Pb}^{++++}$and $2 \mathrm{O}^{--}$. But the tetravalent lead ion being a powerful oxidizing agent easily takes up from its supporting electrode two electrons and is thereby reduced to $\mathrm{Pb}^{++}$. The excess of electrons set free at the spongy lead (negative) electrode flows through the wire to the lead dioxide (positive) electrode where there is a deficiency of electrons, thus constituting the current produced by the cell. The $\mathrm{Pb}^{++}$ions formed unite almost completely with $\mathrm{SO}_{4}{ }^{--}$ions of the acid to form nearly insoluble lead sulfate which deposits on the two electrodes. The $\mathrm{O}^{--}$ions of the $\mathrm{PbO}_{2}$ unite practically completely with $\mathrm{H}^{+}$ions of the acid to form water.

The storage cell is so called because it is readily recharged by passing a current (from a power circuit) through it in reverse direction to the normal. In the charging process the changes described all take place in the reverse sense. The extent to which a cell is charged is indicated by the density of the sulfuric acid; since when the charge is complete, that is, when all lead sulfate has been changed to metallic lead, lead dioxide and sulfuric acid, the density of the solution is at a maximum. On the other hand when the active materials are completely changed to insoluble lead sulfate and water, the cell is discharged and the density of the liquid is at a minimum.

The batteries of electric cars and the starting batteries of gasoline cars are in general lead storage cells. Such batteries deteriorate if they remain uncharged for a long time, owing to the fact that the lead sulfate hardens in a compact mass which is not easily acted upon by the current. It is therefore advisable 
to make occasional measurements of the density of the battery fluid (by means of a hydrometer) to ascertain when the battery needs recharging.

It may be well to point out here that a storage cell does not store electricity. It stores chemical energy. The formation of two formula weights of lead sulfate, as shown by the foregoing equation, liberates 87,000 calories (see Chap. XVI) if the reaction takes place directly (as in a beaker). In the cell this energy is changed into electrical energy (508), no appreciable heat being produced. When the cell is charged, electrical energy is changed into chemical energy (509).

915. The Occurrence and Production of Zinc.-Zinc (technically called spelter, $\mathbf{1 4 8}$ ) ranks third in point of tonnage and value among the metallic products of the United States, being preceded by iron and copper and closely followed by lead. (88I). The United States production, which amounted to but I6,000 tons per annum in I875, reached 685,000 tons in I9I 7 . In I9I3, with a production of about half that of I9I7, the United States made over 30 per cent of the world's output of zinc. In I9I 8 the domestic proportion was much larger.

Over 85 per cent the domestic zinc ores came from six districts; these with their respective shares of the total output were as follows: Joplin district (Missouri and near-by parts of Oklahoma, Kansas and Arkansas), 30 per cent; Montana, 20; Oklahoma-Kansas district, I5; Colorado, 8; Wisconsin, 8; Idaho, 5. The remainder came from a number of other states, including New Jersey, Utah, Nevada, California and Tennẹssee.

The principal ore is sphalerite, the sulfide, $\mathrm{ZnS}$, popularly called blende or black-jack. Smithsonite, the carbonate, $\mathrm{ZnCO}$; willemite, a silicate, $\mathrm{Zn}_{2} \mathrm{SiO}_{4}$; and franklinite, a complex ore containing oxides of zinc, iron and manganese are also of some importance.

Ore to be commercially valuable must contain a large percentage of zinc. In general, some process of concentration (ore dressing, 900) is used to bring the zinc content up to the required percentage. In many cases sphalerite (blend) is 
associated with galena, $\mathrm{PbS}$, from which it can also be separated mechanically (900).

916. Principles of the Metallurgy of Zinc.-The first step in the treatment of either carbonate or sulfide ores is the conversion of these compounds into oxide. In the case of the carbonate this is readily accomplished by heating the ore, thus causing the elimination of carbon dioxide.

$$
\mathrm{ZnCO}_{3} \rightarrow \mathrm{ZnO}+\mathrm{CO}_{2}
$$

The sulfide is changed to oxide by heating it to a red heat with free access of air (roasting, 902).

$$
2 \mathrm{ZnS}+{ }_{3} \mathrm{O}_{2} \rightarrow 2 \mathrm{ZnO}+{ }_{2} \mathrm{SO}_{2}
$$

This process of oxidation may also go further and yield zinc sulfate as the result of the catalytic oxidation of sulfur dioxide to trioxide (615) and the union of the latter with zinc oxide. The roasting of blende is carried out somewhat like that of sulfide ores of copper (902). Or by a newer process, the oxidation may be made more rapid by use of an air blast so that a higher temperature is reached and the product is partly fused so that the small particles are bound together in larger porous lumps, a process called sintering. Roasting reduces the sulfur content of the ore or concentrate from 20 per cent or more to about I per cent.

Zinc oxide is readily reduced to metal by the action of carbon at a high temperature.

$$
\mathrm{ZnO}+\mathrm{C} \rightarrow \mathrm{Zn}+\mathrm{CO}
$$

Since the boiling point of zinc, $918^{\circ}$, is not far from the temperature at which this reaction takes place, the zinc is volatilized at the same time and thus freed almost completely from impurities. The vapors upon cooling deposit metallic zinc.

917. Details of the Smelting Process.-The calcined carbonate or roasted sulfide ore is mixed with powdered coal and placed in specially constructed fire clay retorts. The latter are 4 or 5 feet in length and 8 or ro inches in diameter, and have an opening at one end through which the gaseous products escape. The retorts are set in a specially constructed furnace, 
Fig. 128, in several superimposed tiers. Each retort is fitted with an attached cylindrical pipe which extends beyond the furnace and in which the zinc vapors cool sufficiently to condense. During the reaction in the retort, carbon monoxide escapes and burns at the outer end of the condenser. A small part of the zinc separates in the condenser in powdered form, known as zinc dust. This is a mixture of metal and oxide, which is usually added to the next charge.

Since the charge of each retort is only about rooo pounds of ore, a zinc smelting works requires several thousand retorts.

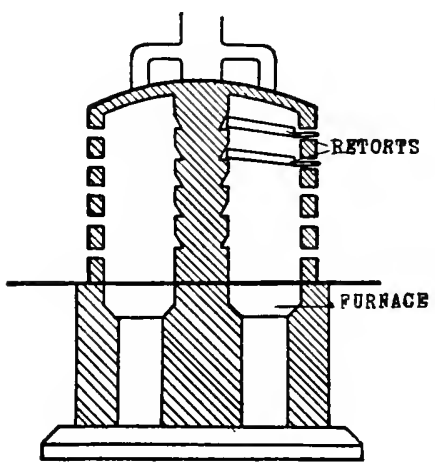

FIG. I 28

It would thus appear that the art of zinc smelting is in a very backward state of development in comparison with the present methods of smelting iron, copper, and lead. In fact, the method of zinc smelting here described (the Belgian process) has undergone no essential change in many decades.

918. Electrolytic Zinc.-Although the retort distillation process is the one by which the bulk of the zinc is at present produced, another process that looks very promising is coming into rather extensive use. In this process the zinc content of the ore is brought into water solution as sulfate and electrolyzed in lead lined wooden vats with graphite anodes and aluminum cathodes, on which the pure zinc deposits. At the anode oxygen is set free and the corresponding amount of hydrogen from the decomposed water yields with the $\mathrm{SO}_{4}^{--}$ions of the solution sulfuric acid. After this zinc has been removed from the solution, the latter is used to prepare a fresh zinc sulfate solution.

The conversion of the zinc content of the ore into sulfate is carried out in two ways. By one process the ore is smelted in a large reverberatory furnace at a high temperature and with an oxidizing atmosphere. The zinc is reduced to metal; the latter escapes as vapor which burns at once to zinc oxide (r48). 
The furnace gases, carrying a dense cloud of oxide, are cooled and passed through numerous large bag filters in which the white solid oxide is collected. This oxide is then dissolved in dilute sulfuric acid (spent electrolyte) to give zinc sulfate solution. The other process consists in roasting the ore in a furnace somewhat like that shown in Fig. 127, in such a way as to convert as much as possible of the zinc sulfide into sulfate. The roasted material is then leached with the dilute sulfuric acid solution from the electrolytic tanks and the resulting crude zinc sulfate solution purified and later electrolyzed.

919. Uses of Zinc.-Zinc is brittle at ordinary temperatures, but it becomes malleable and ductile at $\mathrm{I} 50^{\circ}$ and may be rolled into thin sheets or drawn into wire. Metallic zinc does not oxidize readily on exposure to air, on which account it is used for containers of various sorts. Far more important is the use of zinc for making galvanized iron. This is iron (sheet, wire, pipe, etc.) cleaned with sulfuring acid and coated with zinc by dipping it in the molten metal. This treatment protects the iron from rusting; since even if the iron is imperfectly covered, the zinc has the greater tendency to pass into the ionic state (492) that is, to oxidize (504). Brass (908) and other alloys of zinc require for their manufacture immense quantities of zinc. As negative electrodes in electric batteries (496) (e.g. dry cells) zinc finds an important use. In the laboratory and in chemical works zinc is used as a reducing agent and to produce hydrogen.

Zinc oxide and zinc sulfide are white solids insoluble in water which are used extensively in making white paints. Lithopone, which is also used for the same purpose, is a mixture of zinc sulfide and barium sulfate made by mixing solutions of barium sulfide and zinc sulfate.

$$
\mathrm{BaS}+\mathrm{ZnSO}_{4} \rightarrow \mathrm{BaSO}_{4}+\mathrm{ZnS}
$$

920. Aluminum.-Although aluminum (I74) is the most abundant truly metallic element, constituting over seven per cent of the earth's crust (F. W. Clarke), it was also the latest metal to come into extensive use. The reason for its tardy practical development is found in the fact that none of the proc- 
esses that serve to reduce to the metallic state ores of iron, copper, lead, zinc, tin, mercury, nickel, silver or gold are effective in the case of aluminum. This metal was probably first obtained by Wöhler (696) in impure form in 1827 and in a purer form in 1845 , by heating aluminum chloride with metallic potassium.

$$
\mathrm{AlCl}_{3}+3 \mathrm{~K} \rightarrow \mathrm{Al}+3 \mathrm{KCl}
$$

Deville improved the process by substituting for the too easily volatile $\mathrm{AlCl}_{3}$ its double salt with $\mathrm{NaCl}$ and made it much cheaper by using sodium in place of the very expensive potassium. It was through the efforts of this great French chemist that aluminum began to be manufactured in 1856 . In the year $x 859$ the metal sold for 90 cents an ounce. ${ }^{1}$ This high price, which was largely due to the cost of the sodium required for Deville's process, made this very desirable metal too expensive for very extended use. It was therefore a great advance when in I 886 Castner invented a new process for the manufacture of sodium by which the latter could be produced for 25 cents per pound. Castner's process consisted in reducing caustic soda at a red heat with iron and carbon. By r889 the price of aluminum made by use of Castner's cheap sodium had dropped to $\$ 4.00$ a pound ( $\$ 8.80$ a kilo). But only two years later aluminum, made by an entirely different process which did not require metallic sodium, was put on the market in quantity at $\$ 1.00$ a pound. How this came about will be shown in the following section.

92 r. The Electrical Production of Aluminum.-At the beginning of the Igth century none of the metals of the alkalies or the alkaline earths had been isolated, although the bases and salts of these elements were well known. The preparation of these new metals by Davy at the Royal Institution (London) in 1807 was the most important advance in our science since the discovery of oxygen and the explanation of its rôle in chemical change, more than thirty years earlier. Davy made sodium by passing an electric current from a large battery of galvanic cells

1 This corresponds to $\$_{14.40}$ a pound or $\$_{31}$.70 a kilo. In the year I9I 3 the price in the United States was $2 \mathrm{I}$ cents a pound, or 46 cents a kilo! 
through molten (water free) sodium hydroxide. Although dry solid sodium hydroxide at room temperature is a very poor conductor of electricity, the fused base conducts readily. Metallic sodium collects at the cathode where hydrogen is also set free, while oxygen comes off at the anode. In a similar way potassium is obtained from its hydroxide. In the following year Davy tried to make aluminum by electrolysis, but was not successful. In the course of the succeeding three-quarters of a century other chemists at times considered the possibility of making aluminum by electrolysis; but this method was not practical because the electric generator (dynamo) was not yet developed and all electric power was obtained at great cost from batteries, which derived their energy from metallic zinc.

The era of electro-chemistry began about I 880 with the development of the power-driven dynamo to the stage where electric power was readily produced at moderate cost. The first even partially successful electrical method for aluminum was that invented in 1884 by the Cowles brothers of Cleveland. In this process a powerful electric current was passed through a mixture of aluminum oxide and charcoal in the presence of metallic copper. At the extremely high temperature produced in the Cowles electric furnace the aluminum oxide was reduced by the charcoal and was dissolved by the molten copper and so protected from further change. The product was aluminum bronze, an alloy with copper containing 15 to 20 per cent of aluminum. This was not an electrolytic process since it worked equally well with an alternating current. The first real electrolytic process for aluminum was developed by Hall of Oberlin, Ohio, in 1886.

At nearly the same time a process practically identical with Hall's was patented by Hérault in France. This process, which since its invention has displaced all others for the manufacture of aluminum, is commonly known as the Hall-Hérault process, to the description of which we may now turn.

922. The Hall-Hérault Process.-In order to make clear the principles of the Hall-Hérault process, a few lines must be devoted to the matter of the electrical behavior of fused salts. 
Although dry solid salts are very poor electrical conductors (384) they usually conduct even better than most water solutions when they are in the fused molten state. It seems reasonable to suppose that fused salts are highly ionized (4II) and that their electrical conductivity is to be explained by the migration of charged ions just as in the case of solutions. It. is now well known that many metals (e.g., sodium, potassium, magnesium, calcium, barium, etc.) can be obtained in metallic form by the electrolysis of certain of their fused salts, especially their chlorides.

Hall found that the electrolysis of the fused double fluoride of sodium and aluminum, ${ }_{3} \mathrm{NaF} \cdot \mathrm{AlF}_{3}$, which is found in Greenland as the mineral cryolite, gave melted metallic aluminum. $\mathrm{He}$ also found that if he added to the molten cryolite (M.P. I000 ${ }^{\circ}$ ) aluminum oxide, $\mathrm{Al}_{2} \mathrm{O}_{3}(\mathrm{I} 74)$, that the latter dissolved abundantly, giving a more fusible mixture When this was electrolyzed with a carbon rod anode it gave aluminum as before, and, at the anode, set free oxygen which united with the carbon to form carbon dioxide. In this case since the oxide of aluminum, instead of the cryolite, is decomposed, it is only necessary to add the former from time to time and to draw off the melted metal to make the process a continuous one.

Hall's apparatus consists of a large iron vessel lined with a hard, compact form of carbon which serves as the cathode and within which the molten electrolyte is contained. The anode is a stout rod of carbon which dips into the electrolyte. The strength of current depends on the size of the cell, specifically on the area of anode surface exposed to the electrolyte. The electrolysis takes place at $900^{\circ}$ to $1000^{\circ}$; the electrolyte is maintained at this high temperature by the current alone, without being heated externally.

\section{The Occurrence and Preparation of Materials for the} Hall Process. - The cryolite needed is obtained either from the natural deposits of this mineral in Greenland or it may be made artificially from aluminum hydroxide, soda and hydrofluoric acid (269). Although aluminum minerals are both numerous and abundant everywhere, it has been found that only one 
mineral, bauxite, can be used as a technical source of the metal. Bauxite (first found at Beaux in France) is a hydrated oxide of aluminum, $\mathrm{Al}_{2} \mathrm{O}_{3} \cdot 2 \mathrm{H}_{2} \mathrm{O}$. It is rarely found pure and contains usually oxides of iron, silica, clay, sand, etc. About 80 per cent of the domestic supply of bauxite comes from Arkansas, the remainder coming from Georgia, Alabama and Tennessee.

To prepare the alumina needed for the Hall process, bauxite is digested with concentrated caustic soda to dissolve the aluminum as sodium aluminate (I77). The latter gives by hydrolysis (436) aluminum hydroxide, which in turn gives the oxide when heated.

924. The Production of Aluminum.--Since the invention of the Hall-Hérault process, the manufacture of aluminum has increased prodigiously. In Igr 8 the world's production was nearly 500 million pounds, of which the United States made about 45 per cent. Only four metals, iron, copper, zinc and lead are made in larger amounts than aluminum.

925. The Uses of Aluminum.--Two hundred million pounds of aluminum were used by the allies in aeroplane manufacture in I9I8. Over one-third of the material of the Liberty motor is aluminum. The automobile industry uses a large quantity in the form of castings and in sheets for chassis and paneling. Aluminum, used for castings, is usually alloyed with copper or zinc to improve its workability, etc., since the pure metal shrinks badly in the mold and sticks to the cutting tool when it is machined. "Duralumin"-aluminum and copper with traces of manganese and magnesium - has been much used for rolled and forged parts. Another big supply of aluminum is needed for cooking utensils of various kinds. It is interesting to note that though this metal is more costly than copper if measured by weight, it is less costly if measured by volume. Hence where articles of a certain size are required aluminum may well be substituted for copper. Aluminum is coming into extensive use for electrical transmission wire and also for other electrical purposes. A wire of aluminum of the same weight and length as a copper wire is twice as good a conductor of the current (half the resistance). 
We have already spoken of the thermite reaction of Goldschmidt (330) in which iron oxide is reduced by aluminum at high temperature and free iron and aluminum oxide form. By this method small quantities of pure iron are made for heavy iron and steel repair work. The molten metal formed is used to weld broken parts together. The thermite reaction is also employed to prepare chromium, manganese and vanadium respectively from their oxides and also for the manufacture of many ferro-alloys.

Much aluminum is also used in the steel industry to prevent blow holes in castings. Aluminum powder, mixed with oil, is used as a silvery paint.

926. Gold and Silver.-The most important processes for the recovery of gold from ores that have little or no value on account of other metals present have already been briefly described (8I9). The amalgamation process for gold is also suitable for silver if the latter is present in the free state. Ores in which the silver is present as sulfide, arsenide, or a more complex form are given preliminary treatments of various sorts before amalgamation. In the cyanide process for gold, if silver is present it accompanies the gold to a greater or less extent by reason of its ability to undergo similar reactions. This process, however, is primarily one for the recovery of gold.

Silver (but not gold) is recovered from ores containing no other valuable metals by a process based on the conversion of the silver present into chloride by roasting the ore with common salt and then extracting (leaching) the material with a solution of sodium thiosulfate $(626)$. The silver dissolves as soluble salt of a complex ion and is recovered by precipitation with sodium sulfide which throws down extremely insoluble, black silver sulfide, $\mathrm{Ag}_{2} \mathrm{~S}$. From the latter, metallic silver is easily made.

Many ores of gold or silver or both also contain lead, copper or zinc in valuable amounts. Such ores are smelted in the ways already described in this chapter. In smelting processes the gold and silver are almost completely concentrated in the base metals. It is frequently advantageous to smelt gold or 
silver ores, free from copper or lead, along with ores of these base metals, especially if the former ores contain iron which is needed to make a suitable slag. About 75 per cent of the silver produced in the United States is obtained from the refining of copper, lead and zinc.

Since neither gold nor silver is oxidized at high temperatures, the refinery residues are strongly heated under oxidizing conditions until other metals have been converted into oxides - and thus eliminated. The metallic product, which contains as a rule a large excess of silver, is called doré silver. The alloy of the two metals is usually treated with hot sulfuric acid which converts the silver into sulfate and leaves the gold unchanged. The silver sulfate is dissolved in water and reduced to metal by the action of copper or by electrolysis. The gold residue is melted and cast into bars.

The uses of silver and gold are so well known that no comment on this topic is necessary. 
Y 
INDEX 
? 


\section{INDEX}

[References are to sections, not pages]

Absolute, temperature, 6

Absorption, 73I

Acetamide, 659

Acetates, precipitation by sodium acetate, $45^{2}$

Aceteldehyde, 652

Acetic acid: as a dissolving agent, 457 ; effect of acetates on ionization of, $43 \mathrm{I}$; effect of hydrochloric acid on, 432; ionization of, 409; failure to precipitate acetates, 452 ; graphic formula, 654; general properties, I 57, 653; and sodium hydroxide, 435; titration of, 440

Acetone, 656

Acetylene: composition, 5०; and copper oxide, 83 ; heat of combustion, 357 ; preparation, 49; series, $66_{3}$

Acheson process, 630

Acid anhydride, 313

Acidimetry, 137

Acids: and carbonates, 163 ; dibasic, I02; fatty, 655; ionization of, 4 10; monobasic, I02; parts of, 377 ; properties of, 90 ; strength of, 428 ; tribasic, I59; strong: little soluble salts of, and acids, $45^{8}$; and salt of weak acid, $4.28,43 \circ$; salts of, and weak acids, 457 ; titration of, $44 \circ$; and weak acid, 432; and weak base, 437; weak, I77; little soluble salts of, solution by strong acids, $45^{6}$; as precipitating agent, 452; and strong acid on, 432; and a strong base, 434; suppression of ionization of, 43I ; titration of, 440; and a weak base, 438

Adhesion, 726

Adsorption, 728, 731, 732, 763; accompanying precipitation, 739; and catalysis by finely divided metals, 73r; by colloid, 739; of gases by charcoal, 728; from solution, 732 ; from solution, explanation of, 763

Affinity, 259

Agate, 804,806

Air: adsorption by glass, 729 ; composition of, Iо, 765,766 ; ionization of, 854 ; liquid, 776,777 ; weight of, 3

Aitken, 769

Albumin, 685; and the phosphoric acids, $59 \mathrm{~S}$
Alcohol: absolute, $64 \mathrm{I}$; denatured, $64 \mathrm{r}$; from fermentation, 640, 641; wood, 645

Alcohols: aromatic, 670 ; triatomic, 679

Aldehydes, $65_{2}$; aromatic, 6;0

Alfalfa, assimilation of nitrogen, 5 I 5

Aliphatic compounds, 666

Alkali metals, 823

Alkaline earth metals, 824

Allotropic forms, $582,600,630$

Alloys, fusible, 8 I 2 , 9 I 3

Alpha rays, 480,858

Alum, I75

Aluminium. See Aluminum

Aluminum, I74; acid reaction of salts of, I 76 ; metallurgy of, $920 \mathrm{ff} . ;$ place in Periodic Table, 833; production of, 88I, 924; uses of, 925 ; chloride, I74; hydroxide: gel, 761 ; precipitation by ammonium hydroxide, 452; preparation and properties, I 74,177 ; nitrate, I 75; nitride, 5 I 4 ; oxide, 174 ; potassium sulfate, I 75 ; sodium sulfate, I 75 ; sulfate, 175

Amethyst, 806

Amides, 659

Amine acids, $68_{5}$

Amines, 658 ; aromatic, 674

Ammeter, 400

Ammonia: adsorption by charcoal, 728 ; composition of, 52,53 ; and copper oxide, equation, 84 ; critical temperature of, 775 ; liquid, 5 I 7 ; manufacture from cyanamid, 526; manufacture of synthetic, 525 ; oxidation to nitric acid, 570; properties of, $5 \mathrm{I}, 5 \mathrm{I} 7,527$; sources of, 516 ; theory of the synthesis of, 520-24; uses of, 518 ; and water, 9I; and water, equilibrium, 284.

Ammonium: aluminum sulfate, I 75 ; arseno-molybdate, 810; bicarbonate, dissociation of, 530 ; chloride, 92 ; dissociation of vapor, 529; and sodium hydroxide, ionic theory of, 426,462 ; cyanate, synthesis of urea, 696; fluoride, 269; hydrogen sulfate, IOI; hydroxide: a base, 9I; degree of ionization of, 409; effect of ammonium salts on ionization, 43I; 


\section{[References are to sections, not pages]}

effect of sodium hydroxide on ionization, 432; and nitric acid, 105; titration of, 440; molybdate, 597; nickel sulfate, 817; nitrate, 105; decomposition by heat, $56_{5}$; use as an explosive, $55^{6}, 573$; nitrite, $5^{1} 3$; perchlorate, 355; phosphomolybdate, 597,8 I $_{3}$; picrate, 673 ; sulfantimonate, $8 \mathrm{Ir}$; sulfate, ror; sulfides, 607 ; dissociation of, 530; yellow, 607; sulfocyanate and ferric chloride (ionic equilibrium), 280, 4 I5

Ampere, 400

Amphoteric substances, 177

Analysis, 3I; by means of spectroscope, 786

Anaxagoras, 184

Anhydride of an acid, 3 I 3

Aniline, 674

Animals, dependence on plants, 690

Anions, 389, 39 I

Anode, 295, 389

Anthracene, 698

Antichlor, 6II

Antimony: and its compounds, 8II; place in Periodic Table, 835, trichloride, from antimony and chlorine, 246

Apatite, 580

Aqua regia, 562

Aquadag, $75^{\circ}$

Argol, $66_{5}$

Argon, $51_{3}$; in air, 765 ; discovery and properties, 791; family, 825 ; molecular and atomic weight, 792; place in Periodic Table, 848

Argyrol, $75^{\circ}$

Aromatic series, 666

Arrhenius, Svante, 405, 720

Arsenic: antidote for, 8 ro; and compounds, 810; place in Periodic Table, 835

Arsenious sulfide, 8ro; colloidal suspension, $734,736-38$

Arsine, 810

Atmosphere, 765-99; a disperse system, 774

Atomic-molecular hypothesis, 208

Atomic numbers, $848,878,880$. See flyleaf at back of book

Atomic structure, 880

Atomic volume, 823

Atomic weights, 216; determined by law of Dulong and Petit, 229; oxygen basis, 223; of radioactive substances, 869; relation to symbol weight, 216 ; table, 800 . See also inside of back cover of book
Atoms: Greek conception of, 184 ; modern conception of, 185 ; number in a molecule, 214,215 ; relative weights of, 208-13; structure of, 470,880

Attraction, molecular, 202, 726

Atwater, 687

Avogadro's hypothesis (or law), 193; application to suspensions in liquids, 707; applications, 210; exactness of, 226

Avogadro-van't Hoff hypothesis, $7 \mathrm{r} 6$

Azote, 5 I 2; (nitrogen) I 5

Babbitt metal, 8r r

Baking powder: phosphate, 593; tartrate, 665

Balloons: helium, 797; hydrogen, $3 \circ 4$

Barium, 824 ; flame, 784 ; in Periodic Table, 832; chloride, 164 ; peroxide, 310,319 ; salts, reaction with sulfates, 380 ; sulfate, I64

Base: diacid, I46; ionization of, 410; monacid, I46; parts of, 378 ; strength of, 429 ; strong: and salt of weak base, 429-30; titration of, 440; and weak base, 432; weak, 176 ; and strong acids, 437; and strong base, $43 \mathrm{I}$; suppression of ionization of, $43 \mathrm{I}$; titration of, 440 ; and weak acid, 438

Basic nitrates, 564

Batteries, electric, 496

Baumé, scale of specific gravity, 6 r 8

Bauxite, 923

Bé, 618

Bead test, metaphosphate, 596; borax, 803

Becquerel, 852 ; rays, $8_{53}$

Beets, sugar, 682

Bell metal, 809

Benedict, 687

Benzaldehyde, 670, 697

Benzene, 667; ring, 668

Benzene sulfonic acid, 672,697

Benzoic acid, 67 I

Benzyl alcohol, 670

Beri-beri, 689

Beryllium: and compounds, 826 ; in Periodic Table, 832

Bessemer process, $891-92$

Beta rays, 860

Betts process, 9 I 2

Birkland-Eyde process, manufacture of nitric acid, 568

Bismuth: compounds, 812; place in Periodic Table, 835; nitrate, 564; subnitrate, 564

Bivalent, I 46

Blast furnace, 886 
[References are to sections, not pages]

Bleaching, by chlorine, 249

Bleaching powder, $35 \mathrm{I}$

Blende, 9I 5

Blotting paper, functioning of, 726

Blowpipe, oxyhydrogen, 300

Bluestone, 497

Body: living, need of food, 686; use of word in chemistry, 22

Boiling-point: molar elevation, 718 ; of solutions, I28; of water, 112

Boisbaudran, 845

Bonds: double, 66I; triple, 663; valence, 323

Bone ash, 158

Bone black, use as adsorption agent, 732

Boracic acid, 801

Borax, 801,803

Boric acid, 801,802

Boron: flame, 784 ; in Periodic Table, 833; properties, 8 o I

Boyle's Law, 4; explanation of, I 88

Bragg, W. H., 879

Bragg, W. L., 879

Brandt, 577

Brass, I 48, 908, 919

Breakfast, example, 688

Bredig, 744

Brin's process, for oxygen, 310

Britannia metal, 8I I

British Thermal Unit, $35^{8}$

Bromic acid, 822

Bromides: action of chlorine on, 259 ; insoluble, 257

Bromine: liquid, 255; occurrence of, 254; in Periodic Table, 837 ; preparation and properties, 255,258 ; uses of, 260 ; water, 255

Bronze, 809

Brownian movements: of particles in liquids, 705; of smoke particles, 703

Brownlee apparatus, 44

B.T.U., $35^{8}$

Bullion, base, 9 I 2

Bunsen burner, $78 \mathrm{I}$

Burning of substances, 10-20

Burns: from nitric acid, I0.4; by steam, 11 7; from sulfuric acid, 93

Butyric acid, 680

Butyrin, 680

Cadmium, 832

Caesium, 823 ; most active metal, 842 ; in Periodic Table, $83 \mathrm{r}$

Calcium, 150,832 ; flame, $78_{4}$; spectrum of, 786 ; bicarbonate, 156 ; carbide, 49, 63I ; carbonate, I 50 ; dissolved by hydrochloric acid, 456, 461 ; precipitation of, 448 ; chloride,
I5I; action with carbonic acid, 449; cyanamid, 5I4; fluoride, 267 ; hydroxide, I5I, I66; hypochlorite, 35I; nitride, 514 ; oxide, I $5 \circ$; phosphate, I58; precipitation of, $45^{2}$; silicate, 270 ; sulfate, $I_{53}$; tungstate, 814

Calomel, I82

Calorie, I I I

Calorimeter, bomb, 357

Candle, burning of, 20,780

Cane sugar, 682

Caprilic acid, 680

Caproic acid, 680

Carat, 810

Carbides, 631

Carbohydrates, 682

Carbolic acid, 672

Carbon: compounds, 629-701 ; "fixed," in fuel, 359 ; formation in flame, 782 ; heat of combustion, 357 ; and hydrogen, 631 ; place in Periodic Table, 826, 834 ; properties of, 630,631 ; as reducing agent, 328 ; valence, 648 ; weight in one liter of gaseous compounds, 6o; bisulfide (or disulfide), 546 and 63 I ; dioxide, I9, 30, 633; adsorption by charcoal, 728 ; from calcium carbonate, 150 ; composition of, 39; constancy of concentration in air, 766; critical temperature of, 775; and limewater, $15 \mathrm{I}$; solid, as a refrigerant, 633,775 ; and water, equilibrium, 285 ; disulfide, 631 ; monoxide, 632,665 ; critical temperature of, 775 ; heat of combustion, 357 ; liquefaction of, 777 ; reducing agent, 329 ; oxychloride, 695; tetrachloride, 644

Carbonates and acids, $\mathrm{I}_{3}$

Carbonic acid, I 52 ; action with calcium chloride, 449; equilibrium, carbon dioxide, and water, 285 ; ionization of, 409 ; and sodium hydroxide, I 6 I

Carborundum, 631

Carnotite, 874

Caro's acid, 628

Castner's sodium process, 920

Catalysis by metals and adsorption, 73 I

Catalytic agent, or catalyzer, 239; for ammonia reaction, 522; for decomposition of hydrogen peroxide, 320 ; manganese dioxide in preparation of oxygen, 306; manufacture of sulfuric acid, 61 5 ; platinum $\times 303$

Cathion, $389,39^{\circ}$

Cathode, 295, 389 ; rays, 475,478

Caustic soda, absorbs carbon dioxide, 19. See Sodium hydroxide

Cavendish, 292, 544 
[References are to sections, not pages]

Cells, galvanic, 496

Celluloid, 694

Cellulose, 684 ; products, 694

Cementation process, 894

Cementite, 897

Cerium, 820, 834

Cerium oxide, use of, in gas mantles, $30 \mathrm{I}$

Chalcopyrite, 899

Chamber acid, 6 I 6

Chamber process, 616

Chaptal, 572

Charcoal: adsorption by, 728, 732; burning of, I 8

Charge on a suspension, test for, 749

Charles's Law, 5

Chemical activity of elements, order of, in Periodic Table, 842

Chemical reactions, rate of, $275^{-7} 8$

Chile saltpeter, ro4; iodine from, 26 I

Chlorates, preparation of, 353

Chloric acid, 354; and lead sulfide, 354

Chlorides, insoluble, $25^{2}$

Chlorination process for gold, 8I 9

Chlorine: and aluminum, $174 ;$ and bromides, 259; critical temperature, 775 ; discovery of, 234; from electrolysis of hydrochloric acid, 43; electrolytic preparation, 237, 238; and ferrous chloride, 173 ; and hydrogen, 44, 243; liquefaction of, 242 ; and mercury, 179 ; and methane, 644 ; minimum weight, 63 ; occurrence, 233; oxidation products of, $35^{2}$; an oxidizing agent, 332 ; in the Periodic Table, 827 ; and phosphorus, 247 ; physical property of, $24 \mathrm{r}$; poisonous gas, 236 ; preparation from hydrochloric acid, 235, 239; and sodium, theory of union of, 485 ; and turpentine, 248; union with metals, 246 ; and uses of, 249; and water, at ordinary temperatures, 245 ; at high temperatures, 240; dioxide, 354; monoxide, $35^{2}$

Chloroform, 644

Chlorophyl, 69 I

Chloropicrin, 695

Chromates, 345 ; as oxidizing agents, 346

Chromium: and its compounds, 344 ; place in the Periodic Table, 836 ; preparation of, 925; hydroxide, precipitation of, $45^{2}$

Cinnamic ald ehyde, 697

Cinnamon, oil of, 697

Citric acid, 665

Clark, F. W., 884, 920

Claude, 776
Clay, I 77, 804

Cleveite, helium from, 794,867

Clouds, 768-7I; functions of gaseous ions in their formation, $77 \mathrm{I}$

Clover, assimilation of nitrogen, 515

Cloves, oil of, 697

Coal, distillation of, 634

Coal tar, 667

Cobalt: and its compounds, 8 I6, 817; place in Periodic Table, $8_{3} 8$

Coefficient, in chemical equations, 76

Cohesion, 726

Coins, composition of, 908

Coke, 630 ; as a reducing agent, 328

Collodion, 694

Colloids, 735; adsorption of agent which precipitates, 739 ; chemistry, importance of, 764 ; protecting agent for suspensions, 745 ; test for charge on, 749

Columbium, 835

Combining volumes, Gay Lussac's Law, 220,221

Combustion: heat of, $35^{6-58}$; in nitric oxide, 546; spontaneous, 364. See also Burning of substances

Common ion law, 432

Complex ions, $53^{8}$

Composition, law of definite, 46, 99

Compound, 3 I

Concentration and speed of reaction, 280

Conductivity: change during neutralization, 423; effect of dilution, 406; molecular, 407

Conservation of energy: for bodily processes, 687; law of, $37 \mathrm{I}$

Conservation of matter, $2 \mathrm{I}$

Constant heat summation, law of, 363

Constant of equilibrium (example), 283

Constant proportion, law of, 46,99

Contact process for sulfuric acid, $6 \mathrm{I} 7$

Cooledge, 8 I 4

Copper, $1_{5}$; bead test for, 783 ; blister, 907 ; burning of, 32 ; electrolytic, 907 ; flame, 784 ; from hydrogen and copper oxide, 33 ; metallurgy, $900 \mathrm{ff} . ;$ and nitric acid, 550, 561; production, $88 \mathrm{I}$, 899; properties and place in Periodic Table, 831 ; refining of, 907 ; uses of, 908; (see also Cupric and Cuprous); ammonium ion, 538 ; chloride, 165 from chlorine and copper, 246; hydroxide, 165 ; nitrate, 165 ; oxide, I65; and acetylene, equation, 83 ; and ammonia, equation, 84 ; composition of, $32,3^{8}$; and hydrogen, 33,82 ; sulfate, 165 
[References are to sections, not pages]

Cordite, 693

Corn sirup, 639

Corrosive sublimate, 178

Cotton, 684; bleaching of, 35I; soluble, 694

Coulomb, 400

Cowles brothers, 921

Cream of tartar, 665

Critical pressure, 775 ; temperature, 775

Crookes, 477,859 ; tube, 475

Cryolite, 267, 923

Crystals: melting of, 205; theory of structure, 204; X-ray and structure of, 879

Cupric compounds (see also Copper compounds), 333; bromide, color of, in solution, 396 ; chloride, electroly'sis of solution, 386 ; as catalyst, 239; oxide, 325; potassium chloride, I75; sulfate, electrolysis of solution, 386 ; sulfide, precipitated by hydrogen sulfide, $45^{2}$

Cuprous compounds, 333 ; oxide, 325

Curie, Mme, 855

Current, electrical: by chemical action, 493-95; direction of, 472; electron theory, 469; how carried through a wire, 471 ; nature of, 468 ; strength of, 400

Cyanates, $66_{5}$

Cyanide process for gold, 8I9

Cyanides, 665

Dalton: Atomic Hypothesis, 208; Law of Partial Pressure, 192

Daniell cell, 496

Davy, 234, 92 I

Deacon's process, 239

Decomposition, 25 ; of sal soda, 26

Degree of ionization, 408-10

Deliquescence, 130

Democritus, I 84

Density, I IO

Depilatory, 607

Detonator, 573

Developers, photographic, manufacture related to dye industry, 699

Deville, 920

Dew, 767

Dewar, 777, 779; vessels, 777

Dew point, 767

Dextrose, 639

Dialysis, 743

Dialyzer, 743

Diamonds, 630; and gamma rays, 86 I

Diastase, 682

Diatoms (infusorial earth), 732
Dichlor diethyl sulfide (mustard gas), 695

Dichromates, 345 ; oxidizing agents, 346

Dietetics, 688

Diffusion: of gases, 191 ; in liquids, 704

Dinner, example, 688

Disintegration hypothesis, 481,865

Disperse systems, 725-64, 774

Displacement: electronic interpretation of, 489; metallic, electronic interpretation of, 49r; of metals by one another, 490; of non-metals by one another, 488

Dissociation: electrolytic (see Ionization); hydrolytic, 436 ; of volatilized ammonium salts, 529-3I

Distillation, 23

Dithionic acid, 628

Double-decomposition, $337,38_{3}$; and electrical conductivity, 384 ; and the ionic hypothesis, $4 \mathrm{I} 3$

Drying agents, 130

Dulong and Petit, law of, 229-30

Duralumin, 925

Durion, 539, 804

Dust: in the air, 768 ; counting of particles, 769 ; explosions, 365 ; function of, in cloud formation, 768

Dynamite, 692; preparation, of 726

Effervescence, $\mathrm{I} 63$

Efflorescence, I3I

Egg white, 685

Ekaboron, 845

Ekaluminium, 845

Ekamanganese, 845

Ekasilicon, 845

Electric cells, oxidation-reduction, 502

Electric current, 468, 469; oxidation and reduction by means of, 507

Electricity: frictional, 474 ; nature of, 466

Electrochemical equivalents, law of, 403

Electrodes, potential difference of, 499

Electrolysis, 27; of cupric chloride solution, 386; of cupric sulfate solution, 386; Faraday's laws of, 399; of hydrochloric acid, 385 ; of silver nitrate, 387 ; of sodium chloride solution, 385 ; terms used in, 389 ; theory of, 398,487 ; of water, 27

Electrolytes, 389 ; molecular weights of, 720 ; precipitation of, by common ion, 453; soluble, equilibrium between, 44I; solution of little soluble, 455

Electromotive force, 499

Electromotive series of metals, 492, 499 


\section{[References are to sections, not pages]}

Electrons, 465, 466; mass of, 479; proof of existence, 467 ; vibration, cause of light, 788

Element, 3 $\mathrm{T}, 35$; meaning of term, $873 ;$ total number, 800

Elements, activity of, 842

Emery, I74; powder graded by time of settling in water, 733

Emulsifying agents, 755

Emulsoids, $735,75^{1-57}$; general properties, 756; importance, 757

Energy: chemical, 372; chemical, conversion into electrical energy, 508; conversion of, $37 \mathrm{I}$; electrical, 500; electrical, conversion into chemical energy, 509; forms of, 372; kinetic, of molecules, 198

Enthodermic changes, 366

Enzymes, 682

Epsom salts, I 44

Equations, chemical, 76 ; balancing of, 86 ; balancing of oxidation and reduction, 561 ; meaning of, 77,85 ; problems of, 79,87 ; review table, 86

Equilibrium (general): chemical, 274; constant (example), 283; criterion of, 282 ; effect of changes of concentration on, 280; effect of pressure on hydrogen and nitrogen, 523; effect of pressure on system in, 287 ; effect of removing one product of the reaction, 289; between electrolytes (see Equilibrium between electrolytes); and heat production, 367 ; hydrogen and nitrogen, effect of temperature on, 288, $52 \mathrm{I}$; kinetic hypothesis applied to, 279; liquid and vapor, $20 \mathrm{r}$; between molecules and ions, 405; physical, 273

Equilibrium between electrolytes: and gas evolution, 459; graphic representation of, $417,418 \mathrm{ff}$., 444 ; between soluble electrolytes, 44I; in solution, $4{ }^{\mathrm{I}} 3^{-} 39$; in solution and solid substance, $443 \mathrm{ff}$.

Equivalent, electrochemical, 403

Equivalent weight, 403

Esters, 657 ; of glycerine, 679

Ethane, 643

Ether, 642

Ethyl compounds: acetate, 657 ; alcohol, 64I; structural formula, 649; ammonium iodide, 658 ; iodide, 660

Ethylene, 660; series, 662; structural formula of, $66 \mathrm{I}$

Ethylene chloride, 660

Evaporation, theory of, 199

Exothermic changes, 366
Explosion, 302

Explosives, 571, 692, 693

Fajans' Law, 872

Falk, 722

Families: of elements, $822 ; A$ and $B, 83$ I

Faraday, $242,389,403,775$; law of electrolysis, 399

Fat soluble A, 689

Fats, 677 ; composition of, 680

Fehling's solution, 683

Fermentation, 640

Ferric: chloride, and ammonium sulfocyanate, 280, 415; and hydrogen sulfide, $50_{3}$; and potassium iodide, 503; preparation, I 73 ; compounds, $33 \mathrm{I}$; hydroxide, I73; colloidal, 74I43; a gel, 76I; and hydrochloric acid, 455; precipitation of, $45 \mathrm{I}$; oxide, 173 ; sulfate, 173 ; and sodium carbonate, 384

Ferro-molybdenum, 8 I 3

Ferro-silicon, 804

Ferro-tungsten, 814

Ferrous compounds, 33I; ammonium sulfate, I75; chloride, I73; hydroxide, I 73; oxide, I 73; sulfate, r73; sulfide: dissolved by hydrochloric acid, 456; precipitation of, 452 ; preparation and use, $339,60 \mathrm{I}$

Fertilizer: phosphate, I60; use of ammonium salts in, 518

Films, photographic, 694

Filters, adsorption by, 763

Filtration, 23

Fire extinguisher, 633,644

Firefly, 584

Flame, 780 ; colored, 784 ; reactions in, 783 ; spectra, 786

Flint, 806

Flotation process, 900

Flour, bleaching of, 563

Fluorides, 269

Fluorine: most active non-metal, 842; in Periodic Table, 837 ; preparation and properties, 267

Fluor-spar, 267

Fluosilicates, 272

Food, 676

Formaldehyde, $65^{2}$

Formalin, $65^{2}$

Formic acid, 665

Formula: calculation of, $80,8 \mathrm{r}$; chemical, 62; of elementary gases, 75, 218; graphic, 323; involatile substances, 72 ; making of, 67 ; structural, of ethyl alcohol, 649; structural, importance of, in organic chemistry, $65 \mathrm{I}$; struc- 
[References are to sections, not pages]

tural, of methyl ether, 649; use of, 68; volatile, liquids and solids, $7 \mathrm{I}$; weight, relation to molecular weight, 217 ; weight, and symbol weight, 74

Fox fire, 584

Franklinite, 915

Frasch process, 602

Fraunhofer lines, 790

Frazier, 712

Freezing-point, molar depression, 718

Frost, 767

Fruit sugar, 682

Fuel: composition of, calorific power, 359 ; for steam production, 360

Fullers earth, 732

Gallium, discovery of, 845

Galvanic cells, 498

Galvanized iron, 148

Gamboge, Brownian movements of, 705

Gamma rays, 861

Gas: calorific power, 359; collected over water, calculation of pressure, I 1 3; diffusion, I9I; evolution of, factors governing, 463 ; evolution of, and ionic equilibrium, 459 ; illuminating, 634 ; natural, 643; pressure, cause of, I 88; pressure, law of partial, I 92 ; statistics, 194-97

Gas laws: accuracy of, 225 ; application to dilute solutions, 715 ; problems on, 7

Gases: the inert, or noble, 825 ; ionization of, 770; liquefaction of, 775; mixing of, 190; standard conditions for, 7

Gasoline, 643

Gay Lussac's Law, 5

Gelatine: gel, $75^{8}$; a protecting agent for colloids, 745

Gels, 758-6I; plant and animal tissue, relation to, 759

German silver, 816, 908

Germanium: discovery of, 845 ; place in Periodic Table, 834

Glass: adhesion of water to, 726 , adsorption of air on surface, 729; adsorption of water vapor on, 730; composition, 270, 808; etching of, 27 ; optical, 803 ; quartz, 808 ; various kinds, 808

Glucose, 639; fermentation of, 640

Glycerine, 679

Gold: colloidal, 746; compounds and alloys, 819; extraction from ores, 819 , 926; place in Periodic Table, 831 ; production, 881 ; solution in aqua regia, 562 ; world-production of, 819
Goldschmidt process, 330, 925

Graham, Thomas, 735

Grain alcohol, 64I

Granite, 804

Grape sugar, 639

Graphite, 630

Gravity batteries, 497

Grindstone, 806

Groups: of atoms in organic chemistry, 651 ; of elements, $830 \sim 38$

Guncotton, 693

Gunpowder, 326, 571, 572

Gypsum, I 55

Haemoglobin and oxygen, 314

Hall-Hërault process, $92 \mathrm{I}-23$

Halogens, 231,822

Hampson, 776

Harkins, 754, 849

Hartshorn, 516

Heat: atomic, 230; of combustion, $35^{6-58}$; of formation, 361 ; of ionization, 439; latent, of evaporation, 115 ; latent, fusion of ice, I1 8; law of constant heat summation, 363 ; mechanical equivalent of, 360 ; molecular, 792-93; of neutralization, 362, 439; production and equilibrium, 367 ; production in physical and chemical changes, summary of, 366 ; of reaction, $36 \mathrm{I}$; of solution, 127 ; of solution, 288; of solution and solubility, 134, 288; theory of, I 89

Heliotrope, 697

Helium: and the alpha rays, 795,858 ; balloons, 797; discovery of, 794 ; and family, 825 ; properties of, 796

Hematite, 328,884

Henry, Law of, I 26

Hess, Law of, 363

Hexathionic acid, 628

Hexoses, 682

Hillebrand, 794

Humidity of air, 766

Hydrates, 97

Hydrazine, 53I

Hydriodic acid, 265, 339

Hydrobromic acid: oxidation of, 258 ; preparation of, 256 ; as reducing agent, 34I; and silver nitrate, 257

Hydrocarbons, 643; aromatic, 668; isomerism of, 664

Hydrochloric acid: and aluminum, 174 ; and aluminum hydroxide, 175; and calcium carbonate, $46 \mathrm{r}$; and caustic soda, $4 \mathrm{I}$; and copper oxide, 165 ; electrolysis of, 43,385 ; and iron, 173 ; 


\section{[References are to sections, not pages]}

and lead, I67; and lead dioxide, I57; and magnesium, 149; and magnesium hydroxide, 143, 455; and magnesium oxide, 145; preparation, 103, 250; properties, $25 \mathrm{I}, 252$; as reducing agent, $34 \mathrm{I}$; and sodium acetate (ionic theory of action), 424; and sodium hydrogen sulfate, 253, 289; and zinc, 149

Hydrocyanic acid, 665

Hydrofluoric acid, 269

Hydrofluosilicic acid, 269, 272

Hydrogen: adsorption by charcoal, 728 ; critical temperature, 775 ; discovery of, 292; from electrolysis of water, 27; flame, temperature of, 299; heat of combustion, 357 ; liquid, 779 ; in nature, 292; percentage in water, 36 ; place in electromotive series, 492, 499; place in Periodic Table, 847; preparation, 27, 28, 33, 293-95; properties of , 27, 296, 297; use of, 295, 304 ; weight in one liter of gaseous compounds, 56 ; weight in 22.4 liters of gaseous compounds, $56,63,211$; reactions of: burning in chlorine, 244; and chlorine, 44; and copper oxide, 33,82 ; and iodine, chemical equilibrium, 28I; and iodine, heat of reaction, 367 ; and iron oxide, 290; from magnesium and steam, 28 ; and nitrogen, 298; and nitrogen, equilibrium, 521,$523 ;$ and oxygen, 299-303; chloride, 44; composition, 48; critical temperature of, 775; physical properties of, 25 I (see Hydrochloric acid); preparation of, 44; fluoride, 268; action on quartz, silicon, glass, 270; iodide: preparation, properties, 264; sulfide: aqueous, as reducing agent, 609 ; precipitation of sulfides, $45^{2}$; preparation of, properties, 339, 605-9; peroxide, 318-20; detection of (reaction with chromic acid), $32 \mathrm{I}$; as oxidizing agent, 347 ; as reducing agent, 348 ; use of, 320

Hydrolysis of salts, 436

Hydrosulfurous acid, 606

Hydroxides, preparation of insoluble, 166

Hydroxylamine, 53I

Hypo, 625, 627

Hypochlorites, $35^{\circ}$

Hypochlorous acid, 349; from chlorine monoxide, 352

Hyposulfurous acid, 628

Ice: density of, II9; latent heat of, II8; manufacture of artificial, 5 I9

"Icy-Hot" bottles, 777
Ignition temperature, 302

Illuminating gas, 634

Indestructibility of matter, law of, 2 I

Indicators, 440

Indigo, synthesis of, 698

Infusorial earth, 732

Ink: sympathetic, 81 7; India, $75^{\circ}$

Insulator, 473

Intumescence, $8 \circ 3$

Iodic acid, 822 ; and sulfurous acid, 277

Iodides, 265 ; uses of, 266

Iodine, $26 \mathrm{I}-65$; heat of reaction with hydrogen, 367 ; and hydrogen, chemical equilibrium, $28 \mathbf{I}-83$; place in Periodic Table, 848 ; and starch, 263 , 637 ; uses of, 266

Ionic equilibrium, 405, 4I 3 ff.; and gas evolution, factors governing, 463

Ionic hypothesis, $4 \mathrm{II}$; criticism of, $4 \mathrm{I} 2$; value of, 464

Ionization: cause of, 486; degree of, conductivity method, 408-10; degree of, freezing-point method, 721, 722; degree of, graphic representation, 4I7; of gases, 770; heat of, 439

Ions: in solution: charges on, 404 ; and chemical reactions, $39^{2}$; complex, 538 ; color of, 395; migration of, 397; nature of, 483 ; positive and negative, 393; union of, 394 ; gaseous, $77 \mathbf{I}$

Iridium and its compounds, 818,838

Iron, 173 ; allotropic forms, 897 ; burning of, I2, I7, 29, 8I ; cast, 883, 889; economicimportance, 882; galvanized, I 48, 919; and hydrochloric acid, I 73; magnetic oxide of, 173; metallurgy, $883-98$; ores, 884 ; pig, $886-88$; place in Periodic Table, 838 ; preparation of, from hematite, 328 ; production, $88 \mathrm{I}$; and steam, 29, 290; and sulfuric acid (concentrated), 62I; and sulfuric acid (dilute), r73; welding, 895, 925; wrought, 883,890

Iron compounds (see Ferrols and Ferric): iron oxide, I 73

Isatine, 698

Isomerism, 647,650

Isomorphism, 810

Isoprene, 700

Isotopes, $87 \mathrm{I}, 873$

Joule, mechanical equivalent of heat, 370 ; unit of electrical energy, 500

Kelly, William, 89I

Kerosene, 643

Ketones, 656 
[References are to sections, not pages]

Kieselguhr, 732

Kinetic energy, 368; of molecules, I 98

Kinetic theory, I 87 ; of the liquid state, I98, 704

Kipp, apparatus, 294

Kraft, 577

Krypton, 798; and its family, 825

Lactic acid, 665

Lactose, 682

Lake copper, 906

Langmuir, 754

Lanthanum, 820; in Periodic Table, 833,846

Latent heat: of evaporation, II5; of fusion, I I 8

Laughing gas, $55^{6}$

Lauric acid, 680

Lavoisier, 13

Lead, I67; metallurgy, 9roff.; place in Periodic Table, 834; production, 881, 909; smelting, 910; uses of, 913; acetate, I67; chloride, 167 ; dioxide, 167; oxidizing agent, 326; fluosilicate, 272 , 912; nitrate, 167; decomposition by heat, $565 ;$ oxide, r67; salts, reaction with sulfates, 38 r; sulfate, 167 ; sulfide and chloric acid, 354; and hydrogen peroxide, 347

Lead pencils, 630

Leather, artificial, 694

Legumes, assimilation of nitrogen, $5^{\text {I }} 5$

Levulose, 682

Light, ether wave hypothesis, 788

Lightning, cause of, 773

Lime, slaking of, 150

Limelight, $30 \mathrm{I}$

Limestone, 150

Limewater, I50; test for carbon dioxide, is, I $5 \mathrm{I}$

Liquid: equilibrium with vapor, 201; state, I98; supercooling of, 206

Litharge, 167

Lithium, 823; flame, 784 ; spectrum of, 786

Lithopone, 9 rg

Litmus: reaction to acids, 89 ; reaction to bases, 88 ; sensitiveness, 440

Lubricating oils, 643

Luminosity, cause of, in flames, $7 \mathrm{~S}_{2}$

Luncheon, example, 688

Lye, I 62

Lynde, 776

Madder, $69 \mathrm{~S}$

Magnesia, milk of, $\mathrm{I}_{42}$

Magnesium: burning of, II, 28, 30, 80 ; in Periodic Table, 832 ; bromide, 255 ; chloride, I43; fluosilicate, 272; hy- droxide, I42, I43, I66; nitrate, I45;

oxide, I 42 ; sulfate, I 44

Magnetite, 884

Malachite, analysis of, 34, 899

Maltose, 64I, 682

Manganese and compounds, 342, 837; preparation of, 925 ; dioxide: catalyst, 306,320 ; oxidizing agent, 326; preparation of chlorine, $234 ;$ of bromine, 258

Maple sugar, 682

Marble, I 50

Marine acid, 305

Marsh gas, 643

Marsh's test, Sio

Martensite, 897

Matches, 586

Matte, 903, 9 I I

Matter: change of form with temperature, 9; conservation, law of, $2 \mathrm{I}$; electrical nature of, 482 , 880 ; forms of, 2

Meat, 685

Medicinals, manufacture related to dye industry, 699

Meker burner, $78 \mathrm{I}$

$M \in m b r a n e$, semipermeable, t 10

Mendelejeff, 843

Mercuric compounds, 333; chloride, I 78 ; nitrate, I78; basic, 564 ; oxide, 73, I 78, I 8 I; red ash of mercury, 30 ; sulfate, 179

Mercurous compounds, 333; chloride, I8o, I 82 ; nitrate, I80; oxide, I $8 \mathrm{I}$; sulfate, $I 80$

Mercury, I 78 ; ash, decomposition by heat, I4; and chlorine, I79; oxides, I $8 \mathrm{I}$; and place in Periodic Table, 832; properties, I $78-82,832$; from red oxide, I4; bichloride, 178

Mesothorium, 870

Metaboric acid, 802

Metallography, 897

Metallurgy, $88_{1} \mathrm{ff}$.

Metals, 35; cathions, 390; displacement by one another, 490; oxidation and reduction of, 504; place in Periodic Table, $84 \mathrm{r}$; production, 88r

Metaphosphoric acid, $5 \mathrm{So}, 500$; and albumen, 597

Metastannic acid, 800

Metathesis, 383

Methane: analysis, 55; properties and composition, 54

Methyl compounds: acetate, 657 ; alcohol, 645; ammonium iodide, 658; chloride, 644; ether, 646; structural formula of, 649; iodide, 660 ; naphthalene, 669 


\section{[References are to sections, not pages]}

Methyl orange, I37, 440

Methyl violet, adsorption from solution, 732

Methylene chloride, 644

Meyer, Lothar, 844

Mica, 807

Micoderma aceti, 653

Microcosmic salt, 596

Milk sugar, 682

Millikan, 467

Minimum and multiple weights, explanation of law, 2 I 2

Minimum weights, $6_{3}, 64,212$

Mitscherlich, 810

Mixed metal, 820

Moissan, 267, 630

Moisture, in air, 766

Molecular heats, 792-93

Molecular hypothesis, 186,208

Molecular weight, 217; and depression of the freezing-point, 718 ; of electrolytes in solution, 720 ; and elevation of the boiling-point, 718; and lowering of vapor pressure of solution, 718; from osmotic pressure, data, 717; relation to formula weight, 217

Molecules, I84, 185; attraction of, 202; colors of, in solution, 396 ; kinetic energy of, 198; motion of, I87; perception of, 703; reality of, 702; solubility of, 445; velocity of, 197; velocity of, and temperature, I 89

Molybdenite, 8I 3

Molybdenum: and compounds, 81 3 ; place in Periodic Table, 836

Molybdic acid, 8I 3

Monatomic gases, ratio of molecular heats in, 793

Monazite, 820

Mond process for nickel ores, $8 \mathrm{I} 6$

Monel metal, 8I6

Mordant, 809

Morse, 7 I 2

Moseley, 848,878

Moth balls, 669

Mother of vinegar, 653

Multiple weights, law of, 2 I2

Muriatic acid, 250

Mustard gas, 695

Naphthalene, 669

Natural gas, 643

Nature of matter, 880

Neodymium, 820

Neon, 798; and its family, 825

Neutrality, of solutions, 433
Neutralization: acid and base, 89, 379; change of conductivity during, 423; heat of, 362,439 ; ionic theory of, 421,423 ; of nitric acid by ammonium hydroxide, I05; simplified equation of, 422

Newlands, 844

Newton, first law of motion, I 87

Nickel: and compounds, 816, 817; place in Periodic Table, 838

Nickel ammonium ion, 538

Nickel coin, $8 \mathrm{r} 6$

Nickel steel, 8 I 6

Nilson, 845

Niobium, place in Periodic Table, 835

Niton, 825

Nitrates: properties of, 564, 565; source of, $54 \circ$; test for, 549

Nitric acid: from air, 567; and aluminum hydroxide, I75; from ammonia, 570; and ammonium hydroxide, I05; anhydride, 555; chemical solvent for salts, 560 ; and copper oxide, 165 ; and magnesium hydroxide, 145; and magnesium oxide, 145 ; and metals, 558; from nitric oxide, 569 ; and non-metals, 559 ; oxidation by, 557 ; oxidizing agent, 542; properties of, I04, 541; from sulfuric acid and niter, 104; test for, 549; uses of, $57 \mathrm{r}$

Nitric oxide: combustion in, 546 ; conversion into nitric acid, 569; critical temperature, 775 ; equilibrium with nitrogen and oxygen, 566 ; and ierrous sulfate, 548 ; from ferrous sulfate and nitric acid, 547 ; preparation of, 543 ; properties of, 545

Nitrides, 5 I 4

Nitrites, 553

Nitro compounds, aromatic, 673

Nitrobenzene, 673

Nitrocellulose, 693

Nitrogen: assimilation by plants, 51 5 ; critical temperature, 775 ; cycle in nature, 574; discovery of, 512; fixation of atmospheric, 575; and hydrogen, 298; and hydrogen, equilibrium, 520-25; inert part of air. I5; liquid, from liquid air, 777 ; minimum weight, 63 ; occurrence of $5 \mathrm{II}$; and oxygen, 544; and oxygen, equilibrium, 566; place in Periodic Table, 835; preparation of, 513; properties of, 514; pentoxide, 555; tetroxide, 545; physical properties of, $55^{1}, 55^{2}$; preparation of, $55^{\circ}$; two forms of, $55^{2}$; trioxide, 554 
[References are to sections, not pages]

Nitroglycerine, 692; adsorption in infusorial earth, 726

Nitrolime, 5 I4

Nitrophenol, 673

Nitrosyl chloride, 562, 563

Nitrosyl sulfuric acid, 6 I 6

Nitro toluene, ortho, meta, and para. 673

Nitrous acid, 553; anhydride, 554

Nitrous oxide, 556 ; critical temperature, 775

Nonconductor of electricity, 473

Non-metals, 35; displacement by one another, 488; oxidation and reduction of, 505; place in Periodic Table, $84 \mathrm{I}$

Normal solutions, $136-38 ; 595$

Noyes, A. A., 722,737

Nutrition, 688

Oil: animal and vegetable, 657 ; of bitter almonds, 670, 697; essential, 697; hardening of, 681; hydrogenation of, 681; mineral, 643; of vitriol, 93

Oildag, $75^{\circ}$

Oleic acid, 680

Olein, 680

Oleum, $6 \mathrm{r}_{3}$

Onyx, 806

Opal, 804,806

Open hearth process, 893

Ore dressing, 900

Organic materials, sources of, $70 \mathrm{r}$

Orthoclase, 807

Orthophosphoric acid. See Phosphoric acid, or tho

Osmic acid, 838

Osmium, 818, 838 ; oxide, 838

Osmosis, 7 Ir ; in nature, 724

Osmotic pressure: measurement, $7 \mathrm{II}$; of sugar solution, 712 ; theory of, 713 , 7 I4

Ostwald, Wolfgang, $7+6$

Oxalic acid, $66_{5}$

Oxldation, 325; change of valence, 331, $332,334,336$; intensity of, 338

Oxidation and reduction, $50 \mathrm{I}-5$; cells, 502; electronic explanation, 501; by means of electric current, 507; method of balancing equations, $56 \mathrm{r}$; potentials, 506

Oxides: graphic formula of, 324; of metallic elements, 313; of nonmetallic elements, 313

Oxyacetylene torch, 3 I5

Oxygen: constancy of concentration in air, 766; critical temperature, 775 ; discovery, 305; liquid, 309, 31 2, 777; minimum weight, 63 ; in nature, 305 ; and nitrogen, 544; and nitrogen, equilibrium, 566; in Periodic Table, 836; from plants, 3II; preparation of, 306-10; properties of, 16, 31 2, 31 3; uses of, 315

Oxone, 307

Ozone, 316; as a germicide, 317

Paint: drying of, $36_{4}$; luminous, 859

Paintings, restoration of, $3+7$

Palladium, 838

Palmitic acid, 678

Palmitin, 679, 680

Paraffine, 643 ; series, 643

Parkes process, 912

Pentathionic acid, 628

Perchlorates, 355

Perchloric acid, 355

Perfume, 697

Period of radioactive substance, 864

Periodic law, $843, S_{44}$

Periodic system, $822-50$

Periodic Table, 829; anomalies of, 848 ; arrangement of Harkins', 849 ; history of, 844

Permanganates, 343 ; color of, in solution, 395

Permonosulfuric acid, $62 \mathrm{~S}$

Peroxides, 322; graphic formula of, 324

Perrin, 478, 703,707

Persulfuric acid, 628

Petroleum, 643

Phenol, 672

Phenolphthalein, 137, 440

Phosgene, 695

Phosphate rock, 160,580

Phosphates: precipitation of, $45^{2}$; qualitative test for, 597; and silver nitrate, I 7 I , 597; use and production, 598

Phosphine, 588

Phosphonium chloride, 588

Phosphorescence, $58+$; caused by X-rays, 852

Phosphoric acid, ortho: and albumin, 597; failure to precipitate phosphates, $45^{2}$; from hydrolysis of phosphorus pentachloride, 247 ; ionization of, 592; normal solution of, 595; preparation, I $58,589,590$; properties, I59, 590-92, 597; and silver nitrate, 597; titration of, 595 . See also Metaphosphoric acid; Pyrophosphoric acid

Phosphorous acid, 588; from hydrolysis of phosphorus trichloride, 247 


\section{[References are to sections, not pages]}

Phosphorus: amorphous, $5^{8} \mathrm{I}$; and bromine, 256 ; and chlorine, 247 ; discovery of, 577; effect on iron and steel, 896; and iodine, 264; manufacture of, 580 ; place in Periodic Table, 835 ; red, $579,58 \mathrm{I}$; slow oxidadation of, $58_{3}$; white, danger in use of, $5^{8} 5$; white, physical properties of, 578 ; yellow, 578 ; compounds, 588 ; pentabromide, 256 ; pentachloride, 247; pentoxide, 247,588 ; sulfide, 586; tribromide, 256 ; trichloride, $2+7$; tri-iodide, 264 ; tri-oxide, 588

Photosynthesis, 69 I

Phthallic acid, 698

Picric acid, 673, 693

Pig boiling, 890

Piperonal, 697

Pitchblende, 874

Plaster of Paris, 155

Platinum: adsorption of hydrogen and oxygen, 73I; and aqua regia, 562 ; and compounds, 8I8; properties and place in Periodic Table, 838

Polymer, 6I3

Potash, I62; caustic, I06; monopoly of Germany, 353. See also Potassium hydroxide

Potassium, 106; action on water, 106; aluminum sulfate, 175 ; cupric chloride, I75; flame, 784 ; place in Periodic Table, 831, 848; acid tellurate, 815 ; antimonyl tartrate, $8 \mathrm{II}$; bicarbonate, I62; bichromate (see Dichromate); bromide, 255; use of, 260; carbonate, I62; chlorate: oxidizing agent, 326; preparation of oxygen from, 306 ; preparation and use, 352 ; precipitation of, 447 ; chromate, 346 ; dichromate, $3+6$; and hydrogen peroxide, $32 \mathrm{I}$; and hydrogen sulfide, 346,609 ; and sulfurous acid, $3+6$; ferricyanide, $66_{5}$; ferrocyanide, $66_{5}$; hydrogan tartrate, $66_{5}$; hydroxide, Io6; iodide, and ferric chloride, electronic explanation of, 5०3; nitrite, from potassium nitrate, 305; perchlorate, 355; preparation of oxygen, 306 ; permanganate and ferrous sulfate, $3+3$; and hydrogen chloride, 235, 343; and hydrogen sulfide, 609; oxidizing agent, 326, 343; preparation from potassium manganate, 837 ; and sulfurous acid, 343 ; pratinic chloride, 8I8; tellurite, 8I5; uranyl sulfate, rays from, 852

Potato starch, preparation of, 636

Potential energy, 368
Potentials, oxidation-reduction, 506

Powder: black, 57 I, 572; smokeless, 692

Precipitates, relative stability of different forms, 762

Precipitation, I54; cause of, 446 ; classification of, 452 ; conditions favoring, 454 ; of electrolytes, 442 ; by substance having a common ion, 453

Pressure: critical, 775 ; effect of, on gases, 4; effect on system in equilibrium, 287 ; partial, I I 3

Priestley, 305

Proof spirit, 64I

Propane, 58, 643

Proportions, law of definite, 46

Protecting agents for colloids, $7+5$

Protein, 685

Prout, hypothesis, $85 \mathrm{I}$

Prussic acid, $66_{\vec{j}}$

Ptyalin, 682

Pure substance, 23

Pyrite, 6Io

Pyrophoric alloy, 820

Pyrophosphoric acid, 590

Pyrosulfates, 624

Pyroxaline, 694

Quartz, sand, 804

Quartzite, 806

Quicklime, I5O

Radical, I47; acid, 377; acid, anions, 39I; basic, 378; phenyl, 670

Radioactive change, theory of, 865

Radioactive substances: atomic weights of, 869 ; arrangement of, in Periodic Table, 873 ; valence of, 872

Radiothorium, 870

Radium: discovery of, $855-56$; emanation, 863,864 ; heat from, 862; how measured and sold, 875 ; origin of, 867; place in Periodic Table, $8+5$, 857 ; rays of, 480 ; series, 866 ; technical production, 874 ; total world-production, $87+$; use in therapeutics, 876

Radium clock, 860

Rain, formation of, 772

Ramsay, 791, 845

Raoult, 7I8, 7 I9

Rare earth elements, 820 ; place in Periodic Table, $8+6$

Rayleigh, $79 \mathrm{I}$

Rays of radium, 480, 858-61. See also X-rays

Reaction velocity, kinetic hypothesis applied to, $275^{-78}$ 
[References are to sections, not pages]

Reducing agents, intensity of, 338

Reduction, 327; and change of valence, 335,336 ; electronic explanation of, 50 I; of metals, 504; of non-metals, $5 \circ 5$

Refractory substance, 568

Respiration, 3 I4

Reverberatory furnace, 890

Rhodium, 838

Roasting, of ores, 902 , 916

Rock crystal, 806

Rock salt, 24

Roentgen rays, 476

Rose's metal, 8I 2

Rowland, 468

Rubber: a colloid, 760; synthetic, 700; vulcanization of, 604

Rubidium, 823

Ruby, I 74

Ruthenium, place in Periodic Table, 838

Rutherford, D., 512

Rutherford, E., 48r, 865

Safrole, 697

Salicylic acid, 697

Sal soda, 26

Salt, common: composition, 45 ; electrolysis of, 238 ; preparation, $4 \mathrm{I}$; purification from rock salt, 24

Salt, parts of, 376

Saltpeter, Chile, 104; occurrence, 540

Salts: acid, IO2; active parts of, 376 ; a class of chemical substances, 92 ; hydrolysis of, 436; ionization of, 410; little soluble of strong acids, not dissolved by acids, $45^{8}$; neutral, 102; primary, secondary, and tertiary, 159

Sand paper, 806

Sandstone, 806

Saponification, 679

Sapphire, I 74

Sassafras, oil of, 697

Scandium, discovery of, 845

Scheele, 305

Scurvy, 689

Seaweeds, iodine from, $26 \mathrm{I}$

Selenium, and its compounds, 815, 836

Series: displacement of metals, 492 ; of non-metals, 488

Siedentopf, 706

Siemens-Martin process, 893

Silica, 806

Silicates, 807

Silicic acid, $807 ;$ a gel, 761

Silico-chloroform, 805

Silico-ethane, 805

Silico-mesoxalic acid, 805
Silico-methane, 805

Silico-oxalic acid, 805

Silicon: compared to carbon compounds, 805 ; and compounds, $804-8$, 827; place in Periodic Table, 834; dioxide, 806; fluoride, 270; hexachloride, 805 ; tetrachloride, 805

Silk, artificial, 694

Silver, I68; colloidal, 744; metallurgy of, 926; place in Periodic Table, 831 ; production of, $88 \mathrm{I}$; test for, I69; acetate: precipitation, 452 ; solution by nitric acid, $45^{6}$; ammonium ion, 533-34; effect of acids on, 536 ; ammonium salts, 535, 537; azid, 531; bromide, 257; use, 260; chloride, 169 ; solubility in ammonia solution, $532-35$; hydroxide, I 72 ; nitrate, I68; electrolysis of solution, 387 ; and hydrochloric acid in the presence of gelatine, 745 ; oxide, 172 ; phosphate, 171 , 597; salts, reaction with chloride, 382 ; sulfate, I 70 ; sulfide, precipitation of, $45^{2}$; thiosulfate, complex ion, 626

Slag, $885,888,903$, 9II

Smithsonite, 915

Smoke screens, 587

Soap: cleansing action, $75 \mathrm{x}-54$; composition, $\mathrm{I} 62,678$

Soda, 26; caustic, and hydrochloric acid, 89; caustic, from sodium and water, 40 ; washing, 26

Soda water, 633

Sodamide, 527

Soddy, 48I, 865

Sodium: and chlorine, theory of union of, 485 ; flame, 784 ; place in Periodic Table, 831 ; spectrum of, 786 ; and water, 40; acetate, 157 ; action of water on, 435; and hydrochloric acid (ionic theory), 424; aluminate, I 77 ; aluminum fluoride, 267 ; aluminum sulfate, 175 ; antimony fluoride, $8 \mathrm{II}$; benzoate, $67 \mathrm{I}$; bicarbonate, I6I; bromide, 255; use of, 260; carbonate, I6I; chlorate, 353 ; chloride, action on sulfuric acid, 460 ; electrolysis of solution, 385 ; precipitated by hydrochloric acid, 453 ; fluoride, 269, 270; fluosilicate, 272; hydrogen sulfate, 98, 100; action of hydrochloric acid on, 289; hydrosulfite, 628 ; hydroxide, 88 ; and acetic acid (ionic theory), 434; and ammonium chloride, 426, 462; and hydrochloric acid, 42I; hypochlorite, 350; metaphosphate, 594; nitrate, 


\section{[References are to sections, not pages]}

I04; decomposition by heat, 565 ; nitrite, 513 ; oleate, work of Harkins on, 754 ; perchlorate, 355 ; peroxide, 307 ; phosphates, 159,594 ; pyrophosphate, 594; silicate, 270,807 ; silver thiosulfate, 626; stannite, 809; sulfate, 94; hydrate of, 94, 96, 97; sulfides, 607; tetraborate, general properties, 80I, 803; tetrathionate, 625 ; thiosulfate, 625 ; tungstate, $81_{4}$; zincate, 177

Solder, 809,913

Solid state, theory of, 203

Solubility: curves, I32; effect of crystalline form on, I33; effect of temperature on, $132-34$; gases in liquids, I 25; and heat of solution, I34; liquids in liquids, I24; of molecules, 445 ; of solids, 122 ; two kinds, 135

Solution, I20; compared to gas, 708; comparison with suspension, 708; concentration of, I2I; electrolysis of, 385 ; heat of, 127 ; kinetic theory of, 443 ; of little soluble electrolytes, 455 ; normal, 1 $36-38$, 595 ; saturated, I 22 ; supersaturated, I 23 ; van't Hoff's theory, 7 I 5

Soot, formation in flame, 782

Spark spectra, 786

Specific gravity, I IO

Specific heat, I I I; and symbol weight, 229

Spectra: bright-line, 786 ; continuous, 793; dark-line, 789 ; of gases and volatile substances, $78_{7}$

Spectroscope, 785

Spectroscopic analysis, 786

Spelter, 148,915

Sphalerite, 9 I 5

Spinthariscope, 859

Spirit, proof, 64I

Stannic compounds, 809

Stannous compounds, 809

Stannum, 809

Starch: glucose from, 636; occurrence of, 636 ; properties of, 637,638 ; test for, 637

Steam, 8; action on iron (chemical equilibrium), 290; burns by, I I 7; production, fuel for, 360 ; use for heating, I 6

Stearic acid, 678

Stearin, 680

Steel, 883 ff.; alloys, 898 ; Bessemer, 801 ; crucible, 894; electric, 894 ; high spced, 898; stainless, 898; tempering of, 897
Stibine, 8I I

Stoney, 466

Storage battery, 9I4

Strontium, 832 ; flame, 784

Strutt, 860

Sublimation, I 79

Subscripts, chemical, 6 I

Substance, 22, 23; properties of a, 108

Sugars, structure of, $68_{3}$

Sulfates, 624 ; test for, I64, 380

Sulfides: precipitation of, 6o8; precipitation by ammonium sulfide, 452

Sulfites, 6 I 2

Sulfocyanates, 665

Sulfur: commercial importance of, 604; consumption of, 604; effect on iron and steel, 896; flowers of, 603 ; heat of combustion, 357; monoclinic, 600; place in Periodic Table, 836; plastic, 600 ; properties of, 600,601 ; rhombic, 600 ; rock, 603; roll, 603; sources of, 602; dioxide: critical temperature, 775; preparation and properties, 610; and water, equilibrium, 286; monochloride, 600 ; trioxide, 613 .

Sulfuric acid: and aluminum hydroxide, I75; anhydride of, 61 3 ; and copper oxide, I 65 ; dilute, action on iron, I 73; dilute, action on magnesium, I 49; dilute, action on zinc, I49; fuming, 613; importance of, 614; and magnesium hydroxide, I44; and magnesium oxide, I45; manufacture of, 6I5; neutralization of, 94 ; properties, 93, 620-23; and sodium chloride (ionic theory), 460

Sulfurous acid, 340 , 6I I ; equilibrium, sulfur dioxide and water, 286; and iodic acid, 277

Sun: composition of, 790; source of energy, 374

Supercooling, 206

Superphosphate, I 60

Surface, increase with subdivision, 727

Suspensions, I 20; laws governing, 707

Suspensoids, 735,747 ; of commercial importance, 750 ; influence of the charge on stability of, 740

Symbol weights: accuracy of, 222; how found, 224, 228; oxygen basis, 223; product of, and specific heat, 229

Symbols: chemical, 61 ; and minimum weights, 66

Synthesis, 31 ; organic, 696,698

Table sugar, 682

Talc, 807 
[References are to sections, not pages]

Tannin, used in making red gold solutions, 746

Tantalum, 835

Tantiron, 804

Tartar emetic, 8I

Tartaric acid, 665

Tellurium and its compounds, 8 I $_{5}, 836$, 848

Temperature: absolute, 6; critical, 775 ; effect of change of, on equilibrium, 288; effect of change of, on gases, 5 ; effect of change of, on solubility, I 34,288

Tetramethyl silico-methane, 805

Tetrathionic acid, 628

Thermal Unit, British, $35^{8}$

Thermite, 330, 925

Thermometer: centigrade, 6 ; gas, 6

Thermos bottles, 777

Thiocyanate (sulfocyanate), 665 ; and ferric chloride, 280, 4I 5

Thomas-Gilchrist process, 892

Thorium: and its active products, 870 ; and its compounds, 820 ; in monazite, 820; place in Periodic Table, 834; properties, etc., 834

Thorium oxide, use in gas mantles, 301

Thorium X, 870

Tin: and its compounds, 809, 834 ; analgam, foil, plate, ware, So9; place in Periodic Table, $8_{34}$

Titanium, $8_{34}$

Titration, I 37 ; indicators for, 440

T.N.T., 673, 693

Transition point, 600

Transmutation of elements, $85 \mathrm{I}$

Trimethyl amine, 59, 658

Tri nitro phenol, 673,693

Tri nitro toluene, 673,693

Tripoli, 806

Trithionic acid, 628

Tungsten: and its compounds, 8I4, 836 ; use in incandescent filaments, 814

Turkey red, 698

Turpentine, 248

Tuyere, 886

Tw. See Twadell

Twadell, scale of specific gravity, 6 18

Tyndall, J., 748 ; effect, 748

Type metal, 8II

Ultramicroscope, 703, 706

Unit volume, the chemical, 65

Univalent, I 46

Unsaturated compounds, 527,663
Uranium: parent of radium, 867; properties and compounds, 836; series, 868

Uranyl nitrate, 836

Urea, 659; synthesis of, 696

Valence, r46; atoms and radicals, I 83 ; change of, in oxidation and reduction, 33 I-36; electrical nature of, 484 , 880; and Periodic Table, 850; positive and negative, 840 ; of radicals, 147; and the structure of inorganic molecules, 839 ; table of, I 83

Valeric acid, 680

Vanadium, 835

Vanilla, 697

Vanillin, 697

Van't Hoff, 7I5, 7I9

Vapor, equilibrium with liquid, 201

Vapor pressure: correction of volume of gas for, II 4 ; of hydrates, I3I; liquids and solids, II4; lowering of, by dissolved substance, I29; theory of, 200 ; of water, 112

Varnish, drying of, 364

Vaseline, 643

Velocity of reactions, 275

Vinegar, $x_{57}$; cider, 653 ; plant, 653

Vitamines, 689

Vitriol: blue, 497 ; oil of, 619

Volt, 499

Volume, atomic, 823

Warfare, materials for, chemical, 695

Water: adsorption by glass, 730; composition of, 36 ; distilled, 23 ; electrolysis of, 27, 295; formation of, I39; formula of, 70 ; hard, 156,803 ; ionization of, 420; a product of combustion, 20; properties, 108-I 2 ; reactions of, I40; and sodium, 40; synthesis of, 37 ; vapor, in air, 766 ; vapor pressure, I 12

Water glass, 808

Water soluble B, 689

Weight, equivalent, $4 \circ 3$

Weights: atomic, 800 (sce also inside of back cover); law of minimum and multiple, 64,212 ; minimum of elements, 63; minimum and symbols, 66; symbol and formula weights, 74

Whetstone, 806

White lead, 9 I 3

Willemite, 807, 9I 5 ; phosphorescent, 86 I

Winkler, 845

Wintergreen, oil of, 697

Wöhler, 696

Wollaston, 790 
[References are to sections, not pages]

Wollastonite, 807

Wood alcohol, 645

Wood's metal, 8 I 2

Work, 368

Wulframite, $8 \mathrm{I} 4$

Xenon: discovery and properties, 798 ; and family, 825

$\mathrm{X}$-rays, 476 ; and crystal structure, 204, 879 ; spectra, 877

Xylene, 668

Yeast, 640
Zinc: and concentrated sulfuric acid, 622 ; and dilute sulfuric acid, I49; electrolytic, 9r8; and hydrochloric acid, 149; metallurgy of, 916-I8; and nitric acid, $56 \mathrm{I}$; place in Periodic Table, 832; production, 88I, 915; properties and use, 148, 919; chloride, I48; nitrate, I48; oxide, I48, 919; sulfate, I48; sulfide, 9 I9; and alpha rays, 859 ; precipitation from solution, $45^{2}$

Zirconium, and compounds, 834

Zsigmondy, 706 

PERIODIC TABLE ACCORDING TO ATOMIC NUMBERS, H:I

\begin{tabular}{|c|c|c|c|c|c|c|c|c|c|}
\hline$\underset{\text { riod }}{\mathrm{Pe}}$ & o & $\mathrm{I}_{\mathrm{B}}$ & ${ }_{A}{ }^{\text {II }}{ }_{B}$ & ${ }_{A}$ III. $_{B}$ & IV & $\mathrm{V}_{\mathrm{B}}$ & $\mathrm{A}_{\mathrm{A}} \mathrm{VI}^{\mathrm{VI}}$ & VII & VIII \\
\hline I & $\stackrel{2}{\mathrm{He}}$ & $\mathrm{Li}^{3}$ & $B e^{4}$ & $\stackrel{5}{\mathrm{~B}}$ & $\stackrel{6}{\mathrm{C}}$ & $\stackrel{7}{N}$ & $\begin{array}{ll}8 & 0\end{array}$ & $\begin{array}{ll}9 & \mathrm{~F}\end{array}$ & \\
\hline 2 & $\begin{array}{l}\text { Io } \\
\text { Ne }\end{array}$ & $\mathrm{Na}^{\mathbf{I ~ I}}$ & $\mathrm{Mg}^{\mathrm{I} 2}$ & $\begin{array}{l}\text { I3 } \\
\mathrm{Al}\end{array}$ & $\begin{array}{l}\text { I } 4 \\
\mathrm{Si}\end{array}$ & $\stackrel{\mathrm{I}_{5}}{\mathrm{P}}$ & I6 $\mathrm{S}$ & ${ }^{17} \mathrm{Cl}$ & \\
\hline \multirow{2}{*}{3} & $\begin{array}{l}\text { I8 } \\
\text { A }\end{array}$ & $K^{\text {I9 }}$ & $\mathrm{Ca}^{20}$ & $S c^{2 I}$ & $\mathrm{Ti}^{22}$ & $\mathrm{~V}^{23}$ & $\mathrm{Cr}^{24}$ & $\operatorname{Mn}^{25}$ & $\begin{array}{lll}26 & 27 & 28 \\
\mathrm{Fe} & \mathrm{Co} & \mathrm{Ni}\end{array}$ \\
\hline & & ${ }^{29} \mathrm{Cu}$ & ${ }^{3 \circ} \mathrm{Zn}$ & ${ }^{3 \mathrm{I}} \mathrm{Ga}$ & ${ }^{32} \mathrm{Ge}$ & ${ }^{33} \mathrm{As}$ & ${ }^{34} \mathrm{Se}$ & ${ }^{35} \mathrm{Br}$ & \\
\hline \multirow{2}{*}{4} & $\begin{array}{l}36 \\
\mathrm{Kr}\end{array}$ & $\mathrm{Rb}^{37}$ & $\mathrm{Sr}^{38}$ & $\mathrm{Y}^{39}$ & $\mathrm{Zr}^{40}$ & $\mathrm{Cb}^{4 \mathbf{I}}$ & $\mathrm{MIO}^{42}$ & $?^{43}$ & $\begin{array}{lll}44 & 45 & 46 \\
\mathrm{Ru} & \stackrel{2}{\mathrm{Rh}} & \mathrm{Pd}\end{array}$ \\
\hline & & ${ }^{47} \mathrm{Ag}$ & ${ }^{48} \mathrm{Cd}$ & ${ }^{49}$ In & ${ }^{50} \mathrm{Sn}$ & ${ }^{5 \mathrm{I}} \mathrm{Sb}$ & ${ }^{52} \mathrm{Te}$ & 53 & \\
\hline \multirow{2}{*}{5} & $\begin{array}{l}54 \\
\mathrm{Xe}\end{array}$ & $\mathrm{Cs}^{55}$ & $\mathrm{Ba}^{5^{6}}$ & $\begin{array}{l}57 \\
\mathrm{La}\end{array}$ & $\begin{array}{l}5^{8} \\
\mathrm{Ce}\end{array}$ & $\begin{array}{l}59 \\
\mathrm{Pr}\end{array}$ & $\begin{array}{l}60 \\
\mathrm{Nd}\end{array}$ & $\begin{array}{c}6 \mathrm{I} \\
?\end{array}$ & \\
\hline & & $\begin{array}{l}62 \\
\mathrm{Sa}\end{array}$ & $\begin{array}{l}63 \\
\text { Eu }\end{array}$ & $\stackrel{64}{\mathrm{Gd}}$ & $\begin{array}{l}65 \\
\mathrm{~Tb}\end{array}$ & $\begin{array}{l}66 \\
\text { Dy }\end{array}$ & $\begin{array}{l}67 \\
\text { Ho }\end{array}$ & $\begin{array}{l}68 \\
\text { Er }\end{array}$ & \\
\hline \multirow{2}{*}{6} & & $\begin{array}{l}69 \\
\mathrm{Tm}\end{array}$ & $\begin{array}{c}70 \\
?\end{array}$ & $\begin{array}{l}7 \mathrm{I} \\
\mathrm{Yb}\end{array}$ & $\begin{array}{l}72 \\
\mathrm{Lu}\end{array}$ & $\mathrm{Ta}^{73}$ & $w^{74}$ & $?^{75}$ & $\begin{array}{lll}76 & 77 & 78 \\
\text { Os } & \text { Ir } & \text { Pt }\end{array}$ \\
\hline & & ${ }^{79} \mathrm{Au}$ & ${ }^{80} \mathrm{Hg}$ & ${ }^{8 \mathrm{I}} \mathrm{Tl}$ & ${ }^{82} \mathrm{~Pb}$ & ${ }^{83} \mathrm{Bi}$ & 84 & 85 & \\
\hline 7 & $\begin{array}{l}86 \\
\mathrm{Nt}\end{array}$ & $?^{87}$ & $\mathrm{Ra}^{88}$ & $?^{89}$ & $\mathrm{Th}^{9 \circ}$ & $? \cdot 9 \mathrm{I}$ & $\mathrm{U}^{9^{2}}$ & & \\
\hline
\end{tabular}


INTERNATIONAL ATOMIC WEIGHTS, I9I7

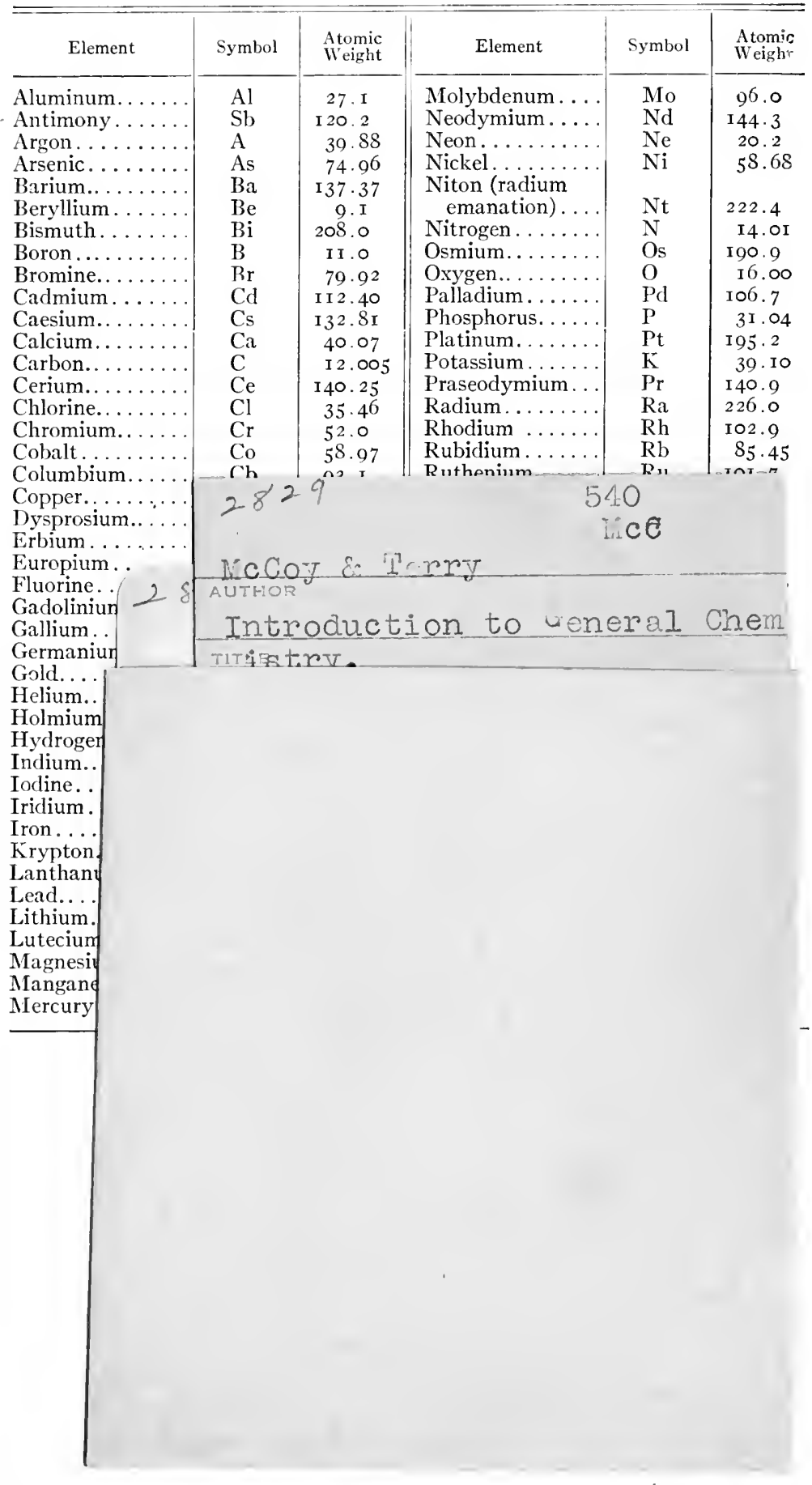


